
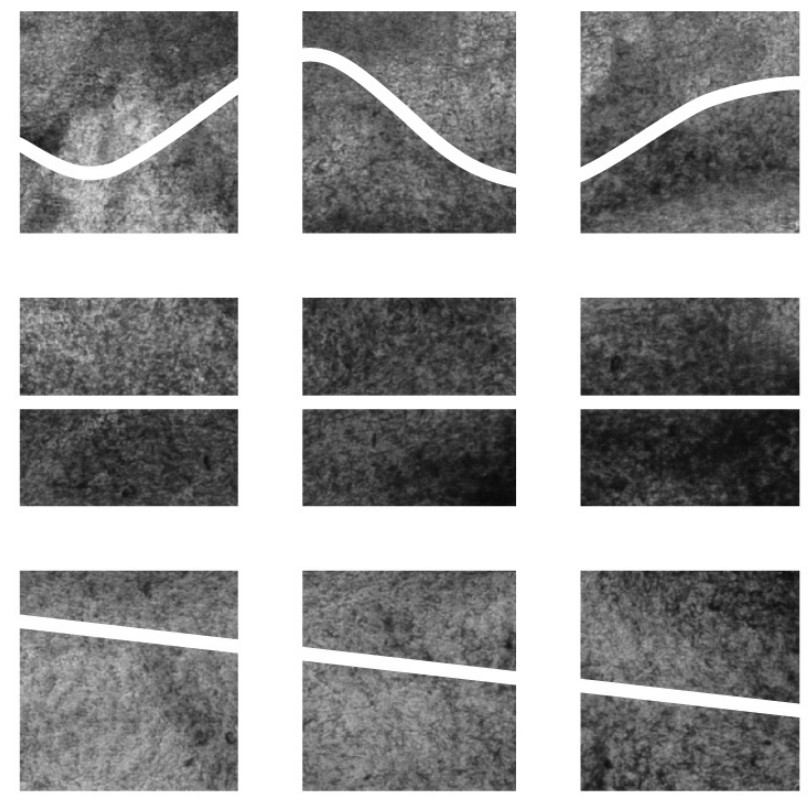

\title{
ARCHITECTURE'S TIGHTROPE
}

Investigating an Alternative Trajectory for Critical Architecture: Critical

Spatial Practices and a Polycontextual

Engagement with an Urban Thick Edge Condition

Angus Robert McDonald Earl

A thesis submitted to the Victoria University of Wellington in partial fulfilment of the requirements for the degree of Master of Architecture

(Professional) 

RIGHT
Figure i: Clifton Street car Park,
NZTA engineers plan laced in
the context 


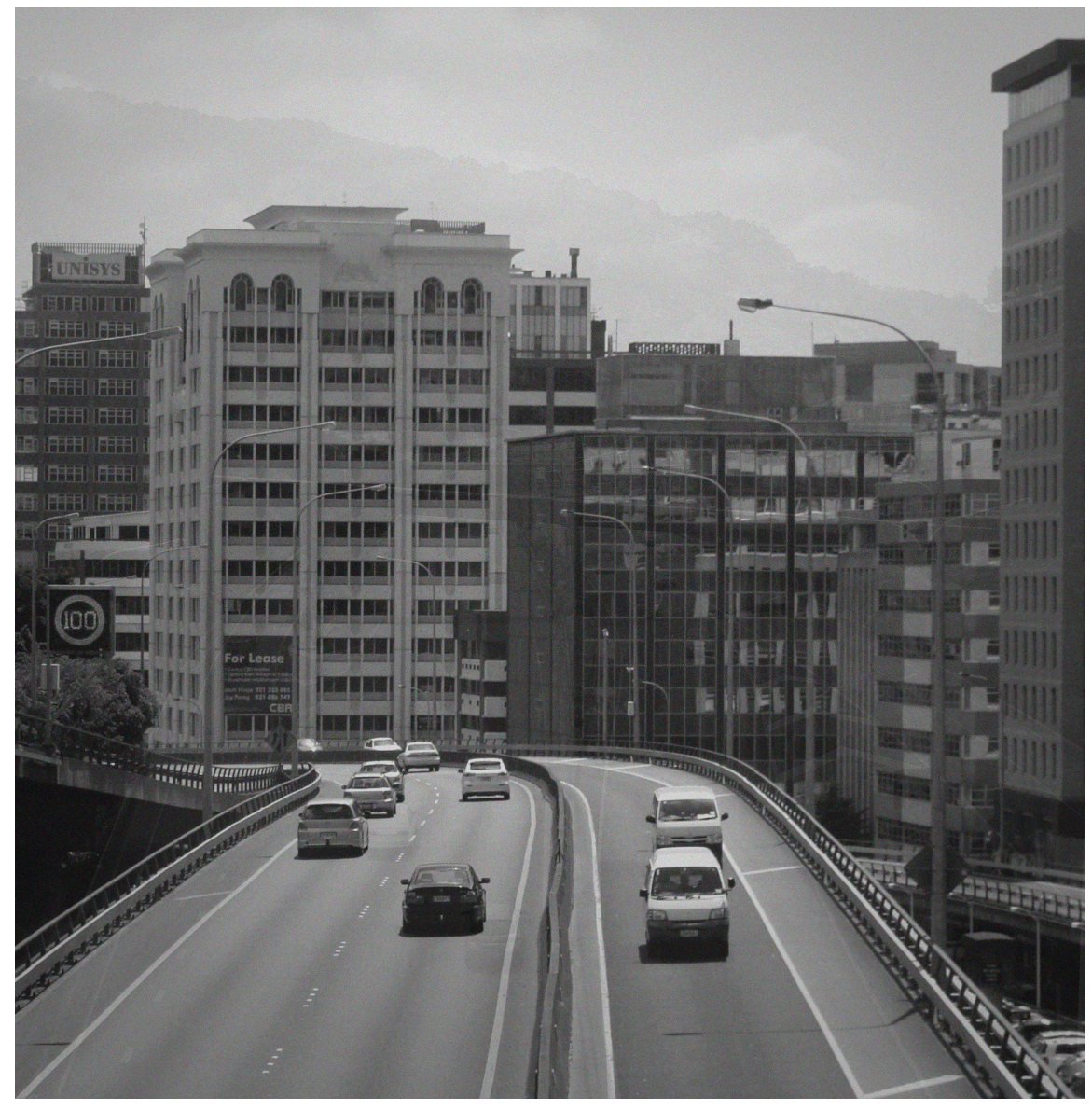


While some consider the unfinished parts of the city as ugly or disturbing, others feel they make an area more interesting than the picture perfect urban fabric. The city's scars are stimuli for the mind. They raise questions, about memories and imaginations of a foregone past, and of potential futures. They visualize the passage of time and the inevitability of collapse. On a smaller level, they show traces of faded lives, moved communities and shrunken economies. The voids provide space for the observer to interpret them as he likes, to fill

them with imaginations and meanings.

Mark Minkjan, "Failed Architecture"

Today's cities, however, look quite different from the cities of the future imagined by our predecessors a century ago. City dwellers are too often confined within monotonous grids, their connections to other people are severed, and they are condemned to an isolated existence. By now, those who migrated to the cities dreaming of a life of freedom and abundance have lost their spirited expressions and been reduced to a crowd of alienated individuals. Modernist architecture built a wall between itself and nature and relied on technology to create artificial environments with no connection to nature. It privileged function and efficiency, and cut itself off from the unique history and culture of its local settings. This kind of isolation from nature and rejection of the local community is to blame for the uniformity of today's cities and the people who live in them.

Toyo Ito, Pritzker Prize acceptance speech 


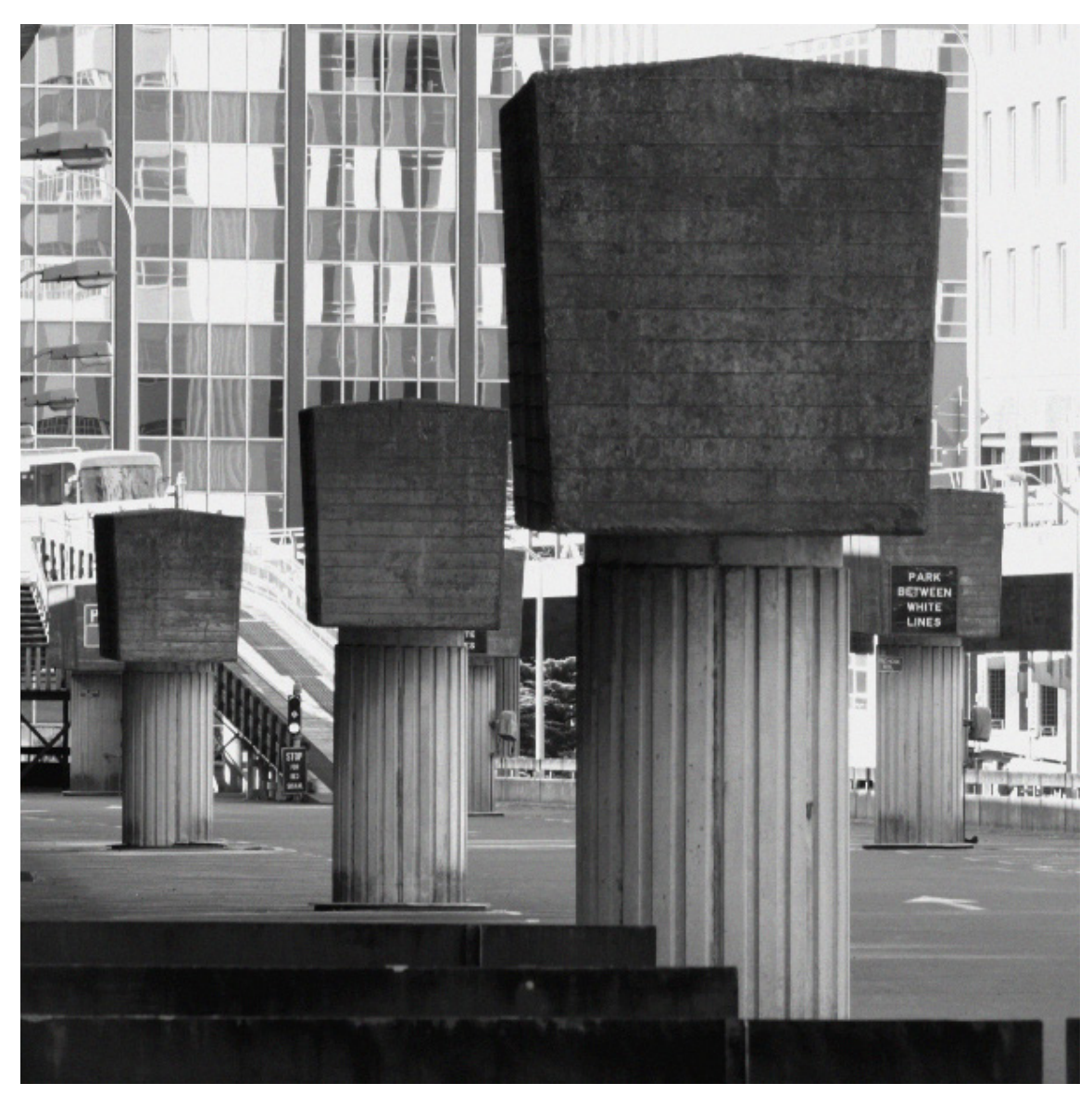




\section{ABSTRACT}

This thesis investigation engages two contemporary interrelated problems - one theoretical and one practical - both of which are interrogated, interwoven and tested through a critical lens. The theoretical context framing the design-research reconsiders the vitality of 'critical architecture' in relation to contemporary discourse, in particular, the so-called 'crisis of criticality' and the implications of this ideological landscape within the built environment. Foregrounding a position to test this theoretical framing, the practical context of the design-research is distinctly urban - engaging one of the contemporary negative outcomes of rapid urbanisation.

The practical problem investigates the 'thick edges' (places of singular and/or impermeable identities) that manifest around and below new urban motorway infrastructural developments, a condition that creates barriers to cultural, social and spatial flows between communities in urban settings. This thesis argues that by engaging with the complex and multiple cultural conditions of urban sites, the rigidity and singular nature of these impermeable thick edge spaces can be opened to diverse flows relating to multiple contexts. Through processes of design intervention, the thesis proposes a 'polycontextual' approach to introduce flows of wider contextual dimensions within an urban site promoting architectural solutions that blur, fray and punctuate thick edges by developing them as threshold conditions between adjacencies.

The theoretical problem analyses the limitations of both the autonomous and post-critical positions; this thesis argues that an alternative trajectory for a contemporary critical architecture has emerged, one that may be used as a theoretical framework for resolving urban thick edge conditions. Jane Rendell, Kim Dovey and Murray Fraser reveal a trajectory to shift architectural practices towards positive and flexible modes of production whilst simultaneously opposing the insufficient positions of the post-critical. They posit that architecture remains an inherently cultural proposition - created through constructive 'relays' that can mediate between theory and design - elucidating strategies of resistance through an engagement with practices that are both critical and spatial. Jane Rendell further argues that strategies for such 'critical spatial practices' can be elucidated through an examination of processes that are: site-specific, socio-spatial, and temporal. Adopting these three categories as the theoretical framework of this thesis focuses the design-research, implicating critical spatial practices as a contemporary and alternative position for critical architectural production - providing a framework for positive and critical positions in current discourse.

In response to this two-fold investigation, the thesis tests a synthesis of critical spatial practices and a polycontextual approach through strategic designresearch propositions. Architecture's Tightrope proposes a multifunctional events facility that permanently supports the New Zealand International Arts Festival, and the structuration of a dynamic, relational and non-deterministic public space. The primary aims of this thesis are: to test a contemporary critically engendered framework for architectural design-research that is both culturally and formally negotiated; and to investigate the potential for this framework to invert the negative conditions of urban thick edges through an engagement with multiple contexts.

Key words: critical spatial practices, thick edge, polycontextual. 


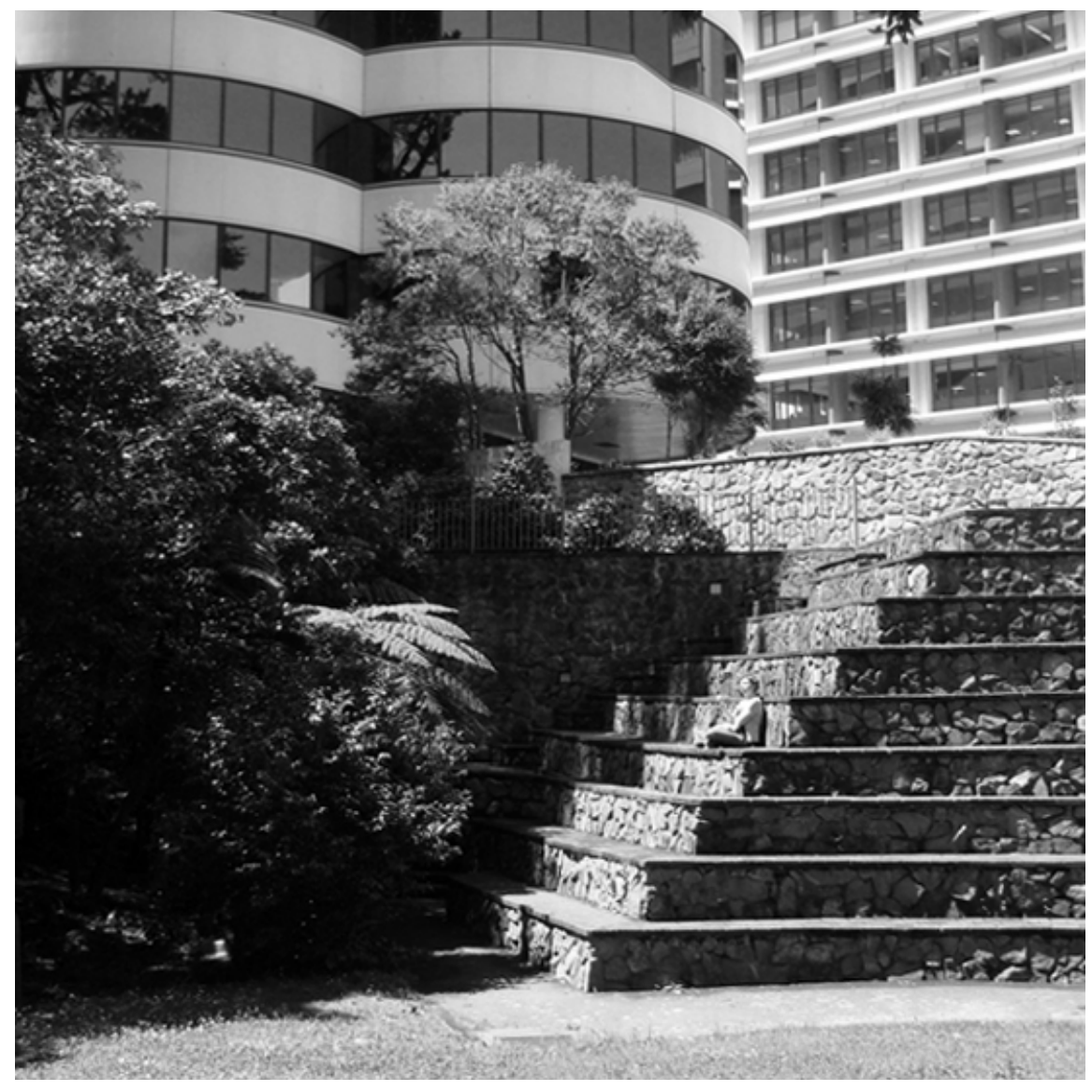




\section{ACKNOWLEDGMENTS}

Firstly, thank you to my parents, Leslie and Debbie, for their unconditional and unwavering support in my choices.

To my supervisor, Daniel Brown, whose patience, intelligence and incredibly generous direction has allowed me to accomplish a difficult achievement in my academic career. Your commitment has never gone unnoticed. Also, to Paul James, Jan Smitheram, and Patricia Bruner - thank you for your advice and inspiration at crucial points throughout my education.

Finally, thank you to my wider family, friends (especially Alex, Eve, Josie and Mijntje), and classmates for helping me through the tougher times and allowing me to enjoy the rest - especially mein Liebling. 


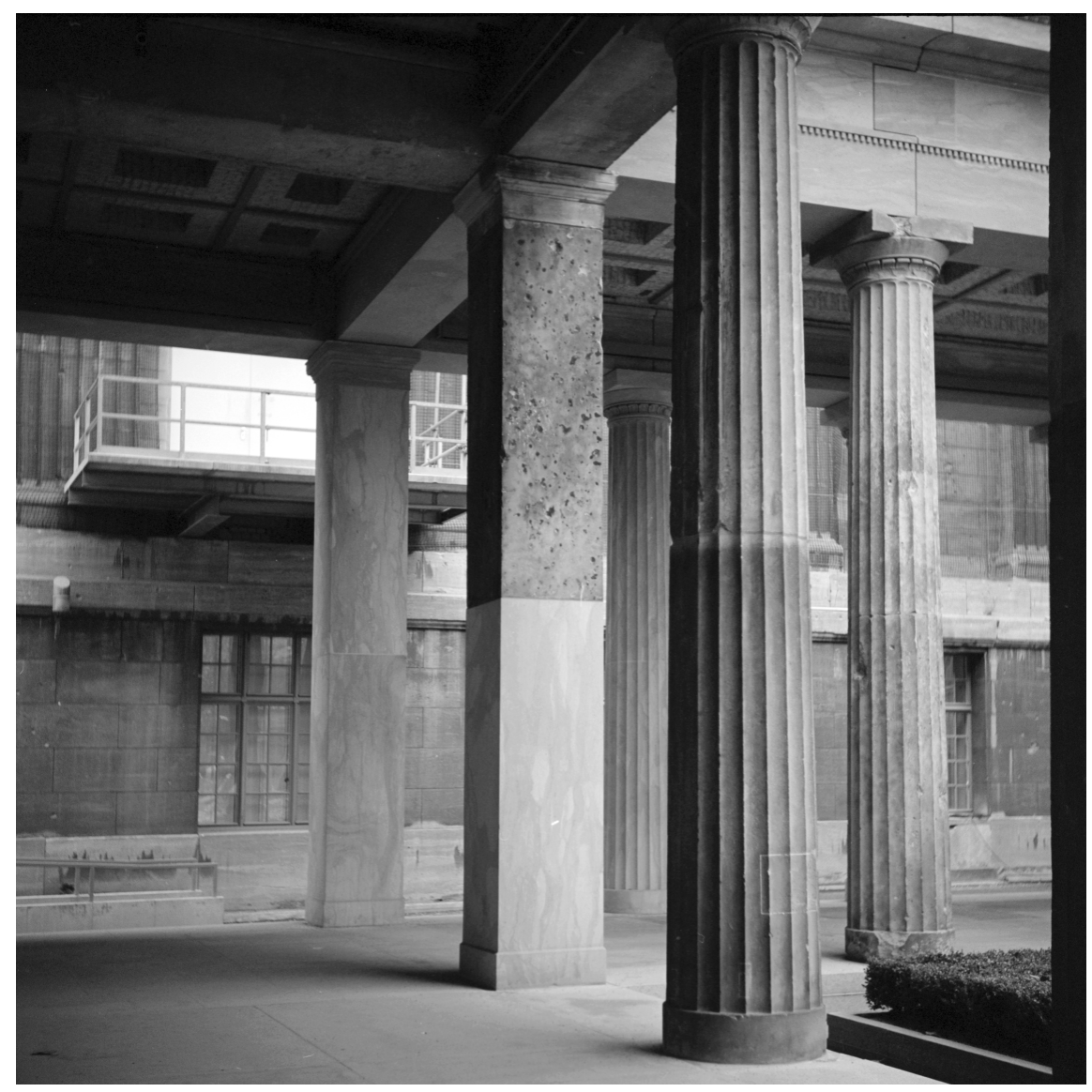




\section{PREFACE}

Throughout my academic education the idea of the 'critical' within architecture has been a constant source of interest; particularly the emphasis that it places on demystifying, resisting and subverting dominant power structures, and how they impact our position - as potential architects - within the context of everyday life. This interest, fundamentally shaped by a selection of writings, teachers, and built projects, has had a significant impact on my ideological outlook not only as an architect but also as a person.

An opportunity to explore these interests was made explicitly clear the first time that I visited the Clifton Street Car Park. The site revealed itself as a series of dualisms and contradictions that are in desparate need of architectural investigation: beautiful yet overbearing; architecturally tectonic yet unresolved; urban and residential; frequently used yet completely forgotten. These dualisms emerged from many site visits, day and night, rain and shine - each visit presenting an even more fertile environment for doing critical practices. Thus, a problem emerged - how can architecture explore the complexities of the existing context and simultaneously reveal it to the wider a public as a site of critical potency?

Fundamentally, this thesis is the result - an attempt to examine and critique the existing theoretical issues encompassed by the site into a rehabilitative design intervention. As the title suggests, the synthesis of these conceptual structures is a delicate balancing act - between idealism and realism - one which through the design-research of this thesis attempts to traverse the tightrope. 


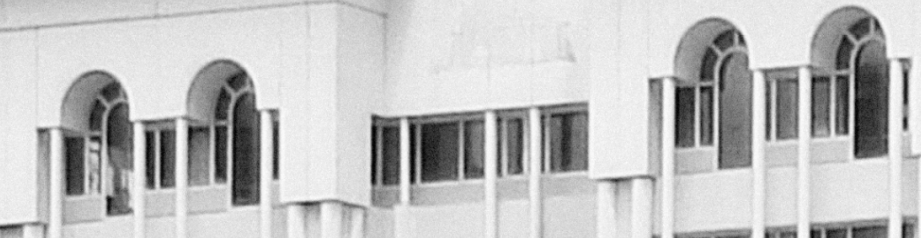

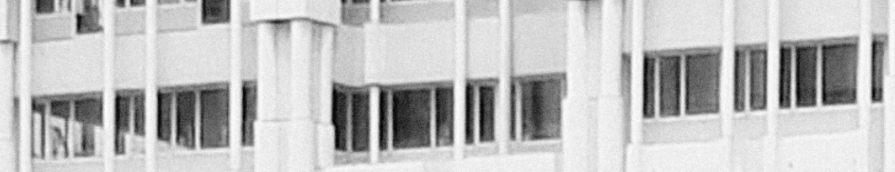

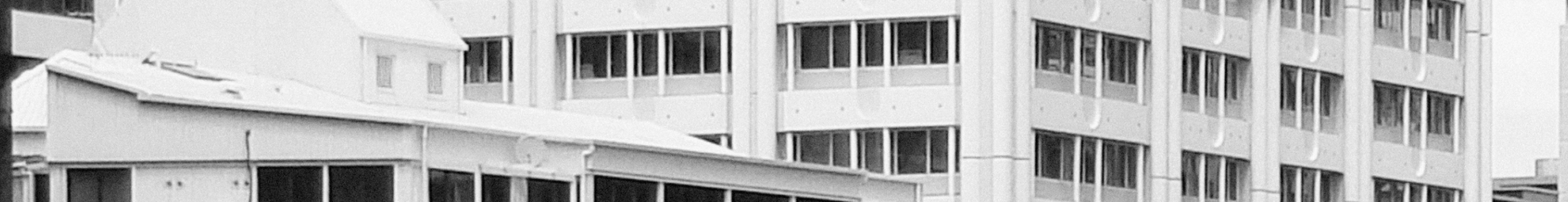

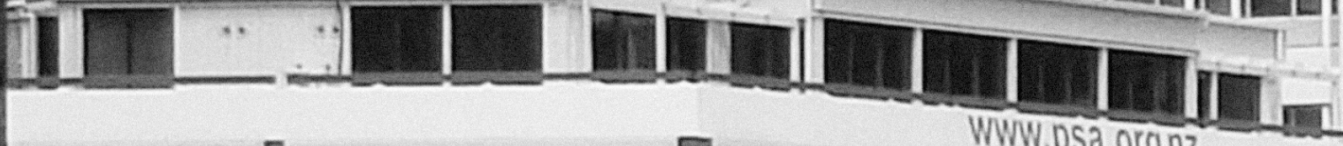
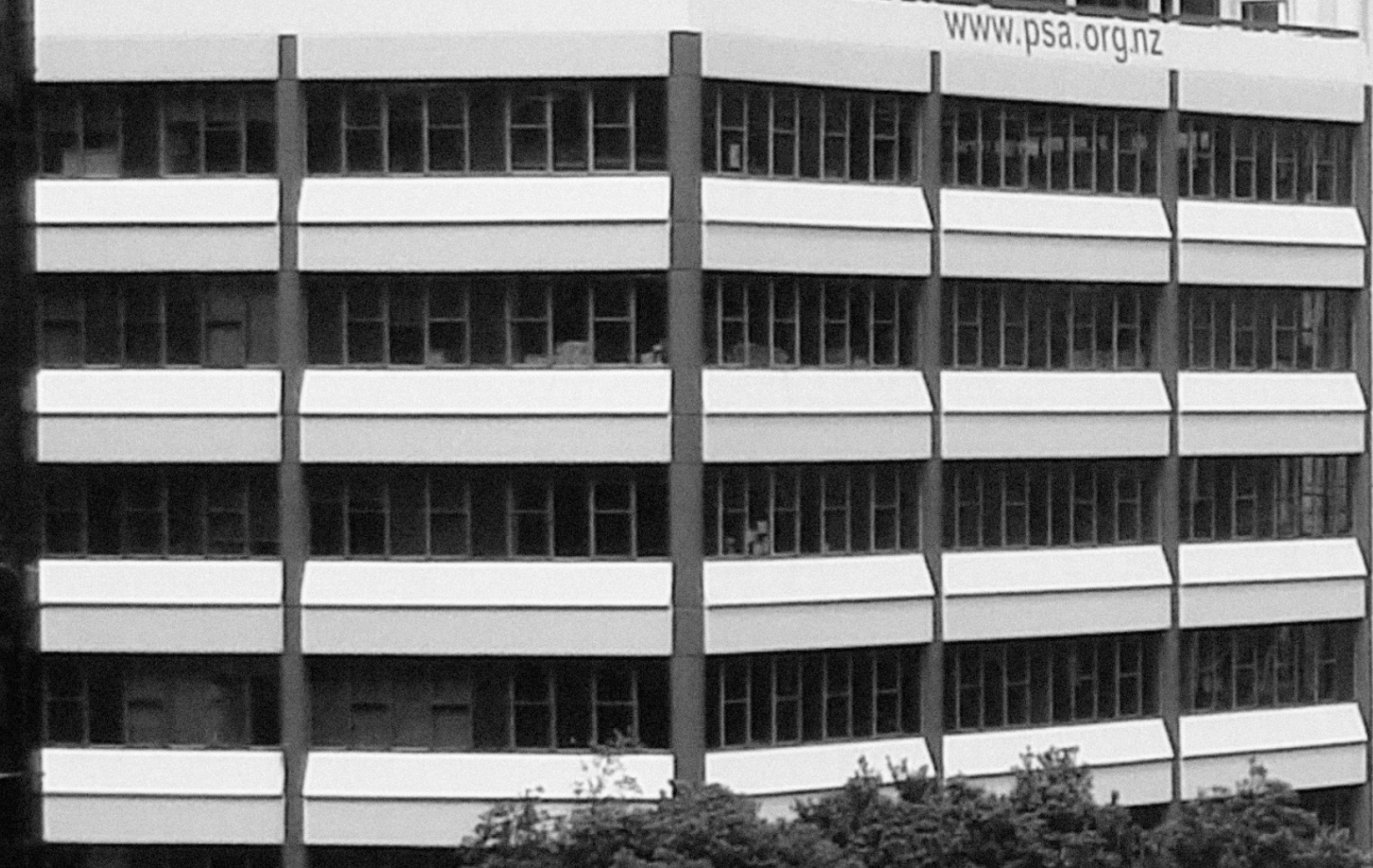

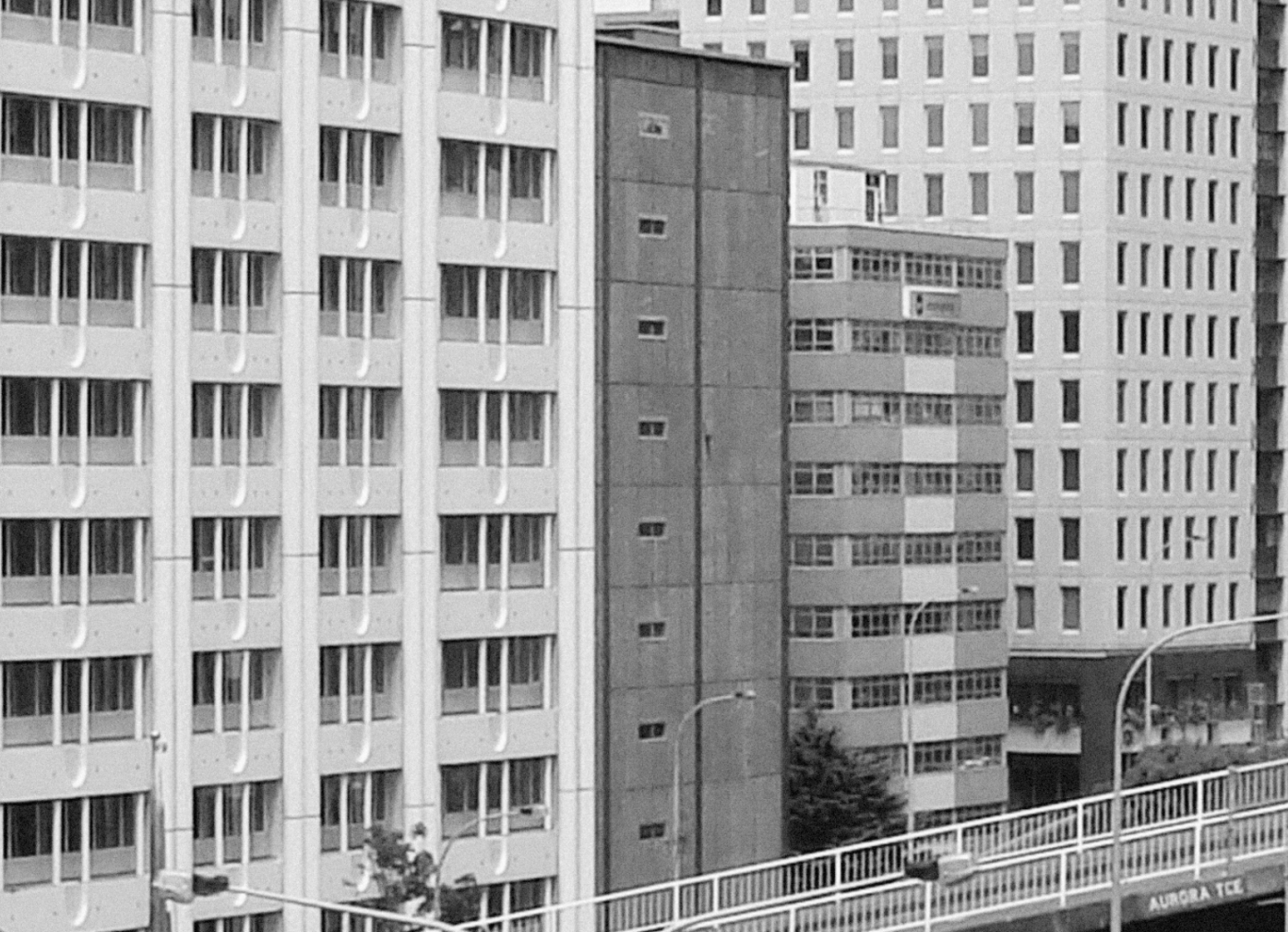

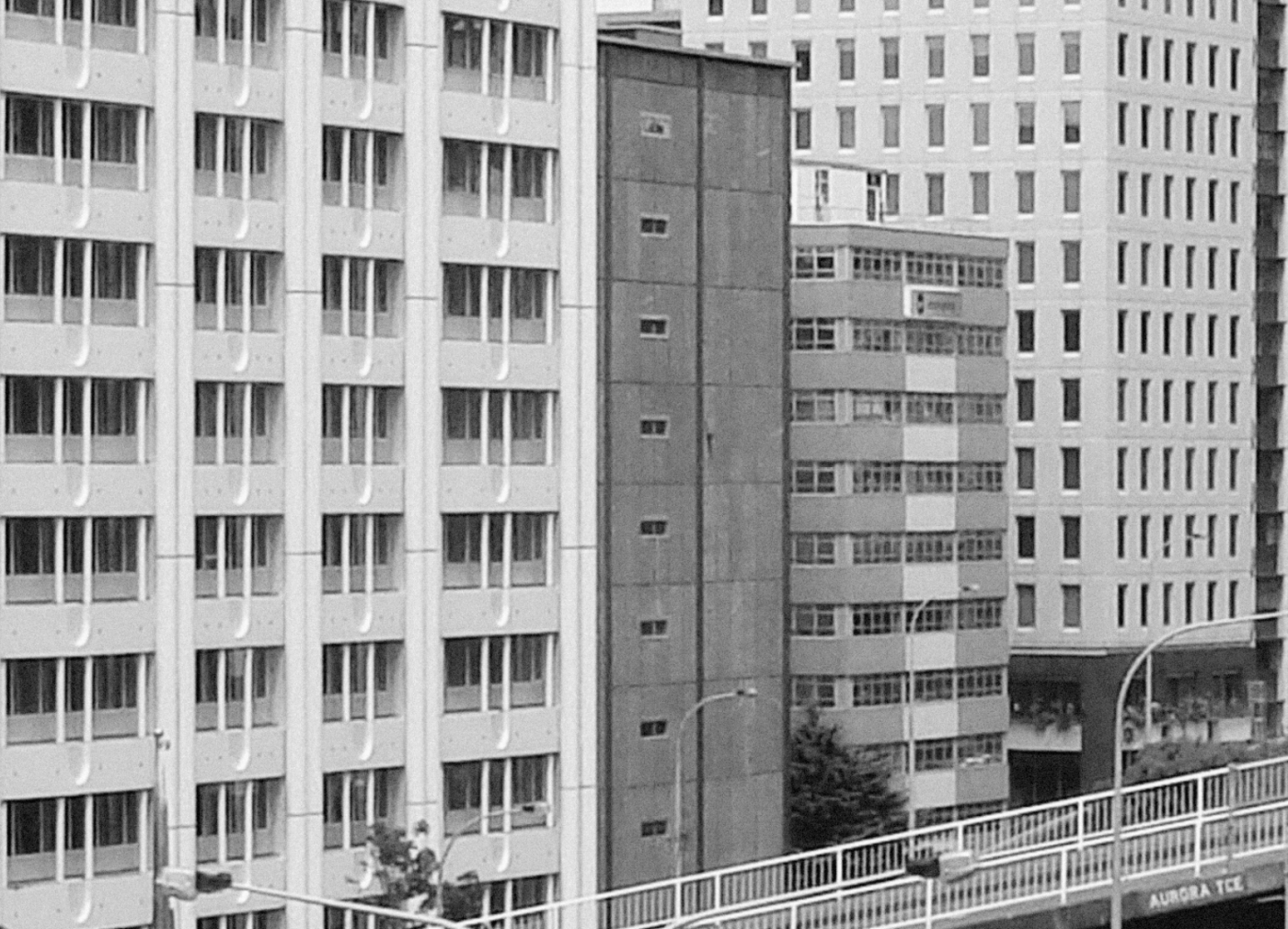

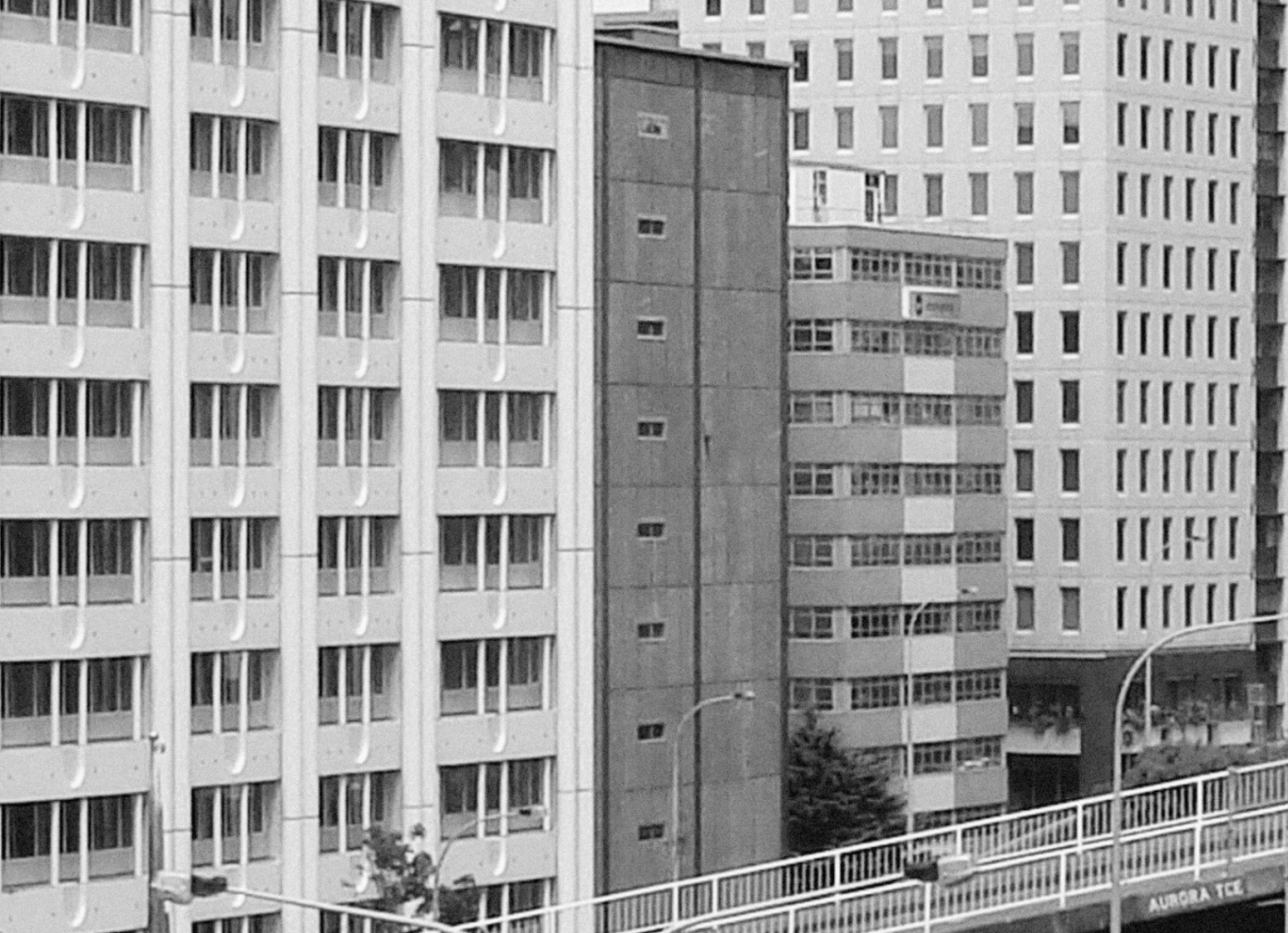

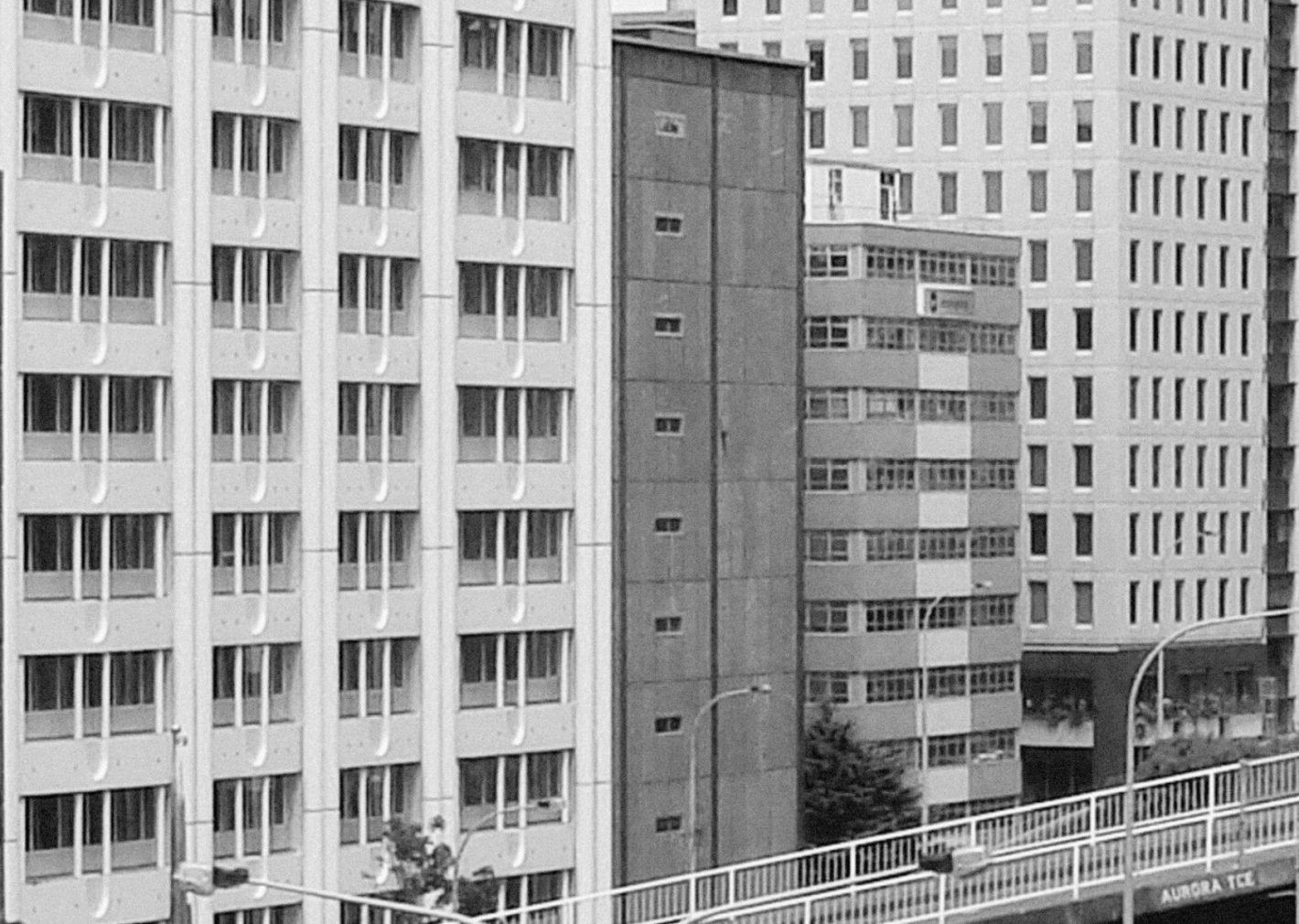

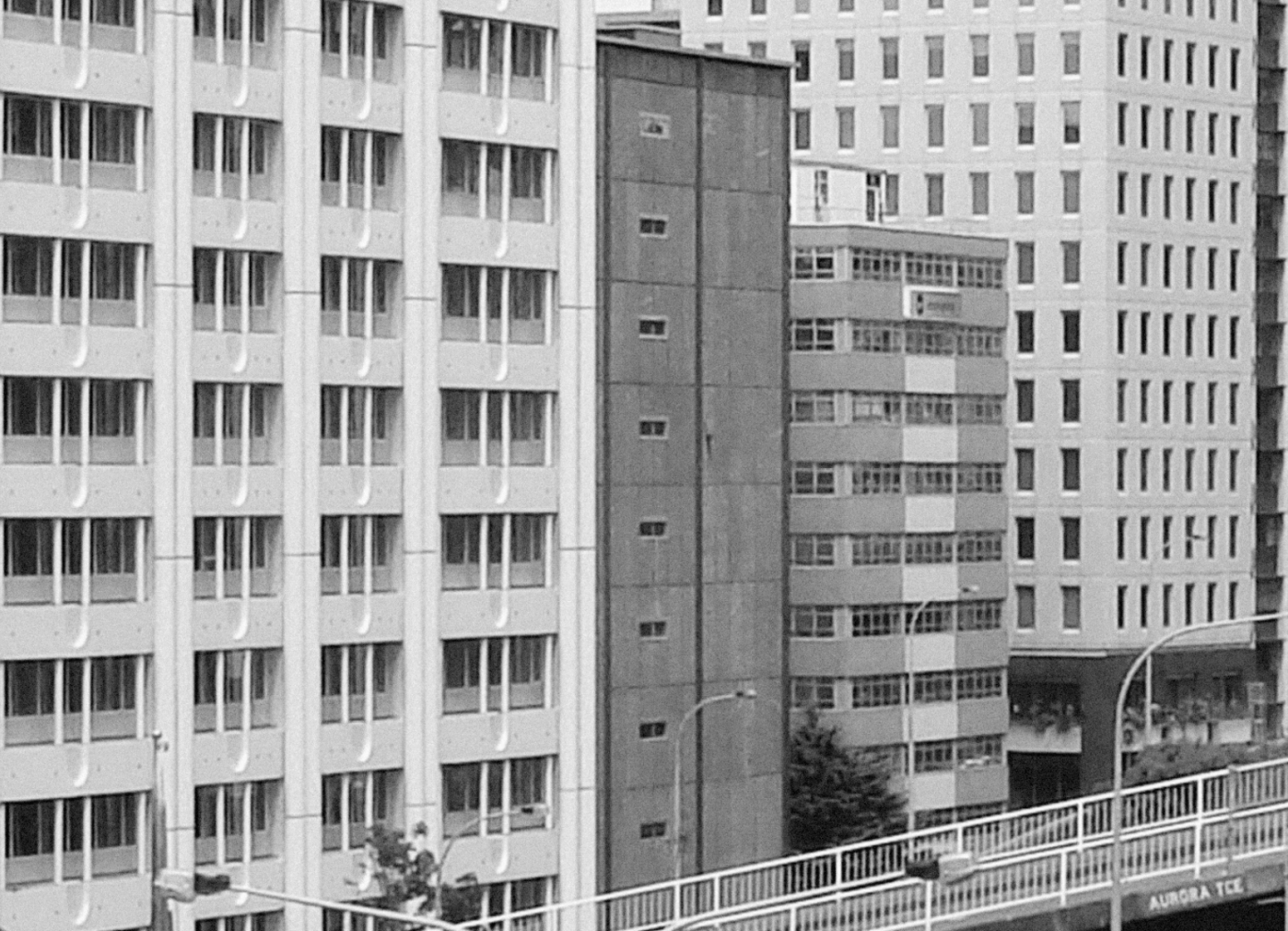

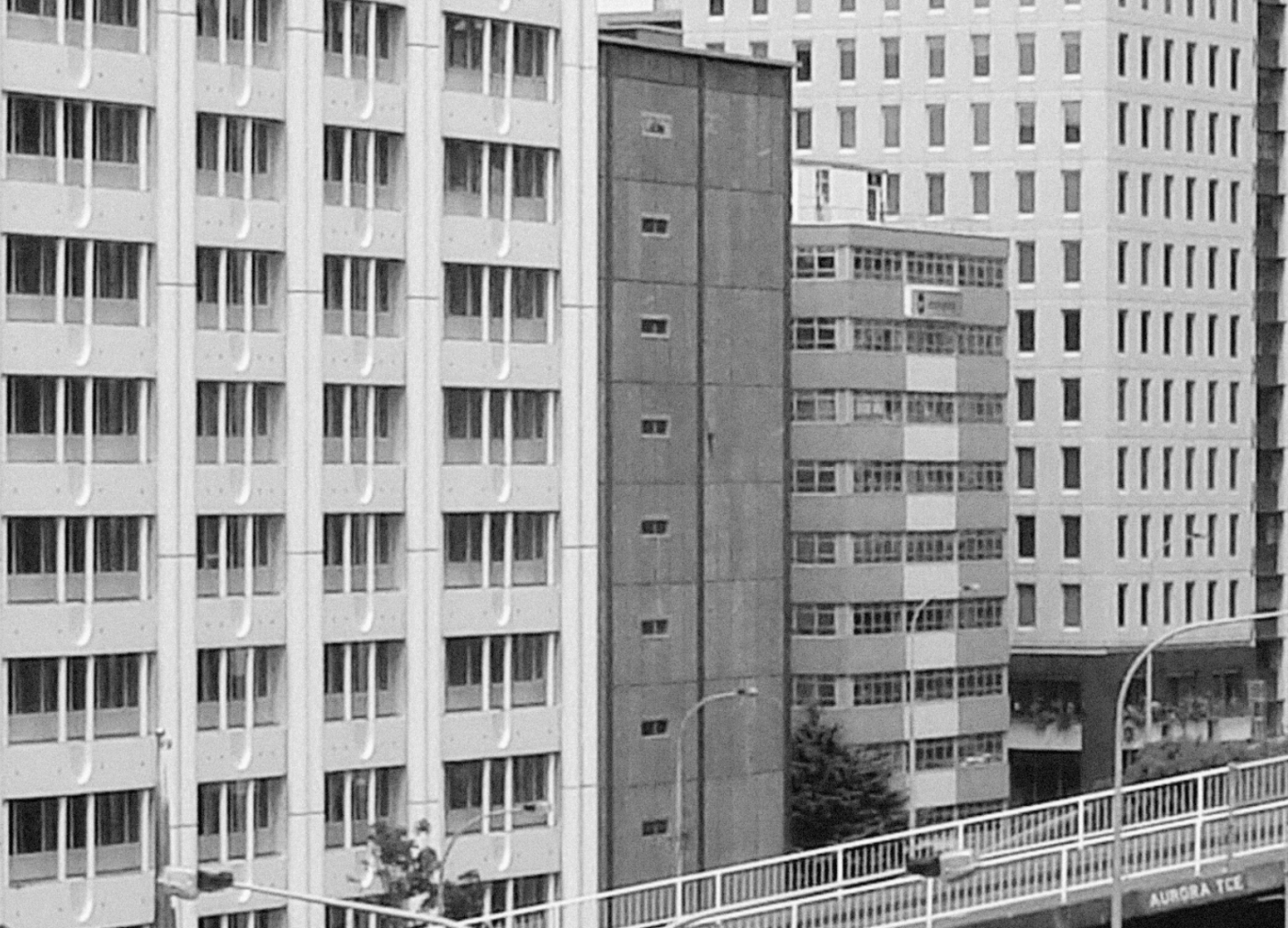
III) III 11

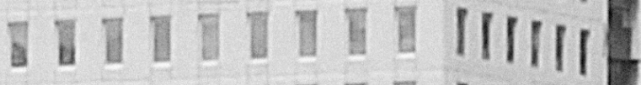
11 IIIIIIIII II

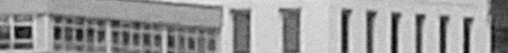
III I:- IIIIIIII IIII)

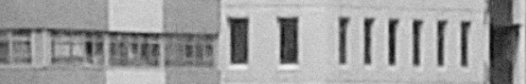

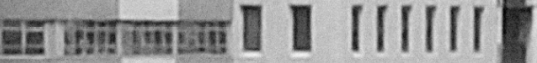

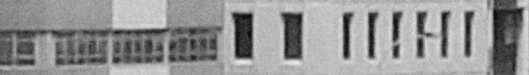

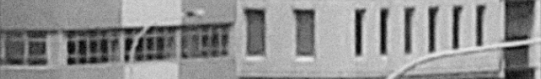

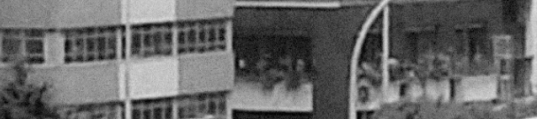

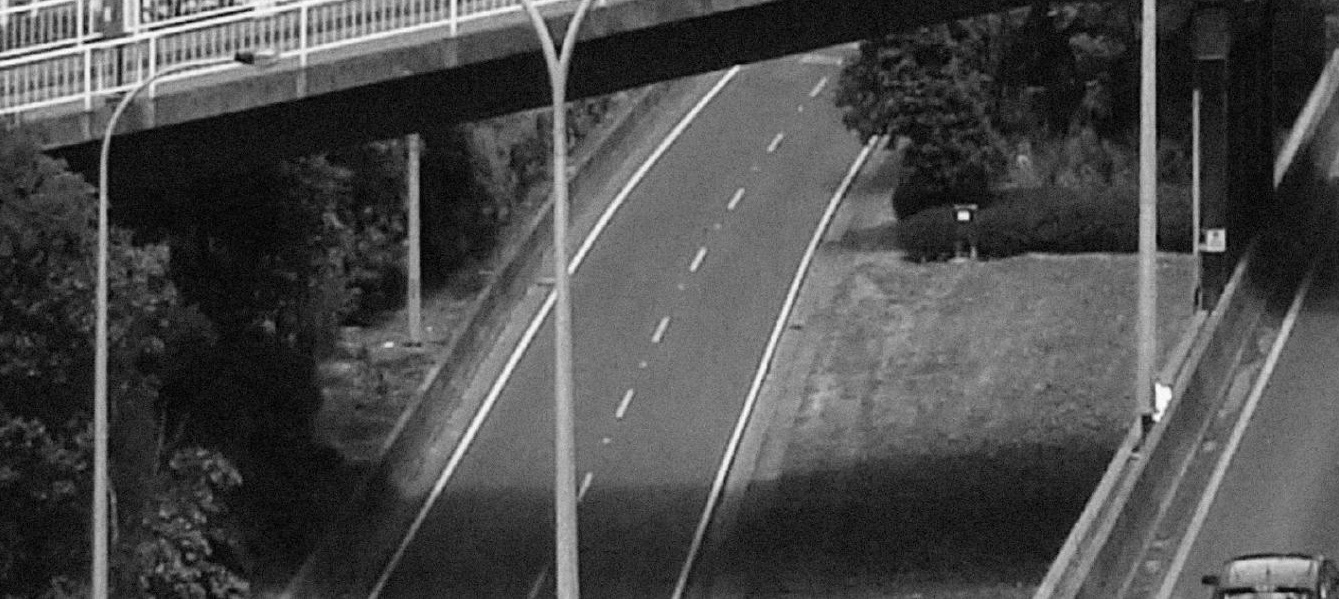




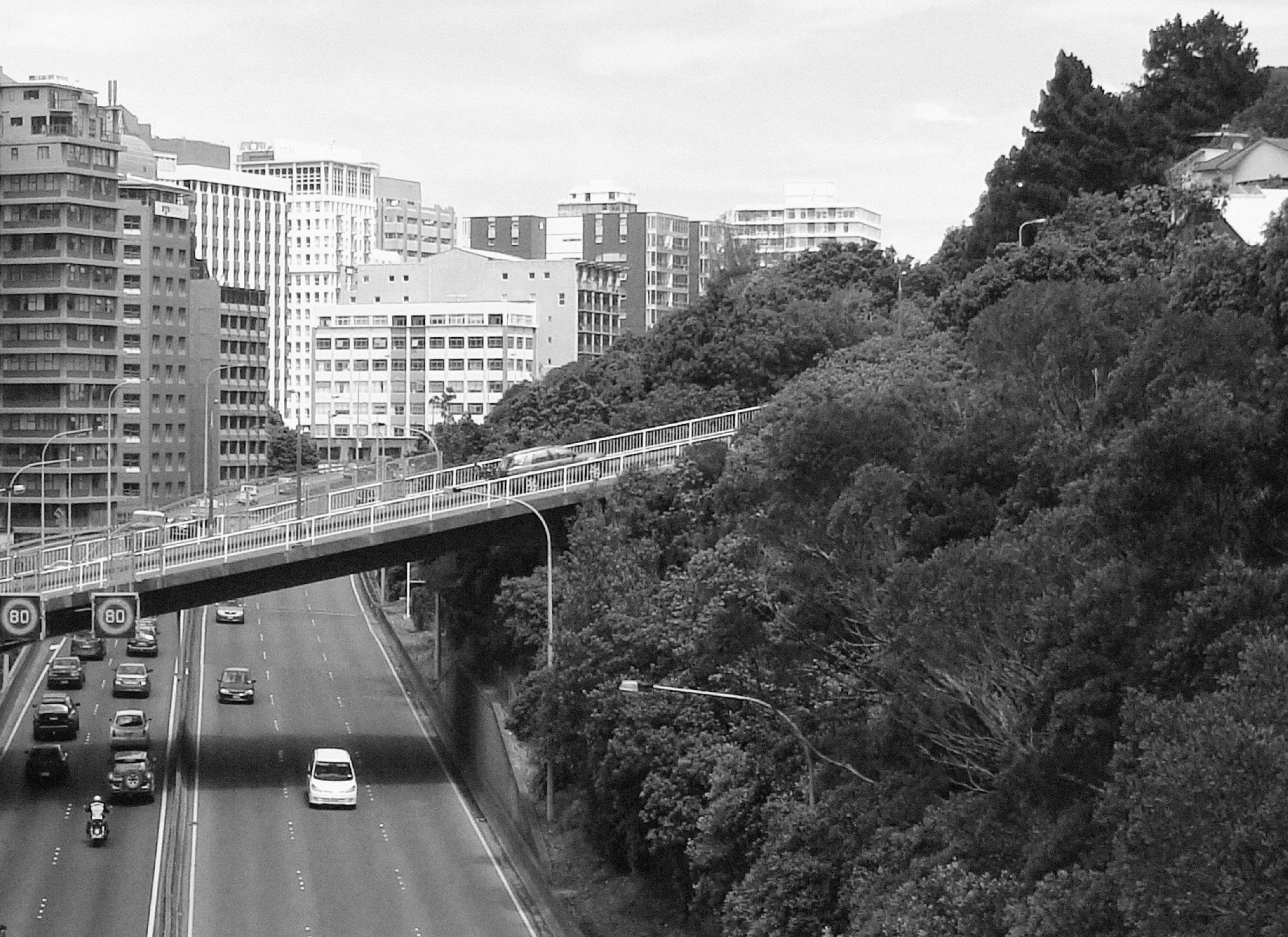




\section{CONTENTS}

Abstract

7

Acknowledments

Preface

9

Contents

11

Introduction:

1 Architecture's Tightrope

Introduction to the Context of Design-Research

Problem Statement

Design-Research Aims and Questions

Scope of Design-Research

Design-Research Approach

\section{SECTION ONE: FRAMING}

(2) Averting the Crisis of Criticality:

2 A (Discursive) Design Problem, Argument and Approach 33

Introduction 34

A Critical Premise: Mediating Between Formal and Cultural Production 36

The 'Late Avant-Garde': Fathers of Critical Architecture's Death Drive. 37

A (Conflated) Crisis? Unravelling the Post-Critical and its Oedipal Discontents 41

Implications of a Post-Critical Ideology: The Technical Perspective and a Loss of Context 44

Architecture's New Desire: Mediation through Critical Spatial Practices 48

Conclusion

3 Negotiating the Thick Edge:

3 A (Real) Design Problem and Approach 53

Introduction

A Sprawling Problem: Modernism's Leftovers

Where Grids Get Cut: Tangible Implications at the Border

A Localised Problem: Three Examples of a Negative Thick Edge

A New Form of Flow: Opportunities for Contextual Positivity 
A Potent(ially) Critical Site:

4 Investigating a Polycontextual Approach to the Clifton Street Car Park

Introduction

An Urbanised Context

A Historical Context

A Political Context

An Economic Context

A Social Context

A Physical Context

A Visual Context

Conclusions

\section{SECTION TWO: MEDIATING}

Expanding Place as Critical Practice:

5 Elucidating Strategies for a Site-specific Practice

Introduction

Between 'Here' and 'There': Continuity and Discontinuity Through Site-specific Strategies

Bernard Tschumi's Discontinuity: An Anti-contextual Approach

Enric Miralles's Continuity: A Contextual Approach

Conclusion: Implications and Strategies for Design

Social Space as Critical Practice:

6 Elucidating Strategies for Socio-spatial Flows

Introduction

Between 'One' and 'Another': 'Loose' Space Through Socio-spatial Strategies

The Palais de Tokyo: Social Space as Appropriated Space

The Metropol Parasol: A Canopy for Multiple Publics

The Rolex Learning Centre: Horizontality, Transparency and Porosity

Conclusion: Implications and Strategies for Design

Dialectical Space as Critical Practice:

7 Elucidating Strategies to Construct Temporal Images 
(8) A Potent(ially) Critical Programme

8 Elucidating Programmatic Strategies for Clifton Street Car Park 173

$\begin{array}{ll}\text { Introduction } & 174\end{array}$

An Economic Opportunity for Thick Edges: Cultural Nodes $\quad 175$

Event Spaces: Performing Arts Facilities

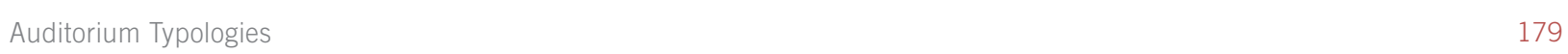

Three Local Examples $\quad 182$

An Exemplary Client: The NZIAF $\quad 192$

Conclusion: Programme Brief 192

\section{SECTION THREE: TESTING}

Design Case Study:

9 Architecture's Tightrope: a Multifunctional Performance Facility for the NZIAF

Introduction: Design Descriptions 204

Functions

Adjacencies

218

Circulations

Critical Strategies

220

222

Implementation of Strategies: Ground

239

Implementation of Strategies: Middle

Implementation of Strategies: Upper

10 Design-Research Reflections:

10 Conclusions and Implications

Reflections on Design-Research

Evaluations

Discursive and Practical Implications

266

Conclusion

266 
17

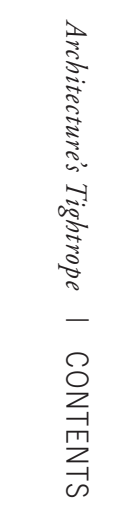



(2) (1)

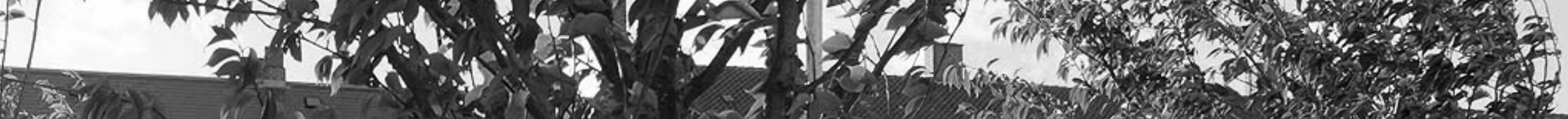

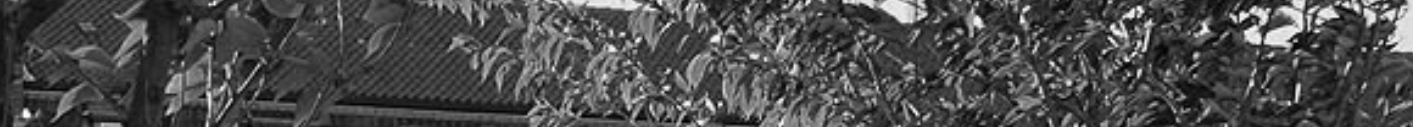

11

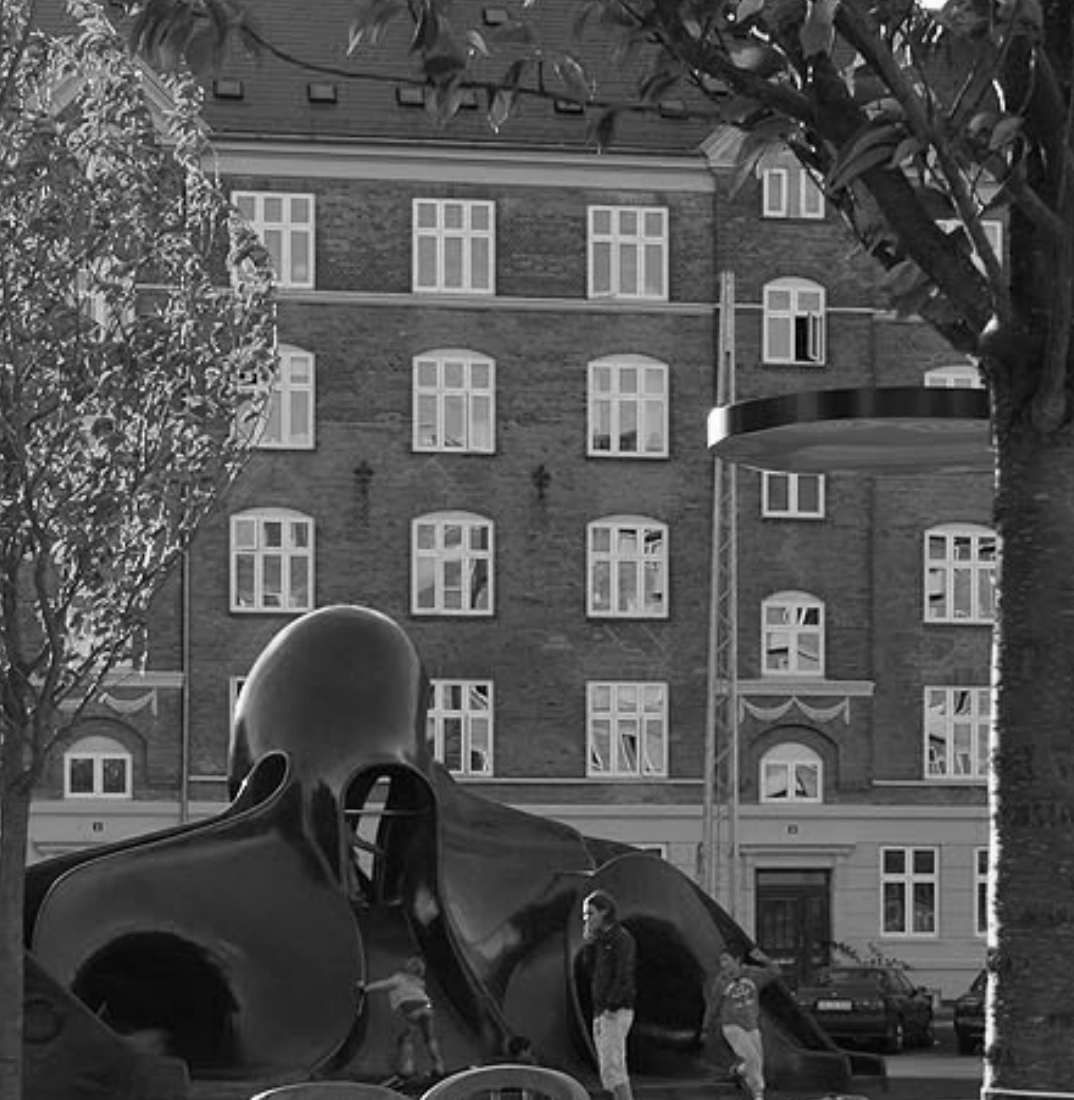

3.

$4.1+2$

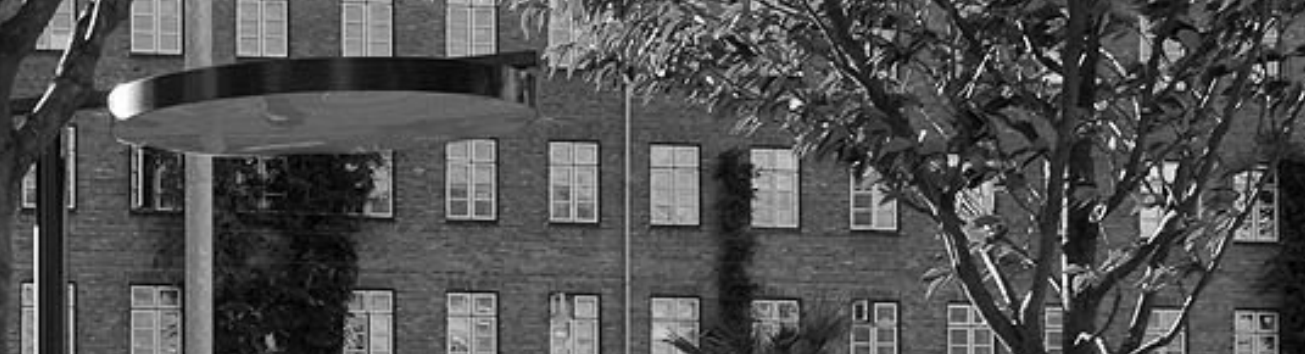

600

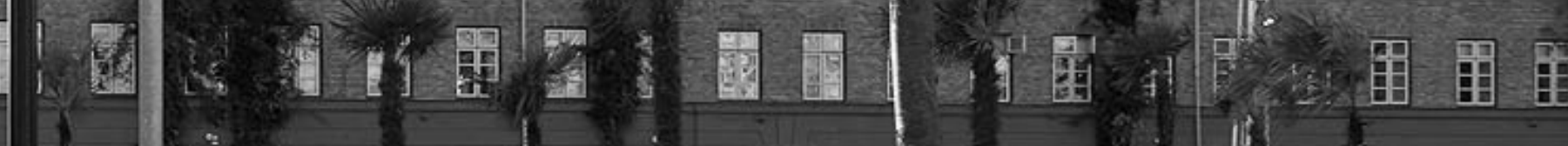

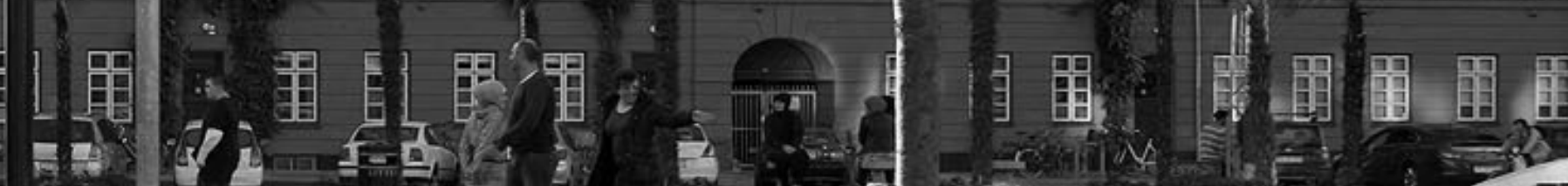

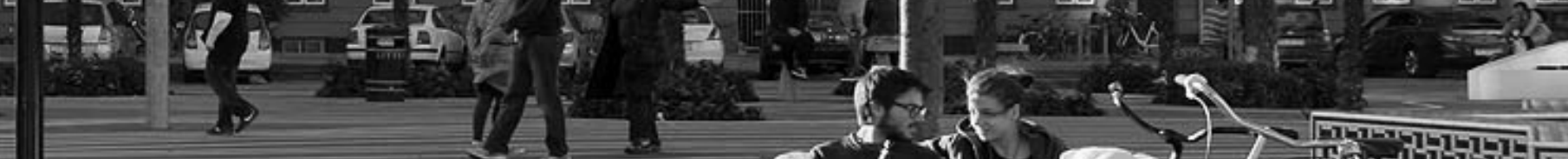

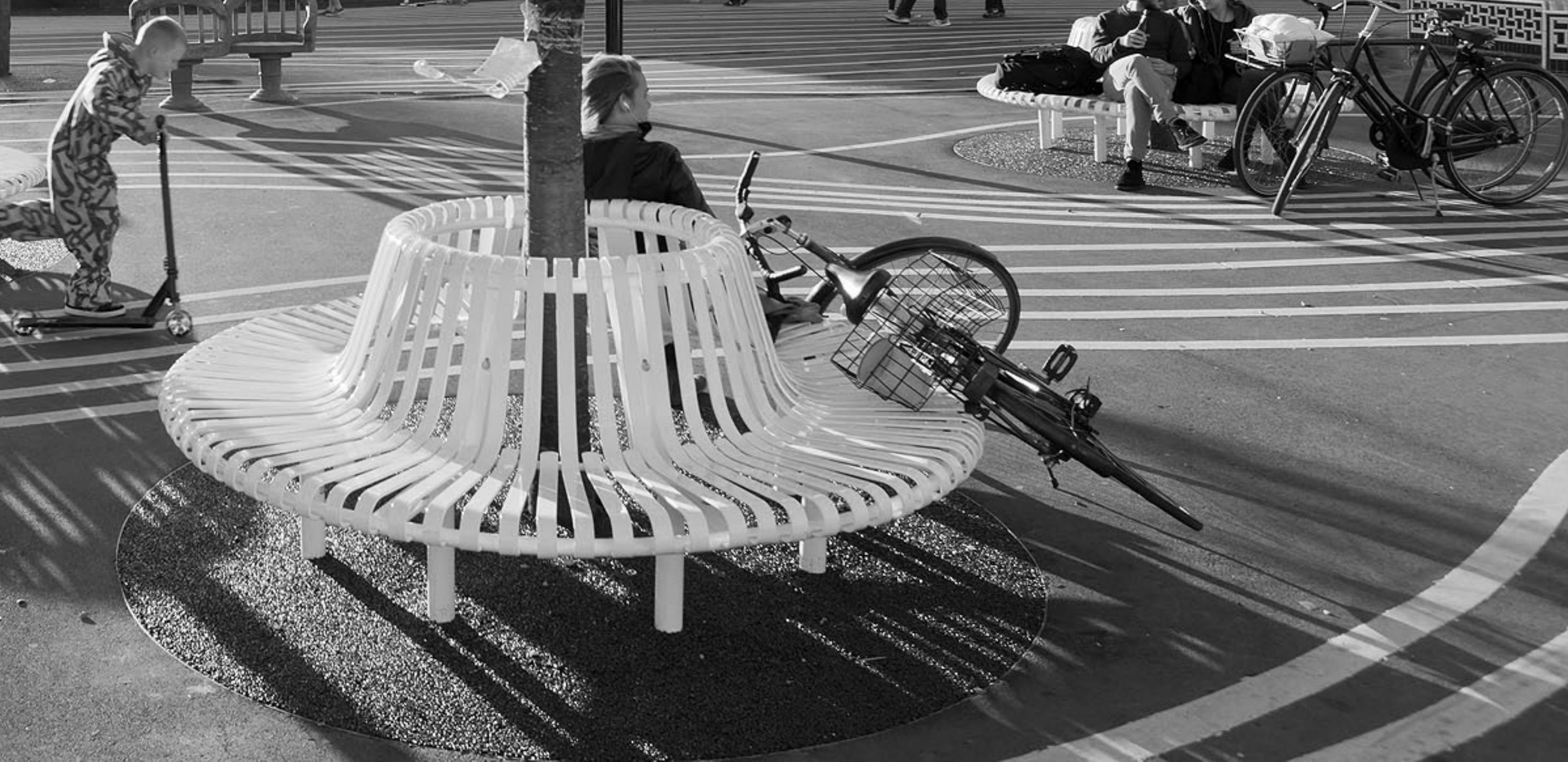




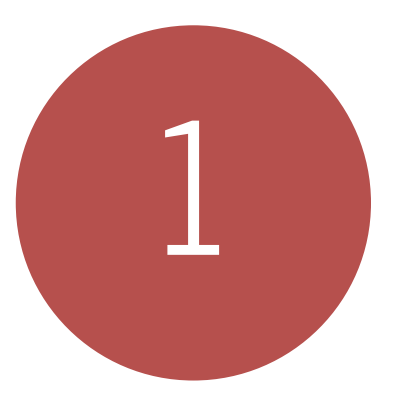

\section{INTRODUCTION}

Architecture's Tightrope

Introduction to the Tightrope

Problem Statement

Design-Research Questions, Aims and

Objectives

Scope of Design-Research

Design-Research Approach 

regard is heavily indebted to the 'deconstruction' practices by authors such as Jacques Derrida and Roland Barthes.
Walking a tightrope is a negotiation, a balancing act, a performance. It requires operating between various dimensions, left and right, up and down. Architectural design practices can be seen to follow a similar mediation of dualities, 'balancing': theory and practice, formal autonomy and cultural instrumentality, barriers and flows. Critical theory provides a useful framework in this regard, offering a multitude of approaches for operating between 'two' - ways of thinking in terms of 'both/and' rather than 'either/or.' These approaches are based upon a foundational desire to question and, when necessary, resist common understandings of cultural forces as static or objective, thus attempting to expose opportunities for positive change. However, unlike critical theory, architecture is not an inherently esoteric or theoretical exercise; it is axiomatically implicated in 'real world' concerns, the social, cultural and spatial conditions that shape its production.

Consequently, this thesis is driven by an intention to engage two contemporary interrelated problems - one theoretical and one practical both of which are interrogated, interwoven and tested through a critical lens. The theoretical context framing the design-research reconsiders the vitality of 'critical architecture' in relation to contemporary discourse, in particular, the so-called 'crisis of criticality' and the implications of this ideological landscape within the built environment. Foregrounding a position to test this theoretical framing, the practical context of the design-research is distinctly urban - engaging one of the contemporary negative outcomes of rapid urbanisation. Specifically, the practical problem investigates the 'thick edges' that manifest around and below new urban motorway infrastructural developments, a condition that creates barriers to cultural, social and spatial flows between communities in urban settings. In response to this two-fold investigation, this thesis sets out to explore a specific critical method to mediate between theory and practice: developing critical theory strategies for problem solving, tested through design propositions that encourage alternative frameworks for traversing the tightrope.
Theoretical Context of Design-Research: Critical Spatial Practices

In "Critical Architecture: Between Culture and Form", Michael Hays contends that architectural design differs from critical theory as it is necessarily spatial - mediating dialectically between formal and cultural production. At opposing ends of this dialectical spectrum, two dichotomous positions exist in a constant state of tension: architecture as a purely 'autonomous' formal product, a process of interrogation through the language of architecture itself and its theoretical interiority, and architecture as an 'instrument' of culture, where the architectural object perpetuates and projects the hegemonic values of a particular zeitgeist. The role of 'critical' architecture is to understand and analyse this condition. In so doing, it must 'cut across' architecture's dialectical dichotomy by resisting the potentially negative implications of the dominant culture through the production of architectural form. This definition for critical architecture "cannot be reduced either to a conciliatory representation of external forces, or a dogmatic, reproducible formal system". ${ }^{2}$ Consequently, Hays's primary proposition for critical architecture - as mediating between form and culture in a resistant manner - presents a reference point throughout this thesis as the fundamental proposition for critique through design-research.

Following the influence of several canonical figures of the 'late avant-garde' - Bernard Tschumi, Peter Eisenman, Aldo Rossi and John Hejduk critical architecture has become rigidly polarised and one-sided. Rather than mediating between form and culture, the influence of these figures shifted critical architectural practices towards a trajectory primarily invested in purely formal autonomy, dislocating the productive tension from Hays's dialectic by removing its cultural capacity for critique. This autonomous position presents a disciplinarity that is exclusively focussed on architecture as a contextless and isolated practice - a rigorous investigation of its conceptual languages through form, structure, and technique. 
In the wake of the late avant-garde's trajectory for a purely autonomous architecture, a new generation of architects opposing the limitations of this context has emerged - expressing a positive intention to re-engage architectural discourse in 'real world' concerns and pragmatic, flexible methods of production. A dominant proponent of this new vanguard is a faction provocatively termed the postcritical'. However, a discursive impasse created by the post-critical position has holistically conflated the proposition of critical architecture, limiting it to practices solely associated with autonomous disciplinarity. In doing so, post-critical architects such as Michael Speaks, Robert Somol, and Sarah Whiting have drawn the discipline into a supposed 'crisis of criticality' - claiming that it is now in fact beyond any necessity to embody a critical intention. This crisis has fostered an ideological climate of submission to the complexities of external forces, supplanting critical processes with a focus on design experimentations based on effects, innovation and technological advancements. Consequently, prominent architects such as Frank Gehry and Zaha Hadid, who can be seen to align with post-critical intentions, legitimize architectural production methods that re-enforce complicity with forces of globalisation - producing iconic and decontextualized structures that avoid the importance of contextual engagement.

Analysing the limitations of both the autonomous and post-critical positions, this thesis argues that an alternative trajectory for a contemporary critical architecture has emerged. Investigating the theoretical contentions of Murray Fraser, Kim Dovey and Jane Rendell reveals a trajectory to shift architectural practices towards positive and flexible modes of production whilst simultaneously opposing the insufficient positions of the post-critical. Fraser, Dovey and Rendell posit an understanding that architecture remains an inherently cultural proposition - created through constructive 'relays' that can mediate between theory and design - elucidating strategies of resistance through an engagement with practices that are both critical and spatial. Heavily indebted to the spatial turn' in cultural studies, these critical spatial practices revitalise Hays's definition for critical architecture through an examination of the earlier investigations of Henri Lefebvre, Michel de Certeau and Marc Augé. These figures of the spatial turn invest the creation of 'place' identity exclusively within the dimensions of social relations - contending that the 'identity of place' arises from conditions that are dynamic, performative and relational insofar as they are understood as a system of interactions between multiple contexts. Importantly, this adaptation of place 'opens' the architectural design process to critical responses relative to the cultural dimensions of a particular site.

Rendell argues that strategies for critical spatial practices can be elucidated through an examination of processes that are: site-specific, sociospatial, and temporal. Adopting these three categories as the theoretical framework of this thesis focuses the design-research, implicating critical spatial practices as a contemporary and alternative framework for critical architectural production. Critical spatial practices suggest a new trajectory for critical architecture, averting the conflating influence of the late avantgarde and the subsequent post-critical impasse, and providing a framework for positive and critical positions in current discourse.

\section{Practical Context of Design-Research: Urban Thick Edge Conditions}

An investigation of patterns of urbanisation that have shaped the fabric of many Western cities including Wellington reveals one of the contemporary urban problems through which critical spatial practices can be tested. Throughout the latter twentieth century, urban infrastructure development processes (referred to as jump-cut' urbanisation when critiqued by Robert Ingersoll in the late nineteenth Century) have contributed to the production of spatial barriers, manifesting as 'negative' leftover urban spaces. These sites are tangible realisations of the concomitant effects of 'global' forces as they impact at a 'local' level. ${ }^{3}$ One specific example of this condition is the creation of what Iain Borden refers to as 'thick edges' - contextually rigid (static and
3. A proposition explored by Bill Hillier and Julienne Hanson in The Social Logic of Space. 
singular) sites, which have emerged around these newly inserted infrastructures. Considered by many as unworthy of architectural attention, thick edges in this context are often left as architectural voids or 'filled in' with singular-use programmes (in particular, car parks) that prevent social flow or engagement. Problematically, as urbanisation has proliferated so has the production of these thick edges, constructing contextually impermeable borders that sever landuse zones and dislocate previously contiguous social, spatial and cultural communities within urban landscapes. Such sites are singular (usually economic) in their motivations - neglecting relations between the surrounding visual, physical, social, political, economic and historical contexts that contribute to the cultural construction of place-identity.

In Wellington, the Clifton Street Car Park (132 The Terrace) vividly exemplifies the problem of the urban thick edge - a residual site that occupies a significant area both underneath and surrounding the Wellington Urban Motorway (1969-1984). This site forms an incision through the residential/commercial threshold between the city centre and its periphery, leaving behind a landscape of anti-social vehicular use that divides communities and prevents social flows. Through its sprawling horizontal concrete parking platforms (three 'datums' at different heights) and large supporting columns, the car park imposes a rigid order to the site - physically and visually separating the green belt to the west from the urban centre to the east. Alongside the isolating social, physical and visual effects of this thick edge, the construction of the car park and motorway has historically been the locus of significant cultural trauma: removing and relocating thousands of graves from the nearby Bolton Street Cemetery; burying the Kumototo Stream, considered an important landmark for native iwi; and destroying hundreds of state homes that were once part of the adjacent Thorndon suburb. The new motorway went ahead despite the cultural calamity that resulted from the destruction of these historic and social references. As a result, the Clifton Street Car Park remains rigidly detached and isolated from its wider contexts, committed to its performance as an economically driven function and a figure of ubiquitous urbanisation, while forming an impenetrable barrier between The Terrace and Thorndon communities.

Many theorists such as Rem Koolhaus denounce these spaces as 'junk', imparting a narrative that suggests thick edges must be ignored and left to deteriorate - as is the case with the Clifton Street Car Park. Consequently, few architectural alternatives for thick edges have emerged. Aaron Betsky and Alexander d'Hooghe have argued for an approach that resists these commonly held assumptions, suggesting that thick edges can become, in fact, sites of theoretical and cultural potency. This thesis argues that by engaging with the complex and multiple cultural conditions of urban sites, the rigidity and singular nature of these impermeable spaces can be opened to diverse flows relating to multiple contexts. Through processes of design intervention, the thesis proposes a 'polycontextual' approach to introduce flows of wider contextual dimensions within an urban site - promoting architectural solutions that blur, fray and punctuate 'thick edges' (places of singular and/or impermeable identities) by creating them as threshold conditions between adjacencies.

Engaging critical spatial practices in a polycontextual approach, this thesis challenges critical architecture to 'invert' the cultural and spatial rigidity of thick edges, presenting them as an urban 'threshold' that is in essence multiple, relational and positive, rather than singular, rigid and negative. In so doing, this thesis looks to create such a shared threshold space to embody the potential to enhance the social and cultural conditions of the adjacent urban environments as places of new, dynamic and positive outcomes.

As such, this thesis intends to test thick edges as an opportunity for critical spatial practices to provide alternative and resistant design possibilities, by engaging critical architectural design in a polycontextual process of inverting existing negative conditions. Consequently this thesis looks to establish a framework of imperatives for dealing with these contemporary urban conditions in the future, to "find civic values and beauty in situations that have been alienated from any trace of community". 


\section{Problem Statement:}

The design-research of this thesis investigates two problems. Firstly, following the post-critical impasse, critical architecture has become maligned, thrusting the discipline into a 'crisis of criticality'. This crisis has fostered an ideological climate that is apathetic to the resistant possibilities and productive capacities of architecture as a critical mediation between formal and cultural production. Secondly, throughout the second half of the twentieth century, the developments of jump-cut urbanisation connecting Wellington's centre to its periphery have resulted in the emergence of a number of thick edges contextually singular and rigid areas that are barriers within the urban fabric, residual and disregarded. These sites, created by the incisions resultant from new urban motorway infrastructure, generate urban conditions that are impermeable to engagements with wider contextual dimensions of relational place identities. In Wellington, the construction of the Urban Motorway (1969-1984) has created several sites that stand as exacerbated embodiments of this condition. This thesis proposes that a critical engagement can negotiate and mediate a number of contextual approaches to resolve such sites - inverting the thick edge from one of negative rigidity to one of positive flows, while resurrecting critical architecture from its crisis of criticality.

\section{Design-Research Questions, Aims and Objectives:}

The primary aims of this thesis are: to test a contemporary critically engendered framework for architectural design-research that is both culturally and formally negotiated; and to investigate the potential for this framework to invert the negative conditions of urban thick edges through an engagement with multiple contexts. The primary objective is to propose a framework for urban development procedures to prioritise multiple contexts in the construction of place identities, re-establishing these thick edges as potent opportunities for critical architectural intervention.
As such, this thesis is framed by the following research questions:

What are the conditions of a 'critical' architecture and what processes have contributed to its currently maligned position and a discursive 'crisis of criticality'?

What are the problematic implications of the post-critical position, and how can critical spatial practices provide a contemporary, flexible and resistant alternative?

What are the negative conditions produced by the proliferation by urban thick edges, and how can architectural intervention provide positive alternatives and approaches to this contemporary problem?

How can critical spatial practices manifest in the built environment, in particular through strategies of architectural design? How can these strategies engage multiple contexts to introduce positive flows to the urban thick edge?

Can the implementation of architectural programmes that have beneficial polycontextual implications facilitate the production of critical spatial practices within the thick edge?

Through the testing of critical spatial practices as a design research framework, is it possible to produce positive solutions to the urban thick edge condition? Further, how does this framework challenge the position of critical architecture in the contemporary context?

\section{Scope of Design-Research:}

This thesis focuses specifically on how critical architecture might be implicated in resolving thick edge conditions caused by the incisions of urban motorway infrastructure development. By testing the role of architectural design in a localised context (Wellington's urban centre), this thesis tests the implications of applying a critical framework within a highly focused urban contextual problem occurring at a site-specific level. 
Positions on the critical capacities of architectural production are varied and vast, presenting a huge field of research and argument beyond the scope of a Master's thesis. To limit this field to a relevant scope, the following key authors have informed the core institutional voices that structure the thesis argument and design framework:

\section{Section One}

K. Michael Hays, Eliot Noyes Professor of Architectural Theory at Harvard University's Graduate School of Design

Murray Fraser, Professor of Architecture and Global Culture at the Bartlett School of Architecture, University College London

Kim Dovey, Professor of Architecture and Urban Design at the University of Melbourne

Jane Rendell, Professor of Architecture and Art, and Vice Dean of Research at the Bartlett School of Architecture, University College London

\section{Section Two}

Henri Lefevbre (1901-1991), was a French Marxist philosopher and sociologist, who studied at the University of Paris.

Michel de Certeau, (1925-1986) was a leading intellectual member of France's Freudian school, Michel de Certeau, and Professor of sociology at the department of ethnology at the University of Paris-VII

Marc Augé. is a French anthropologist, essayist and author.

Nick Kaye, Professor of Performance Studies, and Dean of the College of Humanities at the University of Exeter

Walter Benjamin (1892-1940), was a German literary critic, philosopher, social critic, translator, and essayist associated with the Frankfurt School Institute of Social Research.

Gevork Hartoonian, Professor of Architecturem faculty of Architetcure and Design, University of Canberra.

These authors can be seen to operate outside of the mainstream, opposing and resisting from the periphery. As a result they may possibly be considered less susceptible to the influence of architects that have shaped the impulses of the post-critical. Instead, all of these authors have their roots in critical social theory, particularly in the work of Walter Benjamin who provides a conscious and subconscious influence throughout their work as well as throughout the argument of this thesis.

Additionally, as design-research, the discursive mediations and arguments of this thesis are contextualised and implicated within the realities of contemporary design practices. Built environment precedents are considered and discussed with equal importance to text, drawing upon investigations and built-works that resonate between practices directly (and not-so-directly) related to architectural design (as well as landscape art, sculpture, urban design, etc.). Design is approached in the thesis as a critical, malleable, and multi-contextual process that is capable of embodying both intentions and solutions for architectural research.

\section{Design-Research Approach}

The design-research of this thesis is structured around three sections: Section One 'framing'; Section Two 'mediating'; and Section Three 'testing',

Section One investigates a discursive position for the thesis argument and theoretical framework. It begins with Chapter Two, which conducts an investigation into the central proposition of critical architecture and the reasons for its currently marginalised position. Upon illuminating the context through which the post-critical impasse and subsequent critical crisis has emerged, the core ideological tenants behind the post-critical position are identified and critiqued. In doing so, Chapter Two introduces an alternative trajectory for critical architecture and a resistant position to the 
post-critical project. Engaging three authors who are currently addressing this discursive problem, Murray Fraser, Kim Dovey, and Jane Rendell, critical spatial practices are identified as a contemporary framework through which the design-research can be tested.

Chapter Three focuses the inquiry on a 'real' problem for the design case study - discussing the conditions of late-twentieth century urbanisation that have shaped the production of negative urban spaces, such as thick edges. As a driver of urban sprawl, 'jump-cut' urbanisation is examined to explain the processes by which an urban centre is connected to its periphery through new infrastructure. The conditions of urban thick edges that result from such processes are identified and a positive, polycontextual, alternative approach is established. Chapter Three describes three thick edge exemplars embedded within the urban fabric of Wellington: the Westpac Stadium Car Park, the Ballantrae Car Park and the Clifton Street Car Park. As the most extreme and particularly problematic example of a thick edge condition, the Clifton Street Car Park is selected as the context for the design case study.

Chapter Four conducts a thorough contextual analysis of the site, the Clifton Street Car Park, critically examining the historical, economic, political, social, physical and visual contexts that have shaped its identity as a thick edge. Consequently, Chapter Four produces information that is developed through polycontextual design strategies applied in the design case study.

Section Two mediates between the theoretical framings and testing of the thesis research, implicating critical spatial practices in processes of architectural design by expanding upon Rendell's initial positions. Building upon the research conducted in Section One, the 'spatial turn' is discussed and a theory of place identity is outlined through Lefebvre, de Certeau and Augé. As a thick edge, the Clifton Street Car Park is identified as closer to an identity of 'non-place' - a positioning that informs the focus and expansion of Rendell's framework as it relates to a polycontextual approach. As such, the research framework based on Rendell's critical spatial practices is pursued through a three-fold process of critique that integrates the work of three contemporary theorists: site-specific (Nick Kaye, Chapter Five), socio-spatial (Kim Dovey, Chapter Six), and temporal (Gevork Hartoonian, Chapter Seven) processes.

In Chapter Five, Rendell's position on sitespecific processes as a form of critical spatial practice is developed in further detail through the work of Nick Kaye. Exploring an alternative to traditional notions of 'site', Kaye considers practices that engage the 'performative' possibilities of site-specific intervention. For Kaye, performative practices embody the possibility for architectural design to embed sites with contextual information, creating a dialogue between built form and the identity of a particular place. Analysing two case studies, the Parc de la Villette (1982-83) by Bernard Tschumi and the Igualada Cemetery by Enric Miralles (1994), strategies of continuity/discontinuity are elucidated.

Chapter Six expands the framework of criticalspatial practice through socio-spatial processes, explored through the work of Kim Dovey. Taking a position on public space that resists deterministic appropriation, Dovey argues that architecture enables and/or constrains spatial practices through particular dimensions that can be classified within a spectrum of 'tight' and 'loose'. These dimensions empower architects to explore the possibilities of social flows through programme and form - creating dynamic spaces within which multiple publics can engage. Through three case studies - the Palais de Tokyo (2001) by Anne Lacaton and Philippe Vassal, the Metropol Parasol (2011) by Jürgen Mayer, and the Rolex Learning Centre (2010) by Ryue Nishizawa and Kazuyo Sejima - strategies of addition, subtraction, horizontality and verticality are investigated.

Chapter Seven investigates critical temporal processes in further detail through the work of Gevork Hartoonian. Re-visiting Walter Benjamin's theory of the dialectical image, Hartoonian presents a position that engages the contextual histories of particular places in the visual production of architectural form. The Jewish Museum Berlin (1999) by Daniel Libeskind is analysed as a relevant case study in this regard, demonstrating how strategies of juxtaposition 
are capable of subverting and destabilising the physical and visual context of architectural intervention.

Chapter Eight proposes an architectural programme for the Clifton Street Car Park site relating to an exemplary client - the New Zealand International Arts Festival (NZIAF) - introducing 'real world' architectural components to be implicated in the design case study experiment.

In Section Three the thesis documents, discusses and reflects upon the design-research case study experiment, a multifunctional events facility and public park sited within the Clifton Street Car Park. Chapter Nine presents the design, investigating the intervention of an events facility that has been derived from a polycontextually framework of critical spatial practices. The design is analysed and presented in relation to the research problems and research questions framed in Section One, and the strategies implicated and demonstrated in Section Two.

Chapter Ten concludes the thesis by reflecting upon the design case study relative to the research aims and objectives. The design case study is not presented as a singular solution or outcome, but rather as a provocation and opportunity for further development and reflection. Based upon this understanding, the potential implications of the thesis are discussed as: a testing of critical spatial practices as a discursive framework, and an opportunity to engage urban thick edges as architectural opportunities that can make a positive contribution to the urban fabric. The proposed new Basin Reserve Flyover project is presented as yet another thick edge about to emerge in the urban fabric of Wellington, evidencing the urgency of addressing this contemporary problem, and reinforcing the relevance and vitality of this designresearch thesis proposition in the contemporary context. 
29

$\frac{1}{1}$ 


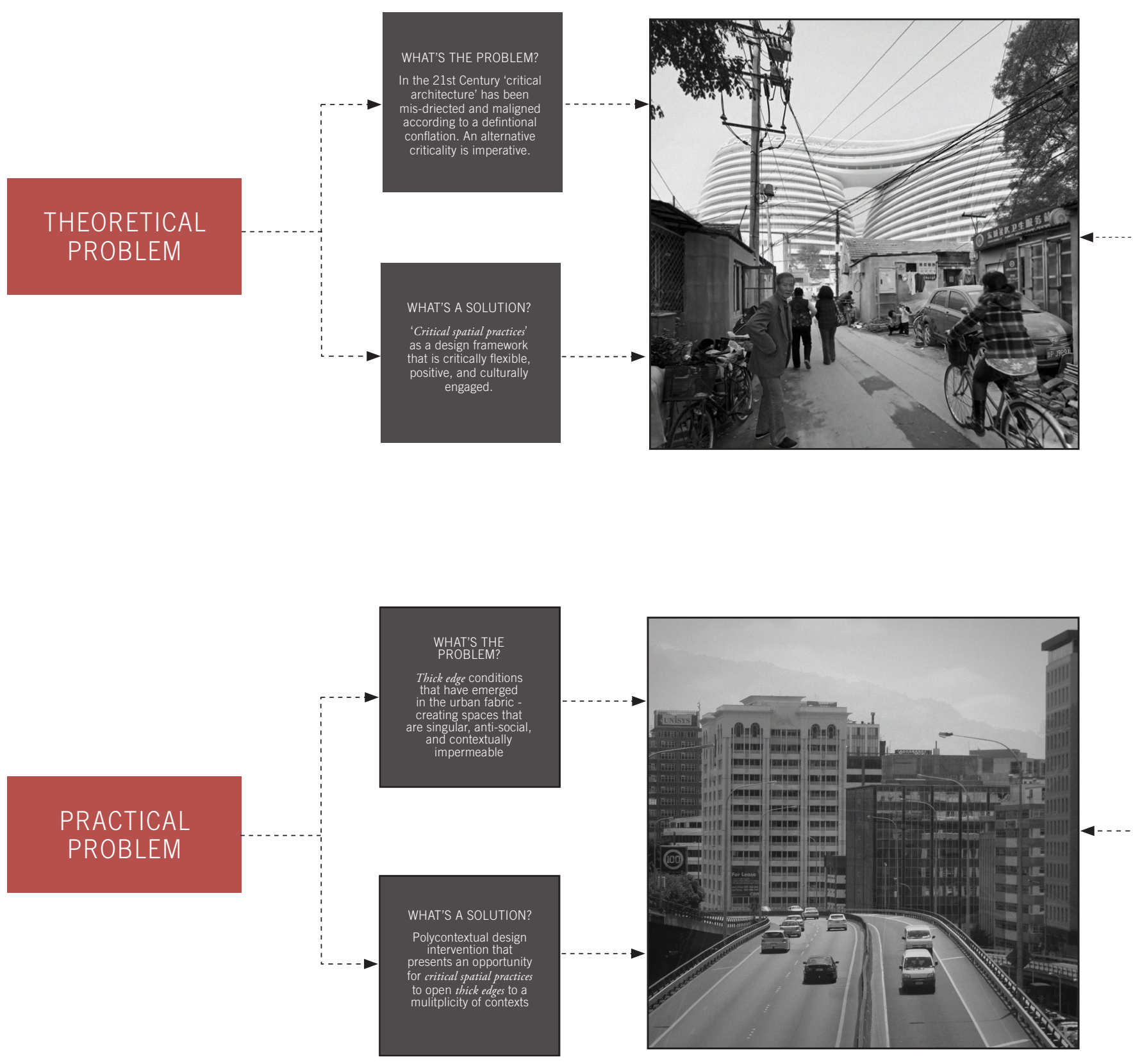

SITE-SPECIFIC

Nick Kaye: Establishing 'site' as a performative index

SOCIO-SPATIAL Kim Dovey: Public space stuctured by a negotiation between 'tight' and 'loose' image 

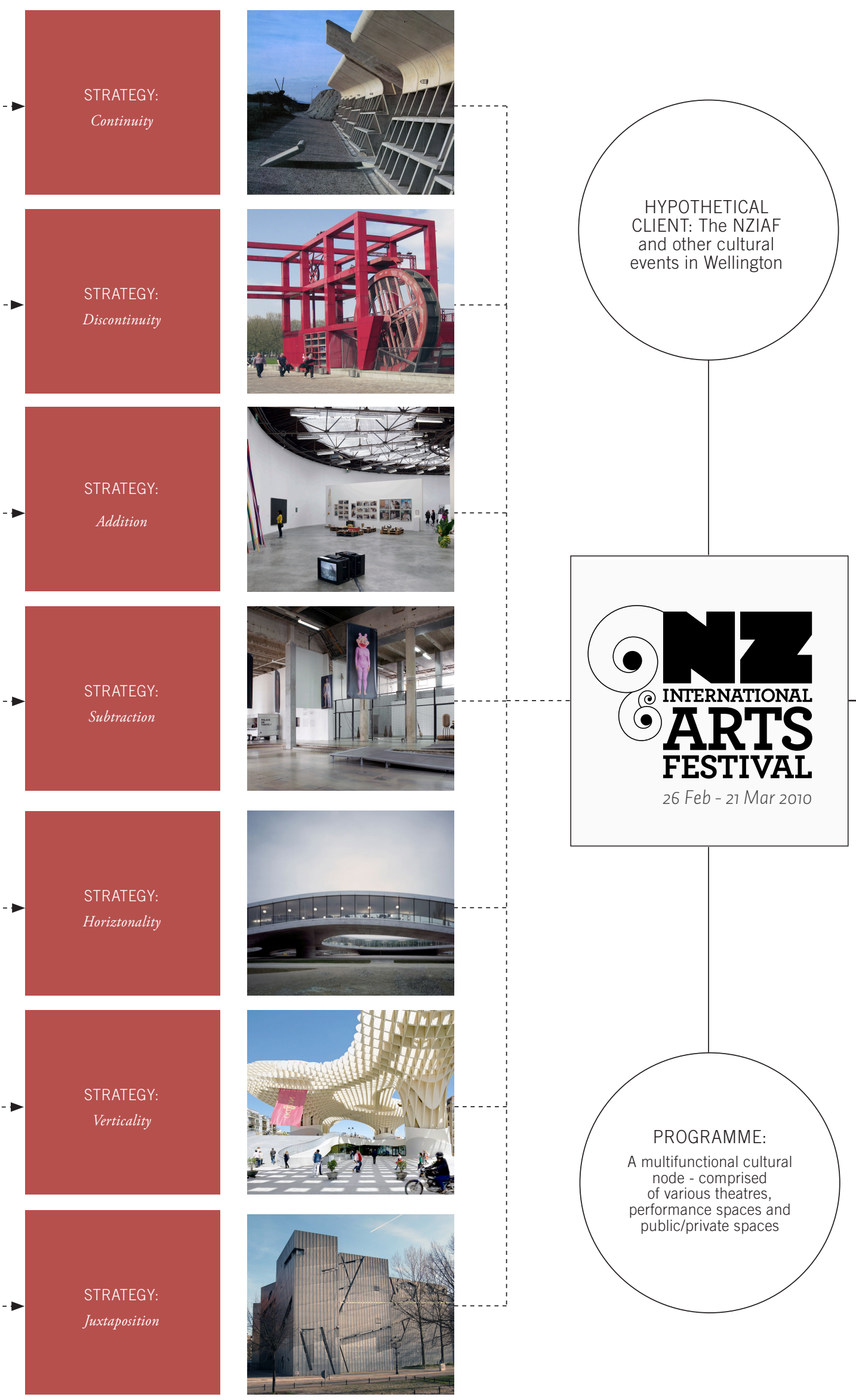

A critical discussion

of design-research
conclusions and

mplications to emerge

from 'Architecture's

Tightrope' 


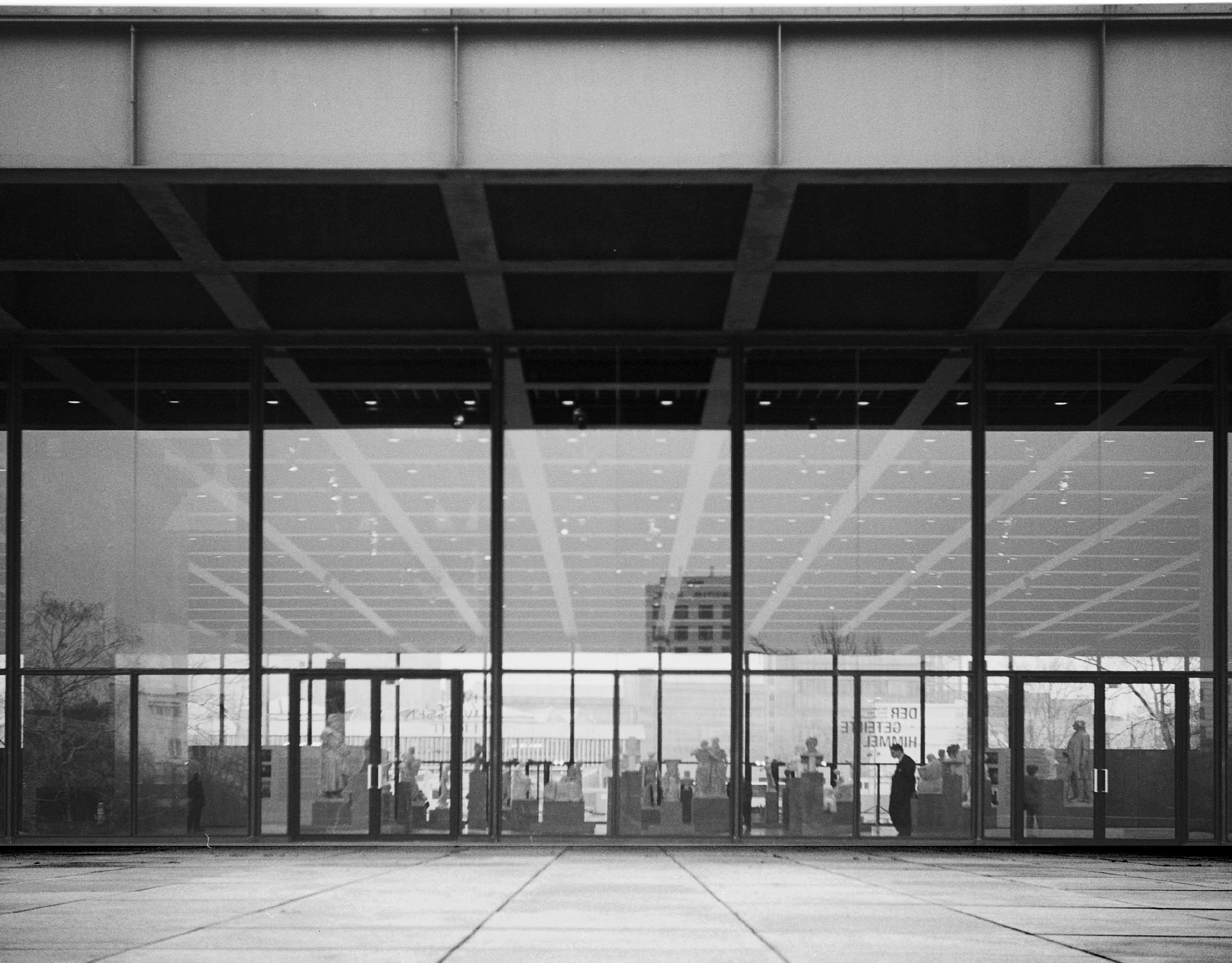




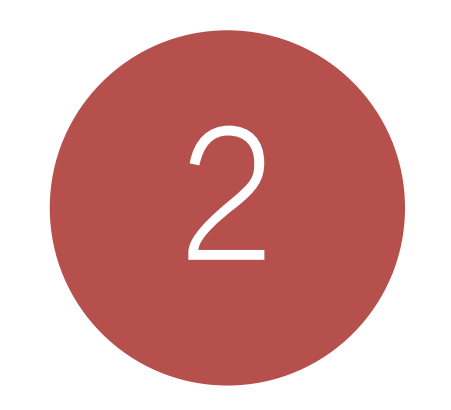

\title{
AVERTING THE CRISIS OF CRITICALITY:
}

\author{
A (Discursive) Design Problem, \\ Argument and Approach
}

\begin{abstract}
Introduction
A Critical Premise: Mediating Between Formal and Cultural Production

The 'Late Avant-Garde': Fathers of Critical Architecture's Death Drive.

A (Conflated) Crisis? Unravelling the PostCritical and their Oedipal Discontents

Implications of a Post-Critical Ideology: The Technical Perspective and a Loss of Context

Architecture's New Desire: Mediation through Critical Spatial Practices
\end{abstract}

\section{Conclusion}




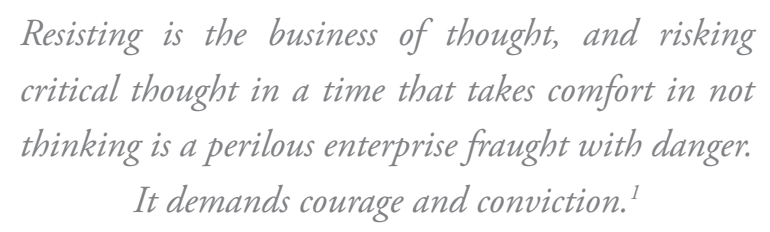

Nadir Lahiji

This chapter examines the theoretical problem and framework for this thesis by discussing the 'critical' faculties of architectural production. In particular, through K. Michael Hays, Chapter Two isolates the tenants of a 'critical architecture', tracing its dominant ideological trajectory from the late avant-garde to it's currently maligned position within a discourse that is considered by many to be postcritical'. An alternative trajectory is argued for and illuminated - framing the first of the primary aims for design-research.

Following the influence of Critical Theory on the spatial disciplines (indebted to the works of cultural theorists from the 'Frankfurt School') the possibility that architecture is capable of bridging the gap between discursive criticism and built

2. Rendell, 2007, p. 2. environment practice emerged as a prominent force in the 1970 s. $^{2}$ This chapter considers the subsequent and essential definition for 'critical architecture', as resistant and oppositional, through the lens of architectural theorist K. Michael Hays. In so doing, the first question for the design-research of this thesis is engaged:

What are the conditions of a 'critical' architecture and what processes have contributed to its currently maligned position and a discursive 'crisis of criticality'?

Building Hays's proposition, the dominant trajectory for critical architecture since the 1970 s is investigated. This follows the influence of a group of significant architectural theorists and practitioners primarily operating within an experimental practice of 'absolute autonomy'. These figures (Peter Eisenman, Bernard Tschumi, John Hejduk, and Aldo Rossi) titled the 'late avant-garde' by Hays, have contributed to a shift in focus for critical architecture - away from dialectical critique as a form of resistance - and towards design strategies invested in isolating architecture as an independent, formal exercise. This trajectory can be seen as producing a context throughout the 1990s that has initiated a discursive impasse since the turn of the twenty-first century - the intervention of the post-critical and the subsequent 'crisis of criticality'. Progressing the argument, a second thesis question is addressed:

What are the problematic implications of the post-critical position, and how can critical spatial practices provide a contemporary, flexible and resistant alternative?

Isolating the central propositions of this impasse, Chapter Two argues that the limited responses of the post-critical - in particular the writings of Michael Speaks, Robert Somol and Sarah Whiting - conflate the definition of critical architecture to that of formal autonomy alone, disregarding the possibility for architecture to embody a socially or culturally inflected criticality. Importantly, the reflexivity of the post-critical position has contributed to a contemporary architectural landscape that is apathetic to the investigations of architecture's autonomy as a formal proposition, as well as its implications as a culturally contextual 
discipline. Rather, this chapter argues that under the guise of positive progress through innovation alone, the post-critical facilitates design practices that are conciliatory to the forces of globalisation and its potentially negative consequences.

Acknowledging the necessity for architecture to move beyond its own formal autonomy, whilst also rejecting the 'new disciplinarity' imposed by the postcritical, this chapter argues that a new trajectory for BELOW critical architecture is vital. This alternative focus Figure 2.2: Diagram illustrating the argument structure this chapter, constructing a framework for the design research is built upon the proposition that, in light of the developments of the 'spatial turn' in cultural studies since the 1970s, critical architecture can re-engage
Hays's original dialectic within the contemporary context. In so doing, architectural design can operate through a framework of critical strategies that can produce positive, flexible, solutions to the complex requirements of contemporary discourse. This chapter concludes with a brief discussion of three authors who affirm this position: Murray Fraser, Kim Dovey and Jane Rendell - isolating critical spatial practices as the framework for this thesis that will be investigated.

THE DOMINANT TRAJECTORY

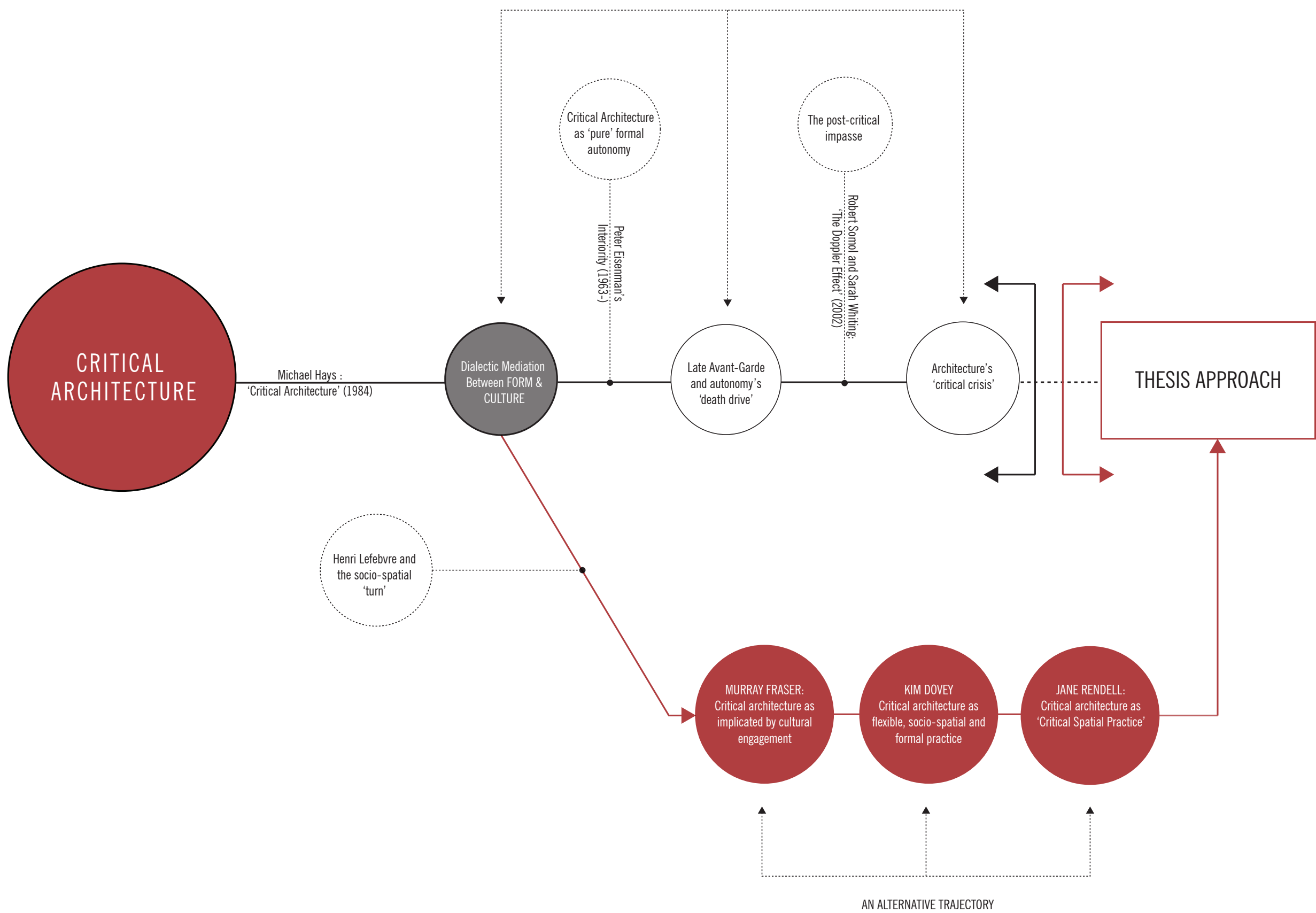


3. Hays, 1984, p. 15. For Hays, culture is understood to be a duality between: "those theoretical and practical systems which authorize, production and use of ideas or a place differentiates itsel and maintains its hegemony"; and, "the artefacts and environments which endure as resourceful physical precedents

or exemplars of systems

of production and become transmitters of culture".

4. Hays, 1984, p. 27. This thesis agrees with this proposition, and argues for

a resistance to particular processes of architectura production throughout Chapter

5. For the purposes of this thesis 'dialectic' is used in the Hegelian sense: as the negotiation between two seemingly opposite, often contradictory, positions engaged in a dialogue with the purpose of negotiating a synthesis.

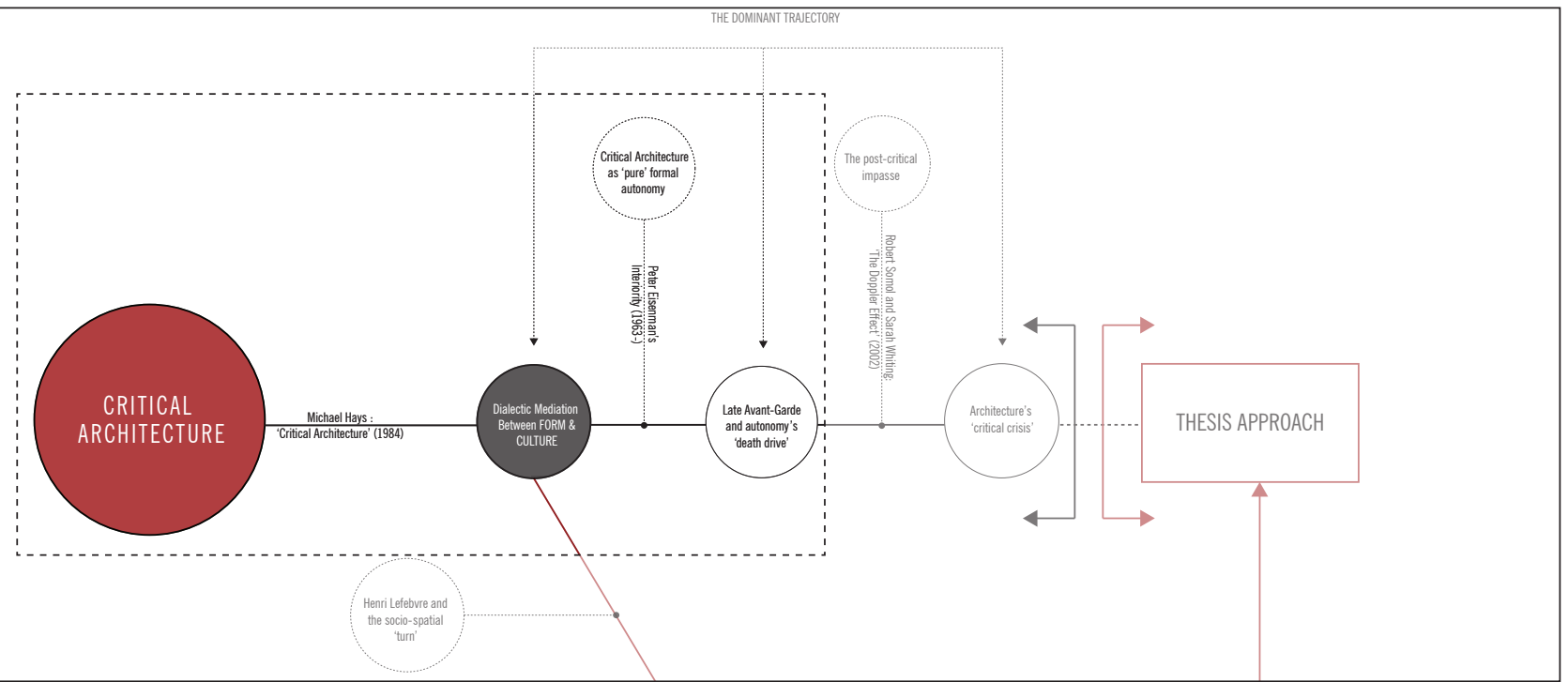

\section{A Critical Premise: Mediating Between} Formal and Cultural Production

In his seminal essay "Critical Architecture: Between Culture and Form" (1984), K. Michael Hays investigates the notion that architectural production can embody an intentional critical faculty. In so doing he develops a progressive method for defining a 'critical' architecture - a method that was perceived as a canonical definition for the generation that followed. According to Hays, the 'critical' in architecture is the means through which architectural production can perform a role that is resistant and oppositional to "the self-confirming, conciliatory operations of a dominant culture and yet irreducible to a purely formal structure disengaged from the contingencies of place and time". ${ }^{3}$ The critical architect emerges as an 'author' of motivations that persistently resist prevailing ideologies, producing "knowledge both about culture and about architecture" ${ }^{4}$ Of particular emphasis to this definition is the identification that critical architecture must operate between modes of formal and cultural production, within which it is in a state of constant dialectic negotiation. ${ }^{5}$ These two modes are identified as: 'architecture as an instrument of culture' and 'architecture as autonomous form'.

The first of these modes, as an 'instrument' of culture, identifies architecture as a cultural product - a system that is capable of confirming and assuring the continuity of dominant and hegemonic valuesystems. The so-called instrumental perspective calls for methodologies that "attempt to restore an architectural object to its original meaning" through a study of "the base material of the historical world". For Hays, the temporality of the instrumental perspective - as perceived at the time of his thesis - was proposed as a historically material construct. Thus the cultural mode of operation for critical architecture is problematically limited:

\section{It is supposed that the only alternative to the strict methodological recovery of the cultural situation at the time of the object's origin is the denial of any historical objectivity and capitulation to the idea that all schemes of interpretation are hopelessly subjective.}

As such, resistance to the 'hopeless' determinism of the instrumental position is presented as an engagement through the second mode: disciplinary 'autonomy' as a critical, formal, procedure. The autonomous position posits that any critique through architecture must be focussed on the systems that

\section{The 'critical' in architecture is the means through which architectural production can perform a role that is resistant and oppositional to "the self-confirming, conciliatory operations of a dominant culture}

produce it (be they structural, linguistic, formal etc.), creating a language of production that is exclusive to the discipline proper. In this regard, the function 
of autonomy is to interrogate the conceptual space of architecture as a 'pure' discipline that can be understood without the complicity of subjectivity 8. Martin L., 2011, p. 181 and/or external forces. ${ }^{8}$ According to Hays, this conceptual space is completely open to interpretation and can be endlessly read and misread at the exclusion of any cultural constraints:

...this second position is characterized by the
comparative absence of historical concerns in favour
of attention to the autonomous architectural object
and its formal operations - how its parts have been
put together, how it is a wholly integrated and
equilibrated system that can be understood without
external references.

9. Hays, 1984 , p. 16

As such, "absolute autonomy" admits to accede to the inefficacy of political and social implications, and by virtue of this admitted powerlessness, maintains a disciplinary purity - a form of critical practice that operates beyond the inevitably compromised influences of culture.

Exemplifying mediation between the autonomous and instrumental positions, Hays cites the 'silent criticality' of Mies van der Rohe, whose projects "abstain from any dialogue with the physical particularities of their contexts" creating architectures that exude a rigorous apriori formal logic (see Figures 2.1 and 2.4). For Hays, Mies's architecture propagates the promise that architectural form alone, through its own order of materials, surfaces and geometries, can resist the dominant order of societal values by for a contemporary re-visiting of Hays's central actively occupying a culturally neutral space. ${ }^{10}$ In so doing, Mies's buildings are resistant to their cultural contexts by intentionally reflecting a formal and visual 'detachment'. Importantly, Hays's identification of the 'silence' of Mies's architecture towards the cultural conditions that produce it forecasts the impending unhinging of the formal/cultural dialectic, and a trajectory for critical architecture as absolutely autonomous - a proposition which continues to inform the dominant perspective for debates surrounding architecture's critical faculties today. ${ }^{11}$

Outlining this trajectory, this thesis argues proposition for a critical architecture, as resistant to processes that are conciliatory to hegemonic cultural value systems, or ideologies, and their negative outcomes. As a result, this thesis understands that critical architecture, operating in dialectical tension, is constantly reflexive - contingent on the 'zeitgeist', the particularities of the context(s) within which it is operating. ${ }^{12}$ Understood in this manner, this thesis contends that 'criticality' occupies a crucial role within the discipline of architecture; regardless of the prevailing values of the discourse, architecture must maintain a constant state of incisive evaluation and re-evaluation in accordance to the situations that produce it at a particular time, in a particular place.

\section{The 'Late Avant-Garde': Fathers of Critical Architecture's Death Drive.}

Following Hays's initial identification of the two modes of operation for a critical practice (instrumentality and autonomy) several dominant voices within the architectural community have contributed to a discursive direction that has favoured a rigorous and 'absolute' autonomy, drawing the definition of critical architecture away from the original, dialectically mediated proposition of Hays.

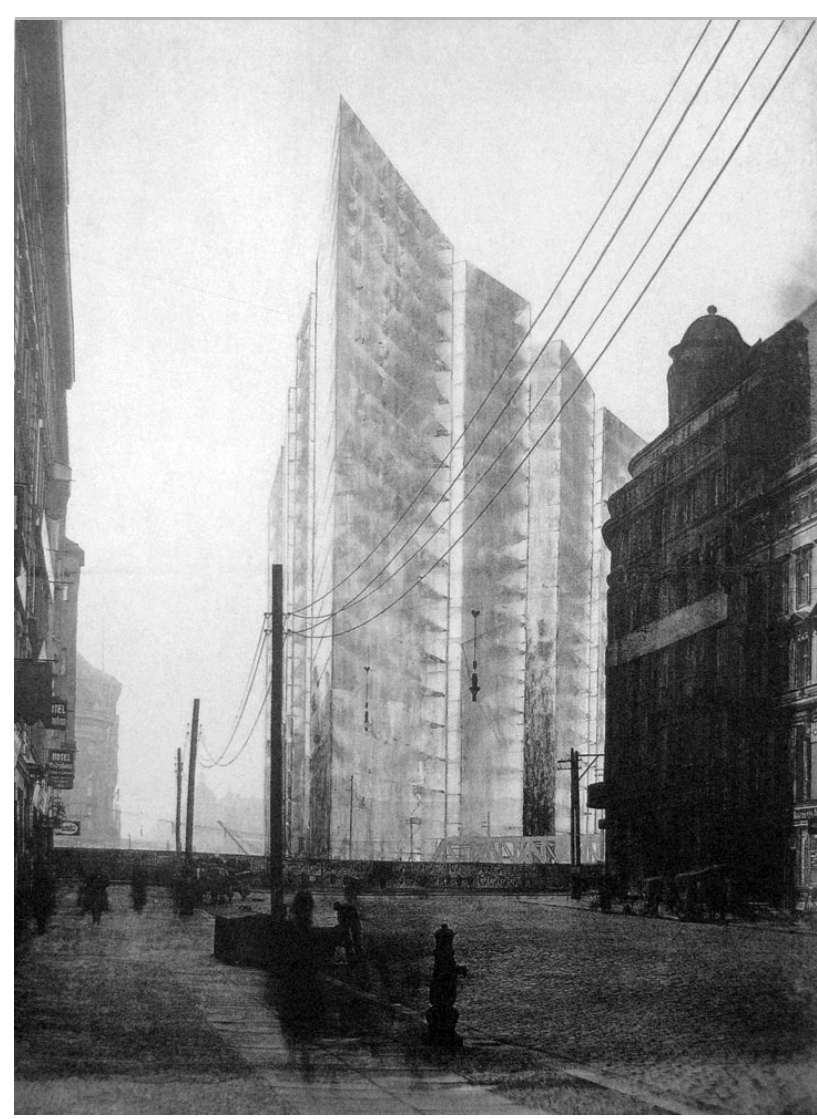

$<$ LEFT

Figure 2.5: Mies van der Rohe Friedrichstrasse Project, charcoal drawing (1922). For Hays, this project represents a 'radical departure' for Mies, 'radical departure' for Mies, physicaly and conceptually relating architecture to the city through volume and transparency. 
In his most recent work, Architecture's Desire: Reading the Late Avant-Garde'(2010), Hays reflects on this trajectory and the drive towards absolute autonomy - citing the works of a group of experimental practitioners operating within the discourse since the 1970s - namely, Aldo Rossi, John Hejduk, Bernard Tschumi, and Peter Eisenman - since considered to be the highly influential 'fathers' of critical architecture. ${ }^{13}$ Hays identifies (with the benefits of hindsight), that this 'late' avant-garde represents the enactment (and, for now, exhaustion) of "architecture's desire"

Architecture's death drive, identified by Hays as encompassing a 'lack of a social need for architecture, architecture's total loss of the real' reached its polemic zenith in the 'post-human' investigations of Peter Eisenman.

to uncover architecture's interiority, its disciplinary

14. Hays, 2010, p. 11 boundaries, through "a reflection on the foundations and limits of architecture itself" ${ }^{14}$ "This desire, has been misconstrued as 'critical', yet, Hays perceives it as grounded in a radical experimentation with architecture's singularity, rather than operating in critically inflected space between form and culture.

As such, the moniker for this particular group is prefixed with 'late', purposefully drawing association with Frederic Jameson's notion of the 'late modern', and implicating them with the post-war experimentations and extreme conceptualisations of avant-garde art practices:

Unlike the fully commercialized postmodernism, the late architectural avant-garde keep's its 15. (Ibid, pp. 11-12) namesake's [Jameson] commitment to rigorous formal analysis... it self-consciously closes in on its own limits rather than opens outwards. ${ }^{15}$

With their 'rigorous' formal autonomy, art practices of this post-war or 'late modern' movement pre-date (as is often the case) the architectural avant-garde in question, reaching a saturation point in the 1950s and 1960s through the minimal abstractions of artists such as Donald Judd, Carl Andre, Frank Stella
16. This process - the shift in art practices from late moder formal autonomy to a more and Richard Serra (see Figure 2.5). ${ }^{16}$ Consigned to the inevitability of a similar fate - architecture's 'death drive' is inscribed axiomatically within the motivations of a pure autonomy, as its detachment from the dialectical tension between form and culture 'freezes' architectural discourse from contact with the real. ${ }^{17}$ This death drive forecasts the inevitability of the downfall of pure autonomy as a misconstrued version of critical architecture's primary focus:

The architecture of the late avant-garde performs the impossibility of architecture's full realization; it stages an architectural project that for historical reasons must be undertaken but ultimately is bought to failure by a dynamic integral to the project itself. Such are the workings of architecture's desire. $^{18}$

Architecture's death drive, identified by Hays as encompassing a "lack of a social need for architecture, architecture's total loss of the real" reached its polemic zenith in the 'post-human' investigations of Peter Eisenman. ${ }^{19}$ Eisenman's critical project can be seen as systematically hijacking any mediation between form and culture in favour of a totalising disciplinary 'interiority'. His body of work is an "unremitting vector" of self-proclaimed interiority that probes into the manifold conditions of architecture as a formal procedure. ${ }^{20}$ Leading to, and following, the identifications made in Hays's "Critical Architecture...", Eisenman's formal experiments directed architectural discourse towards consistently deeper modes of abstraction to the point where it can be perceived as "meaning-free, arbitrary, and timeless". ${ }^{21}$ In this sense, architecture becomes

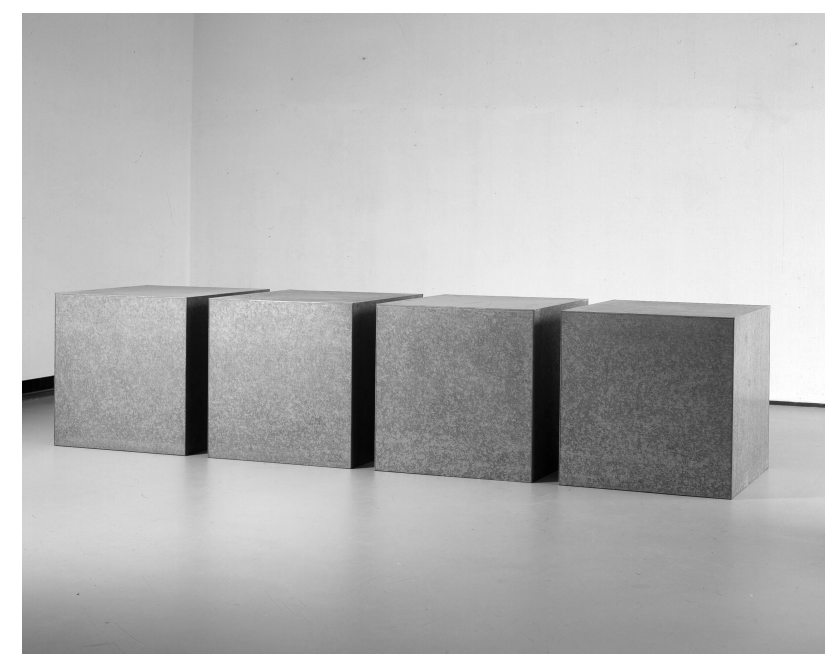

culturally inflected practice - is explored in depth by Rosalind Krauss in (Krauss, 1985) and (Krauss, 1977)

17. This notion, that

architecture's autonomy would lead to a continuous and self-reflexive feed-back loop, was forecast by Manfredo Tafuri in Architecture and Utopia: Design and Capitalist Development (1985). Additionally, this thesis argues for a definition of 'real' space as socially produced, rather than abstract and absolute.

18. Hays, 2010, p. 2

19. Ibid, p. 7.

20. Eisenman, 2004, p. xii.

1. Eisenman, 1986, p. 155.

$<$ LEFT

Figure 2.5: Donald Judd, 'Minimal Myth' (1973). Amongst others, Judd's minimal sculptures can be seen as the distillation of pure formal autonomy in pure formal autonomy in art practices - reducing the logic of sculpture to a core formal logic. 
'post-human' by removing itself from the "humanist nostalgia" of modernism's functionalism, rather, focussed on examining the conceptual language of architecture as a structure and technique. ${ }^{22}$ Thus architecture becomes concerned with "the thing itself", rather than its attachment to the limiting constraints of the human - the functional and the social. ${ }^{23}$ Eisenman's post-human formal experiments 23. Eisenman, 2004. are manifest in his 'House' series - a number of experimental procedures that attempt to engage the structural languages of the notion of 'house' as an architectural object (see Figure 2.6).

Located outside of Cornwall, Conneticut, 'House VI' (designed for Suzanne and Richard Frank between 1972 and 1975) stands as a physical record of Eisenman's formal processes, a collection of orthogonal architectural elements (walls, floors, columns, beams) arranged according to a logic that refines and resolves "relationships within the fractured grid" (see Figure2.7). ${ }^{24}$ In House VI, Eisenman removes the architectural process from its cultural, social and humanistic implications is clearly evident. Kristina Luce, Professor in Architectural History and Theory at the University of Michigan notes:

In other words, Eisenman's goal was to make a kind of sliding puzzle out of House VI. He desired to induce the reanimation of his process of design in the visitor... by reactivating this process, Eisenman undermined the physical object that is House VI, placing it alongside all the other buildings that a visitor might imagine. ${ }^{25}$

As a formal experiment, House VI represents a radical reinterpretation of what a 'house' is in relation to the arrangement and structure of architectural language that constitutes it. However, as an inhabited and thus essentially social object, Eisenman's design has several negative implications. The most prominent of these, are its functional eccentricities (see Figure 2.8) and structural failures. (The clients were required to rebuild it in 1987.)

Crucially, through the construction of House VI, the tenants of post-human autonomy transition across the threshold from conceptual practice into the reality of architecture's functionalism, implicating the distinctly 'human' in the 'post-human'. This transition represents an important moment in Eisenman's investigations of architecture's interiority; a turning point towards the impending finality of pure autonomy that has since been outlined by Hays. As a result, this thesis contends that the autonomous trajectory, and the subsequent removal of the social realities of architectural practice from its role as a critical mediation, has led to the ideological foundations of the post-critical position, and what will now be discussed as architecture's 'critical crisis'.

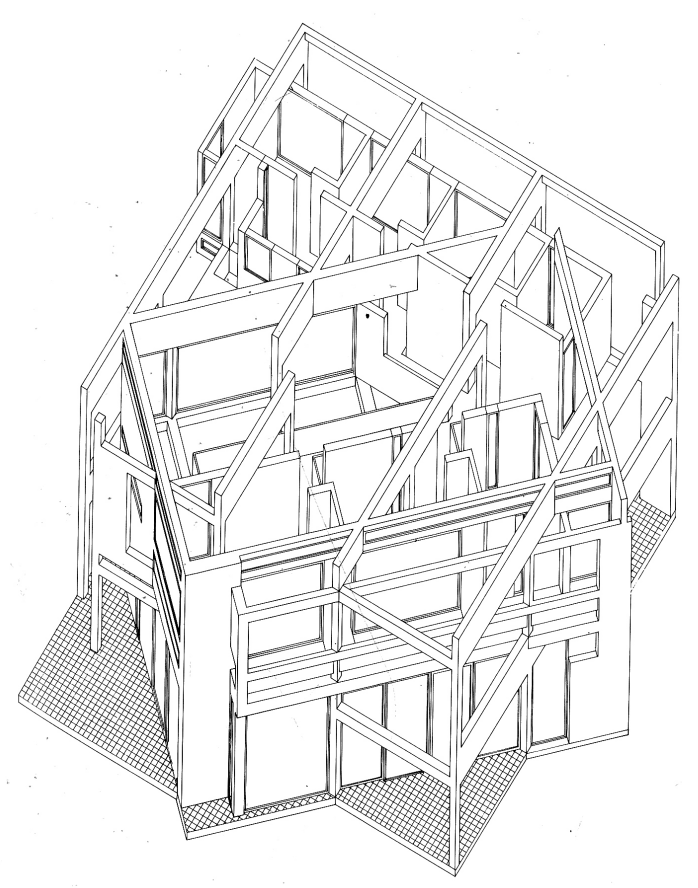

Figure 2.6: Peter Eisenman House III (1972) Axonometric view. 

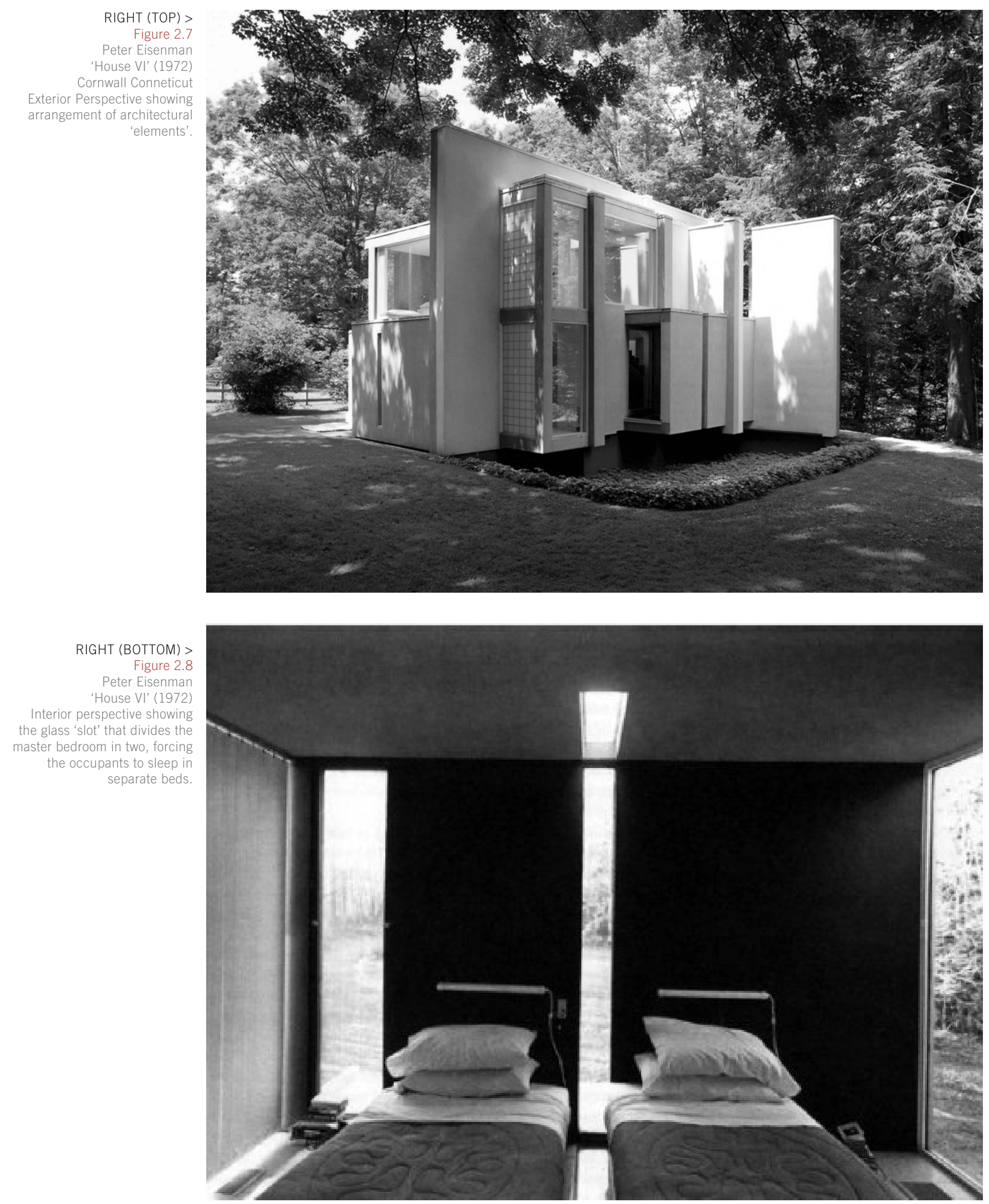
A (Conflated) Crisis? Unravelling the Post-Critical and its Oedipal Discontents

A crisis often seems to explode abruptly, without prior warning. Typically, the forces that feed the crisis intensify and strengthen in the periphery of vision, outside the centre, and their threat to the status quo is not adequately addressed until a later moment-until the crisis erupts. ${ }^{26}$

26. Kaminer, 2011, p. 13) Architecture and Landscape at the University of Edinburgh

27. Ockman, 2003, p. 78 Joan Ockman is Professor of Architecture at the University of Pennsylvania.

28. Doucet \& Cupers, 2009 Dorrian, 2005, p. 229. Stead,

\section{7}

29. 'Crisis' is a time of "intens difficulty, a turning point whic requires an important decision to occur". Simpson \& Weiner, 1989 has been traced back to a conference organised by ANY magazine in 1994, where the highly influential architect Rem Koolhaas was noted as suggesting that there is something "inherently un-critical" about architecture. Koolhaas's statement is symptomatic of a wider trend that was forming within architectural discourse in the mid-1990s as a generation of architects following the late avant-garde attempted to wriggle out from the shadows of autonomy's theoretical negativity. According to William S. Saunders, Editor of the Harvard Design magazine:

Fast-forward to the mid-1990s: many highly intelligent young architects and architectural intellectuals were getting fed up with this [critical autonomy's] detachment, theoretical abstraction, and helplessness... They wanted to be in the game.
Their intellectual elders struck them as overly serious, overly self-important, overly righteous, and "pure". 30

Under the seemingly insurmountable intellectual excursions of theoreticians such as Eisenman, many leading institutions of architecture attempted to explore a discourse that was disguised under the moniker of 'critical architecture' yet propagated a climate of pseudo-intellectual posturing. ${ }^{31}$ This environment was seen by many as contaminating architectural practice with investigations of "bad novels, bad sociology, bad psychology, bad philosophy, and bad movies", ${ }^{32}$ As a result, the motivations of the post-critical's 'new pragmatism' were openly reactionary, positioned against the dislocation of nearly all critical architectural theory from 'real-world' concerns. The intentions of this attitude towards architectural theory did not coherently materialise until 2002 with the publication of two pieces of writing, one from Professor of Architecture at the University of Kentucky, Michael Speaks; and the other from Director of the School of Architecture at the University of Illinois, Robert Somol, and Professor and Dean of the Rice School of Architecture, Sarah Whiting. ${ }^{3}$

In his introduction to a series of interview based articles titled 'Design Intelligence and the New Economy', Michael Speaks sketches the purposes of a distinctly anti-critical position. For Speaks, any discussions around a deeper, critical, meaning in architecture must be brushed aside in favour of a new theory based upon the 'chatter' and 'little truths' that are evident in a hyper-globalised, technology-driven society. His theory of 'Design Intelligence' calls for a professional architectural practice that explicitly denies the "dialectic negativity of Adorno-inspired critical architecture" in favour of innovation through adaptation. ${ }^{34}$ His basis for this conviction is that we are now in a post-capitalist paradigm enabled by globalisation, and therefore must accept that the dominant economic structures are knowledge-based. In such a society, Speaks argues that:

Philosophical, political, and scientific truth have fragmented into proliferating swarms of
31. Interestingly, even Eisenman has accepted that his influence has been inappropriately adopted by many - instead finding safety in a withdrawal from an exclusively theoretical practice. Most recently his textual studies have moved away from an aggressively progressive position and have focussed on the analysis of historically important buildings, see Eisenman, Allen, \& Lourie, Ten Canonical Buildings: 19502000, 2008

32. Ibid, p. ix

33. Baird 2004, p. 16. Martin R. , 2010, p. 348. 


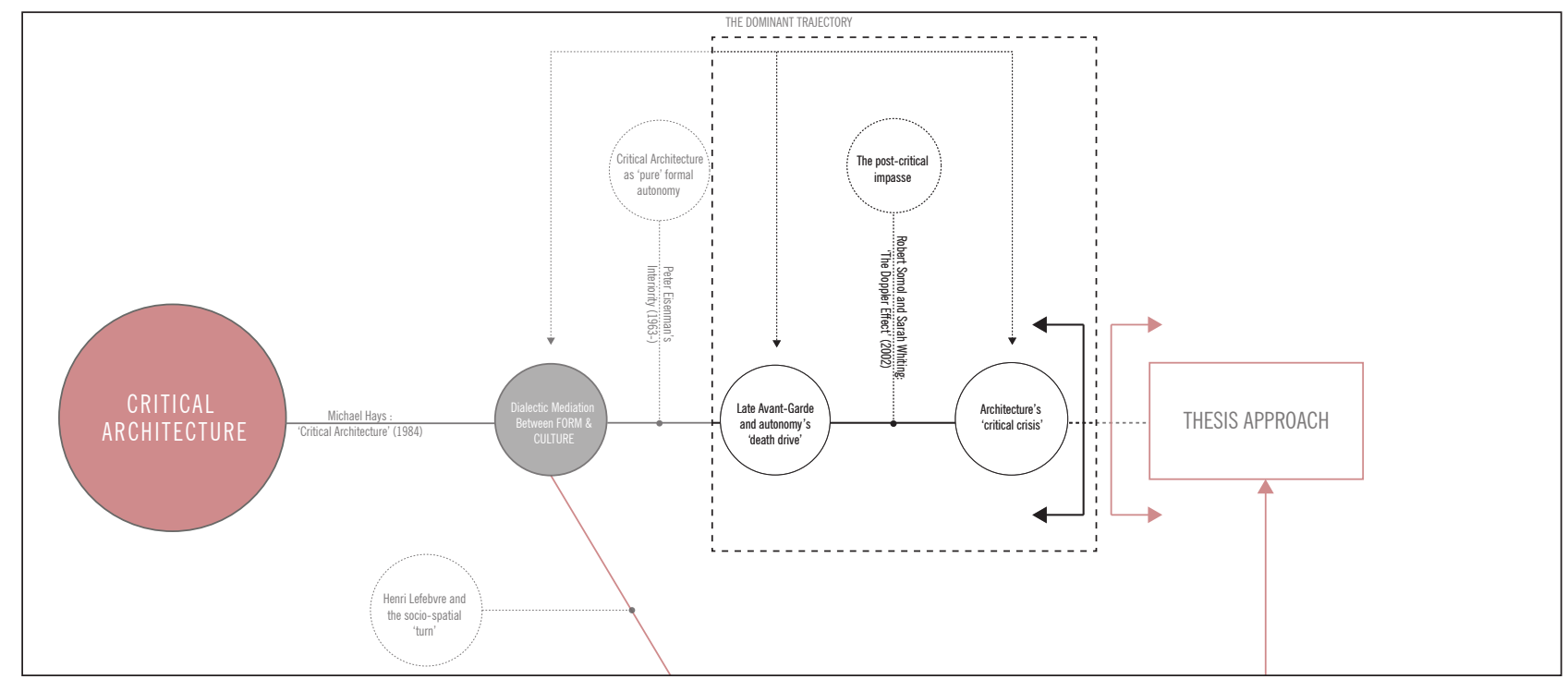

"little" truth appearing and disappearing so fast that ascertaining whether they are really true is impractical if not altogether impossible. No longer dictated by ideas or ideologies nor dependent on whether something is really true, everything now depends on credible intelligence, on whether something might be true. ${ }^{35}$

For Speaks, adherence to critical ideology is 'impractical', in light of which he suggests an alternative - architects should succumb to dominant market forces and embrace the innovations of technological tools such as 3D modelling, rapid prototyping, and generative mass production as means to produce 36. Ibid, p. 105 pragmatic design solutions for their clients. ${ }^{36}$

Almost immediately in the wake of "Design Intelligence and the New Economy", two more American academics - Robert Somol and Sarah Whiting - composed an argument that explores a more philosophically nuanced direction for the post-critical project. In "Notes around the Doppler Effect and Other Moods of Modernism", Somol and Whiting suggest a discursive alternative to the critical, one that is less embedded in the economic concerns of Speaks. Identifying that critical architecture's internally autonomous position is stuck in a 'negative' self-reflexivity that relies on a constant opposition to reification, they propose a 'projective' architectural practice that is based upon performance and emergence:

While reification concerns itself with the negative reduction of qualitativeexperience to quantification, emergence promises that serial accumulation may itself result in the production of new qualities. As an alternative to the critical project - here linked to the indexical, the dialectical and hot representation - this text develops an alternative genealogy of the projective - linked to the diagrammatic, the atmospheric and cool performance. ${ }^{37}$

\section{Accordingly, projective architecture} claims autonomy through expertise and technique whilst simultaneously denying the possibility of interdisciplinarity..$^{38}$ Unlike Speaks, who wishes to disregard the vanguard movements of the late twentieth century altogether, Somol and Whiting instead seek to redefine architecture's disciplinarity in terms of design and effects; projecting forward, rather than looking back or criticizing the status quo:

Rather than isolating a singular autonomy, the Doppler focuses upon the effects and exchanges of architecture's inherent multiplicities: material, program, writing, atmosphere, form, technologies, economics, and so on. It is important to underscore that this multiplying of contingencies differs greatly from the more dilute notion of interdisciplinarity... A projective architecture does not make claim for expertise outside the field of architecture, nor does it limit its field of expertise to an absolute definition of architecture. ${ }^{39}$

Upon outlining a 'looser' definition of what architecture can do - a new approach to design that is multiple and, to some extent, theatrical - Somol

37. Somol \& Whiting, 2002 p. 74

8. Ibid, p. 75 
and Whiting briefly explore what it should do. Here they articulate a simplistic apathy towards the late avant-garde that has come to define the intentions of the post-critical:

Critical architecture is hot in the sense that it is pre-occupied with separating itself from normative, background, or anonymous conditions of production, and with articulating difference... [Projective] architecture is cool, easy, and never looks like work...producing effect without high definition, providing room for manoeuvre and promoting complicity with subject(s). ${ }^{40}$

These closing statements are particularly noteworthy. By refusing to explicitly express why architecture should perform as a malleable, multifaceted practice (other than to divert around its previously rigid autonomy), "Notes Around the Doppler..." simply presents a series of architectural positions and directives (design, innovation, pragmatism). These reflective statements do however implicate an ideological openness, yet do not provide a supporting framework for design.

Contextualising the work of Speaks, Somol, and Whiting reveals that the motivations of the postcritical constitute less a 'crisis' and more a firm appeal for architecture to re-engage with pragmatic and 'real' disciplinary concerns. However, this thesis posits that in spite of valid (albeit intellectually reactionary) intentions, the post-critical has come to inform a misguided position on three interrelated fronts:

+ Firstly, in attempting to deny its forbearers their intellectual potency, it (incorrectly) conflates the crucial, dialectical, definition of a critical architecture with that of pure autonomy.

- Secondly, its consistent request for architecture to replace critical design processes with processes based solely in "efficacy, innovation, and realism" simply constitutes a different kind of reflexive disciplinarity, one that problematically fetishizes the 'newness' of architecture.

+ Thirdly, it attempts to throw critica architecture into a state of crisis, yet it does not deliver a cohesive or resistant substitute, instead occupying a conciliatory position with regards to the hegemonic value systems of globalisation and flows of capital.

Affirming these contentions, Daniel Barber, notes that the fundamental definition of 'critical architecture' set forth, and rejected, by the postcritical is "reliant on a rigid conception of disciplinary autonomy".41 For Barber, the post-critical position is not based upon a rejection of the 'critical' in architecture per se; rather, their attempt to surpass criticality is "based in an attempt to de-legitimise the concept of architectural autonomy that has, for the past thirty years, provided the possibility for the critical model" ${ }^{42}$ Consequently, their position requires the assumption that critical architecture is defined solely by a conflation of the original formal/cultural tension set forth by Hays. A number of post-critical critics have noted that this conflation is motivated by a two-fold bias: a lack of (genuine) interest in social critique; and a drive to reject the influence of the aforementioned critical 'father' figures of Eisenman, Hejduk, et al. ${ }^{43}$ According to Barber:

Somol and Whiting's claim for a new social engagement is on the terms of the potential for 'qualities of sensibility' to impact culture in new, non-oppositional ways... It is left unclear in the 'Doppler Effect' article why these qualities of sensibility are generative of the new, and why they have a positive social function [emphasis added]. ${ }^{44}$

For Barber, the vagueness of the post-critical's social intentions is a consequence of their agenda to place design and innovation as openly, and unashamedly, a priority for the discipline of architecture. As such, the positions elucidated by the Somol and Whiting clearly indicate a wish to construct a different kind of disciplinarity. In so doing they have avoided the more prescient opportunity, that of 'destabilising the relationship' between architecture and its external cultural contexts. ${ }^{45}$

By conflating the terms of critical architecture put forth by Hays, the post-critical erect a theoretical 'straw figure', only to knock it down,
41. Barber, 2007, p. 246 Daniel Barber is Assistant Professor of Architectural History University of Pennsylvania.

42. Ibid, p. 246

43. This impulse has been described as 'oedipal' by critics such as Reinhold Martin, Mark Dorrian, and Jane Rendell. These authors identify that, given the educational proximity of the major post-critical authors to the avant-garde prior (Somol and Whiting were faught by Eisenman) the postFreudian 'Oedipus Complex'. This moniker also refers to the influence of Gilles Deleuze and Felix Guattari's 1972 treatise 'Anti-Oedipus'.

44. Barber, 2007, p. 247 
leaving an ideological vacuum in their wake. As such, efforts by prominent figures to positively express a position have failed to deliver a coherent, affirmative 46. Baird, 2004 intellectual project. ${ }^{46}$

In his essay 'Criticality and its Discontents', George Baird notes that Somol and Whiting attempt to provide easily-digested adjectives - such as "cool", "easy", and "relaxed" - for a new projective design direction, which are only effective insofar as they are an effort to anaesthetise terms associated 47. Somol \& Whiting, 2002, with the critical. ${ }^{47}$ This, in Baird's opinion, begs the question: by what standards, other than a pandering to existing societal, economic, and cultural norms, can the post-critical project, and subsequent crisis 48. Martin, 2010) of criticality, be judged ${ }^{248}$ To present a practice that affirms performance and effect does not express a questioning of the limits within which they operate; neither does the post-critical argument elucidate a strategy to assess its concomitant effects. ${ }^{49}$ Therefore, aside from an omission of the autonomy of Hays (through Eisenman), Somol and Whiting essentially present a resistance to resistance, in much the same way that Speaks's argument requests a submission to the forces of technology and globalisation. This engages the post-critical in a reflexive and essentially directionless position, one that can be manipulated to service the agendas of designers indifferent towards architecture's critical responsibilities.

Reinhold Martin, Professor of Architecture, Planning and Preservation, at Colombia University, further affirms these concerns. In his essay "Critical of What? Toward a Utopian Realism" Martin highlights that the general characteristics of postcritical architectural practices as, "affect-driven, nonoppositional, nonresistant, nondissenting", contribute to the transformation of architecture's potential cultural critical faculties into purely aesthetic, technical ones. ${ }^{50}$ For Barber, Martin and Baird, this

50. Martin R., 2010, p. 348 design-based pragmatism would be less concerning if it did not have wide-ranging implications within the 51. Jane Rendell also built environment..$^{51}$

Implications of a Post-Critical Ideology:

The Technical Perspective and a Loss of Context

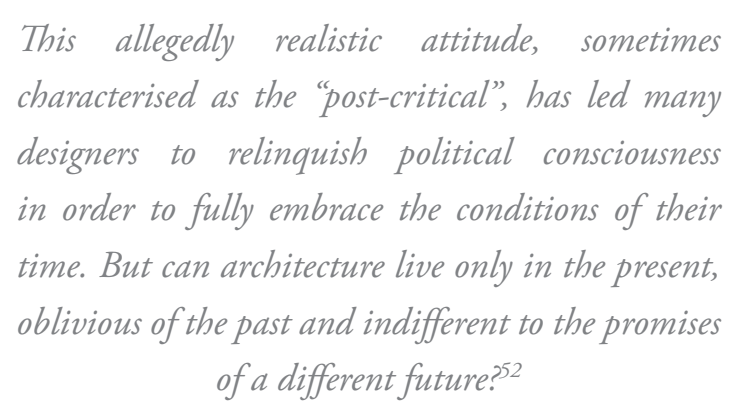

Antoine Picon

Whilst the post-critical may have done architecture a service by wresting it away from the theoretical posturing of the 1990s, the climate of consent and 'perilous' anti-intellectualism that has emerged since is genuinely problematic.

Representative of this shift, the works of highly prominent architects such as Rem Koolhaas, Frank Gehry and Zaha Hadid have aligned with the ethically suspicious ideology set forth by Speaks, Somol and Whiting. Given his recent declaration that design processes should be rooted in 'vigour and skill', Rem Koolhaas has emerged as a positive role model in favour of the post-critical. ${ }^{53}$ Rather than engage with local and geographic issues of context - factors which can complicate and confuse the design process - Koolhaas reinforces the oft-used post-critical analogy of the surfer who rides the 'waves' and 'flows' of globalized capital:

A "new pragmatism", in the face of the overwhelming complexity of undecidable outcomes, informs the choice of ride. The surfer, far from being overwhelmed by the inexorable force of events, instead is skilful enough to ride the wave, to use its massive energy and power to enable his or her own (spectacular) performance. ${ }^{54}$

Analogies such as this call into question a number of worrying connotations. For Graham Owen, the surfer/wave comparison suggests that the role of technology and globalisation is at once a 'force of nature' and simultaneously only able to be negotiated
52. Picon, 2010, p. 14 Antoine Picon is Professor of the History of Architecture and Technology at the Harvard Graduate School of Design.

53. Owen, 2009, p. 1 


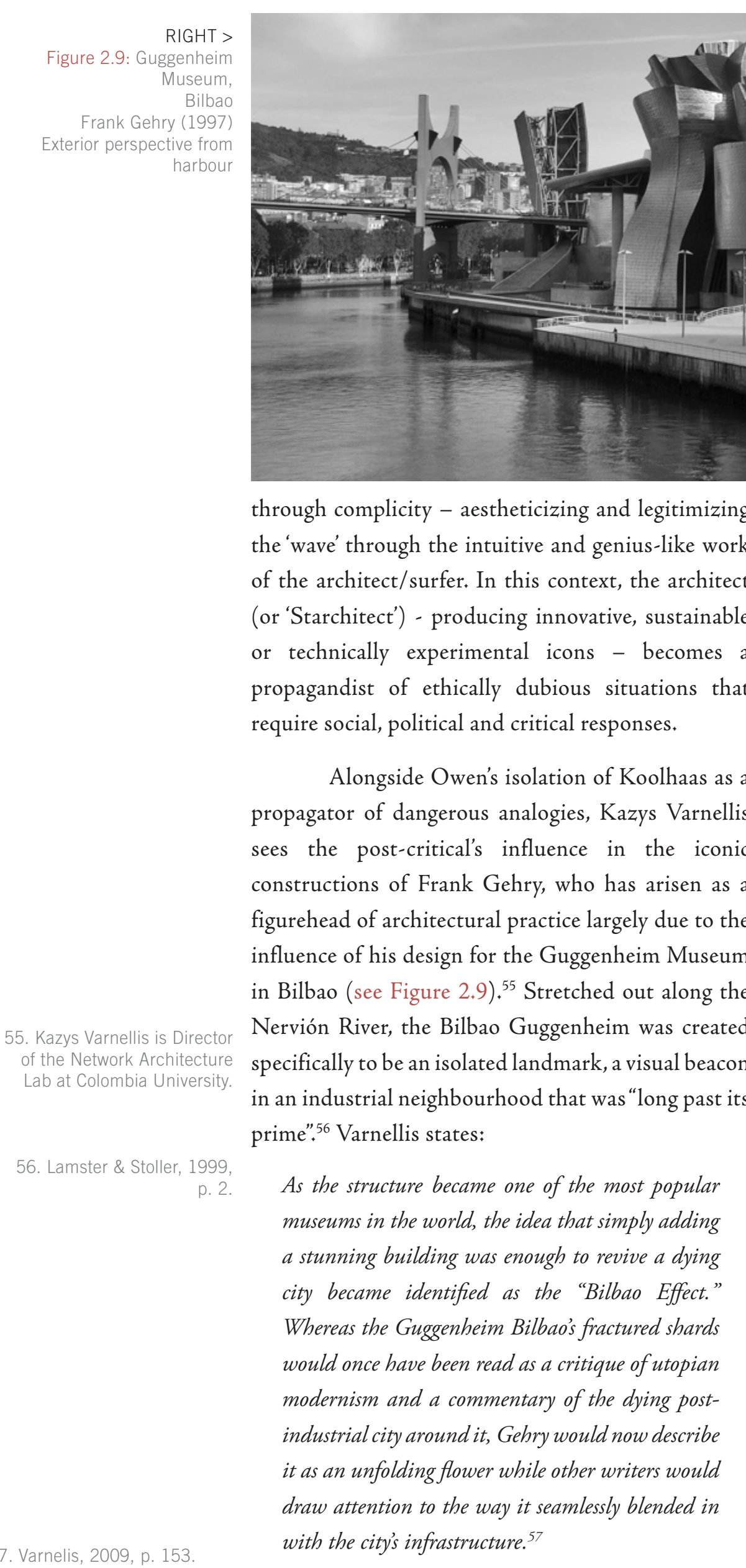

In light of the "Bilbao Effect" esteemed
Western architects have frequently engaged in the construction of 'iconic' structures - large buildings, complexes or even small cities for politically and geographically disparate clients (some of whom may widely be regarded as oppressive or undemocratic). ${ }^{58}$ These impressive technical achievements - although truly novel - are often designed with a disregard to their specific cultural contexts and are therefore divorced from the social implications of their construction. This perspective reinforces the maxim that first and foremost the architect must "get the job" with the only responsibility being to produce buildings with skill and rigour., ${ }^{59}$

Affirming Varnelis's concerns, Antoine Picon contends that these contextually and critically

[I]nnovative technologies must be
critically applied rather than blindly
assimilated as a driving force for
architectural production

indifferent designs that have emerged in the wake of the 'Bilbao Effect' can be seen as directly related to ideologies based in technology innovation. Although recent technological innovations have provided architects with a number of tools that positively advance the ways in which architects design and construct the built environment, these tools "should not however lead to the pitfall of narrow technological determinism" which is infrequently the only explanation "especially in architecture where so
58. Zimmerman, 2009, p. 1. . 2009, p. 1

59. Benedikt, 2009, p. 105 Owen, 2009, p. 1. 
CLOCKWISE FROM TOP >

Figure 2.10: Galaxy SOHO

Beijing

Zaha Hadid Architects (2012)

Interior perspective showing

the 'fluid' and nature-inspired

spaces created by the
architects Exterior perspective from
adjacent 'hutongs'
(2012)

Figure 2.11: Galaxy SOHO,

Zaho Heljing

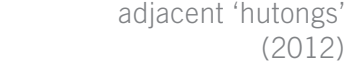

Figure 2.12: Galaxy SOHO, Beijing Exterior Hadid Architects adjacent 'hutongs' (2012)
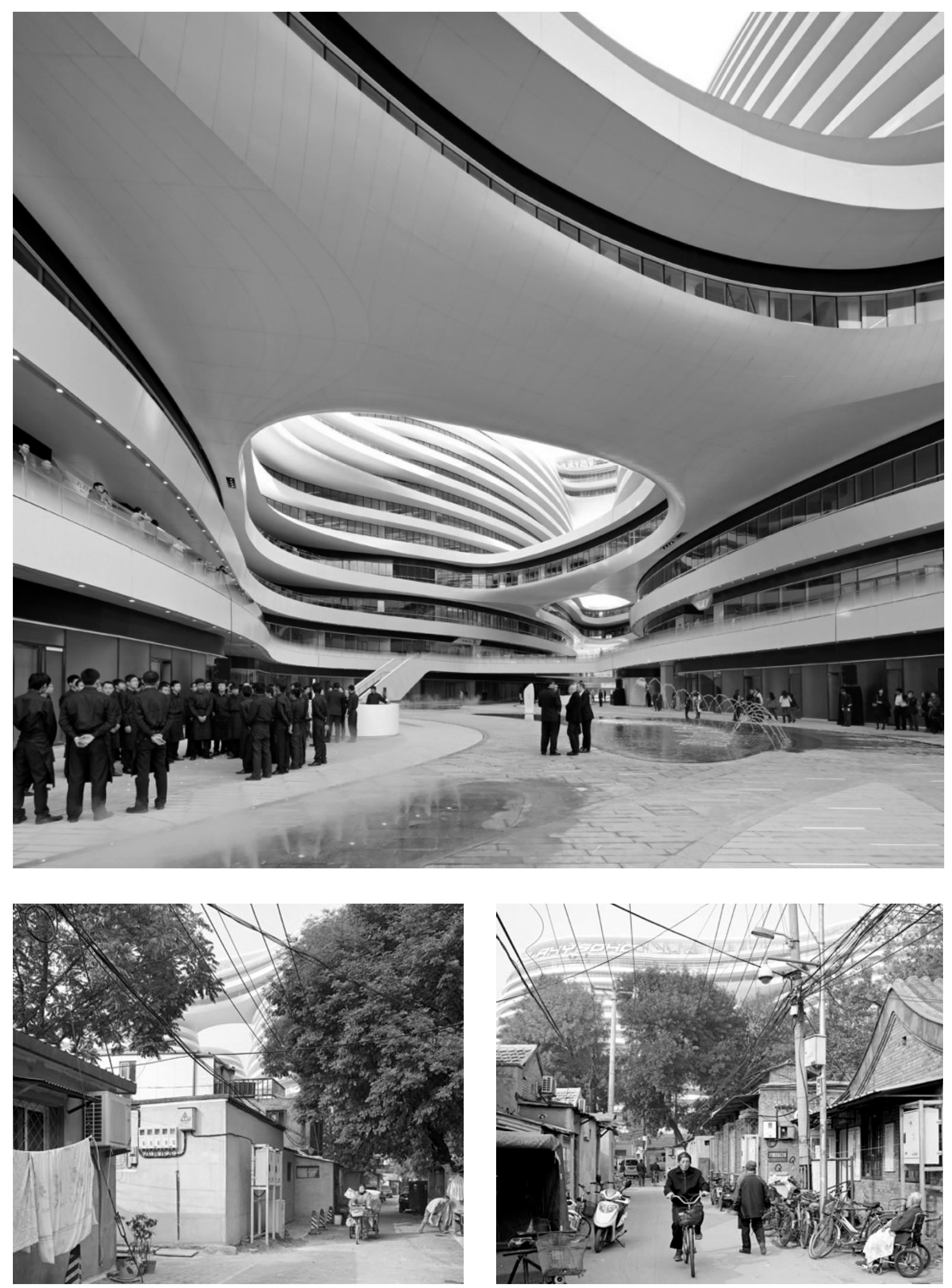


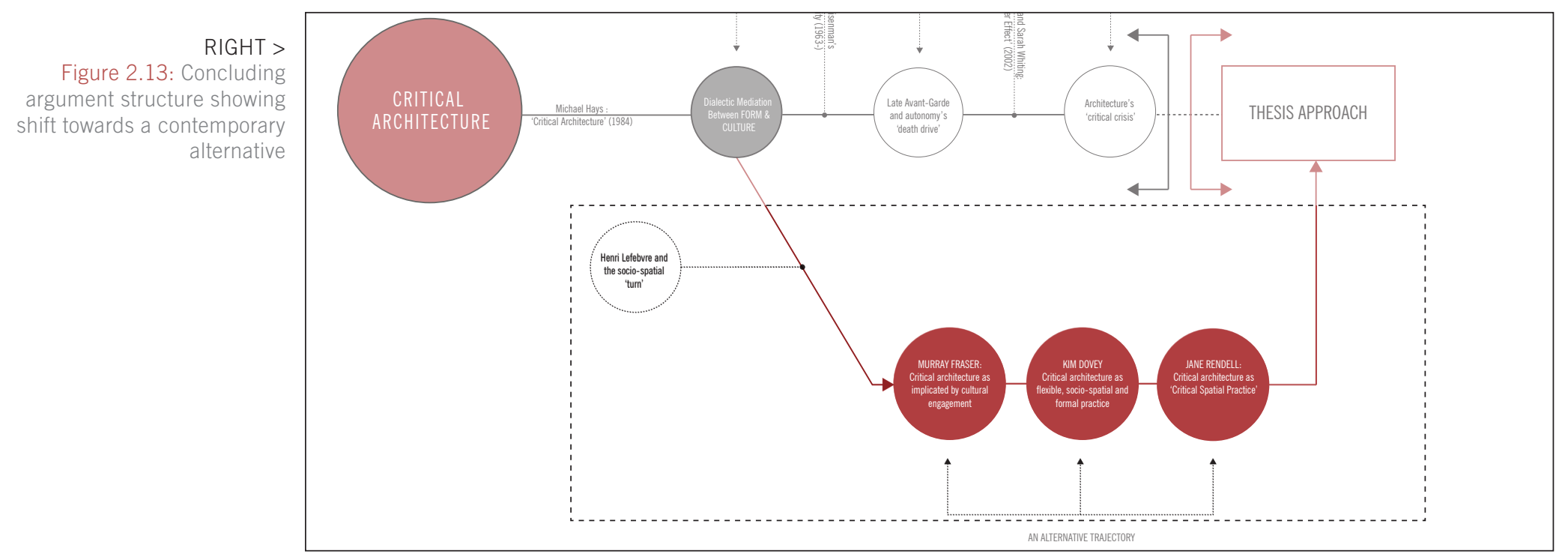

much depends upon economic, social and cultural factors" ${ }^{60}$ Rather, innovative technologies must be critically applied rather than blindly assimilated as a driving force for architectural production. David Brain's perspective is also useful, illuminating several problems that emerge from singularly technologybased perspectives:

First, even the best technical knowledge can produce very well-supported decisions that add up to normatively disastrous outcomes. Second, in the context of planning and design, the technocratic approach narrows the debate in a way that causes many issues simply to disappear from consideration. Third, it undermines our capacity for a productive politics by displacing responsibility for political choice to infinitely contestable claims about the nature of things. Ultimately, the problem with the effort to use technocratic expertise to avoid the messiness of politics is that politics comes back to bite us-typically in its most uncivilized and ultimately undemocratic form, ${ }^{61}$

61. (Brain, 2005, p. 233) University of California, Berkley
An aversion to the 'messiness of politics' and issues of cultural context is evident in the Galaxy SOHO retail and entertainment complex in Beijing, a recently (2012) completed project by Zaha Hadid Architects (see Figure 2.10). The technically impressive $330,000 \mathrm{~m}^{2}$ commercial hub disregards contextual specificity or integration, and refuses to engage with its impact on the social and political history of the region. Instead, Hadid's design intentions operate in a language of detached post-critical jargon, claiming to create movement between spaces that are "inspired by nature". Resulting from her design, the surrounding ancient passages and neighbourhoods or 'hutongs', have become marginalised, fractured and are subsequently in decline (see Figure 2.11 and 2.12). Whilst the decline of Beijing's 'hutongs' is a complex issue that cannot be attributed to architectural intervention alone, the Galaxy SOHO facility is symptomatic of an ideology that positions itself outside of implications that are not internal to the new, pragmatic disciplinarity.

The current ideological climate, fostered by the misguided motivations of the post-critical, has had significant and detrimental effects on the production and design of the built environment. This climate, influenced by the pragmatism and innovative practices of influential architects, has disseminated a further disjunction between critical architecture's imperative to mediate between cultural context(s) and formal autonomy. In light of this, a number of contemporary architectural scholars and practitioners have expressed a desire to re-engage critical architecture's potential by suggesting an alternative intention, one that is engaged with multiple cultural contexts (polycontextual). In so doing, architectural processes can 'open up' spaces for design responses that are "critical, questioning and self-formative in character".62

62. (Taylor and Levine, 2011 p.187) 
Architecture's New Desire: Mediation through Critical Spatial Practices

63. Fraser, 2006, p. 320

64. It is worth noting that these academics all operate outside of the 'dominant' institutions of architectural discourse, namely American Schools such as arvard, Yale and the Cooper Union. As such, their position is innately alternative and removed of bias.

65. Fraser, 2006, p. 317

66. See: Tafuri, Toward a Critique of Architectural Ideology (2000), 1969, and

Tafuri, Architecture and Utopia: Design and Capitalis Development, 1976
The crucial thing, however, is to see these architectural and spatial issues in relation to their specific cultural context, operating simultaneously on global and local levels. ${ }^{63}$

Murray Fraser

While an agreement can be made on the need for architecture to move away from the restricting practices of absolute autonomy, the reactionary attempts of the post-critical to submit criticality to a 'crisis' has done nothing more than prove distracting. As a result, a number of architectural theorists, practitioners, educators have emerged - presenting alternative, critical, approaches to architectural design that are nonetheless built upon Hays's original dialectic. This thesis argues that three contemporary figures in particular - Murray Fraser, Kim Dovey, and Jane Rendell - have outlined a new trajectory for critical architectural practices. These figures express a compatible desire to productively and positively 'open' territory for engagement with the contextual complexities of architectural design, concluding the argument for a contemporary critical practice. ${ }^{64}$

For a moment it is useful to briefly return to the conditions of Hays's proposition from "Critical Architecture...". Responding to Hay's conclusions, particularly with regards to the definition of instrumentality, Murray Fraser has noted that the situating of architecture's possible cultural engagement is heavily influenced by the positions of architectural historian Manfredo Tafuri. ${ }^{65}$ Tafuri, whose historio-political critique of architecture reached prominence immediately prior to the time of Hays's assertions, expresses a position on history that is deeply subjective. Tafuri's critique informs Hays's proposition that the "deterministic instrumentality" of architecture's cultural dimension(s) is directly linked to the temporality of a materialist view of history. ${ }^{66}$ For Fraser, Hay's adherence to this position conveniently negates architecture's critical engagement with culture in a social capacity. Instead Hays ties cultural-architectural production to a pejorative position, rather than maintaining a contextual tension between form/culture. ${ }^{67}$

Whilst Hays's disregard for architecture's cultural engagement can be traced to its death drive, Fraser identifies a second trajectory - a socially orientated criticality that is indebted to (predominantly) Continental cultural studies throughout the 1970s, 80s and 90s. Broadly speaking, a socially inflected criticality builds upon the notion of mediation "between authoritarian power, or capitalist power, and some sort of humanistic aspiration" ${ }^{68}$ Therefore architectural discourse requires a constant negotiation with cultural context, rather than positioning itself 'outside' of culture and critiquing from a detached position:

\section{... it [critical practice] is itself in fact entirely produced and subsumed into cultural practices. So this is why the study of differences between cultural contexts becomes the primary intellectual task... cultural identity offers the location of what might be considered as critical practice, not the other way around. Playing on these issues becomes the way forward in an age of increasing globalisation. ${ }^{69}$}

Additionally, Fraser suggests that the fetishisation of design by the post-critical is another form of "intellectual isolationism" that favours avoidance of culturally engaged positions as concretely as pure autonomy has/does. It is the contradictions and differences that arise from a dialectical understanding of architecture with culture that can inform "different tactics in different situations, whether in terms of their social, economic, or political conditions" [emphasis added]..$^{70}$

Expanding upon Fraser's positioning of critical architectural practice relative to cultural context, Professor of Architecture and Urban Design at the University of Melbourne, Kim Dovey, suggests that a recovery of the formal/cultural dialectic is contingent upon the investigations conducted during the 'spatial turn' in cultural studies - specifically the work of urban theorist and sociologist Henri Lefebvre. ${ }^{71}$ According to Dovey:
71. The 'spatial turn' marks a crucial period of time - the 
1970s - in the development of cultural studies, during which a multitude of scholars began to approach the notion of space from alternative, humanorientated, perspectives. For the implications of this moment, discussed in length, see Warf \& Arias, 2009

72. Dovey, 2006, p. 259
A critical architecture is an unsettling practice, and it may be unsettling for both the architect and the various communities of interest. A critical architecture will destabilise the field of architecture, its boundaries, identity formations and reproductive practices. ${ }^{72}$

Thus, like Hays, Dovey argues that a critical architectural practice is resistant, destabilising and subversive. However, the application of critical architecture can be re-directed, operating through an interdisciplinary perspective related to formal representations and spatial practices. These dimensions purposefully re-define Hays's dialectic through the fundamental redefinition of 'space' undertaken by Henri Lefebvre in his seminal work The Production of Space, "an architecture theory properly understood as

\section{This position expresses a certain positivity, asking that "critical architecture must at least plant seeds of desire for a better future", thus contending that the desires of critical architecture must project forward.}

a mediation between architecture, broadly conceived, and social practice". ${ }^{73}$

Importantly, the influence of the The Production of Space within architectural theory has only emerged recently. This is largely attributed to its delayed translation - not published in English until 1991 - providing some explanation as to why its primary thesis was not considered within the definition of critical architecture until after autonomy had

74. Gottdiener, 1997, 121. Hays, in spite of his adherence to architectura autonomy, recognises that Lefebvre's social critique "has not been fully developed in architecture theory". See: Hays, Architecture Theory Since 1968, 2000, p. 175 hijacked its definition. ${ }^{74}$ Lefebvre saw the normative approach to space as a simple reproduction of the dominant ideas of the dominant class, separating the mind from the inhabitation of public life, asserting that the 'science of space' creates modes of operation that must be critically attacked as forms of dominant value reproduction. Consequently, he argues for a unitary redefinition of space that is non-Cartesian, non-absolute, and therefore non-deterministic.
This new proposition of space encompasses all forms of social experience as constituted within and through it. ${ }^{75}$ As such, space is not only "part of the forces and means of production; it is also a product of these very same relations", a property that makes spatial design different from any other form of social production or commodity. ${ }^{76}$ Lefebvre achieves his 'socio-spatial' redefinition by identifying three modes of production which, when placed in a state of negotiation, establish boundaries for investigation, also known as a 'practised-conceived-lived' triad of: 'representations of space,'spatial practices' and 'spaces of representation.77 Accepting Lefebvre's hypothesis, the realities of architecture are implicated with the interactions of "everyday social life", achieving the flexibility and pragmatism requested of architectura theory by the post-critical, and denied by its rigid autonomy. ${ }^{78}$

Transmitted through this triad, Dovey's first contribution to a culturally contextual critical practice, formal representations, combines Lefebvre's 'representations of space' and 'spaces of representation' into a singular term that concerns the ways in which "built form constructs social meaning as a form of discourse or text" ${ }^{79}$ As such, critical architecture is resistant to submissive identity reproduction (through an examination of social, political, economic, and historic contexts) and accepts a responsibility for the production of identities through architecture.

Dovey's second contribution, spatial practices, concerns architecture's "actions and events through its spatial programmes" ${ }^{80}$ Spatial practices approach questions of identity and subjectivity through a focus on the actions of everyday life, and how these actions are shaped and translated through architecture. Significantly, Dovey identifies the necessity for an emphasis on spatial practices as a means to critically illuminate the structure of social space: the use of borders, thresholds and boundaries to control social interaction, and the impact of standardised spatial fields and building types on the individual. ${ }^{81}$

By manipulating his intentions through the socio-spatial turn in cultural theory, particularly Lefebvre's triad, Dovey expresses the same project for architectural discourse as Fraser, to 'open' and 'unsettle'
75. Lefebvre, 1991, pp. 9-10

76. Gottdiener, 1997, p. 124

77. This triad is used

throughout the thesis as the basic proposition for a social critique of space, and thus the intervention of architecture within space.

78. Dovey, 2008, p. 52 
its critical rigidity. This position expresses a certain positivity, demanding that "critical architecture must at least plant seeds of desire for a better future", thus contending that the desires of critical architecture must project forward - not with the limited disciplinarity and pragmatism of the post-critical but within the framing of everyday cultural life and context. For Dovey (and Fraser), critical architecture must embody a constantly reflective and projective intention to resist, allowing the design process to express a freedom that is nonetheless grounded in an understanding of the cultural contexts that organise it.

Aligning with Dovey, Jane Rendell has suggested an architectural framework that challenges architecture to re-engage its critical faculties in a spatial, social and temporal manner. Identifying contemporary critical theory as necessarily "fragmentary and partial", requiring a self-reflexive and constant process of relay between practices that are both 'critical' and 'spatial' - namely critical spatial practices. $^{82}$

82. Rendell, 2008, p. 7 . As with Dovey, Rendell also recognises the importance of Lefebvre's 'spatial practices' within her defin

In Art and Architecture: A Place Between Rendell situates such practices within three interdisciplinary processes of practical application: 'Between Here and There' (site-specific), 'Between One and Another' (socio-spatial), and 'Between Now and Then' (temporal). Each section contributes towards a unitary process that aims to "negotiate the relationship that theory has to practice and vice versa". ${ }^{83}$ These three categories form the crux of the design-research approach of this thesis - building on Lefebvre through Fraser, Dovey and Rendell to create a contemporary position for critical architecture. In so doing, a structure is presented through which this thesis can examine themes and analyse spatial case studies for design-research.

Significantly, critical spatial practices does not present a specific method for architectural design. Rather, aligning with the motivations and research questions of this thesis, Rendell's work offers a vital and practical framework to guide critical design investigations. This thesis contends that a re-reading of the critical spatial, social and temporal 'themes' from within Rendell's framework presents an opportunity for the construction of a unique and critical design methodology relative to the parameters of a specific set of contexts. As these themes that form the foundation of Rendell's work are expanded upon and explored in detail in Section 2, as a theoretical mediation to the development of the design-research.

\section{Conclusion}

In this chapter an outline for the theoretical argument and approach for design-research has been presented. The work of K. Michael Hays has proven pertinent in this regard - that in order for a work of architecture to embody a critical intention it must be respondent and resonant to formal and cultural concerns.

Arguing that under the dominance of absolute autonomy, Chapter Two has shown that critical architecture has followed a trajectory that has resulted in a discursive conflation - resulting in the cultural dimensions of critical practices becoming disregarded and marginalised by many. Additionally, in an attempt to avert the influence of the late avantgarde, the post-critical impasse has exacerbated this conflation by suggesting an opposition to the notion of critical architectural production. Problematically, the post-critical position has been shown to foster an ideological climate that is disengaged from architecture's capacity to resist contexts of architectural intervention that foster socially, spatially and culturally negative outcomes.

Exposing the inadequacy of the post-critical position on several fronts, an alternative through Murray Fraser, Kim Dovey and Jane Rendell has been presented. These figures consider critical architectural design to require a particular flexibility - an intent to 'open' design processes to a critique and resistance of cultural forces, with the desire to provide positive spatial solutions. This approach considers critical architecture through a new trajectory, one that presents Hays's dialectic through Henri Lefebvre's reconfiguration of the notion of space - challenging architectural design to operate within a framework that is both critical and spatial. 
Upon isolating this position, the details and propositions of a critical spatial practice is expanded upon in Section Two of this thesis. Rather than elaborating on this theoretical framework, this thesis acknowledges that one of the imperative conditions of a flexible critical architecture is to positively engage 'real world' concerns. As such, critical spatial practices are examined in further detail throughout Chapter's Five, Six and Seven - explored as designresearch through its application to a contemporary and practical problem. This problem is now to be examined in Chapter Three. 
atros $=1$

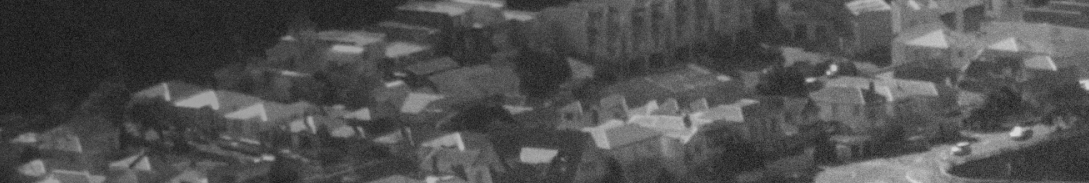

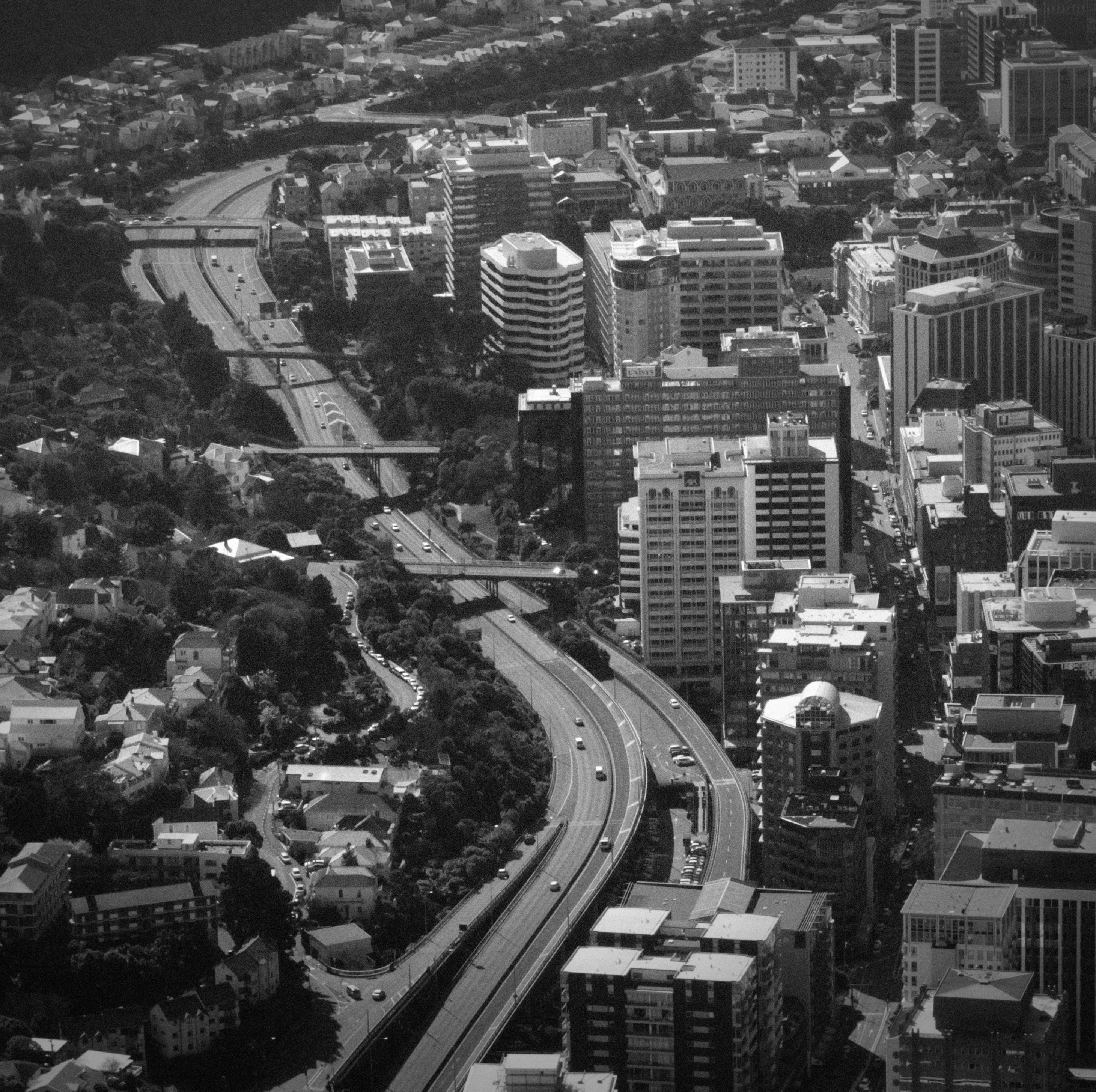




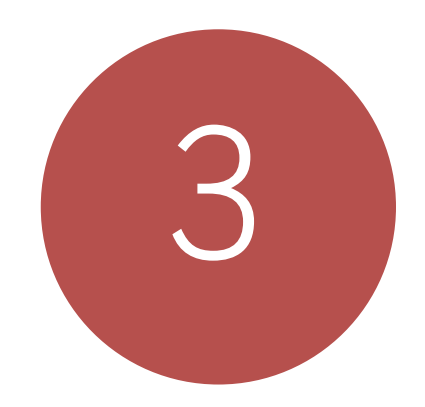

\section{NEGOTIATING THE THICK EDGE:}

A (Real) Design Problem and Approach

Introduction

A Sprawling Problem: Modernism's Leftovers

Where Grids Get Cut: Tangible Implications at the Border

A Localised Problem: Three Examples of a Negative Thick edge

A New Form of Flow: Opportunities for Contextual Positivity

Conclusion: A Polycontextual Approach to the Clifton Street Car Park 


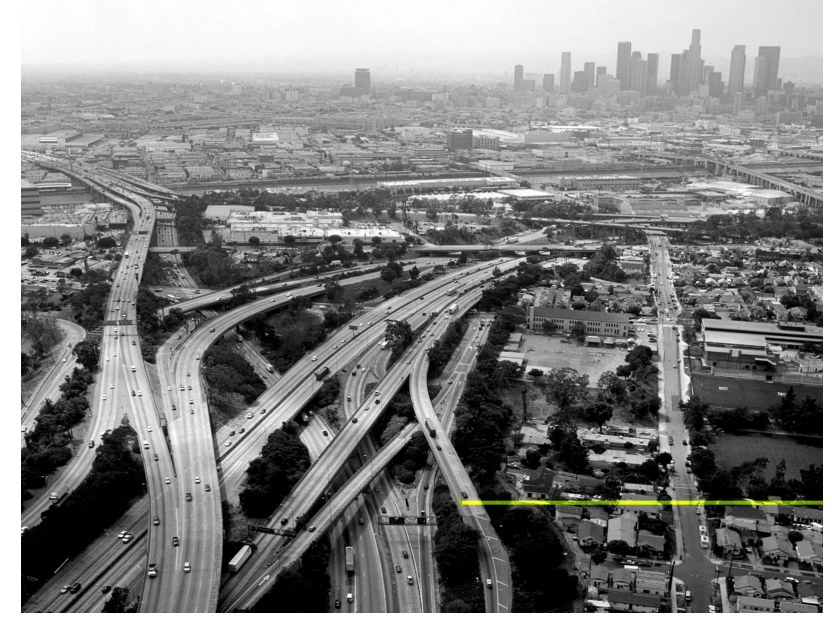

\section{Introduction}

Almost without notice, urbanisation and 'sprawl' have pushed the fabric of the urban centre to its fringe. ${ }^{1}$ The metropolis of the twentieth century has given way to the rapid contractions and expansions of sprawl at a dizzying pace, forcing cities to 'spill out' from their edges (see Figure 3.2). At these edges, sites of distinctly urban dimensions attempt to connect to their peripheries; a desire often fulfilled through the incisions of dense and overbearing highway infrastructures. Around and underneath these infrastructures, unwanted, undesirable, and under-utilised sites emerge as the spatial residues of modern urbanisations singular developmental drives.

A practical and contemporary problem is identified: distinctly negative in character, these sites are frequently 'thickened' by the implementation of pragmatic, singular-use programmes. Problematically, these thick edges divide and dislocate existing communities and cultural contexts - resulting in physically and figuratively rigid conditions that perform as barriers to social and spatial flows.

Pursuing the argument further by localising the problem, three sites operating as thick edges within Wellington are investigated: the Westpac Stadium Car Park; Ballantrae Car Park; and the Clifton Street Car Park. From this analysis, a number of re-occurring conditions are identified that contribute to the rigidity and problematic dimensions of thick edge sites.
This chapter argues that few architectural alternatives for thick edges have emerged beyond the superficial application of recreational landscaping and perfunctorily beautified infrastructures. Through Aaron Betsky and Alexander d'Hooghe, this chapter presents an approach that resists these commonly held assumptions, suggesting that thick edges can be transformed into places of theoretical and cultural potency. Challenging architectural intervention to engage the thick edge through multiple contexts, this chapter proposes that these rigid conditions can be positively inverted - producing flows between various communities, land-use zones and publics. In so doing, Chapter Four addresses the third research question of this thesis:

What are the negative outcomes attributed to the proliferation of urban thick edges, and how can architectural intervention provide positive alternatives and approaches to this contemporary problem? 


\section{A Sprawling Problem: Modernism's Negative Leftovers}

Following the Second World War 'three glorious decades' of growth under the drives of globalised modernism, technological development, and economic prosperity, informed a period of unprecedented urban sprawl and urbanisation, of which our cities are still facing the effects. ${ }^{2}$ In this

2. Bauman, 2000 , p. 16 Wellington is no exception; growing city - the Wellingto City Council predicts that in 2040 55,000 more people will be living in central Wellington, containing $68 \%$ of al Wellington jobs. See: Counci,

3. Frampton, 1983, p. 21 Kenneth Frampton is Ware Professor of Architecture at Graduate School of Architecture, Plannin and Preservation Colombia University.

4. Pope, 2011, p. 143

5. Frampton, 1983 , p. 16 Erampton also notes that this process of development creates a shift in power away from the social realm and towards the institutional confines of economic capital. See: Solomon, 2004, p. 23. methods for the design and expansion of cities period, the influences of progress-driven commercial development and the ease of individual transport created by increased automobile production, encouraged cities to sprawl outwards - to urbanise Kenneth Frampton identifies that this phase of urbanisation in the developed world has radically transformed the fabric of metropolitan centres to such an extent that "with the exception of cities which were laid in place before the turn of the $\left[20^{\text {th }}\right]$ century, we are no longer able to maintain defined urban forms".

Influencing this shift, 'modern' planning emerged as dominant on a global scale - entailing concerned primarily with connecting the gridded order imposed on urban centres to their emerging suburbs through transport. ${ }^{4}$ Built upon promises that technology-based progress could improve the 'health and circulation' of urban environments, this influential urban 'solution' proliferated the "two symbiotic instruments of megalopolitan development - the freestanding high-rise and the serpentine freeway". The genesis of this perspective can be seen in the early models put forth by architects such as LeCorbusier (see Figure 3.3) and Ludwig Hilberseimer (see Figure 3.4). These schemes, which clearly privilege the circulation of the automobile and the scale of the high-rise over human sensibilities, have fundamentally influenced the core logic of urban development ever since (see Figure 3.2).

Richard Ingersoll argues that the intentions of modernism's perspective towards urbanisation are comparable to the 'jump-cut' technique in cinema - a violently fragmented montage that rapidly connects two disparate images. Thus 'jump-cut' urbanism informs a process whereby new highway aggressively 'cuts' between seemingly incongruent land-use zones
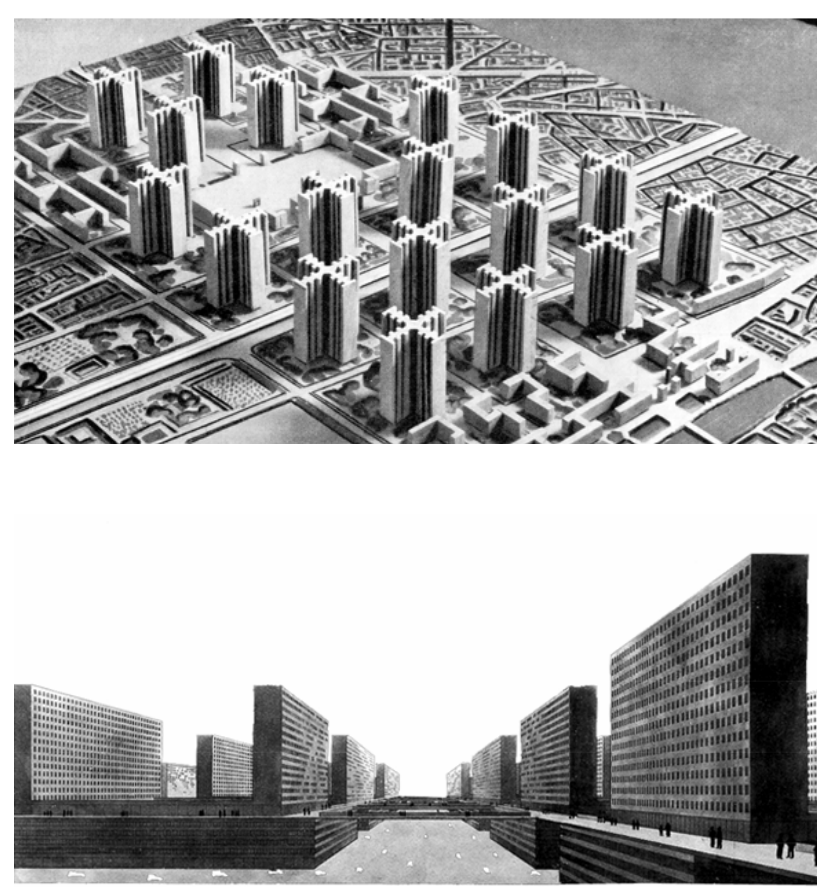

$<$ LEFT

Figure 3.4: 'Grossstadt'

Urban masterplan for Berlin Ludwig Hilberseimer (1927) Charcoal on paper

(industrial, residential, commercial), allowing sprawl to occur at increasingly rapid rates. ${ }^{6}$ Throughout the $20^{\text {th }}$ century, jump-cut urbanism was adopted with such rapacity that it informed the basic structure of what we now understand to be the current identity of the city. Resulting from this technique, urban development became primarily concerned with connecting the dense commercial centres of cities (the urban) to the sprawl of their ever-expanding peripheries (the regional, suburban), disregarding the conditions by which they would normally meet their borders (see Figure 3.5). ${ }^{7}$ These connections have been predicated on the rise and impacts of developments in infrastructure as rail and roading networks cut across, through and around such borders. ${ }^{8}$

In the The Social Logic of Space, spatial geographers Bill Hillier and Julienne Hanson

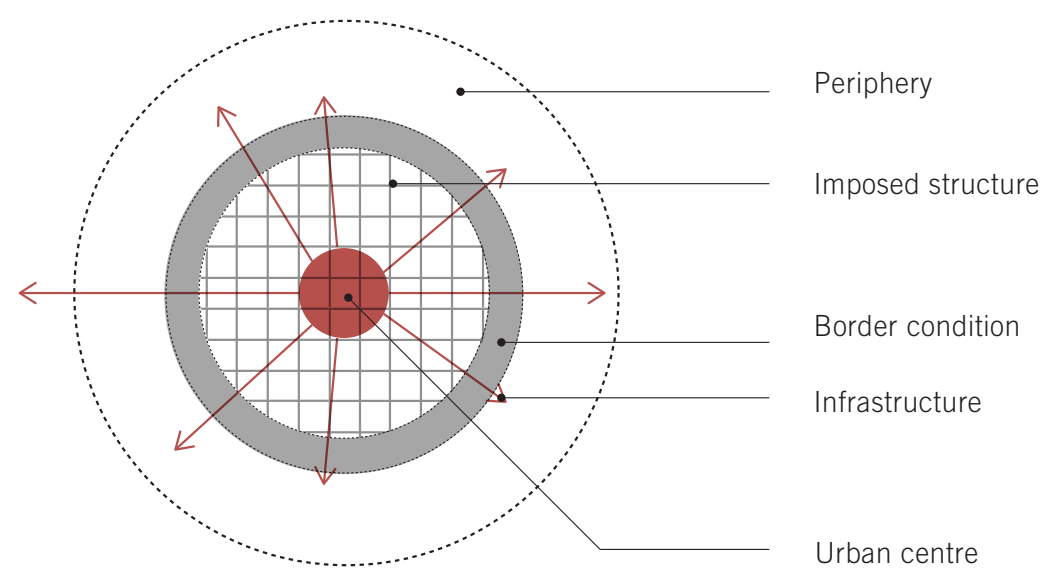

6. Ingersoll, 2006, p. 91. Richard Ingersoll is Professor of Architecture and Urbanism at the Syracuse University of Florence.

7. Solomon, 2004, p. 22

$<$ BELOW LEFT Figure 3.5 Author's diagram showing generalised relationship

between an urban centre and ts periphery as it connects

Urban centre 
8. Ibid. Jonathan Solomon is Associate Professor and Associate Dean at the Syracuse School of Architecture.

9. Hillier \& Hanson, 1984 Bill Hillier is Professor of Architectural and Urban University Colloge Landon; University College London; of House Form and Culture, and Director of Teaching at the Bartlett School of Graduate Studies, University College London. Conversely, a positive a engage local systems as they refer to the social relations within and around such a territory - between the cultural conditions and individuals as they perform within everyday outline that this form of urbanisation represents a fundamental position based upon what constitutes positive and negative spatial relations. For Hillier and Hanson, architectural engagement with urban space relies on mediated relations between global and local forces and interests. In this relationship, the spaces of global systems (in this case jump-cut urbanism) are a projection of a unified ideology and authority across a specific territory (that of the automobile). Consequently, spaces that are predominantly globalto-local manifest as singular in motivation, detached from the cultural complexities of the contexts associated with local culture. (in a capitalist system these motivations are generally economic and/or political.) The more that a global spatial structure acts to realise its aims (becomes spatialised), the more the local system is dominated by a system of ideologically defined formal structures, and social spatial inequality ensues. ${ }^{9}$ Following this observation, when operating as one such system, the globalised forces of jump-cut urbanisation have contributed significantly to the rise of negative spaces within the local built environments of their implementation.

\section{Where Grids Get Cut: Tangible \\ Implications at the Border}

Peripheral or 'edge' areas of cities and other leftover urban spaces have traditionally been considered unworthy of design attention. Such places are usually ugly and out of the way; they are also places where urban and architectural scales collide and raw social and economic divisions are manifest. ${ }^{10}$

Phoebe Crisman

In the context of jump-cut urbanisation, global-to-local systems of spatial negativity are particularly evident. In many cases they have manifested as the left-over spaces created by urbanisation - singularly motivated by the automobile - and used for purposes that are distinctly anti-social. These connecting spaces exist at the collision between structurally-defined land-use zones and are predominantly left disregarded, 'lost' or 'leftover' as the residues of capital interest. Crisman has suggested that urbanisation's residual spaces are complex socio-spatial zones, informing globally ubiquitous conditions yet fundamentally unique local relations which have been perceived as 'unworthy' of architectural focus. ${ }^{11}$ Professor Emeritus of City and Regional Planning at Cornell University, Roger Trancik, notes:

Lost spaces are the surface parking lots that ring the urban core of almost all American cities and sever the connection between the commercial center and residential areas. They are the no-man's-lands along the edges of freeways that nobody cares about maintaining, much less using ... They are the residual areas between districts and loosely composed commercial strips that emerge without anyone realising it. ${ }^{12}$

Peter Newman and Jeffrey Kenworthy agree, suggesting that these spaces are the outcomes of modernism's economic imperatives, produced when the processes of urbanisation are in 'full flight.' ${ }^{13}$ As such, these spaces are ill-defined, incoherent and make "no positive contribution to the surroundings or users", re-enforcing the conviction that they constitute an undesirable and unavoidable spatial 'negativity.' ${ }^{14}$

\section{Spaces that are predominantly global-to- local manifest as singular in motivation, detached from the cultural complexities of the contexts associated with local culture (in a capitalist system these motivations are generally economic and/ or political)}

Building upon this notion, Aaron Betsky identifies negative urban spaces as, "not pretty places or real places, only background noise that threaten to drown out the polite forms of a built polity" and arise from the inherent lack of control expressed by contemporary urban centres. ${ }^{15}$ For Betsky, cities are now developing fluidly creating a disjunction when architects, planners, and urbanists attempt to 'solve' these conditions by implanting a pre-designed order. These imposed ordering systems often take the form
14.Trancik, 2007, p. 64

15. Betsky, 1998, p. 465. Aaron Betsky is an architectural critic, curator, educator, lecturer, and director of the Cincinnati Art Museum 
of modernist-driven grids and masterplans or the linearity of infrastructure (see Figure 3.6). Lost and left-over spaces arise not in definable, controllable, limits but as certain characteristics and conditions:

It [negative urban space] is profoundly modern in a simple, stylistic sense: it is planar and not volumetric; it is based on a divorce between its context, girded by machined materials, and the artificial space it opens up; and it is extensive in its tendencies. Its planarity is predominantly horizontal and gridded, and dissolves at the edges [emphasis added]. ${ }^{16}$

16. Betsky, 1998, p. 465 .

Crisman argues that such conditions have perpetuated throughout the urban fabric and can be regularly identified in their most potent form in sites adjacent to transportation infrastructure:

\section{Even within these admittedly challenging} conditions, however, one kind of edge stands out for the design problems it poses: the zones created by linear cuts incised through the morphological continuity of the contemporary city by rail lines and highways. ${ }^{17}$

17. Ibid p. 115

These sites, prevalent throughout the contemporary city, are a prescient issue and must be investigated by architects if urbanisation is to impart positive social effects within the city.

Rem Koolhaas has suggested that these sites can be categorised as 'Junkspaces', the bi-products of a consumer-driven culture, the "body double of space, a territory of impaired vision, limited expectation, reduced earnestness". ${ }^{18}$ Aligning with the propositions of negative urban space put forth in this chapter, Koolhaas argues that junkspaces appear wherever capital interest is rampant, even suggesting "the entire highway system is junkspace." ${ }^{19}$ However, upon identifying the conditions of their 19. Ibid, p. 181 production, responses to these conditions are frequently problematic. Koolhaas, a figure-head of the post-critical's desire to go with capital flows, is dismissive- arguing that as junk they must be treated thus, disregarded and left to decay. This narrative is distinctly post-critical; it identifies the driving

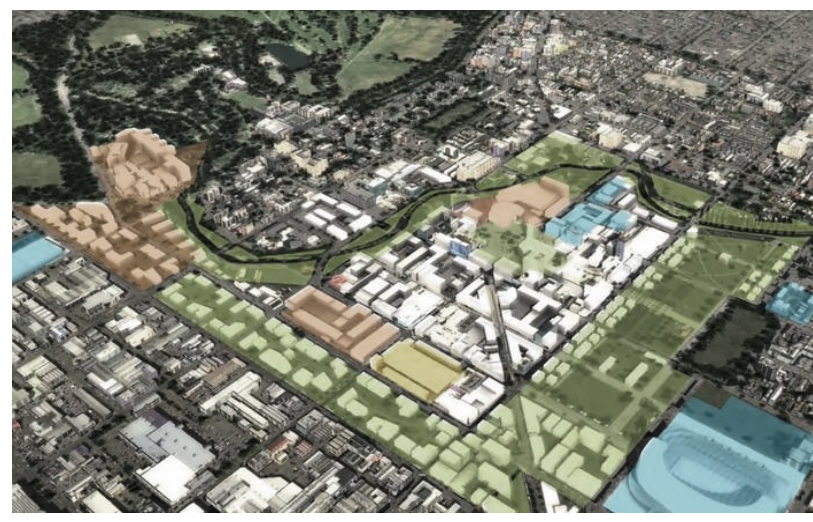

$<$ LEFT

Figure 3.6:

Aerial view of proposed master plan for Christchurch re-build following the earthquakes of 2011. The plan shows the continual desire of architects and urban planners to impose regular and structured order systems within the urban fabric.

force and locus of negative urbanisation and yet concedes defeat. Instead of resisting or opposing, this position perpetuates the problem, permitting capital appeasement and promoting the axiom that whatever does not work should be thrown away or cosmetically upgraded. ${ }^{20}$ Koolhaas's attitude is contagious and continual, facilitating the misuse and degradation of such spaces, treating them as 'junk' and cosmetically filling them with anti-social programmes. Thus, instead of utilising negative spaces, exploiting them for public or social uses, they are often 'filled in' with economically pragmatic programmes, "we make them into parking lots or feeble patches of grass - no man's lands between the scale of the region and the locality". ${ }^{21}$

Resulting from these singularly-motivated approaches, a binary rigidity - a 'thickness' - is formed, manifest as impermeable conditions that 'cut' or separate two spatial conditions (see Figure 3.7). This point is put forth by Iain Borden, who contends that thick edges present spatial and formal barriers, limits "between here and there in and out", impeding the right of passage and therefore restricting positive social and spatial flows. ${ }^{22}$ Treated as undesirable

000 , p. 222 ain Borden is Professor of Architecture and Urba Culture at the Bartlett School of Architecture

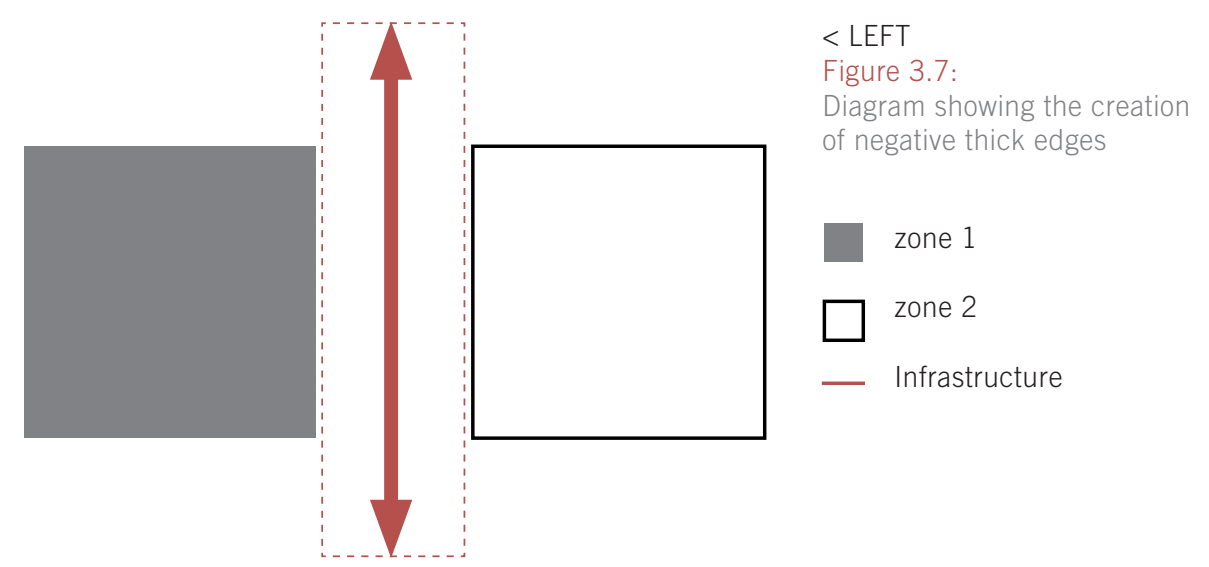




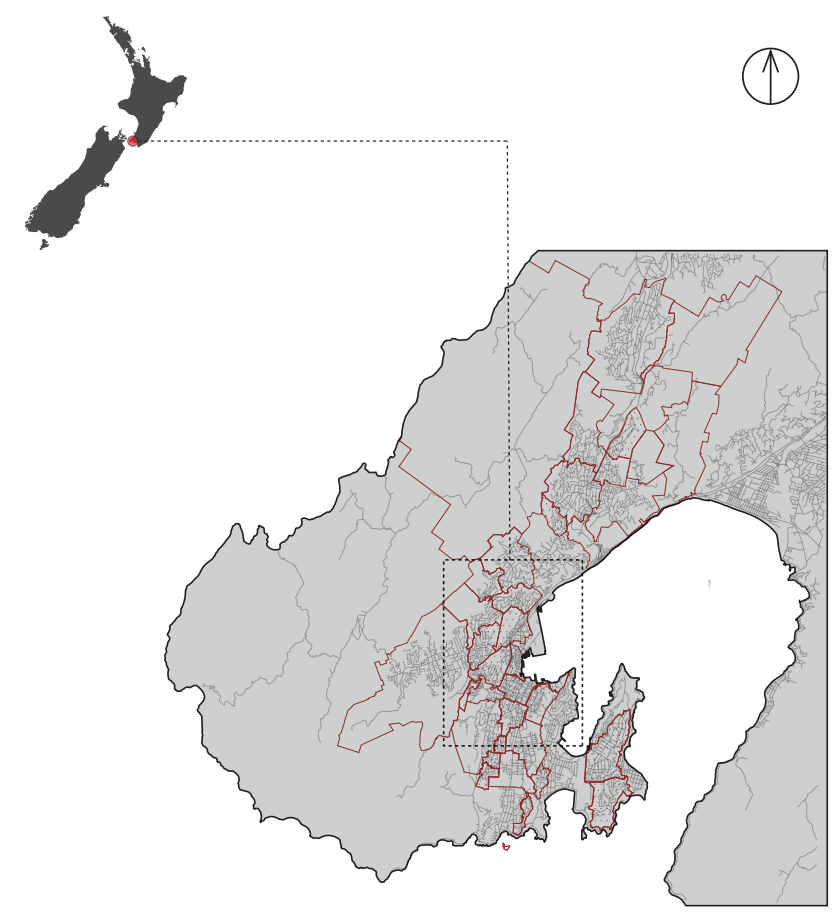

and impractical, and filled with functions that are indifferent to human use, the thick edge condition reproduces and reinforces the spatial negativity that constitutes it - in a vicious urban cycle.

\section{A Localised Problem: Three Examples of a Negative Thick Edge}

Based upon the understandings of jumpcut urbanism's consequential urban conditions, it is pertinent to examine how 'thick edges' have come to emerge in a localised context - specifically, Wellington (see Figure 3.8). Analysing the city's urban centre reveals three relevant patterns: firstly, the urban (centre) is bisected by dense infrastructure - motorways and rail - that connects the outer-lying suburbs to the city (see figu 3.9); secondly, the CBD is additionally bounded, geographically, to the west by a dense greenbelt; and thirdly, examining the figures of the built fabric (see Figure 3.10) these infrastructural passages have carved out significant borders between the formal structures of the high-density CBD and the low-density inner-suburbs. Following the junkspace approach, many of the leftover sites within these borders have been renovated into car-parks, creating impermeable barriers - thick edges. Three sites in particular have been identified: the Westpac
Stadium Car Park, 83 Waterloo Quay; Ballantrae Car Park, 1 Ballantrae Place; and the Clifton Street Car Park, 132 The Terrace (see Figure 3.11).

The Westpac stadium Car Park is situated underneath an expansive, elevated public walkway that leads to the city's largest stadium to the northeast of the urban centre. The site is somewhat of a city gateway, positioned between the industrial waterfront/harbour zone and the Pipitea commercial precinct (See Figure 3.12). Additionally, the car park is wedged between the infrastructure of the Wellington rail system and the Waterloo Quay road system. As a result, the facility 'fills in' a residual strip of space, deemed necessary for parking. Given its proximity to the adjacent sports stadium the need for parking space is evident. However, this should not eliminate the possibility of further developing the thick edge to engage contexts outside of its economics. The occupied space underneath has been positioned in such a way that it creates a vehicle-orientated zone at ground level, and thus presents an almost entirely anti-social space that resists the interactions of a variety of publics (see Figures 3.13-3.15).

Moving to the western periphery of Wellington's urban centre, the Ballantrae Car Park is more inconspicuous. Positioned at the intersection of three roads: the urban motorway (above); Bowen Street (adjacent); and accessed via Ballantrae Place, the site's location at the northernmost point of the

\section{Following the junkspace approach, many of the leftover sites within these borders have been renovated into car- parks, creating impermeable barriers - thick edges.}

Bolton Street Cemetery informs its condition as a leftover space - created by the roading infrastructure's negotiation with the natural topography (see Figure 3.16). Consequently, the parking space is submerged, informing a physically slumped condition between the greenbelt to the west and the sporadic high-rise buildings to the east. 


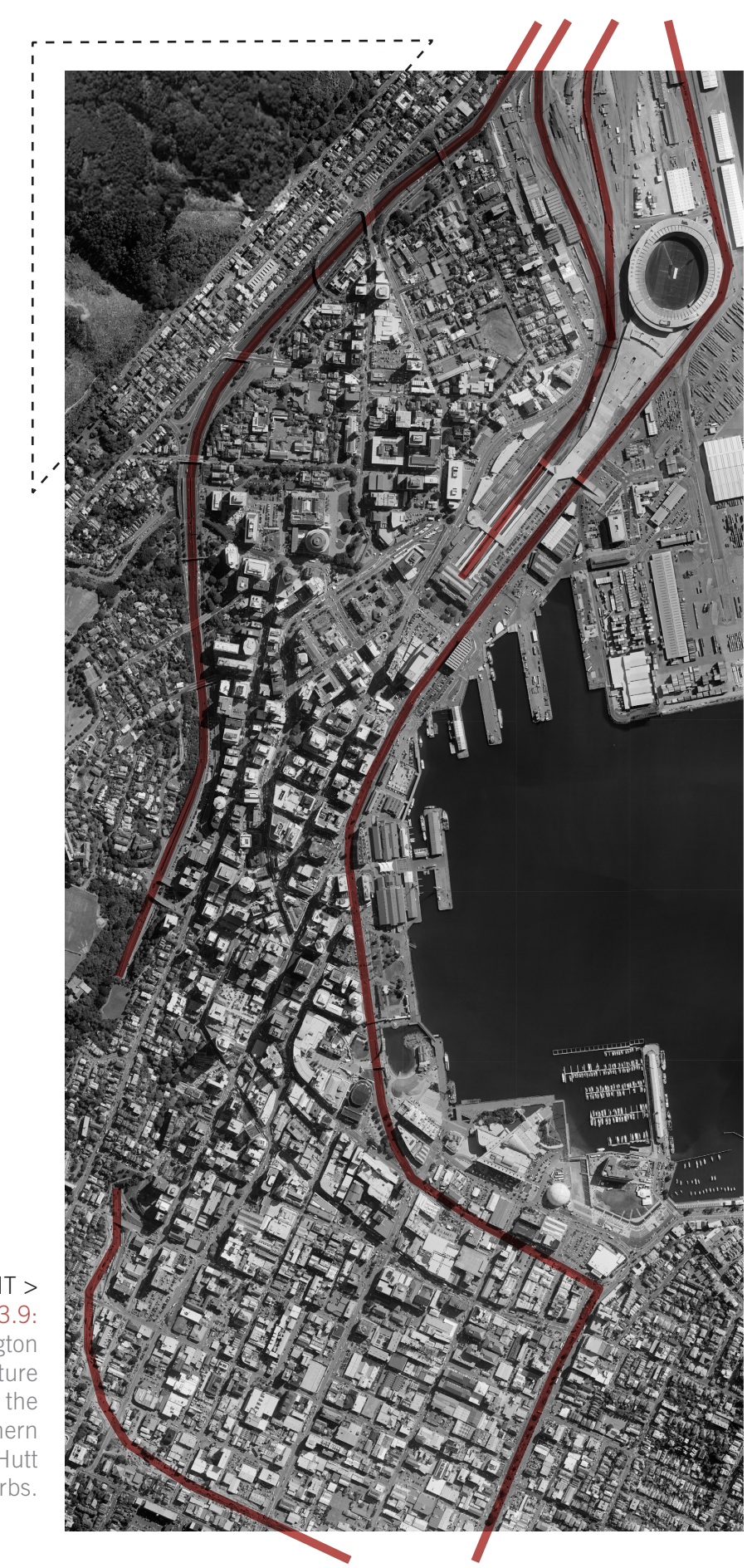

Interestingly, attempts have been made to reduce the orthogonal nature of the infrastructure's architecture by chamfering the columns into visually varied geometries (see Figures 3.17-19). This is an attempt to engage the visual rigidity of the thick edge, however, there are no architectural references that engage the materials, typologies or histories of the surrounding fabric - displaying a resistance to the site's context within the local built environment.

South of Ballantrae Place and located at the western edge of Wellington's downtown financial core, the Clifton Street Car Park is a council-owned

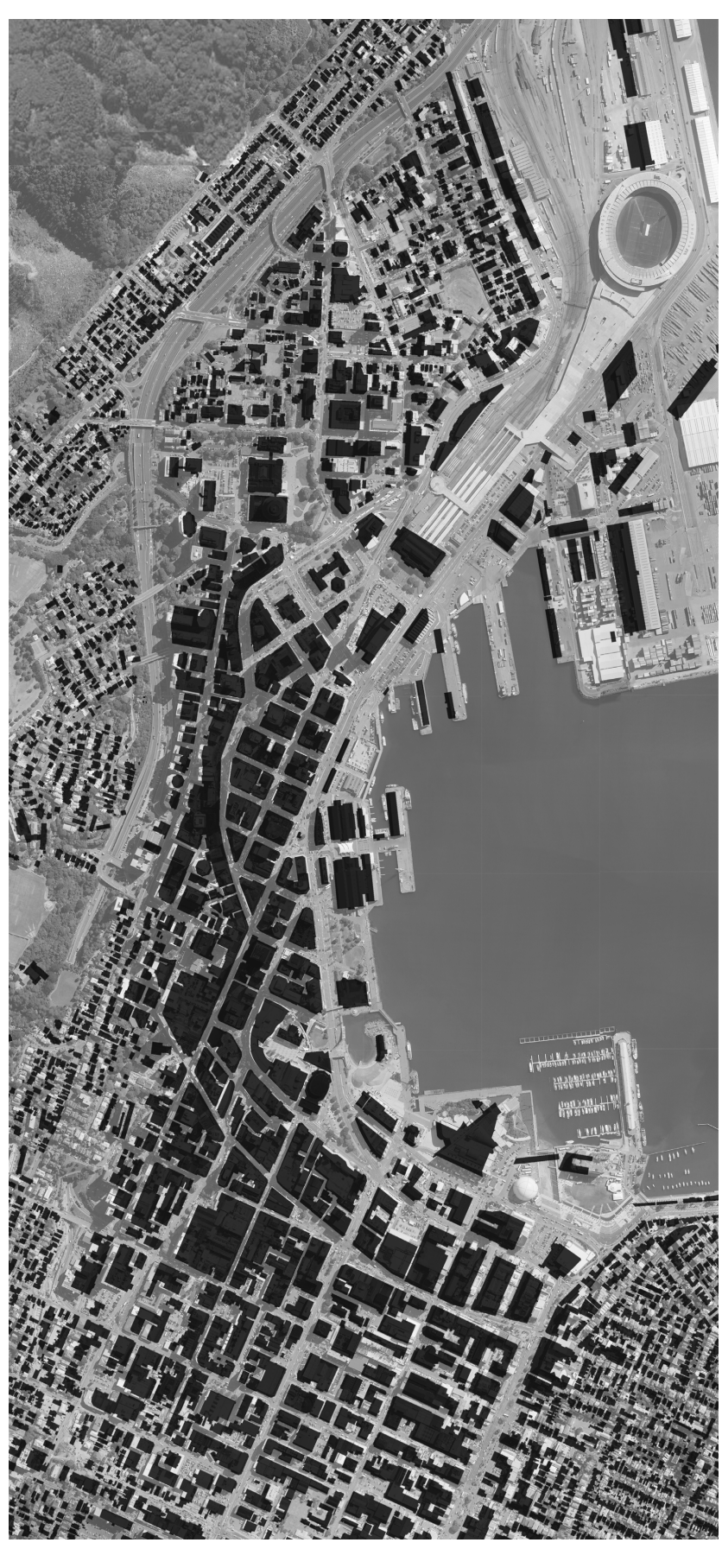

$<$ LEFT

Figure/ground map of central Wellington showing the incresed density and structure of the centre. Residual empty spaces appear where the city grid meets the incision of infrastructure, creating distinct borders between the centre and its periphery. pay-and-display parking facility at 132 The Terrace. Located directly below and adjacent to the southbound Wellington Urban Motorway, the car park consists of a $335 \mathrm{~m}$ curvilinear north-south axis that simultaneously follows the topography of the 'Wellington Town Belt' to the west and the dense commercial fringe of the CBD ('The Wellington Terrace') to the east (See Figure 3.20). According to the Wellington City Council, the car park is officially at the periphery of the Wellington 'Central Area' and forms a zoning border with the Thorndon 'Inner Residential' sector. The site has been excavated within the foothills and dense vegetation of the 


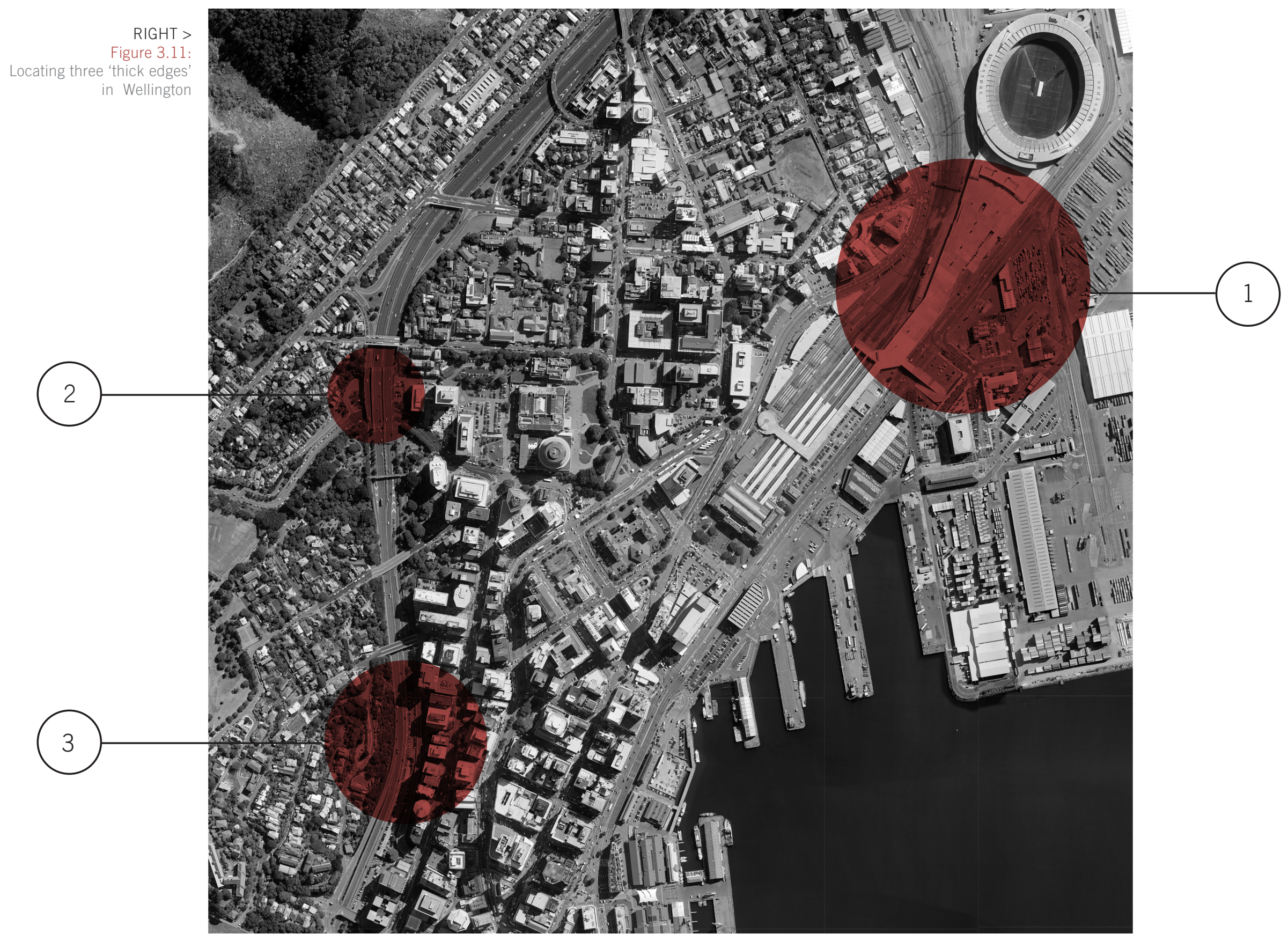

KEY:

1. Westpac Stadium Car Park, 83 Waterloo Quay

2. Ballantrae Car Park, 1 Ballantrae Place

3. Clitfton Street Car Park, 132 The Terrace 
greenbelt, forming steep concrete terraces adjacent to a gently sloping and regular ground plane (see Figure 3.22). Within and behind the town belt lie the urban suburbs of Thorndon and Kelburn, containing many of Wellington's residential heritage properties. North of the site cuts through the historically significant Bolton Street Cemetery, as well as the houses of many of the city's earliest settlers. To the south the car park is diagonally dissected by the Wellington Cable Car, a prominent tourist attraction which connects the retail sector of Lambton Quay with the Wellington Botanical Gardens and nearby Victoria University Kelburn campus (see Figure 3.23). Additionally, of the three sites, the Clifton Street Car Park is the largest - encompassing a vast space underneath the Urban Motorway directly adjacent to the greenbelt. It is also the most complex, comprised of several horizontal datums that have been adopted for parking (see Figure 3.21). Resulting from the car park's size and position,

Congruent with the conditions of leftover border spaces elucidated in this chapter, these three sites display consistent attributes that inform a condition that is impermeable and resistant to various contexts. As such, a number of factors contribute to their condition as a thick edge. These include:

A lack of social activity: driven by the imperatives of vehicular use, the spaces are singular in their anti-social, economically driven function. As such they act as social barriers that are rigidly mono-programmatic and inclusive only of their wider context as it relates to infrastructure. They promote vehicular use over social flows, preventing engagement and interaction between multiple publics by physically dislocating and isolating adjacent communities and land-use zones.

Continuous, rigid materiality: each site portrays a visual and tactile 'hardness' due to the dominant techniques of construction for infrastructure. Concrete is the dominant material and has been used in various forms (poured in-situ, precast, shot-crete).

Continuous, orthogonal typology: reductive orthogonal features are consistent throughout, resultant from walkways and motorways creating horizontal canopy conditions that are supported in a regular verticality by the column systems. This has emerged from the minimal post-and-beam construction techniques used. Consequently, each site has had a consistent and regular order imposed upon it - carved out spaces to support the structures of motorways and other infrastructures.

Excavated ground planes: facilitating the desirability of flat ground planes for parking, supporting columns and motorway datums each site has constructed an artificial (asphalt) ground surface by excavating the existing site. This 'tabula rasa' technique requires the removal of significant volumes of earth as well as the excavation of existing natural and cultural contexts, leaving behind visually and physically ubiquitous landscapes.

Disolcated zoning adjacencies: as they relate to building densities, incisive infrastructure has informed conditions where each site is between various zoning attributes (industrial, residential, commercial), informing polarised adjacent contexts. This bi-product of infrastructural incision forms a barrier to surrounding communities, rigidly dislocating land-uses.

Disproportionate scales: driven by the requirements of infrastructure, the car parks are neither highrise nor human in scale. Importantly, this has left significant volumes of space underneath and around the infrastructures - unused and available for architectural intervention. 
CLOCKWISE FROM TOP RIGHT

Figure 3.12: Aerial photograph
of Westpac Stadium Car Park,

$$
\begin{array}{r}
\text { of Westpac Stadium Car Park, } \\
83 \text { Waterloo Quay }
\end{array}
$$

Figure 3.13: Photograph From elevated walkway above

parking facility

Figures 3.14 \& 3.15 : Photographs of parking facility

from underneath walkway
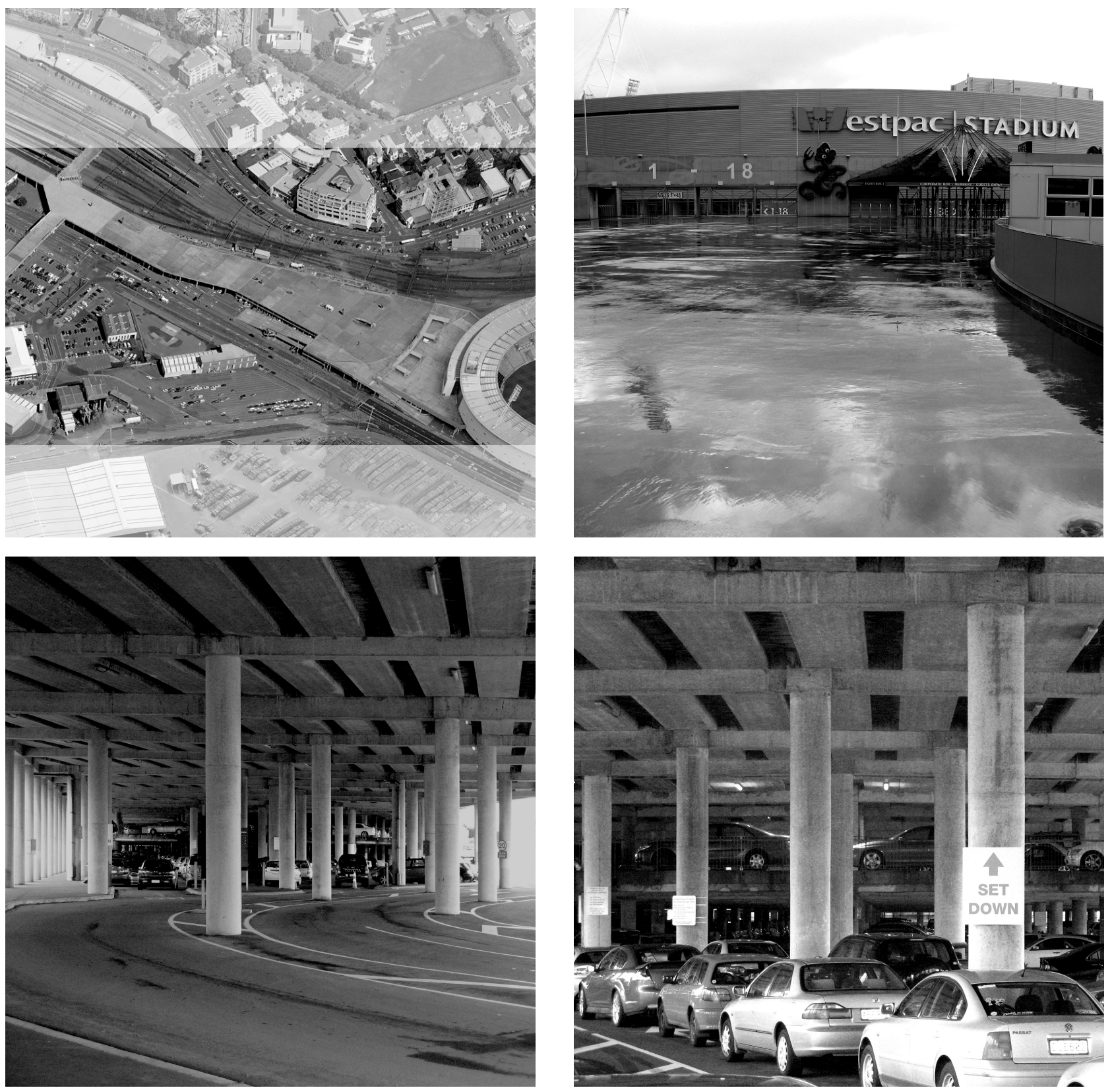

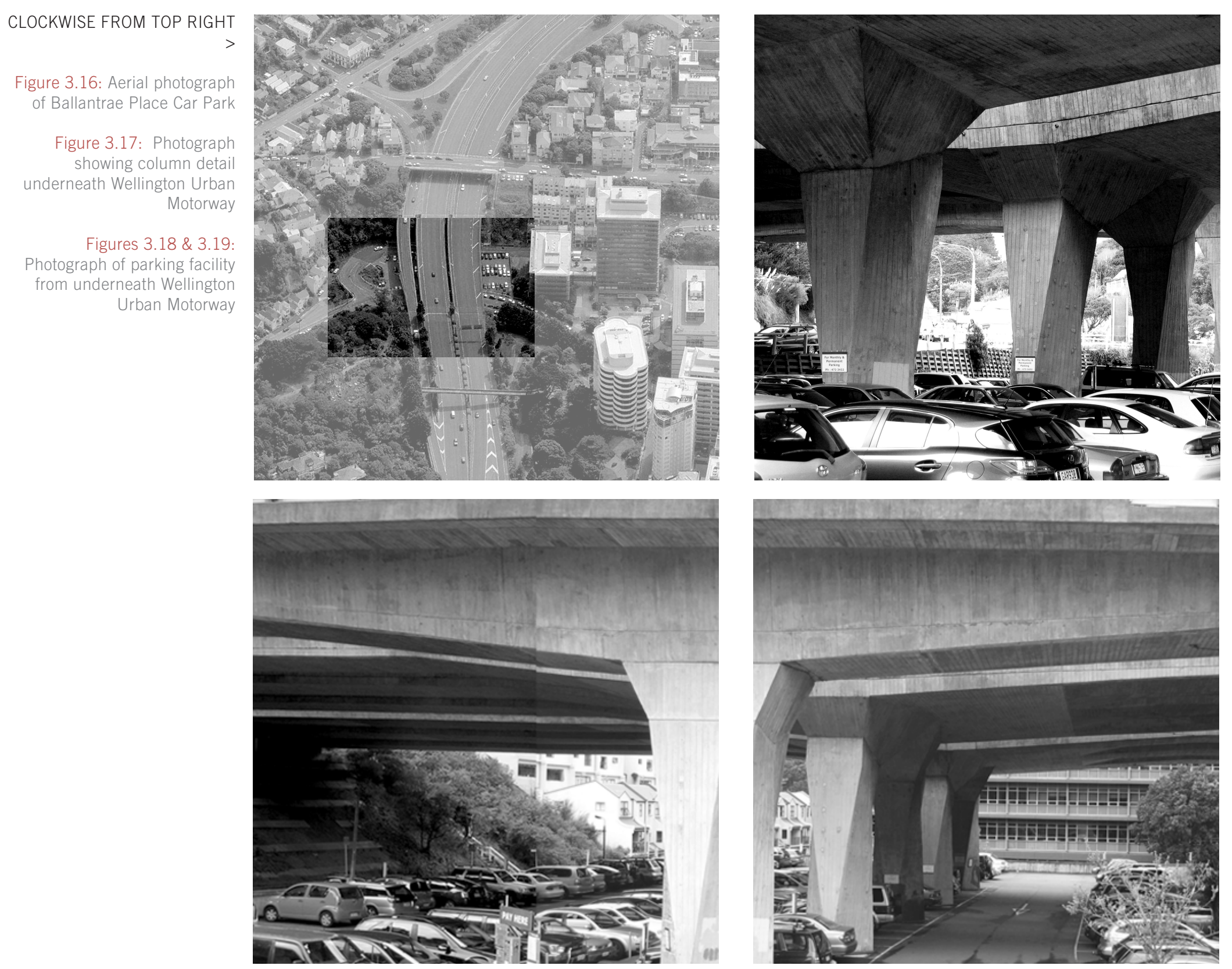
CLOCKWISE FROM TOP LEFT

Figure 3.20: Aerial photograph of Clifton Street Car Park situated between The Terrace and Thorndon

Figure 3.21: Photograph from middle level of parking facility

Figure 3.22. Photograph of Wellington Cable Car a Southern end of site
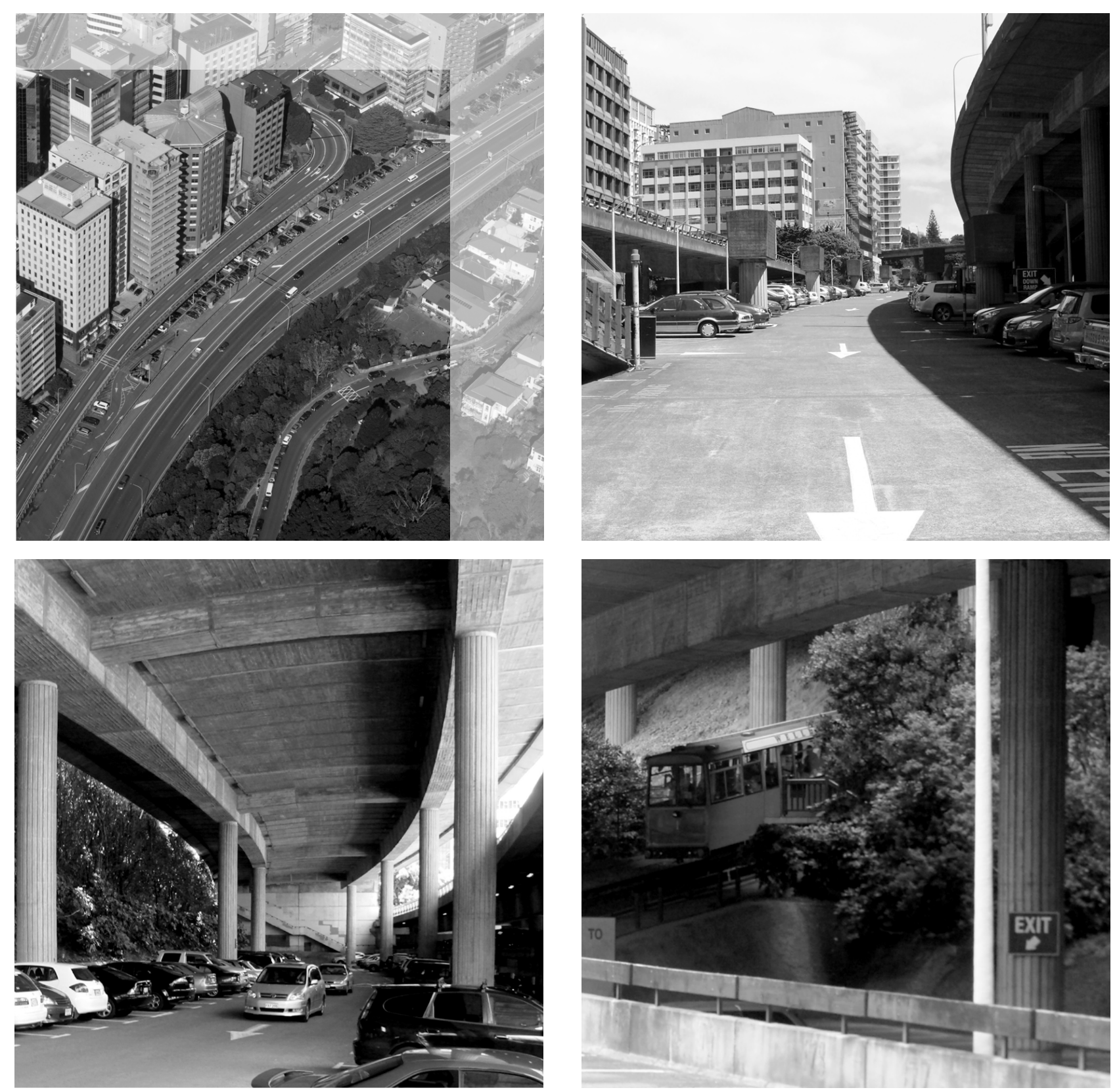
A New Form of Flow: Opportunities for Contextual Positivity

Highway construction and renovation continues unchallenged, with few exceptions, as do new flyovers and parking structures. ${ }^{23}$

\section{Richard Ingersoll}

I would argue that nothing can be accomplished urbanistically until these assumptions regarding the inherently negative qualities associated with contemporary urban space are challenged and

24. Pope, 2011, p. 162 Albert Pope is the Gus Sessions Wortham Professor of Architecture at the Rice University

25. See (Stoll, Lloyd, \& Allen, 2010), (Solomon, 2004), and (Shannon \& Smets, 2010)

In order to arrive at positive architectural solutions for urban thick edge conditions, it is necessary to challenge, alter and resist the current approaches used by governing bodies. Many architectural endeavours have attempted to treat existing infrastructures as an architectural object in an effort to mitigate their negative effects, with mixed results. ${ }^{25}$ In such instances, architecture is 'applied' to these sites - polishing and beautifying their austerity

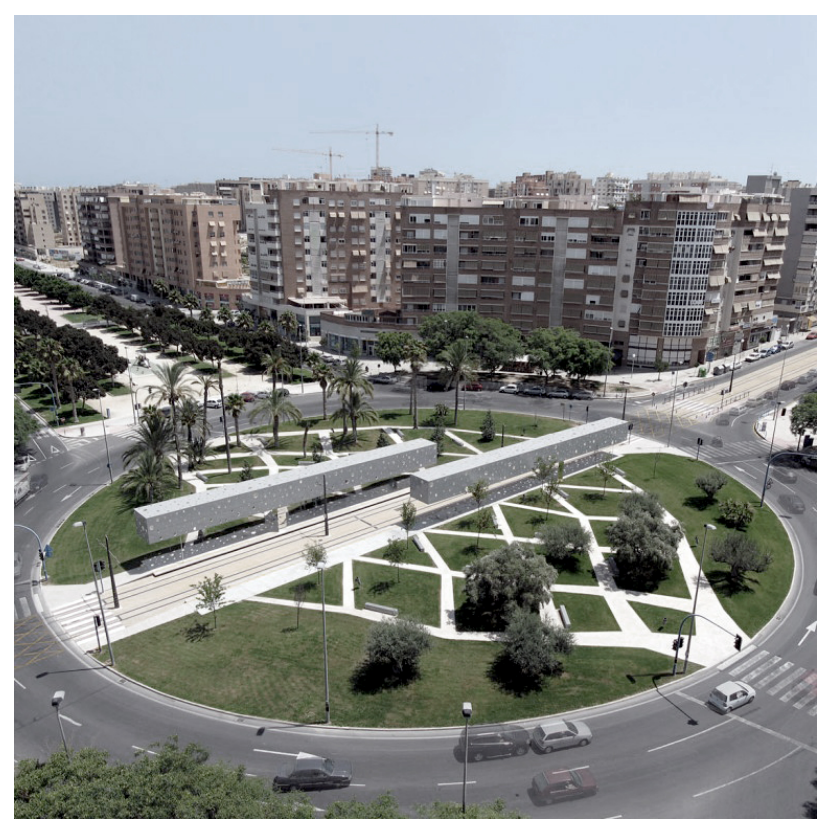

$<$ FROM TOP LEFT

Figure 3.24

Subarquitectura Architects

'Alicante Tram Stop'

(2005-2006)

Figure 3.25

Hargreaves Associates

'Louisville Waterfront Park'

(1999-2009)

Figure 3.26

Jean Nouve

'Plaça de les Glòries Catalanes' (2005) as formal objects through creative interventions. This approach is evident in projects such as the 'Alicante Tram Stop' (2005-2006) by Subarquitectura Architects (see Figure 3.8), Jean Nouvel's 'Plaça de les Glòries Catalanes' project in Barcelona (see Figure 3.9), and the 'Louisville Waterfront Park' (1999. 2009) by Hargreaves Associates (see Figure 3.10). In all three instances architectural intervention is used as a means to adjust the existing conditions of the infrastructure - enhancing their attractiveness to the public.

As a result, the economic and develpomentorientated motivations of infrastructure are passively superimposed with a sceneographic context of 'publicness' created through recreational landscaping. Consequently, the motivations and rigidities of the spaces are only slightly adjusted, introducing new visual, physical and social contexts but overlooking the historical, political and economic potential of the site(s).
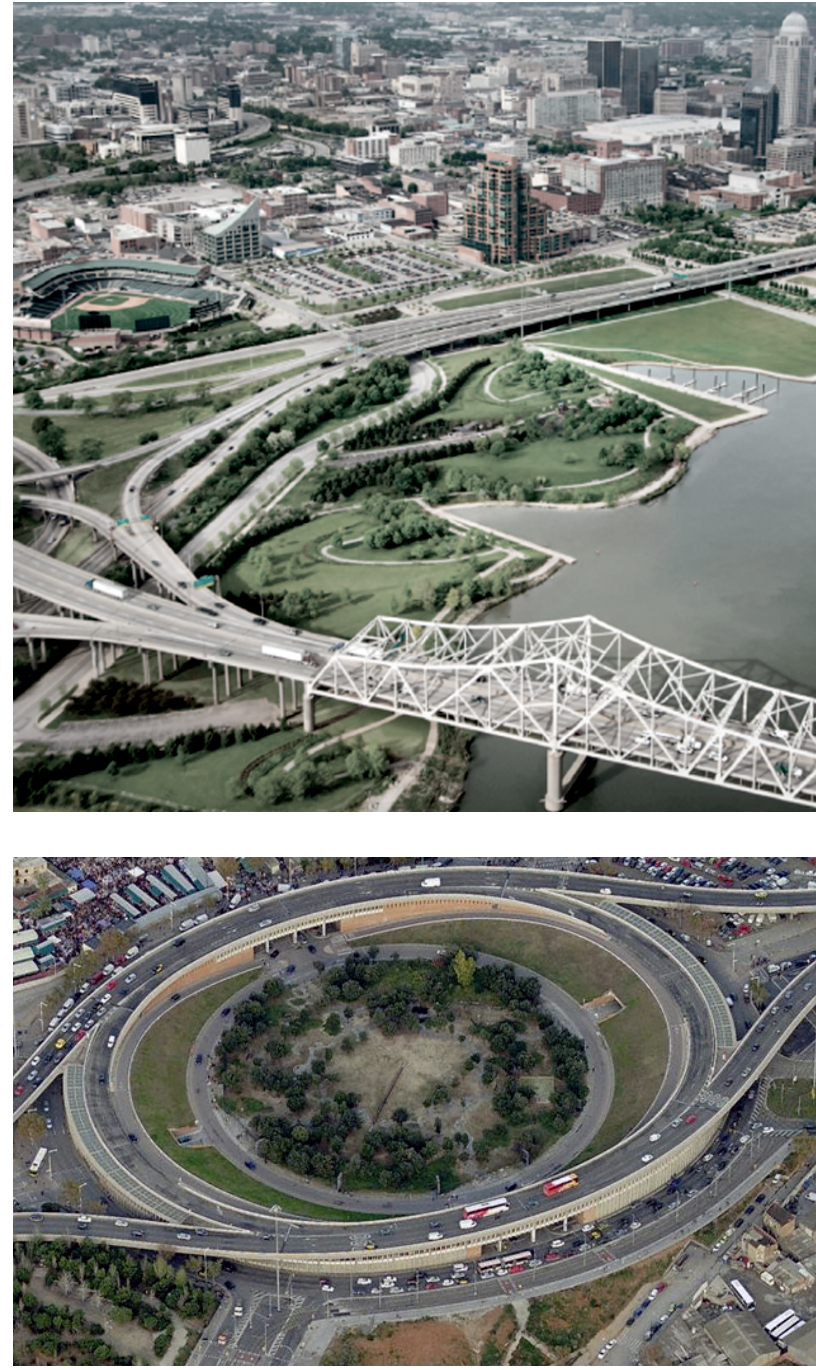

Importantly, Aaron Betsky identifies that an alternative 'flow' through leftover urban sites can be established, one which maneuvers around the positions of approaches that engage a limited range of contextual concerns. For Betsky, this form of flow is not aligned with the movements of capital, 
26. Betsky, 1998, p. 466. This is an essentially Derridean proposition, the resistance f identifiable and objective of identifiable and objective binary conditions. globalisation and urbanisation - rather, it follows the organic and fluid growth of a city - a process which reveals negative spaces as they emerge over time. From this perspective, negative urban spaces are opportunities that allow architects to construct sites of critique and resistance ${ }^{26}$ According to Betsky:

We do not need to build connective structure. Instead we need to weave our structures together by burrowing into them, destroying the false separations between inside and outside, reality and appearance, function and form, and between places by turning them into an amorphous web or landscape... To design this space means to direct the self-organising systems of the city and to wander through the real city, along its real spaces - the formless blobs of streets and parking lots. ${ }^{27}$

Aligning with Betsky's alternative view of negative urban spaces, Alexander D'Hooghe believes that sites of infrastructure embody opportunity to create moments of conflicting, contradictory, and radical critique. In "The Objectification of Infrastructure", D'Hooghe suggests that:

\begin{abstract}
Infrastructure, instead of being about one direction of movement becomes about the resolution of conflict between opposing flows and modes. Infrastructure, instead of being subservient to smooth flows, would instead take advantage of moments of friction and begin to articulate those into delineated public spaces... [A] critical design project will then consist of the creative appropriation of these codes, forms and the entire syntax of bureaucratic order, in order to re-invent and re-build them as authored systems with a human poetic element... Using the same technocratic elements that gave rise to the original form of the infrastructure system, but re-arranging them in a way that multiplies their functionalities and directions of potential use, will enable a consciousness of their existence as cultural objects. ${ }^{28}$
\end{abstract} 82. Alexander D'Hooghe is Associate Professor of Architecture at the Department of Architecture at the Massachusetts Institute of Technology.
This thesis identifies Betsky and D'Hooghe's approach as implicating with a contemporary, flexible criticality - aligning with the framework of critical spatial practices presented in Chapter Two. Importantly, by engaging the rich possibilities, the 'flows and modes' of multiple cultural contexts, architectural intervention can invert the negative conditions of the thick edge created by infrastructural incisions. Here, an 'inversion' of the thick edge becomes a two-fold gesture.

On the one hand, a polycontextual inversion of the thick edge refers to an alternative understanding of its condition at the border. Rather than a static and impermeable barrier, the border condition presents an in-between space of flow and possibility, fraying, and blurring. The thick edge by this definition is a space where identities collide, a potent space of questioning and undoing. ${ }^{29}$ Linda Pollak notes that in such cases, thick edges are not impermeable barriers they are latent thresholds, fertile and complex borders of potential social engagement:

\section{Boundaries can function as thresholds as well as barriers, to support and enable difference in social space... Because boundaries are where things meet, they have potential, when approached and represented from different sides, to function as spaces of debate and ambiguity, where it is possible both to call identities into question and to reveal their independence, whether these are identities of subjects or spaces. ${ }^{30}$}

On the other hand, inversion refers to the turning 'inside-out' of the standard intentions of thick edges. Thus they are redefined, not as a formal and spatial negativity, but, as a cultural positivity. Through polycontextual flows these sites can become rich spaces of complex historical, economic, political, social, physical and visual engagement and interaction. Rather than single-use junkspaces, embracing the flows of context presents a critical and vital flexibility - replacing the static and largely singular conditions of this urban problem. Thus the intervention of critical architectural production within thick edges emerges not as a physical barrier, but as socio-spatial event space - "not a division of things but a negotiation of flows." ${ }^{31}$

Approaching the thick edge from this dual inversion, allows architects to explore new and subversive architectural territory. Reconceptualising
29. Elizabeth Grosz,

Philosopher and Professor of the Women's Studies Program at Duke University, has talked about this notion - as it relates o architecture - in great ength. See: Grosz, 2001, p. 92.

30. Pollak, 1999 p. 77. Linda Pollak is a Lecturer in Landscape Architecture at The University of Pennsylvania architect, landscape architect and partner at Marpillero Poll Architects in New York City.

31. Borden, 2000, p. 240. 
these sites provides a potent alternative to the default sprawling action of much contemporary development - an opportunity to intensify and engage the unseen contexts, and to "conceive of the public realm, urban structure and architectural typology as interconnected". ${ }^{32}$

\section{Conclusion: A Polycontextual Approach to the Clifton Street Car Park}

Engaging the third research question of this thesis, thick edges - the condition that forms as a result of new highway infrastructure and expanding urbanisation - has been illuminated as a contemporary urban architectural problem, spatially and culturally separating communities, land-uses and publics.

This chapter has argued that these sites, although difficult and often unsightly, present architects with potent opportunities. Approached critically thick edges offer a chance to regenerate negative and anti-social spaces within the localised urban fabric, whilst simultaneously contesting and critiquing the global forces that produce them. ${ }^{33}$

Additionally, Chapter Three has argued that engaging with the complex and multiple cultural conditions of urban sites, the rigidity and singular nature of these impermeable spaces can be opened to often disregarded flows of context. These contexts may be historical, economic, political, social, physical and visual; contributing to the culturally engaged dimension of critical architectural production, and providing bases for the inverting of the impermeability of thick edges.

This thesis proposes that it is the task of the critical spatial practices to not only adopt these found sites as grounds for fertile re-engagement, but to expose the unique contextual information that is latent within each site proper. In so doing, architectural intervention can initiate positive cultural flows through contextually rigid sites, enabling new community interactions, experiences and spatial practices.
Of the three thick edge sites identified in this chapter, the Clifton Street Car Park presents the most extreme and exaggerated example of the thick edge condition - therefore posing the most challenges, and strongest opportunity, for the design-research to test this problem. This site in particular provides a number of fertile contexts, explored in Chapter Four, through which critical spatial practices can be tested and critical architectural processes can be engaged. 

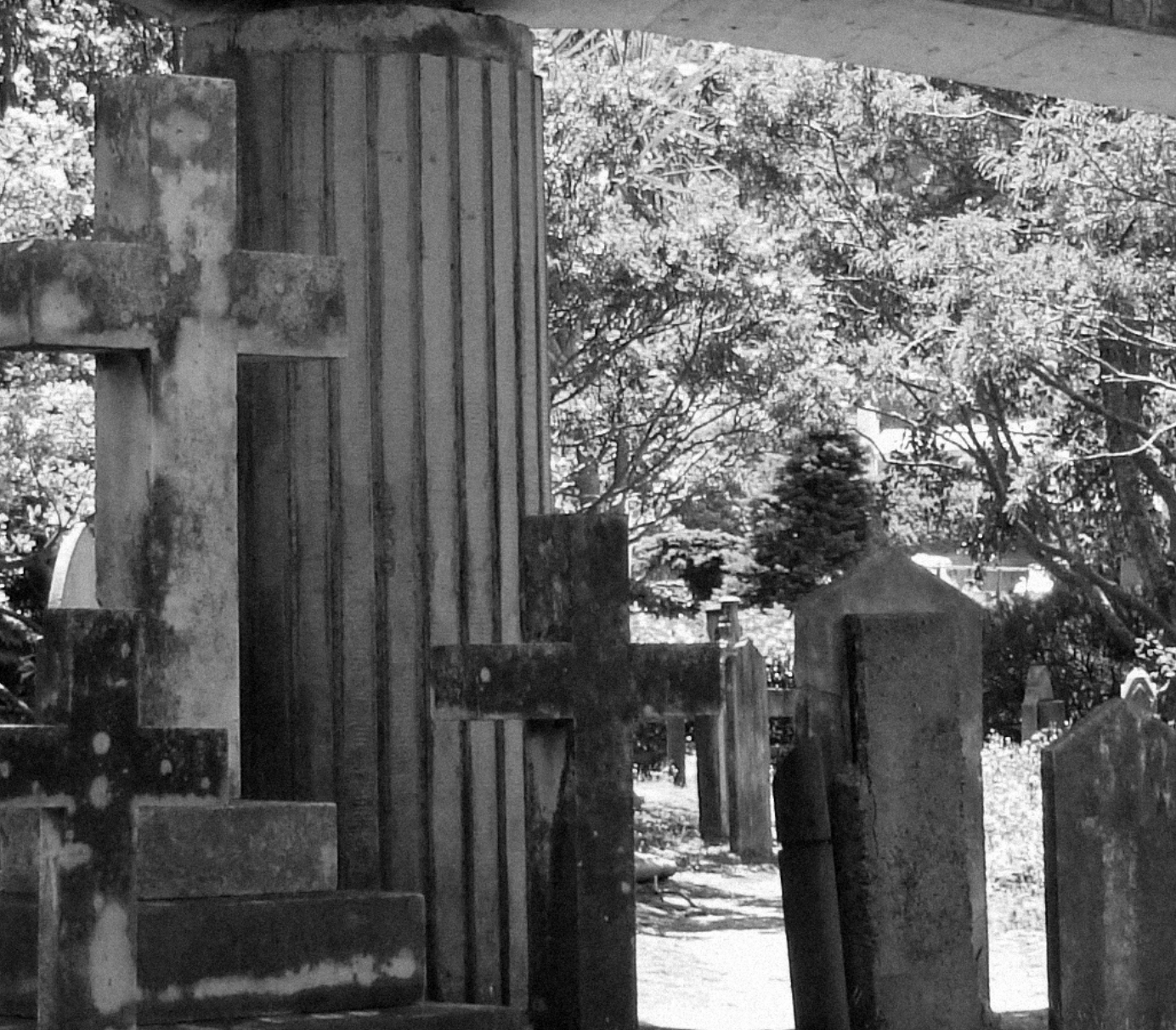

7.

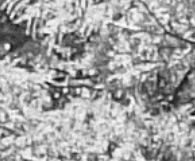

s.

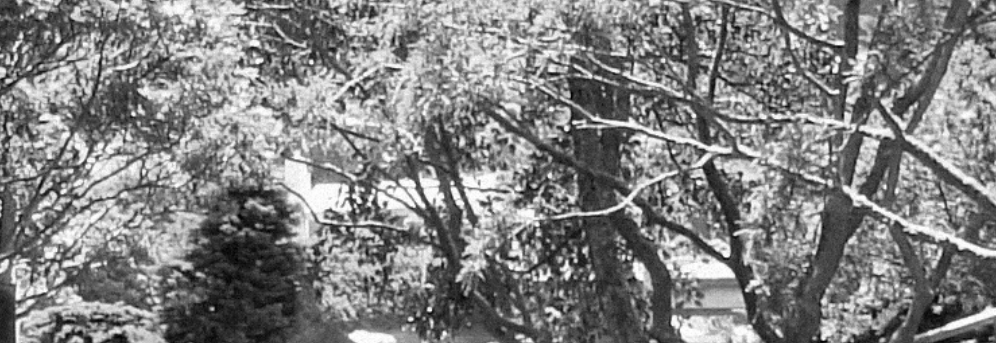
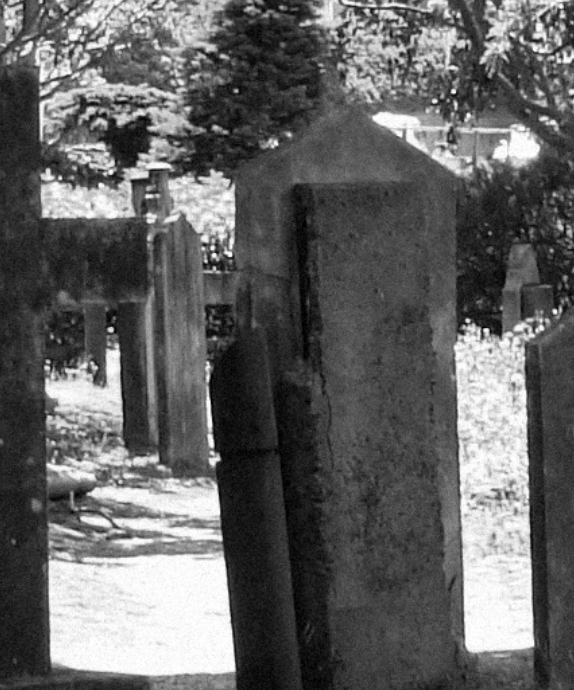

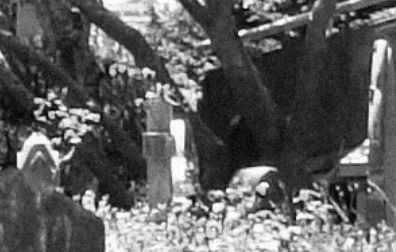

तs
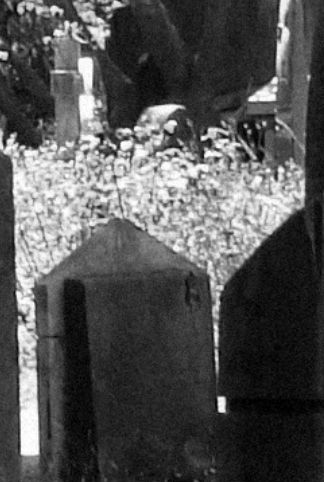

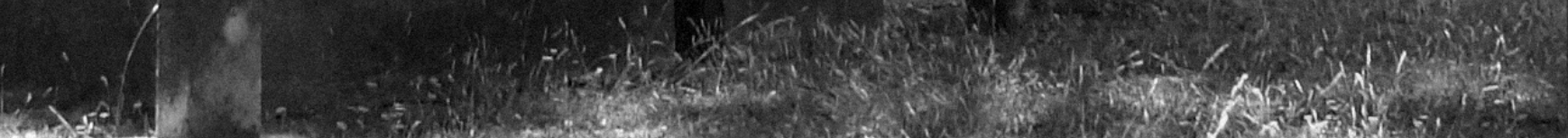

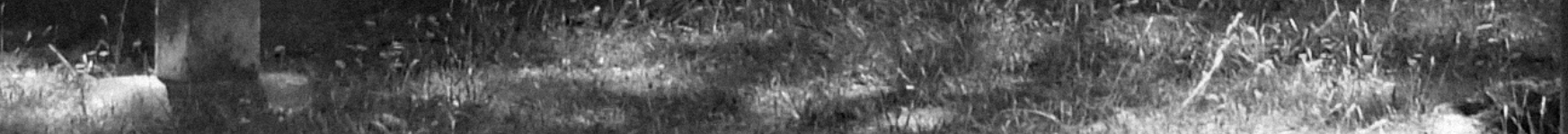

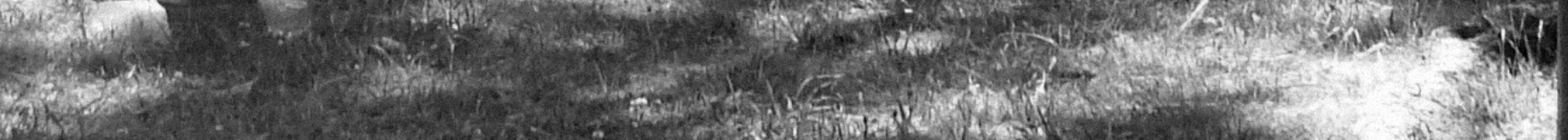

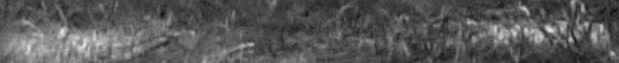

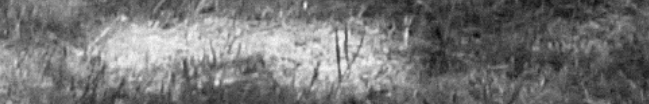
Q

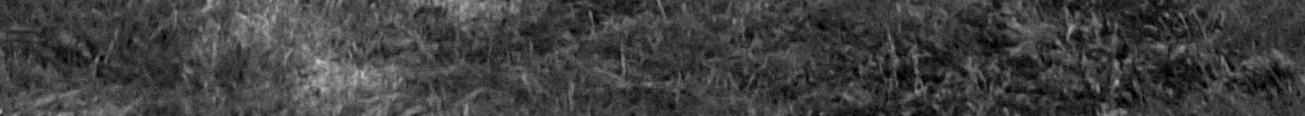
$\lim _{\rightarrow \infty} \rightarrow x^{2}+x^{2}$

\section{$-1$}
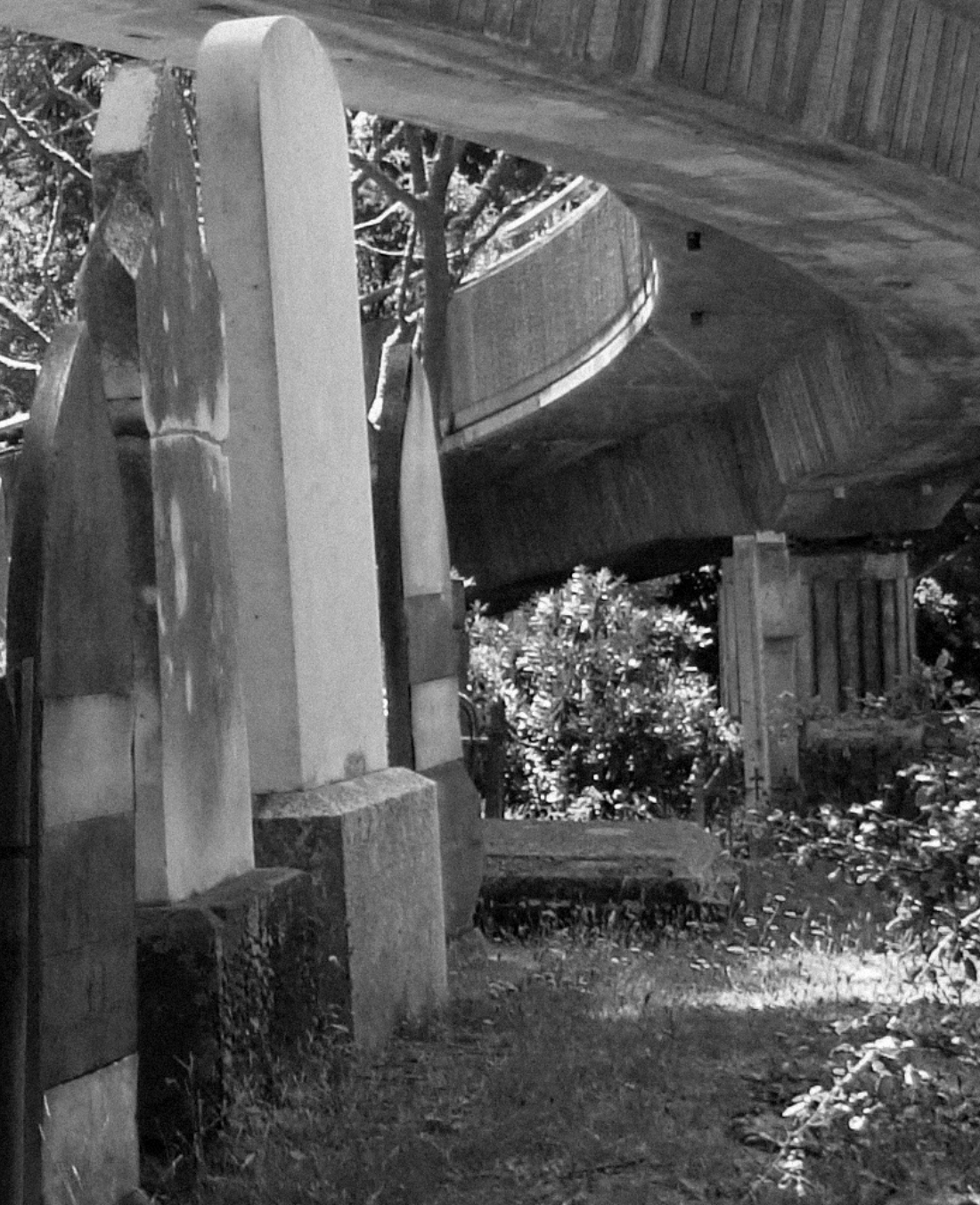

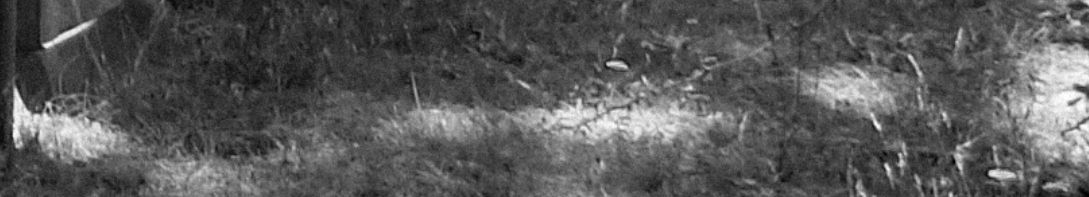

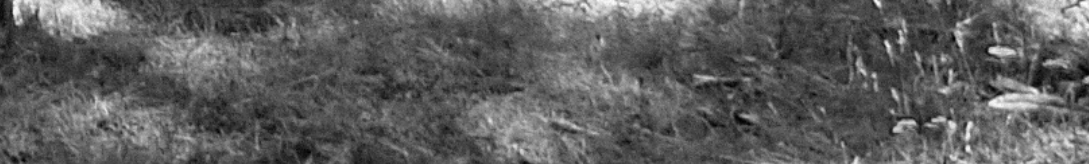

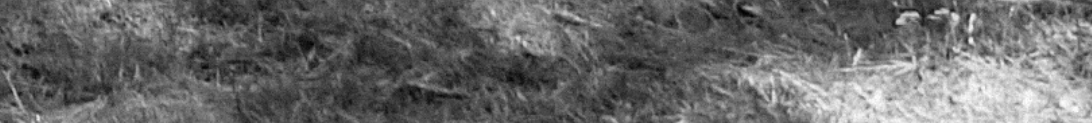

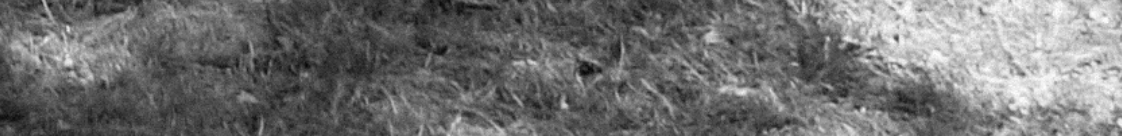




\section{4}

\section{A POTENT(IALLY) CRITICAL SITE}

Investigating a Polycontextual

Approach to the Clifton Street Car

Park

\section{Introduction}

An Urbanised Context

A Historical Context

An Economic Context

A Political Context

A Social Context

A Physical Context

A Visual Context

Conclusions 
In Chapter Three, it was established that within the contemporary built environment urban thick edges, a condition of new infrastructures imposed upon a pre-existing urban fabric of integrated communities, present opportunities for positive critical responses.

Through investigations of three sites, the Clifton Street Car Park has been isolated as the focus for design research in this thesis. Following the argument put forth in Chapter Two - that critical architectures will be resistant to submissive reproduction under global-to-local forces, mediating between formal and cultural production - this chapter considers the design opportunities presented by a polycontextual investigation of 'site' as encompassing historical, economic, political, social, physical and visual processes.

In so doing, this chapter investigates how these contexts are related to production and reproduction of the current identity of Clifton Street Car Park as a thick edge. A polycontextual framework is established for the testing of critical spatial practices within these conditions - lluminating knowledge with regards to contexts that have been historically disregarded, impaired or marginalised by the forces of jump-cut urbanisation. 


\section{An Urbanised Context}

The Clifton Street Car Park figures as both a product of, and symbol for, the impacts of $20^{\text {th }}$ century jump-cut urbanisation in Wellington. The histories of this urban complex - notably the impacts of automobile dependence and the rapid high-rise development of The Terrace - present a background for the production and representation of Clifton Street Car Park as a negative urban space. Additionally, these histories illuminate culturally relevant information that foregrounds the construction of critical responses and practices in the design case study.

The post-war period, 1950 onwards, exemplifies the desire for Wellington to engage with institutions of globalisation such as American planning techniques and economically-driven development (See Figures 4.2-4.12). The city, and consequently the Clifton Street Car Park, has been subject to two notable 'boom' periods - both occurring in the period between 1960 and 1990.

The first of these periods of rapid growth occurred with the introduction of significant infrastructure to accommodate both Wellington's growing population and the rise of the automobile. This resulted in the construction of the Wellington Urban Motorway - a network of roads connecting the urban centre to its peripheral suburbs. Predicting further growth, a two-phase car park development was put forth and initiated as an economically pragmatic solution to the leftover space from the motorway The first phase, completed in 1970, initiated the construction of the Clifton Street Car Park as it exists today. The second phase of this development was abandoned as infrastructure requirements and population growth slowed, leaving the facility unfinished. ${ }^{2}$ 1960 and 1970 , this rate halved in the decade following. (New Zealand, 2013)

The second boom period, instigated by the rise and affluence of the private sector throughout the 1980s, saw the construction of economicallymotivated office towers along The Terrace. 'Facing' towards the harbour aspect, these office buildings further implicate the car park as a residual urban space, and as a thickened edge - informing a polarised position between the densely urban terrace and the natural topography of the town belt. Both of these boom periods, driven by economic imperatives, have contributed to the site performing in a contextually reduced capacity, acting as an incisive barrier to the surrounding area(s).

\section{A Historical Context}

Further investigation reveals four additional historic contexts that contribute to the Clifton Street Car Park as embedded within a culturally complex area: the Bolton Street Cemetery; the Thorndon suburban area; the Kumototo Stream; and the Wellington Cable Car. Each of these contexts has been affected by the construction of the Urban Motorway and subsequently has become segregated or removed by the thick edge created by the car park.

\section{Bolton Street Cemetery and the Clifton Street Car Park}

The history of the Bolton Street Cemetery and its partial relocation through the construction of the Urban Motorway is considered to be one of Wellington's most culturally traumatic and controversial planning decisions. The cemetery is New Zealand's earliest formal burial site, accommodating graves from as early as 1849 (although it is suspected there are older graves as this is merely the date that the cemetery records began). ${ }^{3}$ Occupying a large geographic area north of the Clifton Street Car Park the cemetery documents the earliest colonial and religious settlement of Wellington City as well as providing inner-city green space and a connection to the Town Belt (see Figure 4.13).

The Urban Motorway enters the city directly through the centre of the Bolton Street Cemetery, requiring the excavation of $14,973 \mathrm{~m}^{2}$ from the existing cemetery plot (see Figure 4.14). ${ }^{4}$ This controversial procedure of urbanisation caused much public backlash throughout the 1960's, culminating in the formation of the Bolton Street Cemetery Preservation Society in October 1964. Alongside broadcasts on radio and television, public resistance to the cemetery's desecration was conducted through photographs, letters, as well as newspaper articles and editorials.
4. Alington, 1978, p. 162. This s a conversion of 3.7 acres to metres-squared. 


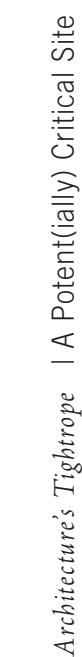

\section{$\longmapsto$}

RIGHT > Figure 4.2: Photograph of 'Shell House', 49-55 The

Terrace, Wellington

(1960)

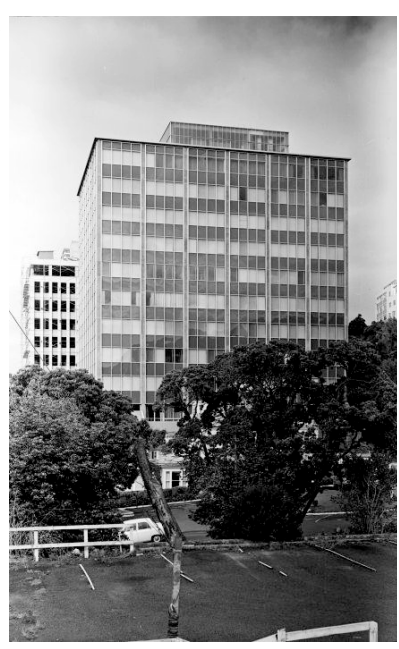

1960: SHELL HOUSE

(49-55 THE TERRACE) IS

CONSTRUCTED. THE UNITED KINGDOM TOW ACT.

In so doing local councils were required to produce development master plans - a clear signal that the country wished to engage with the progressive drives of European modernism, and aim towards the ideals of post-war town planning.
Wellington's first high-rise building is constructed - a 14 storey glass and steel box designed by Stevenson and Turner architects, is seen as a point of departure (for The Terrace in particular) towards downtown Wellington establishing itself as a business centre.

\section{Figure 4.4: RIGHT of the last run of the Wellington trams, Lambton} Quay, Wellington (1964)

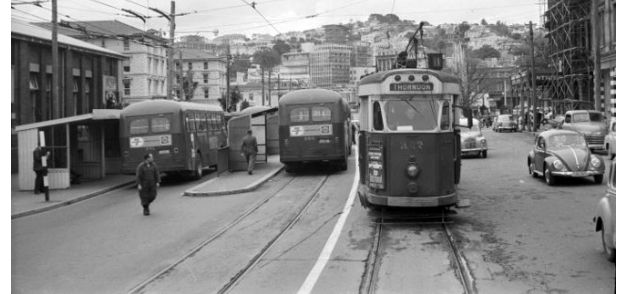

1964: WELLINGTON TRAMWAYS SHUT DOWN

An institution in the city, the removal of the tram system represented a social and economic shift towards a vehicle-based transport infrastructure and a chance to engage with the rest of the world through technological progression and construction. showing construction of the connecting The Terrace to the new infrastructure. This would later form the site of the Clifton Street Car Park. The off-ramp required further excavation of Shell Gully to

occur.

1950

1959: NATIONAL ROADS AMENDMENT BILL INITIATED

Allowing the government to construct networks of freeflowing highways into urban centres, networks that will "enable the free flow of traffic on routes considered essential for national development and defence". ${ }^{5}$ Immediately following a proposal is put forth for a Wellington Urban Motorway that could accommodate the 29,000 commuting vehicles travelling to the city centre every day from the Hutt City suburban periphery. ${ }^{6}$

5. Miller, 1969, p. 33. 6. Yska, 2006, pp. 182-183.

\section{0: CITY COUNCIL} ENGAGES AMERICAN

ENGINEERING firm De Leuw Cather and Company as master planning and infrastructure consultants. Figure 4.3: Photograph of the construction of the following the De Leuw Cather infrastructure master plan. Entering from the north of th city, the motorway excavated significant incision within the

landscap

August 1963: DE LEUW CATHER AND COMPANY RELEASE 'MASTER

TRANSPORTATION PLAN'

Involving significant excavation and land acquisition as well as cutting through the centre of Bolton Street Cemetery and behind The Terrace through Shell Gully (one of the remaining few inner city green spaces).

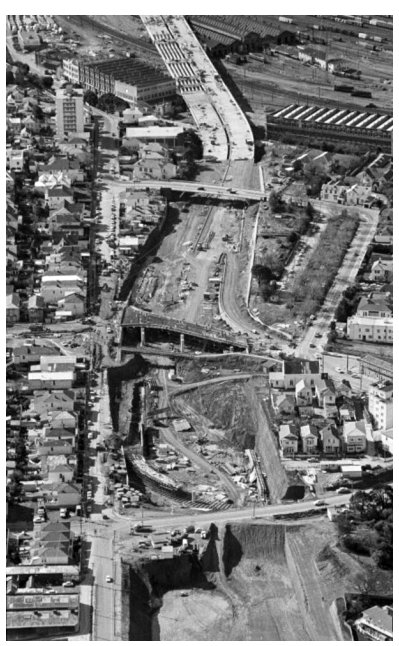

Throughout the 1960's THE DE LEUW CATHER PROPOSAL RECEIVED MUCH RESISTANCE from the public, much of which focussed on the negative visual and cultural outcomes of the motorway. Resisters felt that the motorway represented the direct importation of American planning ideals into a context that required more subtle and local solutions. Local publications were flooded with letters from concerned citizens as details emerged of the potential desecration of the existing cultural landscape,

in particular the Bolton Street Cemetery.

RIGHT > Figure 4.6: Cartoon by Neville Lodge depicting public resistance to the Urban Motorway proposal.

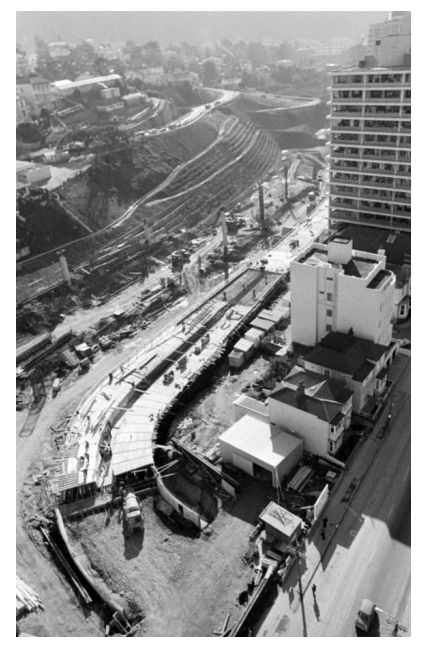

969: FIRST STAGE OF THE DE LEUW CATHER TRANSPORTATION PLAN OPENED WITH THE CONSTRUCTION OF THE 'FOOTHILLS MOTORWAY'

The first development of the De Leuw Cather plan saw the construction of the Wellington Urban Motorway - connecting Lower Hutt City to TeAro and The Terrace. The motorway carved through the foothills of the Town Belt, contributing to "the changed face of The Terrace with its commuter-

supported office buildings and huge car park facility".

7. Kernohan, Wellington's Old Buildings, 1994, p. 19
"... it is felt that 637 car spaces approximates the maximum that can be accommodated in the Shell Gully car park, having regard to the additional private parking development in the area. 's

A. D Martin, Deputy City Engineer 8.Martin, 1970, p. 5

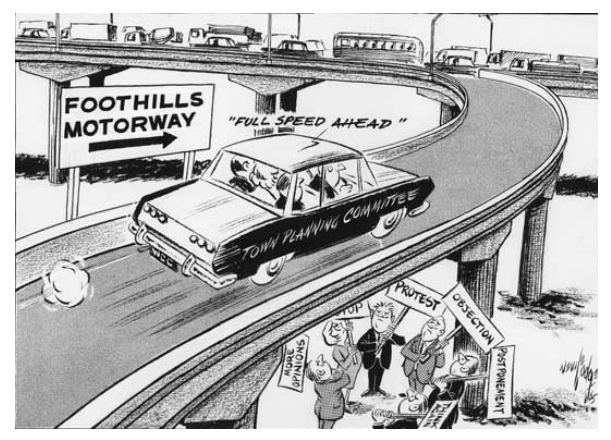




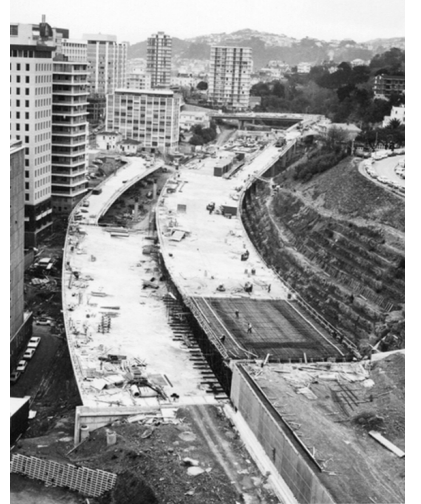

1970: CAR PARK PROPOSED IN SHELL GULLY

Following the perceived 'success' of the Urban

Motorway by the City Council, schemes put forth by the Wellington City Corporation for the "investigation into the economic feasibility of

incorporating a parking building into the elevated section of the motorways between Aurora and Everton Terraces", an area known as Shell Gully. ${ }^{9}$ This site was perceived as having high intrinsic value given its proximity to The Terrace.

9. Martin, 1970, p. 1

9. Martin, 1970
LEFT

Figure 4.7: Photograph, excavation through Shell Gully. Terracing of the Townbelt is becoming evident (to the right) and the concrete platforms that will form the Clifton Street Car Park are under construction.
1961-1981: RAPID

EXPANSION AND

DEVELOPMENT OF THE

TERRACE

Upon the construction of the

Urban Motorway - connecting

the urban centre to its

peripheries - 'spectacular'

commercial growth along The

Terrace ensued, to the extent that "in the first five years

following the De Leuw Cather study, the actual expansion exceeded more than three

times that allowed in the study over the 20 year period to $1981 " .{ }^{10}$

10. Martin, 1970, p. 4-5.

11. Kernohan, Wellington's Old

Buildings, 1989, p. 1 .

"On The Terrace a continuous growth of anonymous earthquake-proof structures has sprung up to serve this [development] need. Purely additive and cost-measured, with no elegance or style, they come alive symbolically in the halflights, with changing banks of lights forming fascinating kinetic patterns... Glimpsed through the jagged rampart, old Wellington waits to disappear, old bush, bare hills, small scale colonial buildings. "I

1981-1991: SECOND PHASE OF DEVELOPMENT PRIVATISATION

The Terrace irreversibly transforms into a privatesector commercial zone coinciding with an economic 'boom' period in the stock exchange. This exacerbated the economic mandates of urban development, resulting in the production of "anonymous, bland office buildings". ${ }^{12}$

\section{Ibid}

\section{(a)}

1

1970: CAR PARK APPROVED

Wellington City Council approved a scheme for 660 670 16'x8' spaces beneath the motorway in Shell Gully, 'Scheme IIIA', with the addition of two parking 'platforms' and the potential for future extension. This scheme was a two-phase development costing an estimated $\$ 1,075,000$ and $\$ 5,144,000$ respectively $(\$ 14,985,500$ and $\$ 71,707,360$ in Q4 2012). Phase One entailed regrading and excavating the land to accommodate 475 parks underneath the southbound section of the Urban Motorway as well as The Terrace off-ramp. Phase Two allowed for further decks, parking platforms that would separate the site into several levels, to be constructed to support a further 803 spaces. Automobile and population expansion slowed, and Phase Two was cast aside, the extra parking deemed as uneccessary. As a result a number of footings remain in preparation for Phase Two.
1977: SECTION 301A OF THE MUNICIPAL CORPORATIONS ACT INITIATED

The 301A Act entitled developers, flourishing in the private market, to legally enforce the demolition of a number of heritage buildings that were deemed to be 'earthquake prone'. A significant proportion of these buildings occupied the desirable real estate of The Terrace and their removal prompted developers to erect 'earthquake safe', orthogonal, office towers.

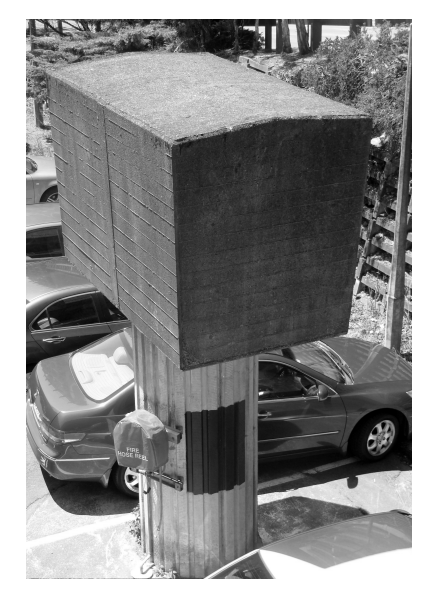

BELOW

Figure 4.12: Photograph,

looking north, showing thick edge condition created by the Clifton Street Car Park - the back of The Terrace is to the right, the Townbelt is to the

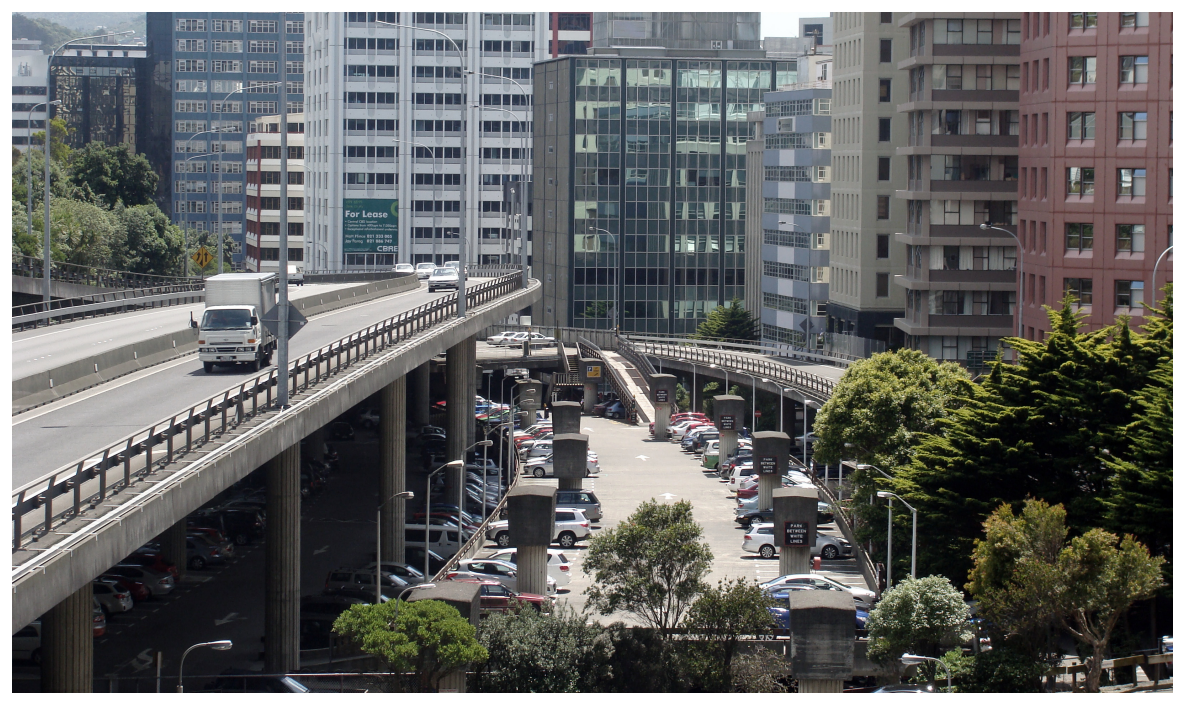

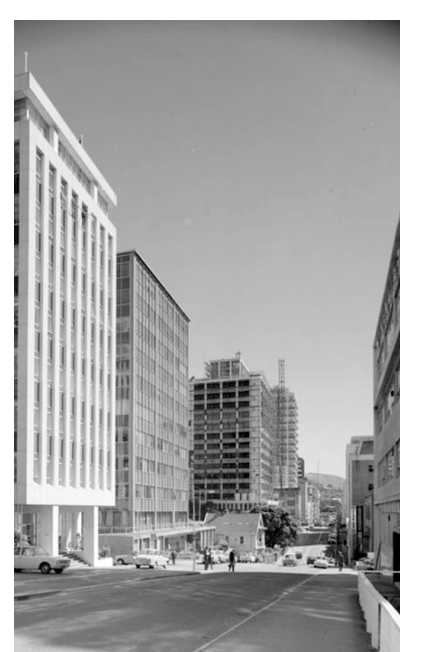

1981 onwards: HIGH CITY/ LOW CITY

\section{Facilitated by the}

infrastructural addition of the Wellington Urban Motorway, The Terrace has come to form a visual and economic backdrop for the city.

Through dense commercial development, The Terrace has created a visual skyline for Wellington, informing the high city/low city structure put forth by the City Council. ${ }^{13}$

13. Council, 12. Central Area, 2012, p. 13.

\section{$\wedge$ ABOVE}

Figure 4.9

Photograph of The Terrace c. 1970.

\section{$<$ LEFT}

Figure 4.8:

Photograph of remaining footing from incomplete Phase Two of Clifton Street Car Park proposal.

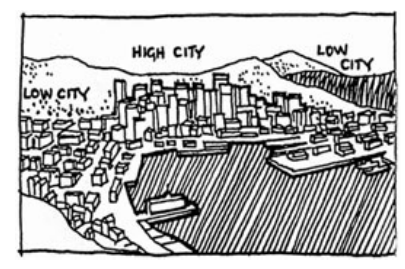

$\wedge$ ABOVE

Figure 4.10.

Sketch of 'High-city/low-city' skyline created by The Terrace RIGHT > Figure 4.11: Diagram showing 'High-city/ low-city' skyline created by The Terrace
2013: CLIFTON STREET CAR

PARK

The high city/low city

development imperative has informed a physical context where The Terrace faces towards the Harbour aspect, visually rejecting the Clifton site. This additionally implicates the defintion of the site as situated between two land-use zones.

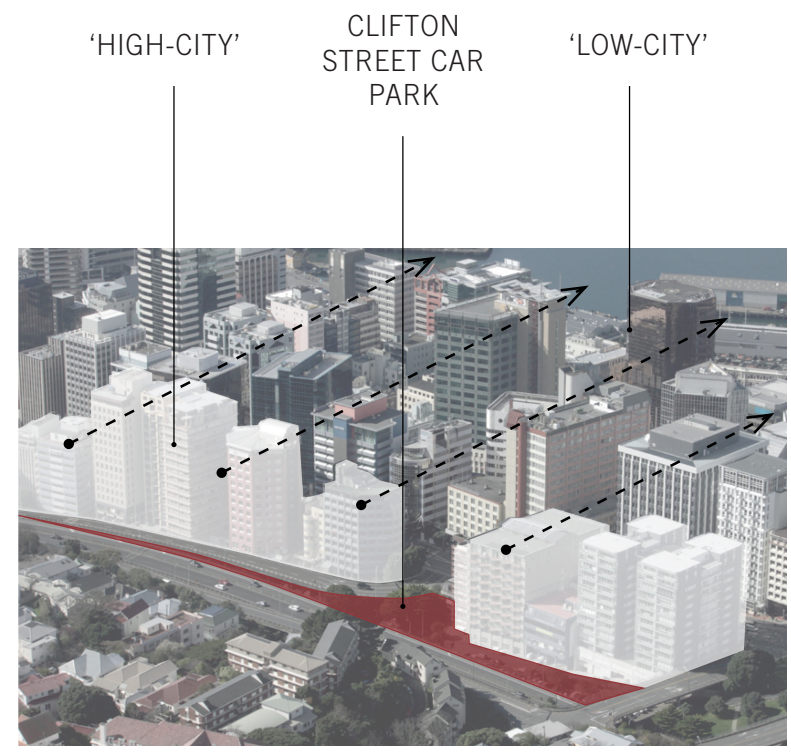


Figure 4.13: diagram/map showing proximity of Bolton Street Cemetery to the north of the Clifton Street Car Park

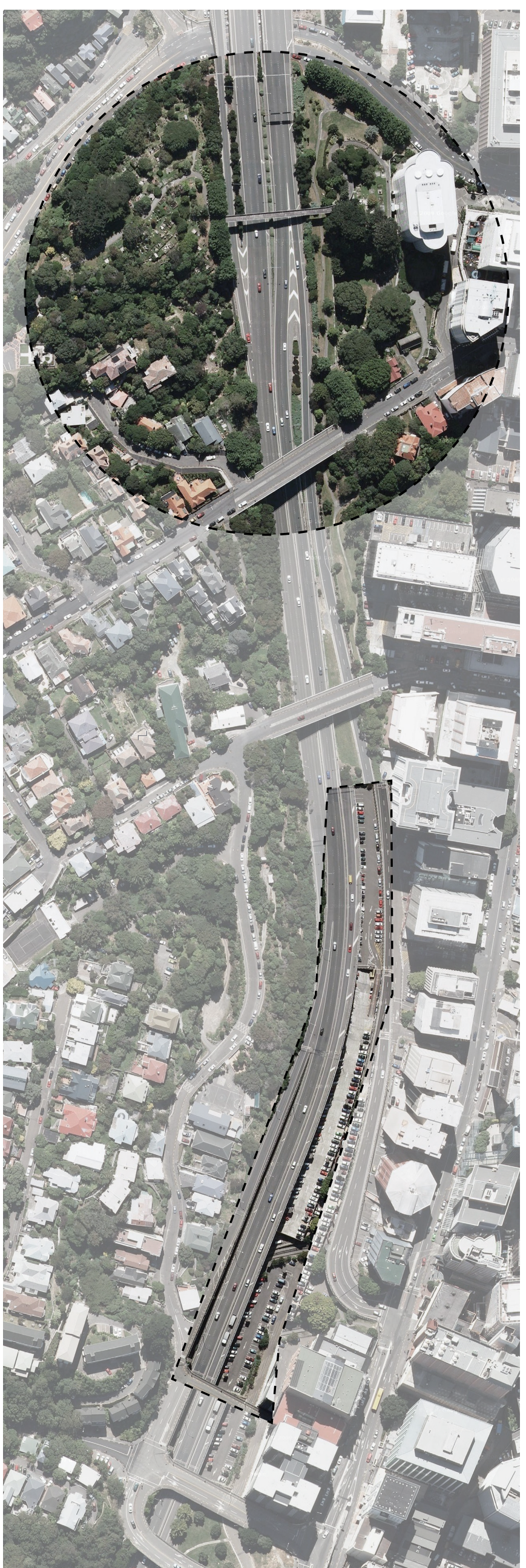

In spite of public upheaval in 1969 the Ministry of Works began the process of removing human remains from the cemetery that lay in the motorway's path. In 1965, an estimated 700 graves were to be affected; however, between 1968 and 1972 a number of unknown burials were revealed. By the motorway's completion, the disinterment of 3,693 buried corpses and some 900 memorials occurred, relocated in cardboard containers to nearby mass graves within the cemetery (at the 'early settler's lawn') or to the suburban Karori Cemetery (see Figures 4.15 \& 4.16).

No other event better expresses incisive processes of jump-cut urbanisation than the Bolton Street Cemetery's bisection. Negatively impacting the cultural fabric of the city, the construction of the Urban Motorway (including the Clifton Street Car Park) expresses how global-to-local forces of urbanisation can act through contextually singular motivations (economic).

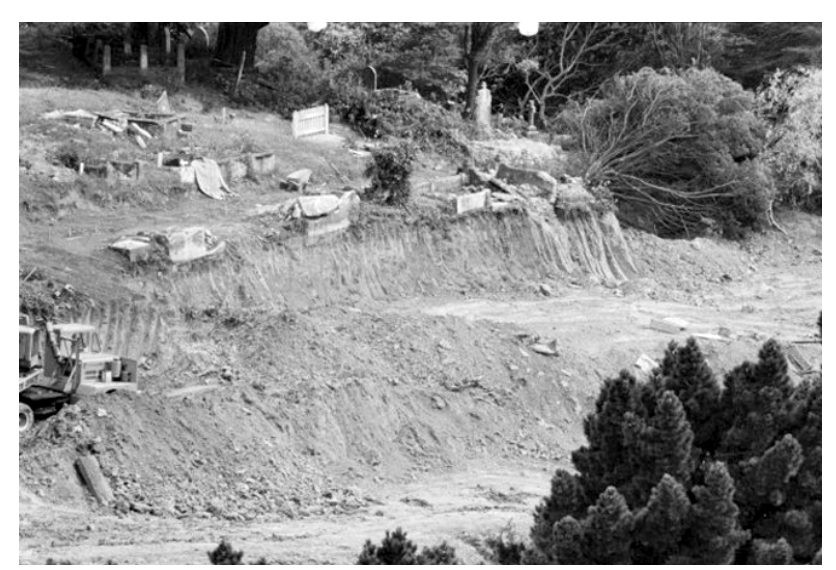

$<$ LEFT

Figure 4.14: Photograph showing the partial excavation of Bolton Street Cemetery during the Urban Motorway construction (1969)

The history of the Bolton Street Cemetery associates the Clifton Street Car Park in the process of identity-destruction, shifting the emphasis of development towards the reification of inequality between global and local interests. A critical response in this thesis will engage in mediation between the intervention of architectural form within the negativity of the site and the multiple contexts of this identity destruction. As such, the desecration of Bolton Street Cemetery is an important cultural history for the production of critical design responses to the site, and the opportunity to invert the thick edge to the possibility of positive polycontextual flows. 


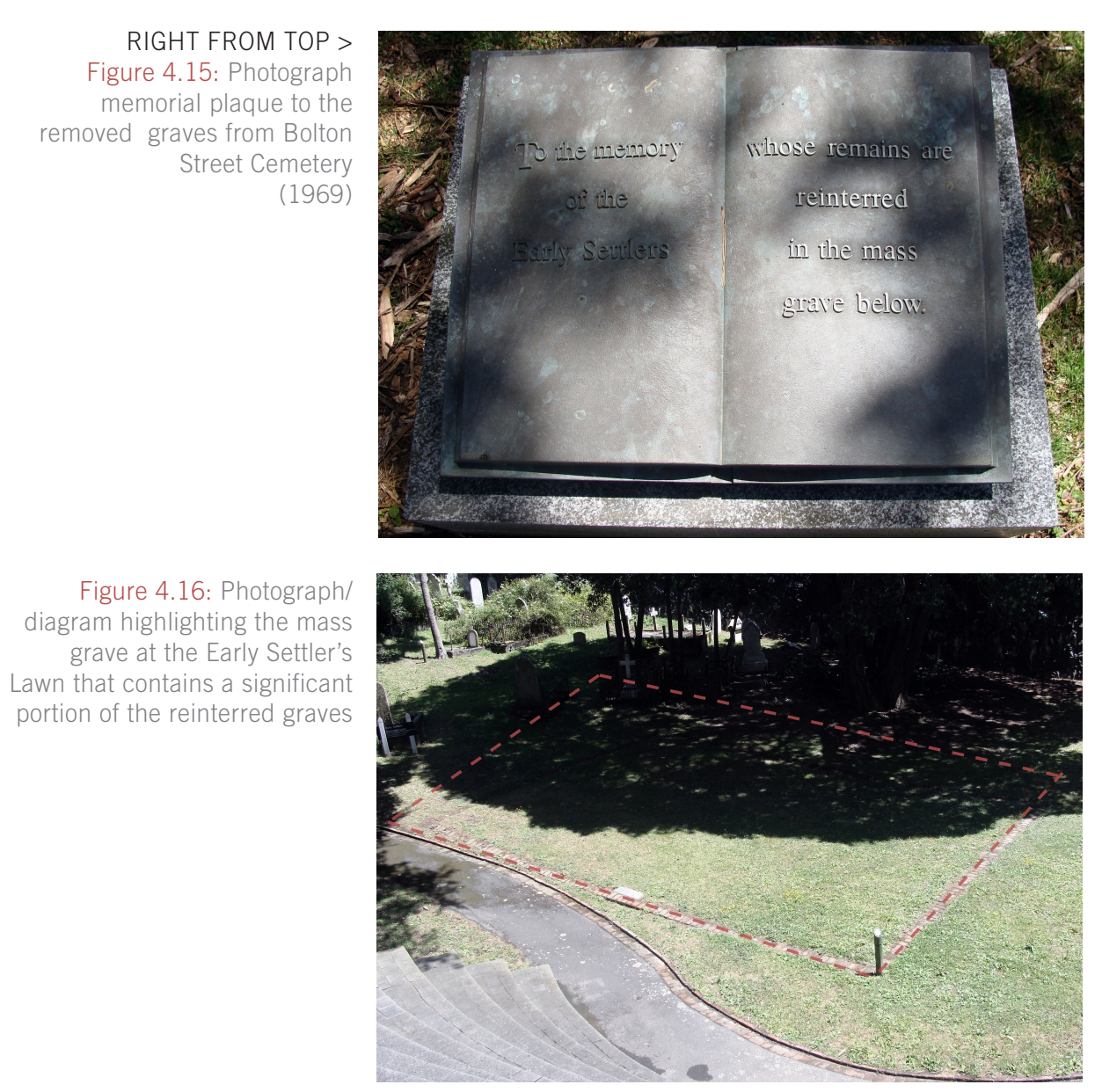

The Thorndon Suburb

In terms of Victorian and Edwardian housing, Thorndon is one of New Zealand's most historically significant suburbs. As home to the country's first settlers in 1840 , Thorndon was established as the location of many of the country's important historic events - many of which are associated with native iwi as Thorndon Flat was a 'favoured settlement for local Maori.'. ${ }^{14}$ Architecturally, the suburb is characterised by the size and variety of its housing types (see Figure 4.17). Towards the end of the early settlement period, at the end of the $19^{\text {th }}$ century, Thorndon had established two distinguishable housing areas - one of wealthy elites in the northern sector and the densely populated working class area to the south. There has been great effort since the 1960 s to maintain Thorndon's heritage fabric and value:

It is a popular tourist destination, and has been the subject of numerous books, three heritage trails, online guides, drawings, paintings and photographs, illustrated talks and, in the case of the Thorndon Society, hundreds of newsletters. Few other historic suburbs in New Zealand have roused so much consistent community interest and support over such a long period. ${ }^{15}$

At the time of the Urban Motorway proposal, areas of Thorndon outlined for demolition were considered to be in decline - accommodating mainly low-middle socio-economic families. As such, the potential demolition of housing stock within the area "would not be a great loss." ${ }^{16}$ Although much of the public outcry revolved around the exhumation and relocation of Bolton Street Cemetery, the motorway construction "forced relocation of hundreds of people". Between 1966 and 1971 as many as 400 dwellings were destroyed, causing the relocation of an estimated 1000 people (and directly affecting 2000). ${ }^{17}$

Following the destruction to large areas of the suburb during Urban Motorway construction, the Thorndon Trust was formed - initiating a renewed interest in heritage value and a resistance to the "formidable adversary of developers." ${ }^{18}$ From 1973 onwards local volunteers and community bodies pressured the city council to regard Thorndon as a legally protected area. These actions resulted in the dislocation of the area from the nearby commercial expansion along The Terrace throughout the 1980s. As a result, the car park now exists as a typological vacuum - a border condition between two strongly identifiable land-use zones - the densely structured commercial fabric of The Terrace, and the lowdensity housing of Thorndon.

\section{The Kumototo Stream}

Prior to 1840 the Clifton Street Car Park was bisected by the Kumutoto, a small stream feeding an outlet at Jervois Quay, which has since become covered "in asphalt and car parks" by the intervention of the Urban Motorway. ${ }^{19}$ Culturally significant, the stream once marked the location of early Maori settlement (the Kumutoto kainga, or 'small village'). The original site of the kainga is now occupied by the Wellington Club (88 The Terrace). In memorial to the relocation of the kainga in 1877, a single Pohutakawa tree was planted, occupying a space adjacent to the club ever since. The Pohutakawa tree stands as a symbol of both the natural location and burial of the Kumutoto stream, as well as the local settlement and colonial
5. Ibid.

16. Ibid, p. 33. 


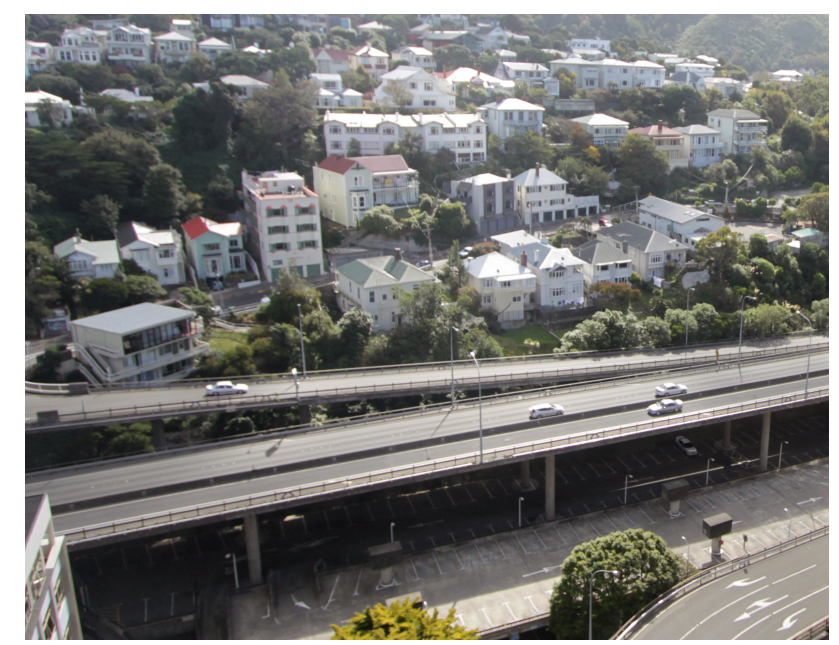

interaction of the Maori people in the area.

\section{The Wellington Cable Car}

Designed by James Fulton, and constructed in 1902 on behalf of the Kelburn and Karori Tramway Company Limited, the Wellington Cable Car provides a direct link between: Lambton Quay, The Terrace, the Clifton Street Car Park, the Botanical Gardens and Victoria University's Kelburn campus (see Figure 4.18). Purchased by the Wellington City Council in 1946, it remains as the only form of preautomobile public transport in the city, remaining as a symbol of Wellington's transition from public to private infrastructure. In 1978 it was converted to a Swiss-designed hydraulic system, and has become advertised as primarily a tourist attraction.

In spite of its economically (tourism) driven context, the cable car currently facilitates a direct transport connection to the Clifton Street Car Park, stopping at the south-western end of the public path that runs Botanical Gardens and is used by a variety of peoples. ${ }^{20}$ Transversely cutting through the site, it exposes users to a cross-sectional view of the border conditions created by the site. Whilst the infrastructure of the cable car has created a decisive 'cut', an end point, to the site - its scale is significantly less imposing than the motorway overhead, reducing the impact of the edge it creates. Additionally, due to its tourist-driven identity, the cable car engages multiple publics passing through the site, and thus presents a potent opportunity to introduce social activity and permeability to the car park.

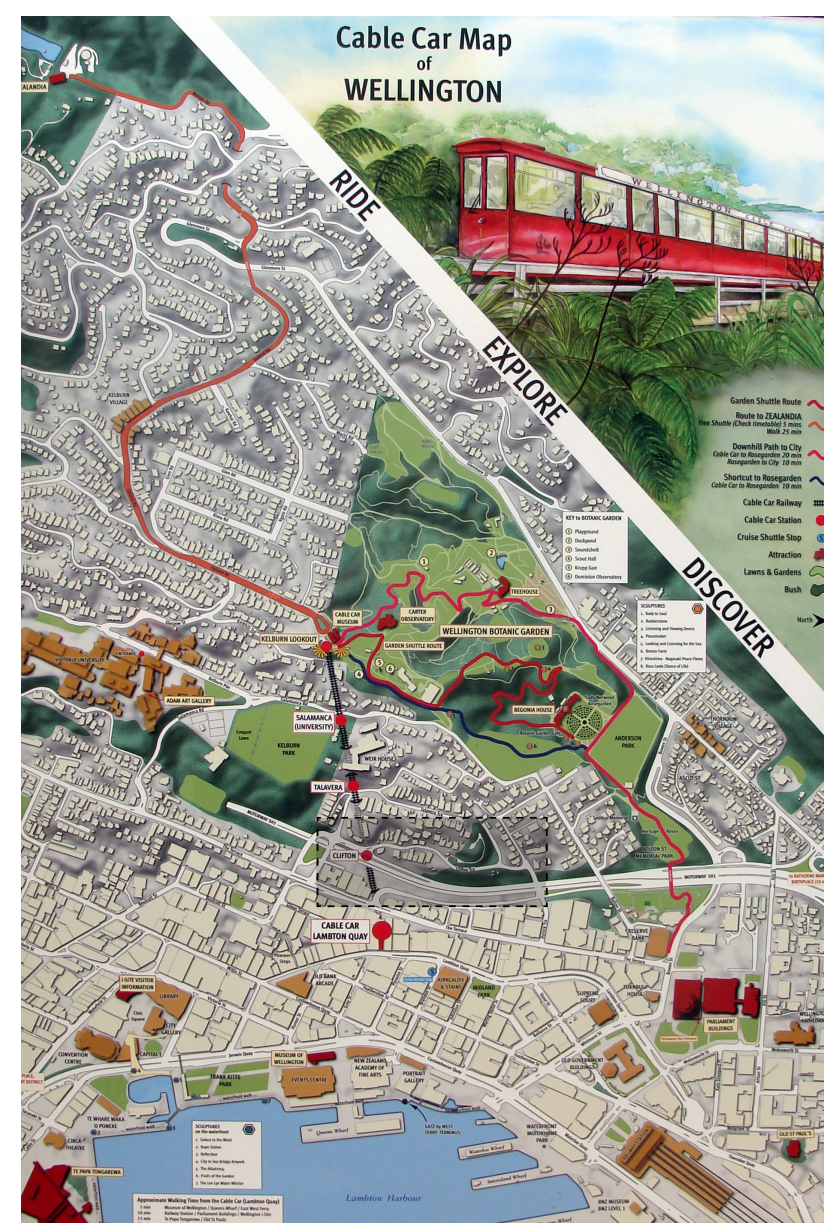

\section{A Political Context ${ }^{21}$}

The political purpose(s) of the Clifton Street Car Park correlates to its position within the Wellington City Council District Plan, which covers strategies and policies of "transport, urban design, heritage, parks and open spaces, and more, delineating future development in the city.". 22 Accordingly, the Central Area is based upon specific zoning allocations which reinforce an urban 'sense of place'.

The desire of the Wellington City Council to prioritise a high-city/low-city urban structure, a visual transition from The Terrace to the waterfront, has left the Clifton Street Car Park in a political 'no-man's land' (see Figure 4.19). ${ }^{23}$ The Terrace forms the backdrop for the 'high-city', leading down to Lambton Quay and Lower Willis Street. It also presents the foothill border for the 'Inner Residential' suburb of Thorndon. In the case of the Clifton Street Car Park, the City Council Design Guides provide little clarity for design as the site is officially located in-between two land-use zones. Importantly, as the thick edge has no zoning itself, it becomes either a non-zone typology or needs to be embraced through
$<$ LEFT

Figure 4.18: Wellington Cable Car map highlighting the location of the Clifton Street Car Park site as it relates to the able car route.
23. Council, "12. Central Area, 2012", p. 13.
21. For the purposes of scope political context within this hesis is limited to provisional nforced by governing bodies such as the Wellington City Council.

2. Council, "1.3 The District Plan in Context", 2012 


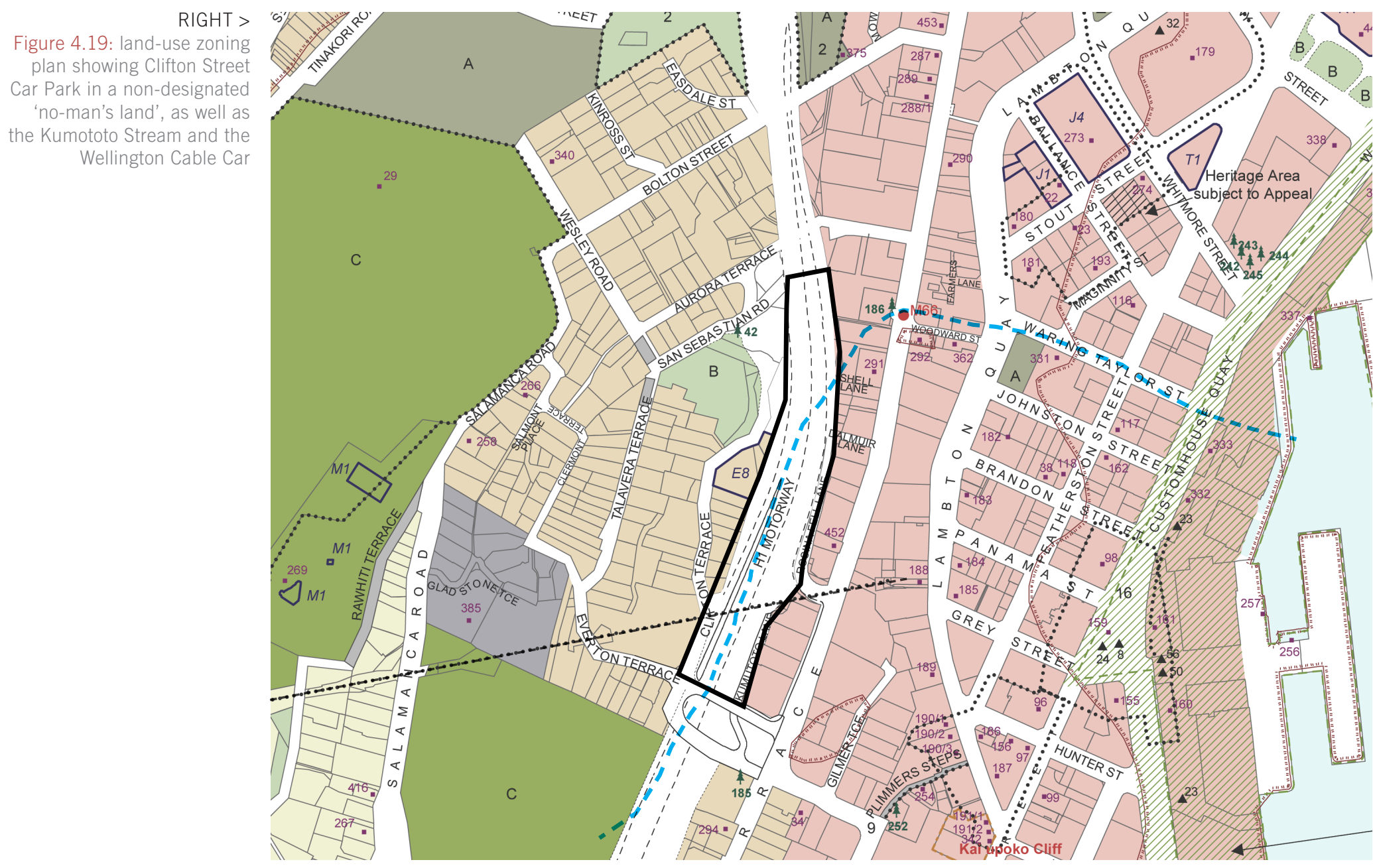

KEY:

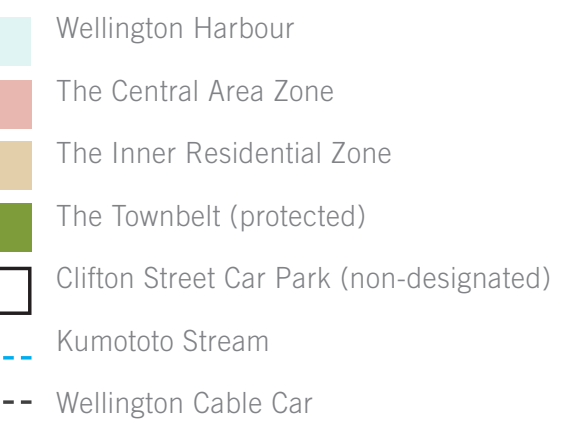

design as a new inter-zone typology. This informs an imperative for the polycontextual approach of this thesis.

Investigating the "Central Area Design Guide" presents the most likely political intention for the site as it isolates the prominence of the built environment in shaping public space in downtown Wellington:

Building design and appearance has a direct bearing on the visual quality of the public environment and distinctive nature of the Central Area. By way proposing design processes that are relational and of example, buildings typically define the edges of public space in the Central Area. This is in contrast to the rest of the city where buildings tend to be surrounded by open space. ${ }^{24}$

24. Council, "Central Area Urban Design Guide", 2012

The guide also provides a number of 'design' specifications that are orientated around context specificity; however, the suggested parameters and design considerations prioritise physical context (height, bulk, form, visual coherency). This approach, although relevant to the visual fabric of the city, is severely limited in scope - suggesting that public space is defined by buildings rather than the confluence of cultural interactions that occur between them.

The Design Guide is a broad document that is inherently limited on a site-by-site basis, especially with regards to Clifton Street Car Park. Posing a critique of this, the thesis proposes to engage critical spatial practices to test polycontextual approaches, flexible. In so doing, this thesis can engage what Nadir Lahiji terms architecture's 'political unconscious', 
25. Lahiii, 2011, p. 4

26. John Key, caused significant controversy with his statement that Wellington is "dying". See: Cowlishaw, Chapman, Hallahan, \&

RIGHT >

Figure 4.20: Diagram illustrating the economic context of high commercial property value instigated by the privatisation of The Terrace. proposing new solutions for the site through a resistance to the political forces that have defined it (or, in this case, left it ill-defined). ${ }^{25}$

\section{An Economic Context}

Recent debate surrounding Wellington's economic prosperity has sparked heated and polarising responses - notably the opinion of Prime Minister John Key that it is a 'dying' city due to the migration of traditional corporate business to Auckland. ${ }^{26}$ Emerging from the vitriol, the need for alternative economic sources was identified as a means to revitalise Wellington's economic frailty - in particular an acknowledgement that Wellington is a "leading centre for creative industries" and therefore needs to expand upon its knowledge-based economic infrastructures. ${ }^{27}$ Relating to this economic vision, the Clifton Street Car Park currently poses an inflexibility - a singular economic function that is service-based.

As one of three council-owned car parks in the Wellington city centre (alongside Civic Square and the Michael Fowler Centre car parks), the multilevel facility has both covered and uncovered parks with reserved parking prices ranging from $\$ 225-300$ per month. ${ }^{28}$ There are 562 casual parks, 250 reserved parks and approximately 10 motorbike parks (822 total). ${ }^{29}$ The park facilitates automobile requirements for offices in the surrounding area, predominantly
Rutherford, 2013; and Parliament, 2013.

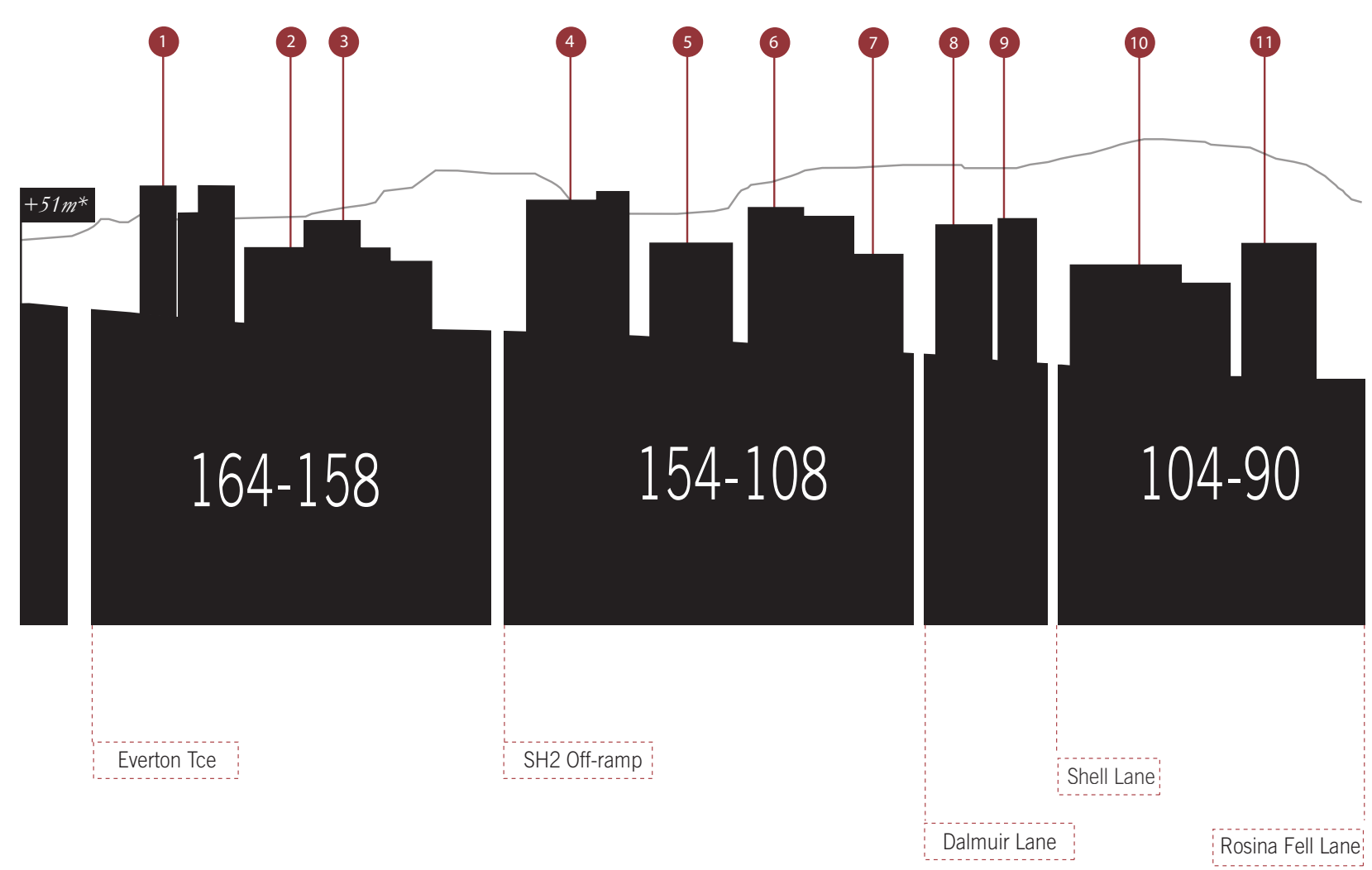

KEY: 'Lot Number (legal desription): Company/function; capital value; rates.'

(1) 164 (LOT 1 DP 349051 AU68): Montreaux Apartments; $\$ 390,000-840,000 ; \$ 1,677.64-\$ 3,462.12$

2158 (LOT 2 DP 52623 - WEDDEL HOUSE): Engineers New Zealand House; $\$ 7,200,000 ; \$ 100,812.97$

3154 (LOT 2 DP 309308): Atrium Tower Apartments; $\$ 200,000-\$ 1,475,000 ; \$ 2,800.37-\$ 20,652.65$

4138 (LOT 1 DP 52252 - SUBJ TO ROW DP 63127): Domainz Ltd; \$15,000,000; \$59,613.60

5132 (LOT 1 DP 52252 - SUBJ TO ROW DP 63127): Joan Stevens Hall; $\$ 15,000,000 ; \$ 59,613.60$

6120 (LOT 1 DP 85832): Quest Serviced Apartments; $\$ 235,000-\$ 280,000 ; \$ 1,063.00-\$ 3,920.50$

7114 (LOT 4 DP 10306): The Terrace Conference Centre $\$ 10,500,000 ; \$ 147,018.92$

8108 (LOT 2 DP 23307): Taupo Central Apartments; $\$ 9,700,000 ; \$ 135,817.46$

9104 (PART LOT 1 DP 7597 LOT 1 DP 23307 - SCIENZ HOUSE): The Terrace Eye Centre; $\$ 4,800,000 ; \$ 67,208.63$

1096 (PT LOTS 12 DP 12193 - 20 M2 SUBSOIL BUILDING ON ROAD RESERVE): Transpower New Zealand; \$29,400,000; \$411,652.92

1190 (LOT 1 DP 67607): The Terrace Lofts Apartments; $\$ 570,000-\$ 1,125,000 ; \$ 7,981.03-\$ 15,752.03$ 
The Terrace. As such, the Clifton Street Car Park is a commercial asset to the city council, contributing to the $\$ 7,699,000$ that the Wellington City Council grossed from public parking July $31^{\text {st }}$ to December $31^{\text {st }} 2012$. The total revenue from public parking for the $2012 / 2013$ financial year is expected to be $\$ 15,630,000 .{ }^{30}$ However, according to reporter Katie

30. Chapman, 2013. Chapman, parking occupancy has fallen from $75 \%$ to $58 \%$ since 2010 , the impact of which is visible, noting that "some inner-city car parks popular with commuters, such as the Clifton Terrace car park, were no longer full by $9 \mathrm{am} .{ }^{31}$

31. Chapman, 2013

Additionally, the car park is positioned next to high-value commercial property that significantly contributes to the identity of The Terrace as Wellington's financial core. Isolating buildings that are directly adjacent to the site (lot numbers 164 90), reveals capital values that range from $\$ 4.8 \mathrm{M}$ to $\$ 29.4 \mathrm{M}$ (excluding individual apartment values) (see Figure 4.20) Most of the adjacent buildings function within commercial usage; however, recently many have been converted into high-end apartment complexes as companies shift towards desirable waterfront locations. In stark contrast to this, the legally protected town belt (directly west of the car park) holds no specific commercial value and is considered to be of 'priceless' cultural value.

In line with this thesis, David Cunningham argues that the emblematic significance of architectural production as a critical investigation has its immediacy in the "seam it shares with the economic"."32 For Cunningham, through economic

32. Cunningham D, 2007, 36. David Cunningham Professor of Philosophy a the University of Westminster,

London. re-configurations of sites, architecture can "articulate the existing social tensions" of a particular context, in a subtle gesture, shifting the urban organisation of a site towards the immediacy of the social..$^{33}$ Thus, architectural intervention within the singular and infrastructure-based economic context of the Clifton Street Car Park Site must reconfigure the economic relations that currently exist - exposing and resisting them - whilst positing a positive 'reconfiguration' towards the social.

\section{A Social Context}

Following the Lefebvrian proposition outlined in Chapter Two, that space is a socially produced construct, the Clifton Street Car Park presents a confluence of possible publics. The potential of the site as a social space is exemplified by the variety of individuals that exist around this thick edge - students, families, business people, homeless persons, tourists (see Figure 4.21). However, the social performance of these individuals is limited by its infill with a distinctly non-social programme - car parking.

Although technically a 'public' site, the car park is characteristic of what Dovey refers to as a private-public condition - it can be accessed by multiple publics, yet its interests are distinctly private. Through economic influence, the site performs a reduced social agency - the capacity for individuals to operate within multiple social contexts as a result of the rules and resources projected into a particular space. ${ }^{33}$ Dovey, through Lefebvre, argues that architecture frames the social structures of everyday life and is therefore capable of 'enabling' or 'containing' the production of relations within space. Based upon this understanding, the social context of the Clifton Street Car Park is particularly constrained, relating directly to the conditions proposed by Hillier and Hanson - that in a system dominated by global-tolocal structures (in this case the urban imperatives of infrastructure and private development) spatial relations are restricted in a negative faculty.

The existing social context of the Clifton Street Car Park poses an opportunity, not only for critical response(s), but for architectural intervention to enable socio-spatial positivity: to create flow between multiple segregated publics that are primarily 'pushed out' by the thick edge.

\section{A Physical Context}

The Clifton Street Car Park expresses a conflicted past of architectural intervention into the naturally occurring geography, namely the incision of infrastructure and developer-driven built 

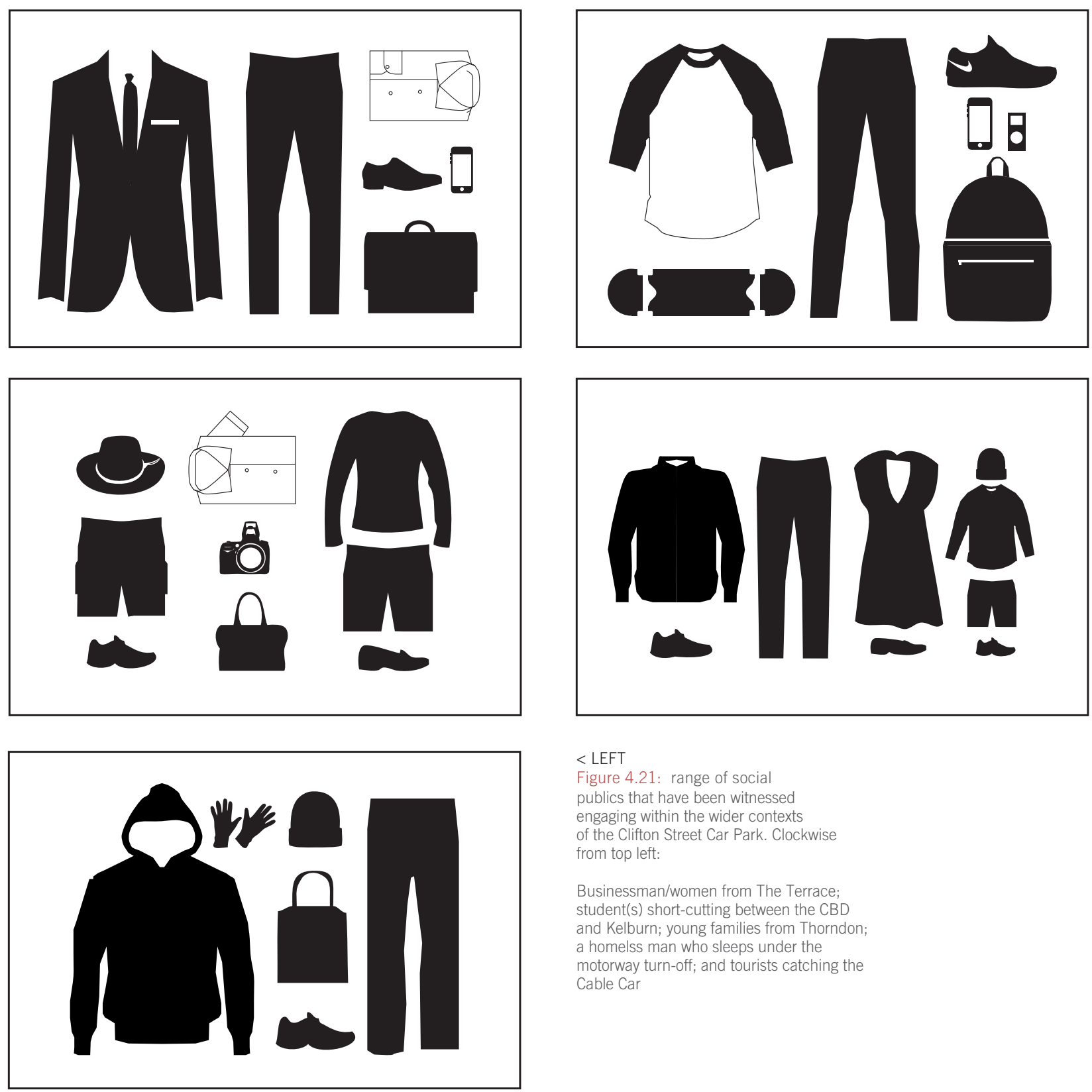

environments. As such, a unique physical landscape has formed, a collection of visual, tactile and structural languages that are in tension with each other.

\section{Typologies and Materiality}

Within the wider contexts of the car park, a number of conditions exist that contribute to the physical language of the site; these are:

Typologies and materials of landscape:

Soil/earth, Pohutakawa trees, Kumototo Stream/ river, foothills topography, bark.

Typologies and Materials of infrastructure:

Shuttered and boxed concrete, shotcrete retaining walls, landscape re-grading and terracing, ramps,

$<$ LEFT

Figure 4.21: range of social

publics that have been witnessed

engaging within the wider contexts

of the Clifton Street Car Park. Clockwise

from top left:

Businessman/women from The Terrace; student(s) short-cutting between the CBD

and Kelburn; young families from Thorndon;

a homelss man who sleeps under the

motorway turn-off; and tourists catching the

Cable Car

parking spaces, excavation, brick follies and toilets, circular columns, strong horizontal platforms, concrete stairs.

Typologies and materials of commercial architecture:

Orthogonal moment-frame construction, steel, expansive glass/glazing, strong vertical forms.

Typologies and materials of residential architecture:

Painted timber, ornamental details, gable rooflines, timber-frame construction.

Alongside typology and materiality, the site exhibits clear and imposing orthogonal order, a strong visual and physical identity that has been created by the infrastructure. 
The existing physical structure of the car park is a complex arrangement of five, vertically stacked, $0.5 \mathrm{~m}$ thick horizontal concrete platforms (used as motorway or parking space) that are supported by a system of $1.50 \mathrm{~m}$ deep two-way beams. There are sixty-nine $1.10 \mathrm{~m}$ diameter vertical concrete columns that support the platforms and motorway, of varying height. Wedged behind The Terrace and running parallel to the Town Belt, these parking areas provide significant gravity-load bearing platforms for architectural intervention and insertion. Additionally, each datum poses a unique relationship to the surroundings, orientating the user to a multiplicity of perspectives.

Moving east to west across the site, Datum 0 is the entry ground plane that is accessed via Dalmuir and Shell Lanes. It has been re-graded into a gentle south-north slope that finishes in a cul-desac (see Figure 4.22). Datum 0 also contributes to the bounded identity of the site as a thick edge - it regraded the ground plane into a slightly sunken slope (see Figure 4.23).

Datum's 1 and 2 are stacked vertically on top of the ground plane at varying heights above Datum $0(3.87 \mathrm{~m}$ and $14.81 \mathrm{~m}$ respectively), with almost half of Datum 1 sheltered above by the unfinished Datum 2. Datum 2 is a completely exposed parking platform, connected (although separated by a fence) to the southbound off-ramp which links to The Terrace (see Figure $4.24 \& 4.25$ ).

The second (raised) ground level, Datum 3, is 2.2-3.8m above Datum 0 (due to Datum 0's decline), and runs directly underneath the southbound Urban Motorway (Datum 4)which is $12.67 \mathrm{~m}$ overhead. This volume creates a canopy effect - informing a gigantic interior space, and creating a visceral verticality and spatial identity above (see Figure 4.26).

\section{Circulation}

There are a number of points of arrival and/or departure that enable or restrict movement through the site. Notably, there are five pedestrian
Cable Car Stop: This 2-way stop is at the southern boundary of the site, connecting users (down) to Lambton Quay, or (up) to the Everton Terrace (second stop) and the Botanical Gardens (final stop). Everton Terrace is at the heart of the Thorndon inner residential zone. The Botanical Gardens, a popular tourist attraction, are within walking distance of Kelburn University.

Northern Stair: This pedestrian (not disabled) access stair is at the northern boundary of the site. Linking to the pedestrian path that follows the Thorndon section of the Town Belt, this stair leads directly to Bolton Street and the Bolton Street Cemetery.

Rosina Fell Lane: A pedestrian tunnel/underpass, this lane allows access from Lambton Quay via the pedestrian Woodward Street.

Shell Lane: The vehicular entrance to the car park, framed by two high-rise structures, it is a precarious pedestrian arrival/departure point. Visually, the entrance is bisected by the motorway off-ramp overhead.

Dalmuir Lane: The vehicular exit from the car park. This exit, similar to Shell Lane, is a vehicle-orientated threshold. It provides a narrow pedestrian corridor and single footpath.

Within the arrival/departure points of the car park, there are several vertical thresholds running longitudinally along the site. Upon entering from the east, the user moves underneath the Urban Motorway off-ramp and through three levels of parking (Datum 0, 1 and 2). Past this point, the site reveals a cavernous void that occupies another ground level (Datum 3) covered overhead by the southbound Urban Motorway (Datum 4). Between these two zones are several up/down ramps that 'tie' the threshold together. Passing through this threshold exposes the vast expanse of the site that runs parallel to The Terraces of the Greenbelt boundary. The volume between Datums 3 and 4 constitutes one of the largest covered spaces in the city; however, it is 
Datum 0; access points to site

for vehicles and pedestrians:

and the remaining footings

from the incomplete Phase Two development

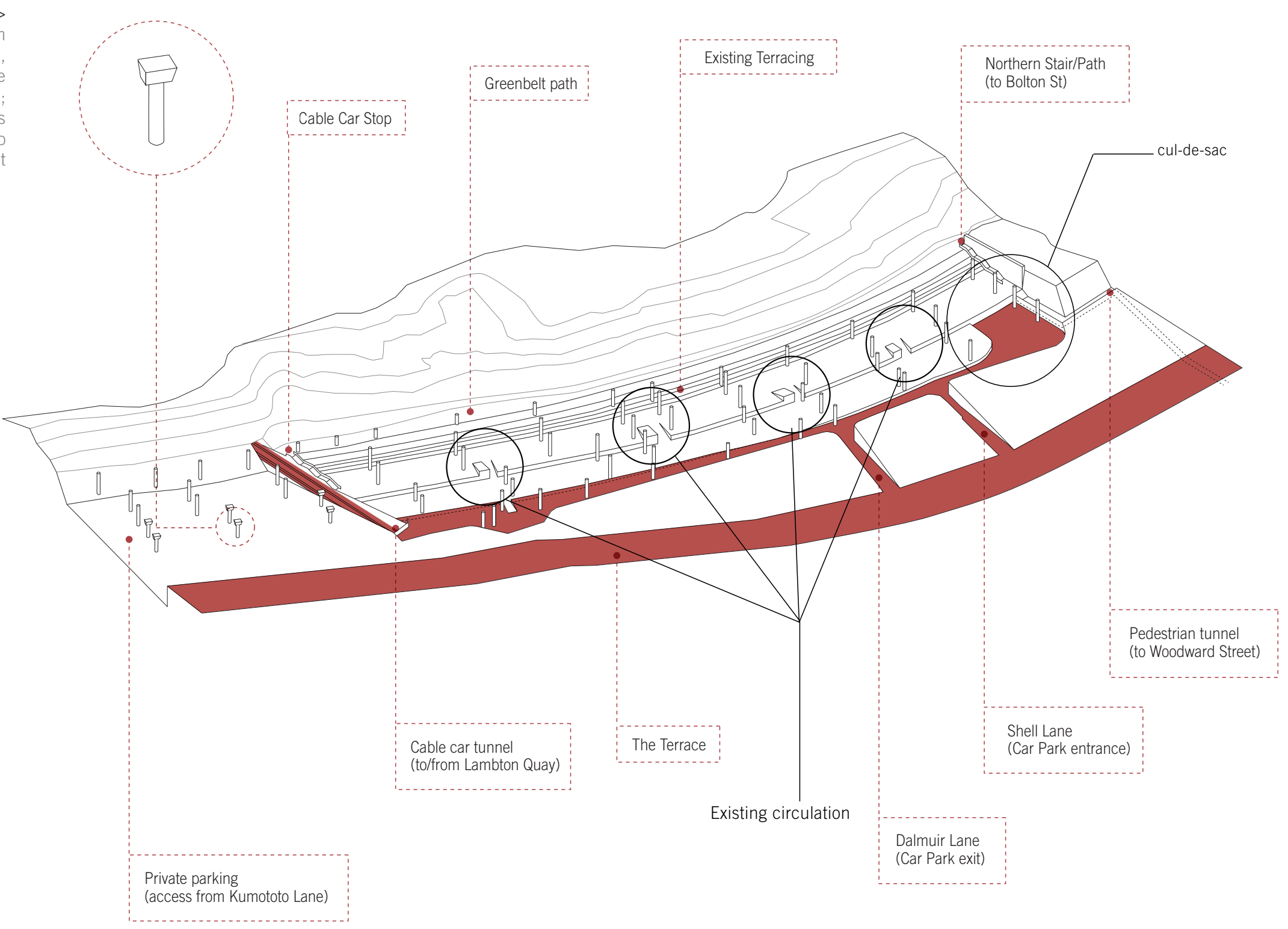

RIGHT > Figure 4.23: Exploded isometric diagram highlighting site as it relates to The Terrace and Town Belt. These two zones inform a visual, physical and zoning 'in-between' condition.

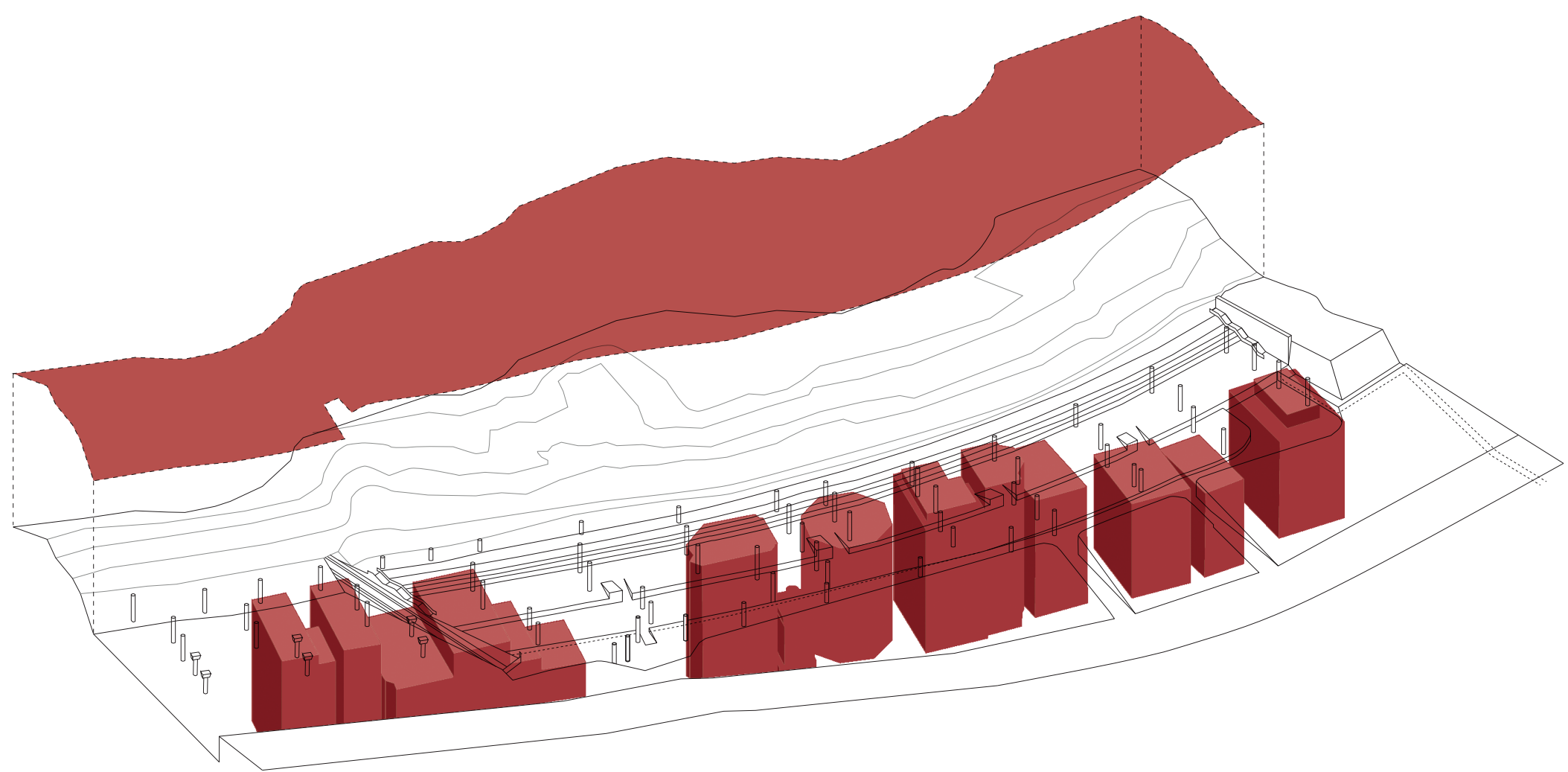


RIGHT FROM TOP >

Figure 4.24: Exploded isometric diagram highlighting site Datum

1 , the partially covered main parking platform that spans the longitudinal length of the site.

Figure 4.25: Exploded isometric diagram highlighting site
Datum 2, the exposed parking platform that is adjacent to the motorway off-ramp.

Figure 4.26: Exploded isometric diagram highlighting site Urban Motorway (southbound), Datum 4 , that forms a canopy above the second ground plane, Datum 3.
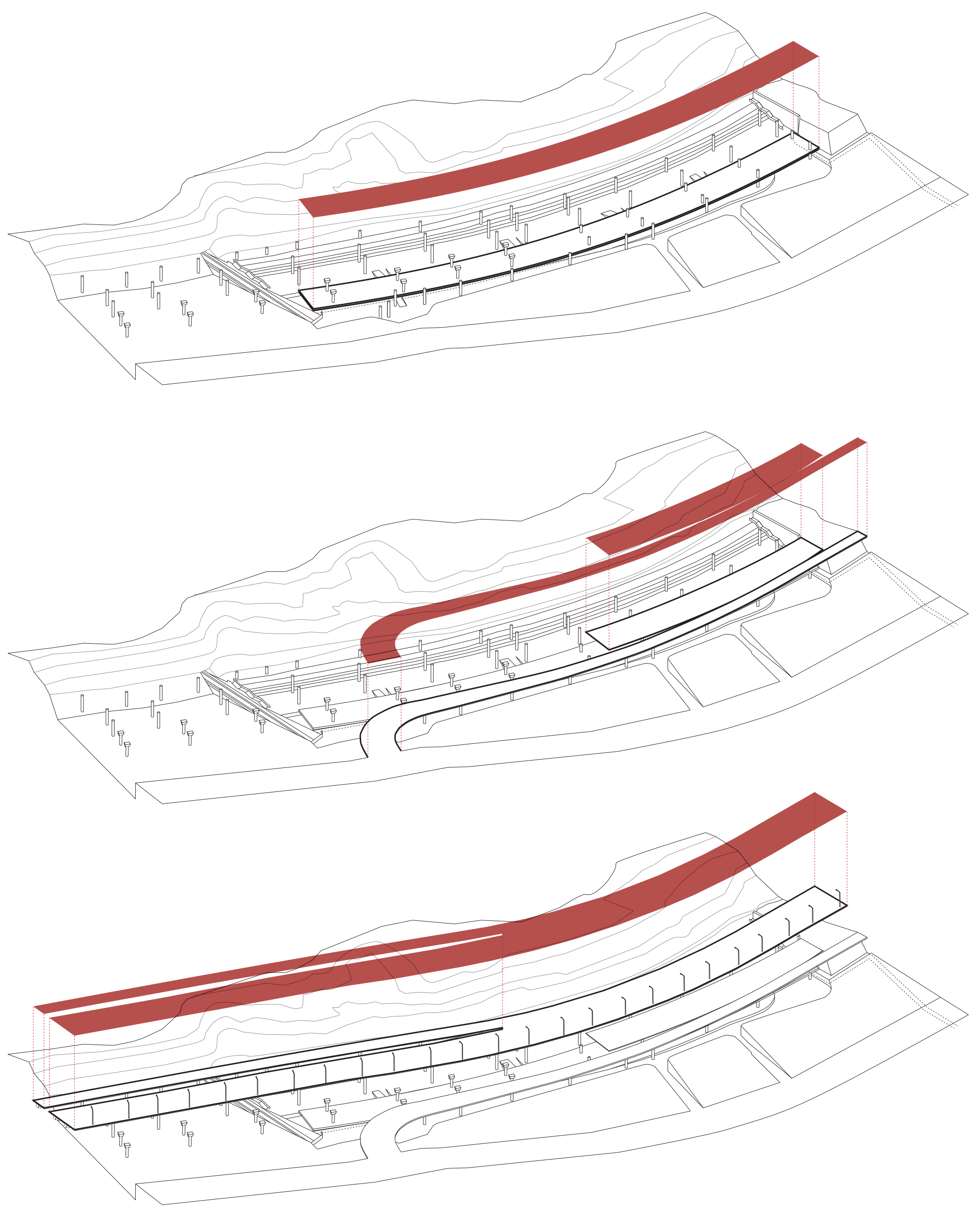
not an 'indoor' space - there is no protection from the elements beyond shelter from precipitation. This volume presents one of the strongest physical opportunities for the site, capable of containing large outdoor events, public activity, and/or small buildings capable of supporting such actitivties. Additionally, this volume forms a strong overhead identity for the site, a large infrastructural canopy (see Figure 4.27).

Infrastructure

Throughout the Urban Motorway and Clifton Street Car Park construction process, intensive excavation and re-grading has flattened, sloped, and terraced the site into an artificial topography. The effects of this are particularly notable on Datums 0 and 3, where the user is in contact with the artificial ground surface. These processes have over-written the contextual histories of the landscape in a tabula rasa maneuvre (the vegetation of Shelly Gully, Kumutoto Stream), informing a relationship between landscape and infrastructure that is dominated by the latter.

\section{Visual Context}

The visual context of the Clifton Street Car Park contributes to the typological identity of the site. Similar to the physical context, the visual constructs the 'image' of the site as an architectural place (explored in Section Two). For the purposes of this thesis, the visual context is considered as a montage - a series of images that are framed by the physical structure (typology and materiality).

When considering the visual context, it is relevant to analyse the Clifton Street Car Park from five montage-based perspectives based upon common circulations within and through the site:

The Entrance Alleys

The Underground Path

The Cable Car

The Northern Stair

Thorndon/Bolton Streets

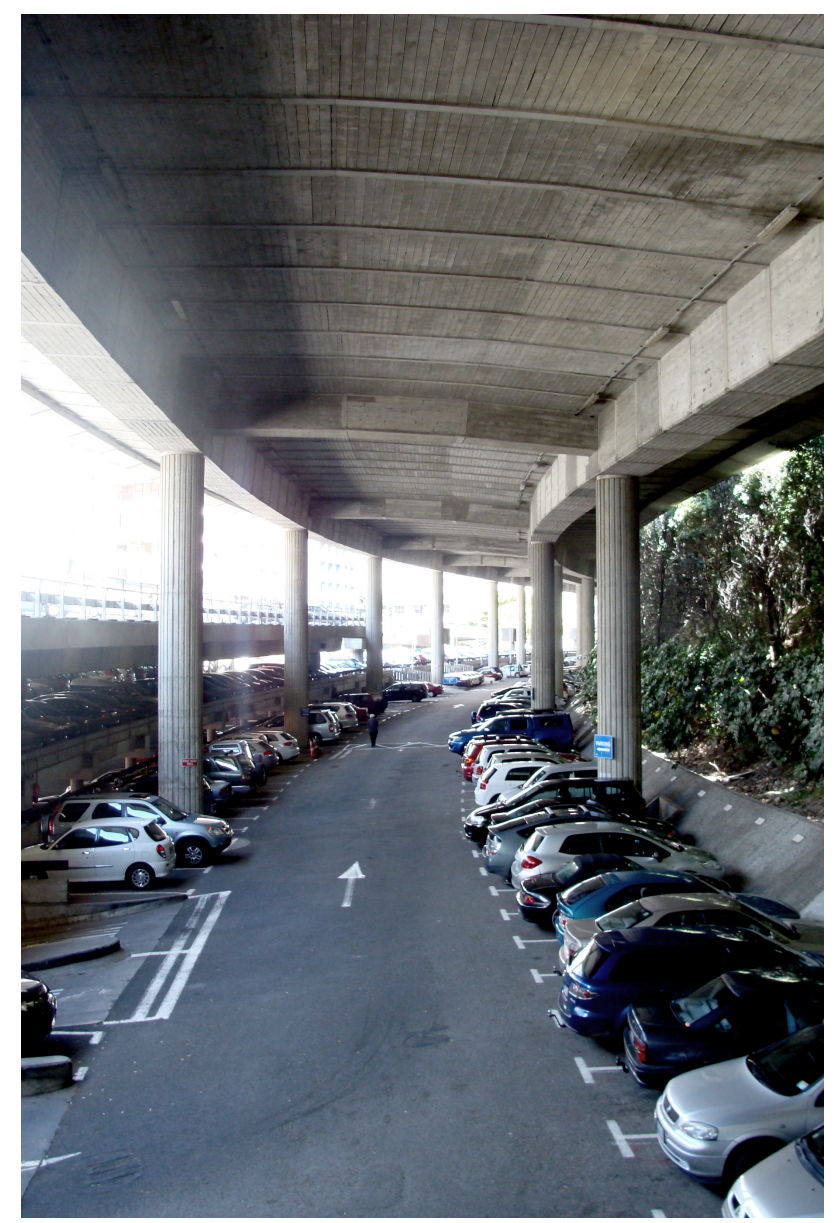

LEFT

Figure 4.27: Photograph ooking south from underneath he spatial volume created between Datums 3 and 4 by the motorway above. The excavated terraces can be seen to the right. Datum 's 1 and 2 can be seen to the left.
Each perspective follows common circulation routes into, through, and out of the site. They show the various typologies, materials, scales and proportions of the existing context.

Additionally, visual analysis attempts to encapsulate social interaction as relating to the site. As such, the five perspectives provided in this chapter are based upon movements of various publics witnessed by the author engaging with the context(s) of the car park in its various dimensions. Within each visual montage, one particular image has been isolated by the author and emphasised. These images relate to the framed threshold points of arrival/departure, framing the visual transition of publics into the site. These perspectives are presented in figures 4.28-4.78. 
Visual Perspective 1: The entrance alleys Tuesday 22/05/2012, 1:37pm

RIGHT, FROM TOP > Figure 4.28: Isometric diagram showing the path followed by visual perspective 1 - beginning in the PWC building, level 8 , and finishing at a car park on

Figure 4.29: technical site plan showing the path (1-1')followed that explores visual perspective
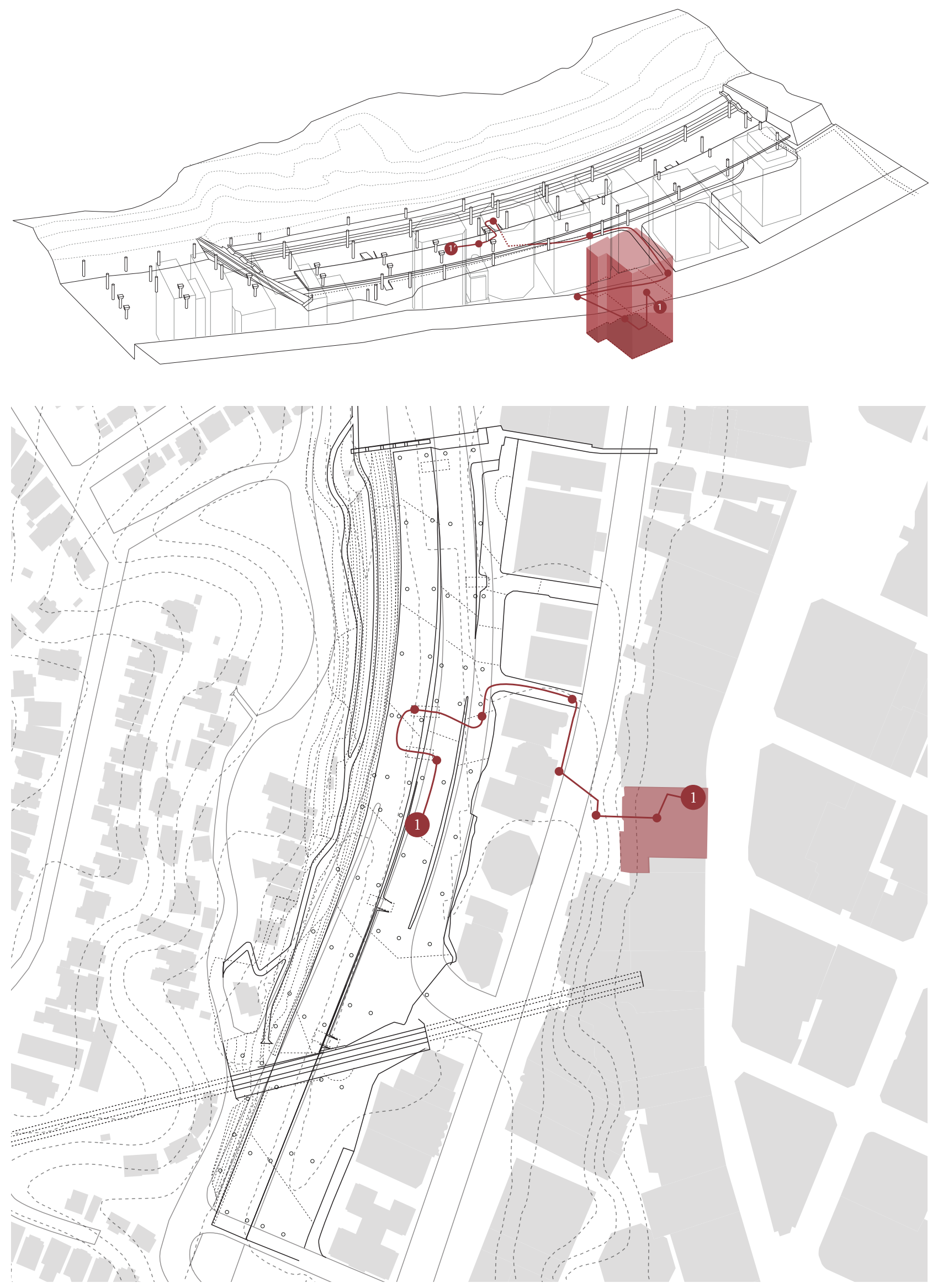

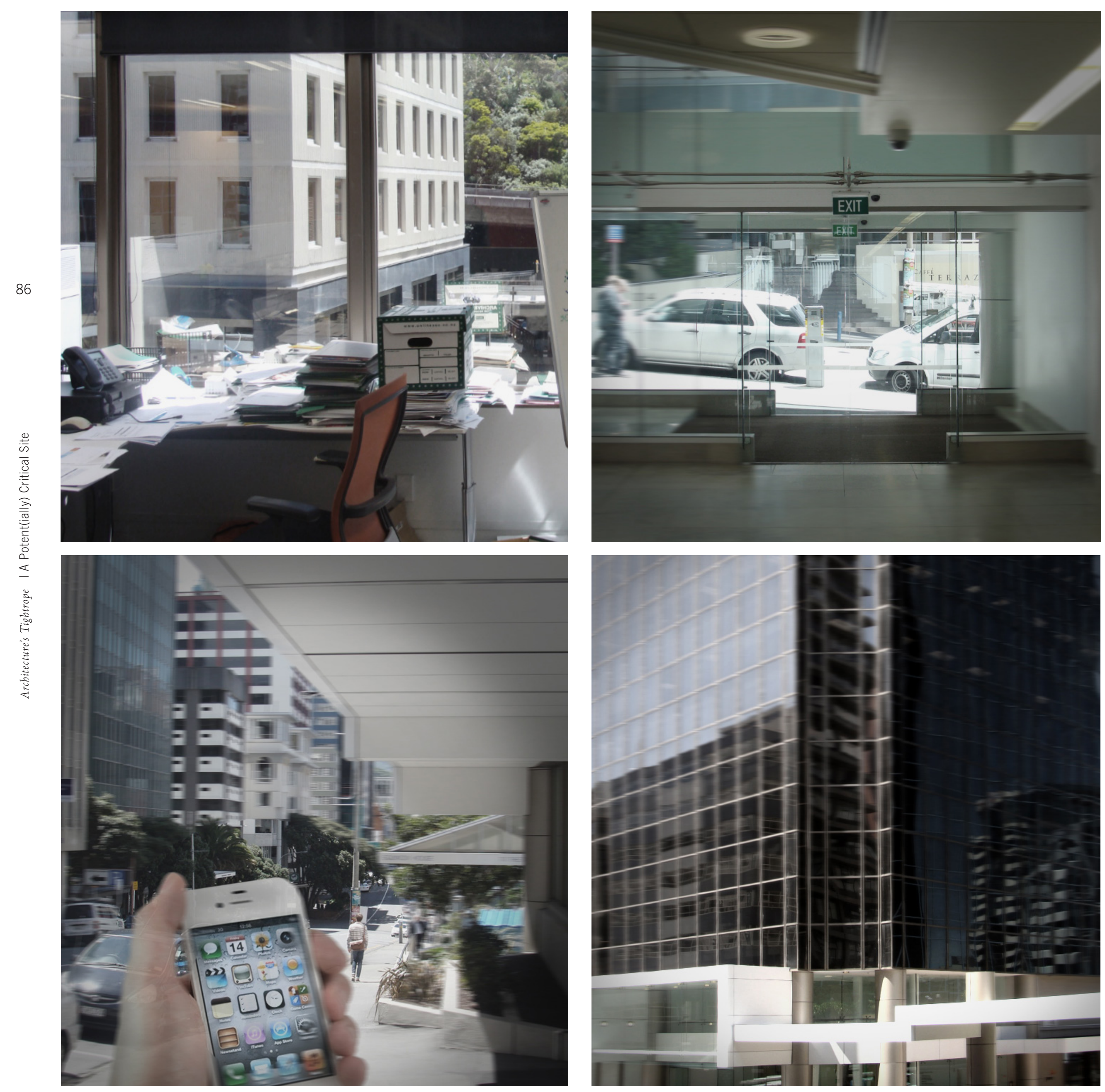
$<$ LEFT, CLOCKWISE FROM TOP LEFT

Figure 4.30-4.33: Photographic

montage of visual perspective

1. The Clifton Street Car Park

(visible in the background

of 4.30 ) appears in flashes

from The Terrace. The strong

orthogonal verticality of the

high rise buildings imposes on

the figure, framing publics at

ground level.

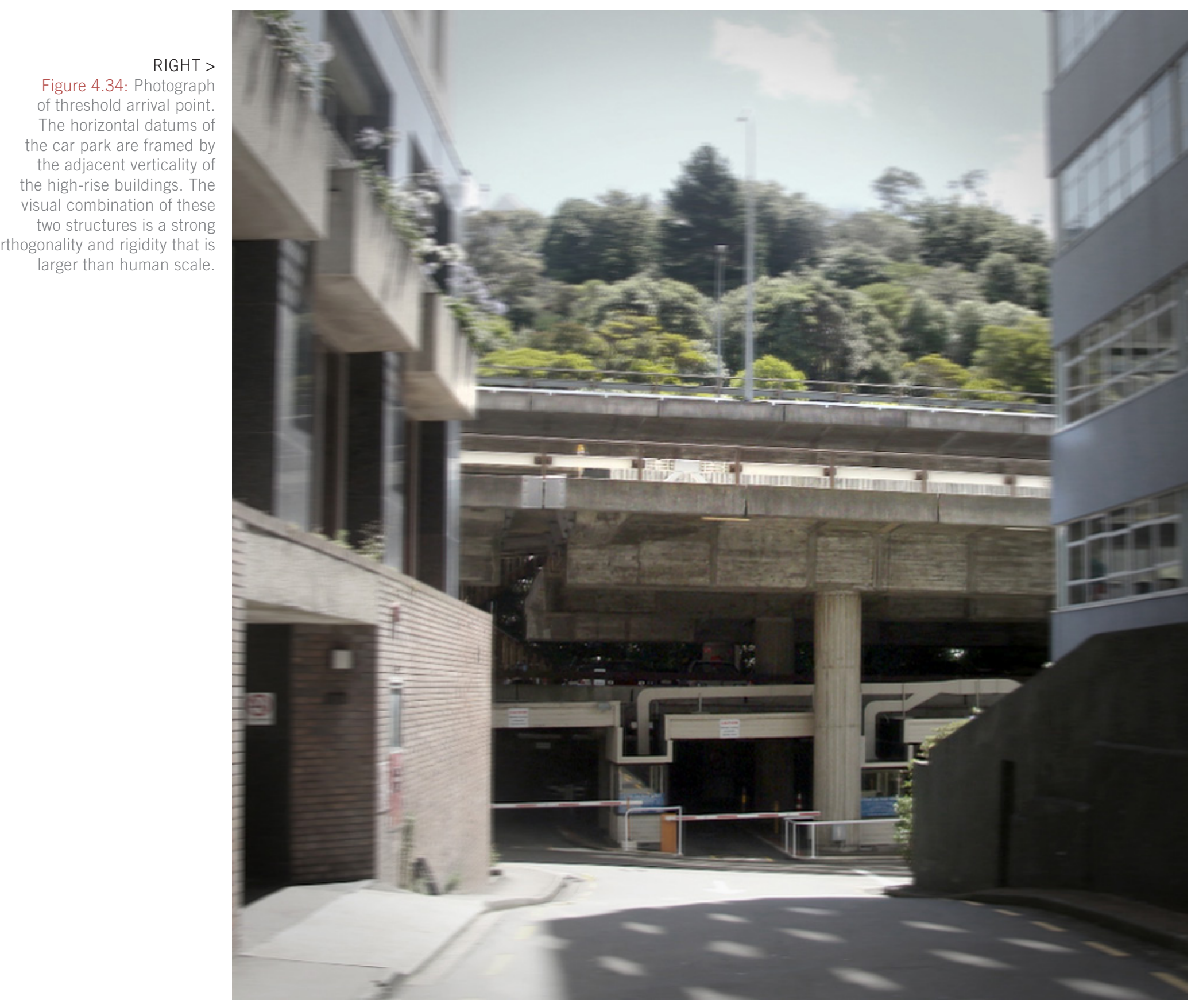

87

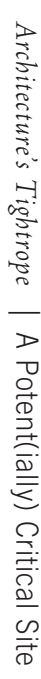

OPPOSITE, CLOCKWISE FROM

TOP LEFT >

Figure 4.35-4.38: Photographic

montage of visual perspective

1 (continued). The orthogonal

rigity and artifical materiality is

continued upon entering the 

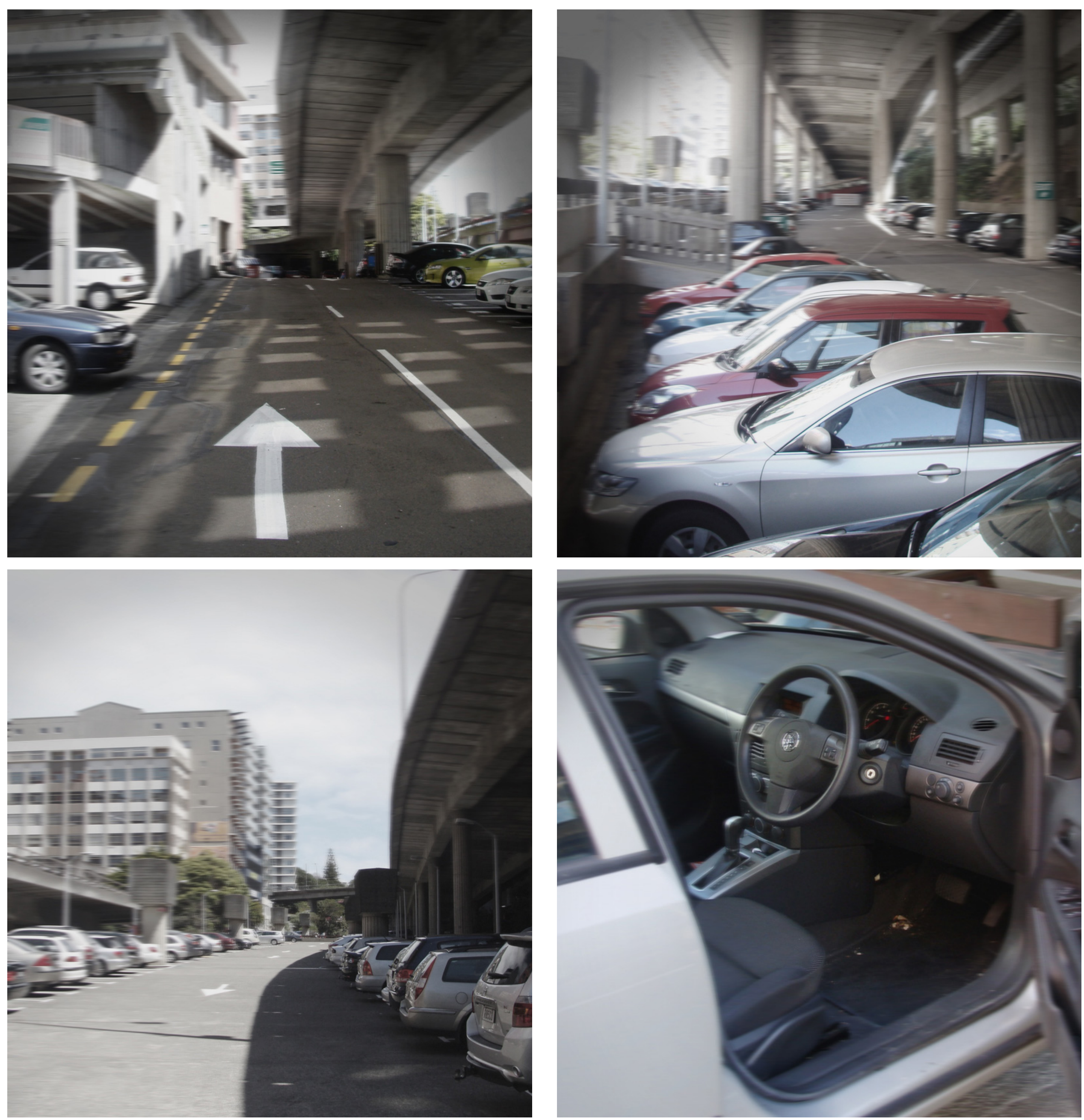


\section{Visual Perspective 2: Underground path Sunday 4/10/2012, 3:51 pm}

RIGHT, FROM TOP >

Figure 4.39: isometric diagram showing the path followed by visual perspective 2 - beginning at the intersection of Lambton Quay and Woodward Street and finsihing on the (empty)

Figure 4.40: technical site plan showing the path (2-2) ollowed that explores visua perspective 2 in montage.
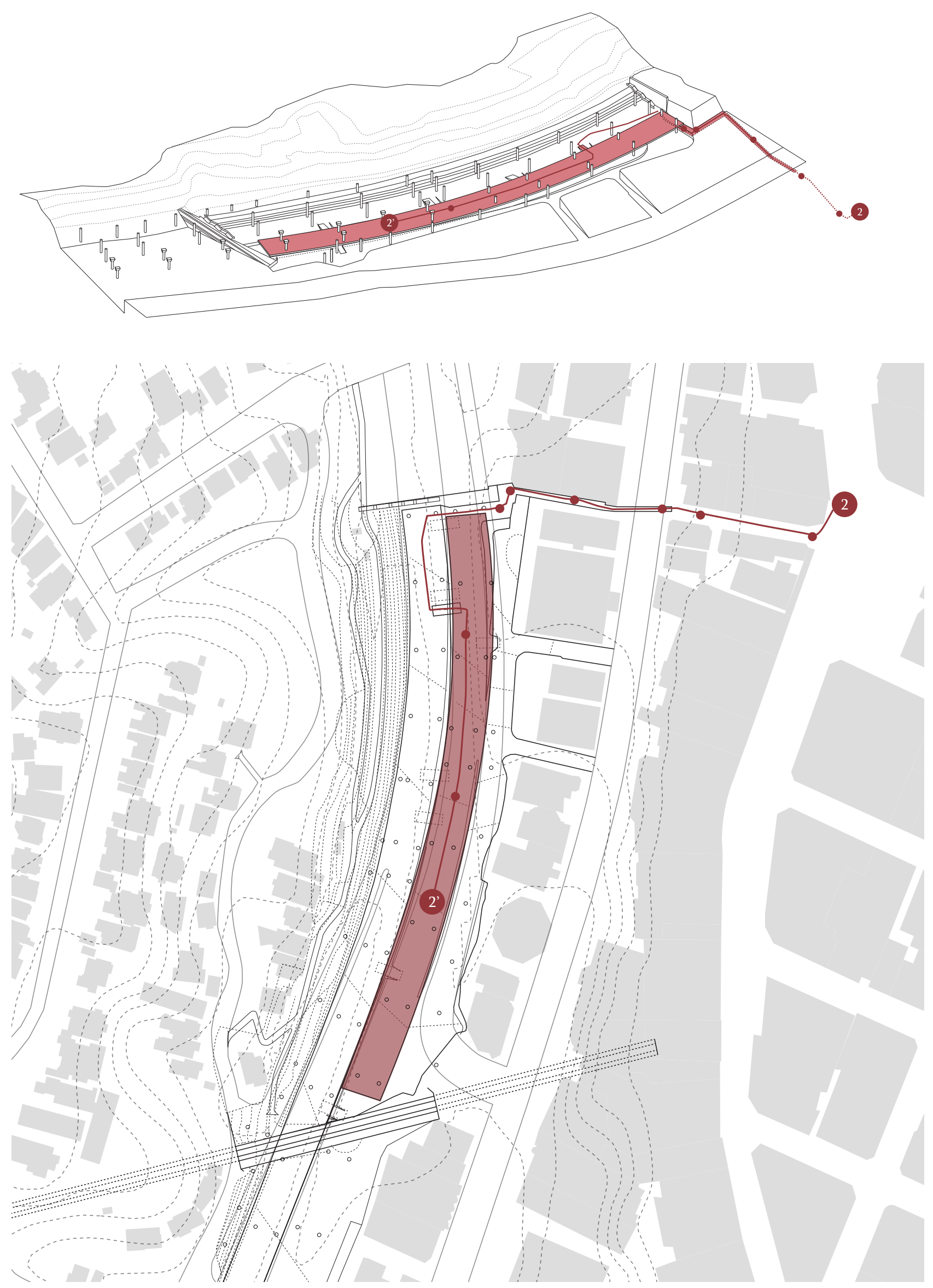

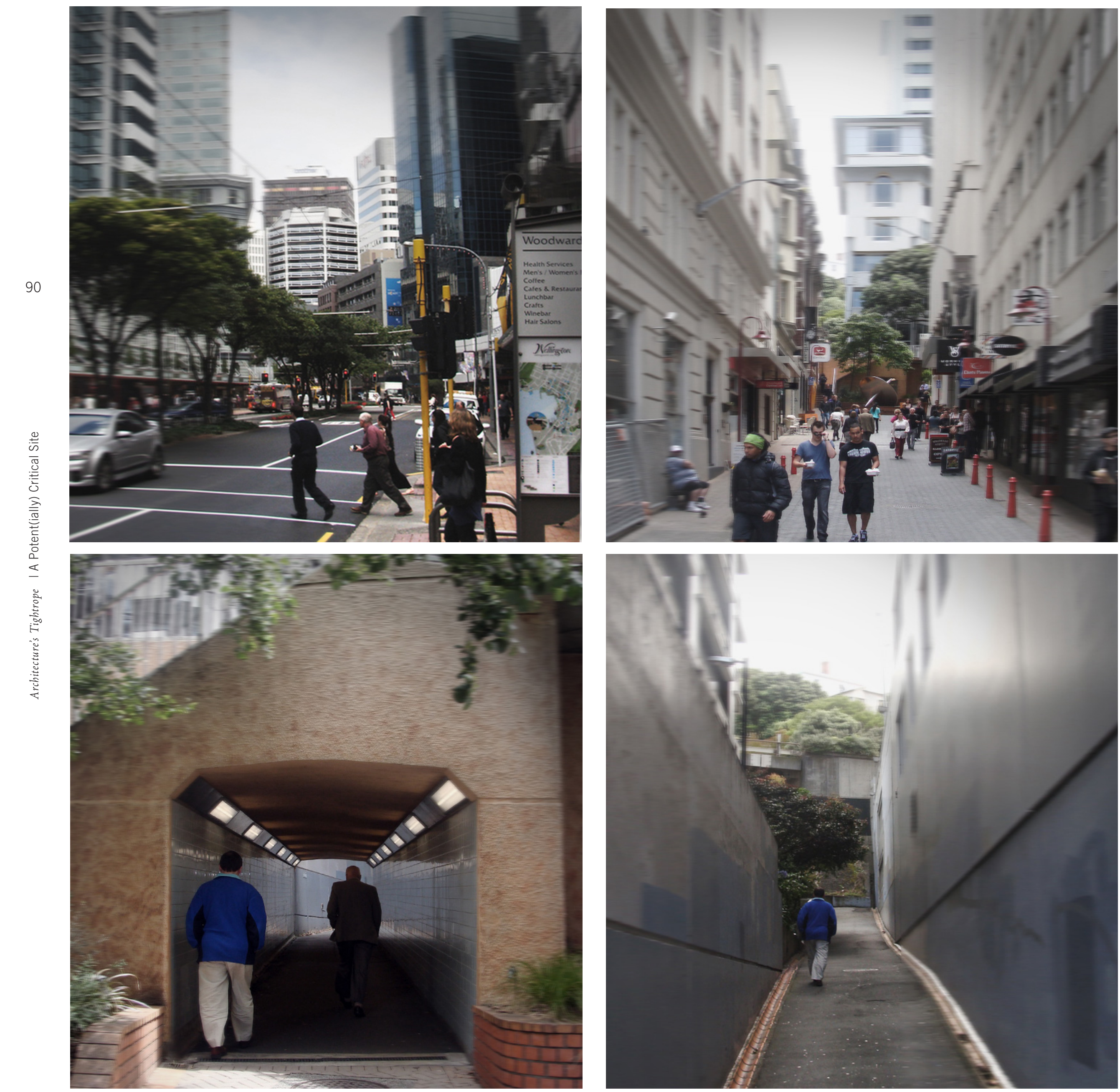
Figure 4.41-4.44: Photographic montage of visual perspective

2. The Clifton Street Car

Park can be accessed via

an underground path that

connects to Woodward Street.

The verticality of The Terrace

is emphasised further in this

perspective as circulation is

residually positioned around

the buildings.

RIGHT >

Figure 4.45: Photograph of threshold arrival point at the northern end of the site.

The horizontal datums of the car park are framed by

the adjacent verticality of the high-rise buildings. The visual combination of these two structures is a strong orthogonality with a visually empty space between them. The infrastructure has clearly 'cut' through the site.

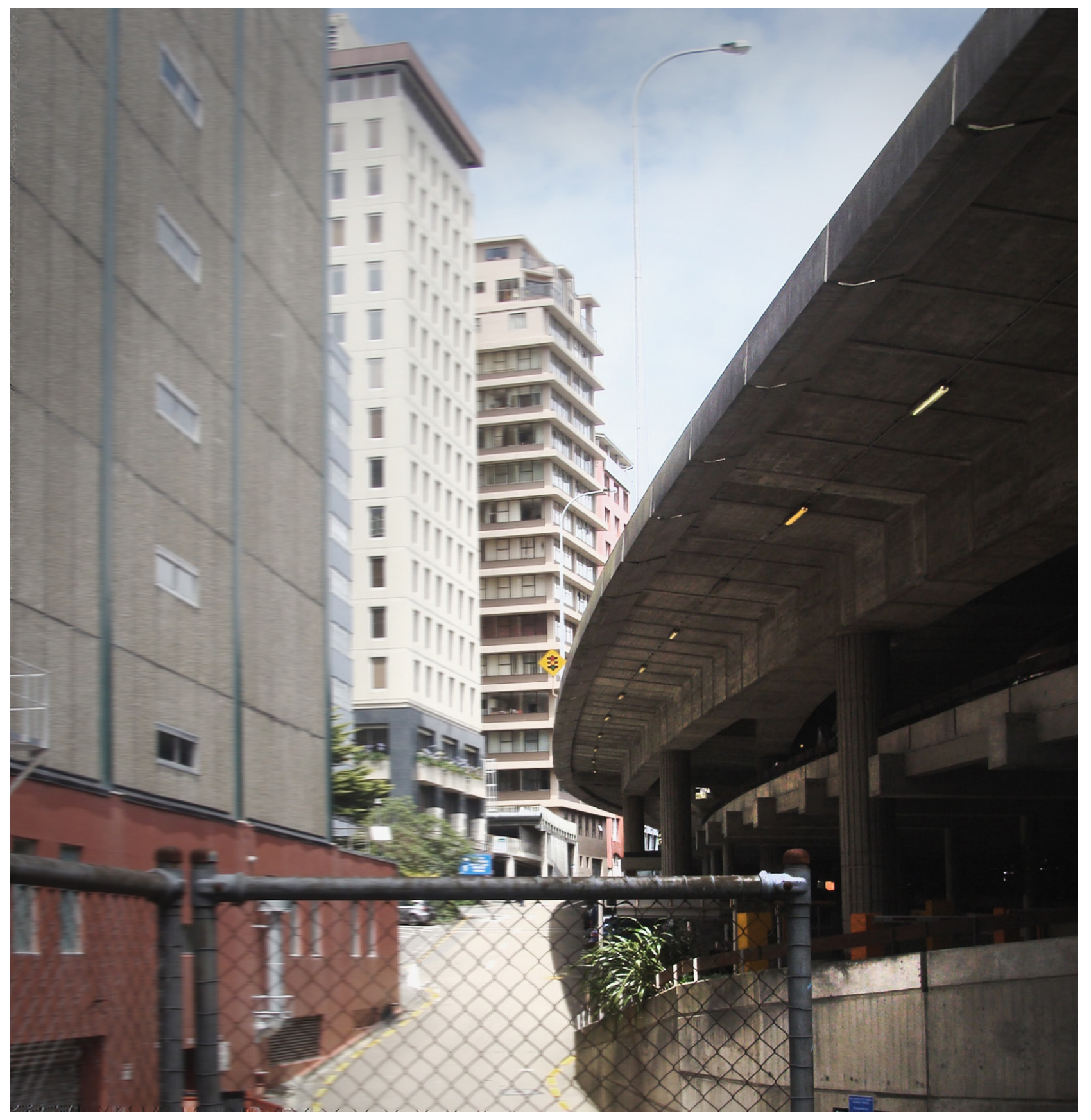



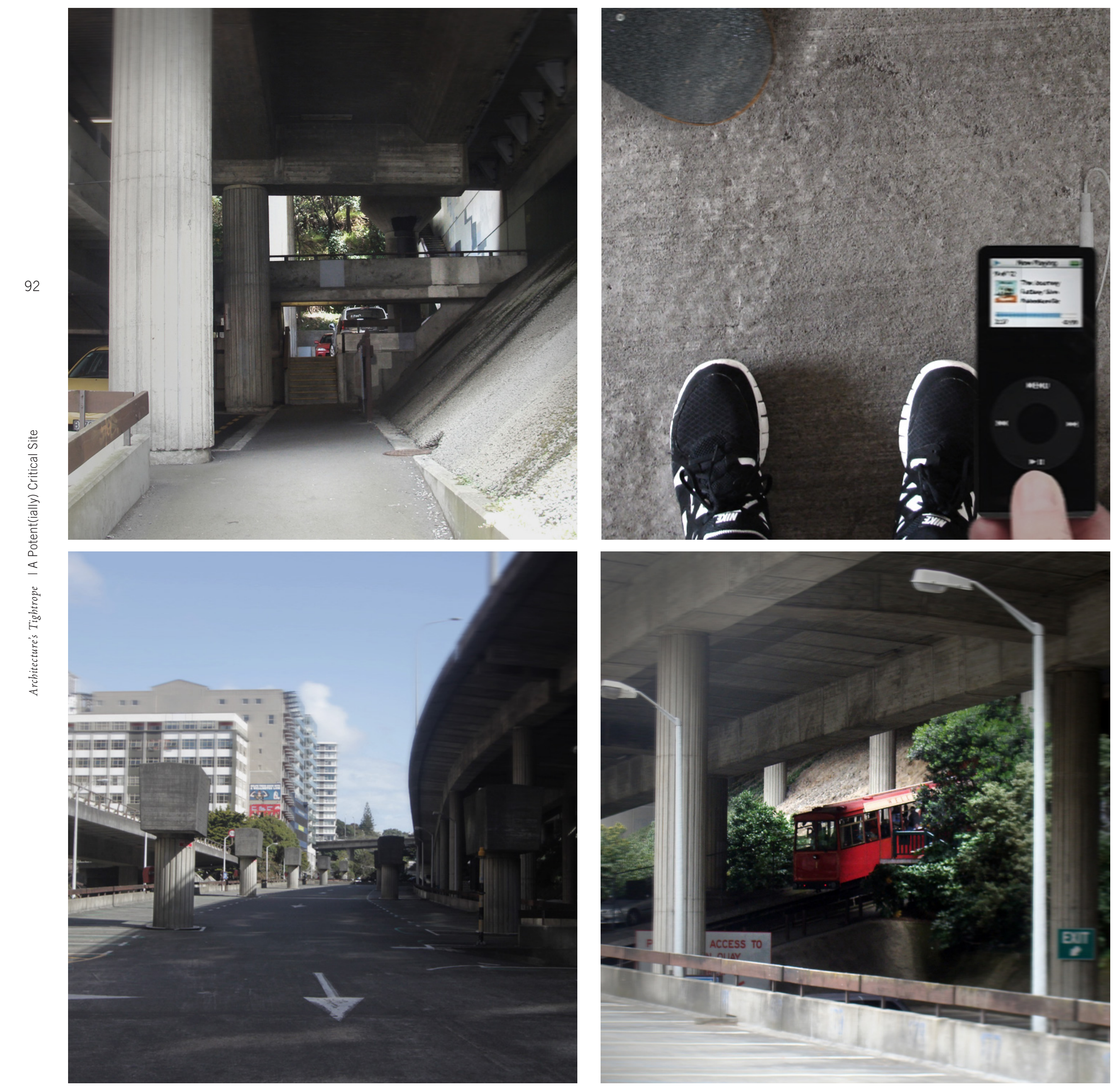
Visual Perspective 3: Cable Car Sunday 03/12/2012, 1:45 pm

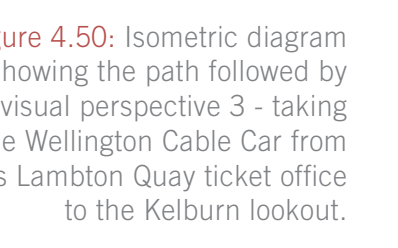

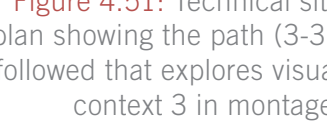
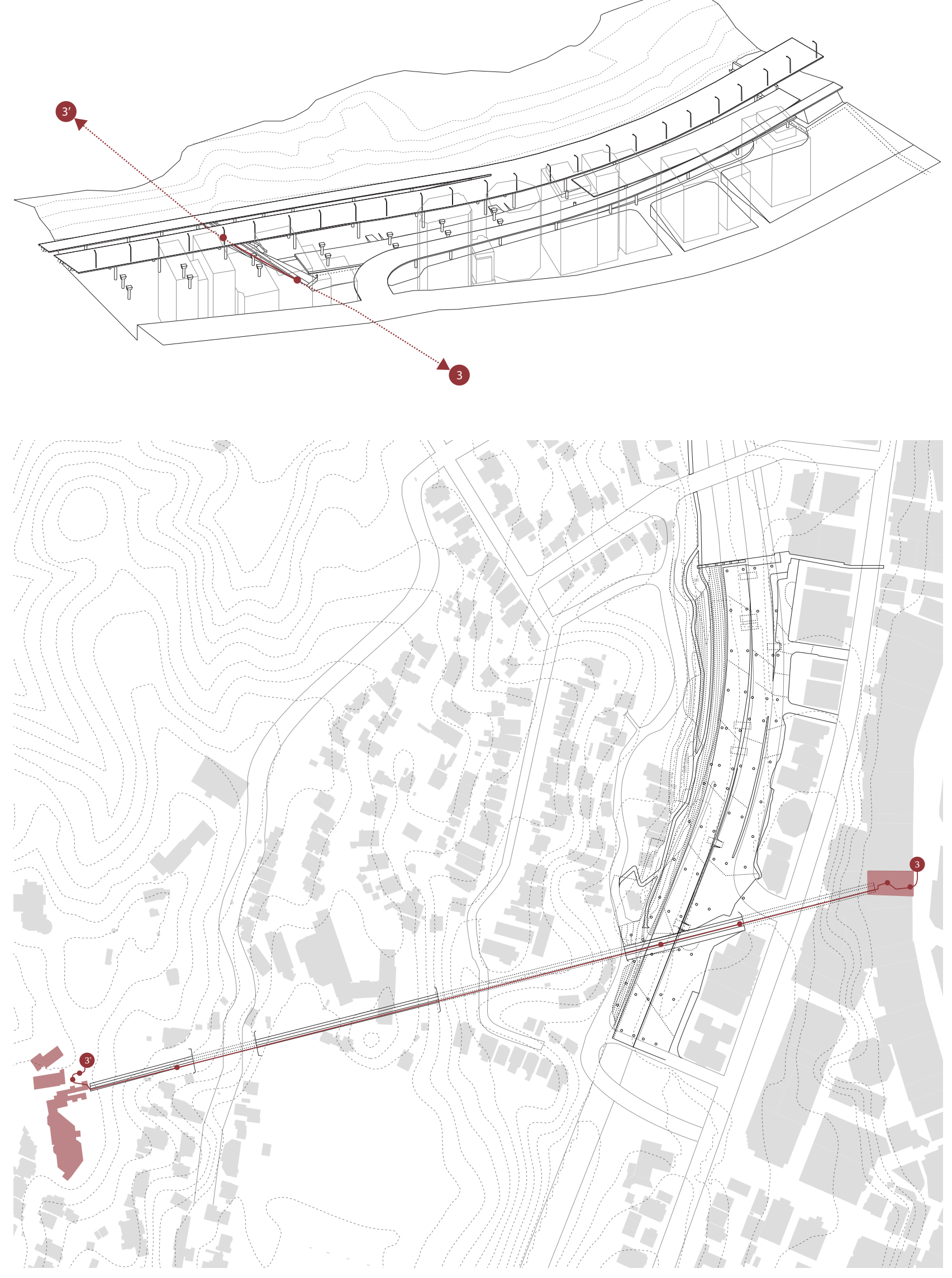

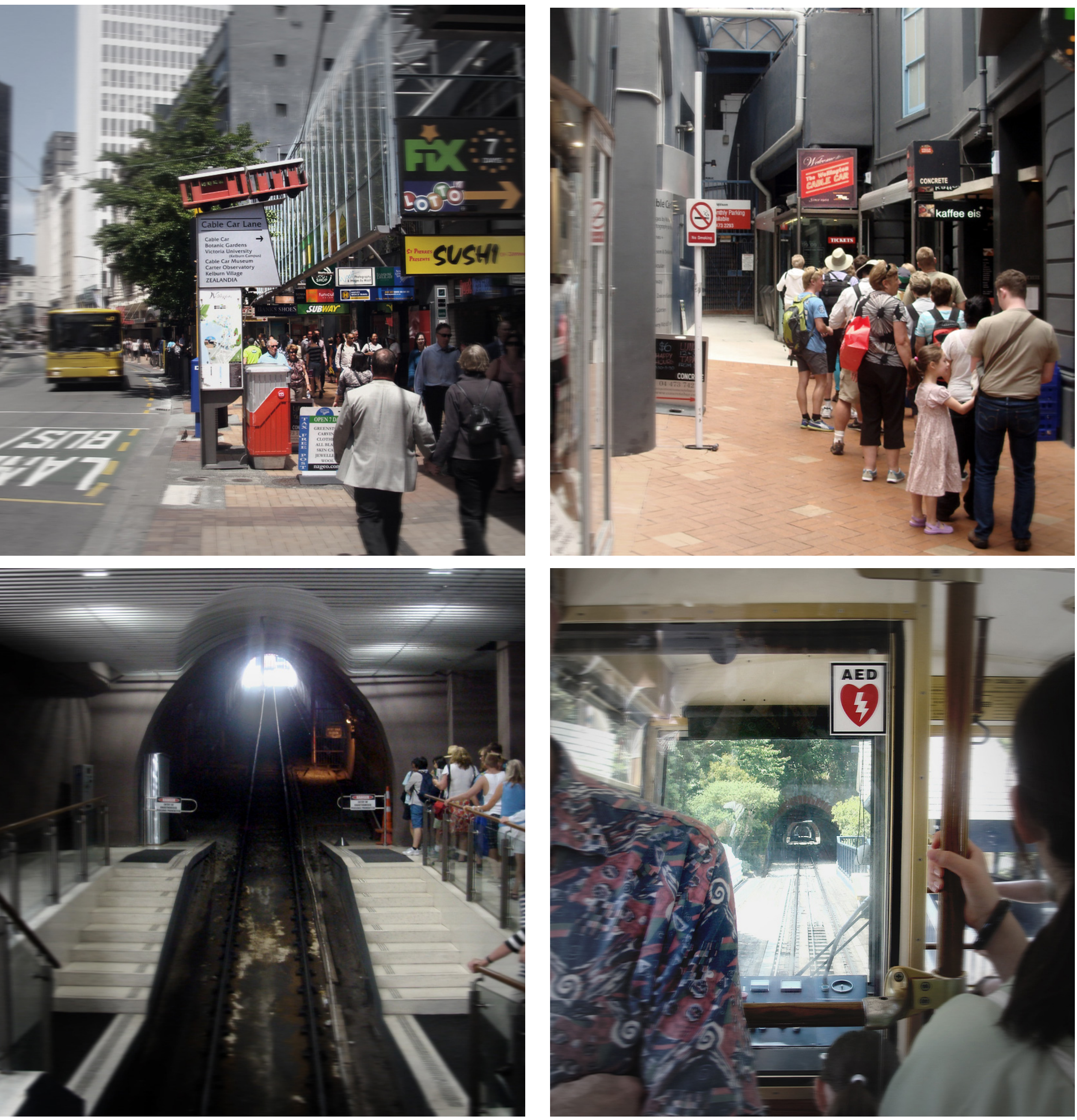
$<$ LEFT, CLOCKWISE FROM TOP LEFT

Figure 4.52-4.55: Photographic montage of visual perspective 3. The Clifton Street Car Park can be accessed via the

Wellington Cable Car. This is

a popoular tourist attraction,

particularly in the summer

months.

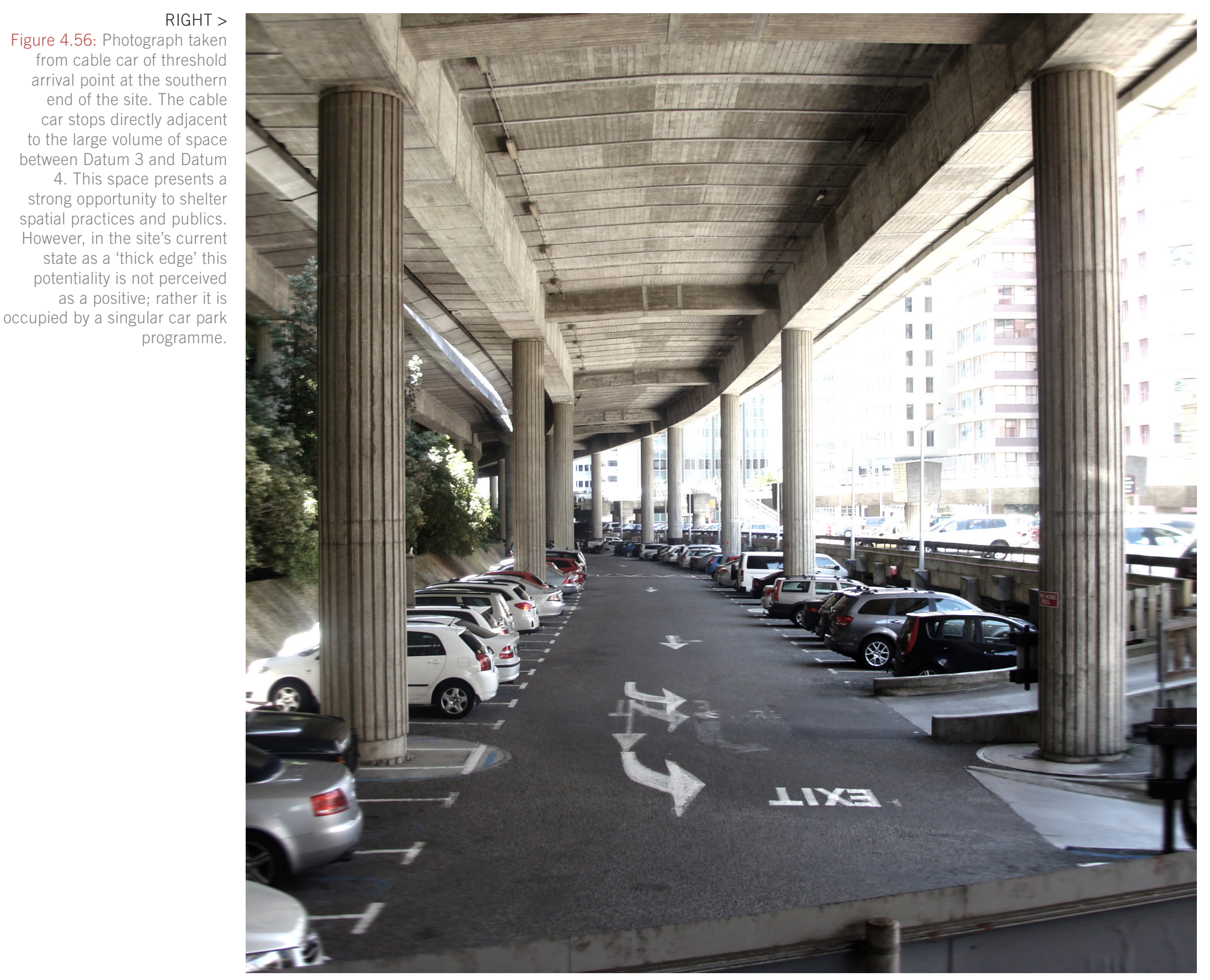



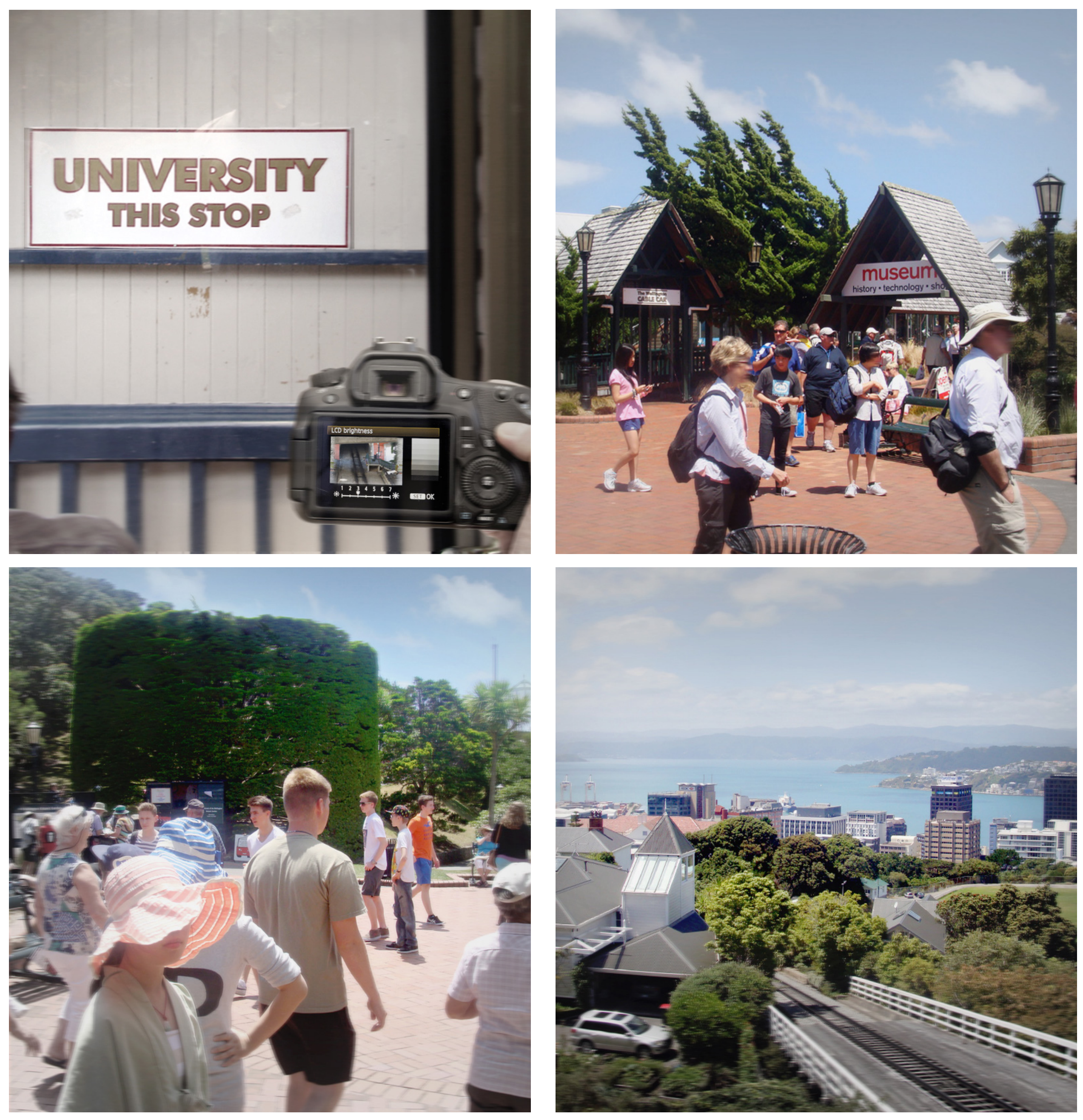
Visual Perspective 4: Northern Stair Thursday 03/04/2012, 8:45 pm
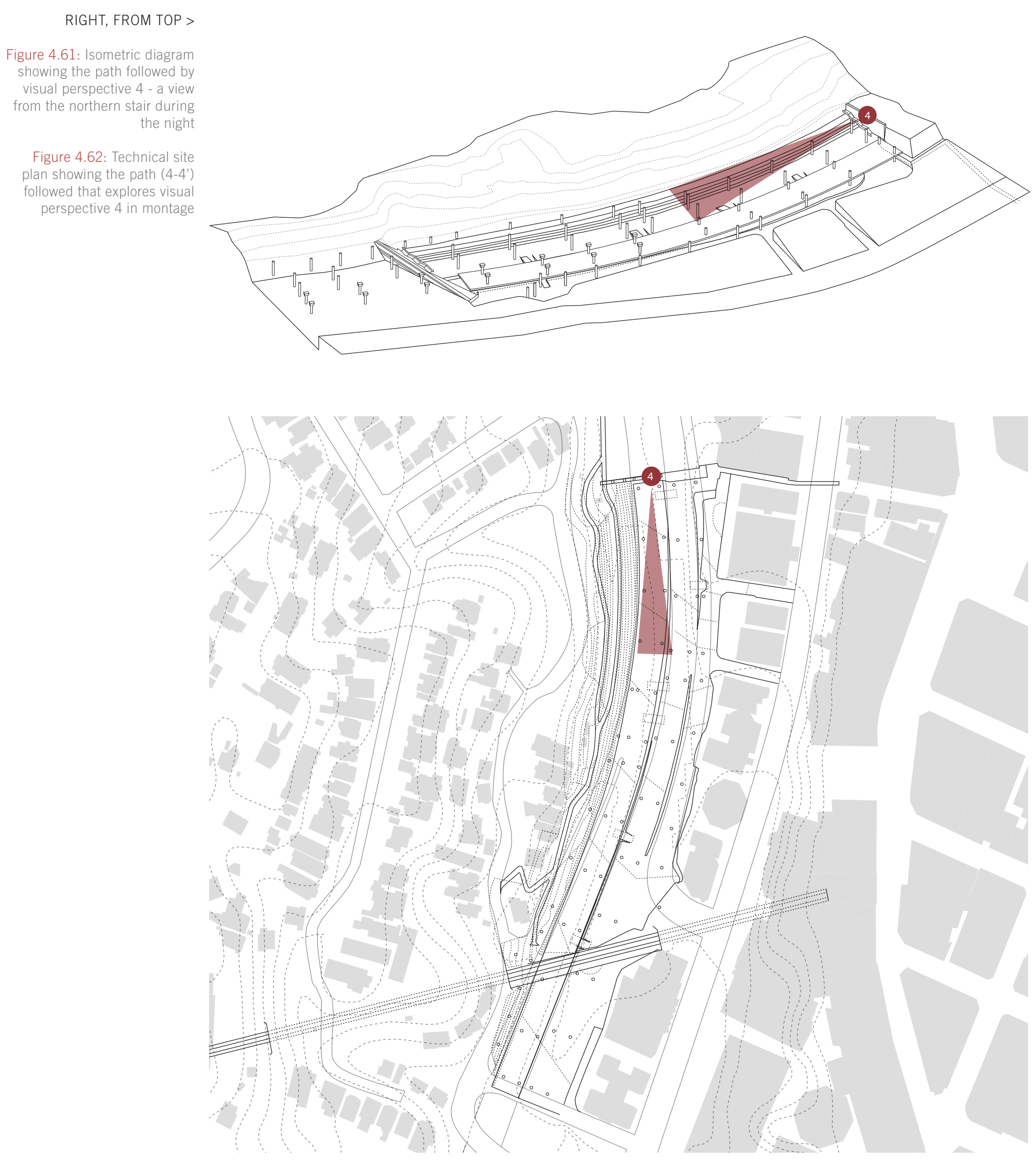
RIGHT > Figure 4.63: Photograph taken from the northern stair in the evening. This threshold shows the relationship between the large, open but sheltered

volume underneath the motorway and the greenbelt (to the right). Additionally, the strong orthogonality of the site

is re-iterated to the left, framed

by The Terrace (vertically) and

Datums 0, 1 an 2 (horizontally).

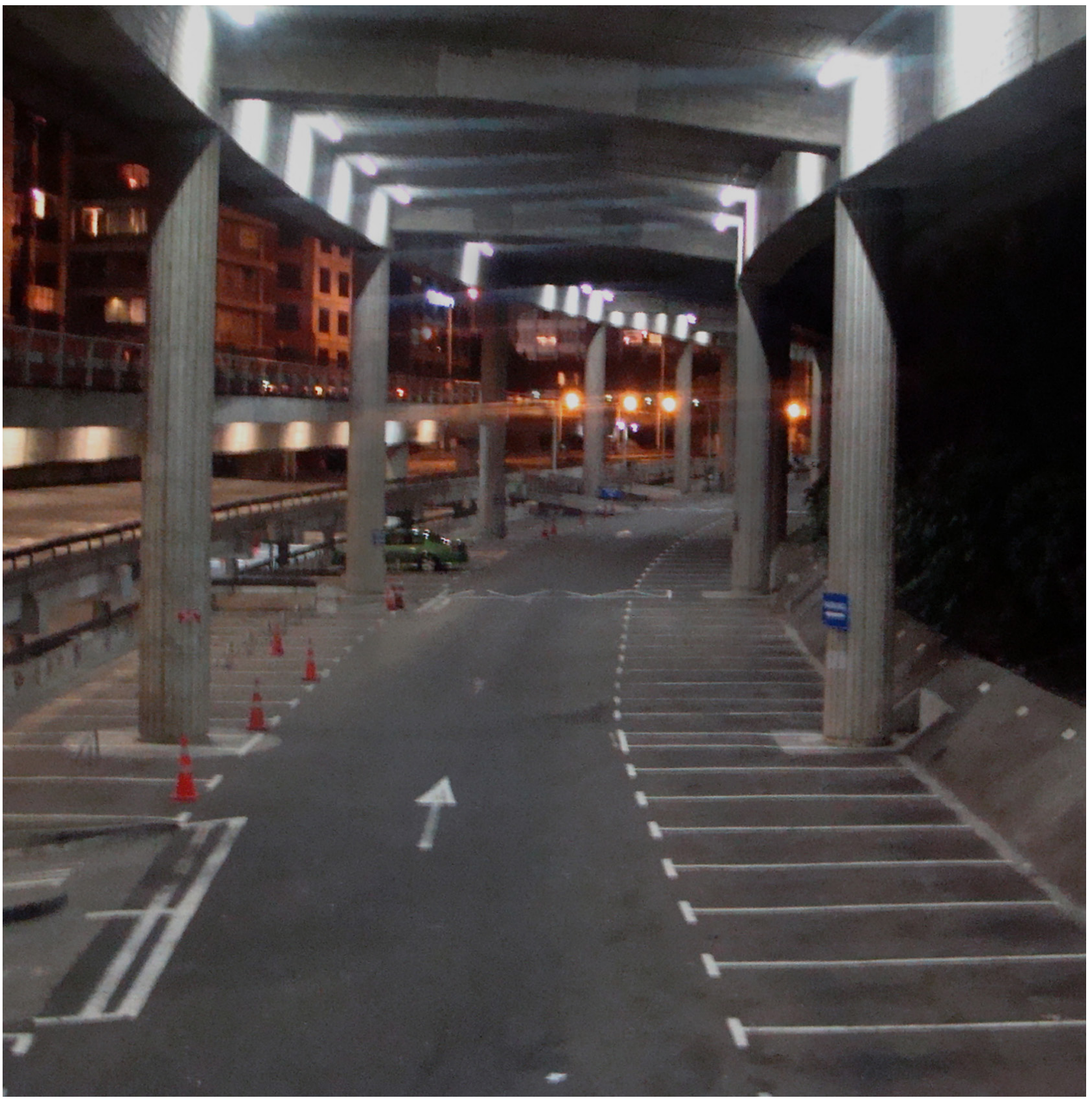


Visual Perspective 5: Thorndon/Bolton Street Saturday 05/04/2012, 1:45 pm

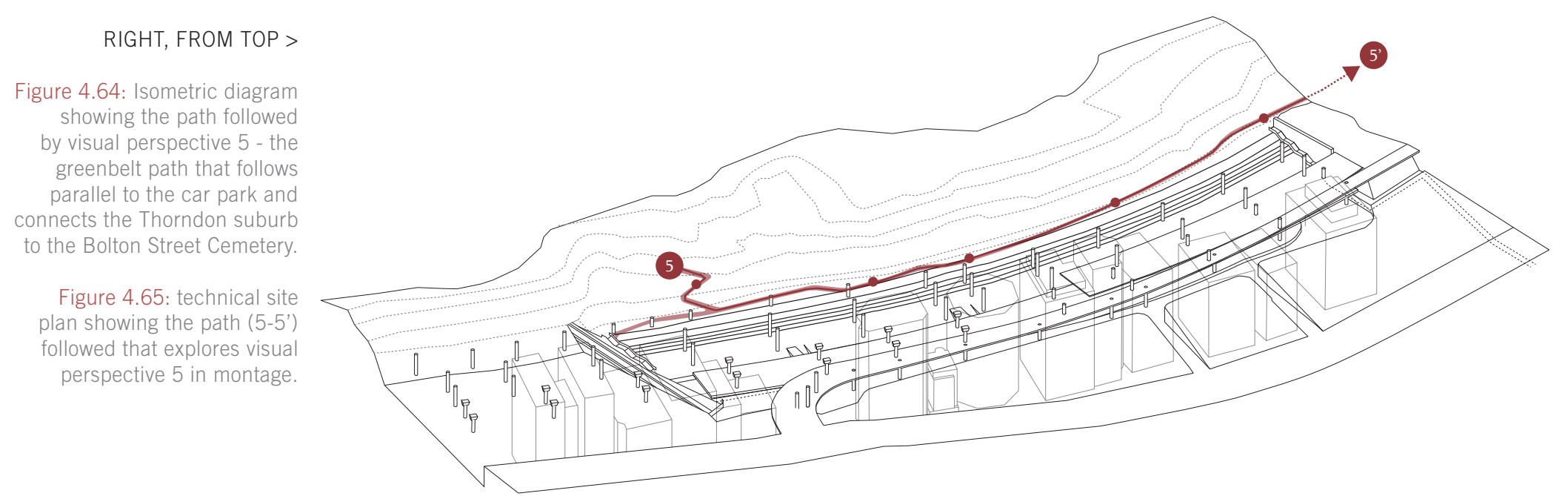

99

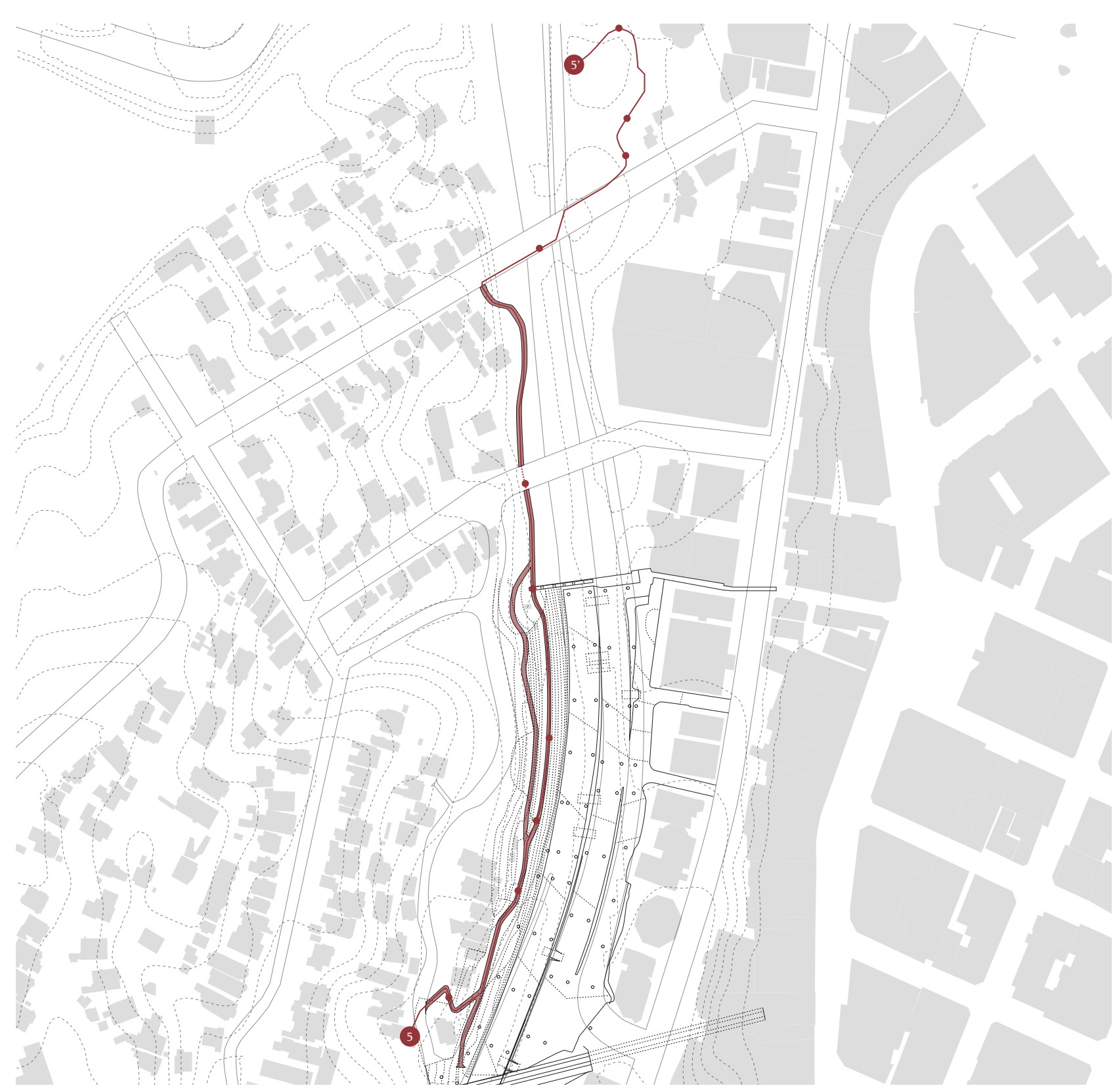



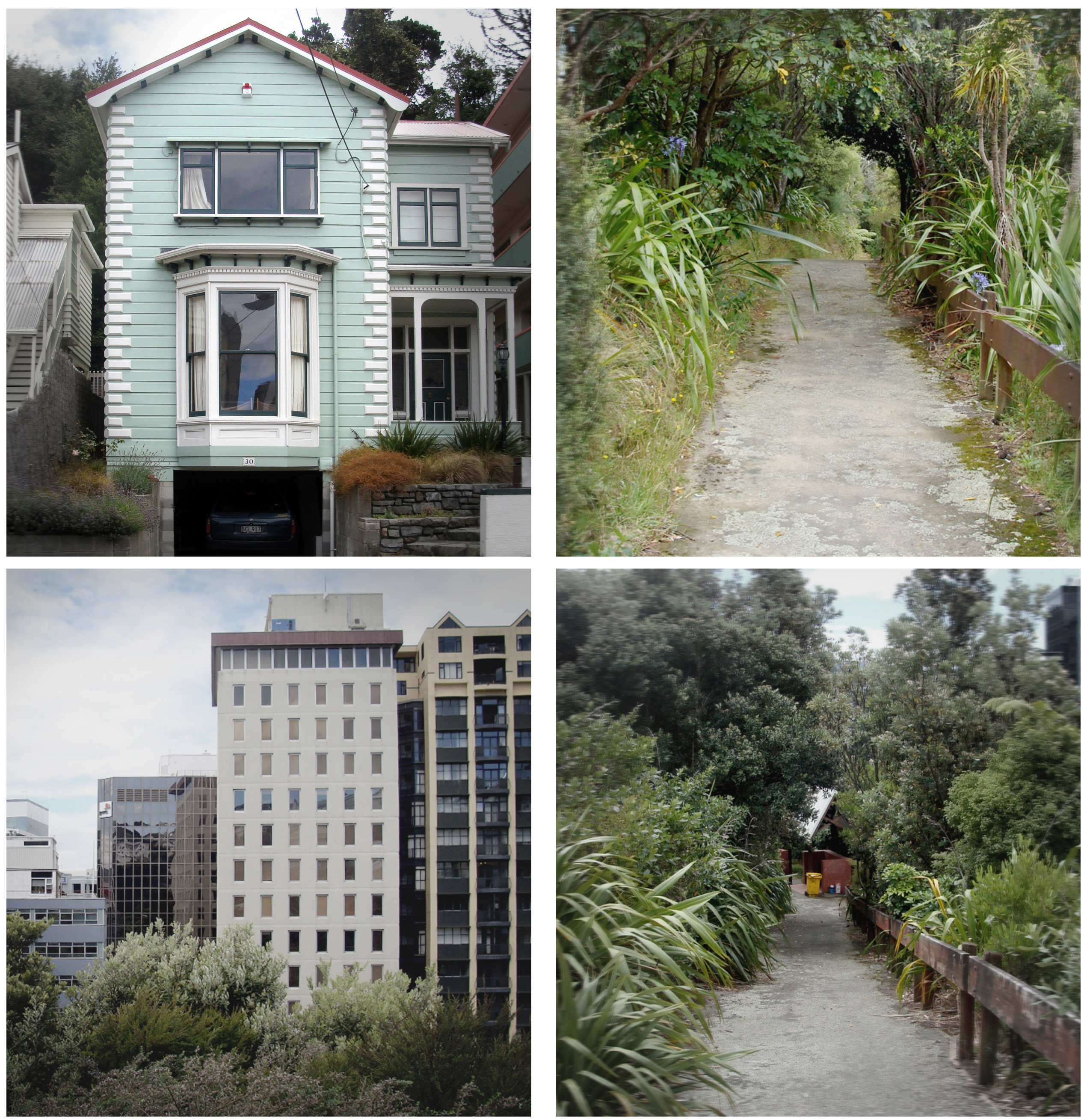

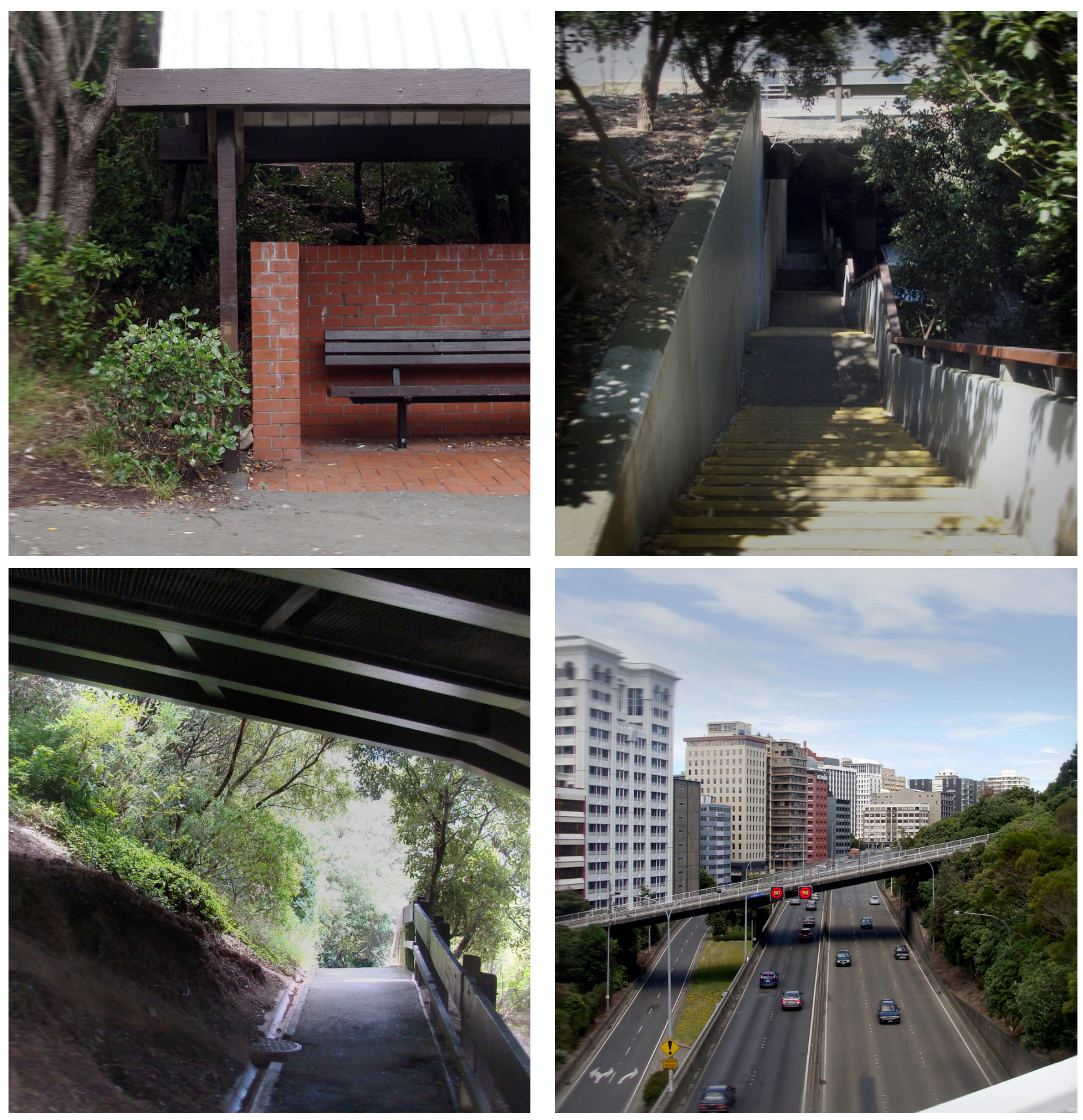

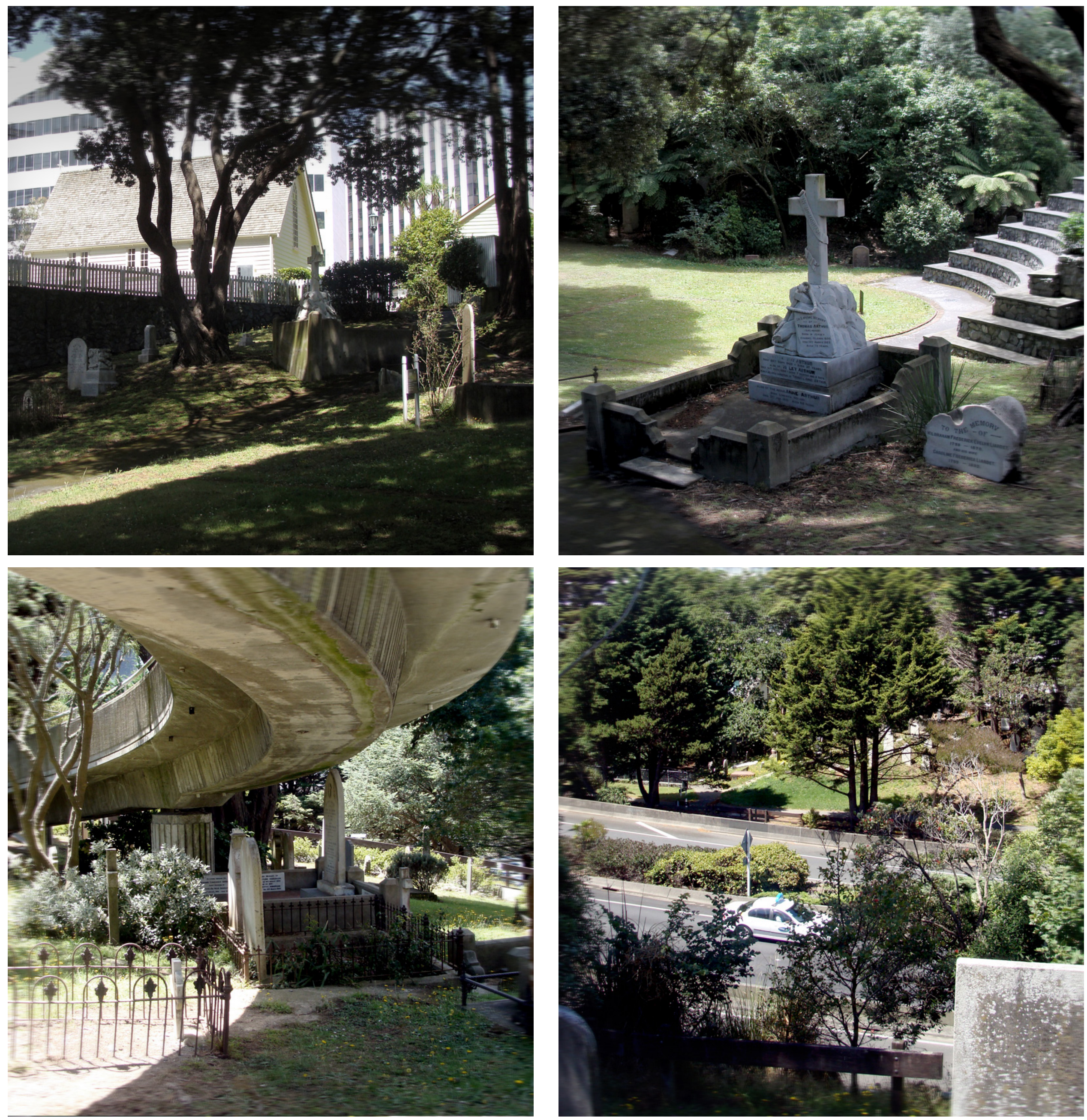
Figure 4.66-4.77: photographic 5. The Clifton Street Car Park is historically and visually linked to the Boloton Street Cemetery. Additionally, the Urban Motorway creates a distinct visual and physical barrier between the Thorndon Suburb and The Terrace.

\section{Conclusion}

Throughout this chapter, a polycontextual analysis of the Clifton Street Car Park has revealed essential information that informs the strategies and critical framework for the design case study. Investigating the urban problem and specific site of research from this lens has opened the design process to the possibilities of a context-based criticality. In so doing, the resultant design case study will resist the notion that architectural practice should exist as purely pragmatic and innovation-based (the postcritical position), or dislocated from cultural concerns (the absolutely autonomous position).

Additionally, a thorough site analysis in the design process - including wider contextual implications for architectural design - reveals negative implications of, and opportunities for, the urban thick edge condition of the car park. Consequently, the specific conditions that have shaped the literal and figurative rigidity of the Clifton Street Car Park have been isolated

Information gathered from an analysis of the urbanised, historical, economic, political, social, physical and visual contexts of the site will perform as generators for design solutions through a test of critical spatial practices.

Following the argument, research, and opportunities outlined in Chapter's Two, Three and Four, Section Two mediates this information through the framework of critical spatial practices to elucidate strategies for how design can be used to resolve the contemporary research problem of the urban thick edge condition. 

1. Kahn, 2005, pp. 288-289 Andrea Kahn is Professor of Urban Planning and Preservation, Colombia University.

2. Keith \& Pile, 1993 , p. 5 Michael Keith is Professor of Media and Communication at the Faculty of Arts and Sciences, Boston College; and Steve Pile, Professor of Human Geography (Geography) in the Faculty of Social Sciences at The Open University

For design, it [context] is a mode of conceptual operation, a process of knowledge formation. More than simply amassing and organizing facts, figures, and impressions of a given condition, the descriptions and analyses that designers produce actually generate the knowledge necessary to engage a given condition as a site. Site representation is not a matter of getting a reality right as much as a matter of constructing forms of knowledge that can cope with multiple realities. ${ }^{1}$

Andrea Kahn

A different sense of place is being theorized, no longer passive, no longer fixed, no longer undialectical... but, still, in a very real sense about location and locatedness. ${ }^{2}$

Michael Keith and Steve Pile

In Section One of this thesis, two problems (and therefore two opportunities) have been established: in relation to critical theory, architecture has been misled to a crisis of criticality, a crisis that can be averted through a flexible critical approach; and in relation to systems of twentieth century urbanisation, driven by singular motivations and new infrastructure that is not responsive to existing urban contextual conditions, have created conditions of spatial inequality and negativity - thick edges - that inhibit meaningful contextual flows through these sites that are necessary for urban social engagement. This section looks to address these conditions, as they exist at the Clifton Street Car Park, through Jane Rendell's framework of critical spatial practices.

Based on these understandings, Section Two focuses the thesis on elucidating specific strategies for architectural design - as the relate to critical spatial practices - strategies that open the design case study to processes that are site-specific, socio-spatial and temporal in their critique. Thus, Chapter's Five, Six and Seven in this section examine Rendell's framework in further detail, engaging the design process in specific theoretical and architectural applications of critical spatial practices. Consequently, this section mediates between the themes put forth by Rendell and the potential for architectural intervention to engender the Clifton Street Car Park to operate as a critical 'place', with the directive of mediating between the discursive framing and the design test.

As is suggested by its phrasing, the proposition of 'space' is crucial to the framework of critical spatial practices. In light of this, it is of particular importance to introduce Section Two by briefly elaborating on it's use since the spatial turn by Rendell - in particular how it has effected architectural notions of place and how this relates the identity of Clifton Street Car Park as a contextually impermeable thick edge.

Traditionally architectural space has been conceived as Cartesian, absolute and abstract, coinciding with its use in planning and architectural practices relative to the identity of a particular site. This notion of how space is conceived is thought to be the geographic 'reality' - real space within which phenomena such as people, objects, and positions, exist. $^{3}$ As such, absolute space in architectural discourse follows three assumptions: space can be represented; space can be seen; and, concordantly, space can be designed. ${ }^{4}$ Under these prescriptions, the complexities of site and context are viewed as an abstract totality - distancing the architect from
3. Lefebvre, 1991, p. 361 Lehtovuori, 2010, p. 20. 
conditions that are external to the contingent (often cadastral) bounds of a project. Treatment of space as an abstract and absolute condition has stubbornly shaped the built environment of many Western centres, rendering focus on the boundaries and zoning of sites according to a limited range of attributes. Alternatively, Lefebvre's redefinition of space as a social construct - rather than simply as absolute and Cartesian - is crucial, and has subsequently initiated debates around the identity and politics of 'place..

'Place' as an architectural principle gained currency as a disciplinary topic between 1970 and 1990, and has primarily relied upon ontological definitions of dwelling. In such cases, the primary

\section{Rather than treating place as an order or a specified and absolute structure, de Certeau suggests that it is an ordering system - where social and spatial practices operate as ordering activities that realise the various possibilities of a site.}

6. See: Heidegger, 1962; Merleau-Ponty, 196 Bachelard, 1969 and Norbers Schulz, 1980

7. Dovey, 2008, p. 53 phenomenologically tied to our sensuous interactions motivations for defining a place are experiential with space - embodying experience as a connecting mechanism between the 'being' and the 'world'. These explorations have frequently been criticised for their nostalgic attachment to bygone concepts of 'community', related to romantic ideas such as the 'harmonious village' and the 'hut', notions that are considered redundant in the current urban landscape. However, this earlier conviction of place has forged the genesis of its use and has since been hijacked in popular architectural discourse, referring to a desire for certain characteristics to (often) identify whole regions:

Wellington's success as a city relates closely to its sense of place. Wellington has a dramatic setting, is compact, and has good public transport. It is at the centre of the nation and is the national capital. It will have distinctive and beautiful buildings connected by high-quality public spaces and recognise the legacy of the past through the protection and conservation of its natural and cultural heritage.

As the Wellington City Council identifies, place in this setting is tied intrinsically to the image of the city - its geography and cosmetic appeal. Lefebvre sees this appropriation of 'place' as removed from the lived practises of everyday life; devoid of social depth. In "Right to the City", he contends that politicised definitions of place are problematic as they implicate the right to appropriation, how place is defined holistically. ${ }^{9}$ This is evidenced in the lack of definition presented by the Wellington City Council for the Clifton Street Car Park. The thick edge displays characteristics that are antithetical to positive place-connotations in this sense, and therefore defy definition in a superficial use of the term.

In light of Lefebvre's critique, anthropologist Michel de Certeau has argued for a definition of place that shifts focus away from simply visual character, and towards a definition that is essentially cultural, social and 'practised'. For de Certeau, identities of a particular 'place' are continually constructed and reconstructed through human actions in everyday life; they are relational insofar as they are understood contextually. ${ }^{10}$ For de Certeau, as traditional descriptions of place become increasingly defined through strategies of power and institutional forces, such as governance and commercial enterprise, possibilities and opportunities for resistance appear. In such situations, specific sites (such as thick edges) become exposed to 'tactics' of subversion and critical interventions. ${ }^{11}$ Additionally, relational places, according to de Certeau, are inherently intertwined to the performance of various publics within them:

Space is a practised place. Thus the street geometrically defined by urban planning is transformed into a space by walkers. In the same way, an act of reading is the space produced by the practice of a particular place: a written text, i.e.: a place constituted by a system of signs... [I]n relation to place, space is like the word when it is spoken, that is when it is caught in the ambiguity of an actualization. ${ }^{12}$
10. See: de Certeau, The Practice of Everyday Life, 1984

11. Dovey, 2008, p. 52 
Thus, rather than treating place as an order or a specified and absolute structure, de Certeau suggests that it is an ordering system - where social and spatial practices operate as activities that realise the various possibilities of a specific location. This definition of place is performative - requiring the actions of individuals to initiate it, epitomised in the activities of occupying the public environment (performing as publics). Even according to de Certeau's proposition of place, the immediate context of the Clifton Street Car Park denies positive engagement. Crucially however, the surrounding contexts of the car park are fertile with social and cultural activity and history that is currently being isolate by the impermeability of the thick edge.

Extending upon this definition, anthropologist Marc Augé illustrates a counterposition: the concept of 'non-places. Augés thesis documents the fleeting and fragmented notion of place as it is transformed by an increasing velocity of modernisation noting that, "if a place can be defined as relational, historical and concerned with identity, then a space which cannot be identified as relational, historical, or concerned with identity will be a nonplace" [emphases added]. ${ }^{13}$ In this environment nonplaces are realised by rapidly travelling through de Certeau's places - experienced in a montage of sites and locations:

Space, as frequentation of 'places' rather than a place, stems in effect from a double movement: the traveller's movement, of course, but also a parallel movement of the landscapes which he catches only in partial glimpses, a series of 'snapshots' piled hurriedly into his memory and, literally, recomposed in the account he gives of them. ${ }^{14}$

Non-places are ungrounded, and de-contextualized. They represent the desires of modernity taken to the extreme - a form of 'supermodernity'. Unlike de Certeau's places, Augés non-places can be readily identified, formed by singular motivations - whether that be transport, leisure, transit etc., and are characterised (but not completely defined by) locations such as motorways, airports, shopping malls and other supermodern homogenous spaces. Non-places attempt to form a global monopoly of place, one that threatens the uniqueness of context through spatial, social and formal homogenisation.

The polarising spectrum of place/non-place is tied directly to the tensions between globalised and local cultures. In the twenty-first century, local/ global tensions have become embedded in all places - placing an end to the 'closure' of the nostalgic local place, yet 'exposing' it to the inauthentic notions of dwelling in an increasingly global commodity culture. ${ }^{15}$ Recognising this point, and based upon the identity politics of sociologist Anthony Giddens, this thesis posits that critical architectures must identify disparity in the dialectic between the places of everyday social activity and the spaces of global production, calling attention to, and resisting the myopia of decontextualized architectural practices. ${ }^{16}$

Place-making thus takes on a mediating framework, "a collagist character; a collision of practices and images rather than one narrative replacing another". ${ }^{17}$ Such an approach gives the experience of a particular place "a phantasmagoric character wherein the global and local, the familiar and the strange become inextricably intertwined". ${ }^{18}$ Critical spatial practices must therefore look to resist 'politicised' and appropriated place descriptions that require architects to gloss over spatial inequalities such as the thick edge conditions of urban space.

Following the logic of de Certeau and Augé, the Clifton Street Car Park expresses a disjunction, a disparity, in the tensions between the global and the local - it is rigidly global in its context. Its identifiable characteristics are therefore closer to an Augéian non-place, resisting the performative character and contextually relational identity of a a de Certeauian place

Importantly, for Rendell this adaptation of place opens certain contexts to critical responses in architectural practice; "it is the ongoing redefinitions of space and place in cultural geography that open up discussions of the differences between such terms as site and context" ${ }^{19}$ By introducing perspectives 19. Rendell, 2008, p. 192
15. This point is put forth by sociologist and critical theorist, David Harvey. See: Harvey, Justice, Nature and the Geography of Difference, 1996.

16. Dovey, 2008, p. 54

7. Dovey, 2008, p. 53.

18. Giddens, 1991. 
from cultural disciplines, Rendell is opening the modes through which architecture can pose critical responses beyond its own autonomy - producing a certain discursive flexibility.

Additionally, for Rendell, critical practices positioned within the place/non-place spectrum initiate a set of mediating processes:

\section{Here/ there (site-specific)}

One/ another (socio-spatial)

Now/then (temporal)

These themes construct a notion of place as both social and locational; a practice of place-making that is capable of exposing, and embedding the multiplicity of dimensions in Clifton Street Car Park through

\section{Critical architectures resist 'politicised' and appropriated place descriptions that require architects to gloss over spatial inequalities - such as the thick edge conditions of urban space.}

This thesis develops Rendell's framework by exploring critical spatial practices through three key influences: Nick Kaye, Kim Dovey, and Gevork Hartoonian - providing critical positions for sitespecific, socio-spatial and temporal architectural intervention that overlap, at times even contradict. Approaching design-research in this manner allows for a critical flexibility - a framework that will implicate the wider contexts of the Clifton Street Car Park in the design process, polycontextually opening the thick edge.

Elucidating strategies for design from Rendell's framework, Kaye, Dovey and Hartoonian are translated through architectural case studies. Each case study focuses on different architectural strategies that critically engage a variety of contexts in the built environment. These strategies implicate criticalspatial practices in the architectural design process. As such, they are not limited to sites of infrastructure, thick edges or specific programmes; rather, they embody the manifestation of the theoretical foundations of this thesis, particularly how such strategies can inform critical design processes that respond to thick edges such as the Clifton Street Car Park.

Based upon these findings, Section Two concludes by investigating and establishing a critical programme for the site - that of a performing arts facility. An exemplary client, the New Zealand International Arts Festival, is established and analysed providing supplementary parameters for the design case study and concluding with a design-research brief. Combined with the strategies for design through critical spatial practices, this programme will contribute to the outcomes of the design research.

This section addresses the following research questions:

- How do critical spatial practices manifest in the built environment, in particular through strategies of architectural design? How can these strategies engage with a polycontextual approach to the urban thick edge?

- Can the implementation of architectural programme facilitate the development of critical design intervention within the thick edge? 


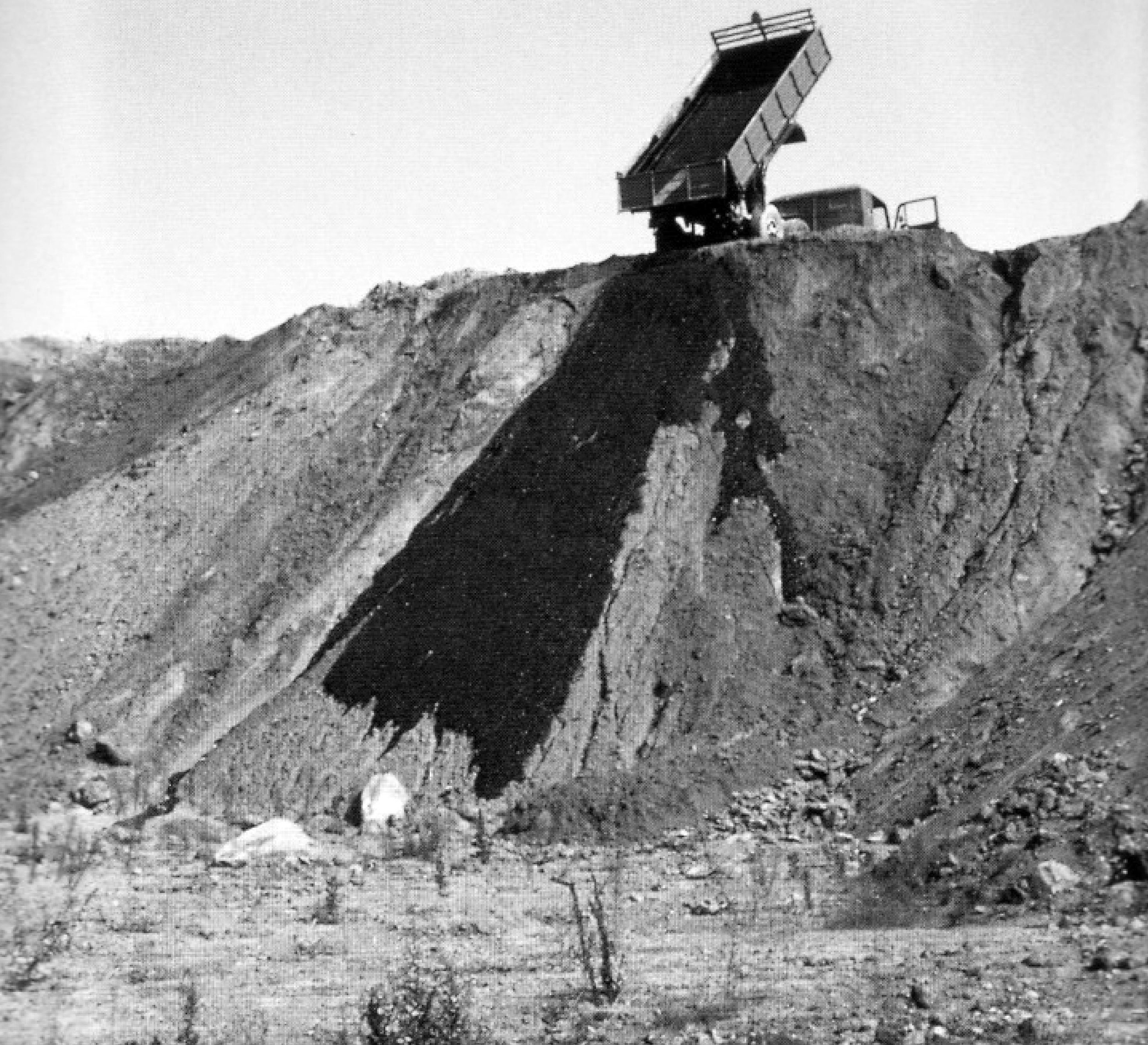




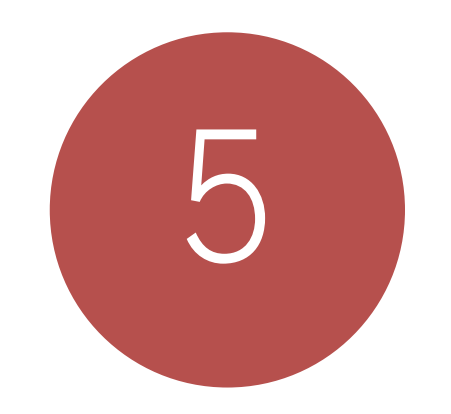

\section{EXPANDING SITE AS CRITICAL PRACTICE:}

Elucidating Strategies for a Sitespecific Practice

Introduction

Between 'Here' and 'There': Continuity and

Discontinuity Through Site-specific Strategies

Bernard Tschumi's Discontinuity: An Anticontextual Approach

Enric Miralles's Continuity: A Contextual Approach

Conclusion: Implications and Strategies for Design 


\section{Introduction}

As Massey notes above, the spatial turn has provoked a relational understanding of space (and its relationship to place) that is intrinsically linked to the contextual dimensions of location. In Chapter Four a polycontextual understanding of one such location, the Clifton Street Car Park, was established. Accordingly, for Rendell, the properties of a given site (such as the car park) are bound by a dual definition: a geographic location, (an un-zoned cadastral parcel of land at 132 The Terrace); and a place condition (a thick edge that is closer in identity to that of a non-place). Informing the framework of critical spatial practices, Rendell suggests that sites can be reconfigured according to this definition as both 'here' (a site, specific) and 'there' (a wider cultural location, or context(s)), allowing architectural intervention to construct a critical dialogue through its relationship to site as a geography (position), and its placement within its contextual relations (location).

Expanding further upon Rendell's framework, this chapter looks to engage these two dimensions of 'site' as a potentially critical construct - elucidating strategies for design through sitespecific practices. Here, the work of Nick Kaye is of particular importance, examining the notion of site as a performative index - an idea that has been shaped by disciplines such as land art and geography. For Kaye performative strategies challenge traditional relationships between architecture and site that engage a location as cadastrally bounded and static. Performative interventions positively destabilize these rigid approaches to site by acting out' contextual information, constructing a sitespecific index of referents, traces and events. These indices adopt the form of dual metaphors, creating a dialogue of continuity or discontinuity between the architecture and its context - strategies that reveal information that may confirm or critique the identity of a particular place.

Two architectural interventions emerge as relevant case studies in the discussion of performative sites: the Parc de la Villette (1982-1983) by Bernard Tschumi; and the Igualada Cemetery by Enric Miralles (1994). Analysing how strategies of continuity and discontinuity are deployed through architectural design within these case studies reveals crucial translations of critical spatial practices within the built environment. As a result, this chapter posits that performative strategies reveal an opportunity for critical spatial practices: to explore an inversion of the Clifton Street Car Park thick edge condition by introducing wider contexts into the process of design intervention.

In so doing, this chapter adds to Rendell's framework and mediates between the theoretical framings of Section One, and the design case study in Section Three; engaging the fourth research question:

How can critical spatial practices manifest in the built environment, in particular through strategies of architectural design? How can these strategies engage multiple contexts to introduce positive flows to the urban thick edge? 


\section{Continuity and Discontinuity: Performative Sites}

'Site': substantive. Local position. The place or position occupied by some specified thing. Frequently implying original or fixed position

'Site': 1. transitive. To locate, to place. 2 .

2. Kaye, 2000, p. 8. See also: Onions, 1973

3. This is evident in the Wellington City Council's designtation of the Clifto Street Car Park 'site' non-designated

4. Kaye, 2000, p. 9.
5. Interventions that question normative site defintoins may be incongruent with the perception of a place by many a fact exemplified by the controversy surrounding sitespecific works such as Richard Serra's 'Tilted Arc'(1981) and Maya Lin's 'Vietnam Veteran's Memorial' (1982) Both of these sculptures are considered highly site-specific, with Serra noting that 'to move

the work is to destroy the work'. After much public outcry regarding its visual relationship to its location, 'Tilted Arc' was removed from its site within a year of its erection. See: Senie,
Intransitive. To be situated or placed. ${ }^{2}$

In Site Specific Art Nick Kaye, Dean of the College of Humanities and Professor of Performance Studies at the University of Exeter, provides a key analysis of critical approaches to the notion of site-specificity. As Kaye identifies above, on the one hand a site is frequently defined relative to an understanding of position - a straightforward entity contained by boundaries that delimit it from the surroundings, implying a 'fixed position'; and on the other hand, as an identity-based location - a place in which its meanings are defined. In many cases, urban planners and architects reduce the definition of site to the strict delineation of cadastral limits (its position) - reducing the notion of site to the first definition as a simple classification of metes and bounds. ${ }^{3}$

Kaye proposes critical "strategies which work against assumptions and stabilities of site and location", and which offer a context of practices that resist or expose the "underlying concept of site". In this sense, site-specific practices will not only critically engage the particularities of a certain location, but, also the very notion of what a site is and can do (as it is portrayed within architectural discourse). 5

This approach to site as a critical construct, emerged within architecture from the influence of wide-ranging examinations of site conducted by art practices throughout the 1970s, particularly in land art and sculpture. Works by Robert Smithson, Richard Serra, Mary Miss and Gordon Matta-Clark began to expand the definition of site in art practice as no longer confined to the gallery system (see Figure 5.2). ${ }^{6}$ These experiments were crucial to architectural discourse as they introduced an argument of site as dynamic, responsive, and a contextual presence within the work. Rosalyn Deutsche argues that these works explore the expression of spatial intervention through

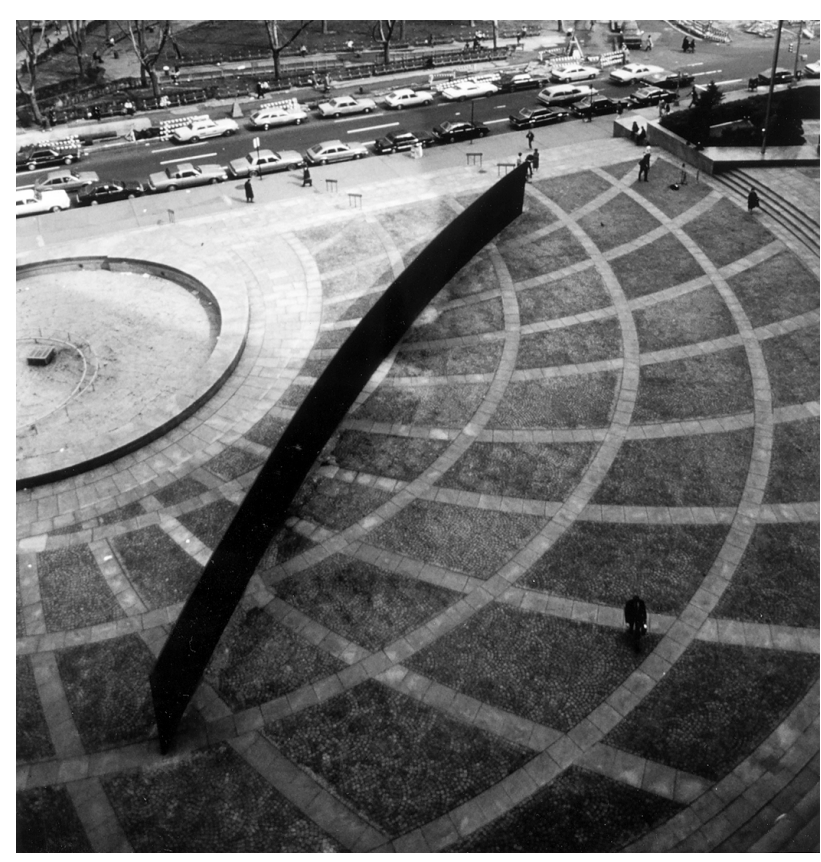

the very processes by which they are themselves being deployed, and as a result:

broadened the concept of site to embrace not only the aesthetic context of a work's exhibition but the site's symbolic, social and political meanings as well as the historical circumstances which artwork, spectator and place are situated.

Intended in this way, site-specific practices become essentially 'performative', inscribing (and inscribed with) layers of contextual knowledge within the place/ non-place spectrum. Performative sites are built upon relations of continuity and discontinuity between intervention and site; they 'act out' the relations of their locations, their contexts, in a perpetual state of constructing a place/non-place. A performative site can act out this role in a state of exposure or resistance according to its position on the place/non place spectrum - 'troubling' expectations as to what a site can and should be:

It is in such contexts that site-specific art frequently works to trouble the oppositions between the site and the work. It is in this troubling of oppositions, too, that visual art and architecture's approaches to site realise or may be read through the terms of performance. ${ }^{8}$

For Kaye, performative interventions ignite this critical opportunity by embedding a particular
Figure 5.2: Photograph

of Richard Serra's 'Tilted Arc' 1981) before it was removed from its site, Foley Federal Plaza, New York City

6. (Prev.) For a further investigation of this impasse see Krauss, "Sculpture in the Expanded Field", 1979.

(Deutsche Evictions: Art and Spatial Politics, 1996, p. 162) 
location with an index of place/non-place operations, a language that creates dialogue between the site and its context. An index in this sense refers to a "type of sign which arises as the physical manifestation of a cause, of which traces, imprints, and clues are examples". As an index, a performative site becomes 'unfixed', informing a relational specificity through a 'field' of architectural signs. ${ }^{10}$ As an index of wider contextual dimensions, the performative site is composed of a series of referents, records of events and traces that seek to undermine the idea that there is any oneto-one relationship between an architectural object and its symbolic meaning. ${ }^{11}$ Rather, a performative site acts out by doing, by colliding the signifier of an architectural element with its function (see Figure 5.3). A performative index, Kaye argues, creates a 'place-event'. A place-event is a field of relations

RIGHT Figure 5.3: Photographic
montage of
of 'Cibachrome', Gordon Matta-
Clark (1977).
In Matta-Clark's spatial
practice, place-making is
created through strategies of
'cutting' and 'tracing'. The
architecture thus becomes an
index that 'acts out' cuts and
traces as a double-metaphor.
These works destabilise the
architectural definition of
specific locations (house,
factory, wall, floor), critically
engaging normative defintions
of a site as fixed and static.

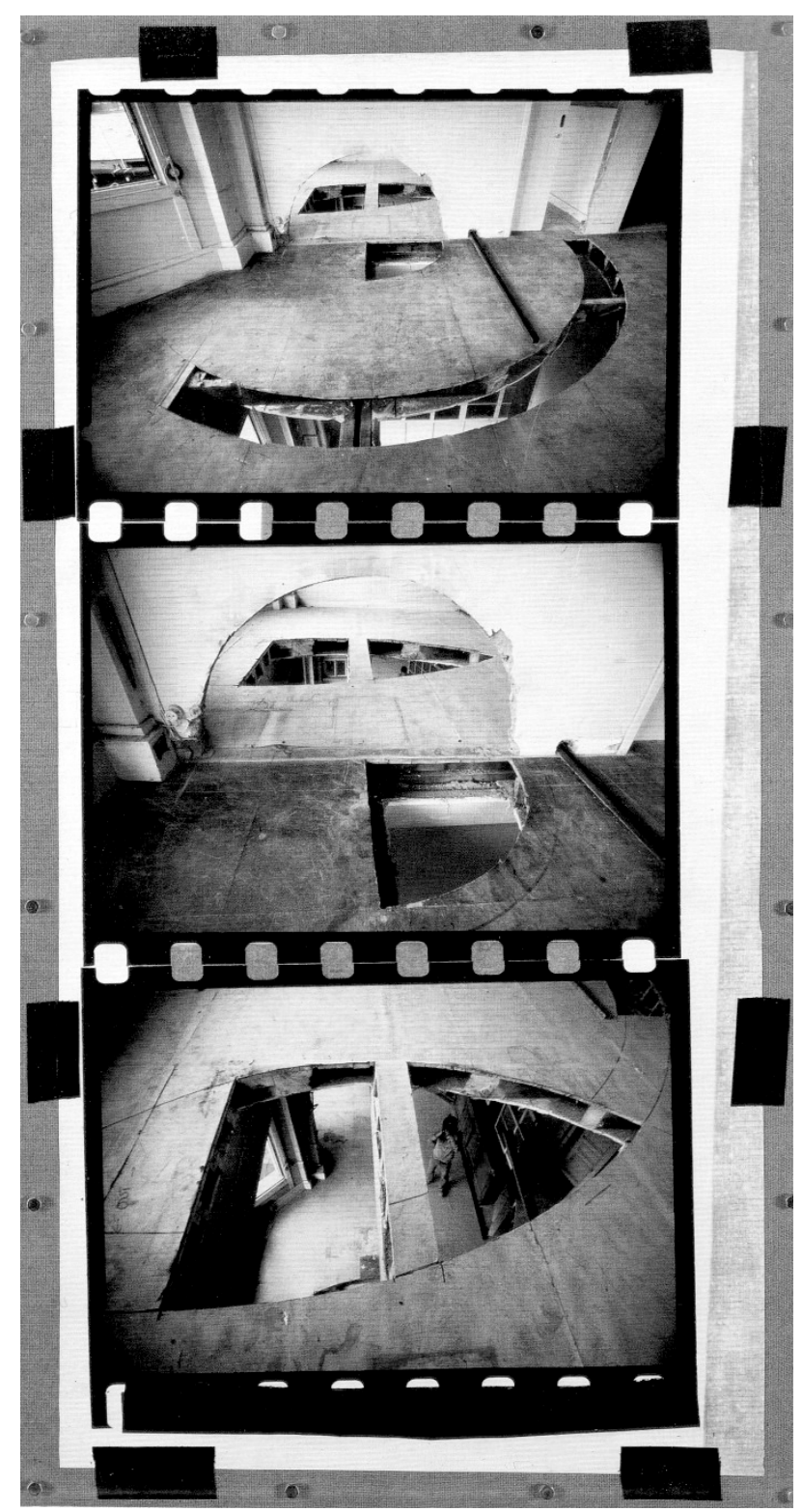

where a "series of interlinked narrative and formal structures, 'architecture' and 'event' are invested one within the other" through strategies that aim to 'upset' the boundaries and conditions of a site. ${ }^{12}$ Architecturally, two projects display critical strategies for performative sites in opposing ways - as an index of discontinuity, and of continuity.

\section{Bernard Tschumi's Discontinuity: An Anti-contextual Approach}

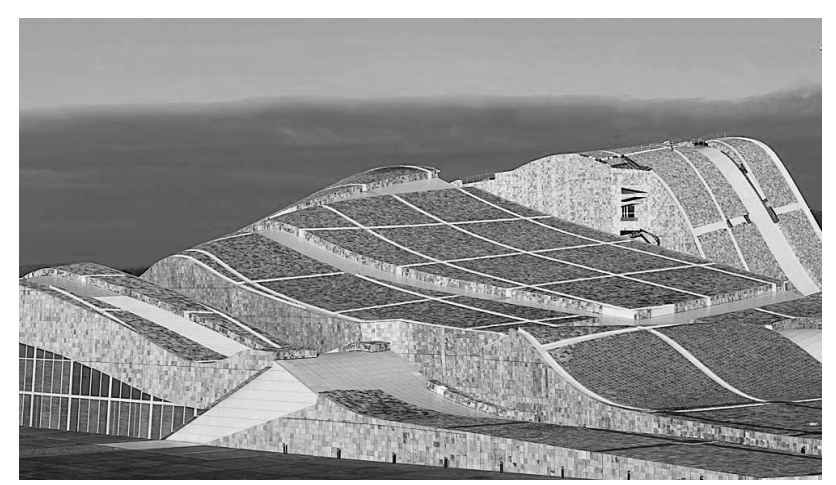

An example of a performative approach to site, operating through strategies of discontinuity, is Bernard Tschumi's Parc de la Villette (1982-83). An 'Urban Park for the twenty-first century' that occupies a large site within the 19th arrondissement on the northeast side of Paris, Parc de la Villette is an attempt to develop a critical relationship between architecture, its site and its use (see Figure 5.5). ${ }^{13}$ The site, consisting of pre-existing structures such as a Museum of Science and Industry, a City of Music, and a Grande Halle for exhibitions and concerts, already expressed a clearly localised environment of social activity and architectural identity with respect to its cultural context (see Figure 5.6). Thus, for Tschumi, a critical intervention required the implementation of architectural elements that perform a discontinuity between each site and its wider context:

Thus in rejecting the imposition of 'a masterly construction', a compliment to the pre-existing structures, or a deconstruction of previous architectural definitions of site, Tschumi sought to deploy an 'abstract mediation' between the site and the project's 'programme' or 'list of required utilities. ${ }^{14}$
2. Kaye, 2000 , p. 56.

LEFT

Figure 5.4: Photograph of The 'City of Culture of Galicia', Eisenman Architect's (2013).

Even Peter Eisenman's most recent projects have displayed performative characteristics that are beyond the confines of pure autonomy. This is evident in the City of Culture where Eisenman has created where Elsenman has 'reated, a superimposed site 'collage' on the buildings surface. This collage contains archaeologica referents - large traces of streets and alleys (historical ontext); and a superimposed grid, a Modernist logic.

13. Ibid, p. 47.

4. Ibid, p. 49.

OPPOSITE> Figure 5.5: Aerial map highlighting Parc de La Villette located in the 19th arrondissement of Paris. 



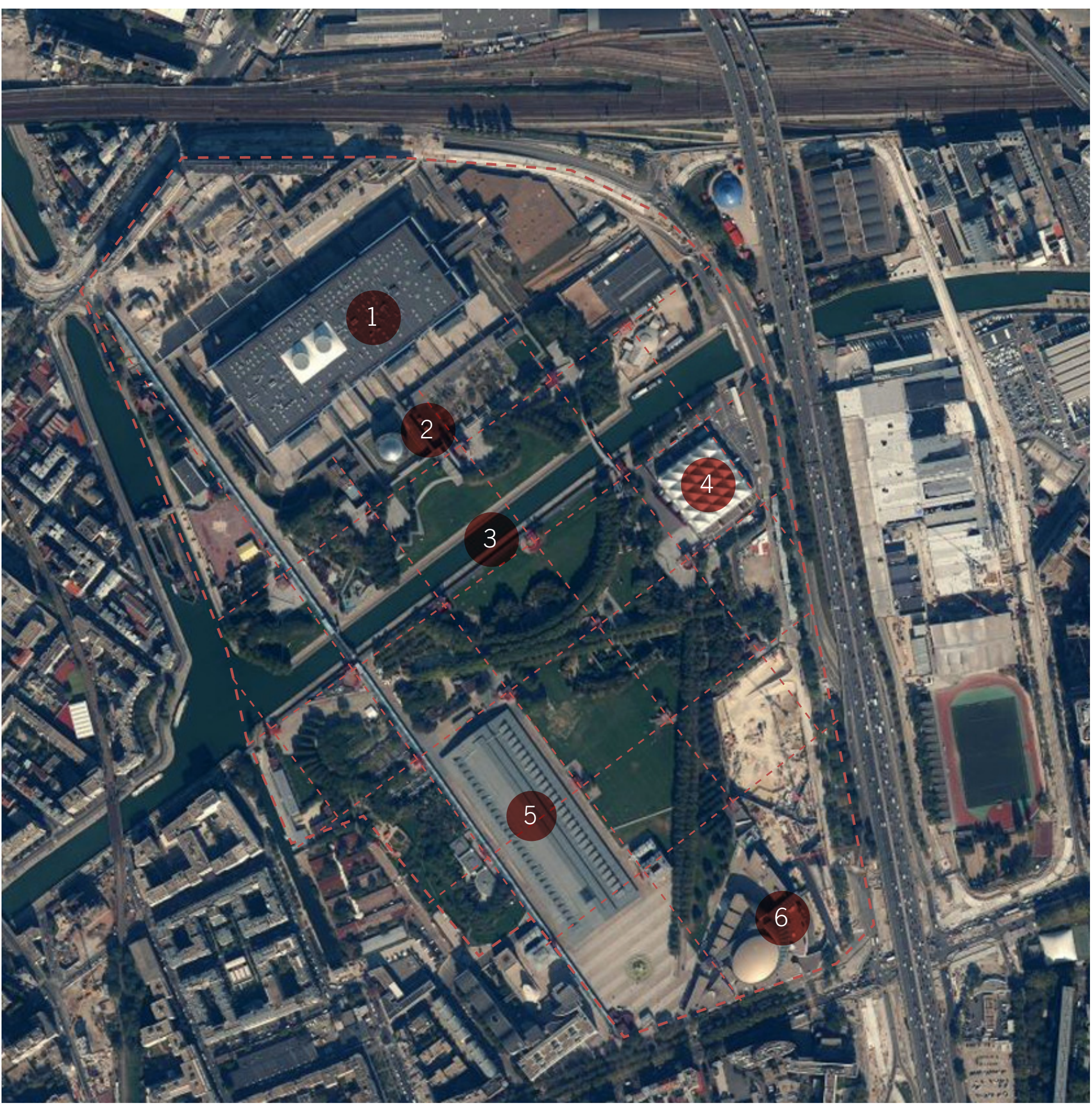

KEY:

1. Museum of Science and Industry

2. La Géode

3. Canal Saint-Martin

4. Zenith de Paris

5. Grande Halle

6. Cité de la Musique 
$<$ OPPOSITE

Figure 5.6: Aerial map showing cultural context of the site; site boundary; and imposed grid of folies

RIGHT > Figure 5.7: Exploded isometric of Tschumi's applied ordering system to the site - throug surfaces, points and lines.

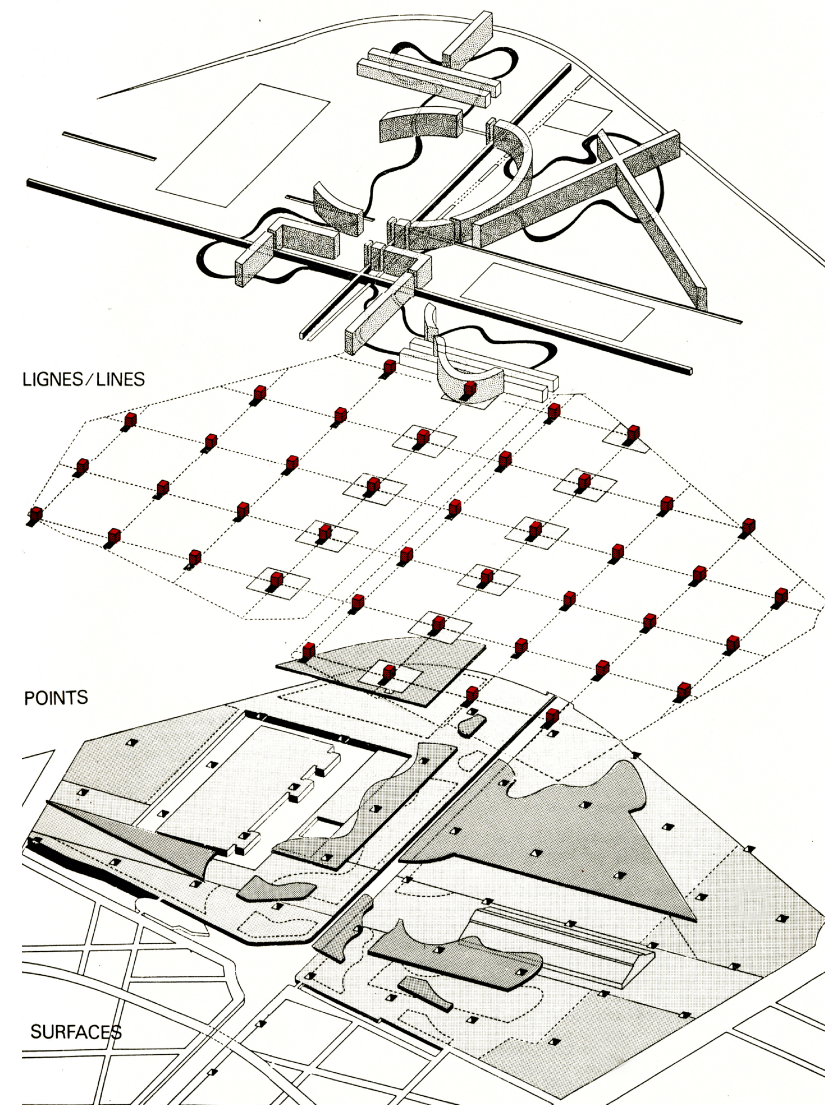

Tschumi's process begins with the application of an abstract system of points, lines, and surfaces that impose a strict order across the entire park. By applying a rigidly logical order, Tschumi is creating a language of discontinuity between the grid and the site "the park became architecture against itself: a disintegration for the addition of the systems' internal coherences is not coherent (see Figure 5.7). The excess of rationality is not rational".

Tschumi's ordering system imposes the grid onto the park and creates its index of collisions serving the purpose of exposing events and moments that emerge where this index and the site meet (see Figure 5.8). These collisions manifest architecturally as 'folies' - unconventionally imposing on the existing site wherever they are forced to meet - rather than embodying any form of specific contextual reference (see Figure 5.9). In the folies, Tschumi is playing on the dual definition of the word - in English as a house of spectacle, or entertainment value; and in French as a form of insanity or madness. Madness provides the performative metaphor for the index across the site, 'acting out' the title of the work (referring to the

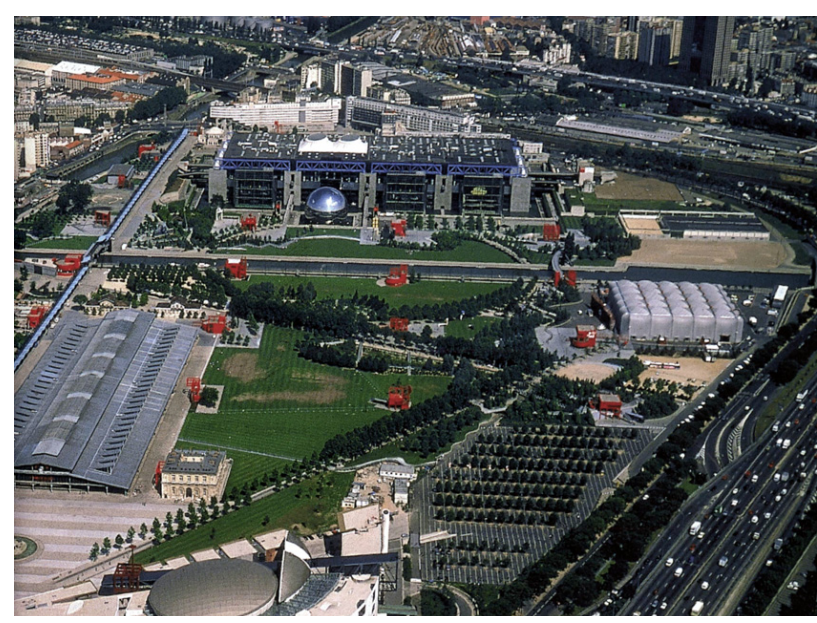

Figure 5.8: aerial photograph showing the imposed index across the site - the structures collide with the existing in an anti-contextual strategy

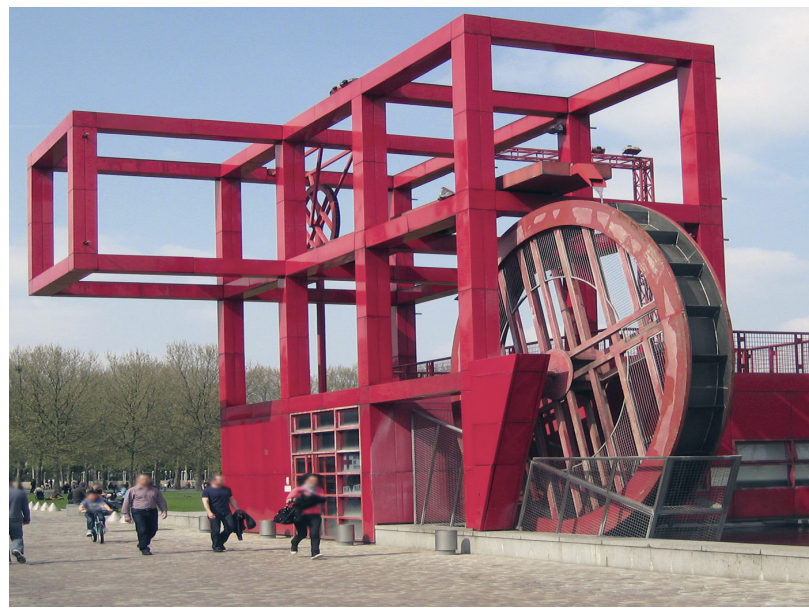

$<$ LEF

Figure 5.9: photograph showing a point of collision between ne of Tschumi's 'folies' and and the park - the folie is coincidentally located in the Canal Saint-Martin

irrational rationality of the imposed grid) and as the 'irrationality' of the 'anti-contextual' imposition of the folies. Through this index, the folies are expressing the critical intentions of the architect - an approach to the overall site as an index of referents that are both in and of madness.

Consequently, the motivations of Tschumi's performativity informs his site-specificity - his aim (as one of the figureheads of Hays's late avantgarde) is to push architecture's disciplinarity to its borders - to explore the production of architecture dislocated from the influence of its cultural context. At the time of its construction this entailed a focus of 'deconstructing' the relation between architecture as a discipline unto itself, and architecture as deployed within the social events of space. By 'upsetting' or 'deconstructing' the perception of these relations, Tschumi's "site-specificity is intimately tied to notions of event"; he creates an anti-thesis to Kaye's place event, an index of anti-place events. ${ }^{16}$

The collision spaces of Tschumi's folies create a system of anti-context, of event and discontinuity (what Tschumi refers to as disjunction), through 


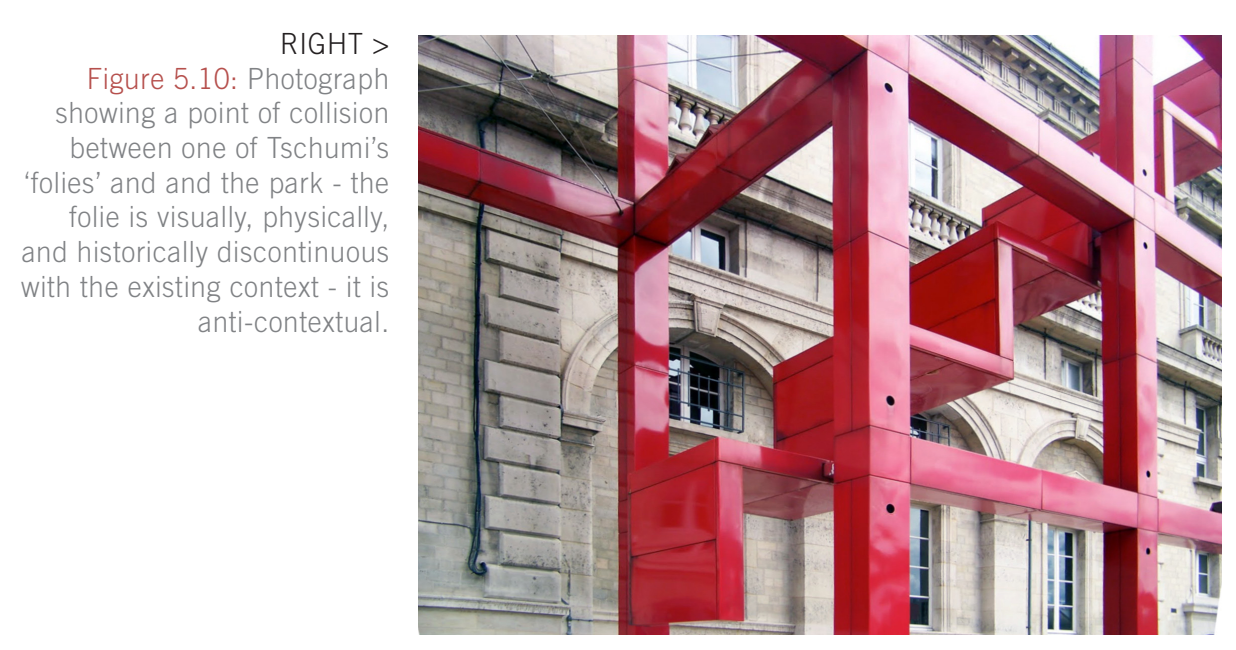

18. Tschumi, 1987, p. 6 . operations of typology, materiality, composition/ order. They physically and visually contrast the soft landscape contexts of the park - starkly orthogonal, bright red and highly polished in their materiality and construction; arranged across the site in a purposefully unnatural and imposed fashion (see Figure 5.10) - the intention of which is to create a language, a physical and visual dialogue that subverts the existing identity of the site as a tourist-focused park, and to create a 'logic of materials' and forms that subvert expectations. ${ }^{17}$ For Tschumi the grid is a crucial operation in the construction of this dialogue as it presented the design process with a series of dynamic negotiations, dialectic oppositions:

We had to design a park: the grid was anti-nature. We had to fulfil a number of functions: the grid was anti-functional. We had to be realists: the grid was abstract. We had to respect the local context: the grid was anti-contextual. We had to be sensitive to site boundaries: the grid was infinite. ${ }^{18}$

Tschumi's work does not de-contextualise its site, it is anti-contextual - aiming to destabilise the proposition of site as a context, and to create 'mobility' between events that may or may not occur at the folies (see Figure 5.11). As a result, the Parc de la Villette projects a certain contextual negativity; it "aims at an architecture that means nothing" one that is "pure trace or play of language". ${ }^{19}$ For Tschumi, sitespecificity at the Parc de la Villette is a resistance to the notion of context itself and thus opens itself to a "multiplicity of meanings" through location and place as sites of events.
In relation to the Clifton Street Car Park Tschumi's design for Parc de la Villette is both useful and problematic. Identifying the site as a performative index, Tschumi engages architectural design strategies of discontinuity as they relate to order, composition, typology and materiality, offering the orthogonality of the grid as a "paradigmatic twentieth-century form. ${ }^{20}$ These processes are referenced in the overlaid index of folies and in the composition of the folies 20. (Ibid) themselves. Thus Tschumi's processes are crucial to the communication and formation of the design as an overall system (index) and for its individual parts. His discontinuity informs the locus of critique - his resistance to the context of the park as a cultural identity and place.

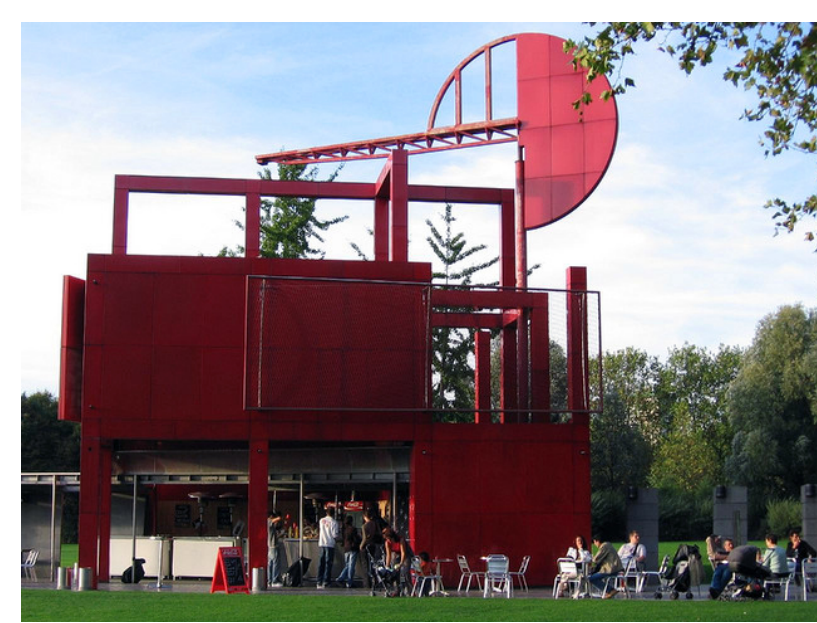

$<$ LEFT

Figure 5.11: Photograph showing a point of collision between one of Tschumi's 'folies' and and the park - the folie creates anti-thesis to what Kaye refers to as a place-event.

In spite of its 'anti-contextual' approach, the Parc de la Villette is unavoidably implicating context. The construction methods of the folies are a reference to the rationality of Modernism, a notion also referred to through his use of the colour red (a reference to the Constructivist design movement). The folies are axiomatically grounded in precedent, and all of the cultural connotations that are associated with them. As a result, rather than producing a complete anti-context, the Parc de la Villette is providing a polarising resistance, a discontinuity that responds to the existing physical, visual and historic contexts of the site and its relationship to the architecture as a constructed intervention. Thus, Tschumi's anticontextual approach is paradoxically polycontextual in its discontinuity.

Designing site-specifically, and critically, within the polycontextual landscape of the Clifton 
Street Car Park, however, requires an opposing process to that of Tschumi. The physical context of the site already exhibits forms of irrational rationality' as the infrastructure has carved an ordering system of orthogonal horizontal and vertical elements throughout. Applying Tschumi's logic at Parc de la Villette to this site would only contribute to an exacerbation of the existing physical, visual and historic issues that have contributed to the negativity of the thick edge.

Therefore a critical design approach will be to adopt a strategy of site-specific discontinuity, following Tschumi, with the elements of infrastructure - with the orthogonal grids and rigid logics of their construction. It will perform this through materiality, composition, and typology, in an opposing trajectory to Tschumi - with the intention of engaging context(s) rather than destabilising them.

\section{Enric Miralles's Continuity: A Contextual Approach}

Offering alternative strategies (through continuity) to Tschumi for the design of a performative site is Enric Miralles's Igualada Cemetery (1994). Located in the outskirts of Barcelona, the Igualada Cemetery is an experimental approach to the relations between architecture and site through its historic al physical and visual identity (see Figure 5.12). The site, an abandoned quarry located within a rough and deeply eroded river valley, has no adjacent commercial or housing areas, isolating it from any adjacent urban context. As a result, the critical intention of Miralles is to engage the identity of the site as a naturally occurring landscape that has been traumatically altered through man-made processes.

Following Kaye, and aligning in contraposition with Tschumi, the cemetery presents an attempt to construct a language - or series of contextual dialogues - across the site. The design engages its contexts through a critical lens of continuity, one that, according to Anatxu Zabalbeascoa, "engages with the pre-existing history of the site by combining a variety of elements from the disciplines of landscape design, architecture, urban design, sculpture, and land art". In so doing, Miralles's design approach resists the proposition that site is a fixed or stable spatial entity: $:^{21}$

21. Zabalbeascoa, 1996, p. 14

At the Igualada Cemetery, a space is provided that allows a multiplicity of ways of viewing and using the architecture... [T] he site becomes a place of interaction - architecture as living art to which the user can personally and physically relate. ${ }^{22}$

For Miralles, the intervention of architecture required a strategy of continuity with the site (as it existed at the time of the design) that performs as an index of 'grounding', through processes of embedding and excavation. This index follows a linear path, unlike Tschumi's grid, terraced at two different levels, and organised by several architectural gestures/ coordinates (see Figure 5.13). The entrance to the

\section{Following Kaye, and aligning in contraposition with Tschumi, the cemetery presents an attempt to construct a language- or series of contextual dialogues - across the site.}

(main) lower terrace is marked with sculptural steel totems that signify an arrival/departure threshold (see Figure 5.14), followed by a series of vertically stacked loculi, or 'niches' - burial areas that guide the visitor through the cemetery and are embedded into The Terrace face (see Figure 5.15). The progression finishes with a cul-de-sac of mausoleums, retained by gabion walls (see Figure 5.15). This linear ordering system informs a 'social landscape' within which the design is located. ${ }^{23}$ Consequently, for Miralles the circulation through the design adopts the spatial form of a street, a procession that emulates the 'path of life', in a spatial (as a physical path) and temporal (as a passage through the lives of the buried) metaphor.

Engaging these notions, Miralles 'opens' the site to a polycontextual interpretation through an approach that is aligned with Kaye's performative position. It is within this action of 'opening' that an opportunity arises for architecture to be "an agent 



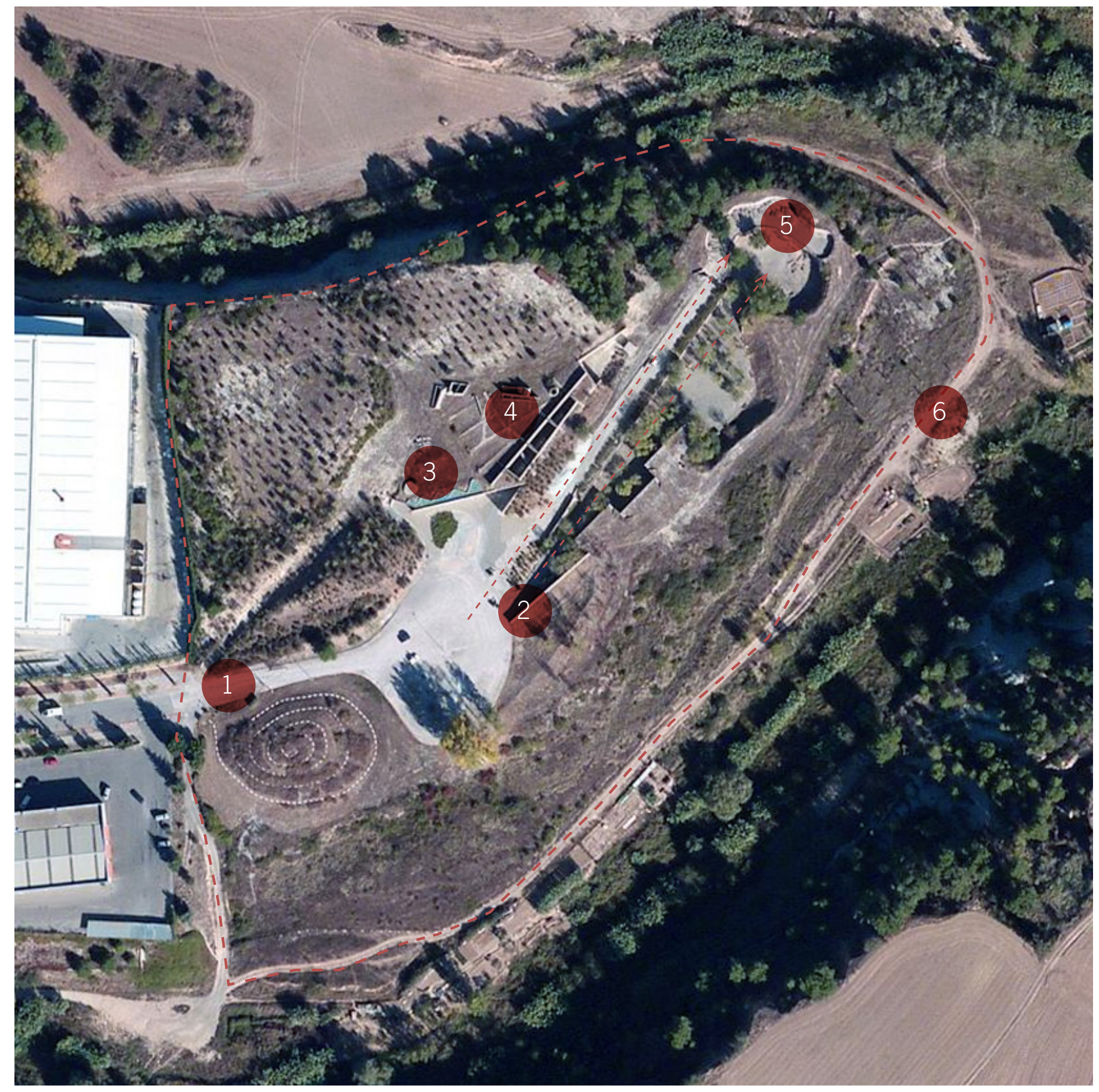

ABOVE ^

Figure 5.13: Aerial map of the cemetery showing, in plan, the major gestures executed by Miralles

$<$ OPPOSITE

Figure 5.12: Aerial map showing urban context of

the site at the periphery of

Igualada, Spain
KEY

1. Cemetery entrance

2. Path 1 entrance, lower terrace

3. Mortuary

4. Chapel

5. Cul-de-sac of mausoleums

6. Naturally occurring terraces 
Berrizbeitia and Pollak identify that processes through which the Igualada Cemetery is 'grounded' creates an index of: the architecture and the physical and landscape; and the architecture and the site's history - subverting historically modern assumptions that site is "merely the ground on which architecture rests" 26
One process which the site 'acts out' as a grounding index is the excavation/terracing of the existing site to conduct the intervention. This operation of grounding performs the "site's history as a quarry and its new use as a cemetery." The most vivid instances of the excavation process occurs in the construction of the niches, where the entranceway, chapel, and procession carve into the hillside, pushing the earth to the sides, creating a valley that frames the occupant and activates the edges of The Terraces as burial positions. ${ }^{28}$ This is recognised by Zabalbescoa as an exchange in the design process that creates a series of continuous and reciprocal dialogues between the past and the present (historical context), the site as a quarry and as a landscape:

\section{Much of Miralless work is concerned with excavating, shifting or marking in some way the sites on which the buildings are constructed. Although in theory this may seem to be an imposition on the land, in practice it enables the architecture to open up a dialogue with its environment, to work with it, even using what was 'replaced' by construction in the architecture itself. ${ }^{29}$}

Within the excavated landscape, Miralles adopts a second design process of grounding embedding/burial. The embedding of the 'cliff-like' niches into The Terraces exaggerates the procession as it moves downwards towards the mausoleum, giving the occupant the impression of being 'buried' by the cemetery (see Figure 5.16). This process also occurs as occupants pass between The Terraces, experiencing the ground above - punctuated by cuts and holes that allow light to filter through (see Figure 5.17). Wooden railway sleepers set into the ground as figurative bodies also 'act out' as performative referents of embedding/burial. Scattered within the main procession through the site, between the niches, these wooden sleepers embrace the natural processes of deterioration that occur by embedding in the landscape, a secondary referent to burial.

The Igualada Cemtetery informs a sitespecific strategy of continuity primarily through operations of materiality and its aforementioned composition/placement. Materiality contributes to 


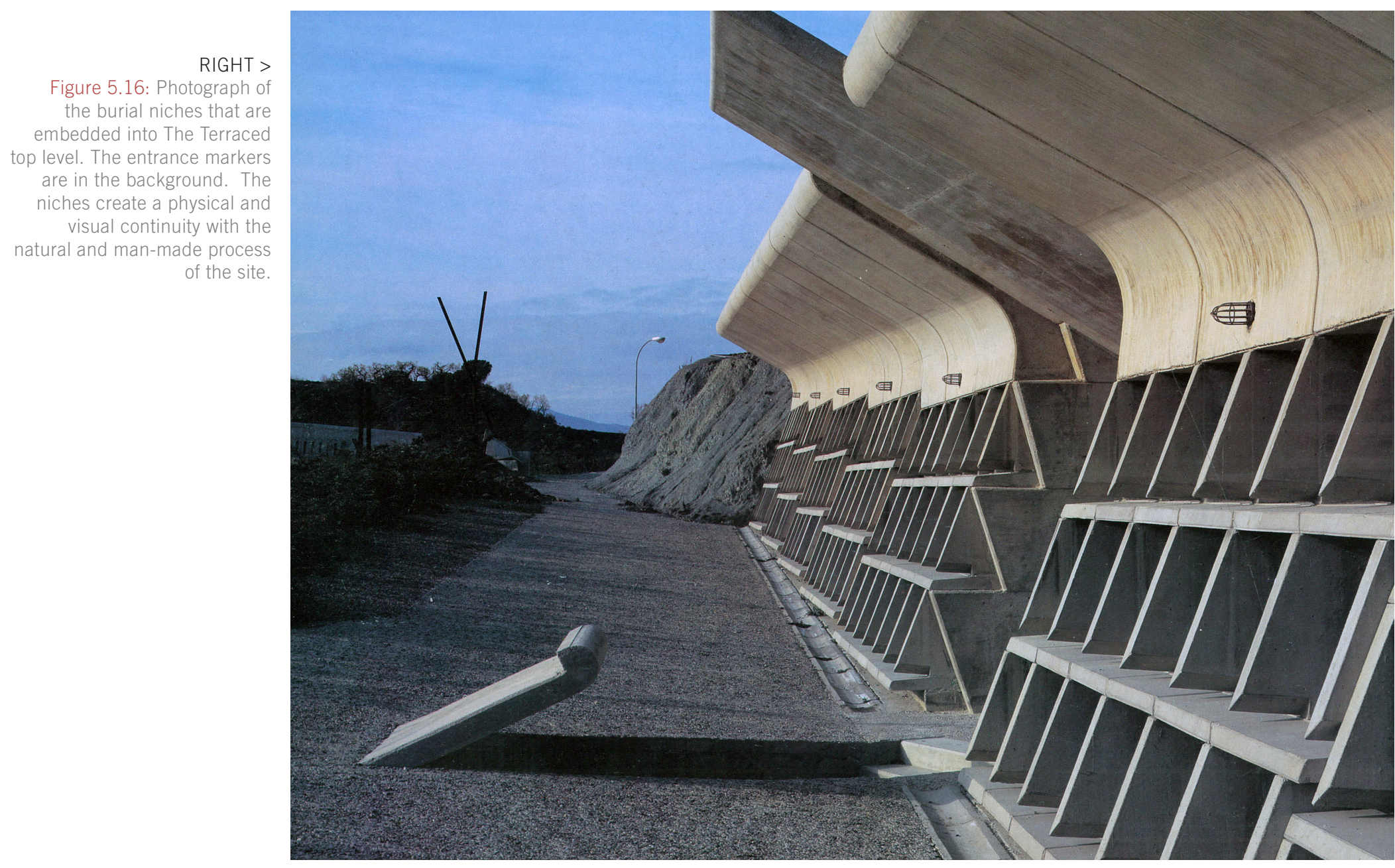

the visual identity of the cemetery within its specific context by generating a language that coordinates with its natural setting. To do so, the design incorporates stone (gabion walls), earth (terraces), and wood (sleepers, seats) to "refer to a process of material continuity between building and site", constructing a strong language between the site's history and the design (see Figure 5.18). ${ }^{30}$ In particular, the use of 30. Rendell, 2008, p. 37 gabion walls (composed of quarried stones collected from the landscape and bound in mesh) exposes a visual referent that "represents a kind of abstraction

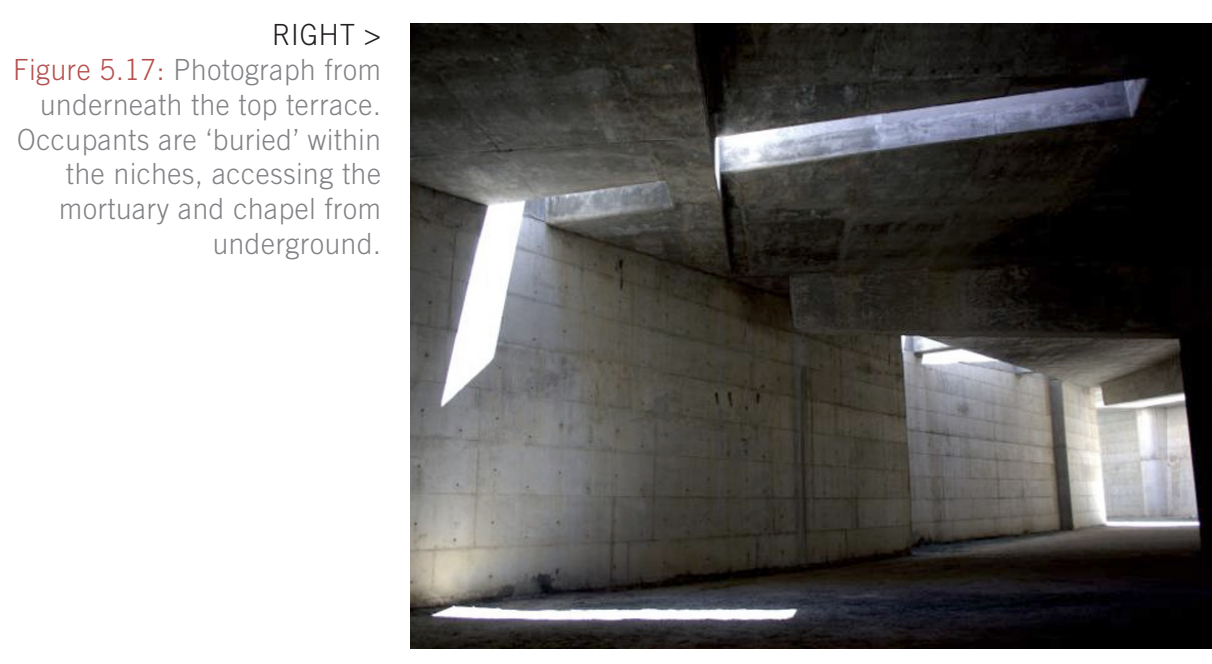

that is not formal, but instead operates through a displacement". ${ }^{31}$ In this sense, the gabion walls are conciliatory with the architecture as it mediates between the natural and built - coding a site-specific practice. Additionally, these walls instruct a visual dialogue between the ground and The Terrace exposing how "the building becomes a part of the surrounding landscape, and also represents that landscape", implicating the topography of the site as the conceptual and physical content of the design. ${ }^{32}$

Miralles's design strategies, as they relate to the architecture's indices of grounding, are of particular use to the design testing of critical spatial practices at the Clifton Street Car Park. The Igualada Cemetery presents a critical examination of site as an index that is continuous with the landscape's history (as a quarry) and naturally occurring setting - performing its contextual dimensions physically, visually and historically. The car park has undergone similar industrial insision - related to the imposition of infrastructure - that can be critiqued indexically by creating continuity with the prior landscape. Whilst
31. (Berrizbeitia \& Pollak, 1999, p. 48) 


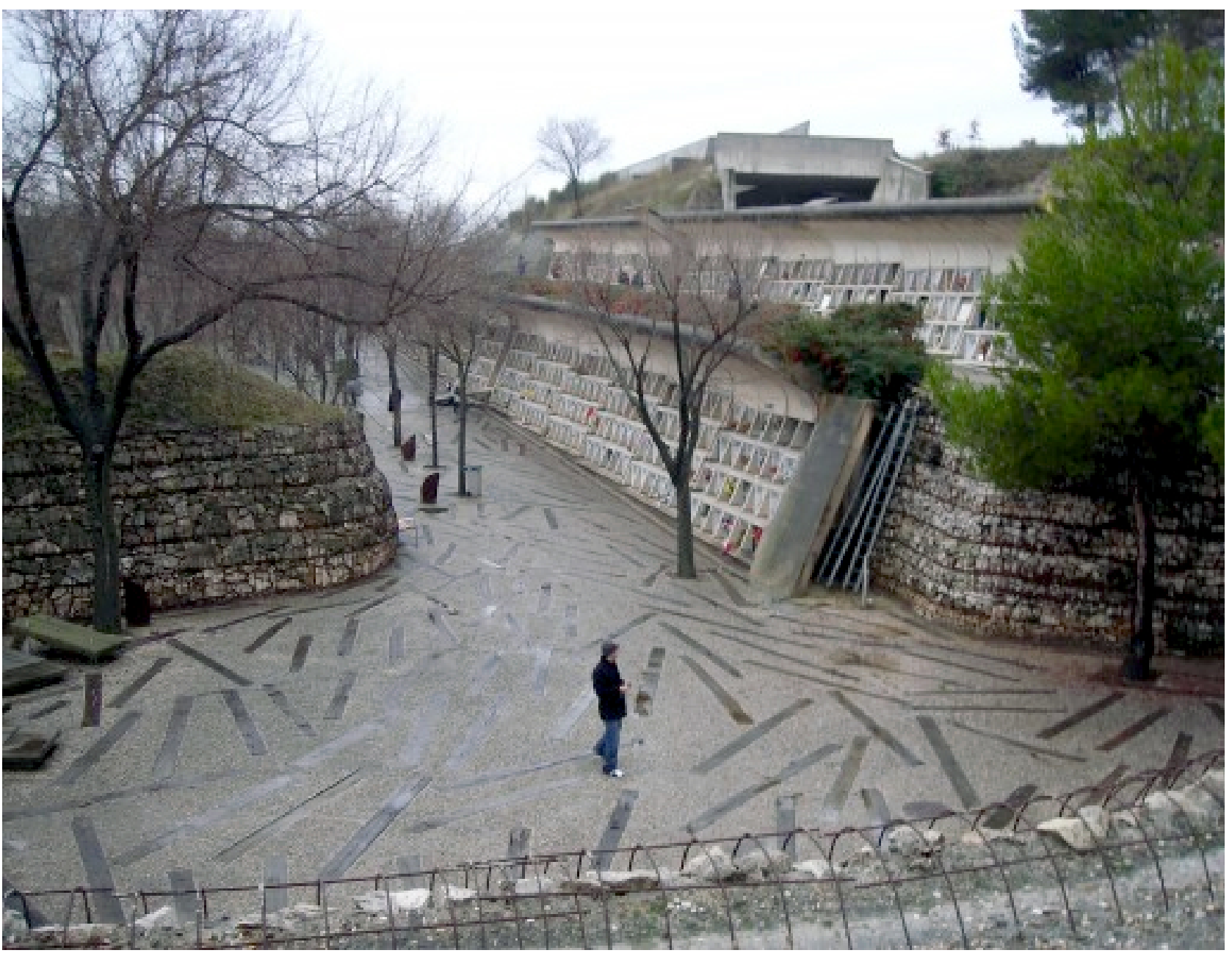

the trauma associated with the Igualada site is less divisive socially and historically than the thick edge condition of the Clifton Street Car Park, Miralles nonetheless explores potent and critical design processes that can be adapted within the design test of this thesis.

Conversely, the cemetery has limitations for the design case study. Primarily, due to its non-urban location, the Igualada Cemtery is concerned with its relationship to the landscape as a naturally occurring condition - its dialogue with the quarry, the materials of the site, and its programme as a burial site. It provides little information to inform critical strategies of resistance as they relate to highway infrastructure and the thick edge condition as a social, political and/ or economic context.
Conclusion: Implications and strategies for design

Throughout Chapter Five it has been posited that, given its dimensions as a thick edge and embodiment of an identity closer to that of a nonplace, the Clifton Street Car Park is an opportunity for a framework of critical spatial practices to explore critical, site-specific, practices.

Following the investigations of Henri Lefebvre, Michel de Certeau and Marc Augé, Rendell's identification of a dual approach to site - as a geographic location and an unfixed place composed of a series of contextual relations - presents a critical position towards the politicised appropriation of site as a defined and 'zoned' region. This stance 'opens' the Clifton Street Car Park to its wider contexts of place, critiquing its current position as a non-defined entity between the central area and inner-residential zones. Implicated by this notion of site, the contentions of Nick Kaye are particularly important - introducing an argument for site-specific strategies that look to 
create a performative index within the design process.

A performative approach will adopt the use of dual metaphors to 'act out' contextual information, constructing a contextual dialogue of continuity/discontinuity through ordering systems, materiality and typology as a critical resistance or exposure to/of the pre-existing condition(s) of a site as a position and a place. These critical practices express an opportunity for the design process to positively reconfigure the site and to create a place of polycontextual flows. The primary narrative of a site (in the case of this thesis, the thick edge) is thus replaced and subverted by multiple narratives, or as Dovey states, "a collision of practices and images rather than one narrative replacing another", enabling "focus on the relationships between elements rather than on the elements themselves". ${ }^{33}$

In relation to the Clifton Street Car Park the performative suggests that the design experiment should concern itself with an inversion, or site-specific discontinuity, with the physical, visual and historical contexts that have informed its rigidity. This rigidity operates through:

A lack of social activity

Continuous infrastructural materiality

Continuous infrastructural typology/ordering system

Excavated ground planes

Zoning adjacencies (residential sites, commercial sites)

Disproportionate scales

Additionally, the design experiment will test how it can perform in site-specific continuity with the landscape as a site of physical, visual and historical trauma. These contexts have operated through:

\section{The burying of the Kumototo}

The removal of graves from Bolton Street Cemetery

The incision, terracing, and excavation of Shell Gully

\section{The kainga Pohutakawa tree}

The demolition of low socio-economic housing in Thorndon

Experiments testing how these strategies can be deployed, prior to additional information at this point, are shown in Figure's 5.19-5.25.

In the anti-contextual discontinuity of Parc de la Villette, and the highly contextual continuity of Igulada Cemetery, Kaye's definition of site-specific practice is made evident. Thus a polycontextual approach to architectural design can inform a critical dialogue of continuity and/or discontinuity. Kaye argues this through an engagement with physical context (typological, material), visual context (compositional, order), and political context (the normative definition of 'site'). How these contexts are engaged through architectural design speaks to a critical intention to construct specific identities of place insofar as they are continuous (contextual) or discontinuous (anti-contextual).

All of these categories (investigated in Chapter Four), when encompassed by performative operations, 'open' the locational notions of site within an expanded field of relations. Through this lens, critical architectural design will respond to a multiplicity of influences that are connected in parts - rather than imposing a singular identity across the site. The design experiment for the Clifton Street Car Park will treat the site as a polycontextual field of relations, with the architecture as the index through which multiple contexts can flow. Whether the intervening architectures are continuous or discontinuous with the existing site will determine its critique with the aim of inverting the contextual rigidity of the current thick edge condition. 


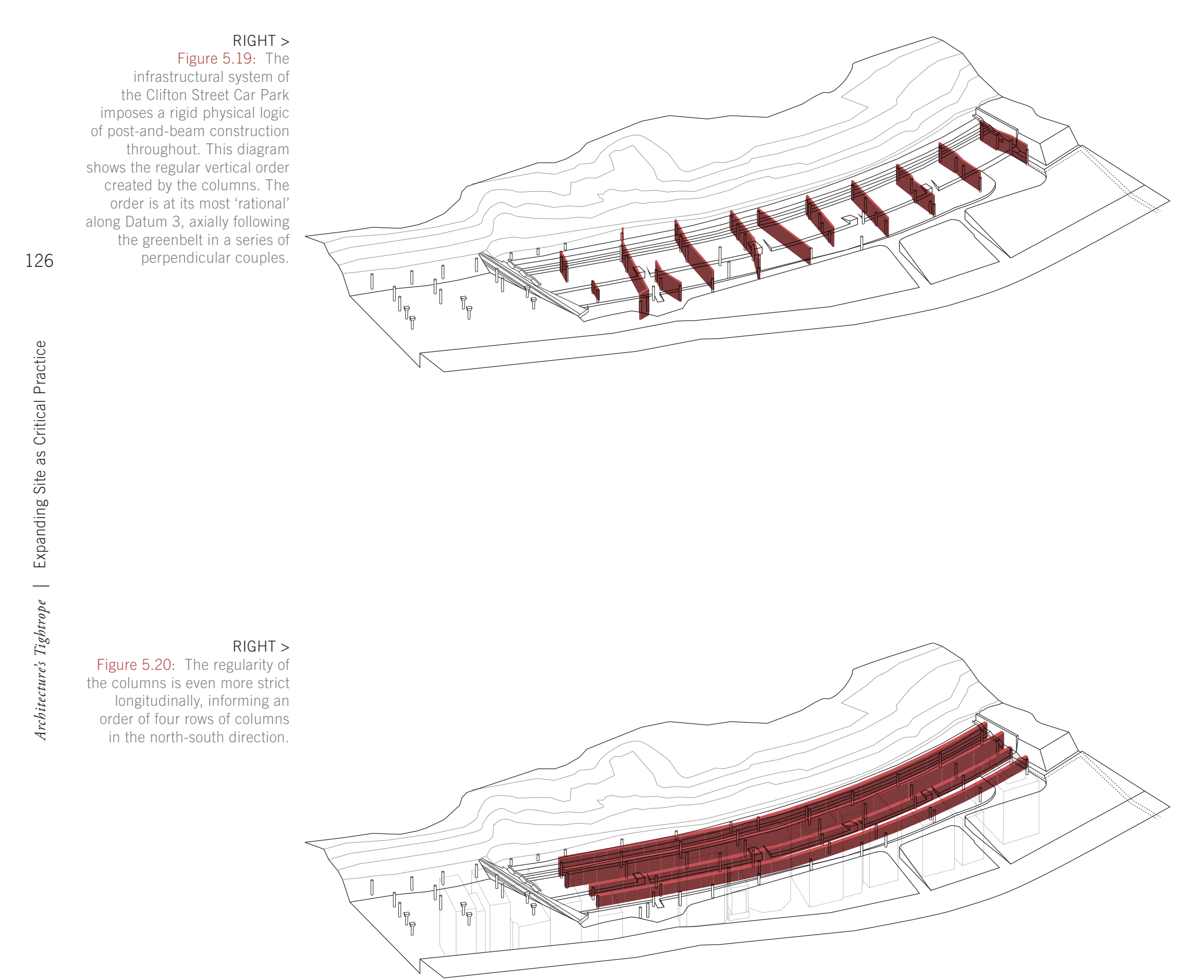


<smiles>CCCC</smiles> 


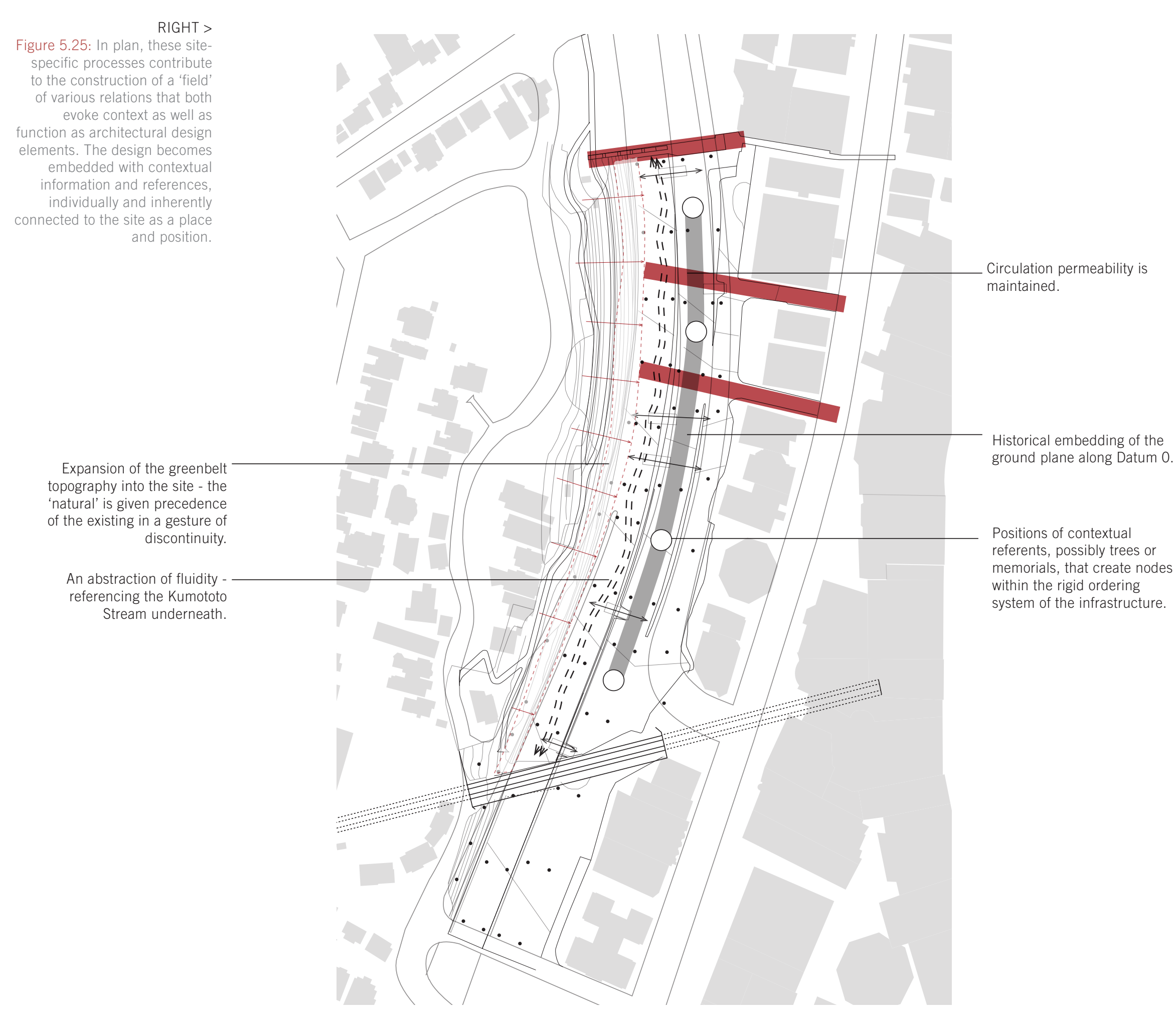




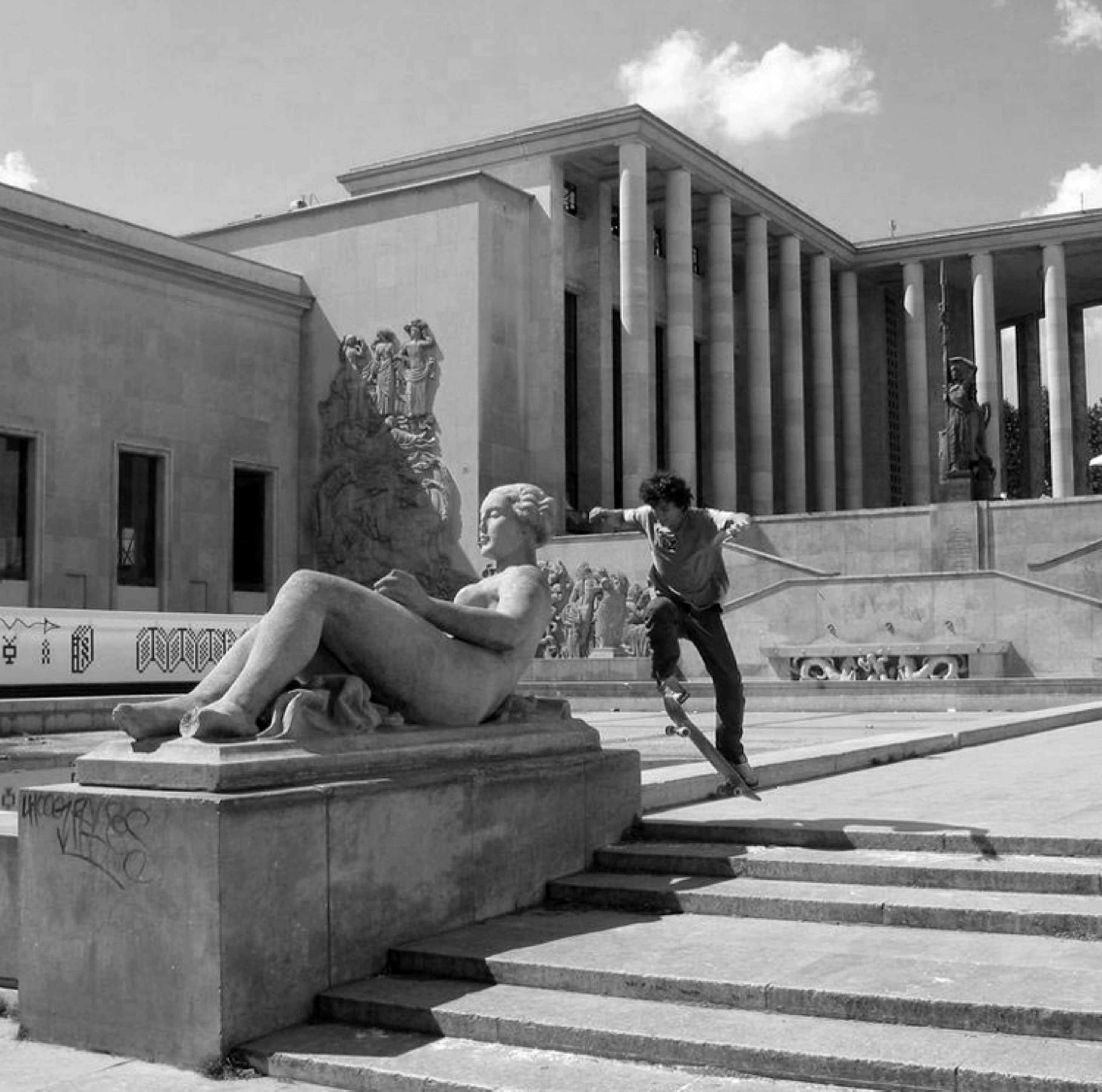




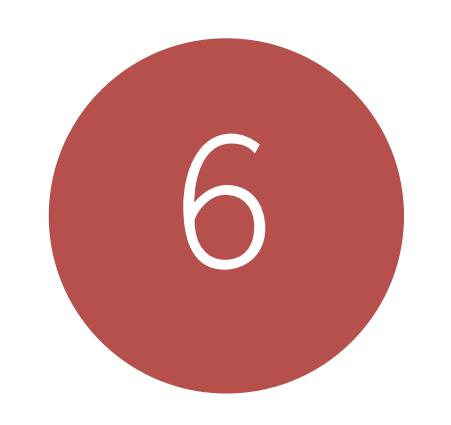

\section{SOCIAL SPACE AS CRITICAL PRACTICE:}

Elucidating Strategies for Sociospatial Flows

Introduction

Between 'One' and 'Another': 'Loose' Space Through Socio-spatial Strategies

The Palais de Tokyos: Social Space as Appropriated Space

The Metropol Parasol: A Canopy for Multiple Publics

The Rolex Learning Centre: Horizontality, Transparency and Porosity

Conclusion: Implications and Strategies for Design 
'Public Space': any space to which the public has generally unrestricted access becomes public - in effect even if not in law. For this reason, this appendix defines public space as all areas to which the public has access - including streets and accessways, pedestrian routes, squares and parks that are part of a private development [emphasis added]. Similarly, public carparking buildings, elevated plazas, walkways, bridges and underpasses are classed as public spaces. ${ }^{1}$

Wellington City Council

\begin{abstract}
In Chapter Five a performative understanding of the notion of site was explored and expanded upon through the contentions of Nick Kaye. In particular, the relationship between critical spatial practices and the creation of site-specific indices was investigated, elucidating strategies of continuity and/ or discontinuity to construct a dialogue between architectural intervention and its relative contexts.
\end{abstract}

Operating within the dynamic and social place/non-place spectrum outlined within this section, this chapter explores and develops the second process of critical practice outlined by Rendell's framework. Arguing that critical spatial practices will approach assumptions of space between interests that are private ('one') and public ('another') from a resistant position of 'opening', a critical position will engage space as relational, as both public and private - rather than defined as either/or where one term is privileged over the other and becomes susceptible to 2. Rendell, 2008, p. 9. processes of control and alienation. ${ }^{2}$

Developing Rendell's framework further, Chapter Six implicates the work of Kim Dovey who posits that architecture 'frames' the practising of a de Certeauian place; it provides a system of structures through which space is activated and produced engaging social and political contexts in combination with the physical, visual and historical flows created by site-specific practices. For Dovey, this 'structuration' empowers architecture and the built environment to provide the tangible spatial structures through which the performance of spatial practices by individuals or 'publics' are enabled or constrained. Critically negotiating several dimensions of 'tight' and 'loose' spatial structuration, architecture can positively resist politically controlled and socially deterministic approaches to public sites. These processes contribute to the 'opening' of design processes to strategies that allow spaces to be appropriated by multiple publics through multiple contexts.

The importance of these processes in the development of critical spatial practices is revealed through three pertinent and contemporary architectural case studies that critically engage public space as structured by architecture: the Palais de Tokyo (2001) by Anne Lacaton and Philippe Vassal; the Metropol Parasol (2011) by Jürgen Mayer; and the Rolex Learning Centre (2010) by Ryue Nishizawa and Kazuyo Sejima.

Analysing how strategies of addition, subtraction, horizontality and verticality are deployed through architectural design within these case studies reveals crucial translations of critical spatial practices within the built environment. As a result, this chapter posits that strategies of tight and loose public space reveal opportunities for critical spatial practices -the potential to introduce flows of social and political context to the Clifton Street Car Park thick edge condition.

In so doing, this chapter adds to Rendell's framework and mediates between the theoretical framings of Section One, and the design case study 
in Section Three; engaging the fourth research question:

How can critical spatial practices manifest in the built environment, in particular through strategies of architectural design? How can these strategies engage multiple contexts to introduce positive flows to the urban thick edge?

\section{Between 'One' and 'Another': 'Loose'} Space through Socio-spatial Strategies

The definition of public space presented above by the Wellington City Council expresses a widely-held desire to represent public space as topographical, an approach that is representative of traditional approaches to 'the public' as well as the concept of 'public space'. Topographical public space asserts that space itself is defined abstractly by borders, and therefore clearly demarcates access to particular urban contexts for 'the public' or 'the private' realms. ${ }^{3}$ In this situation, 'the public' is defined collectively as the entirety of citizens within the public sphere.

\section{Architecture must treat space as relational, as both public and private, rather than defined as either/or where one term is privileged over the other and becomes susceptible to processes of control and alienation.}

architectural discourse through the 'new urbanism' movement.

Such arguments are primarily built upon Hannah Arendt's idea of public space as it relates to the Greek Agora - a place of public gathering, instigating new urbanists to call for 'legible' public space, defined areas that can be recognised as exclusively public'. See: Arendt, The Human Condition, 1998 [1958].

4. Iveson, 2007, p. 5. Kurt Iveson is Senior Lecturer in Urban Geography at University of Sydney.
Problematic outcomes of these attitudes arise when spatial intervention appears incongruent with the ideals of the democratic masses, when social activity within defined public space impedes upon the 'order' and 'quality' of 'good public space'. According to Kurt Iveson:

Here, it is argued that exclusion from public spaces is the product of so-called 'anti-social' and criminal behaviour. Planners and law enforcement agencies charged with the responsibility of improving public space argue that the exclusion of a troublesome minority will make public space more accessible for the well-behaved majority.

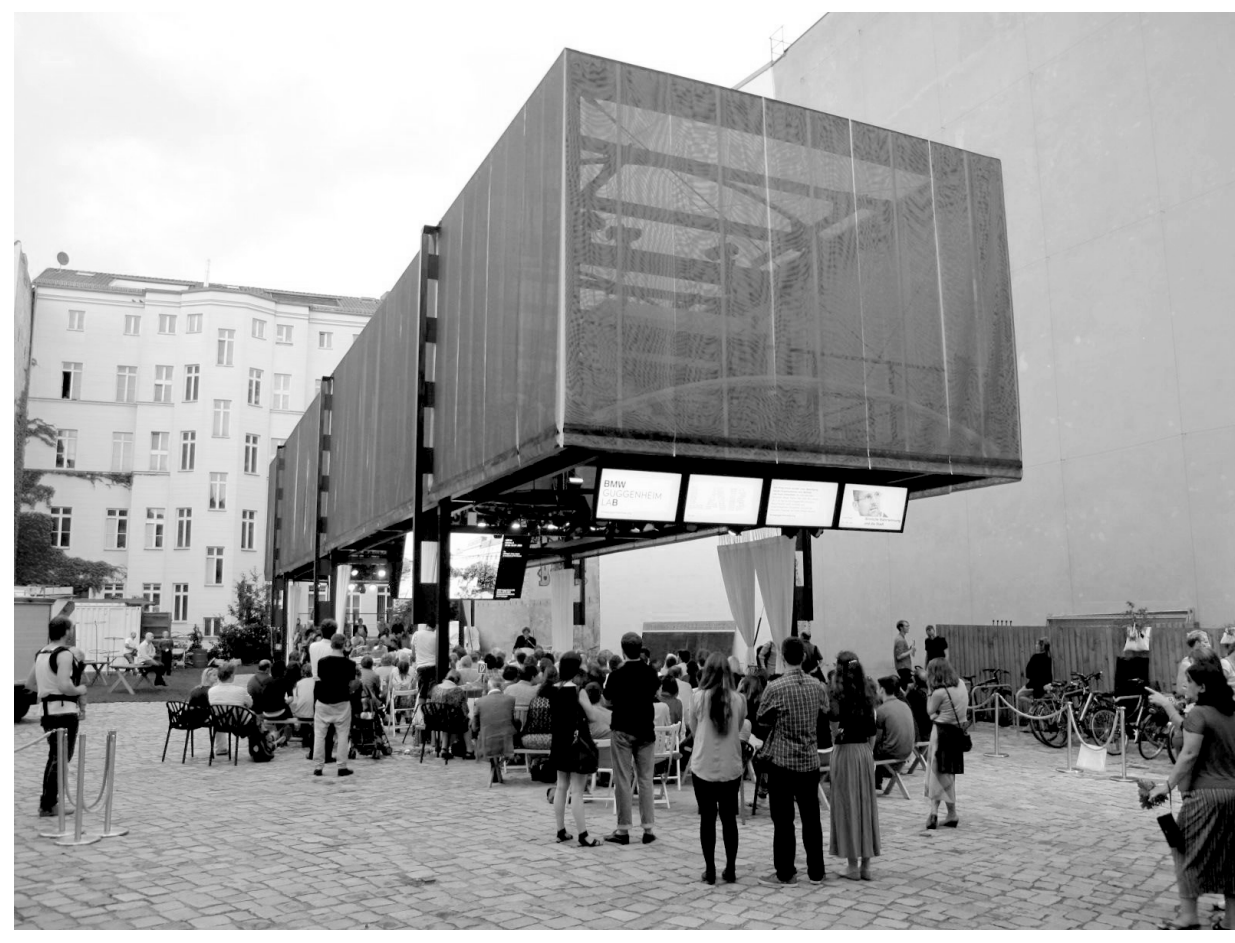

Bruce Robbins argues that the common $\wedge$ ABOVE idea of the all-inclusive 'public sphere' is a phantom, Figure 6.2: The BMW -

a nostalgic view that is the catch-cry of those who Bow Wow, Berlin, 2012. wish to control access to topographically defined public spaces. ${ }^{5}$ For Robbins this (phantom) public space is generally based upon narratives that the urban landscape has previously been quantifiably 'more public', and therefore must be 'retrieved' as a place of dialogue, co-existence and access. ${ }^{6}$ However, in many instances this view instructs environments that are fertile places for tactics of control, security, and surveillance - negating the desires of certain citizens to privacy in the public realm - they produce environments which reduce the city to "a script that we must follow", a linear and deterministic view. ${ }^{7}$

Kim Dovey's contribution to critical spatial practices explores an alternative requirement for the production of public space to resist mechanisms of power and control through architecture's ability to "frame space both literally and discursively" ${ }^{8}$ Framed by architecture in particular ways, public space is capable of resisting tactics of social and political appropriation - it can display, explore, and produce socio-spatial flows. Dovey's approach to space, as contributed to by architectural form, is built upon the relationships between 'agency' and 'structure' investigated by cultural theorists such as Michel Foucault, Anthony Giddens, and Pierre The 'roaming' LAB travels to various city centres to present architecture to the public through various interactiv modes (video, lecture, display, exhibition). Various architectural strategies inform a buildings that enable sociospatial flow where the $L A B$ is sited. Architecture is 'framing' 'Ioose' public space through spatial practices (programme) and structure (the architecture)

5. See Robbins, The Phantom Public Sphere, 1993, Bruce Robbins is Professor in the Humanities, Departin the Literature at Columbia University.

6. Robbins, 1993, p. viii

7. Betsky, 1998, p. 458. See also: Deutsche, "Art and Public Space: Questions of Democracy", 1992

8. Dovey, Boyer, Fraser, \& Morrow, 2013, p. 11. of English \& Comparative 
9. See: Giddens, Central Bourdieu. ${ }^{9}$ Through these figures, Dovey identifies Problems in Social Theory: Action, Structure, and Contradiction in Social Analysis, 1979; Bourdieu, 1990; Foucault, On Power 2000 [1988]

RIGHT > Figure 6.3: Diagram showing the dimensions of tight/ loose space as identified by Kim Dovey and applied to the Clifton Street Car Park.

Many of these dimensions are discursive and/or structural - contributing to the overall "structuration' of a design, its capcity to enable or constrain critical socio-spatial agency. The current condition(s) of the Clifton Street Car Park are positioned on these scales by a red circle. that 'agency' expresses the capacity for a system (in this case multiple publics) to perform within the organised properties of a wider 'structure' (in this case the dimensions of public space). The relations between agency and structure are those of 'enabling' and 'constraining' - agency can either be restricted or allowed to flow according to the nature of its structure:
From this view architecture can be considered as a form of structure, and the social action it 'frames' as a form of agency. Architecture evokes and enables certain forms of life while constraining others with both walls and sanctions...this complex interaction of structure and agency is what Giddens calls structuration. ${ }^{11}$

11. Dovey, 2008, p. 19.

Applied architecturally, spatial practices and spatial structure contribute particular properties to the design of the built environment, properties that exist in constant mediation between the polarised

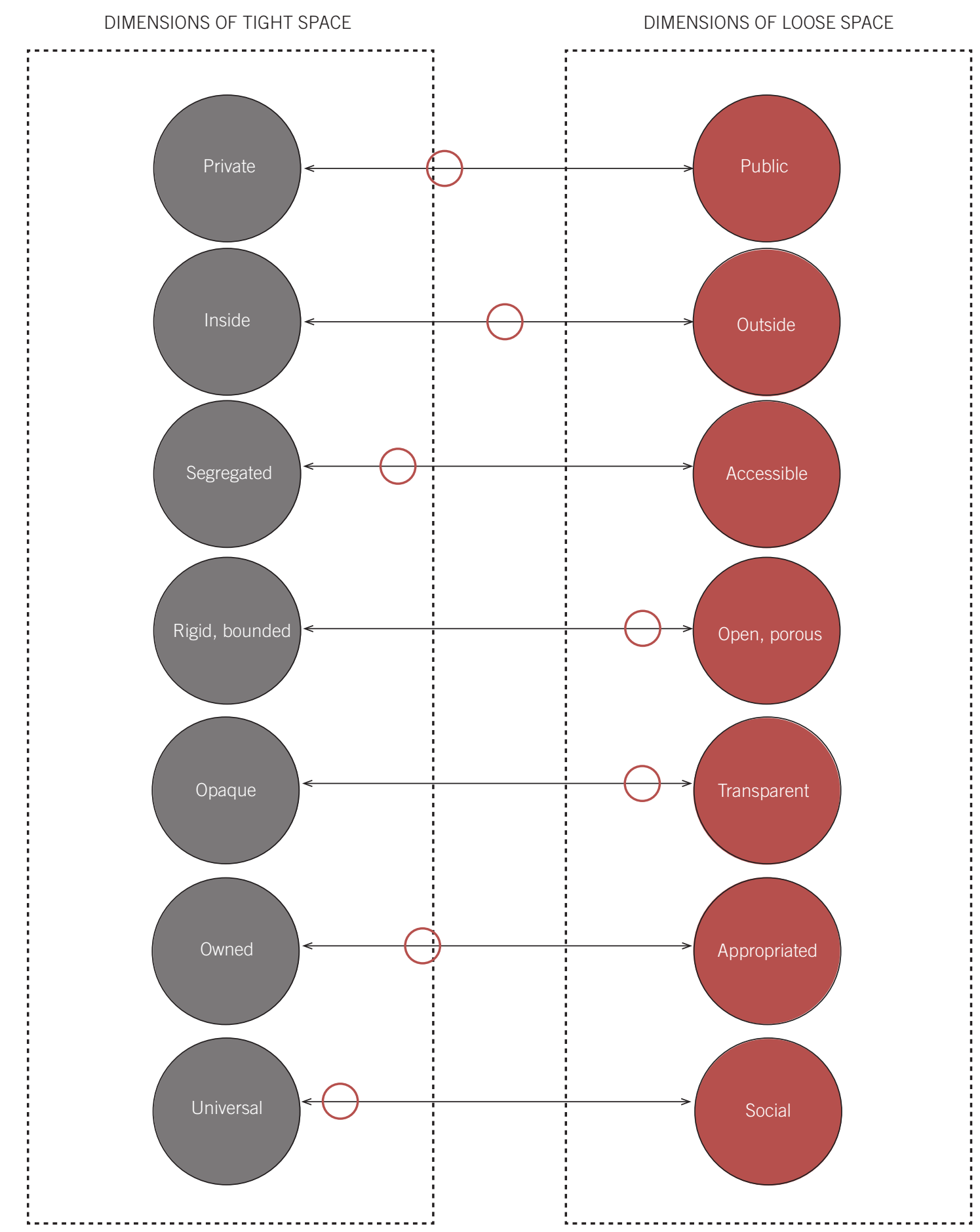




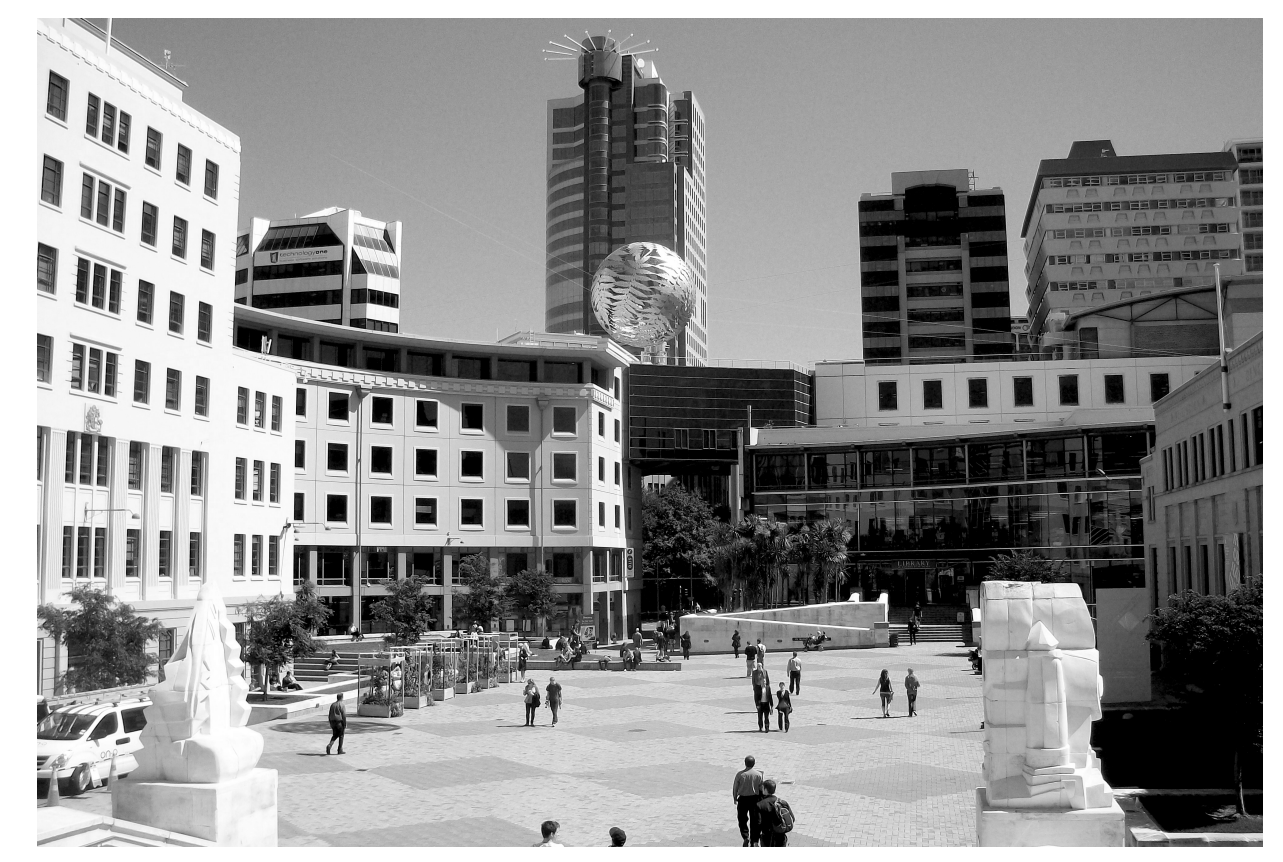

$\wedge$ ABOVE dimensions of tight and loose space (see Figure 6.2). Figure 6.4: Photograph of Wellington's Clvic Square during a time of low activity.

The site is bounded, static an constrained by the structures
that surround it. movement and instability through which stable territories are erased and new identities and spatial practices become possible"; it represents a desire for an urban condition that resists certain dimensions of socially and politically defined contexts. ${ }^{12}$ Socially, loose

12. Ibid space posits that multiple publics are deployed as individuals, relationally interacting in the processes of spatial production. Loose space therefore resits the politically appropriated and deterministic definition that public space is singularly defined by ownership/ access, in favour of architectural structures and programmes that facilitate possibilities for exchange. Additionally, loose spaces invest and embed places with social context, exposing possibility and opportunity 13. Franck \& Stevens, 2007, for social activity where it may not already exist. ${ }^{13}$ pp. $15-18$

Conversely, loose space intentions are polarised by social and political tight spaces, "where identities and spatial practices have become stabilised

14. Ibid, p. 22. in strictly bounded territories with choreographed spatial practices and socially controlled identities." ${ }^{14}$

As such, a critical design approach will resist the "mindless repoduction of socio-spatial practices" by constructing "spatial flows" between literal and discursive dimensions of tightness and looseness

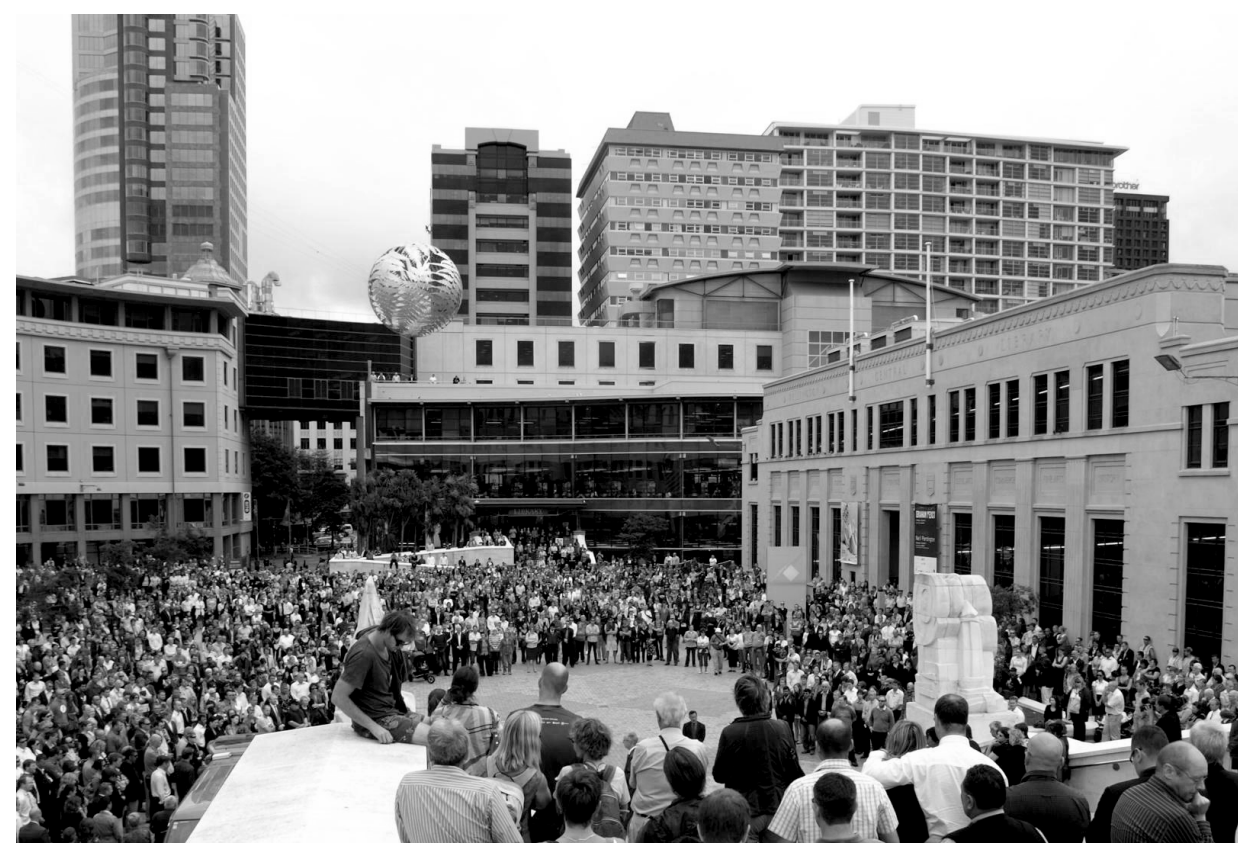

Tight spaces follow the same logic as the negative $\wedge$ ABOVE

urban spaces highlighted previously in Chapter's

Three and Four, as sites of global-to-local forces, singular use, and limited approaches to engagement with context(s).

Properties of loose/tight spaces contribute to the structure within which multiple publics can perform. Importantly the dimensions of looseltight are in a state of constant mediation, and - much like the place/non-place spectrum put forth by de Certeau - are never fully realised as absolutes. Rather, for Dovey, the role of critical spatial practices is to acknowledge the dimensions that are at play in any given situation and to facilitate positive synthesis between existing and intervening architectures.

Exploring the means through which the loose/ tight spectrum may be deployed architetcurally, Dovey analyses critical architectural production as inherently informing "the structure of social space, the use of boundaries to mediate social encounter and standardised spatial fields and building types. ${ }^{15}$ For Dovey, architetcural form and structure negotiates between a variety of spatial dimensions along which the social and political instruments of power in places are played out. ${ }^{16}$ As such, a critical design approach will resist the "mindless repoduction of socio-spatial practices" by constructing "spatial flows" between literal and discursive dimensions of tightness and looseness (see Figure 6.3). ${ }^{17}$
Figure 6.5: Photograph of

during a time of public address and demonstration. The space is recognised by publics as one of civic power. In this sense it is capable of supporting a social 'looseness' as it becom

appropriated, social and practiced as 'outside'. 


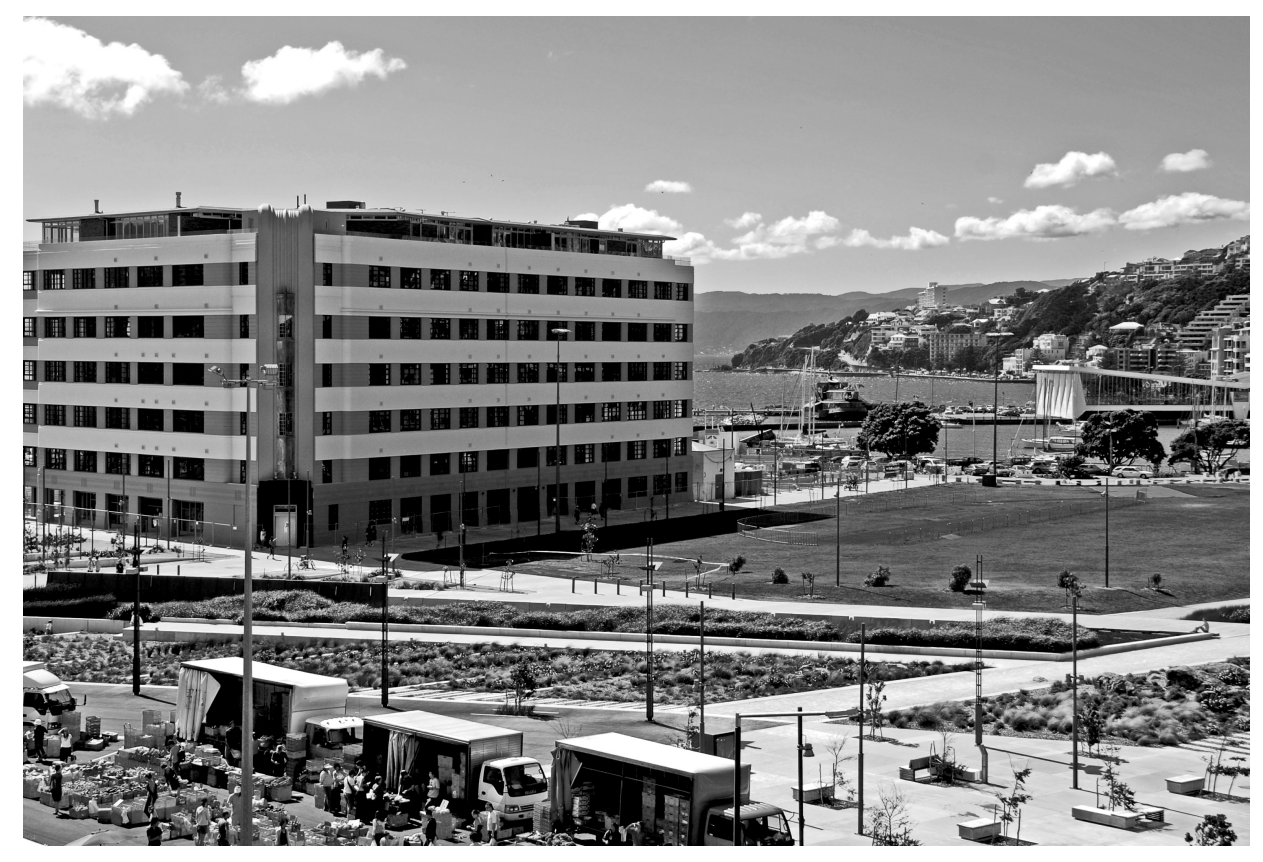

$\wedge$ ABOVE Figure 6.6: Photograph of Wellington's Waitangi Park. The public transparency and accessiblity of the park allows for multiple publics to engage and for multiple spatial practices to occur. These include farmers markets, skateboarding, concerts, recreation etc.

Dovey's dimensions of tight/loose public space are readily found contributing to public spaces in Wellington. Civic Square - although overtly accessible to multiple publics - presents a public space which displays socio-spatial tight properties. The square is a fundamentally bounded territory, defined rigidly by the buildings and infrastructure that surround it, with little inside/outside, public/private permeability or flow (see Figures 6.4). Although Civic Square is internally 'open', it is constrained by its outside barriers - its facades. This informs a defined public space that is enclosed by its architecture. However, spatial looseness arises when the square is appropriated by publics for events and civic upheaval - acknowledging the square as a gathering place for 18. Ibid, p. 254. protest and event (see Figure 6.5). ${ }^{18}$

Another of Wellington's prominent 'public' spaces, Waitangi Park, displays properties that

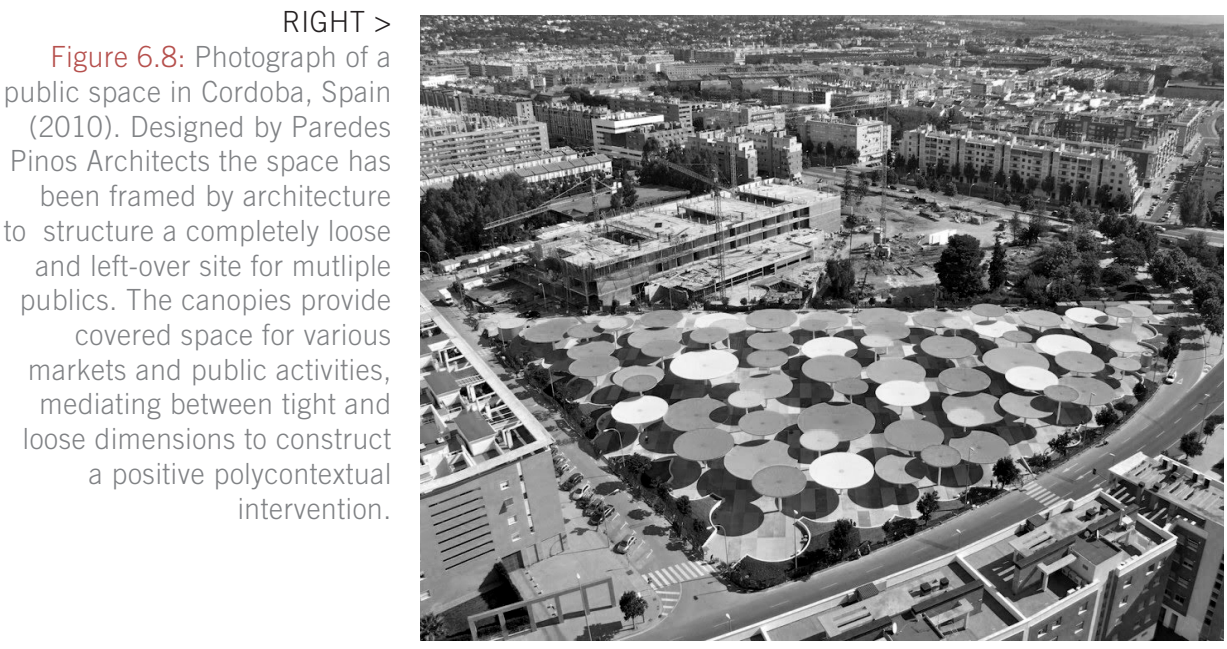

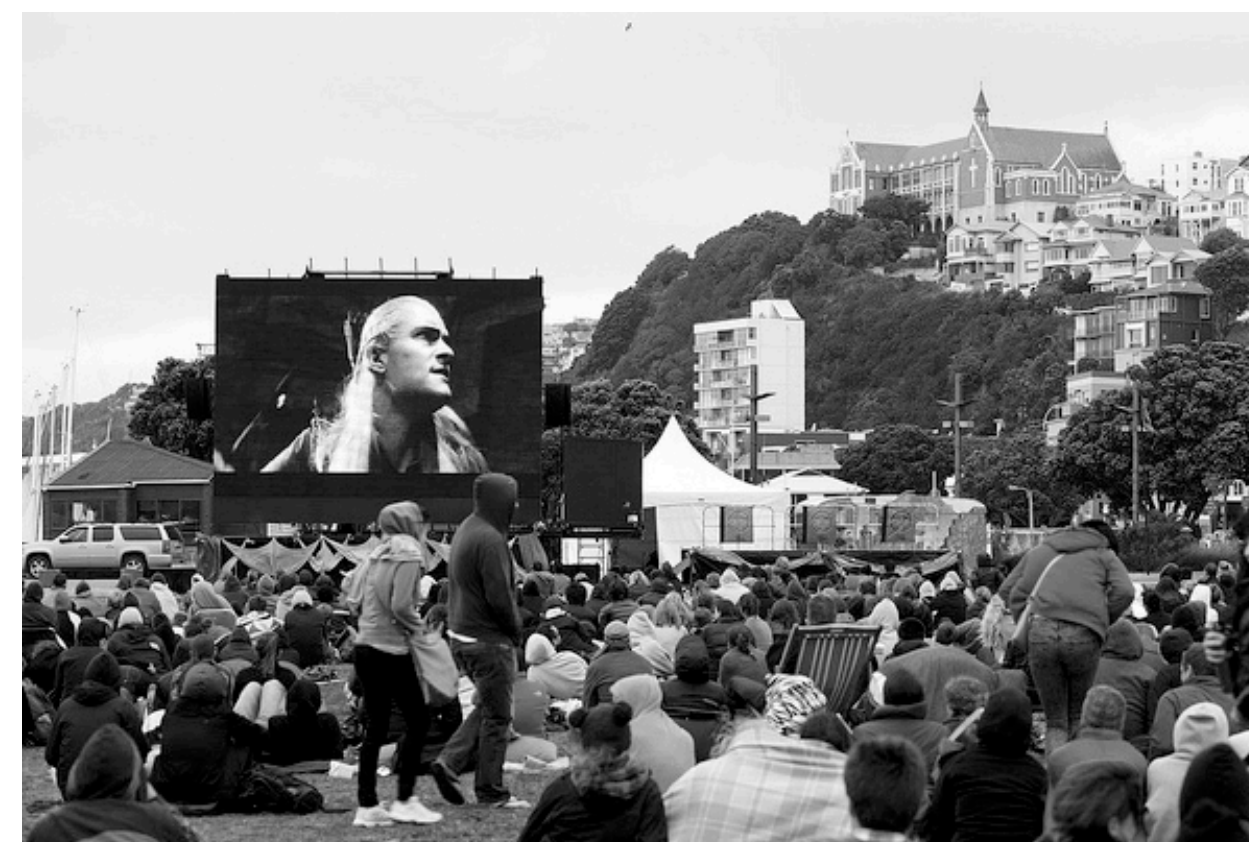

exaggerate socio-spatial looseness. As an urban park, the site is almost entirely unbounded and porous it is outside - presenting a space of accessibility and transparency for multiple publics (see Figure 6.6). The site has been facilitated through circulation and public activity spaces, but offers little privacy. The park's looseness and openness positively facilitates engagement with multiple publics; however, it is architecturally unstructured and unsheltered, requiring significant infrastructure to support specific events, and lacks public/private diversity (see Figure $6.7)$.

Importantly for the design case study of this thesis, the Clifton Street Car Park displays properties that are closer to that of a tight space - a socially constrained and 'strict' site that is politically defined as public, yet performs in a limited capacity as a public space. Engaged for the singular purpose of car parking, the Clifton Street site is highly segregated, fixed, and socially restricting. Dovey's dimensions of loose space, although not deterministic, provide a series of properties for the discursive and architectural 'structuration' of this spatial condition. They impart a framework through which the thick edge can be politically (as a defined public space) and socially (as confluence of social actors) mediated, even inverted. This follows the aim of acknowledging the "messy and dynamic urban geographies of publicness", opening the site to its wider social context(s) (see Figure $6.8) .{ }^{19}$ Consequently, three contemporary projects are

\section{ABOVE}

Figure 6.7: Photograph of Wellington's Waitangi Park. During outdoor public events . (he teral outside nature of the park becomes problematic.
9. Iveson, 2007, pp. 8-9.

RIGHT, OPPOSITE > Figure 6.9: Aerial hotograph of Paris highlighting the location to the Seine river of the Palais de Tokyo adjacent 


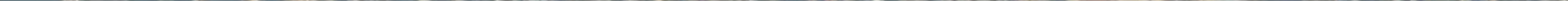




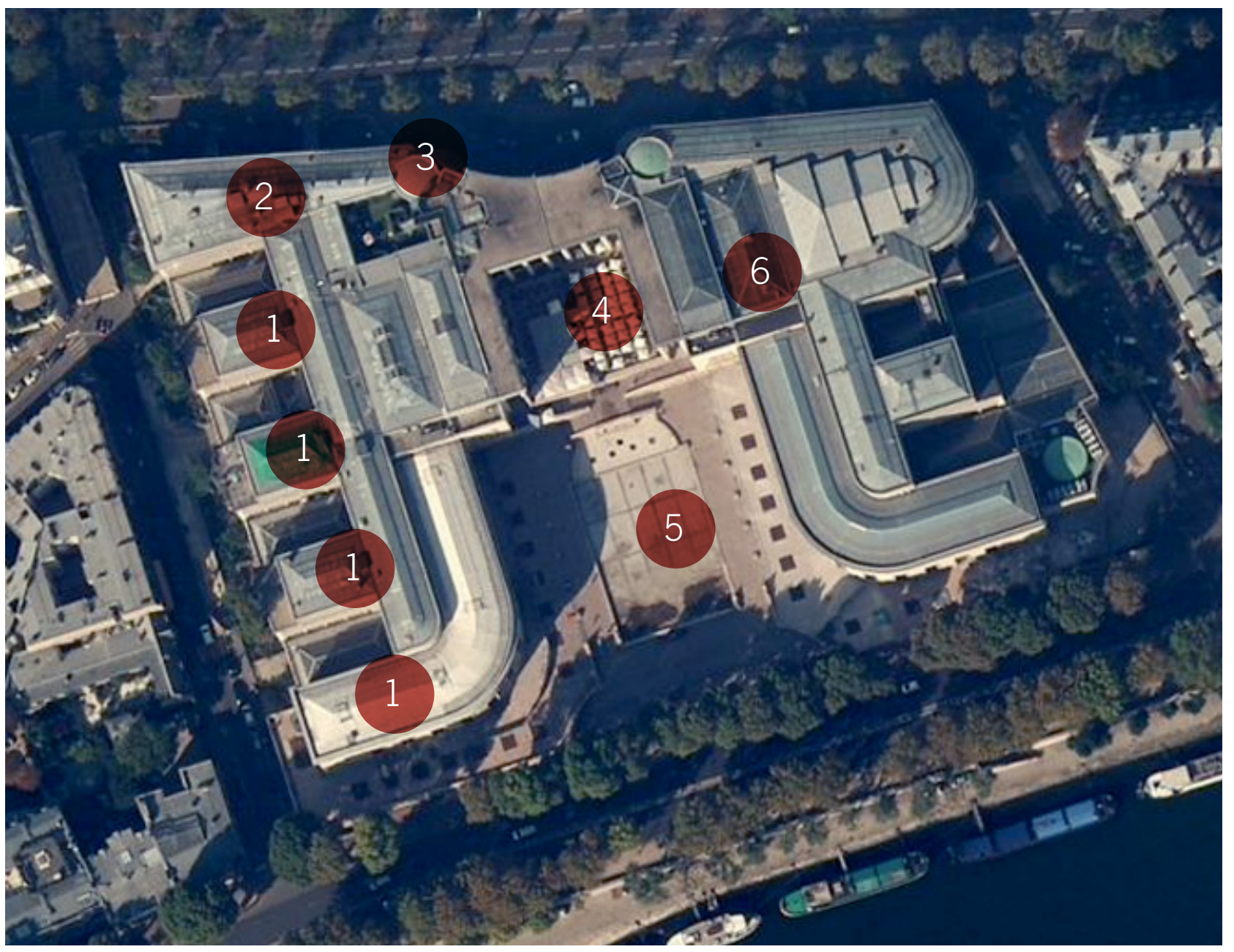

$<$ LEFT

Igure 6.10: Aerial map of the Palais de Tokyo showing, In plan, the significant spatial areas of the centre. The site is bounded by the Seine River and arterial roads that form its circumference. To create permeability, Lacaton and Vassal have stripped back the interior structure of the old building - introducing visual and social transparency between interior and exterior.

KEY:

1. Multiple overlapping gallery wings 2. Cafeteria, bookstore

3. Level One entrances

4. Colonnade

5. Public square

6. Musée d’Art Moderne de la Ville de Paris

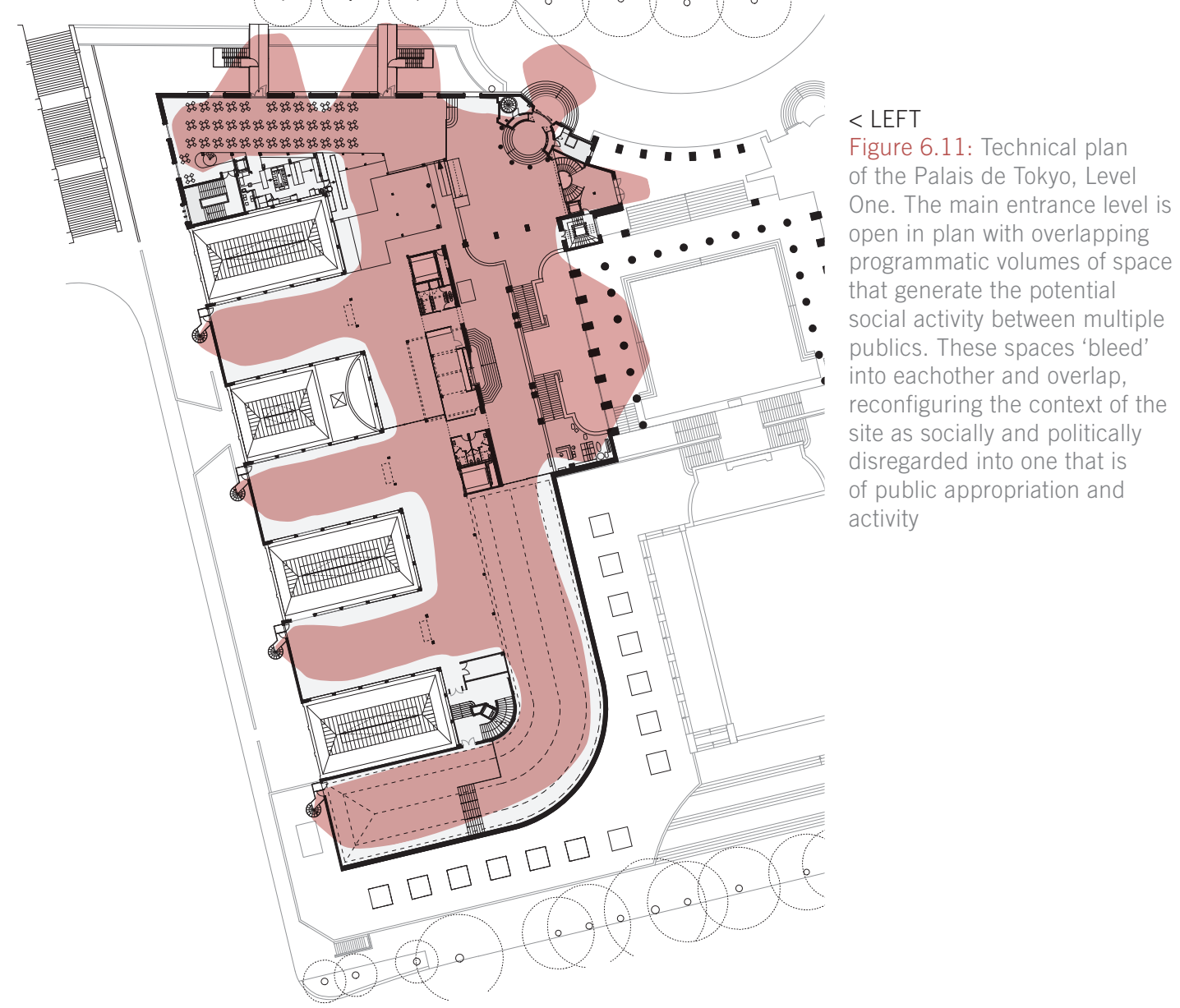




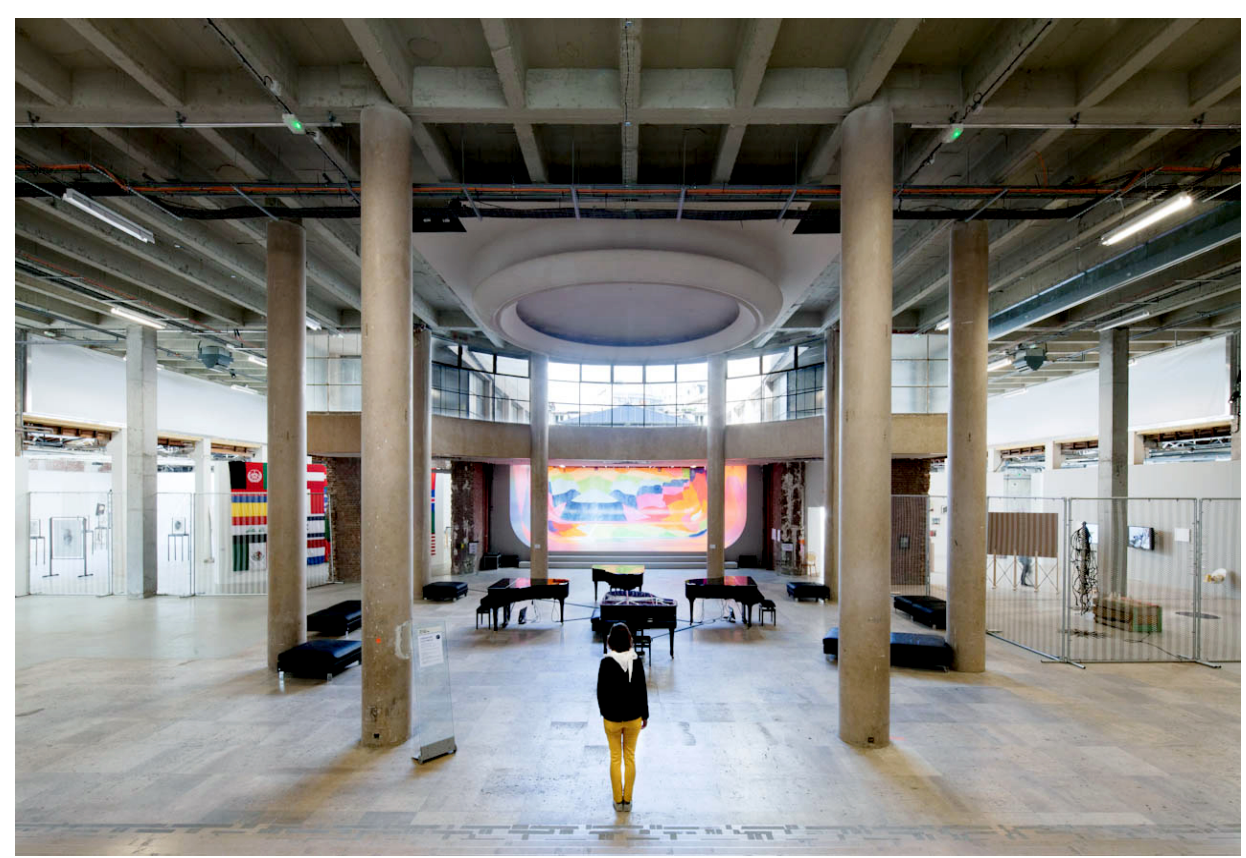

$\wedge$ ABOVE investigated in this chapter as exemplars of Dovey's Figure 6.12: Photograph of an interior 'volume' of social space in the Palais de Tokyo's level one. The orignial structur has been stripped back and walls removed to inform an environment of 'spatial excess' Concrete frames have been added where necessary fo structural support, creating a visual and physical motif of intervention throughout. Overlapning areas between the gallery wings are activated through performances and alternative spatial practices such as piano and skateboarding. The space is appropriate by multiple publics engage or disengage as they wish

20. Lacaton \& Jean-Phillipe, 2010

21. Ayers, 2012, p. 4 critical strategies for the social and political inversion of thick edges - exploring dimensions of tight and loose space through architectural design. These are: the Palais de Tokyo (2001); the Metropol Parasol (2011); and the Rolex Learning Centre (2010).

\section{The Palais de Tokyo: Social Space as Apropriated Space}

Whilst public space can be hijacked and its defintion appropriated for political motivations, conversely it may be appropriated by architectural intervention for social means. One such example is the 'Palais de Tokyo' (2001) by Lacaton+Vasal Architects - an art gallery, performance and event space that has been inserted within a neo-classical museum on the north bank of the Seine in Paris (see Figure 6.9). The design intervention re-creates and re-imagines the original venue as a 'place of contemporary creation' which encourages a dialogue between the previous building (left in ruin) and the addition of the new structure as an appropriated, relational social space. ${ }^{20}$

Originally built in 1937 to house art collections for the Exposition Internationale des Arts et Techniques', the Palais de Tokyo fell into disrepair following the relocation of its collections in 1976 to the Centre Pompidou. ${ }^{21}$ The structure remained abandoned until 1999 when the French Ministry of Culture decided to develop Anne Lacaton and Phillipe
Vassal's proposal for a flexible, public, arts facility and museum. Following the instructions of contemporary curator, art critic and theorist, Nicolas Bourriaud, the Palais de Tokyo explores the notion of appropriation - opening a space that is widely perceived as negative and obsolete for the purposes of relational social interactions between multiple publics. For Bourriaud, this meant employing architectural design as a framing device, to mediate a place for "the realm of human interaction and its social context, rather than the assertion of an independent and private symbolic place"; for the space to seek and establish intersubjective encounters. ${ }^{22}$ To achieve this looseness, Lacaton and Vassal adopt strategies of addition and subtraction through: the addition of space as a socially produced, public proposition; and the subtraction of physical structure to create transparency, porosity, and blur the occupation of outside and inside.

Socially, Lacaton and Vassal acknowledged the abandoned building as privately defined, segregated, and owned - a predominantly tight space (by Dovey's definition) which has become disengaged BELOW

from the public sphere. Inverting this condition, the architect's superimpose the addition of "loose spaces that are constantly in flux, redefined by their users with temporary, often virtual boundaries". ${ }^{23}$ The addition of space with this intention introduces social volumes' that overlap and can be accessed through multiple entrance points by multiple publics: "No need to look for an entrance - just walk in anywhere.

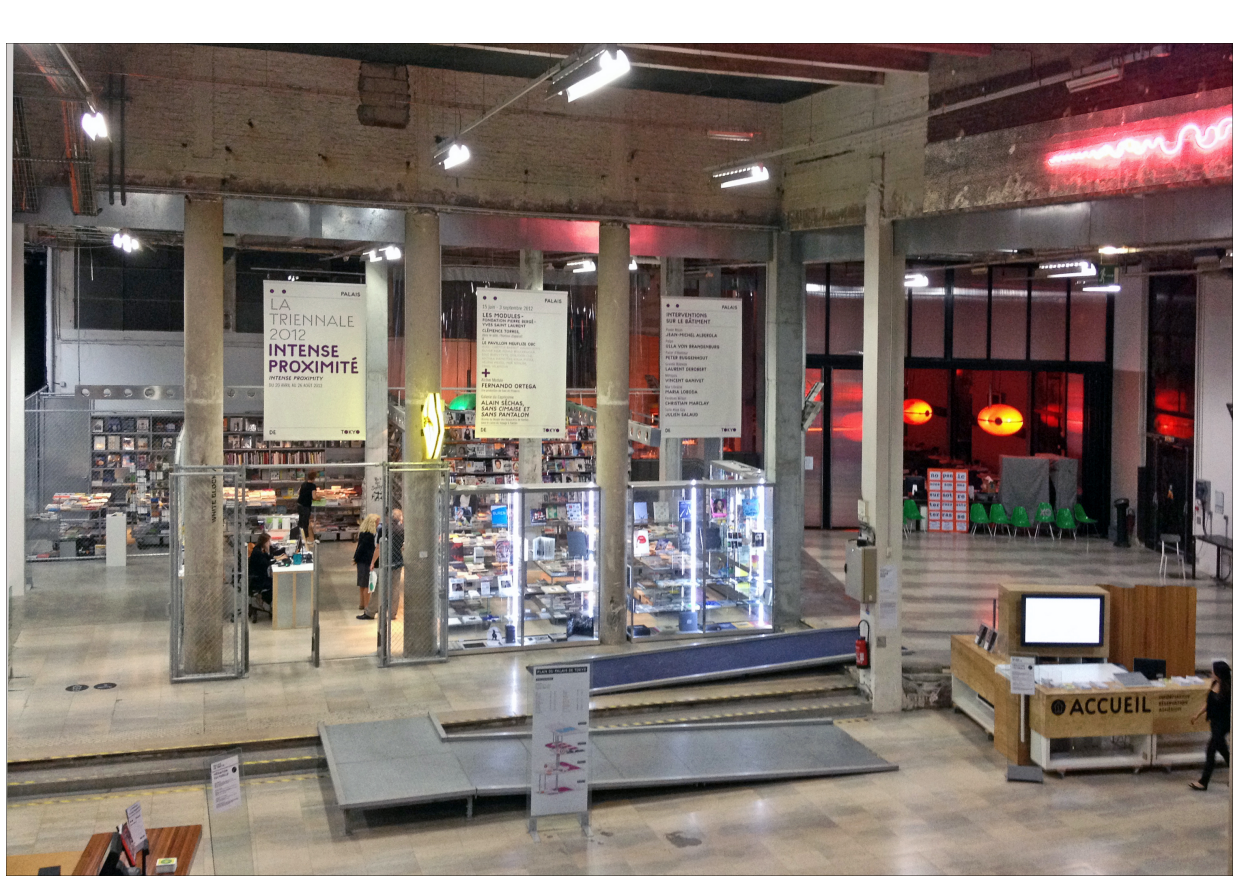




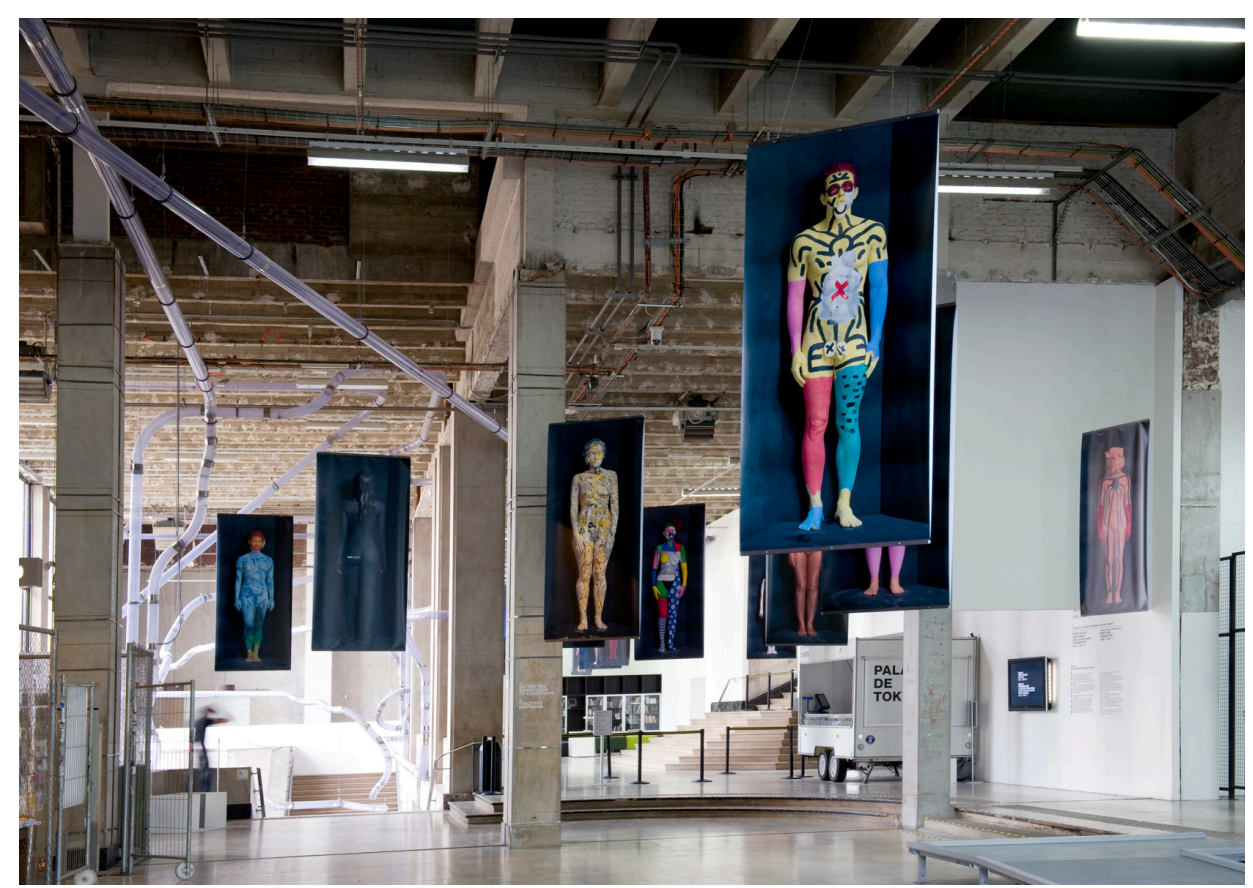

$\wedge$ ABOVE No doors, foyers, queues or commissionaires: it's Figure 6.14: Photograph of up to you how you use it" (see Figure 6.13). ${ }^{24}$ To an interior 'volume' of socia space in the Palais de Tokyo's level one. The spatial flexibility created by Lacaton and Vassal's design strategies works harmoniously with the curation of Bourriaud. Exhibitions and performances 'spill out' into the circulation spaces and refuse

to be submitted to defined locations - to be simply huns on the walls.

24. Lacaton \& Jean-Phillipe, 2010.

25. Rendell, 2008, p. 94

The addition of space creates transparency, visual and physical, between inside and outside. This is evident in the deployment of multiple programmes introduced through the architecture - igniting a variety of different spatial practices. On top of the exhibition spaces, the Palais consists of two cafes, a book shop, a skate ramp, and eight-metre high circulation volumes that can be used as sites for performance (see Figure 6.12). The circulation created by inserting programmes in this way is conditioned by Bourriaud's curation - he attempts to create 'events' rather than to merely "hang art on walls". ${ }^{26}$ This has the effect of instigating a dynamic programmatic flow between each of the galleries, connecting them through people and art, and blurring the normally defined conditions through which the art and architecture is experienced (see Figure 6.14)

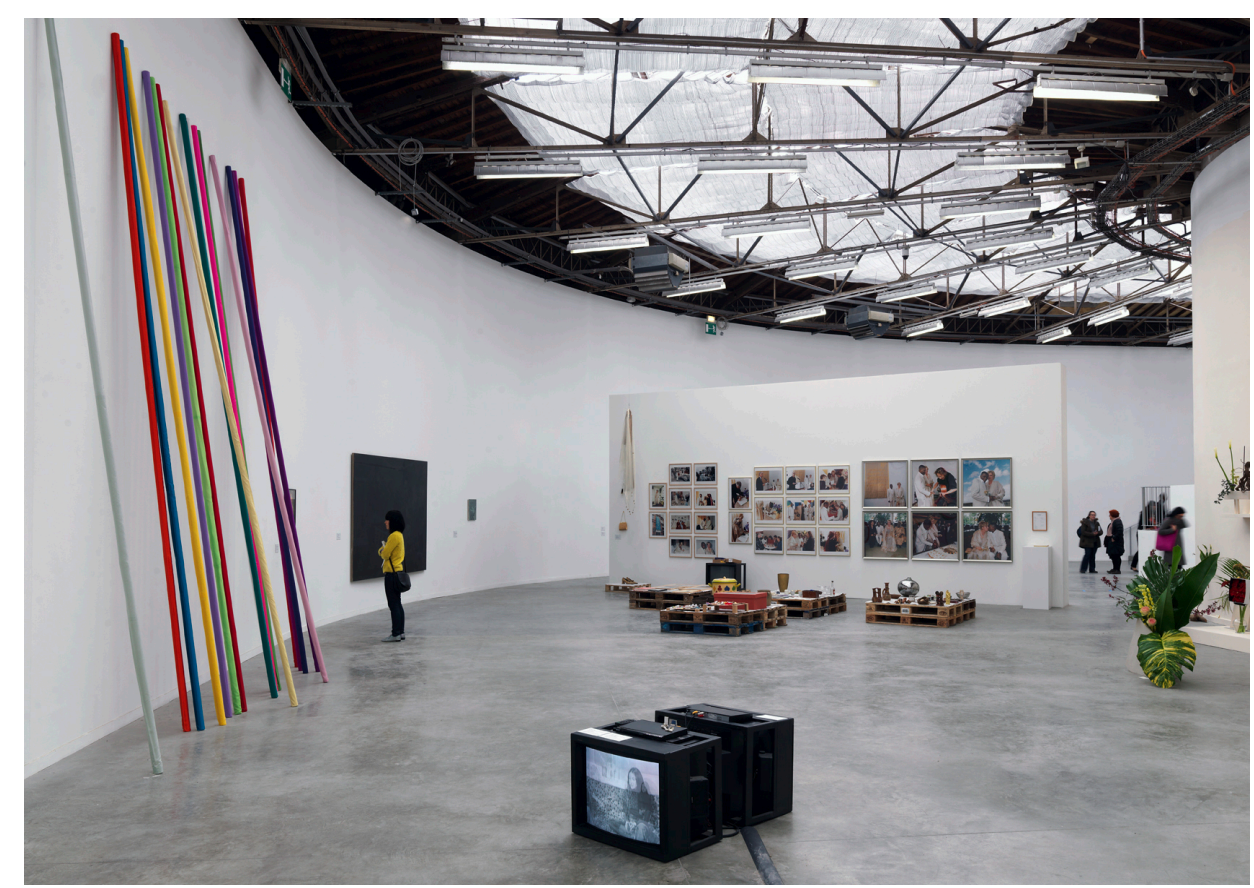

The addition of social space correlates $\wedge$ ABOVE

directly to the means through which the physical Figure 6.15: Photograph of architecture and additional design mediates between the level one southern gallery tight and loose through processes of subtraction. The giant structure and land area occupied by the Palais presented the architects with the potential to introduce luminosity and openness, stripping back excess structure to flood the gallery spaces with natural light. Combined with the new programme and spatial intentions, this required the removal of much of the existing, introducing a new transparency and visual porosity to the appropriated building (see Figure 6.15). Moreover, Lacaton and Vassal's design 'inserts' new concrete structure to inform a material discontinuity between the stone and brickwork of the existing, creating a form that physically and visually contrasts the old. The renovation therefore forms a repetition, a motif throughout in dialogue with the inhabitants as the spatial addition is neither a process of conservation nor of economic development. This process informs an experience of the Palais de Tokyo that refuses to fetishize the original structure; rather, the architects expose it as an appropriated ruin that is constant mediation with a dynamic socially produced space.

These design strategies critically embrace the actions of various publics, rather than creating a tightly controlled space. In so doing, transparency, accessibility and porosity are paramount. Through such processes Lacaton, Vassal and Bourriaud
RIGHT, OPPOSITE > Figure 6.16: Aerial photograph of Seville highlighting the

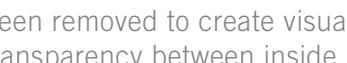

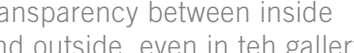
art works are protected shear curtains that loosely separate the visual inside from outside. 



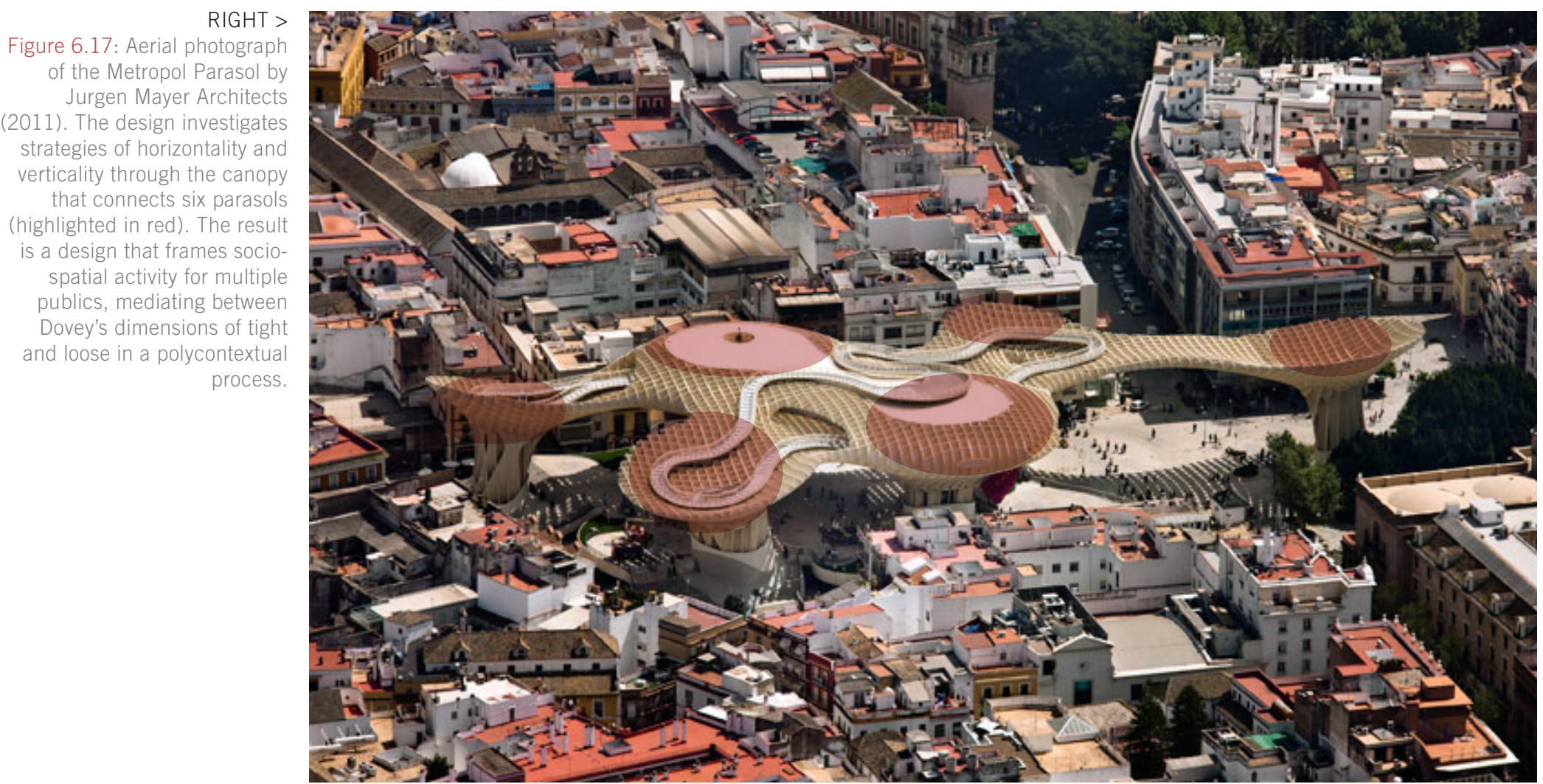

introduce looseness as a socio-spatial imperative. Based upon these understandings, the Palais de Tokyo provides precedent for the design case study of this thesis to engage addition and/or subtraction and successfully create dimensions of looseness. A critical approach to space, and place, at the Clifton Street Car Park will look to introduce these dimensions spatially and physically by reconfiguring the existing structures and to frame practices enabling flow.

The Metropol Parasol: A Canopy for Multiple Publics

Where the Palais de Tokyo has been designed through strategies of appropriation to create loose

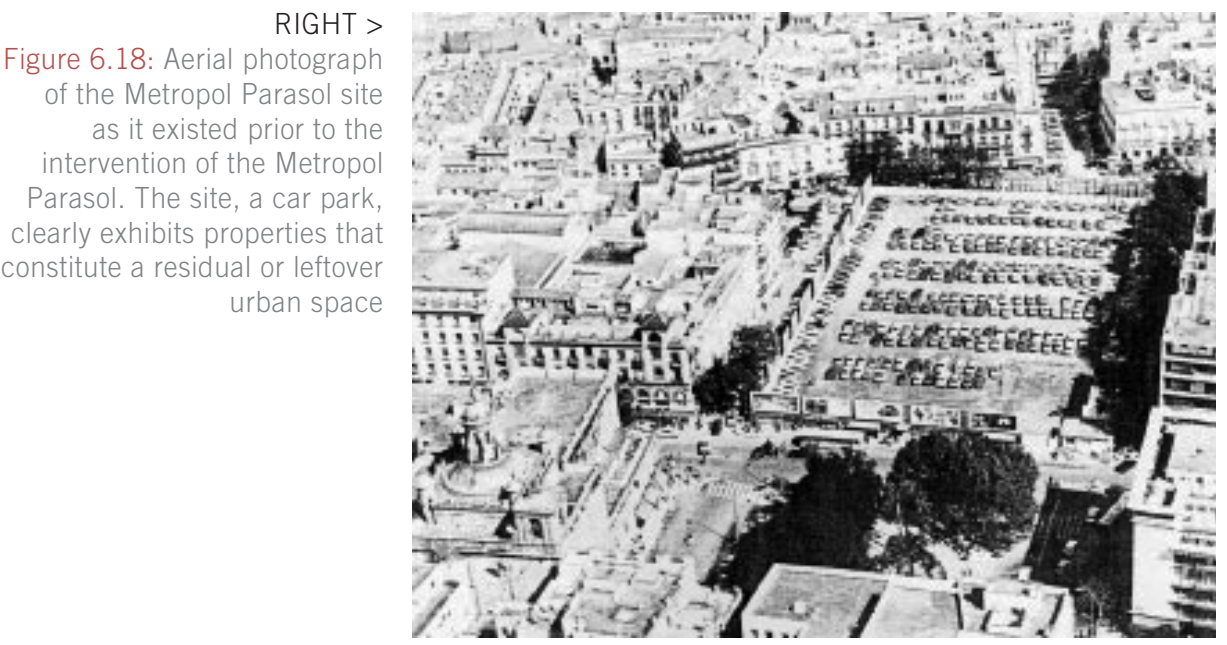

space, adding and subtracting space and structure to allow flow between multiple publics, the Metropol Parasol (2011) by Jürgen Mayer Architects investigates how architecture can frame space through strategies of horizontality and verticality. The Metropol Parasol presents an architectural intervention that covers and encolses a large public space in the centre of Seville, Spain - reconfiguring what existed as a car park into a multi-level series of interweaving public spaces (see Figure 6.16).

The plaza site has a rich and longstanding history in Seville. Defined in the middle of the nineteenth century as a market hall within a medieval monastery, it was converted into a temporary barracks between 1973 until in 1982 it

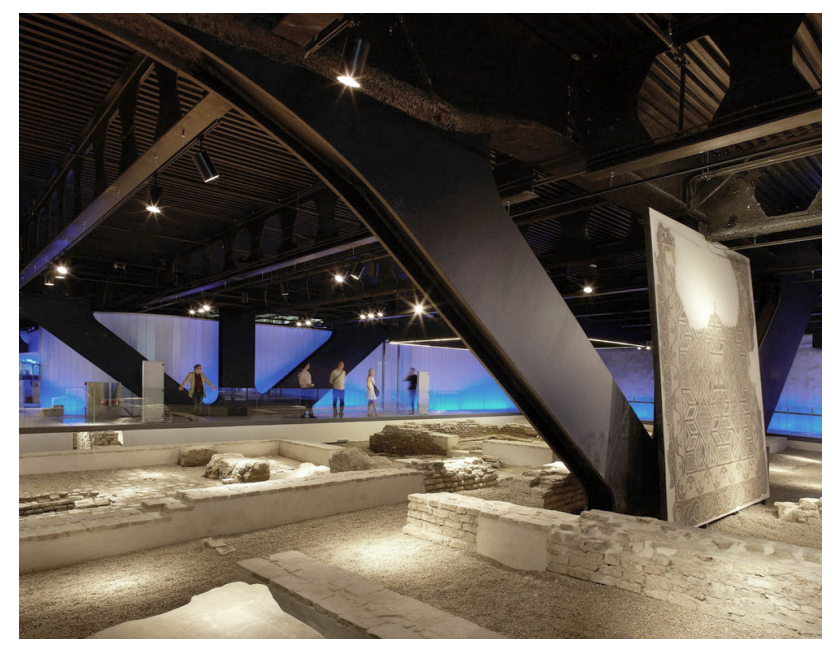

$<$ LEFT

igure 6.19: Interior photograph showing the pherranean museum beneath the Metropol Parasol 

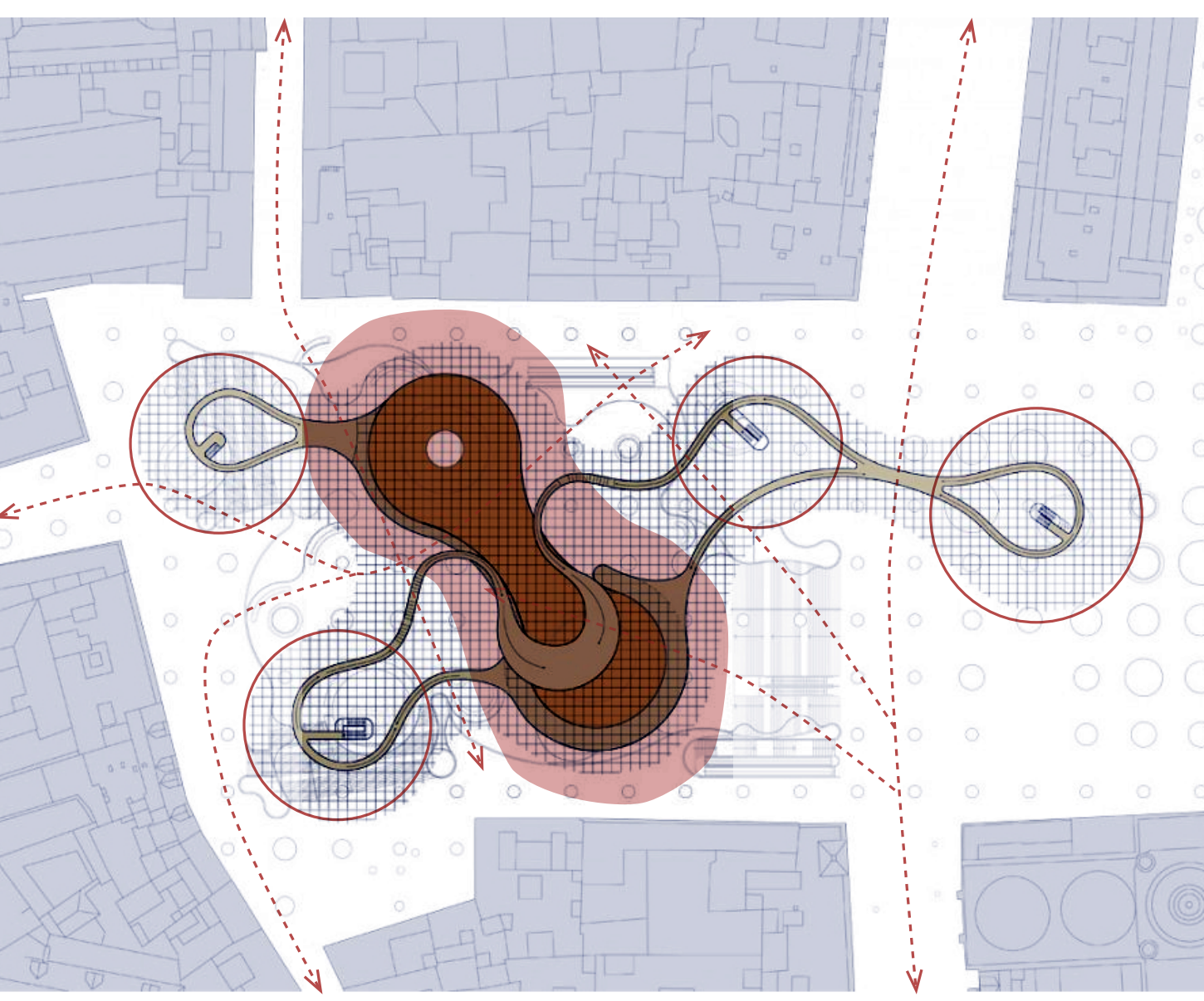

was demolished and used as a car park (see Figure 6.18) ${ }^{27}$ The current design response arose from the exposure of archaeological ruins during excavation for an underground extension of the car park facility. As with the Clifton Street Car Park and other thick edges highlighted in Wellington, the intended parking facility was an attempt by the Common Council of Seville to 'fill-in' the residual void in the urban fabric with a singular private-public programme. However, "digging was halted when mosaic floors and other remains of Roman villas were discovered at a depth of six metres", shifting the context(s) of the development from a negative leftover to a place of historical significance. ${ }^{28}$

In 2004, following the uncovering of the site's latent histories and its recognition by the people of Seville as worthy of conservation, Jürgen Mayer's proposal was presented and accepted as a design alternative. The project looks to engage the existing context(s) of the site to define a "unique relationship 29. Mayer, Metropol Parasol, between the historical and the contemporary city". ${ }^{29}$
For Mayer, the purpose of the insertion of public space in this context, facilitated by architecture, was twofold: firstly, to create a "bustling social centre" that draws in 'locals' to "stroll, sketch and skate", thus 'fleshing out' the publics through activity; secondly, the design must acknowledge and respect the physical landscape as a part of the fabric of the cultural history of Seville. ${ }^{30}$

Mediating between these imperatives Mayer initiated the negotiation of a multitude of programmes - a farmers market, a civic plaza, a panoramic roof terrace, an archaeological museum, as well as several bars and restaurants that are underneath, adjacent to, or inside the design (Figure 6.19). ${ }^{31}$ The variety of

\section{The horizontality of the canopy allows the design to reconfigure the openness of the site, introducing various points of access and permeability - shifting the boundary of the plaza inwards towards the programmes it frames.}

these uses activates the space day and night, enabling 31. Argyriades, 2011 multiple publics to access and appropriate the site at 


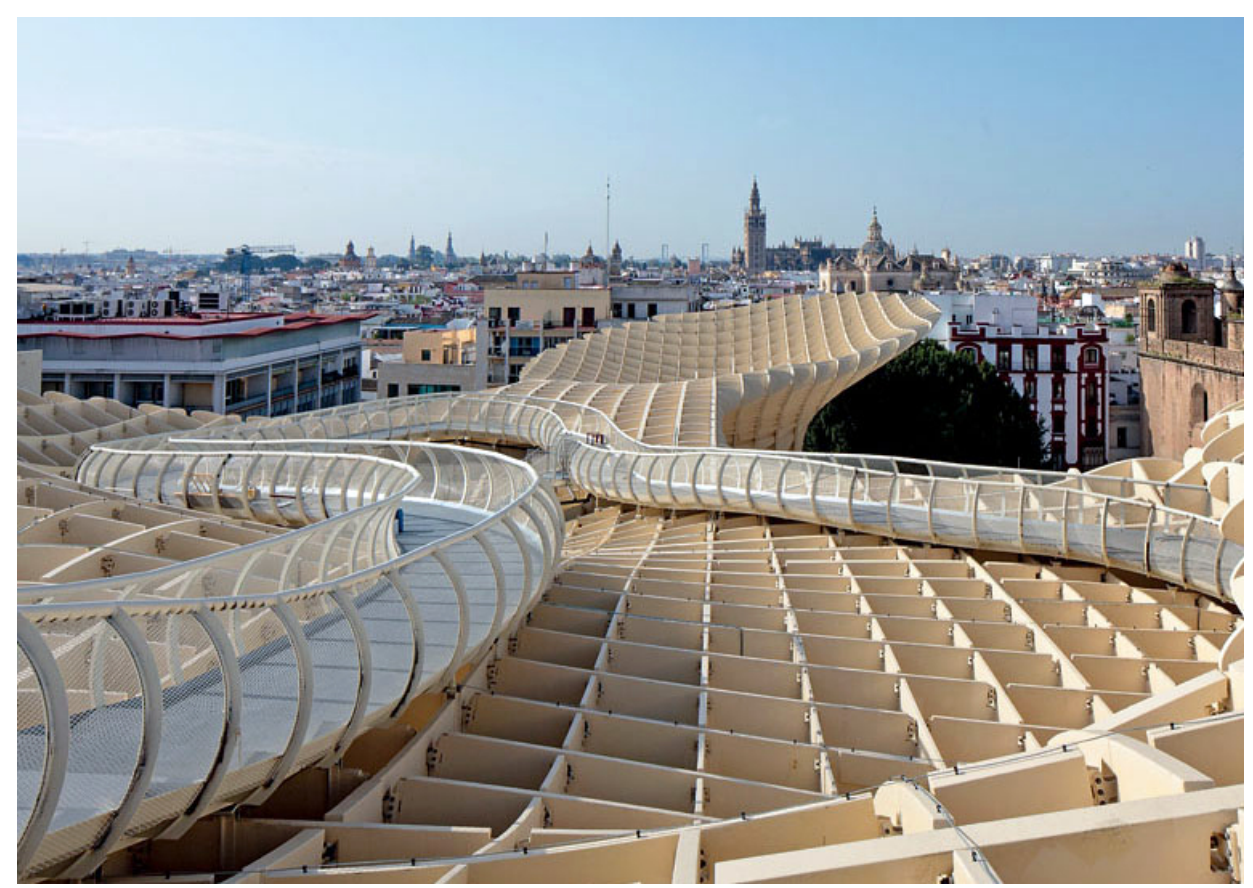

$\wedge$ ABOVE their will. To achieve flow between such programmes, Figure 6.21: Photograph from Mayer employs a canopy design that manipulates the roof terrace of the canopy. Vertical elevators within the parasols reposition publics the process of framing space structurally and socially vertically upwards, engaging (see Figure 6.17).

the wider visual context in discontinuity with the design.

The foremost function of the canopy is to introduce practical infrastructure to the exposed and open dimensions of the space, connecting six vertical'parasols' linked together by a fluid horizontal plane. These parasols, comprised of a complex timber lattice structure, support the canopy which shades

\section{The design projects a positive resistance} to its physical urban context, a critical flexibility that counteracts the negative anti-contextual discontinuity of Tschumi's Parc de la Villette.

occupants from the local weather conditions and manipulates the overhead porosity of the space to control exposure to sun and/or rain. Additionally, each supporting element contains a concrete lift shaft that leads to a rooftop restaurant and walkway, lifting publics above the ground to a view over the city (see Figure 6.21). In so doing, Mayer recognises the lack of shelter presented by sites such as Waitangi Park and the limitations of a purely outside space. Additionally the canopy presents a design device that envelops the

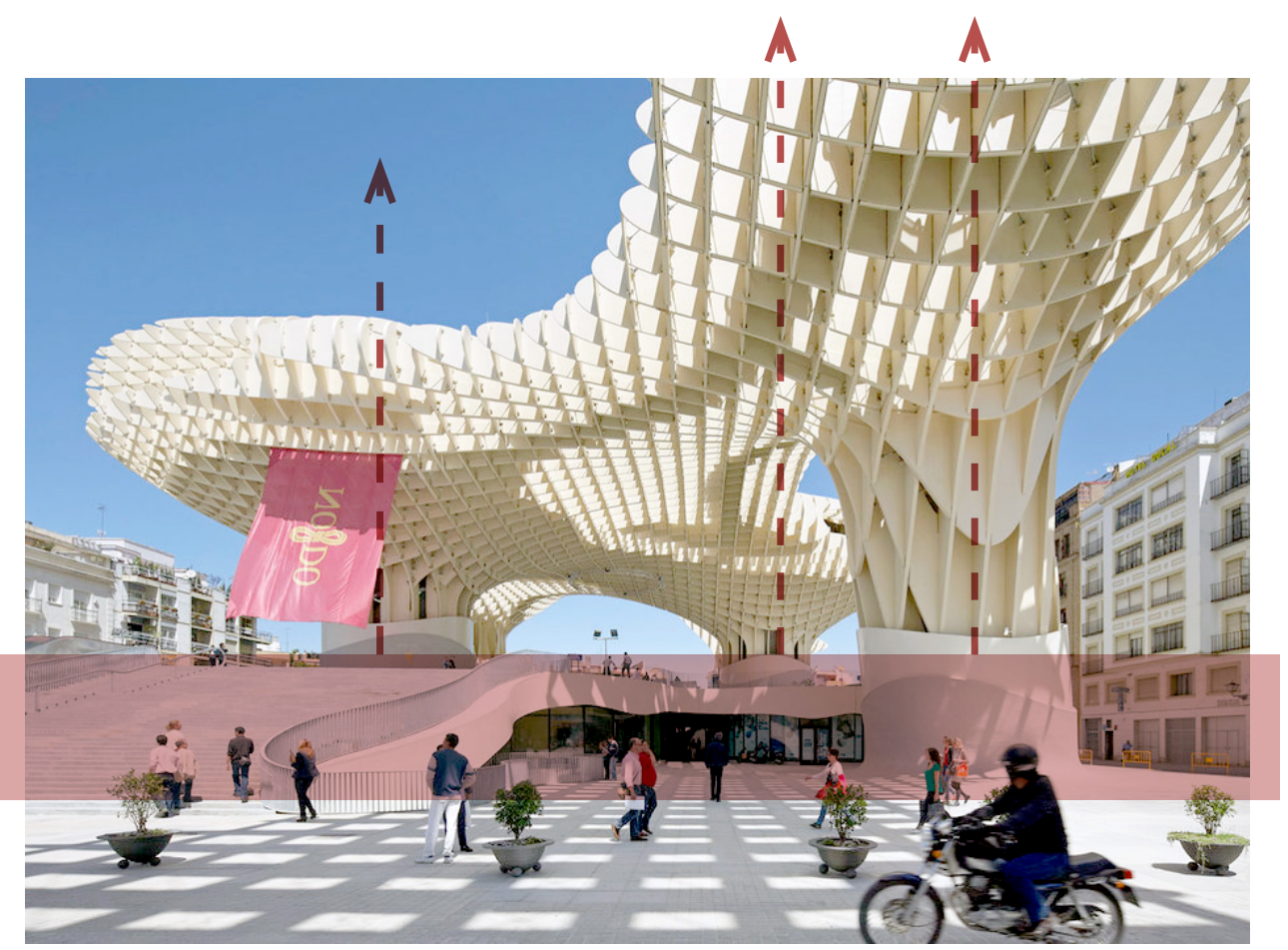

space to facilitate additional programmes, including ^ ABOVE

those that may need to be conducted outside yet Figure 6.22: Diagram/image require the presence of flexible infrastructure (such of the manipulated ground as staged dance, cinema, and other performances). lifted horizontality (dashed the Par

Additionally, the canopy design transforms the dimensions of the bounded (yet completely open) left-over space into a public node - the Plaza de la Encarnacion. Much like Civic Square in Wellington the existing site presented Mayer with an open but segregated space, one which was disproportionately

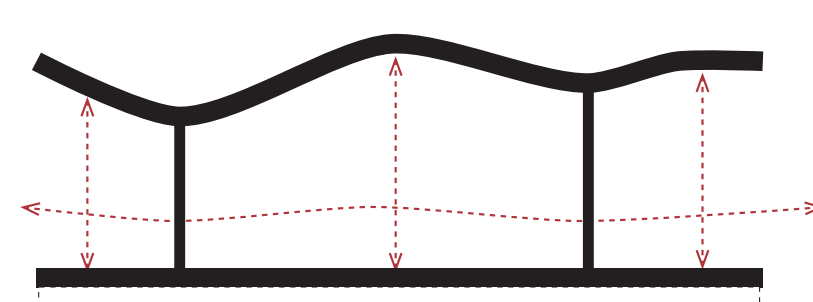

$<$ LEFT

Figure 6.23: Additionally, the verticality of the parasols, connected by the latticed canopy, allow a number of various activites to occur underneath (dotted red arrows). loose in some dimensions (completely outside and unsheltered, openly accessible from all directions) whilst tight in others (rigid and bounded by the surrounding urban structure, singularly programmatic). The horizontality of the canopy allows the design to reconfigure the openness of the site, introducing various points of access and permeability - shifting the boundary of the square 
inwards towards the programmes it frames. The effect is an inversion of the site's boundedness, informing a social permeability and flow (see Figure 6.20). This creates a "hive of activity - a social and cultural hub where both residents, visitors and tourists can gather under the architecturally motivating 'crown-like 32. Argyriades, 2011. waffle',32

Much like the Palais de Tokyo, Mayer embraces the complexity and importance of social space as a collision of multiple publics. Unlike the Palais however, the Metropol Parasol is positioned within a largely open and public site, requiring the addition of structure to mediate between public and private, tight and loose. The gesture of the canopy is crucial to the design in this regard. Its verticality shelters and frames the space at a scale that allows for various activities to occur underneath. Simultaneously, the canopy's horizontality informs a looseness at human scale for various programmes and spatial practices to flow between one and another.

Alongside the social and spatial reconfiguration of the site, Mayer engenders the design with historical context. The ground plane is embedded with its archaeology, shaping a subterranean museum beneath the canopy. In so doing, the architecture "maintains the existing street level for the market and creates a fresh plaza on its [the museum] roof beneath the canopy".33 This gesture allows the design to structurally frame the historical context, providing an infrastructure for certain spatial practices, whilst also maintaining the site's open and porous dimensions, its looseness at the ground level (see Figure 6.22 and 6.23).

Formally, the fluidity of the canopy, as well as its timber construction, creates a physical and visual discontinuity with the surrounding fabric, a "stark contrast to the geometry of the old town, in materiality and even mentality". ${ }^{4}$ This can be observed from the roof terrace which engages the immediate visual context of the design with the wider built environment. The design develops upon strong intentions to introduce local referents, collaged together in a 'primordial soup' of forms such as trees, mushrooms, and references to the organic forms of architects such as Antonio Gaudi. These gestures can be seen to resist the rigid and repetitive urban fabric that surrounds the site, creating "sensuous forms that swell from each trunk, imbued with an inner energy that expresses the vitality of the city". ${ }^{35}$ The design projects a positive resistance to its physical urban context, a critical flexibility that counteracts the negative anti-contextual discontinuity of Tschumi's Parc de la Villette. However, as a critical index, the design is less site-specific than the continuity of Miralles's Igualada cemetery. The canopy's lack of engagement with its surrounding structures, as well as its haphazard arrangement of formal referents, results in a design that is vague in its index and thus fails to 'act-out' its intentions beyond mainly aesthetic concerns.

Using canopy design through horizontall vertical manipulation strategies is pertinent to critical intervention within the Clifton Street Car Park. Positive aspects of canopy design - as a framing and sheltering device for spatial practices - implicates the volume between datums three and four as a potentially useful space for public/private functions. The intervention of a new canopy can facilitate the use of this space as a sheltered, outside, volume whilst introducing looseness to the adjacent levels. As such, the Metropol Parasol explicates strategies for the framing of space where dimensions of looseness are prevalent, introducing an infrastructure for spatial practices to eventuate. As a thick edge, these strategies will require adaptation if they are to be implemented in the design case study. Manipulating vertical and horizontal planes through site-specific processes has the potential to frame the site to new programmes and to invert its infrastructural rigidity in a gesture of discontinuity.

\section{The Rolex Learning Centre: Horizontality, Transparency and Porosity}

Horizontal and vertical volumes are manipulated as strategies for framing public space in the design of the Metropol Parasol. Alternatively, SANAA architects, Ryue Nishizawa and Kazuyo Sejima, use these strategies in the design of the 


showing the location of the Rolex Learning Centre (2010)
RIGHT >
Figure 6.26: Aerial photograph of Lausanne, Switzerland

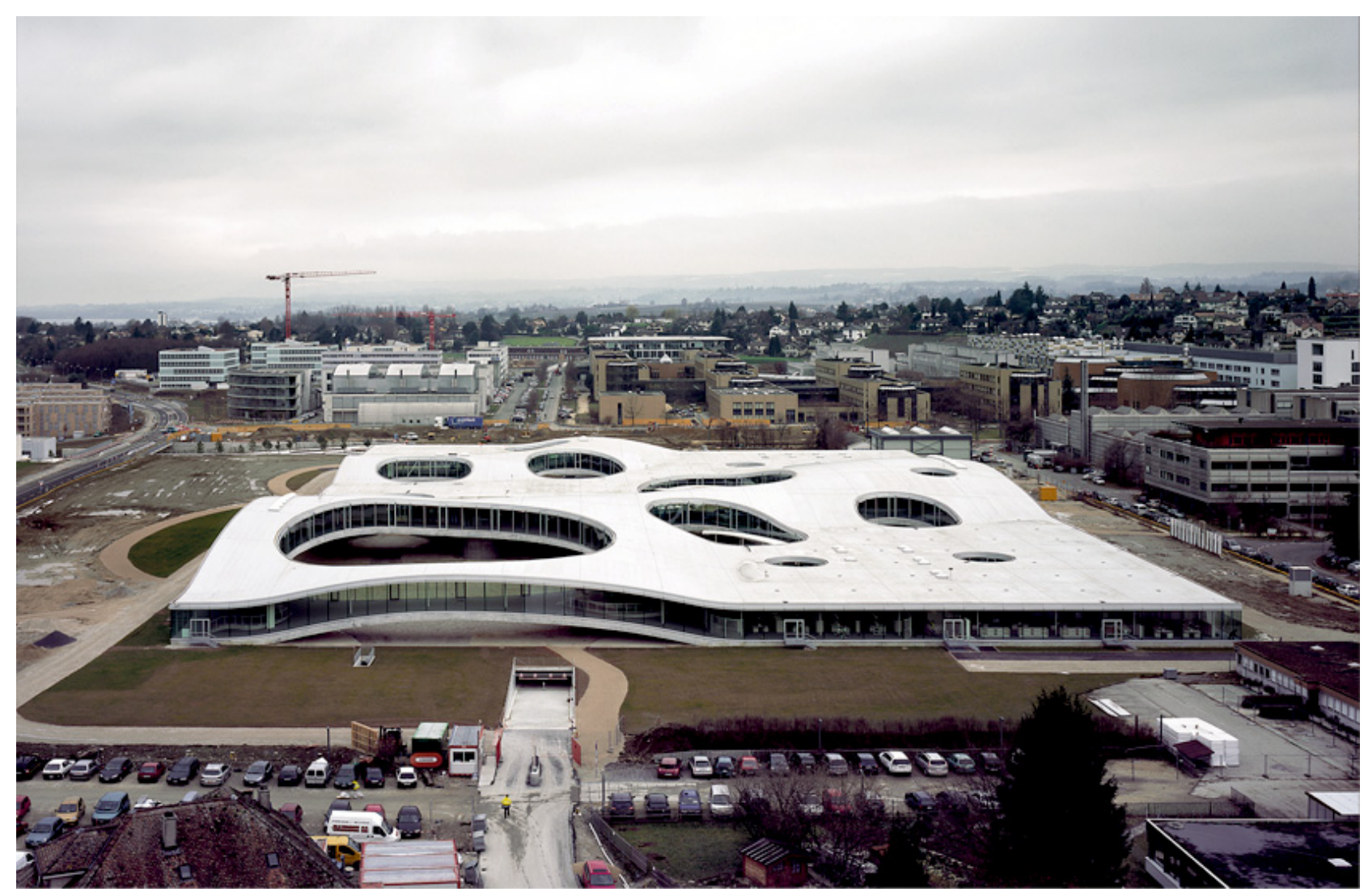

Rolex Learning Centre (2010) to frame loose, private space. The primary aim of SANAA's project to date, although in this case existing within the 'walls' of a semi-private structure, is to explore the degree to which "architecture opens itself to the unplanned chaos of contemporary life" and "creates a maximum of social, cultural and political effects with a minimum number of elements" ${ }^{36}$ In light of such statements, their intentions align clearly with the critical thesis of Dovey - to enable multiple socio-spatial flows through architectural structures.

Built on the University campus of the EPFL (Ecole Polytechnique Fédérale de Lausanne) in Lausanne, Switzerland, the Rolex Learning Centre

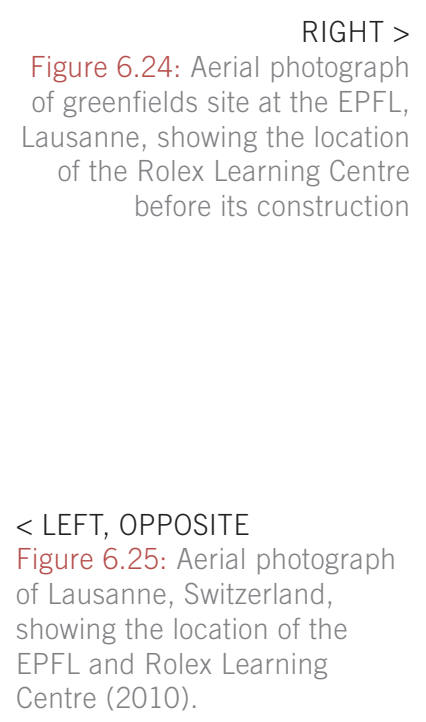

$<$ LEFT, OPPOSITE Figure 6.25: Aerial photograph of Lausanne, Switzerland, showing the location of the EPFL and Rolex Learning Centre (2010).

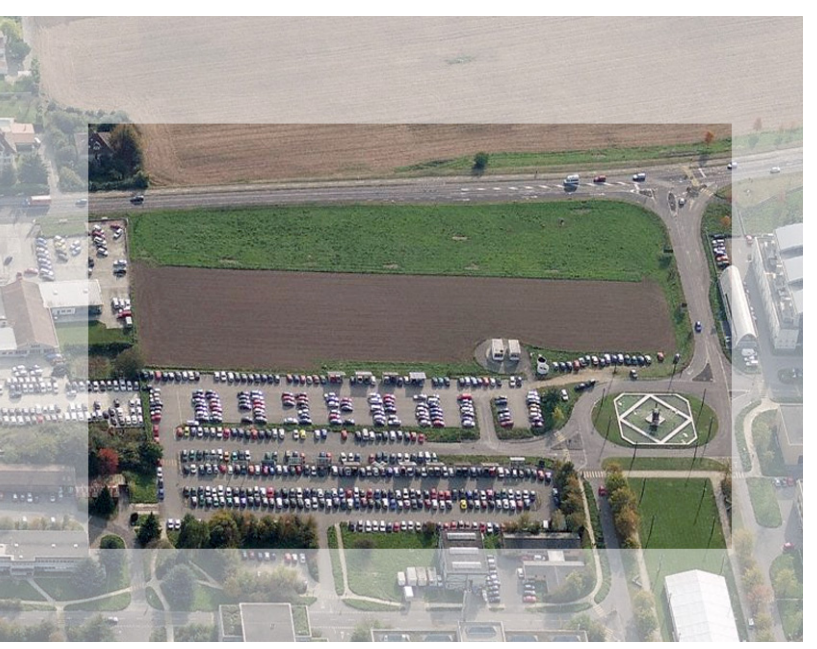

is a necessarily enclosed multifunctional building - a library, language centre, student workspace, career centre, EPFL precious book collection, publication office, cafeteria, restaurant, lobby, and presentation theatre - yet it is not entirely private, engaging multiple publics in multiple activities. Previously an empty greenfields site and (as with the Metropol Parasol) a parking lot, the learning centre is an additional social and educational node that is somewhat of an archipelago in its visual and physical context (see Figure 6.25 and 6.26). As a result, the design is an act of re-framing, internalising the centre within the borders of four walls. Within this re-framing, Nishizawa and Sejima implement strategies to structure spatial flows that mediate

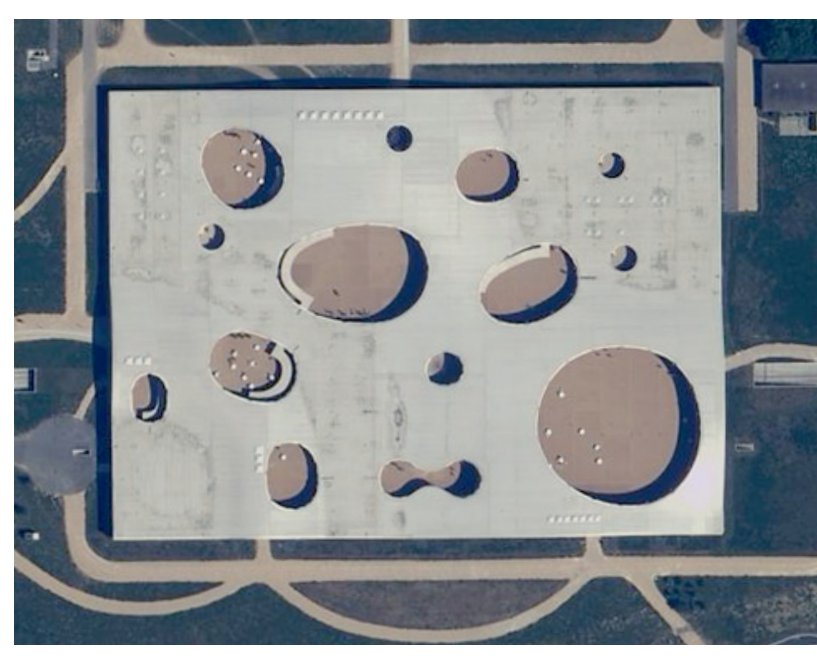


RIGHT >

Figure 6.28: Digrammatic plan of the Rolex Learning Centre showing the anchor voids and circulation flows that connect the buildings' various

RIGHT, BOTTOM > Figure 6.29: Digrammatic plan of the Rolex Learning Centre showing programmatic layout and volumes, structured by the 'inverted box'

KEY:

1. Pedestrian ground level walkway 2. Student study areas

3. Arts and Engineering collections

4. Science and Humanitites collections

5. Precious book collection

6. Car park entrance

7. Restaurant

8. Administration

9. Cafeteria

10. Forum/lecture theatre

E. Entrances
Accès Nord Y. Metro M1
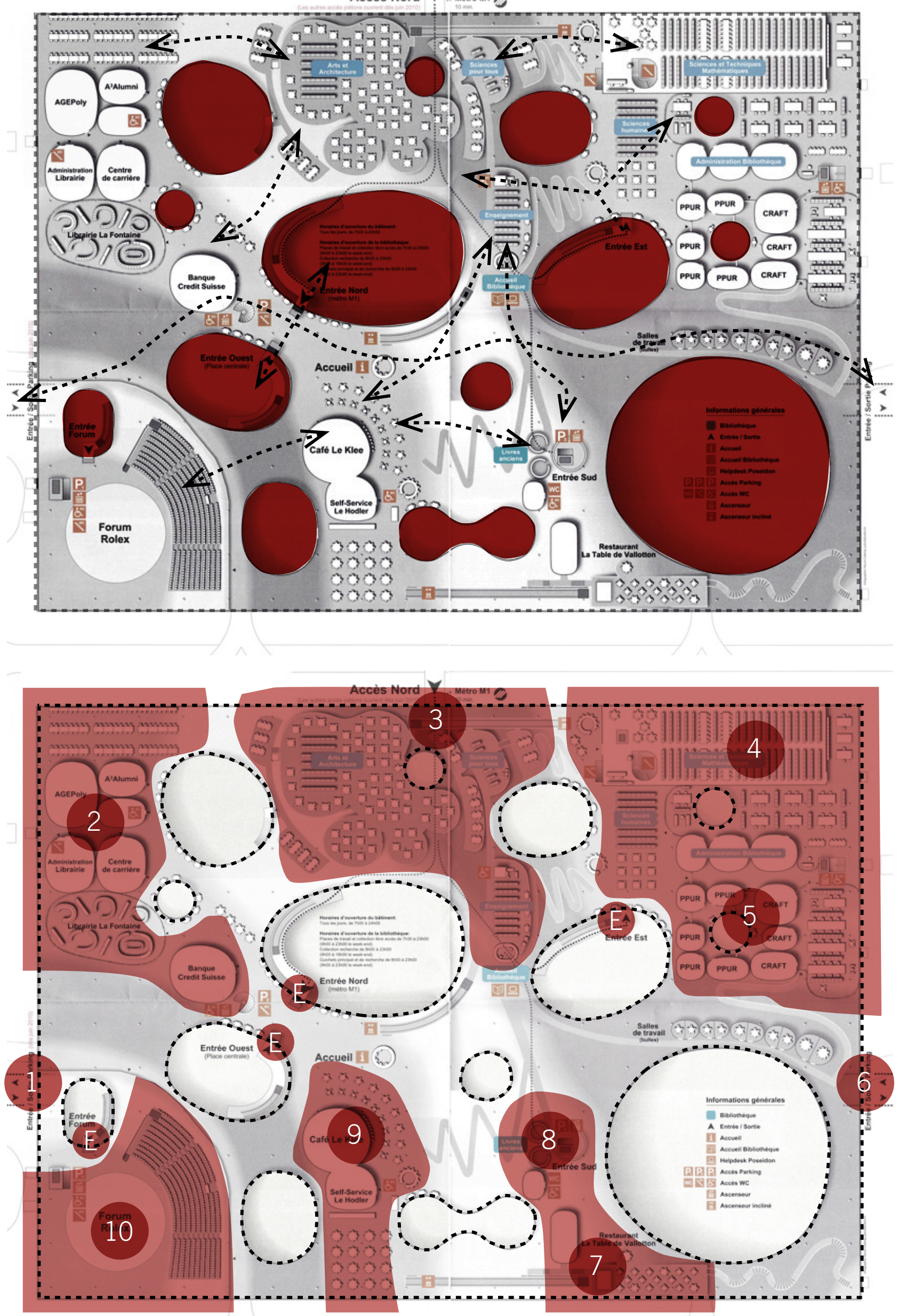


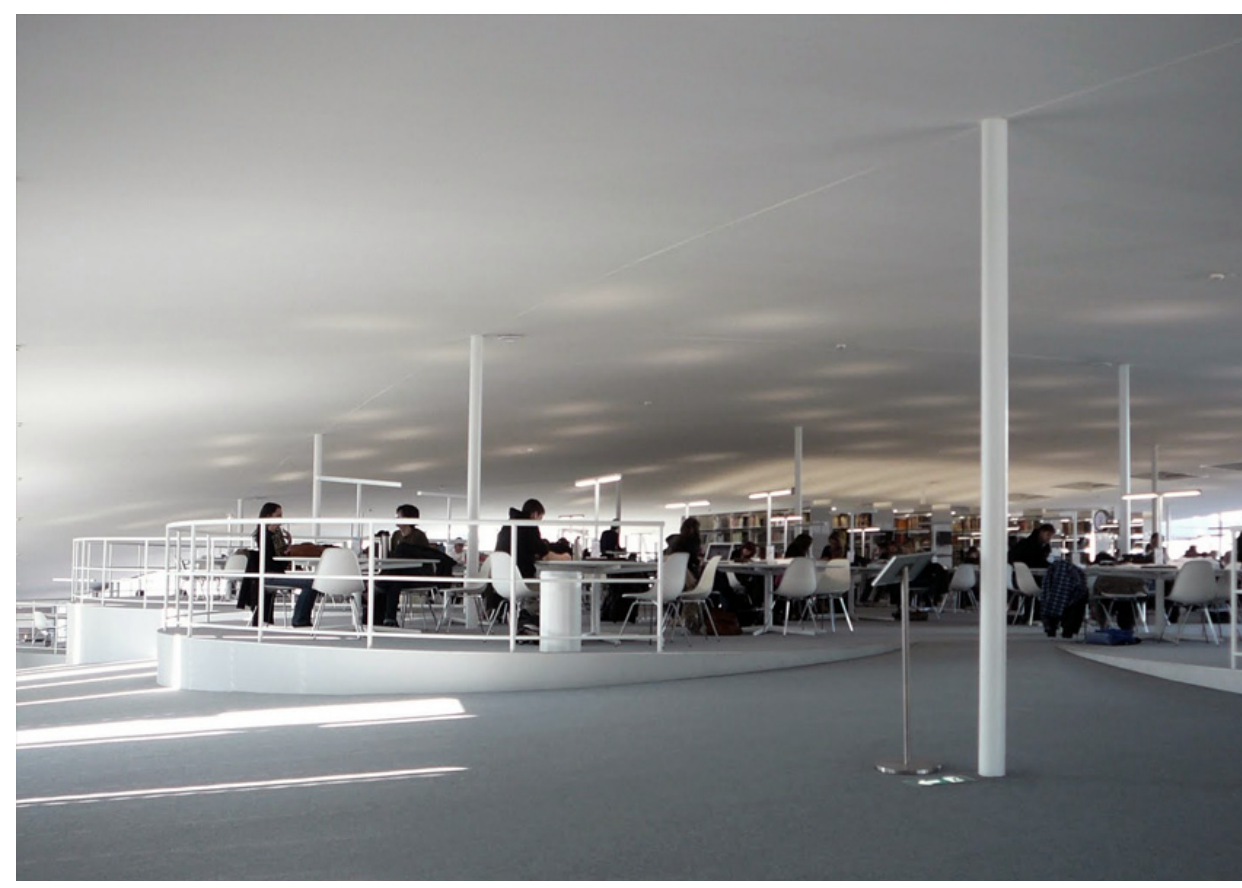

ABOVE $\wedge$ between different aspects of Dovey's dimensions than Figure 6.30: Interior the Palais de Tokyo and the Metropol Parasol. photograph of the studying areas of the Rolex Learning

Centre. The undulating

floor and fluid plane allows programmes and publics to
flow between the anchor voids

several gestures that inform a spatial'fluidity', creating an interior urban landscape that frames "a place where activities are separate but naturally related to each

37. Hasegawa, 2006, p. 148. other... in a single, coherent setting" ${ }^{37}$ This responds 38. Nishizawa, 2013, p. 66

to the notion that space continues seamlessly between locations, framed by architecture, and through what Nishizawa refers to as a 'horizontal infinity. 38

\section{The result is a circulation and arrangement of spatial practices framed by the architecture that allows for non- linear, non-deterministic, flows between spaces and publics.}

The Rolex Centre explores this notion; one of the primary gestures of the design is that it houses all of its programmes within a single storey of uniform height $(3.50 \mathrm{~m})$. The occupants of the building thus move 'seamlessly' through the programmes within a spatial volume that is sandwiched between two undulating concrete planes. For Nishizawa and Sejima, this horizontality creates flow between publics,

39. Nishizawa, 2013, p. 8. programmes, inside and outside, a "fluid dynamism of architecture... a fluid, organic dynamism". ${ }^{39}$ The result is an inverted box that looks to bring the exterior into the interior, to create a continuous spatial experience that merges the social context(s) within the defined space of the building. Additionally, SANAA visually connect the exterior site to the interior of the design by wrapping the inverted box in glazing, refusing to produce an exterior 'image' through the use of a traditional façade.

Analysing the building in plan reveals that the programmes are anchored to several voids or vertical porosities that puncture through the horizontal planes to create points of transparency throughout (see Figures 6.26-6.28). Circulation is equidistant to the various programmes, branching outwards from various entrances that are positioned at the buildings centre to "create smooth lines of flow". 40 The voids seem random at first; however their position is highly specific. The larger central voids act as circulation
Figure 6.31: Exterior ph of the ground plane beneath the Rolex Learning Centre. The undulating horizontal floor plane informs a landscape beneath the building, creating a fluid transition between exterior and interio

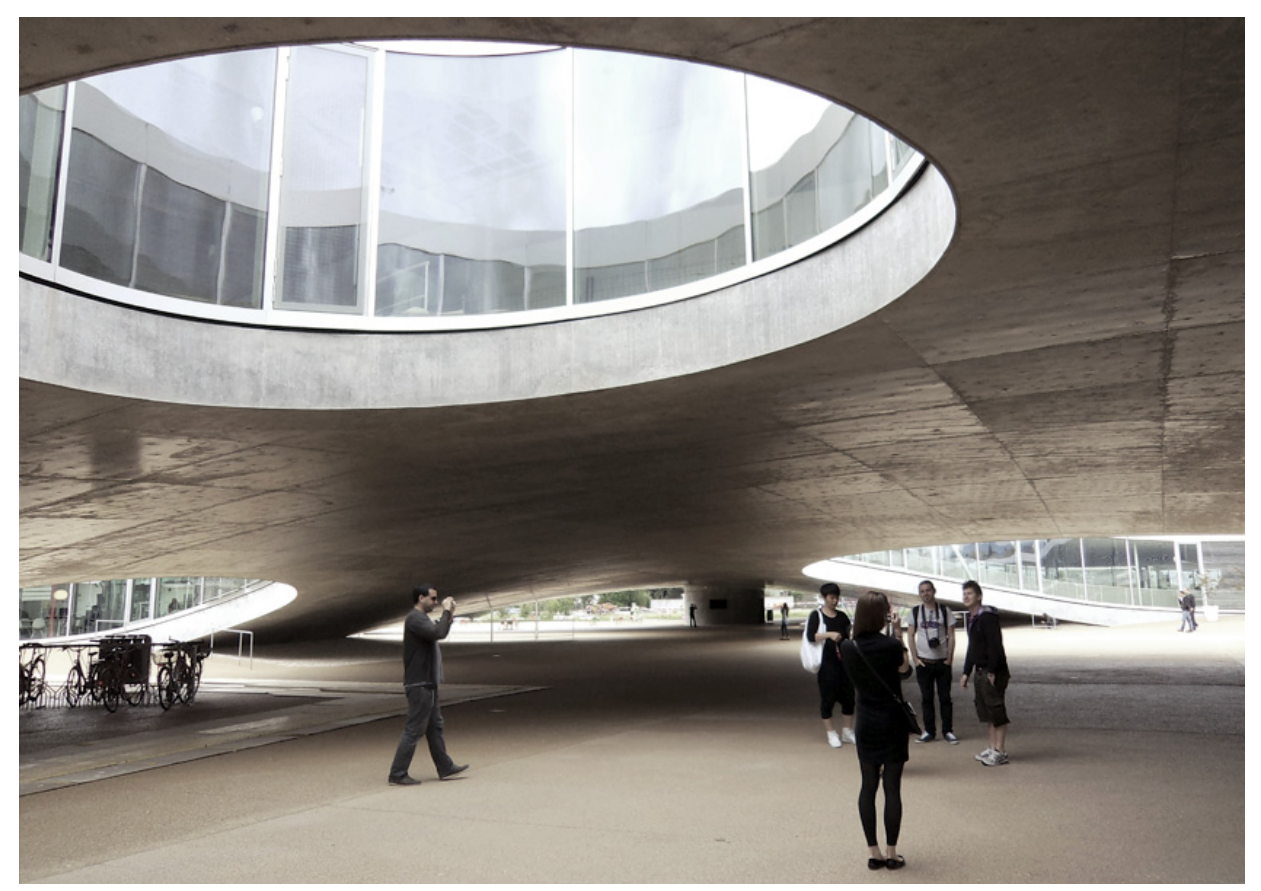

nodes creating thresholds between the exterior ground 40. Hasegawa, 2006, p. 25. plane and the interior landscape. Such thresholds take the form of entrances, connecting publics to the interior environment via ramps and/or stairs. The voids 'push' the programmes outwards from the central circulation areas, towards the glazed skin of the structure. This gesture, although opposite in its outward direction, follows the same logic as the Palais de Tokyo and the Metropol Parasol, creating social permeability within the boundaries of the building/ 


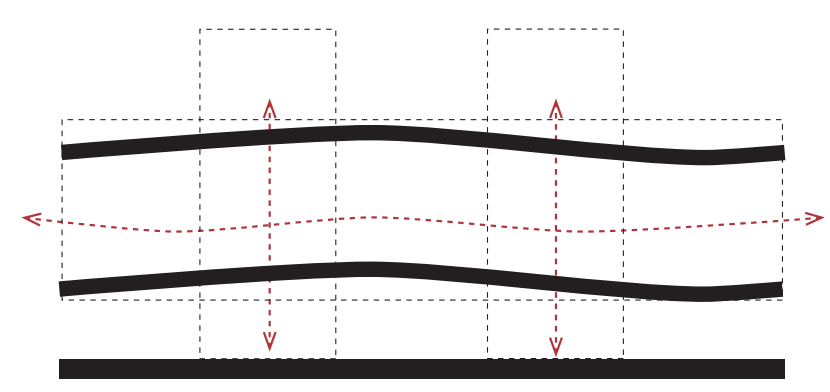

site. The result is a circulation and arrangement of spatial practices framed by the architecture that allows for non-linear, non-deterministic, flows between spaces and publics (see Figure 6.30). Additionally, the voids and manipulated horizontal planes frame activity, resisting the exaggerated 'openness' of a park, square or leftover space.

Unlike the Metropol Parasol, the Learning Centre's verticality is almost entirely non-visual. Verticality emerges in the form of the anchoring voids and shallow undulations in the floor and ceiling planes. This uniformity reduces the building to human scale at all times and emphasises the moments where the vertical punctures occur. Verticality in this sense is a strategy to fluidly connect the occupants on the ground plane, outside, to those within the horizontality of the building, its inside (see Figure 6.31).

Design strategies used by Nishizawa and Sejima in the Rolex Learning Centre, notably the manipulation of the buildings horizontality (in circulation, layout, and structure), are relevant to critical intervention within the Clifton Street Car Park. The design explicates methods for placing and arranging a multiplicity of programmes to inform a social looseness within a bounded territory. This is particularly relevant to the creation of permeability through the car park's social thick edge condition. Additionally, Nishizawa and Sejima's approach explores how looseness can be created in conditions of confined and uniform horizontal planes - such as datum's $0-2$ of the car park - anchored through vertical punctuations that create circulation points and link the above to the below, the inside to the outside (see Figure 6.32). However, the design of the centre does not claim to be polycontextual or critical beyond its socio-spatial strategies. As previously stated, it creates an internal environment, and does not respond to site-specific particularities in that sense. Moreover, the detached physical, visual and historical conditions of the site reduce gestures of continuity (with the physical site) and discontinuity (through materials, typology) critically impotent as they do not engage in any form of specific resistance, limiting the gestures of fluidity and voiding to the performance of structuring the space in a formal and social sense.

\section{Conclusion: Implications and Strategies for Design}

Throughout this chapter the fourth research question has been engaged - expanding upon the parameters and directives of critical spatial practices. Following the imperatives for the structuration of tight/loose public space put forth by Dovey, strategies exhibited by the three case studies have been examined, exploring how critical socio-spatial strategies manifest through architectural design - elucidations that are particularly relevant to the experimentations of the design case study.

Analysing the Palais de Tokyo, the Metropopl Parasol and the Rolex Learning Centre reveals strategic applications of addition, subtraction, horizontality and verticality to illustrate how architectural design can invert the social (and therefore spatial) thick edge condition of Clifton Street Car Park. Based upon these precedents, specific design manoeuvres will empower Section Three, the design testing, to introduce a flow of publics through the site - creating social permeability between the adjacent areas of Thorndon, Bolton Street, Kelburn, The Terrace, and Lambton Quay.

Specifically, following Lacaton and Vassal, strategies of addition and subtraction (to frame both spatial and structural relations) show how a negatively perceived site can introduce new conditions by reconfiguring - rather than 
demolishing or conserving - the existing dimensions of a tight space. As it stands, the infrastructure that comprises much of the car park is already stripped back' to its engineering essentials. However, as a strategy of physical subtraction, the design will cutinto the imposition of non-essential structure where necessary. This strategy is particularly useful along Datum 1 where the concrete platform has created a dark and bounded spatial volume.

The strategic and critical addition of space is equally essential to the design. Negotiating a programme that is capable of instigating multiple uses to the currently singular condition of the car park, space can be appropriated in the form of social volumes. These volumes, following the Palais de Tokyo, can overlap and bleed into each other, introducing a flow between programmes. Appropriating the site as such is a critical gesture as it resists the deterministic intentions of its current use, informing a spatial looseness.

In a similar vein, the horizontal and vertical manipulation of structure and volumes created by the Metropol Parasol is relevant to the translation of Dovey's critical position into design. In particular, the space underneath the Urban Motorway is logically implicated. The canopy created by the motorway provides a rare condition - sheltered outside space that can encompass a variety of spatial practices. A manipulated gesture in this regard, such as Mayer's design, posits that an overhead sheltering device can envelop various activities whilst simultaneously engaging in the creation of an infrastructure underneath (a dimension lacking in the current thick edge). Introducing a manipulated canopy adjacent to the motorway can take advantage of the positive aspects of the existing conditions and the flexibility of a architectural structure to frame various sociospatial practices. Moreover, a dynamic verticall horizontal canopy is typologically resistant (visually, physically) to the orthogonality of the car park - a proposition explored further in Chapter Seven.

Adding to the strategies put forth by the Palais de Tokyo and the Metropol Parasol, the Rolex
Learning Centre exemplifies how undulating datum planes above and below, interspersed with public scale vertical openings can create circulations of socio-spatial and programmatic flows. Primarily a horizontal strategy, the fluidity created by Nishizawa and Sejima's design shows how entrance thresholds and vertical voids can create programmatic anchors. Positioning similar anchor points within the sitespecific index of the design case study can structure the social uses of a space yet allow them to bleed between each other. This approach is an inversion of the current thick edge, refusing to enforce a determined and controlled public/private condition. Following SANAA, this can inform a landscape of dynamic uses that critically mediates the necessary dimensions of tight and loose.

Moreover, the three examined case studies show how spaces between two horizontal planes (such as Datum's 1 and 2 of the Clifton Street Car Park), or large open spaces (such as Datum's 3 and 4) can produce multiple public/private conditions within one bounded site. A combination of these design strategies will determine how the design conducts a socio-spatial critique of the existing conditions, engaging various contexts to provide positive solutions to the thick edge. 


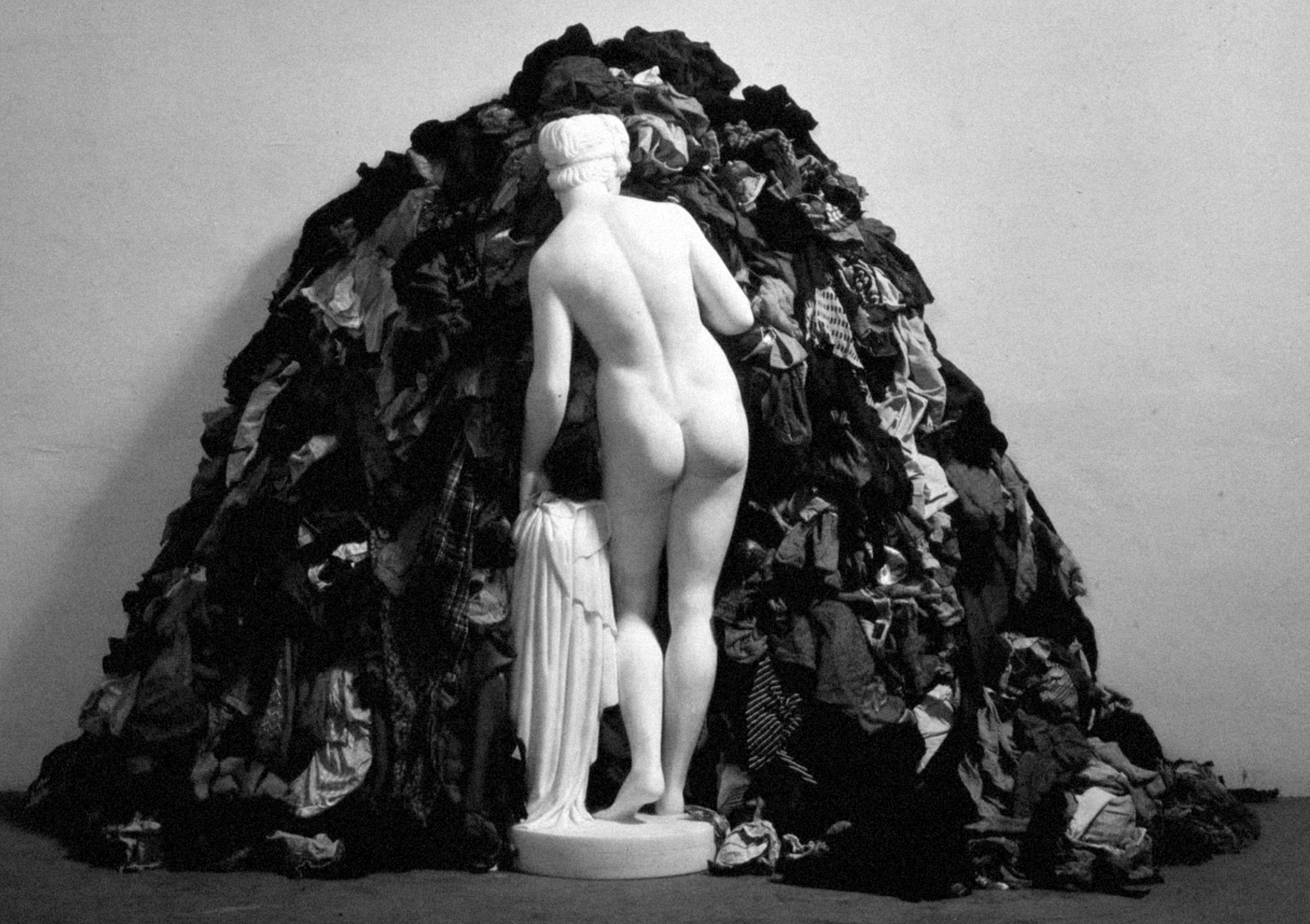




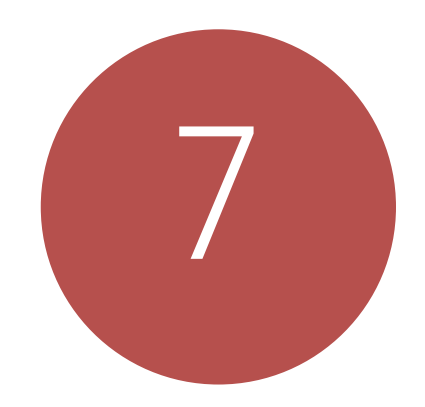

\title{
DIALECTICAL SPACE AS CRITICAL PRACTICE:
}

\author{
Elucidating Strategies to Construct \\ Temporal Images
}

\begin{abstract}
Introduction
Between 'Now' and 'Then': Walter Benjamin's Dialectical Image

Avoiding Spectacle: Framing Dialectics

Through Visual Juxtaposition

The Jewish Museum Berlin: Engaging

Historical Context in the Dialectical Image

Conclusion: Implications and Strategies for Design
\end{abstract}




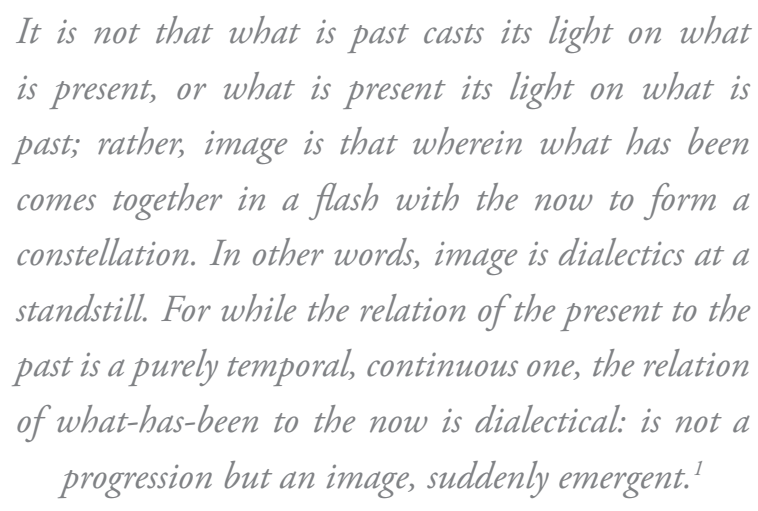

1. Benjamin \& Tiedemann, 1999, p. 462

Walter Benjamin, The Arcades Project

\section{Introduction}

This chapter builds upon the sitespecific and socio-spatial practices that have been investigated in Chapter's Five and Six - turning to a temporal understanding of historical and visual context as contributing to a polycontextual lens for critical spatial practices. For Rendell, the writings of Walter Benjamin contribute to the foundations of such a critique, shifting the research from wider site and socially inflected issues to "examine particular works as new interventions into existing contexts, highlighting the importance of the temporal"- in particular how historical and visual contexts can be interrupted or displaced to positively subvert and resist the identity of a particular place. ${ }^{2}$

Walter Benjamin's work is relevant to such discussions as it dissects and deciphers the urban landscape of the expanding metropolis during the early twentieth century. As the burgeoning development of modern construction began to shape cities into contextually ubiquitous and ahistorical places (reaching its zenith as the Augé -ian nonplace) Benjamin constructed a two-fold critique of the city: architecture as experienced in a distracted montage of images; and history as a dialectical negotiation between 'then' and the 'now'. These two positions have been highly influential, instructing a means for architectural design to embed a place with a critical temporality - a visual engagement with the historical context(s) of a site. This thesis proposes that one solution to reducing the impermeability of a thick edge is to reawaken shared histories of adjacent communities to the Clifton Street Car Park, thereby transforming this condition into a dynamic threshold rather than a static and separating barrier.

In particular Benjamin's examinations of the urban landscape have been positioned as pertinent within the contemporary context through the writing of Gevork Hartoonian. ${ }^{3}$ Hartoonian's practice focuses on a twenty-first century re-interpretation of the writings of Benjamin as it relates to the current state of digital image reproduction.

This chapter contends that through Hartoonian, a distillation of Benjamin's 'dialectical images' presents that architectural design "inserted into a given context aims to critique the construction of the past in the present, drawing attention to repressed aspects of history". ${ }^{4}$ In so doing, architecture positions historical events in relation to the current context in a way that is not static but that operates dialectically. Essentially, in the dialectical image, formal constructions can intentionally frame visual compositions that juxtapose the existing fabric with the insertion of the new, revealing a contextual critique.

The Jewish Musuem in Berlin is analysed as a case study that is of particular relevance to this discussion. Elucidating strategies of juxtaposition from Libeskind's design reveals a translation of the temporal into the visual through the dialectical image. This the case study implicates clear opportunities for the design of Architecture's Tightrope to engage with the visual and historical contexts of Clifton Street Car Park, contributing to a polycontextual flow and inversion of the thick edge condition.
3. Gevork Hartoonian is Professor of Architecture at the University of Canberra. 
This chapter adds to Rendell's framework and mediates between the theoretical framings of Section One, and the design case study in Section Three; engaging the fourth research question:

How can critical spatial practices manifest in the built environment, in particular through strategies of architectural design? How can these strategies engage multiple contexts to introduce positive flows to the urban thick edge?

\section{Between 'Now' and 'Then': Walter Benjamin's Dialectical Image}

In perhaps the most influential of his critiques of the built environment, Benjamin argues that the architecture of the city does not register with its occupant in a conscious or cognitive manner.

5. Elliott, 2011, p. 27

6. Benjamin, 1968 [1936], p.

BELOW Figures 7.2 (LEFT) and 7.3 (RIGHT): The Galaxy SOHO (2011) design (discussed in chapter two) by Zaha Hadid architects (bottom left) has been imitated by a Chines developer (bottom right) rficially replicated based concept images - it was unbuilt
before the copy emerged. This is an example of 'designing for spectacle', where architecture

is treated as a purely visual commodity advanced in his frequently cited essay "The Work of Art in the Age of Mechanical Reproduction". In this seminal work he posits that architecture is experienced through habitual performances of use (spatial practice) and perception (for Benjamin this is primarily a tactile experience), "the reception of which is consummated by a collectivity in a state of distraction". Benjamin argues that as publics move between the largely repetitive typologies of buildings the modern city is viewed in a drawn-out montage - a series of commodified images and visual moments. This process can be seen as directly linked to developments in visual technologies, a parallel evolution with devices of image reproduction, and cultural shifts driven by devices such as photography and film:

If one considers the dangerous tension which technology and its consequences have emerged in the masses at large - tendencies which at critical stages take on a psychotic character - one also has to recognise that this same technologization has created the possibility of psychotic immunization against such mass psychosis. ${ }^{7}$

According to Benjamin, the culture of technological image reproduction has distilled the reception of architecture into a readily absorbed image, an image which decontextualizes the experience of it to that of phantasmagoria - a superficial spectacle of the work as an imitable commodity (see Figure 7.2 and 7.3). Importantly, Benjamin's argument has been updated by many contemporary cultural critics - arguing that this condition has proliferated exponentially through the rise of digital technologies.

Nadir Lahiji believes that through digital media the impact of technology on the decontextualization of the architectural image has become all but instantaneous. ${ }^{10}$ Thus, in the early twenty-first century, the images of the buil environment have valorised far beyond what was
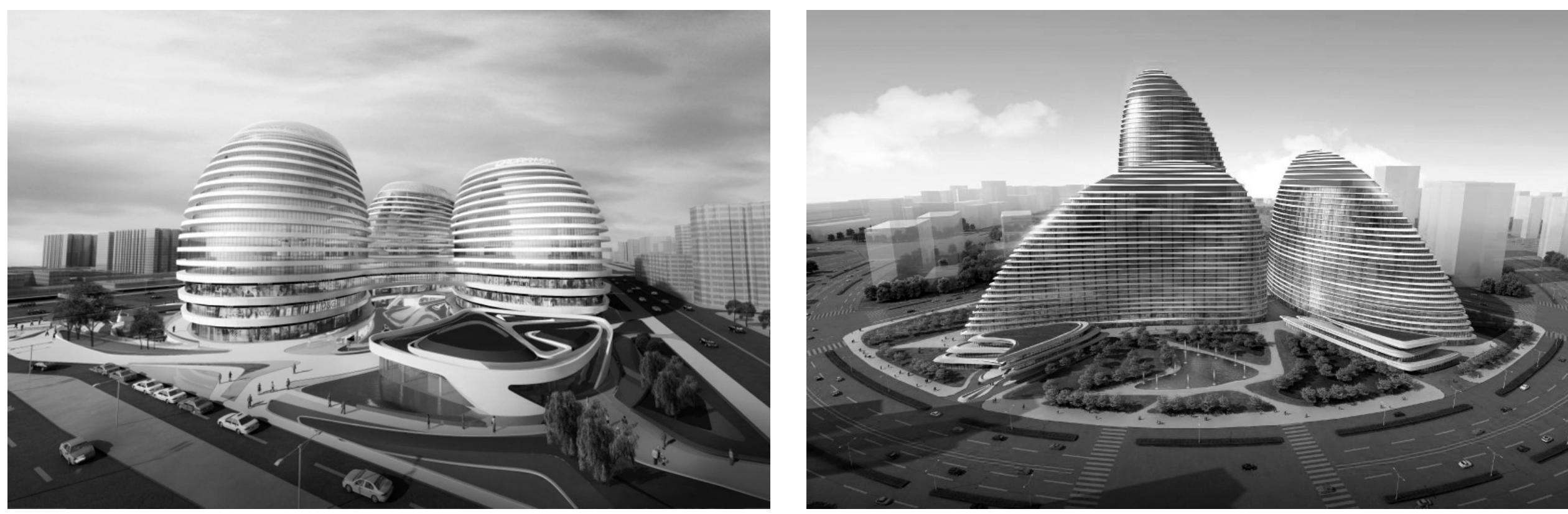

9. See: Foster, Design and Crime (and Other Diatribes), 2004, p. 11

10. For Lahiji, The image of architecture is the external identity of the discipline; it's projection as a commodity into the spectacle of society. 
11. According to Jameson the spectacle of late capitalism informed a situation where depth is replaced by surface or multiple surfaces", see: Jameson, 1991, p. 62; (Foster, "Image Building", 2004

12. Lahiji, 2011, pp. 212-213 See also: Lacan, 1997.

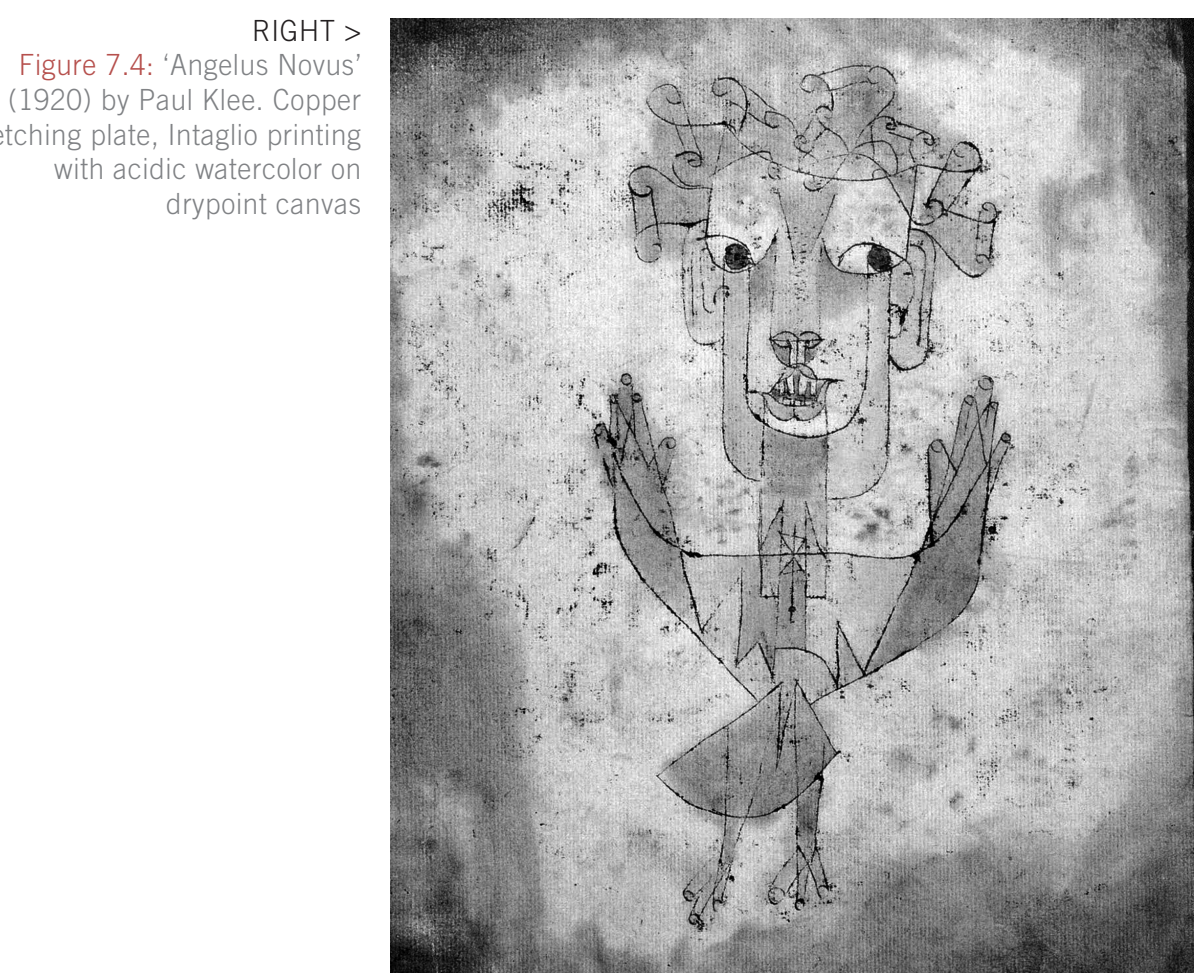

provides Benjamin with an apt metaphor for a critical understanding of notions associated with history. His analogy posits that a 'storm from paradise' (the drives of innovation and technology through notions of progress) is recklessly propelling society into the future. As the angel is blown forwards, back turned, 13. Benjamin, 1968 [1940], he can only look into the past, at the wreckages of p. 249. modernity. ${ }^{13}$

For Benjamin, this links the notion of history as read backwards yet visually represented by the fabric of what persists in the present. His approach avoids the static reification of history, and asks architecture to consider the past as an ever-changing relational construct (between now and then), rather than as an objective event. Engaging contextual histories is therefore perceived as a mediated relationship - unable to be reproduced, yet capable of critically provoking the present - "a destructive and fundamentally redemptive enterprise" that constantly shifts and resonates. ${ }^{14}$ Regarding all moments in time as a constant negotiation between 'catastrophic reality' and 'utopian possibility' is what separates Benjamin's reading of the city as a potentially critical exercise. This view resists submission to both historical reification and the post-critical encouragement of the inevitability of technological progression. ${ }^{15}$

15. Elliott, 2011, p. 73

\section{His approach avoids the static reification of history, and asks architecture to consider the past as an ever-changing relational construct (between now and then), rather than as an objective event.}

Consequently, these two positions manifest in what Benjamin refers to as 'the dialectical image', where "the momentary recognition and illumination of past and present" appears in the built forms of architectural production. ${ }^{16}$ For Benjamin, the dialectical image was visually distilled in the decaying Parisian shopping arcades of the 1930s and 1940s. The arcades represented the 'primal history' of modernism - the phantasmagoria of mass distraction and commodity consumption (for Benjamin, the 'now') in distinct contrast with the historically rich fabric of Paris (the 'then'). Building upon Benjamin's position, the histories of the Clifton Street Car Park, in particular the tabula rasa incisions of the Urban Motorway and private development of The Terrace, can be seen as dialectical - as the desires of Wellington to positively follow the progressive economies of other western cities; and as a contextual thick edge, a site of historical trauma that is now in a state of historic, economic, political, social and visual negativity. 
Avoiding Spectacle: Framing Dialectics Through Visual Strategies

In Architecture and Spectacle: a Critique, Gevork Hartoonian builds upon this positioning of Walter Benjamin in the current context, contending that the experience of a building as an image embodies the 'spectacle' of architecture in the digital age. This has created a historically singular situation where the production and reception of architecture is infiltrated and 'shaped' by technology to the point of

19. Although attributed to number of factors, Hartoonian sees this trend as the logica outcome of the spectacle of late capitalism, dividing mainstream architectural design into two categories: 'theatricalization' and 'theatricality'. Theatricality

ffried Semper - a belief hat architecture has a latent criticality and ability for cultura communication that lies within

the expression of its deep tectonic structure. Conversely theatricalization - most obvious in the recent works of Frank Gehry and parametricism - is the condition of superficia embellishment.

20. Hartoonian, 2012, p. 3 . "total commodification. ${ }^{17}$ Thus, the image has come to pervade the design process, contributing to the aestheticisation of architecture as a spectacle that is apathetic to many of its other contextual dimensions. ${ }^{18}$ For Hartoonian, this emphasis on the visual projection of a building has dislocated the appearance and form of a work of architecture. As a result, the design process is manipulated and exaggerated in order to fulfil a condition of 'superficial delight' in the eye of the beholder. Such a focus represents an attempt to remove individuals from their increasingly distracted experience (a position originally put forth by Benjamin): ${ }^{19}$

In the mainstream of contemporary consumer culture, no one feels at home without access to the cultural products distributed globally... [I]t is the excess, both visual and tactile, that orchestrates today's culture of spectacle for which we have no adequate words except to say, "It's cool!". ${ }^{20}$
The desire to design for visual spectacle has become evident in the production of much of contemporary architecture, where a focus on surface, facade and ornament is once again prevalent. One notable example is the recent construction of the new Supreme Court of New Zealand in Wellington (see Figure 7.5). Designed by Warren and Mahoney, and completed in 2010, the courthouse boasts an extensive (and expensive) façade of tessellating patterns that according to the architects take the form of "a bronze screen with a design strongly influenced by the Maori idea of Pohutakawa and Rata trees symbolising both shelter and leadership". ${ }^{21}$ Beyond the visual, the screen provides little architectural contribution to the function of the building, performing no tectonic integration, and little cultural value outside of abstract symbolic expression. The Supreme Court, alongside other recent buildings in Wellington ${ }^{22}$, expresses an intention to superficially juxtapose its visual surroundings by introducing a projected façade. Problematically, the courthouse does so uncritically submitting the design of the building to the realms of spectacle.

However, in the forces of image production Hartoonian identifies an opportunity for positive critique - 'a potential to resist' - by subverting the spectacle from within:

Of all the art forms, movies we watch and buildings we visit, to mention two artworks with
22. See also: the Cordon Bleu Cooking School (2010) by Archaus architects, lower cuba Street, Wellington.

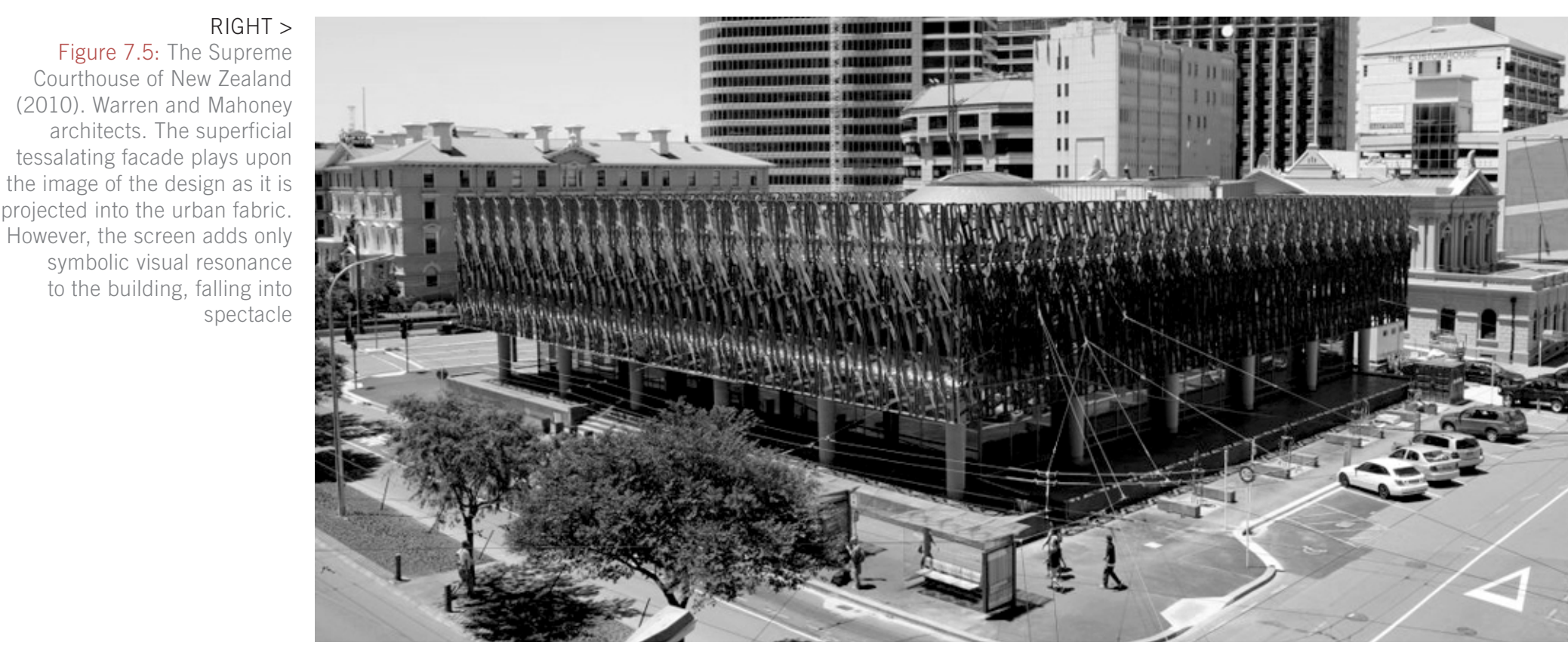




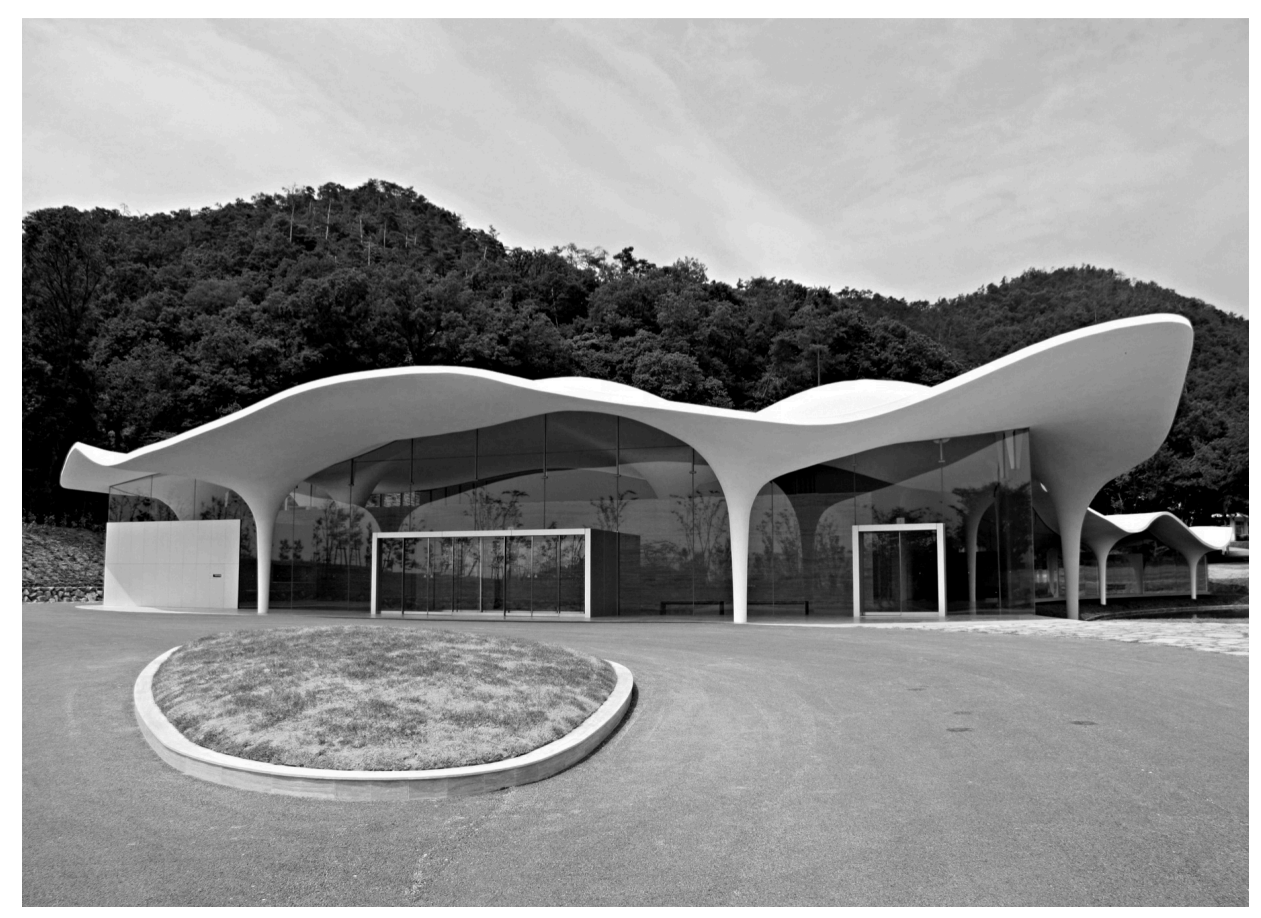

ABOVE ^

Figure 7.6: 'Meiso no Mor Municipal Funeral Hall' (2009) by Toyo Ito architects. This design reflectsthe use f contemporary techniques (fluid-form, parametric design) in a manner that resists spectacle. The design is drawn from the surrounding landscape in combination with the programmatic requirements

- refusing to present a consistent facade and provide an overall image of the design

close ties to capital and technique, architecture still remains the most controversial mainly because it has the potential to resist but also be plunged into the contemporary culture of spectacle. ${ }^{23}$

In the dialectical image, Hartoonian suggests that architectural discourse can productively overcome the limitations of the current culture by plying the past for material, saturating the image of a building

For Hartoonian the dialectical image ignites strategies of juxtaposition, as both a means to engage in the processes of spectacle creation, and crucially as the embodiment of a visually temporal relationship.

beyond the superficial. For Hartoonian, the challenge for critical spatial practices is "how to use digital techniques and yet resist the prevailing culture of the spectacle" (see Figure 7.6). ${ }^{24}$ This implicates the urban visual context as history, not in a nostalgic fashion, but as a means to reveal critical knowledge of the past into the present, 'opening' the design project to positive outcomes:

If the Promethean and forward looking vision of progress is suspended momentarily, then the rubble

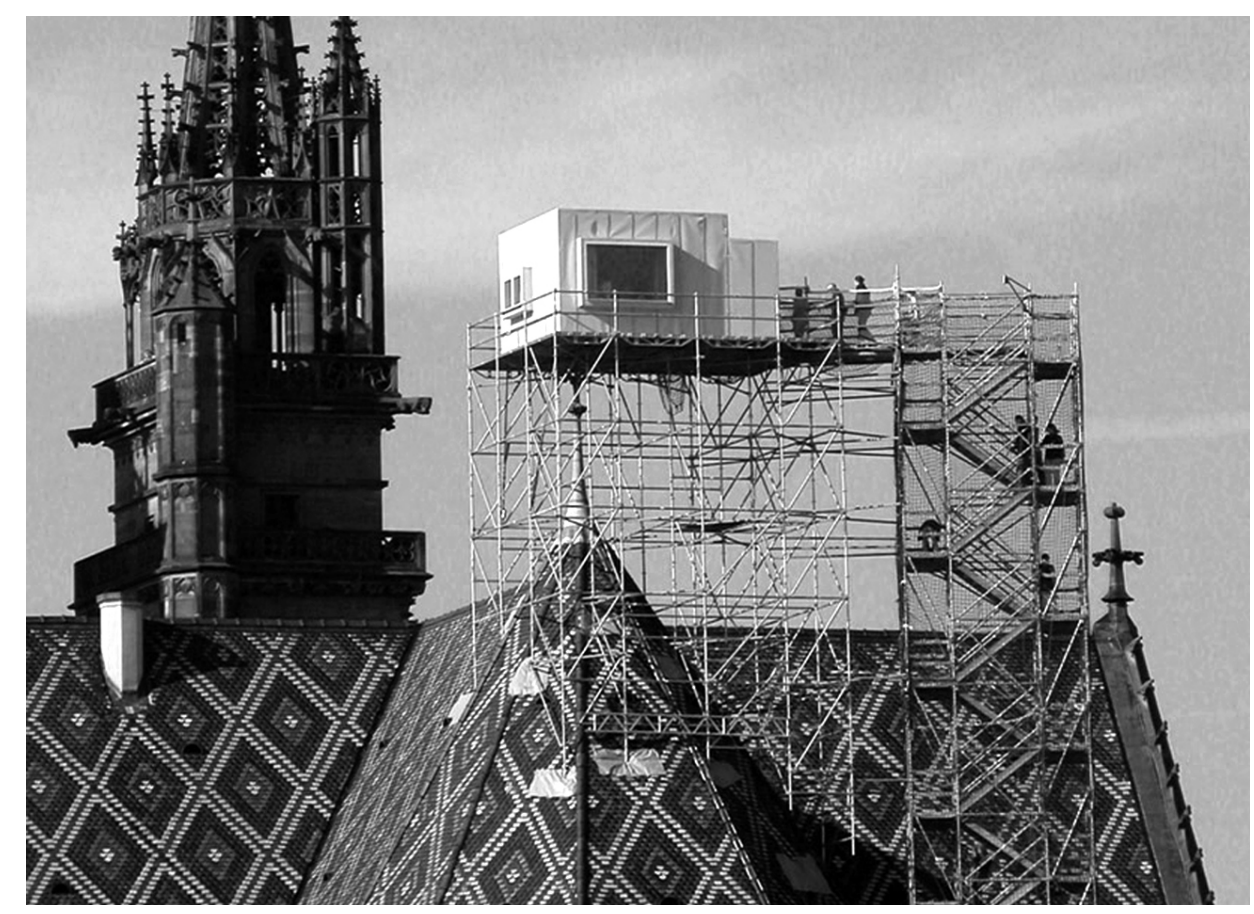

of the history might disclose something different. This is not to lament for the return of the bygone past, but to problematize the now of the present, and rework utopias that had to be submitted to the ashes of history, if capitalism had to survive. ${ }^{24}$

For Hartoonian the dialectical image ignites strategies of juxtaposition, as both a means to engage in the processes of spectacle creation, and crucially as the embodiment of a visually temporal relationship. Here, juxtaposition operates through visual contrast, "to startle, to make manifest that which lies hidden and forgotten, to bring the repressed unconscious to consciousness". ${ }^{25}$ These moments of contrast intend to create contradictory experiences - whether through seemingly inappropriate materials, forms or languages - as a means to interrupt or displace the dominant identities of a particular context.

Juxtapositions disrupt the distracted montages of the urban fabric, constructing a dialogue in a similar manner to the strategy of discontinuity. However, unlike the design operations of discontinuity, which may occur as a wider anti-contextual gesture (as is the case with Parc de la Villette), juxtaposition requires a rich visual context to exist already. In this way, juxtaposition creates an intervention where an "insertion of objects, texts, images and voices into a context already thick with meanings has produced a complex scene". Reconfiguring the existing visual
ABOVE $\wedge$

Figure 7.7: 'Engel' (2002) by Tatzu Nishi. This architectonic sculpture is precariously positioned on the roof of the Cathedral of Basel, juxtapositing the wo architectural typologies o destabilise the historical significance of the building

(n)

25. Gilloch, 1996, pp. 115-116. 


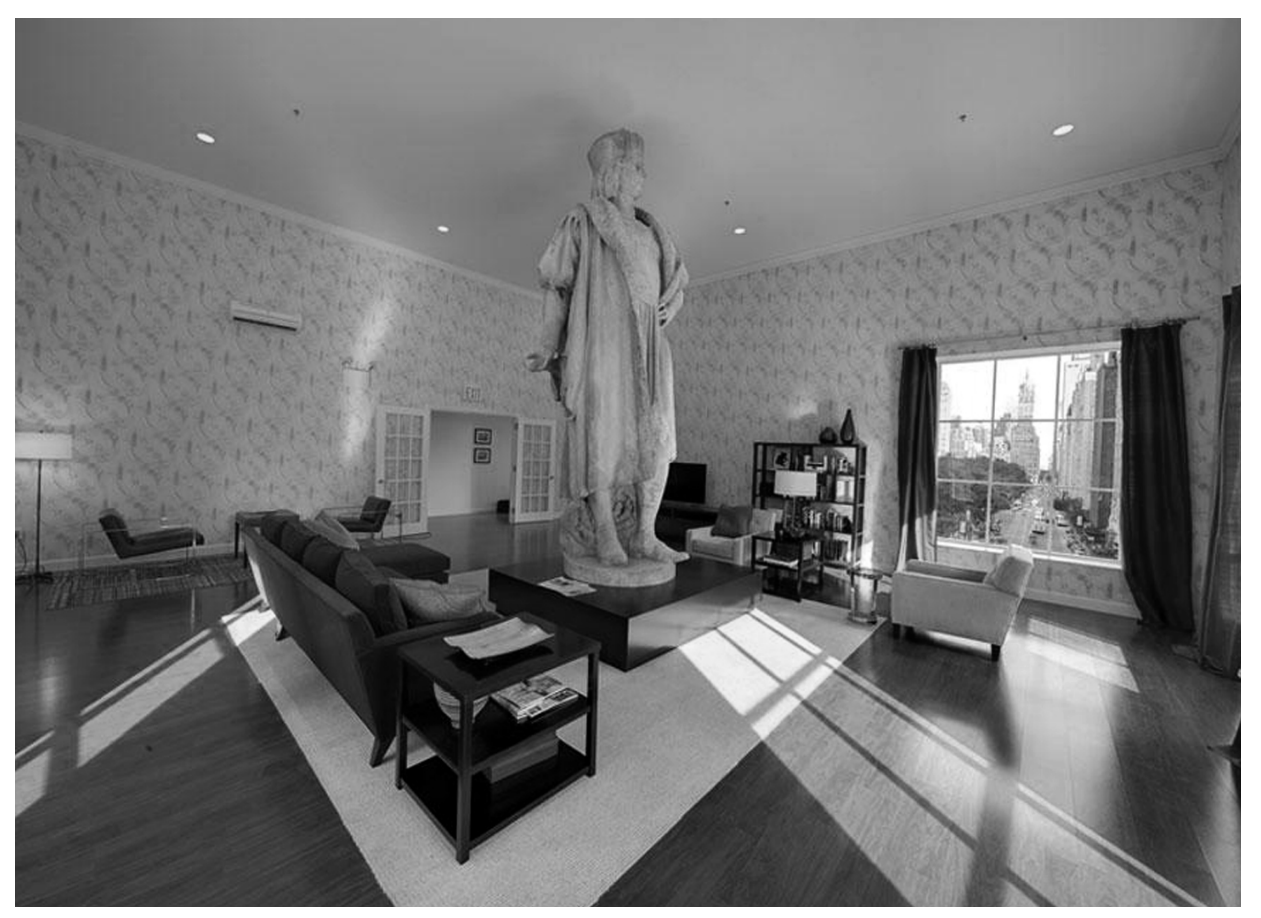

ABOVE $\wedge$ Figure 7.8: 'Discovering Columbus' (2012) by Tatzu spectacle as a purely superficial device. Rather, it Nishi. In this instance Nishi "invites the viewer to move through the work, drawing
surrounds a sculpture of Christopher columbus in New York City - positioning it within a recreated domestic interior space. The effect is a formal juxtaposition that draws attention to the two latent ideologies of the moment and

the domestic out hidden and differing meanings over time, rather than through the one-off shock effect" ${ }^{26}$

Importantly, without critical attention to the context, as related historically to the intentions of its production, juxtaposition befalls to mere spectacle. One such example of this complex negotiation is the effects of juxtaposition explored by artist/architect Tatzu Nishi. Nishi's works attempt to question the languages and identities of particular contexts (predominantly monuments and public sculpture) through the intervention of architecture. ${ }^{27}$ In Engel (2002) Nishi surrounds the weathervane atop a spire of the historic Cathedral of Basel with an inhabitable box (see Figure 7.7), juxtaposing the architecture of the gothic cathedral with languages of building sites (scaffolding, prefabricated components, plastic sheeting etc.). Similarly, in Discovering Columbus (2012), he constructs a 'display' room around a statue of Christopher Columbus in Manhattan, New York. Both rooms are reached via a precarious scaffolding stair, encapsulating the monument within a domestic interior space (see Figure 7.8). In such works Nishi inserts juxtaposing elements, interrupting sites of public history with scenes that are traditionally the forum of personal drama.

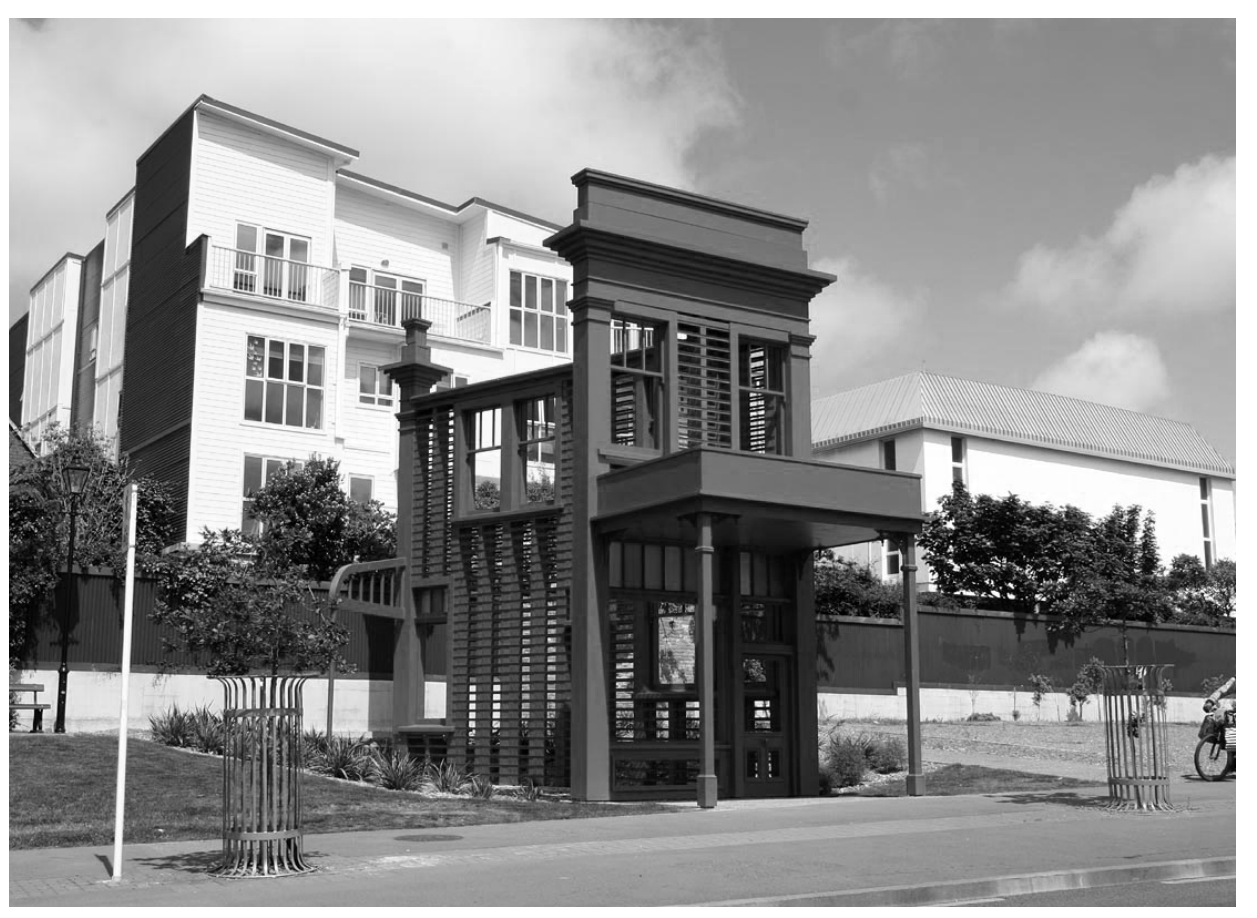

Locally, Regan Gentry's sculpture Subject to ABOVE ^

Change (2009) explores the strategy of juxtaposition Figure 7.9: 'Subject to Change' through the insertion of architecture into a historically the opposite side of Karo. Drive a series of renovated an complex context. Gentry's sculpture critically engages the history of the Wellington Urban Motorway by positioning a bright red structural skeleton of a 'heritage-style building' next to the motorway exit on Karo Drive (see Figure 7.9). The architectonic sculpture informs a contrast with the surrounding villas of Karo Drive - houses that were forcibly relocated by the New Zealand Transport Authority in 2006 to make way for a re-routing of the motorway off-ramp:

The piece is a thoughtful reflection on the buildings that have been removed and those that have been retained. It will itself, we expect, become an embedded and popular part of the Te Aro streetscape. $^{28}$

The sculpture is incomplete - a replica of two sections of an absent house, giving the impression that it is a 29. Gentry, 2009. "slice of building left behind by developers". ${ }^{29}$ Here, Gentry places a contrasting architectural form within the same context as the relocated and renovated villas, deriving its image from the architecture "in 30. Ibid. its immediate vicinity" ${ }^{30}$ In so doing the sculpture critically references the demolition of low-income property through forces of development, and typologically juxtaposes the forms of the nearby villas. 
Additionally, by revealing only the skeleton of the villa's structure, Gentry is destabilising the superficial identity of the work - stripping back the facades of the villa - creating an inverted image, an anti-spectacle. Through this visual juxtaposition (colour, material, 'empty' space), the work is in dialogue with its context as both an anti-spectacle and as a historical referent - it is critically engaging the past in the present as a dialectical image.

The Jewish Museum Berlin: Engaging Historical Context in the Dialectical Image

The works of Gentry and Nishi are examples of small scale architectonic interventions adapting strategies of juxtaposition to create dialectical images. Applied holistically, and as a primary design strategy for a full-scale work of architecture, is Daniel Libeskind's Jewish Museum Berlin(1999). Libeskind's design uses a series of context-specific indices, 'lines, voids,' 'traces', and 'axes' to generate design imperatives for a building that performs as an anti-spectacle.

BELOW, LEFT As such, the museum has been called "one of the

important realized buildings of what can be called the indexical project in architecture". ${ }^{31}$ However, unlike the site-specificity of Kaye's approach, Libeskind uses his performative index to present a series of specific images - a critique of symbolic representations of

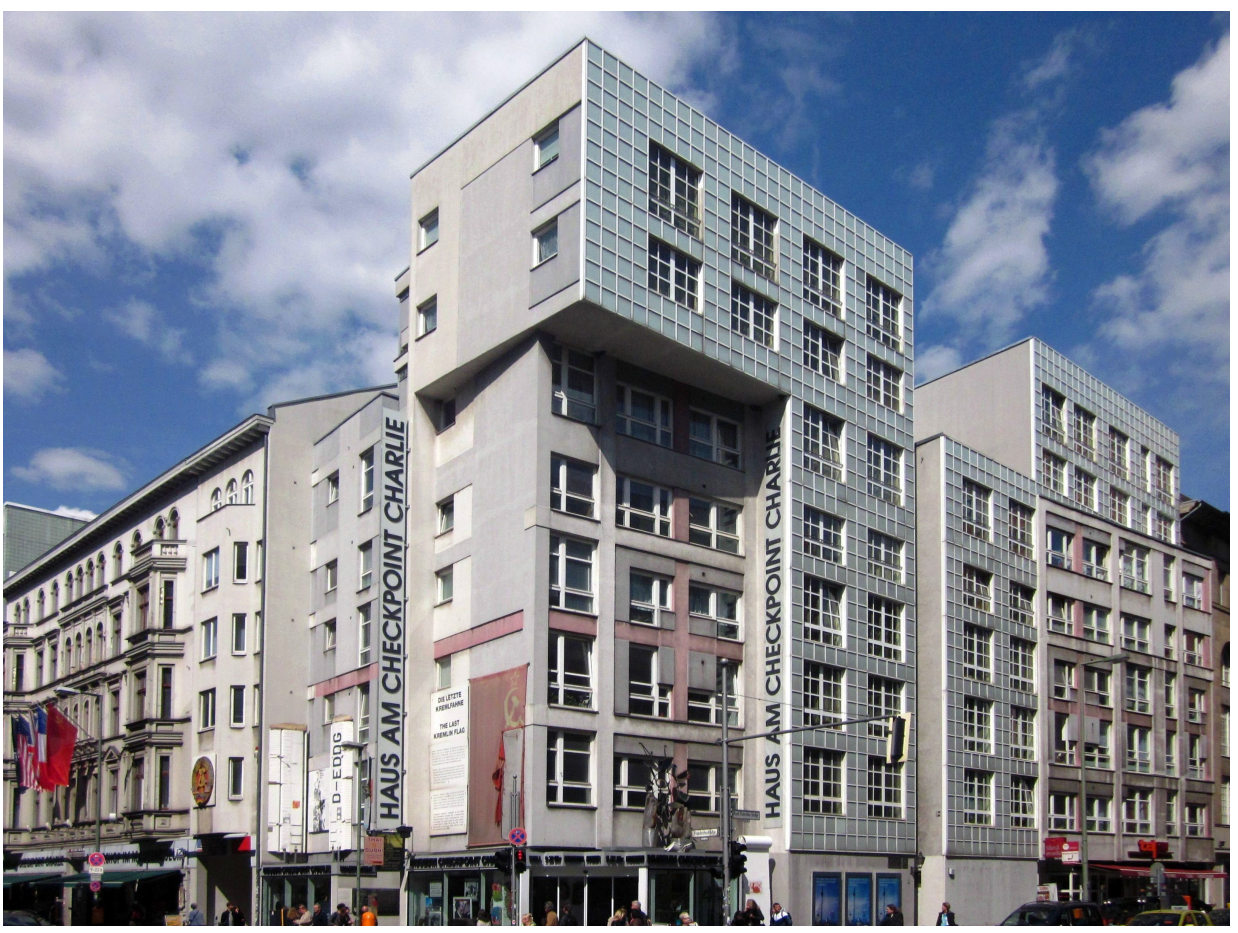

history through various subversive juxtapositions. In so doing, the design aligns with Benjamin's historical method as it denies the problematic assumption that historical context is a stable identity, treating the past and the present as dialectical rather than concrete.

Situated at the intersection of Markgrafenstraße and Lindenstraße in the Friedrichstadt district of east Berlin, the Jewish Museum is directly adjacent to and on the property of the Prussian Collegienhaus (see Figure 7.12). Barely surviving the air raids that destroyed much of Berlin during World War II, the Collegienhaus was restored between 1963 and 1969 under the supervision of Günter Hönow. Originally erected as a Superior Court of Justice for the (then) Prussian state - the Collegienhaus now stands as a Baroque landmark and provides an entrance point to Libeskind's design. Importantly Friedrichstadt has become a locus point for the 'reconstruction' of Berlin since the 1980's, an annex identifiable through 'unrelated' structures of various eras and styles - an archipelago of heterogeneous architectural icons (see Figure 7.10 and 7.11). ${ }^{32}$ This has informed an environment that is rife with spectacle - impressive buildings that are spread out in an assortment of projected architectural images. Within this context the Jewish Museum Berlin was intended to stand out as "devoted above all to a representation of Jewish history as an integral part of the city's history".33

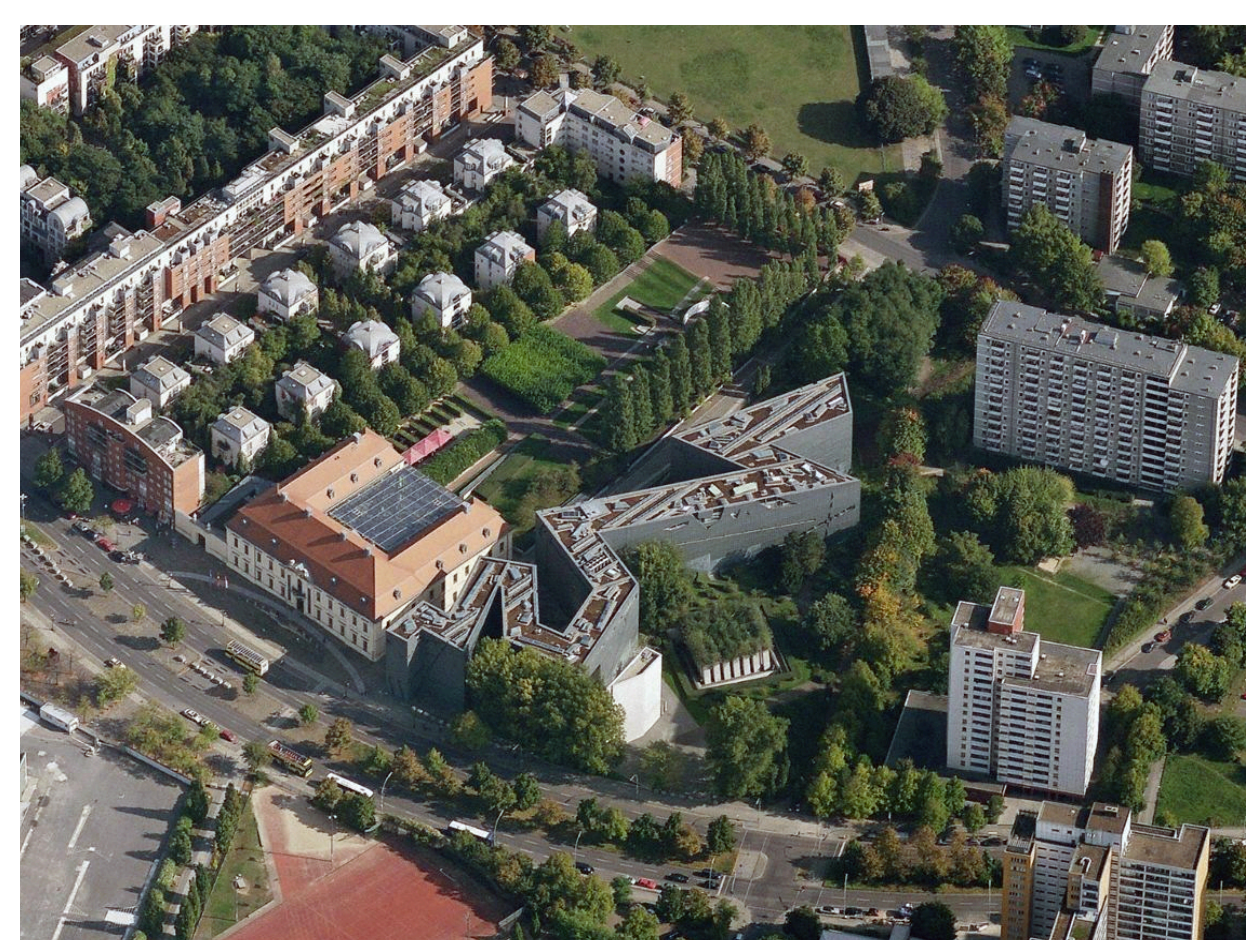




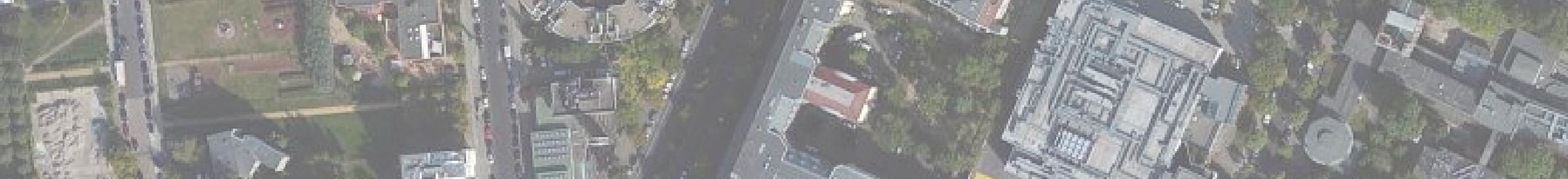
-

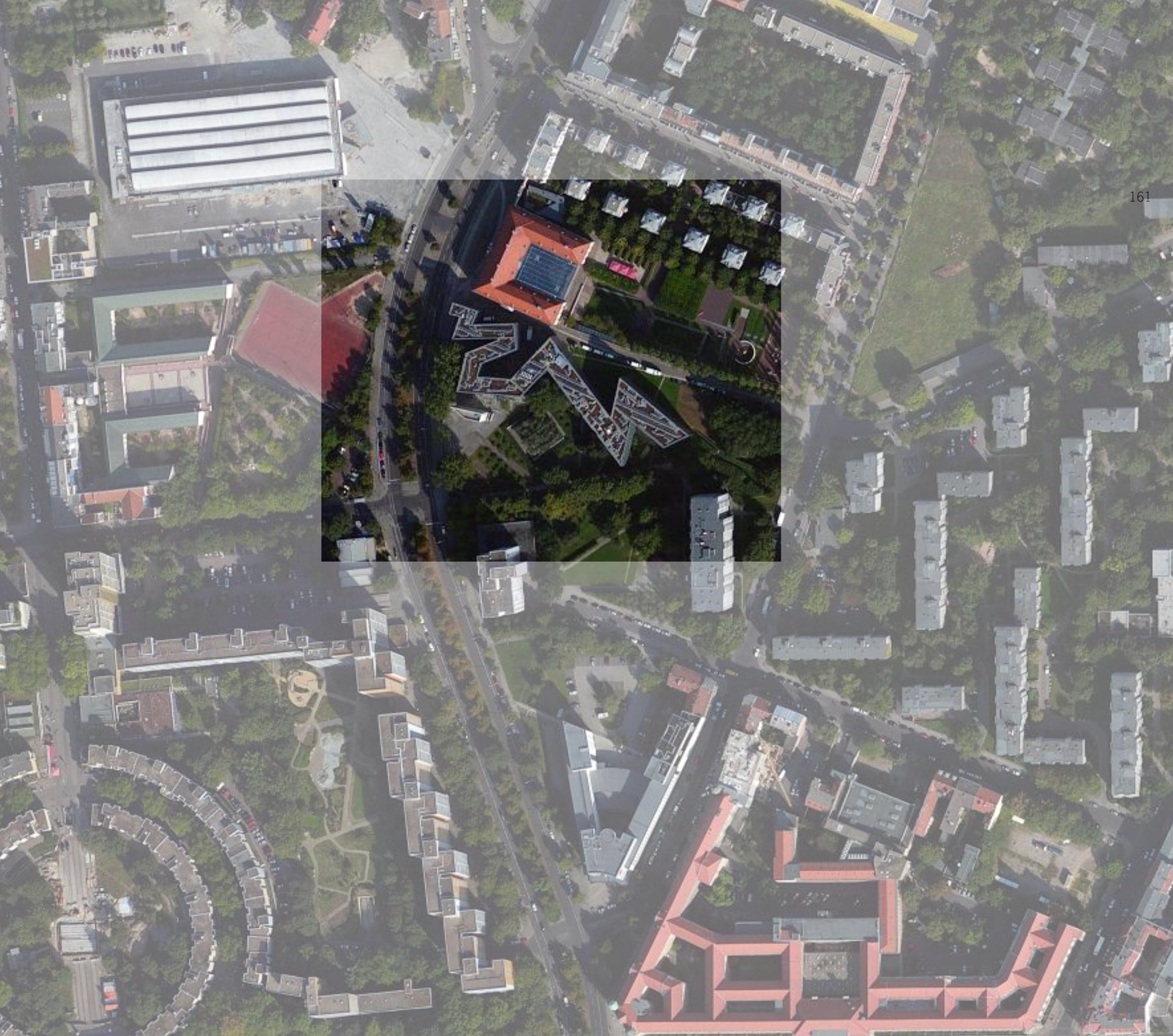


Relative to this sensitive brief, the architecture of the Jewish Museum operates between, on the one hand, an 'uncompromising uniqueness' and on the other, a 'painstakingly' contextual approach with regards to the surrounding urban fabric and architectural histories. ${ }^{34}$ These imperatives are yielded visually through indexical images that are experienced as one proceeds through and/or around the building. This is an attempt by the architect to refuse the construction of an icon or spectacle, instead, implicating an experience of 'open-ended ambiguity' that is "always on the verge of becoming".
Libeskind's proposal for the Museum is titled Between the Lines, thus informing the most visual of his spatio-historical indices. Straight lines, or axes, are used throughout the building - as uninterrupted or fragmentary markers - performing throughout the project as a double metaphor - a division' between the physical gesture and the segregation experienced by the Jewish population in Berlin. ${ }^{36}$ At the core of p. 23 this approach is a series of juxtapositions.

The primary juxtaposition strategy occurs through the building's radically new typological and material gestures that contradict the existing context,
Figure 7.13: Axonometric diagram of the Jewish Museum Berlin and adjacent Collegienhaus highlighting 'zigzag' extruded volumes of the museum in formal juxtaposition with its visual context

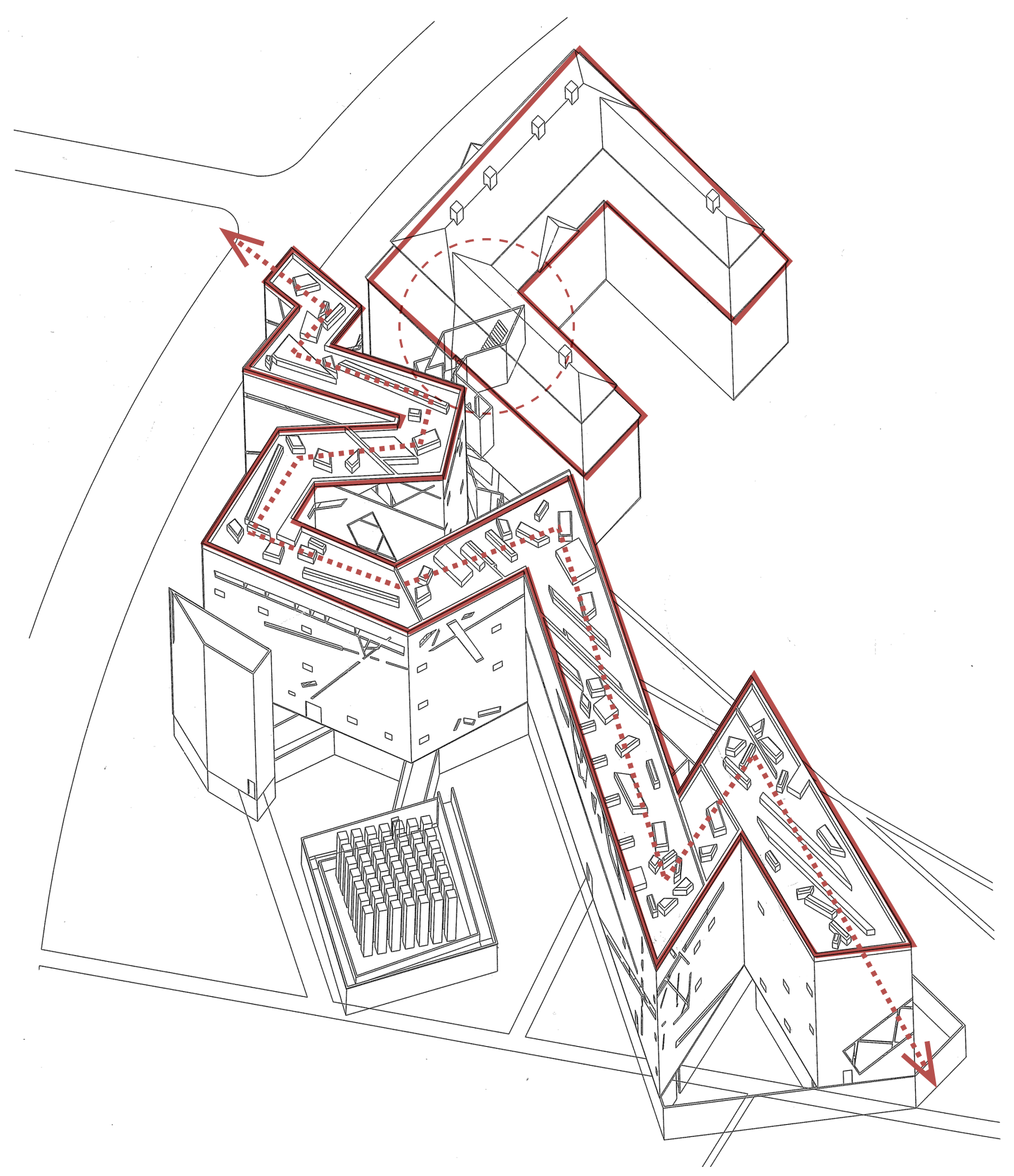


37. Schneider \& Libeskind,

RIGHT

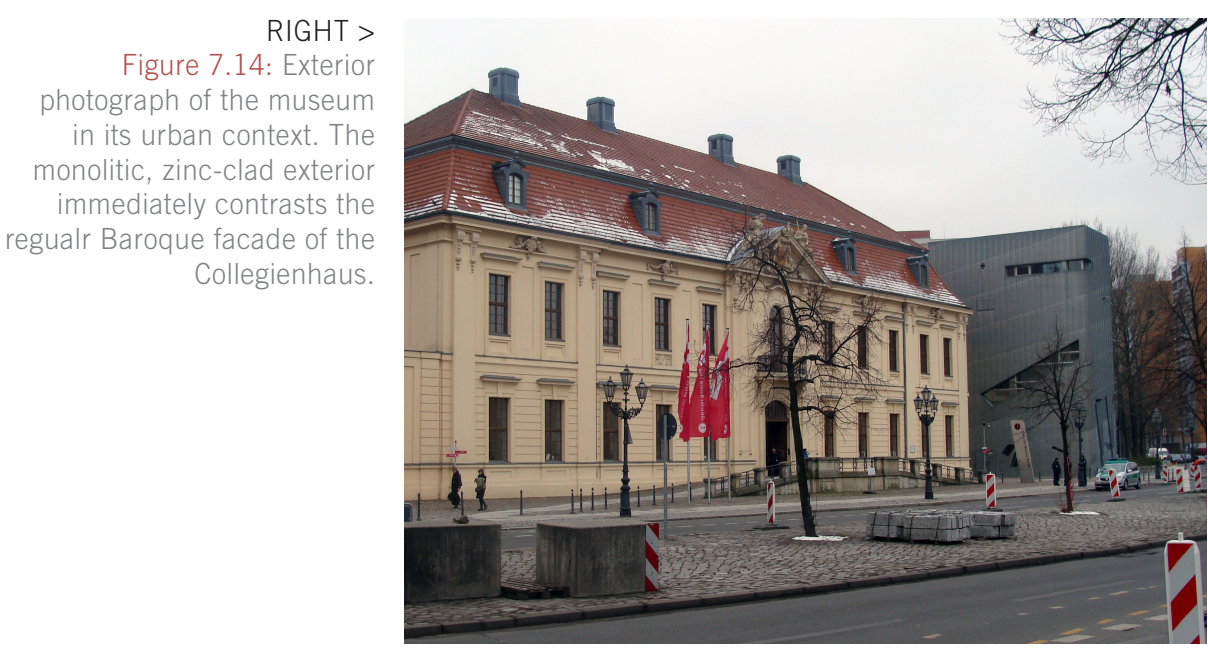

The extruded volumes of the zigzag plan generates an experience of the exterior of the building that is immediately incongruous with its visual context. The visual composition created by Libeskind is striking in its contrast between the old (the 'then') and the new (the 'now'). Analysed from its primary street elevation, the jagged masses and zinc-clad facades of the extension are immediately identifiable as 'other' to the baroque Collegienhaus, a visual disruption in the montage of the streetscape gs position in space" ${ }^{37}$ Facilitating this the building (see Figure 7.13). Conceived in plan, the zigzag is generated "from imaginary lines on the city map which connect the site with the street addresses of great figures in Berlin Jewish cultural history - Heinrich von Kleist, Heinrich Heine, Mies van der Rohe, Rahel Varnhagen, Walter Benjamin, divided into 60 interior sections, referents to each of the "Stations of the Start" described by Benjamin in his notes about Berlin in the essay "One-Way immediately towards the influence of the dialectical image throughout the project: regarding the history of Berlin's oppressed Jewish population as ultimately recoverable in the 'now', experienced through architecture in a series of visual mechanisms, images,

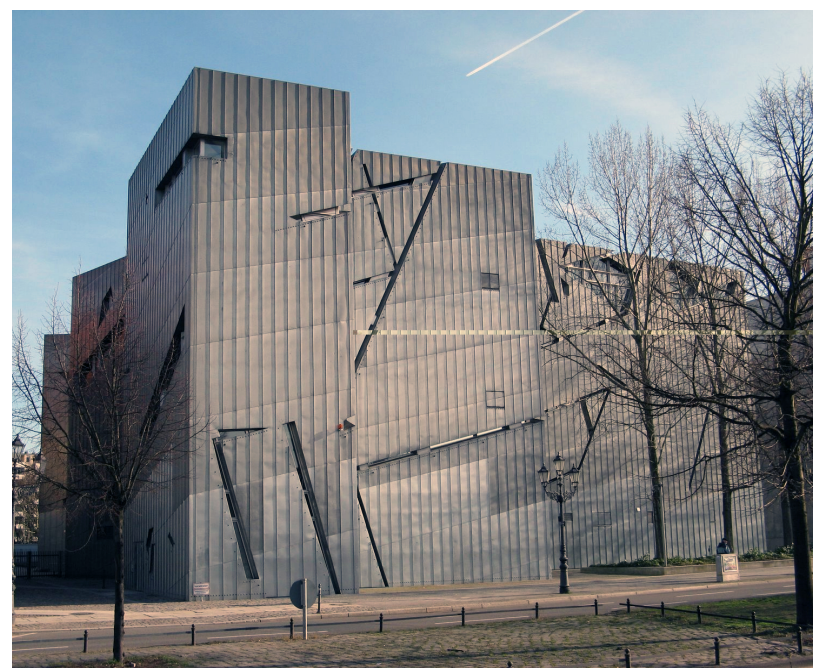

style of the old, yet at the same time [the museum] works as a lateral element that clearly defines the

that at first appears contradictory and intentionally iconic (see Figure 7.14). However, moving around the design, the initial spectacle of the contrast is unable to be fully grasped as the overall geomtery of the building appears as a façade-less mass, rendering the totality of the zig-zag plan unintelligible through visual experience alone (see Figure 7.15 and 7.16). This is evidenced by the occupant's inability to produce an overall exterior image or 'gesalt' of the building. According to Andrew Benjamin:

... [T] he contrast is unable to be fully grasped as the overall geomtery of the building appears as a façade-less mass, rendering the totality of the zig-zag plan unintelligible through visual experience alone.

\footnotetext{
The Museum's external gesalt is deliberately broken and odd in outline, too well disguised by its cladding, and so bunker-like and tangential in its address to its neighbourhood that it resists every attempt to read it as iconic...[T]he design refuses to settle for spectacle. ${ }^{41}$
}

In so doing, the design forms a visual resistance to the wider heterogeneous context - subverting the visually detached and readily aestheticised buildings that are littered throughout Friedrichstadt. Much like the deconstruction of the villa typology by Gentry, this effect is largely due to the subversion of 
its urban context. The 'bunker-

like' exterior is anti-iconic,

(in spite of its contrasting

nature), critically engagin the visual context of the area.

Additionally, the 'arbitrary'

cuts and traces superimposed

across the building's surface destabilise the regularity of the Collegienhaus's facade

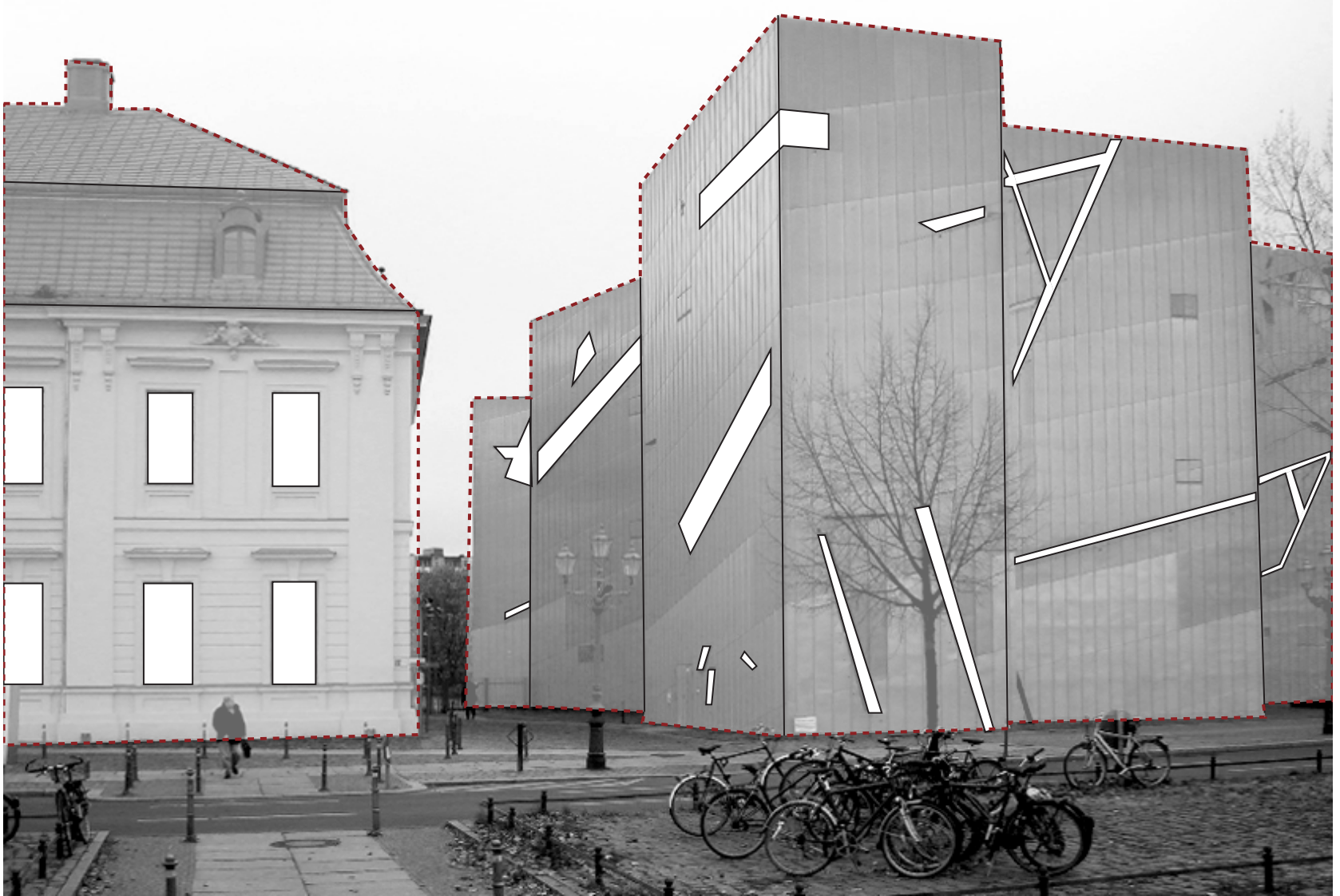

the superficial aspects of the design - the façade. The shifting volumes and consistent zinc cladding create an anti-façade, resisting the temptation to 'plunge into the culture of spectacle. Similarly, the juxtaposition created by the shifting masses of Libeskind's design infiltrate the background perspective, refusing to present a linear frontage. This contrast highlights the two-dimensional projection of the Collegienhaus's façade, placing the two structures in a visually contradictory dialogue, inviting the visitors of the

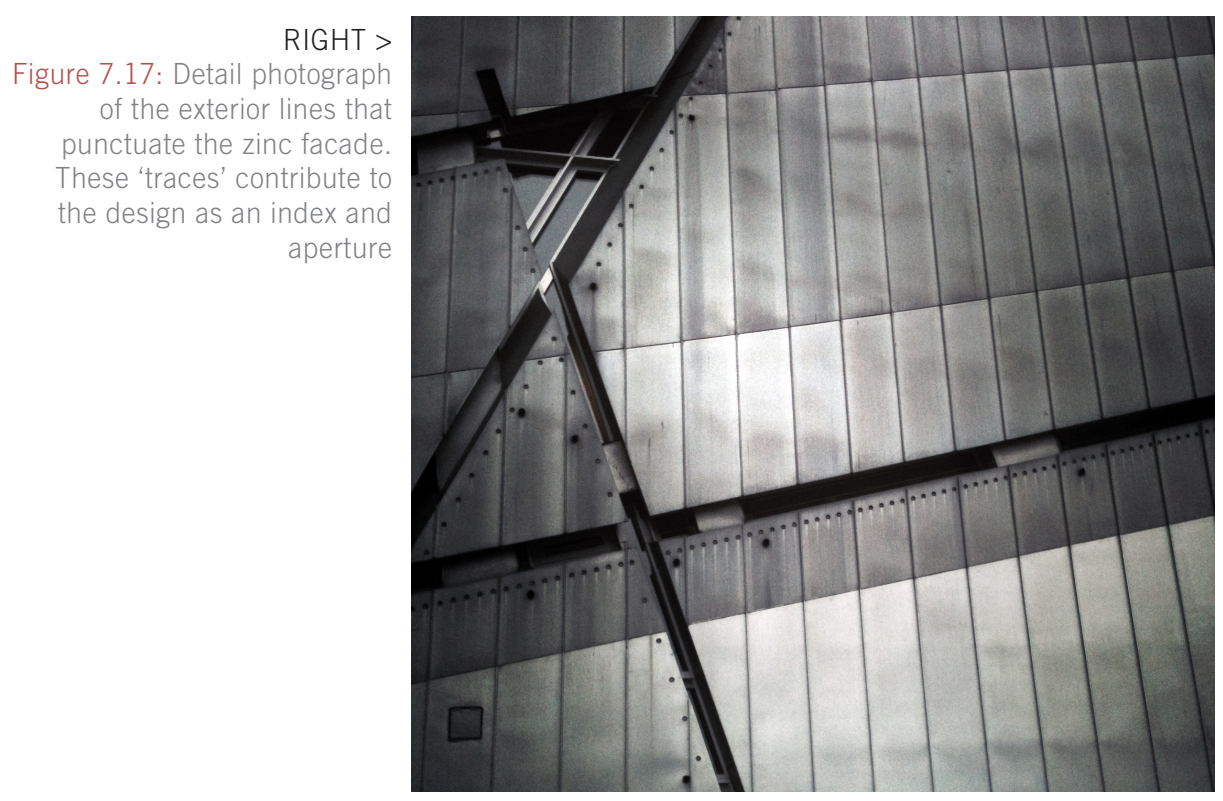

museum to question their relationship architecturally and historically.

Alongside the zigzag plan, an series of lines create an index that is visually superimposed across the distinctive zinc cladding of the exterior. The jagged volume of the building is wrapped with punctuations - lines of apertures that frame views from the inside, and destabilise the visual presentation of the exterior (see Figure 7.17). Additionally, these cuts contribute further to the visual juxtaposition of the two buildings occupying the site - creating a distinct contrast with the regular and symmetrical apertures of the Collegienhaus. As a critical design operation the lines have a simultaneous action - to resist the typical notion that a window should reveal the scale of the interior/exterior relationship, thus avoiding a stable image of the building's volume; and to shift the role of the window to that of an indexical marker. At first this index appears arbitrary, yet it is its arbitrariness that is in keeping with Libeskind's wider motivations, to express the seemingly 'meaningless' execution of Jews in Europe and their subsequent absence in Germany's past. 
Physical exterior separation between the Collegienhaus and the Museum is crucial to the resonance of the overall juxtaposition of the two buildings. Initially it appears that Libeskind's design is dislocated from its adjacent contexts. However, this visual separation is inverted as visitors enter the extension, exposing the insertion of the entrance stairway of Libeskind's design within the foyer of the Collegienhaus (see Figure 7.18). In so doing, the new entrance announces a sharp division between the Museum's overall 'Germanness and Jewishness'. According to Terry Smith the effect of this juxtaposition is:

To vanquish the Museum of Berlin, leaving us to take the entire ensemble [dialectical image], Collegienhaus included, as, now, the Jewish Museum Berlin. This latter Museum certainly seems to be the experience of the visitor: the rococo structure functions as no more than an elaborated entrance. 42

42. Smith, 2010, p. 151.

As Smith identifies, upon overwhelming its exterior image the contrasting new museum infiltrates the fabric of the historical Collegienhaus. The purpose of this gesture is twofold: firstly, through its imposition within the interior of old, the new

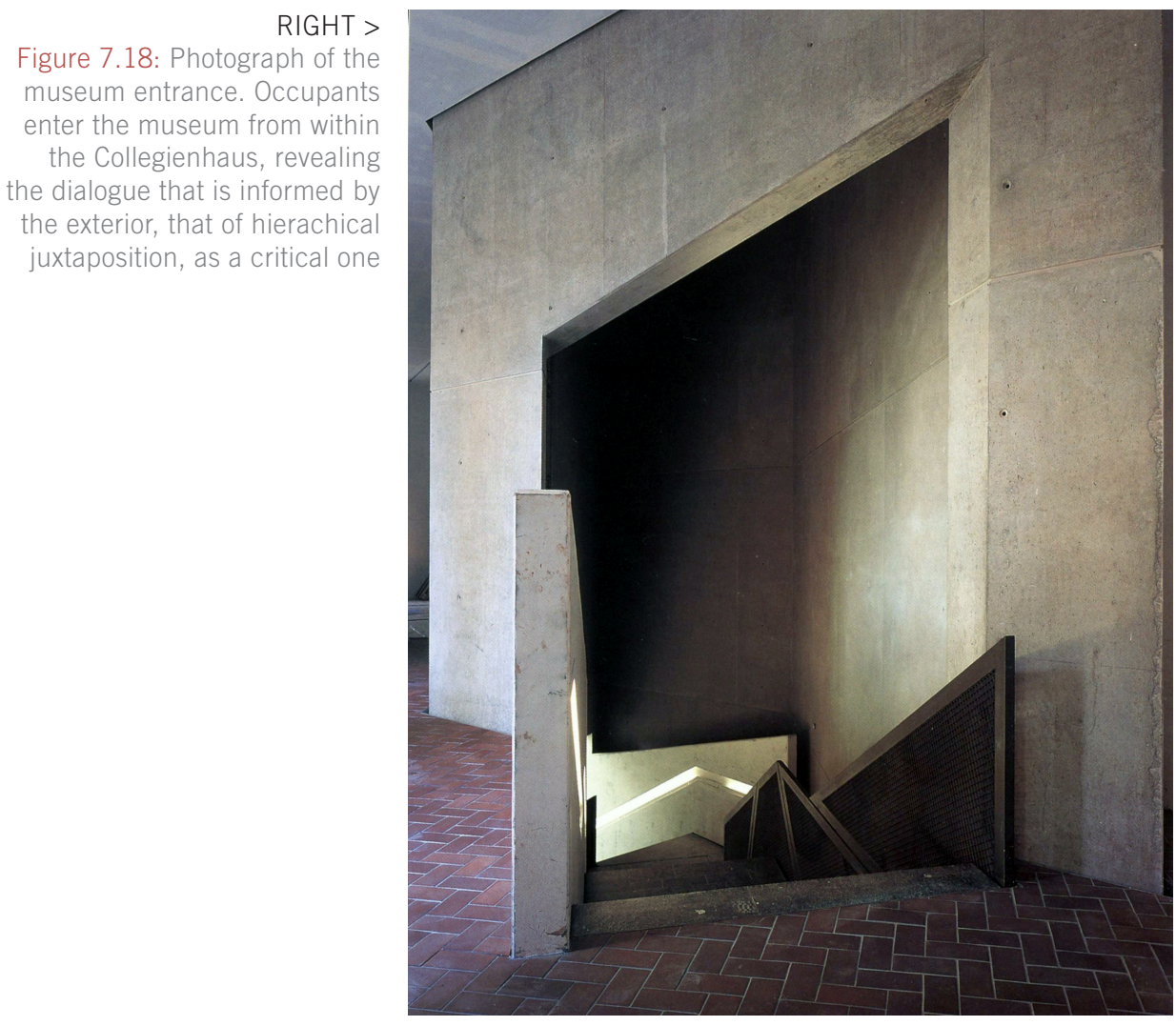

museum adopts a dominant position in the hierarchy of the two juxtaposed buildings, contending that the new face of Berlin must be a projection of positivity and hope for European Jews as accessed through the past; secondly, the connection of the two buildings below the ground datum allows each structure to remain externally separate, subverting notions of façadism, and producing a singular object presence. Thus Libeskind constructs a dialectical image - synthesizing the structure of the past, literally and metaphorically, with the intrusion of the contrasting, new design. In so doing, the architecture 're-inserts' a series of dialectical images within a visual context of Berlin's Jewish past, present and future.

\section{Conclusion: Implications and Strategies for Design}

Following a re-reading of Walter Benjamin by Gevork Hartoonian, a position on the dialectical image has been argued. This critical, temporallyfocused approach contributes to the framework of critical spatial practices by engaging visual and historical context(s) in the design process. Consequently, a temporal engagement will contribute to a polycontextual the 'opening' of thick edge conditions displayed by the Clifton Street Car Park.

Translating this critical practice into design research, the Jewish Museum Berlin displays how strategies related to juxtaposition can implicate the dialectical image in the design process. This is of particular relevance to the design case study, explicating how the current commercial and infrastructural visual context of the car park can be subverted. However, unlike the Friedrichstadt district, the Clifton Street Car Park is located in adjacent to a landscape (The Terrace) that is defined (in Benjaminian terms) by primarily modernist, developer-driven office buildings that construct a repetitive montage of glazed facades as one moves throughout the wider context.

In addition to the visual context of The Terrace, the rigidly orthogonal infrastructure of the 
Urban Motorway contributes to a visual identity that is distinctly 'modern'. These features are shown in Figures 7.18-7.21.

Based upon these understandings, formal intervention within the thesis's experimental design site can be tested to contrast, contradict, and destabilise this visual orthogonality of the visual context. Following the strategies employed by Libeskind, this may involve the intervention of a 'radical' typology that contains an indices related to the contextual history of the site. In so doing, the design case study can frame a series of dialectical images, a series of spectacles that invert the homogeneity of the existing, yet explore dimensions beyond simply the contrast of spectacle.

Chapter Seven concludes the elucidation of strategies for Rendell's framework, and the fourth research question - demonstrating how critical spatial practices manifest in the built environment, in particular through strategies of architectural design. This thesis now looks to develop further information for the design case study by exploring the potential of architectural programme to engage a polycontextual approach to the thick edge condition. 
strong verticality of buildings

either side, bissected by the

motorway's horizontaality-: The

natural irregularity of the greenbelt forms the'backdrop.

The lane is anti-piedestrian,

forcing publics to adcess from

the narrow footpaths either side

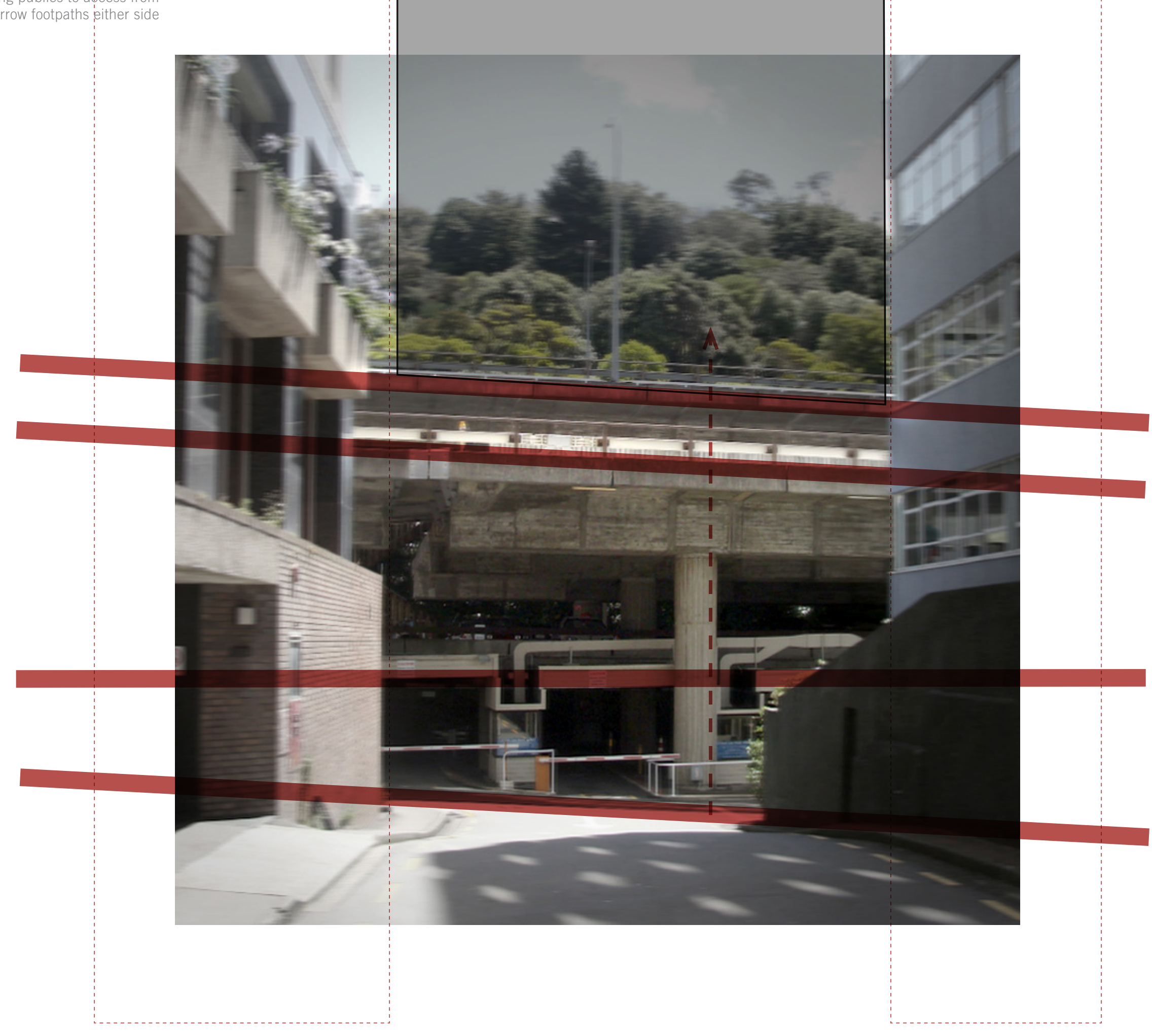




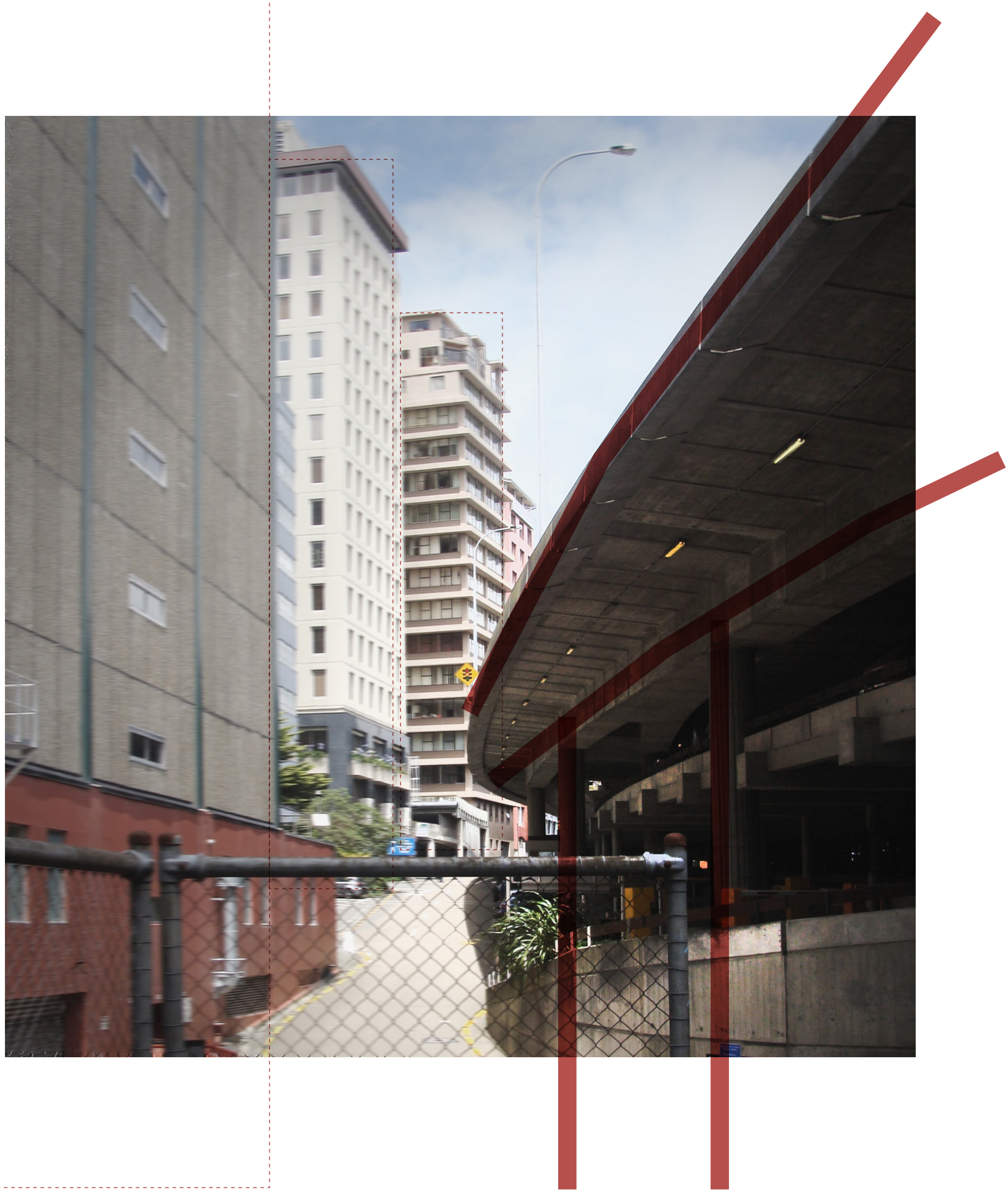



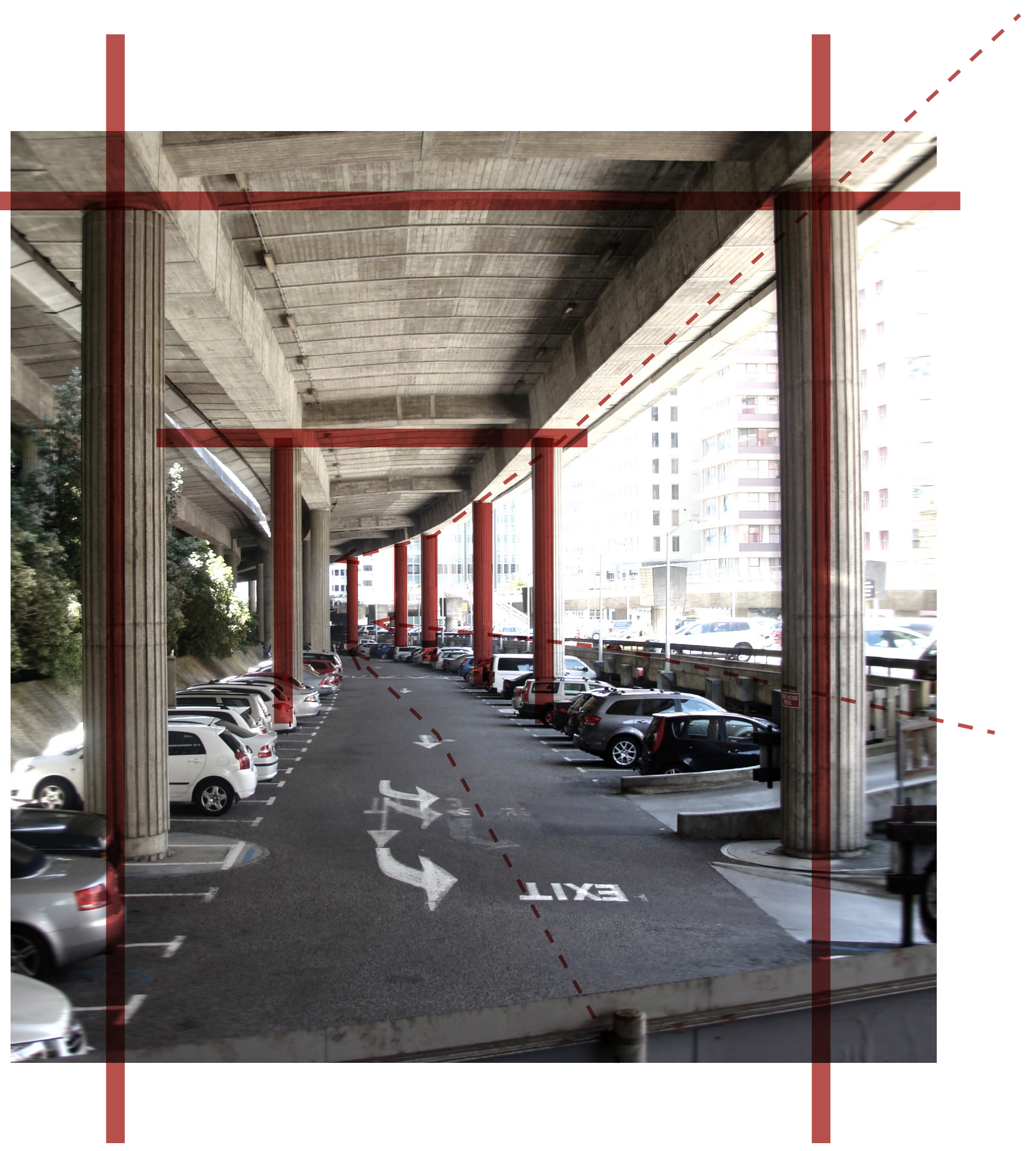
of space between Datum's 3

and 4 is desolate, empty, and

The same language emerges as in figure 7.20 , but is exaggerated when the car park is not actively used.

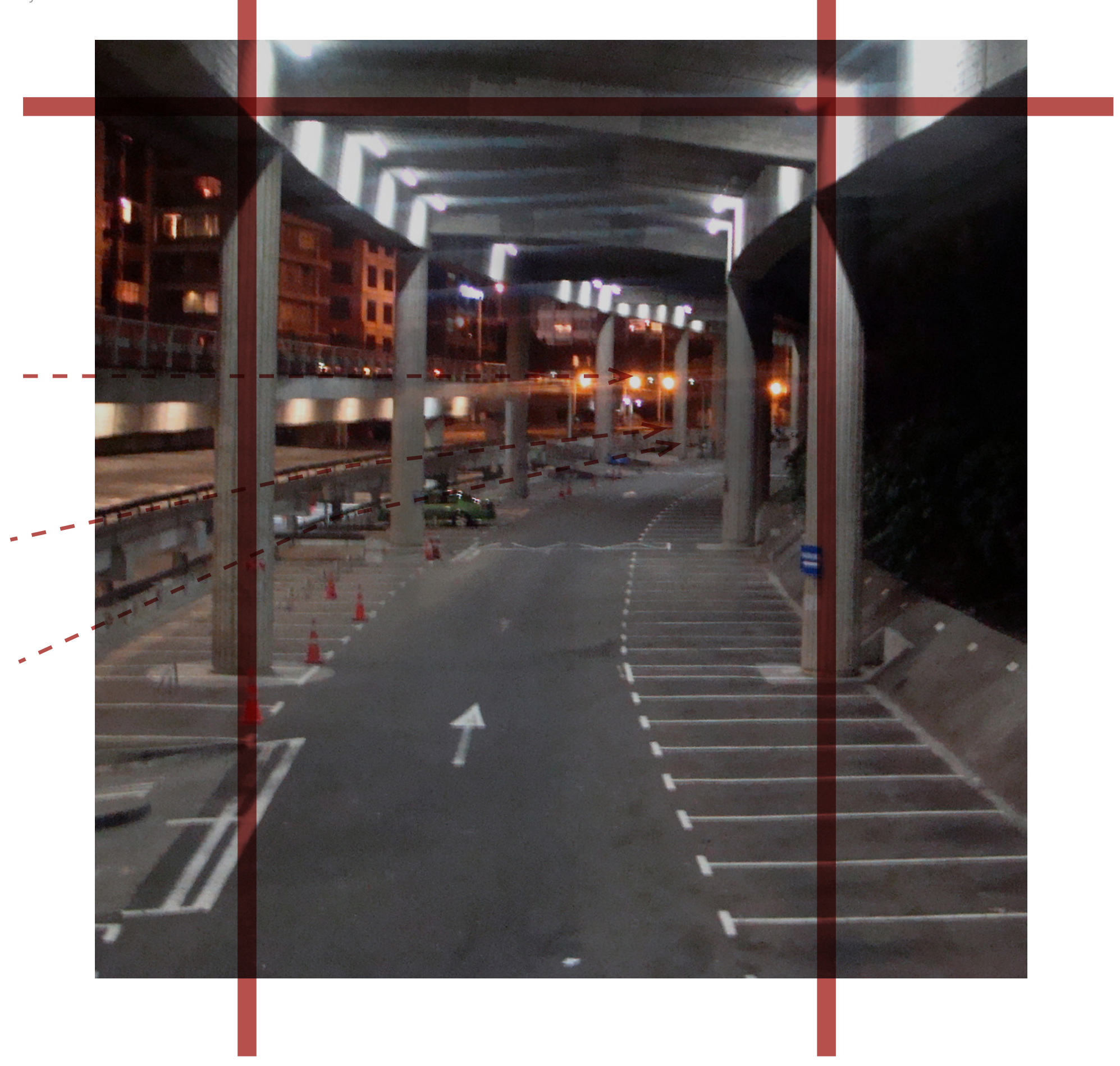


171

$\frac{1}{1}$ 



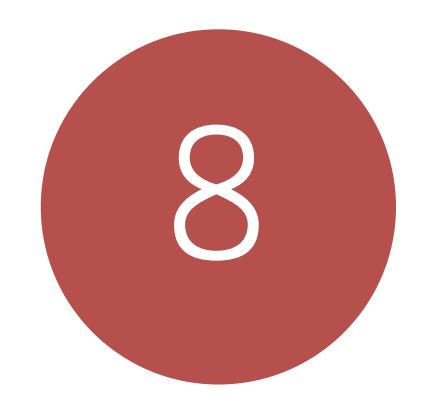

\title{
A POTENT(IALLY) CRITICAL PROGRAMME
}

\author{
Elucidating Programmatic Strategies \\ for Clifton Street Car Park
}

\author{
Introduction \\ An Economic Opportunity for Thick edges: \\ Cultural Nodes \\ Event Spaces: Performing Arts Facilities \\ Auditorium Typologies \\ Three Local Examples \\ An Exemplary Client: The NZIAF \\ Conclusion: Programme Brief
}


The most successful downtowns are populated by residents, workers, shoppers, diners, tourists and business visitors, who occupy buildings and enliven streets and public spaces during different days and hours. In the aggregate, the activities of these groups give the downtown area daytime and night-time vitality on work days, weekends, and holidays. ${ }^{1}$

Based upon the definitions of, and design strategies for, critical spatial practices outlined so far in Section Two, this Chapter Eight now looks to positive strategies for the revitalisation of existing economic, political and social contexts of Clifton Street Car Park specifically through the implementation of architectural programme. As a means to contribute to the inversion of the thick edge, economic diversity and vitality is posited as a crucial component to architectural intervention - and the implementation of an arts-based 'cultural node' is presented.

The contributors to prosperous urban spaces identified above by Kromer (residential, business, retail, tourism) all currently exist within the various adjacent contexts of Clifton Street Car Park. However, the programmatic thickness of the site has constructed constrained conditions that refuse to take advantage of such factors, isolating social interactions and communities adjacent to the site. The intervention of a cultural node presents a response to these conditions that will allow for an economic and social inversion, creating a space of social permeability through the site.

Investigating this proposition further, Wellington's cultural calendar reveals the need for additional venues to support its regular cultural events. An events facility for the New Zealand International Arts Festival as an exemplary client is proposed - a community that could occupy the new events facility design experiment, and for which the core programme brief in the design case study will be structured. It is argued that the NZIAF (and Wellington events planning in general) requires three diverse scales of performance space. Consequently this chapter investigates performance spaces currently available in Wellington, analysed specifically through the CIRCA Theatre, the St James Theatre, and the Wellington Town Hall. This chapter concludes with the clarification of a programme brief, presenting the site as an opportunity for architectural design to introduce positive contextual flows, structuring a space that is both public and private.

In so doing, the thesis progresses the framework of critical spatial practices, presenting the Clifton Street Car Park as an opportunity for architectural design to introduce positive contextual flows to the thick edge, structuring a space that is both public and private. Mediating between the theoretical framings of Section One, and the design case study in Section Three; this chapter engages the fifth research question:

Can the implementation of architectural programmes that have beneficial polycontextual implications facilitate the production of critical spatial practices within the thick edge? 


\section{An Economic Opportunity for Thick Edges: Cultural Nodes}

More so than other sectors, the cultural industries are seen to draw vital resources from city life and from city cultures. The city - and most often those with a compact metropolitan core-emerges as the effective horizon of place for the cultural industries. However complex it might be to theorise, cultural industries, in the main, 'inhabit' the city. ${ }^{2}$

O'Connor, 2004, p. 2. Justin O'Connor is Professor in the Creative Industries Faculty, University of Queensland Australia

3. Council, "Central City Framework: Approach to Implementation", 2011, pp. intervention is most successful when it is driven by distinctly local-to-global intentions and clustered services. which is 'reflected in the urban environment'. The production of this culture - as it is framed by architecture - is a "social phenomenon" that exhibits "intensely local characteristics"; characteristics which can be undermined by global-to-local structures of social exchange and commodification. ${ }^{4}$ This point is significantly reiterated by several critics, historians and urbanists who note that when cultural industry is driven by singularly motivated forces, "culture as a pattern of non-place globalized events and experiences", the repercussions are erosive to local cultures. ${ }^{5}$ Conversely, as highlighted above by Justin O'Connor, positive social production through cultural within close proximity - sharing infrastructure and

Aligning with O'Connor's perspective, intervention within the Clifton Street Car Park through the culture industry can draw influence from 'within' the city - as a bi-product of intentions that are distinctly local. This approach draws economic and political strategy from influences that thrive upon the competitive advantage of "local, tacit knowhow" which is "not accessible globally" ${ }^{6}$ A local-toglobal approach does not deny the positive effects of 6 . Leadbeater \& Oakley, 1999 global economy or policy; rather, it draws influence p. 14. from a social directive that engages specific political and economic contexts as a means to "negotiate a new accommodation with the global market" in which the cultural industry of a particular city can engage with the global market whilst constructing a "distinctive and defensible local base"7 For O'Connor, cultural nodes are of particular economic and political efficiency when induced to create social flows at a $7 . \mathrm{Ibid}$ specific location:

The intimate mapping of city and [cultural] node means that flow is not unidirectional; the culture industries work symbiotically with the city. Cultural production and consumption transform the landscape of the city... This 'revitalisation of the symbolic content' of cities draws in city governments, who link these transformations with 'ambitious public efforts of urban rehabilitation in the attempt to enhance local prestige, increase property values and attract new investments and jobs. ${ }^{8}$

The cultural node concept, and revitalisation through 8. O'Connor, 2004, p. 3. cultural intervention, operates between arts and commerce. In so doing, cultural nodes present the potential to engage the existing thick edge economic and social contexts of the Clifton Street Car Park in an opportunity for productive enterprise. ${ }^{9}$ Locally, a positive synthesis between the arts and economics can be seen in the successes of WETA Workshop and the 9. Roodhouse, 2010, p. 19. associated film industry of the region. ${ }^{10}$ However, in the case of WETA Workshop, there is little focus on the social production of space as an imperative - the industry itself is located outside of the urban centre and is both privately and economically driven. As an alternative approach, the centralisation of cultural and creative activity into a node concentrates 'multiple and diverse' publics into a new urban location. ${ }^{11}$ 

Wellington", 2013

13. Forcast.id, 2013
12. Council, "Profile of

Given recent debates around Wellington's commercial future, this chapter proposes that a cultural node will address the economic context of the site based upon several existing contextual factors: The identification of Wellington as a "leading centre for creative industries" ${ }^{2}$; a projected population increase from 28,724 in central Wellington between 2013 and 2031 , and necessary cultural vitality to support this growth ${ }^{13}$; the requirement for centralised cultural infrastructure, contributing to the knowledgebased sector of Wellington's economy, introducing economic diversity to the central city $^{14}$; and the need for alternative economic infrastructure to revitalise

14. Cultural centralisation and Wellington's status as a "dying city", a process that has

\section{The cultural node concept, and revitalisation through cultural intervention, operates between arts and commerce. In so doing it engages the existing thick edge economic and social contexts of the Clifton Street Car Park in an opportunity for productive enterprise.}

diversity has been identified by the New Zealand Property Council as a potent economic revitalisation policy fo Wellington, see: Council N. Z.,

15. Recently New Zealand's Prime Minister, John Key, caused significant controversy with his statement that

Wellington is "dying". See: Cowlishaw, Chapman, Hallahan, \& Rutherford, 2013; Parliament, 2013

Montgomery, 2003, p 294. John Montgomery is an urban planner, Economist and Managing Director of Urban Cultures Ltd.

17. Ibid. emerged from the migration of traditional corporate business (away from areas such as The Terrace) to Auckland. ${ }^{15}$

This raises a fundamental question, how can the polycontextual revitalisation of a thick edge contribute to resolving this problem? Building upon O'Connor's identification that an area of centralised and shared cultural production can instigate positive economic and social flows - John Montgomery highlights several strategies for their successful implementation. ${ }^{16}$ For Montgomery, the pitfall of cultural nodes is primarily the alienation of low socio-economic groups due to increased commercialisation and property value in surrounding areas. In the context of Clifton Street Car Park, this is less likely to be an issue as the site is surrounded by middle-to-high value residential, and high-value commercial zones. ${ }^{17}$ However, the adjacent suburbs have historically removed and repositioned the activity of low socio-economic groups - a factor that can be critically approached through the design. An events facility has the ability to attract performances and groups that are known to have a high appeal across the social spectra. It is imperative to this thesis that the design case study engages these qualities as interpreted as political and/or socio-economic - qualities that not only create flows between the existing communities (Thorndon and The Terrace), but also introduce marginalised publics to the thick edge.

Upon drawing attention to this, Montgomery outlines several conditions that are necessary for the programmatic success of cultural nodes. These include $^{18}$;

- Diversity of primary and secondary land uses

- Small, medium and large-scale cultural venues

- Presence of an evening economy, including café culture

- Strength of small-firm economy, including creative businesses

- Access to education providers

- Presence of festivals and events

- Availability of workspaces for artists and low-cost cultural producers

- Small-firm economic development

- Managed workspaces for office and studio users

- Location of arts development agencies and companies

- Arts and media training and education

+ Complementary daytime and evening uses

These points direct the implementation of architectural programme towards imperatives of private and public flexibility. Therefore, a cultural node at the Clifton Street Car Park must look to: facilitate multiple events and multiple publics; as well as an infrastructure that can support global and local groups at multiple times - day and night, throughout the year. Based upon these understandings, a successful node requires an examination of cultural events that occur locally and regularly. From the 2012 Wellington events calendar, these events include: 
[Indoor Event]

[Indoor and/or Outdoor Event]

1. ASB Gardens Magic (summer outdoor cinema): 8-27 January

2. Positively Pasifika Festival: 19 January

3. Wellington Cup Carnival: 19-26 January

4. NZ Hertz Sevens Tournament: 1-2 February

5. NZ Fringe Festival: 15 February - 9 March

6. Paniyiri Greek Food Festival; 23 February

7. Chinese New Year Festival: 23-24 February

8. NZ International Arts Festival: 24 February -

18 March

9. Writers and Readers Week: 9-14 March

10. Capital E National Arts Festival: 11-23 March

11. Southeast Asian Night Market: 16 March

12. NZ Dragon Boat Festival: 16-17 March

13. Holi Festival of Colour: 17 March

14. Festa Italiana: 24 March

15. Wellington Fashion Week: 3-7 April

16. Battle of the Bands: 18 April - 15 June

17. Creative Fibre Festival: 25-28 April

18. NZ International Comedy Festival: 26 April

-19 May

19. National Shakespeare Festival: 1 June

20. Wellington Jazz Festival: 6-8 June

21. The Performance Arcade: 8 June - 27 July

22. Wellington Lux Festival: 21-24 June

23. NZ International Film Festival: 27 July - 11 August

24. Beervana: 9-10 August

25. Wellington on a Plate Food Festival: 9-25 August

26. NZ Chocolate Festival: 6 - 8 September

27. Japan Festival of Wellington: 9 September

28. Mexican Food Festival: 12-19 September

29. NZ Improv Festival: 17-21 September

30. NZ World of Wearable Arts: 26-30

September

31. Blossom Festival: 29 September

32. Diwali Festival of Lights: 21 October

33. Wellington Folk Festival: 25-28 October

34. Blow Creative Arts Festival: 10-24 November

Many of these events have overlapping dates and venue requirements, informing a dynamic and ever-changing cultural 'scene' in Wellington (see Figure 8.3). Additionally, as Figure 8.3 reveals, larger and longer events are clustered around January, February and March (summer) due to Wellington's temperamental weather conditions and lack of large,

covered, outdoor spaces.

Although some of these festivals have designated venues, many are disparately located and would benefit from a centralised, flexible location. Of those identified, the New Zealand International Arts Festival is the only event/festival which requires permanent office space - other events are organised independently or through governing bodies such as Creative NZ. ${ }^{19}$ Based upon these understandings, a critical design intervention can test an opportunity for programme to enable a positive inversion to a thick edge condition. Moreover, by providing a facility that is capable of accommodating a number of these events, critical architecture looks to initiate positive economic, social and political solutions - engaging multiple publics in the production of a polycontextual space.

\section{Event Spaces: Performing Arts Facilities}

Of the events identified, many require only portable infrastructure (food markets, etc.), and can be readily superimposed within the Clifton Street Car Park site. Events that require significant infrastructure are primarily performing arts and cinema based. As such, the event spaces will be designed around

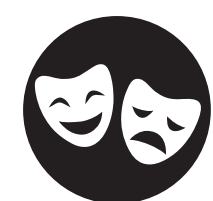

DRAMA
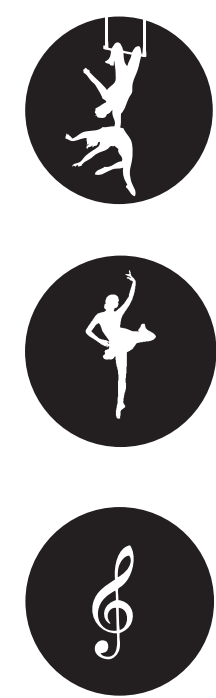

19. CreativeNZ.
$<$ EFT

Figure 8.2: Diagrammatic key of four categories of performance 


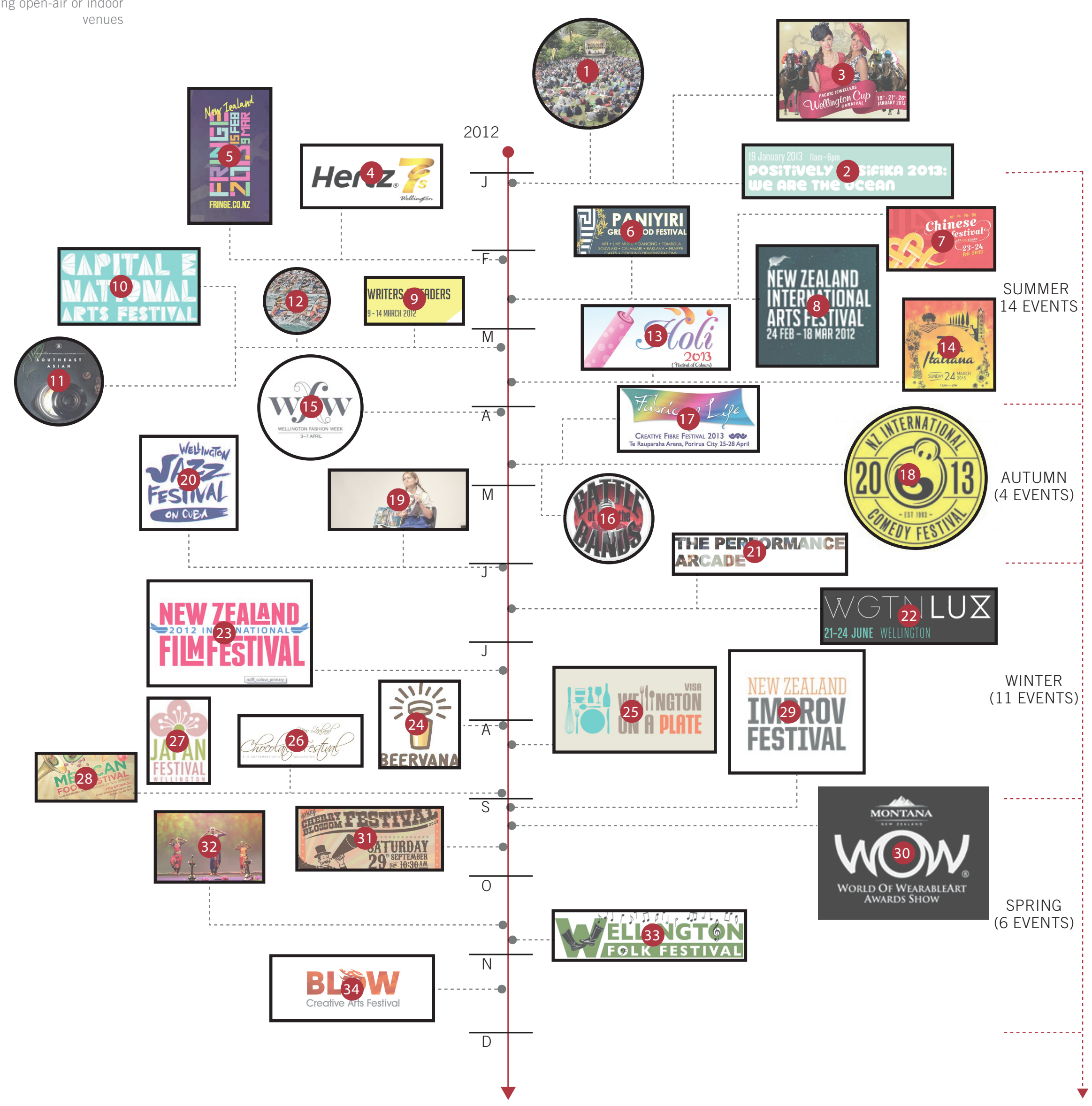


the larger programmatic requirements of a flexible performing arts facility. This requires an analysis of specific requirements for performing arts venues of varying scales.

The performing arts can be generally divided into four distinct categories: drama, entertainment,

20. Strong, 2010, p. 8. dance, and concert (see Figure 8.2). ${ }^{20}$ As performance types vary significantly (from dance, music, opera and theatre, to spoken word, cinema, musical theatre, and circus arts) different events requiring specific spatial and format requirements that may fluctuate in relation to the event/venue size. Venues that encompass these activities can be divided into: 'receiving' facilities, presenting touring shows and require rapid change-over between events; 'producing' facilities, encompassing original productions; and those that do both. To provide maximum opportunity for a variety of publics to engage with the design, the programme brief will provide a series of facilities that are flexible to both produce and/or receive.

Performance venues are designed around three programmatic adjacencies: front-of-house; auditorium (staging and seating); and back-of-house (see Figure 8.4). Traditionally the relationship between each of these components is linear, informing a straightforward separation between performer and spectator (see Figure 8.5). Each of these components has a variety of sub-components which facilitate the various standard functions of the venue (see Figure 8,6). However, the complicated existing physical context of Clifton Street Car Park, and the design intention to construct a variety of spatial practices and social flows, requires a negotiation of these adjacencies - informing how they are dispersed within the context of the site.

\section{Auditorium Typologies}

Regardless of the facility's intentions, the format of the auditorium - the space that encapsulates the relationship between the spectator and the performer - is crucial to the flexibility and experience of any performance. This relationship frames the interaction of spectator and performer, prompting the arrangement of specific front-of-house, staging, and back-of-house functions. Auditoria design follows six formats that are capable of encompassing most performance events (see Figure 8.7). Positioning a performing arts facility within the space of Clifton Street Car Park requires a brief examination of these existing venue typologies:

Proscenium/end-stage: The proscenium stage or 'end-stage' is the most common form of auditoria, prevalent since its emergence as an operatic venue in the seventeenth century. This model is based upon a performance/spectator division by the 'proscenium arch' or 'fourth wall', a threshold between the auditorium and stage that frames and reveals the event. ${ }^{21}$ The audience is positioned end-on with the auditorium to maximise viewing, however, this forms a limiting and passive 2-way relationship between audience and performer(s). The stage is raised, often with an orchestral pit between stage and audience. Performers enter from back-of-house to stage in a linear format.

Thrust Stage: taking cues from the early
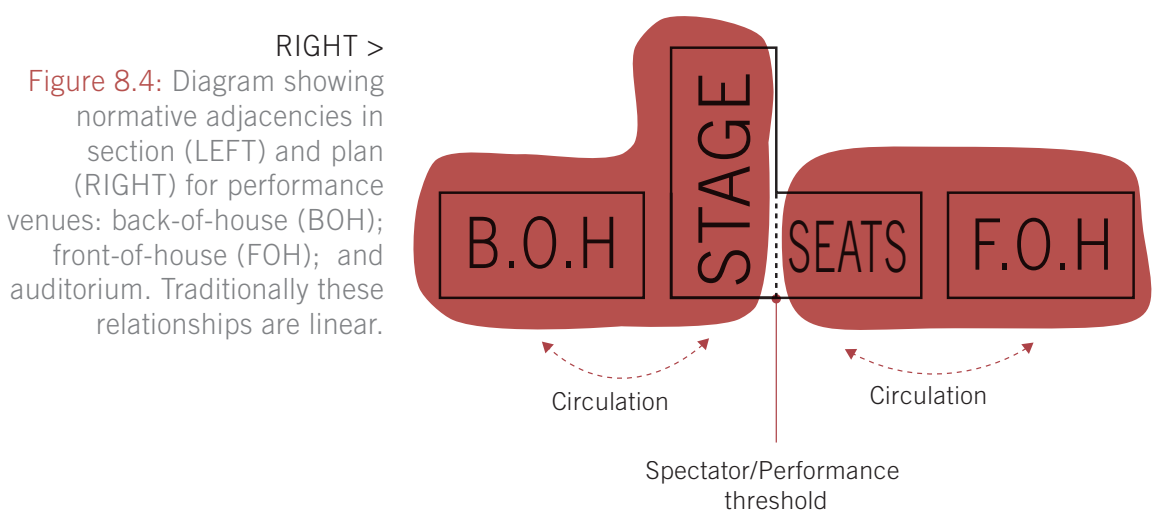

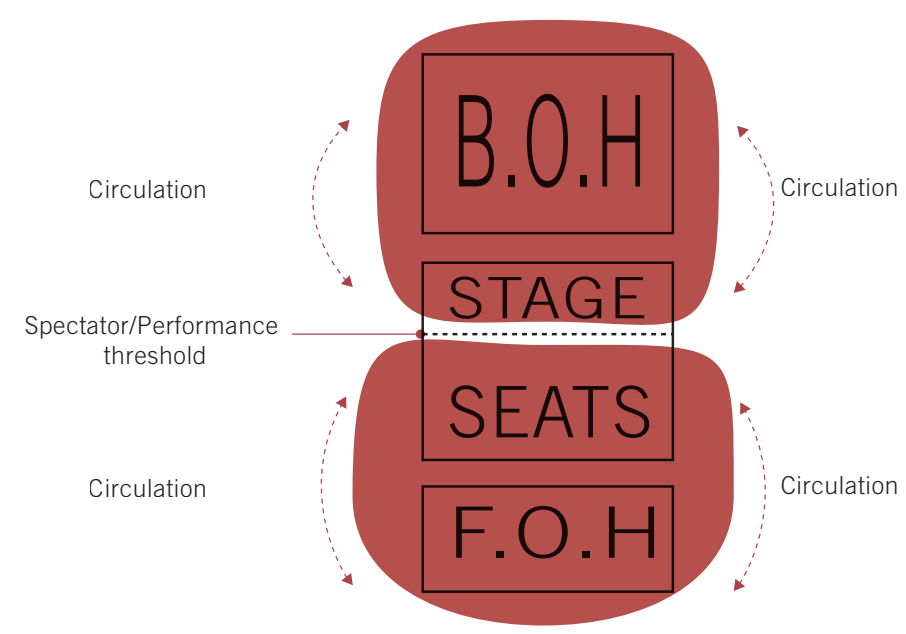



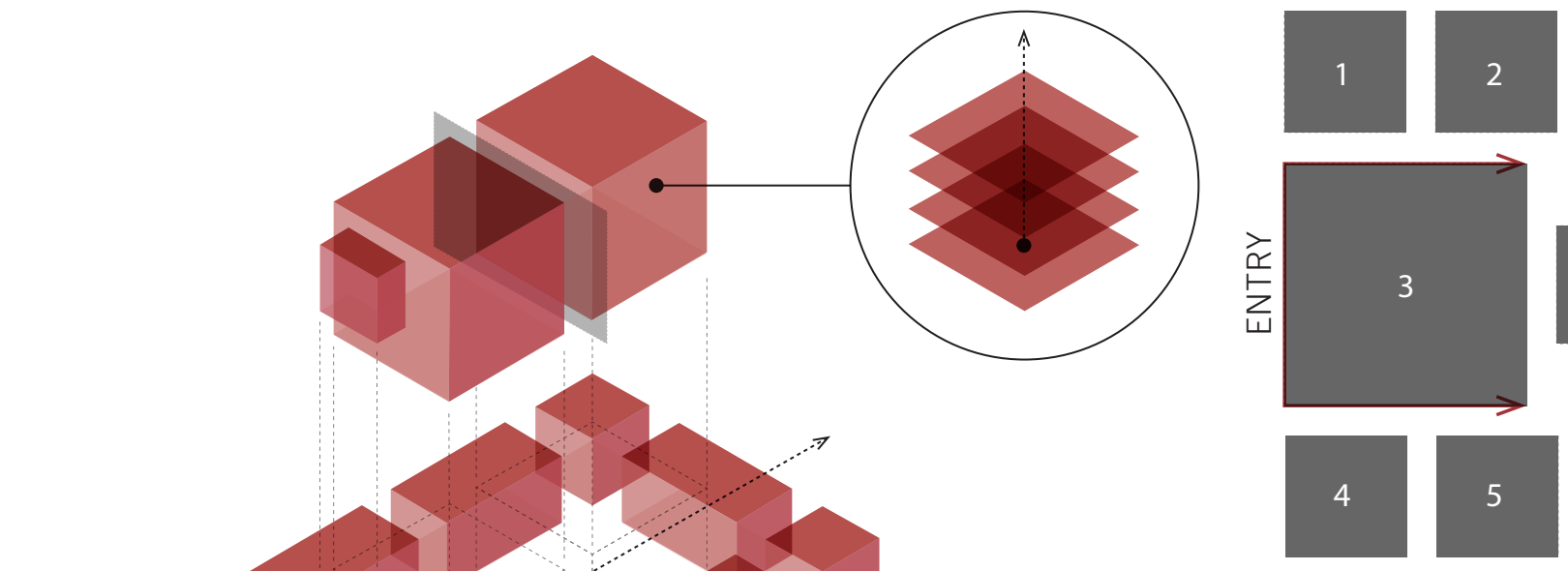

1. Function room(s)

2. Catering, bar/kitchens

3. Foyer/lobby

4. FOH offices and storage

5. Toilets

6. Control room

7. Auditorium

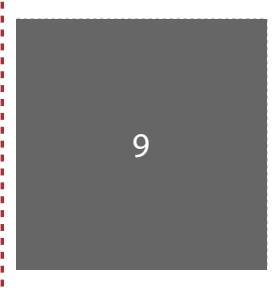

10
9. Stage

10. Administration

11. Production offices

12. BOH dressing rooms, toilets

13. Wardrobe(s)

14. Scene dock/staging

15. Rehearsal room/studio
ABOVE $\wedge$ Figure 8.5: Isometric diagram showing normative adjacencies

in proportionate volume for

a linear performance venue. $\mathrm{BOH}$ activities are often stacked', introducing a vertical adjacency. outdoor Greek amphitheatres, 'thrust' stage venues expand upon the proscenium format, extending the down-stage stage outwards, thus positioning the audience around three sides of the performance. The thrust stage may be backed by an open or closed proscenium arch. Performers enter from the rear of the stage or through the body of the audience. The extended stage invokes a high degree of intimacy between stage and audience, allowing $270^{\circ}$ visual aspect for the performers.

Thru-scenium: thru-scenium staging is a hybrid-format event auditorium. Thru-scenium stages extend down-stage, as well as stage-left and stage-right, into the audience, usually in the form of an arc. Thru-scenium arrangements allow greater performance flexibility to accommodate multiple performance types in one venue.

Traverse/Promenade: this format places the audience adjacent to the stage, informing a linear and parallel performance/spectator relationship. Usually rectilinear, the stage is a 'stretched' thrust, allowing for visibility around three sides of the performance. The audience perceives other spectators as the backdrop, eliminating the need for a traditional end-on proscenium. Performers enter from the rear of the ABOVE $\wedge$ stage, often directly from the back-of-house.

$$
\begin{aligned}
& \text { Figure 8.6: Plan diagram } \\
& \text { showing normative adjacencies } \\
& \text { in proportionate volume for a }
\end{aligned}
$$

Arena: also referred to as 'in-the-round', arena linear performance venue. auditorium space. This constructs an essentially freeform event space as stage direction and proscenium are made redundant. Performers are required to enter through the audience. The intimacy of the relationship between performer and spectator is entirely dependent on the scale of the auditorium.

Black Box: also known as 'flat-floor' or adaptable spaces, black box stages are a variation of the arena format - capable of accommodating a wide variety of indoor small-scale performance types and configurations. They are usually without architectural embellishment and often accompany larger venues as rehearsal spaces. The spectator/performance configuration varies according to the production.

The design case study will look to provide a facility capable of producing/receiving productions that may require any of these typologies. Elucidating the specific requirements for each requires an examination of Wellington's current venues. 


\section{PROSCENIUM/END-STAGE}

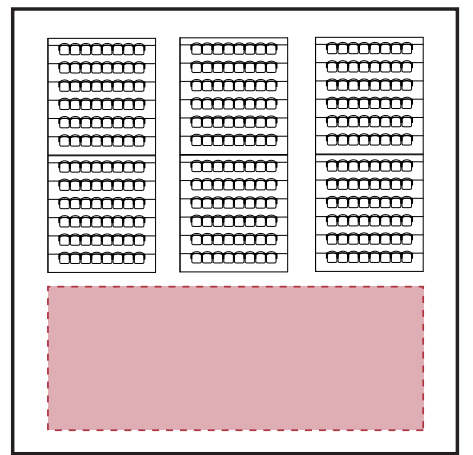

Scale: Any, 50+ seating,

Format: Permanent Stage, 1 option, indoor or outdoor

Staging: Raised stage, usually permanent Down-stage, up-stage, stage left/right

Use: most performance types, theatre, opera, smallscale concert, etc.

\section{THRUST STAGE}

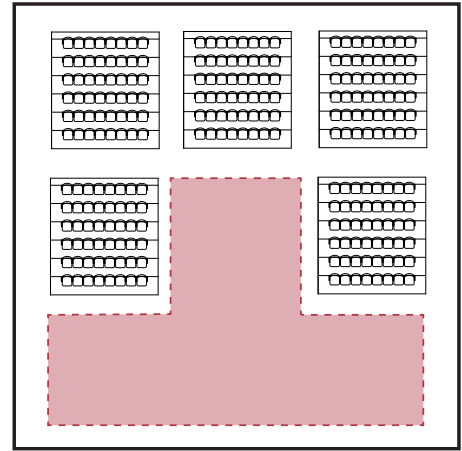

Scale: Any, 50+ seating,

Format: Permanent Stage, 2 options, indoor or outdoor

Staging: Raised stage, usually permanent Down-stage (extended), up-stage (possibly closed), stage left/right

Use: most performance types, theatre, opera, small-scale concert, etc.

\section{ARENA/'IN-THE-ROUND'}

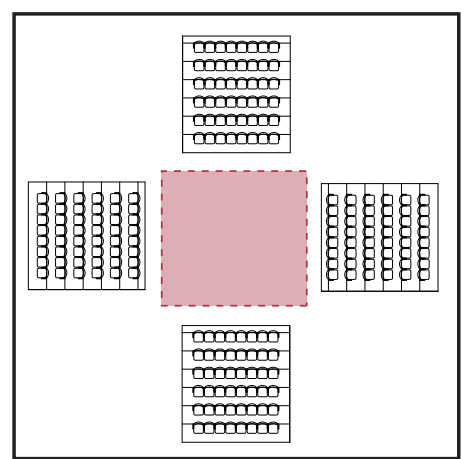

Scale: Any, 50+ seating,

Format: Permanent or temporary Stage, adjacent seating, indoor or outdoor

Staging: centre stage, usually permanent. Only down-stage, up-stage

Use: Runway performance types - concert, fashion, gymnasium, circus, bespoke etc.
Scale: Any, 50+ seating,

Format: Permanent or temporary Stage, multiple seating options, indoor or outdoor

Staging: No discernible stage blocking/directions

Use: Flexible performance types - concert, free-form theatre, bespoke etc.

\section{THRU-SCENIUM}

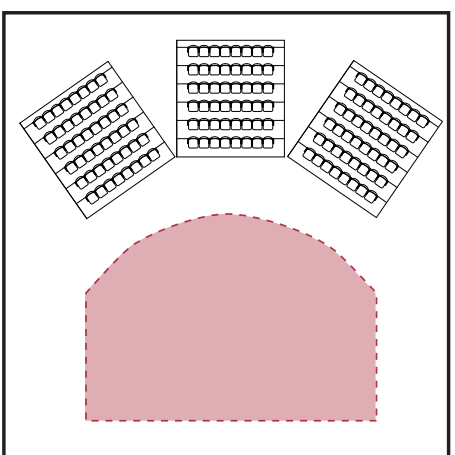

Scale: Any, 50+ seating,

Format: Permanent Stage, 1 option, indoor or outdoor

Staging: Raised stage, usually permanent. Down-stage, up-stage, stage left/right

Use: most performance types, theatre, opera, smallscale concert, etc.

\section{FLAT FLOOR/BLACK BOX}

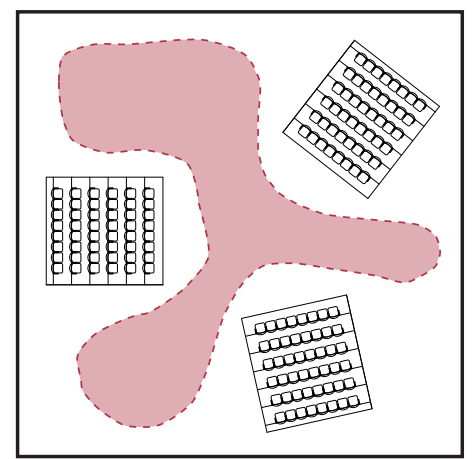

Scale: 50-500 seating

Format: Flexible, indoor

Staging: Flat. No discernible stage blocking/directions

Use: free-form theatre, fringe theatre, open-form 
Three Local Examples

According to the Wellington City Council and the Venue Web Trust, there are 25 spaces in central Wellington that regularly host the performing

22. Council, 2013 arts. ${ }^{22}$ These include (see Figure 8.8):

1. The Botanical Gardens Sound Shell

2. Memorial Theatre

3. Clifton Street Car Park (proposed)

4. Lighthouse Cinema Wigan Street

5. The National Library Theatre

6. City Art Gallery Projection Room

7. Civic Square

8. Wellington Town Hall

9. Michael Fowler Centre

10. Plaza Ballroom

11. Wellington Opera House

12. Gryphon Theatre Ghuznee Street

13. TSB Arena

14. Shed 6

15. Frank Kitts Park

16. Designated Wellington Waterfront Area

17. St James Theatre

18. Reading Cinema (Courtenay Place)

19. CIRCA Theatre

20. Soundings Theatre (Te Papa)

21. Paramount Cinema

22. Hannah's Playhouse

23. The Embassy Theatre

24. BATS Theatre Company

25. Waitangi Park

26. Cuba Street

Mapping the locations of these venues shows that performing arts facilities, much like the events they accommodate, are generally decentralised (see Figure 8.8). Most of these venues can be grouped according to four positional patterns: the Civic Square node; the Cambridge/Kent Terrace node; the Courtenay Place passage; and a curvilinear axis that follows the Waterfront. This also identifies that there is a significant lack of performance facilities within close geographic proximity to the Clifton Street Car Park - the site exists within a vacuum of performancebased cultural activity.

Of Wellington's 25 main existing performance spaces, scale, seating, flexibility and shelter vary significantly. Figure 8.9 demonstrates that these spaces range in seating size from small
$(0-500)$ to medium (500-1500) to large (1500+). Of these 25 spaces: three venues are limited to cinema projection only (Embassy, Readings, and Lighthouse); two venues have been marked as earthquake prone (Paramount Cinema, Wellington Town Hall); and there is currently a total lack of open-air, outdoor performance spaces that are sheltered.

A number of existing venues are small-scale, capable of seating up to 350 persons in a flat-floor, black-box format. However, none of these seat more than 322 people, requiring events with capacity seating allowances of 350-500 people to occupy a medium-sized venue - primarily the St James Theatre, or a partitioned section of the TSB Arena.

Examining the medium-sized venues shows a problematic shortage of 500-2000 seat facilities. Events with seating capacities in this range are restricted to the St James Theatre which is a heritage end-on theatre with limited staging formats. In addition to the St James, these events may occupy: a partitioned section of the TSB Arena; the lower levels of the Michael Fowler Centre (Concert Hall); or the earthquake prone Wellington Town Hall.

Within the large existing venues, there is a sufficient range of auditoria typologies and seating flexibility. Events with seating capacities in this category are generally distributed according to performance type: concerts accommodated by the Michael Fowler Centre; opera and dance accommodated by the Opera House; and entertainment and recreation events accommodated by the TSB Arena. Although the TSB Arena is considered to be a 'flexible' venue with multiple size/ seating arrangements, it is widely acknowledged that it is an acoustically inappropriate venue for small to medium capacities. Alongside Central Wellington's large auditoria, there are several uncovered outdoor performance venues. Events which are specifically designed for open-air performance (large ceiling height, flexible open space) are required to provide temporary canopy/tenting shelter as all of Central Wellington's large outdoor performance spaces are un-sheltered. Additionally, most of these venues do not provide permanent seating of any kind.
RIGHT, OPPOSITE > Figure 8.8: Map of Wellington showing the location of the 25 central city performance spaces

OVERLEAF > Figure 8.9: Diagram of 25 performance venues/ spaces in central Wellington. These venues vary in size, infrastructure and shelter. This diagram evidences that there is a shortage of 500-2000 seat venues 


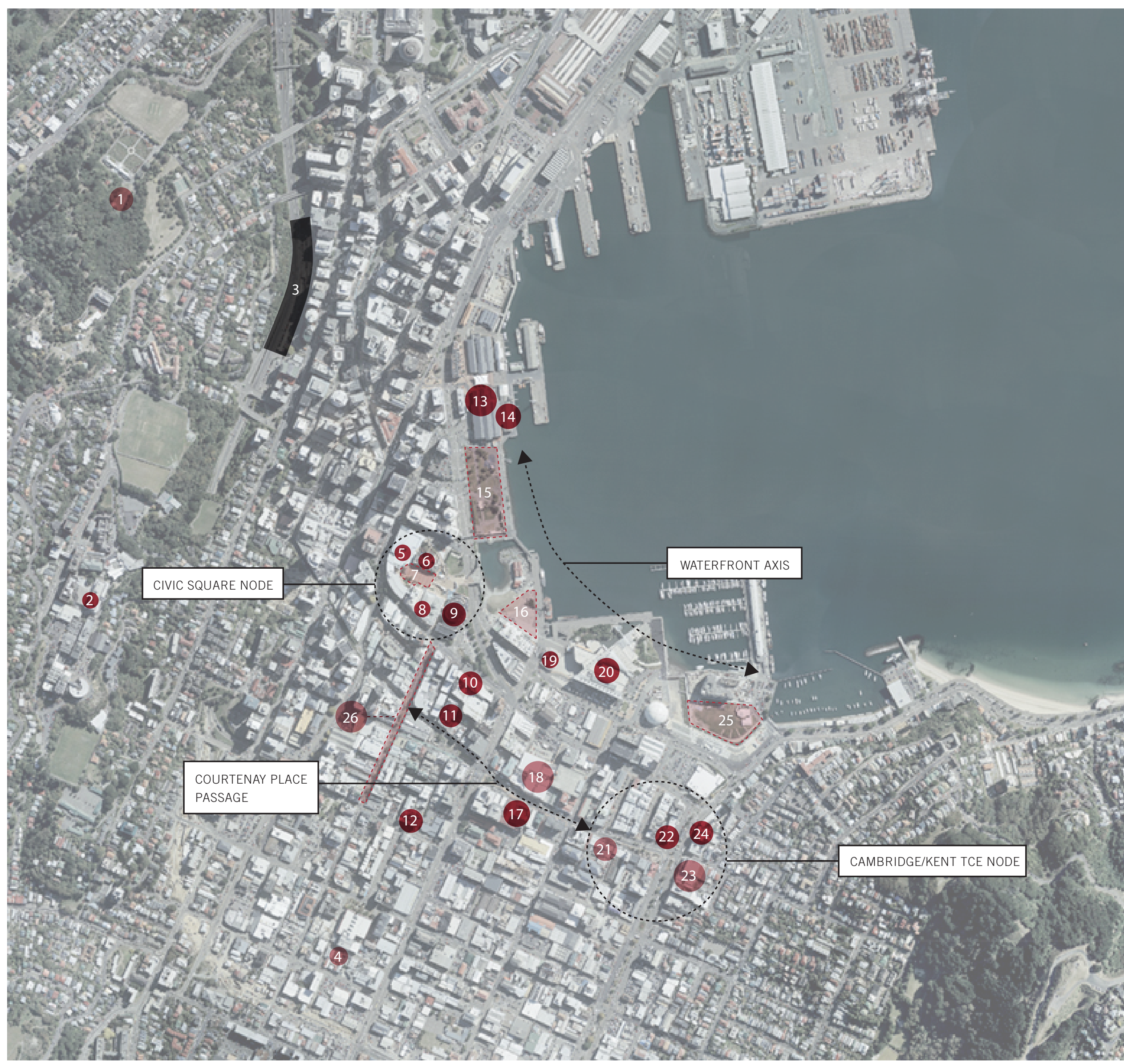

PERFORMANCE VENUES: Local Context

Performing Arts Venues

Cinema Venues

Outdoor Venues

Site/Clifton Car Park 

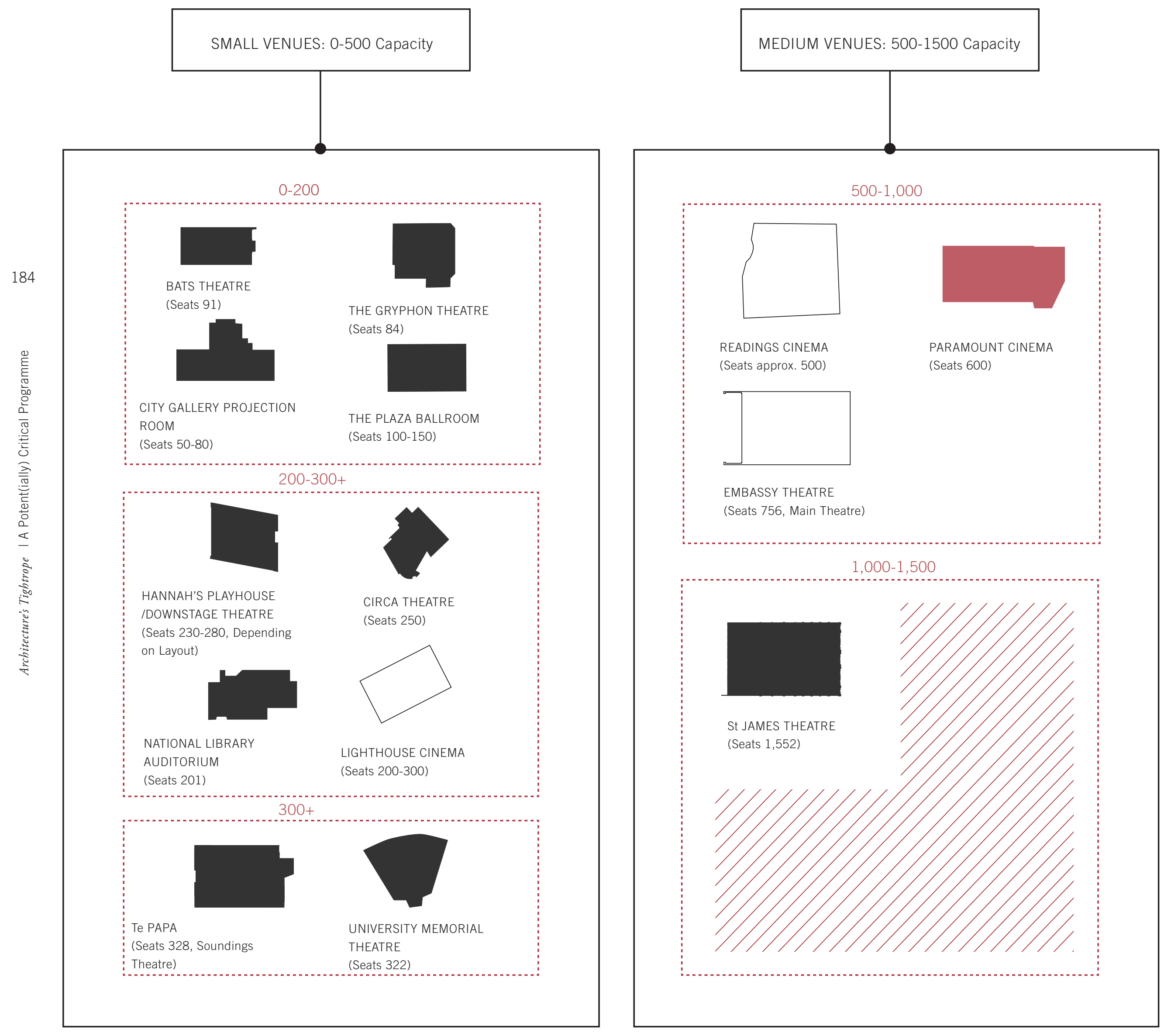

PERFORMANCE VENUES: Local Context

Performing Arts Venues $\bigcirc$ Cinema ONLY Venues

Earthquake-Prone Venues

Outdoor (Greenspace)

Outdoor (Paved) 

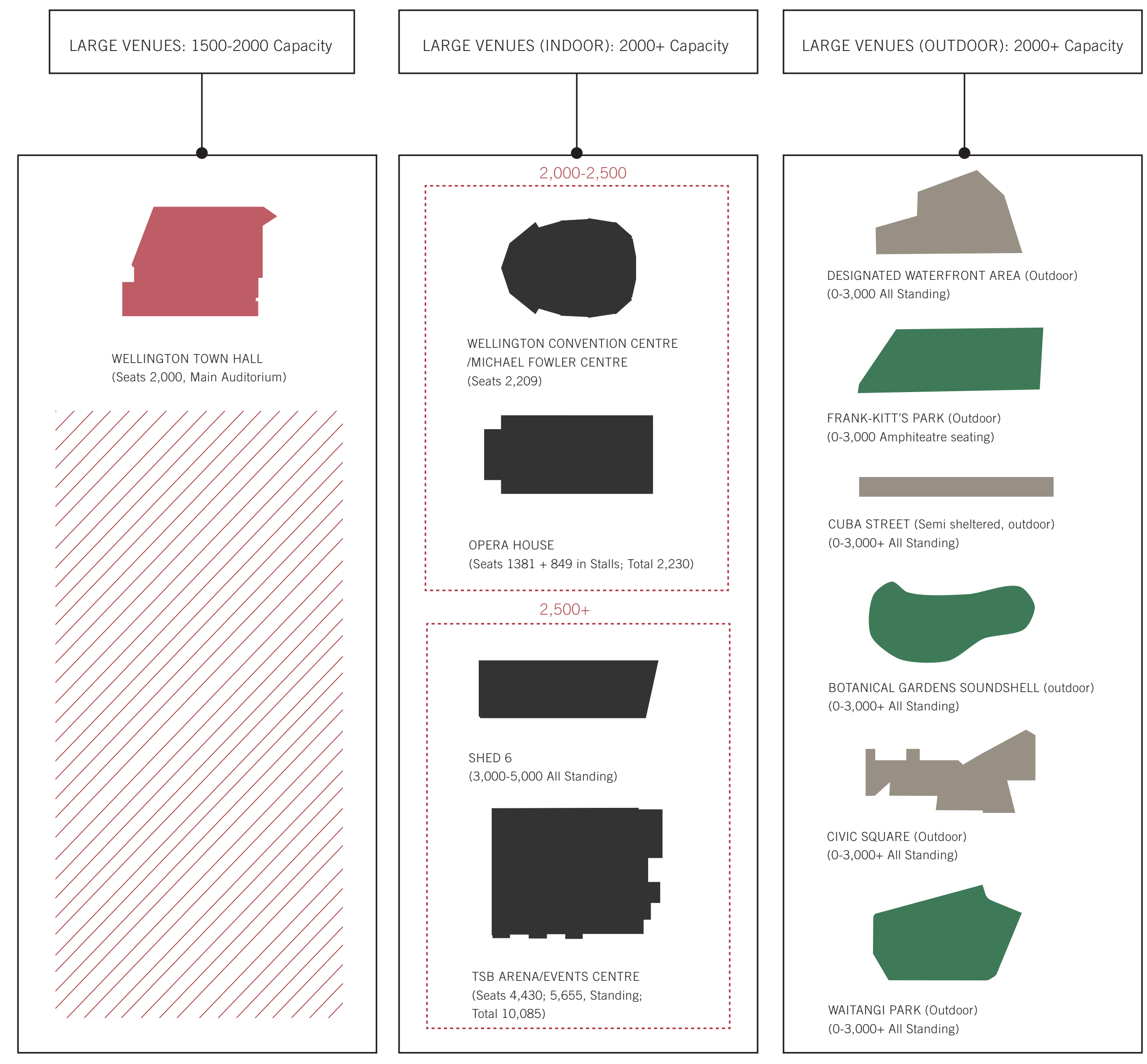
This thesis proposes that there is a clear opportunity in Central Wellington for a multipletypology and scale performance facility at the Clifton Street Car Park to provide:

Small, indoor, flexible black-box theatre space, capable of hosting events with capacity seating of 350-500 persons;

Medium, indoor, venue space with flexible fronton staging formats, capable of hosting events with capacity seating of 500-1500 persons;

Medium, indoor, venue space with flexible sideon (traverse) staging formats, capable of hosting events with capacity seating of 500-1500 persons;

Large, open-air, sheltered outdoor venue space capable of hosting events with capacity standing/ seating of 1500-2000 persons.

Multiple 'event' spaces, overflow areas where publics can perform or spectate.

A flexible range of staging/auditorium typologies to accommodate multiple typologies.

These spaces will be arranged in such a way that several of their adjacencies can be shared, recognising the benefits of implementing an architectural infrastructure for multiple venues in one location. As such, three of the local venues highlighted in this chapter can be analysed as examples that elucidate specific programmatic outlines to partially fulfil these design requirements: the CIRCA theatre; the St James Theatre; and the Wellington Town Hall (see Figure 8.10). Elements will be taken from each of these examples, combined with standard theatre typological requirements, to inform a design that is appropriate to the needs of the Wellington cultural environment.

\section{The CIRCA Theatre}

Located at 1 Taranaki Street, the CIRCA Theatre is one of Wellington's premiere small-scale theatres. Constructed in 1994, the theatre was a reaction to the lack of flexible black box venues in the city. The venue encompasses two stages: a main auditorium, designed to seat approximately 250 people, taking the form of a 'kite' shape; and a secondary studio theatre, that is capable of seating 100 people in a variety of formats and provides rehearsal space.

Custom seating in both theatres allows for flexible arrangements according to the requirements of the performance. Lighting is connected to a movable steel grid that surrounds the stages. Analysing the theatre in plan reveals that the programmatic adjacencies are slightly irregular to the normative linear format as $\mathrm{BOH}$ activity is split and circulation is not linear (see Figure 8.11). Important requirements elucidated from this example include:

$\mathrm{BOH}$ : dressing room space for 5-10 performers; separate parking/loading bay; significant staging area(s); a workshop; storage and a kitchen to facilitate both performers and publics. $\mathrm{FOH}$ : significant gathering/overflow space in the entrance foyer; 6 separate toilet cubicles ( 3 male, 3 female); a bar.

AUDITORIUM: $49 \mathrm{~m}^{2}$ stage (identical for both); pipe grids over stages to accommodate lighting; flexible seating layouts; limited entrance points.

From this exemplar it is clear that a 350-500 seat black box theatre incorporated into the design case study will require larger floor areas for all three adjacencies, maintaining similar area proportions.

\section{The St James Theatre}

The St James Theatre, built in 1912, is recognised as one of Wellington's most valued heritage buildings. Located at 77-87 Courtenay Place, the venue is a traditional proscenium theatre, with linear programme adjacencies. Of the medium-sized venues in Wellington it is the most flexible, seating up to 1552 people and hosting events from all four performance categories.

Given the time of its design, the St James is limited in its production flexibility - providing no permanent lighting, rigging, or sound equipment. The spectator/performer relationship is front-on, divided 


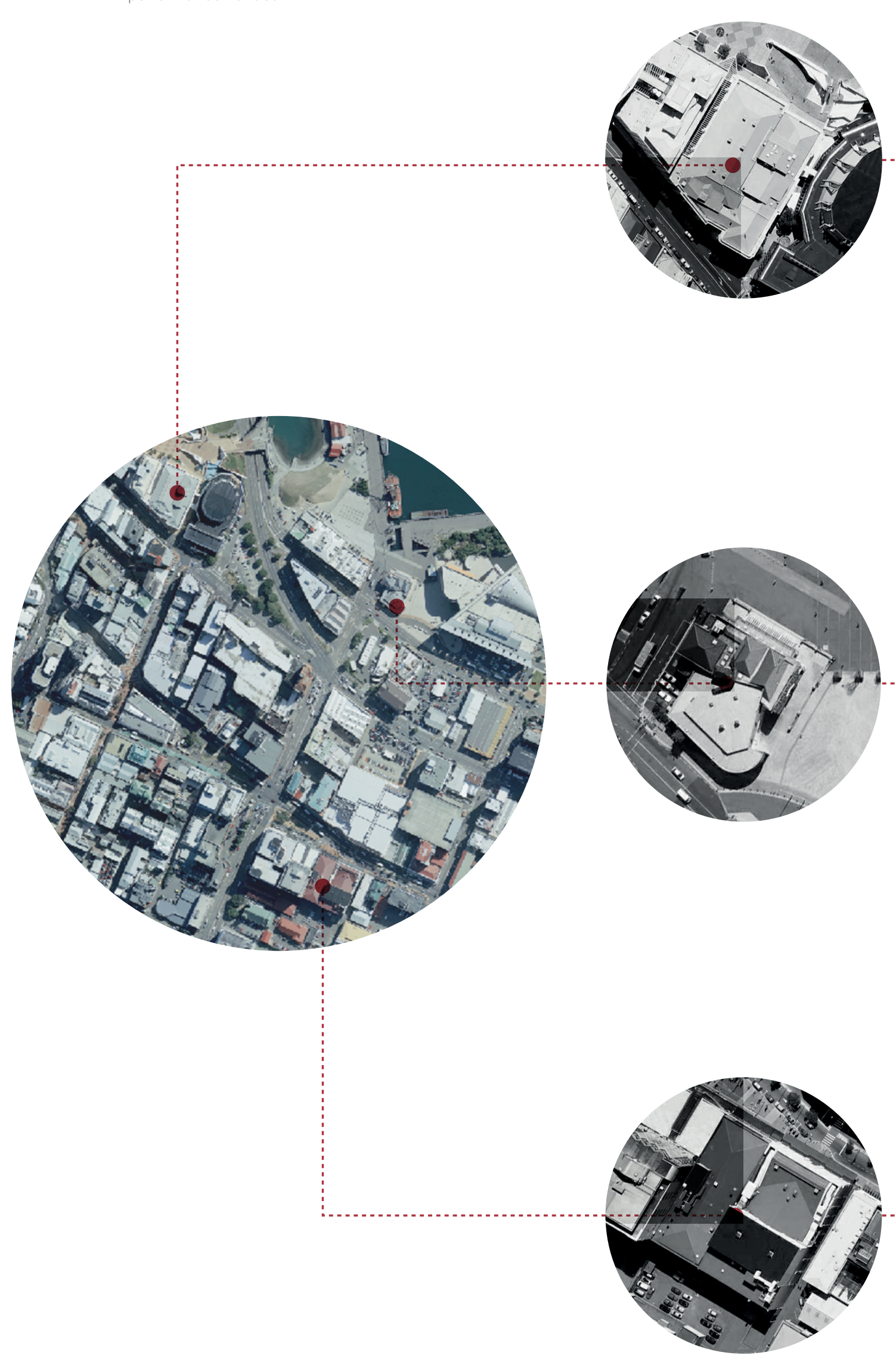

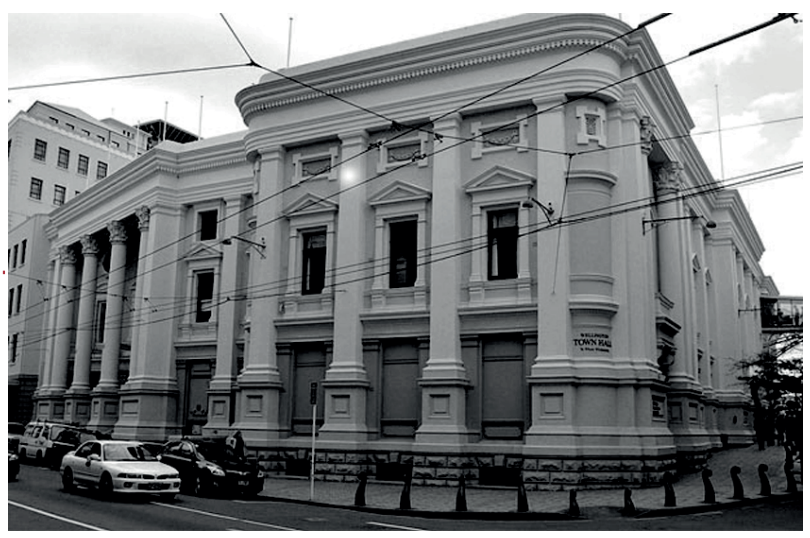

LOCAL EXEMPLAR 1: Wellington Town Hall

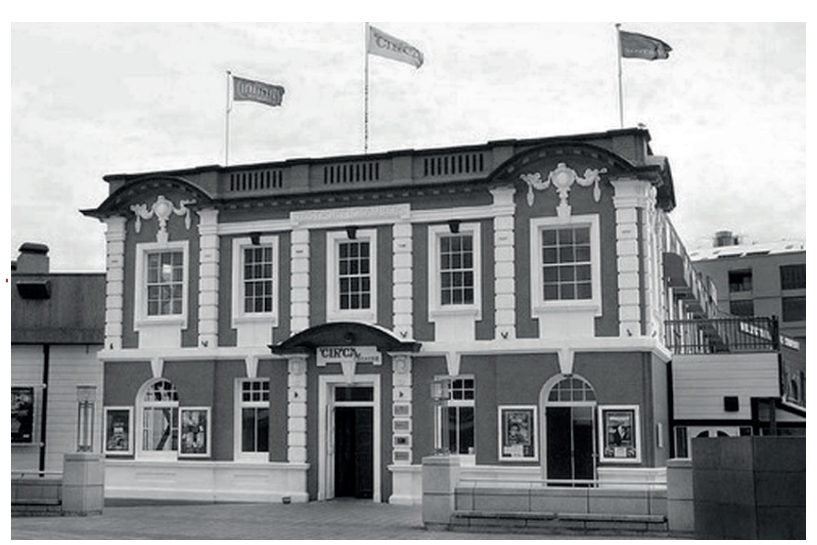

LOCAL EXEMPLAR 2: Circa Theatre

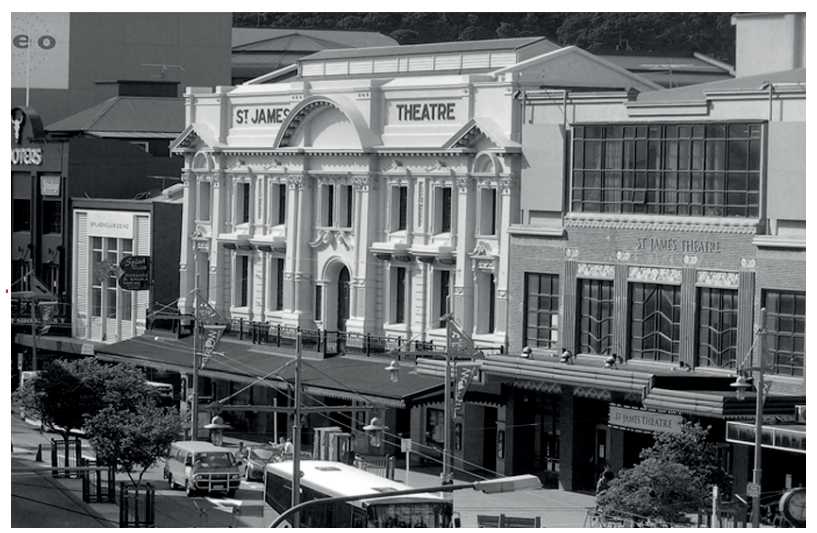

LOCAL EXEMPLAR 3: St James Theatre 
LOCAL EXEMPLAR 1: THE CIRCA THEATRE

Ground Floor Layout Plan (NTS)

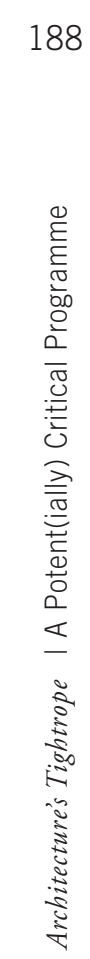


LOCAL EXEMPLAR 2: THE St JAMES THEATRE

Ground Floor Layout Plan (NTS)
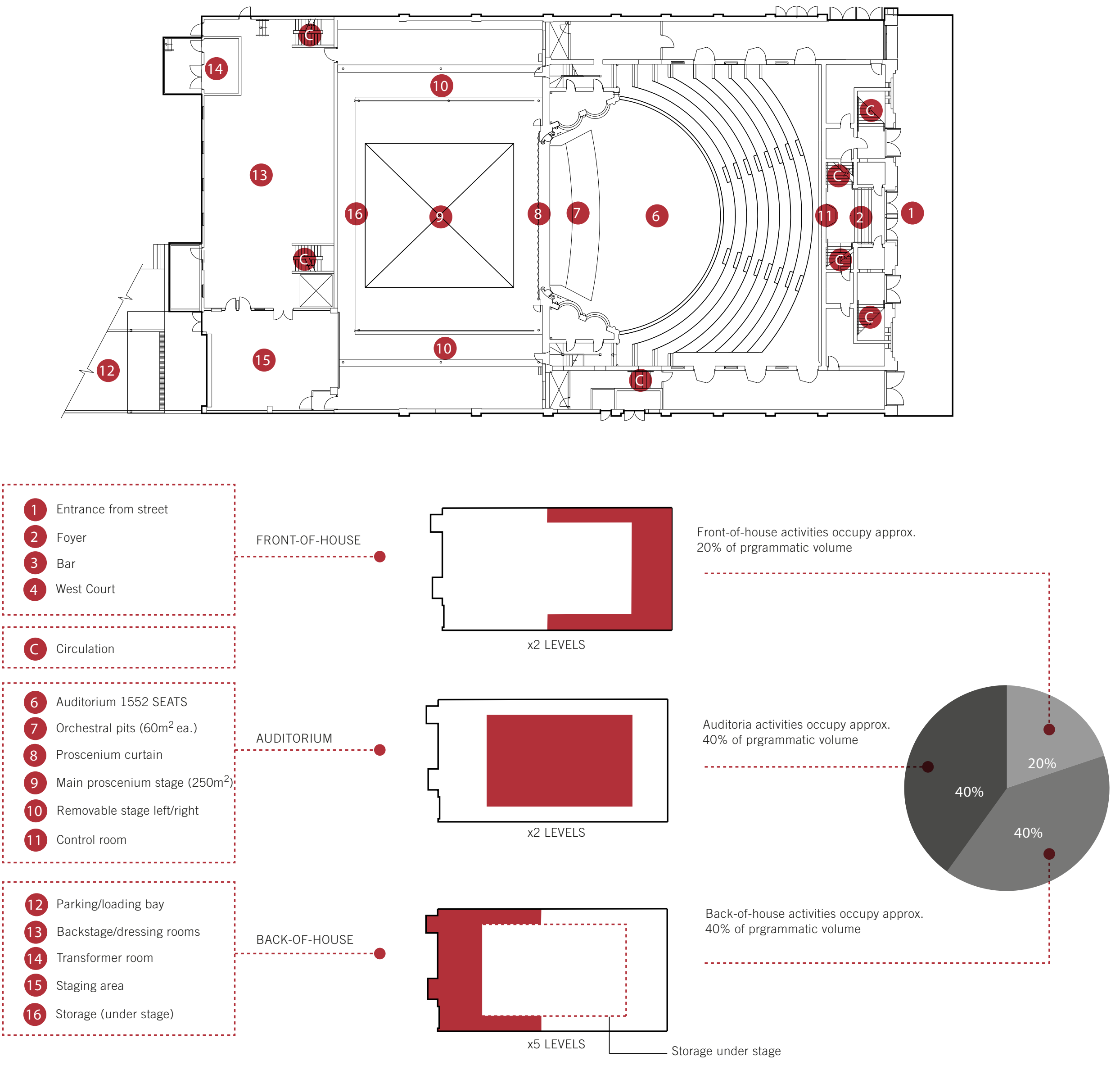


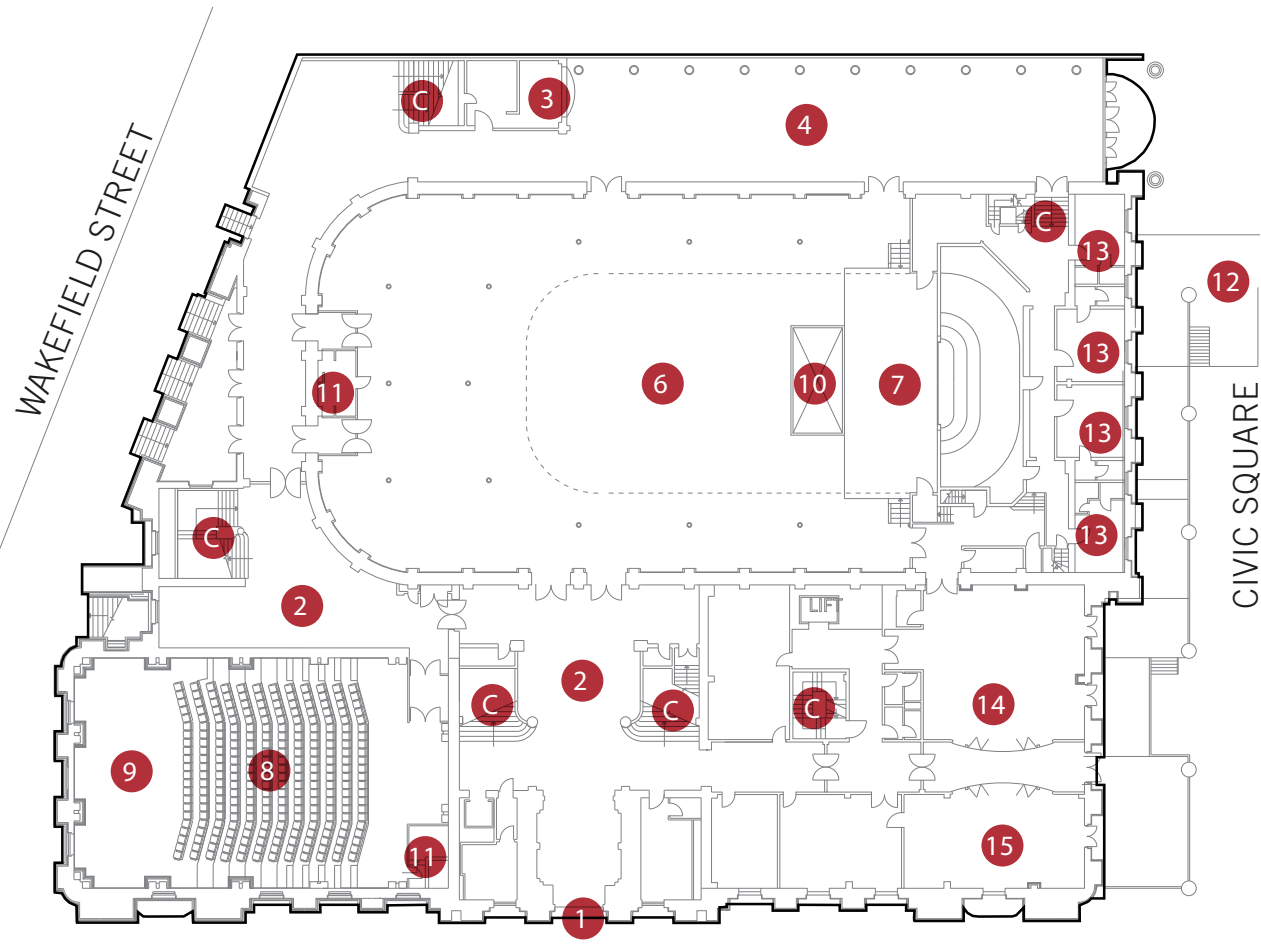

Ground Floor Layout Plan (NTS)

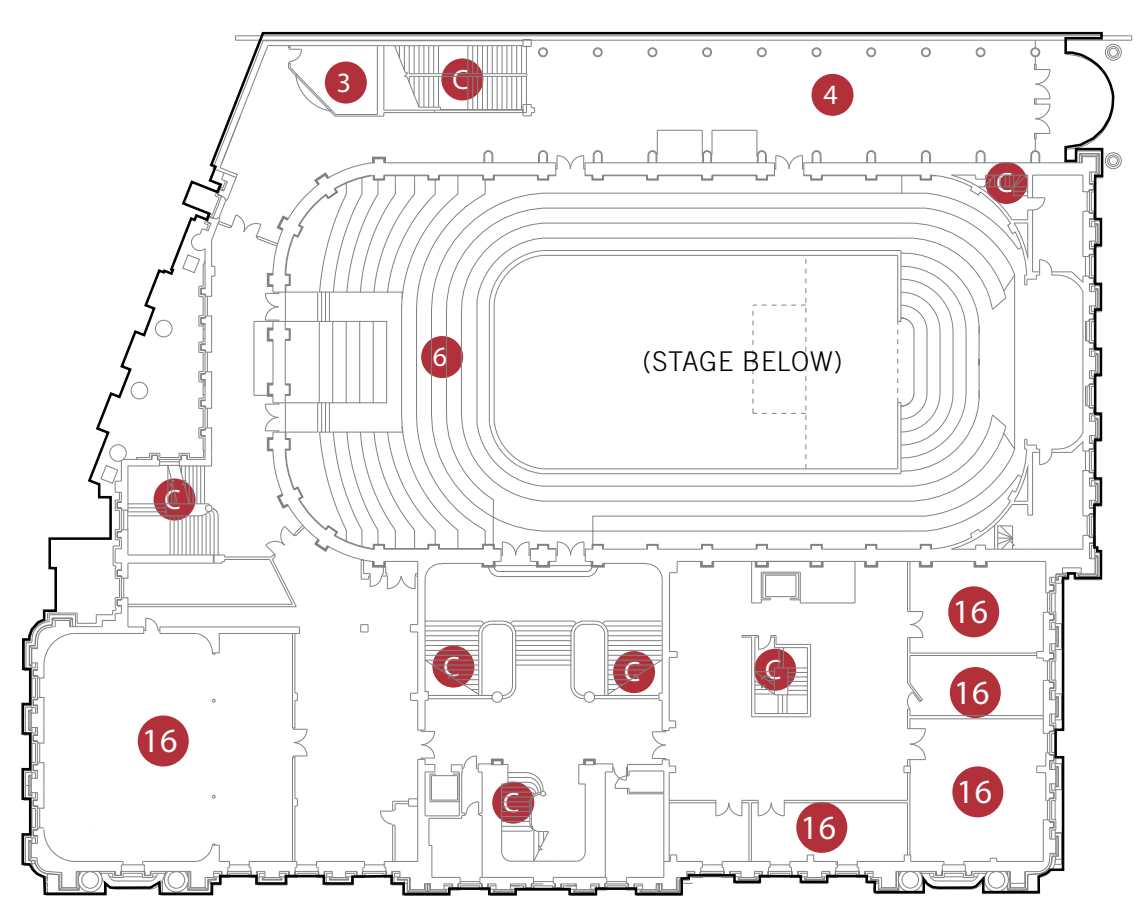

First Floor Layout Plan (NTS)

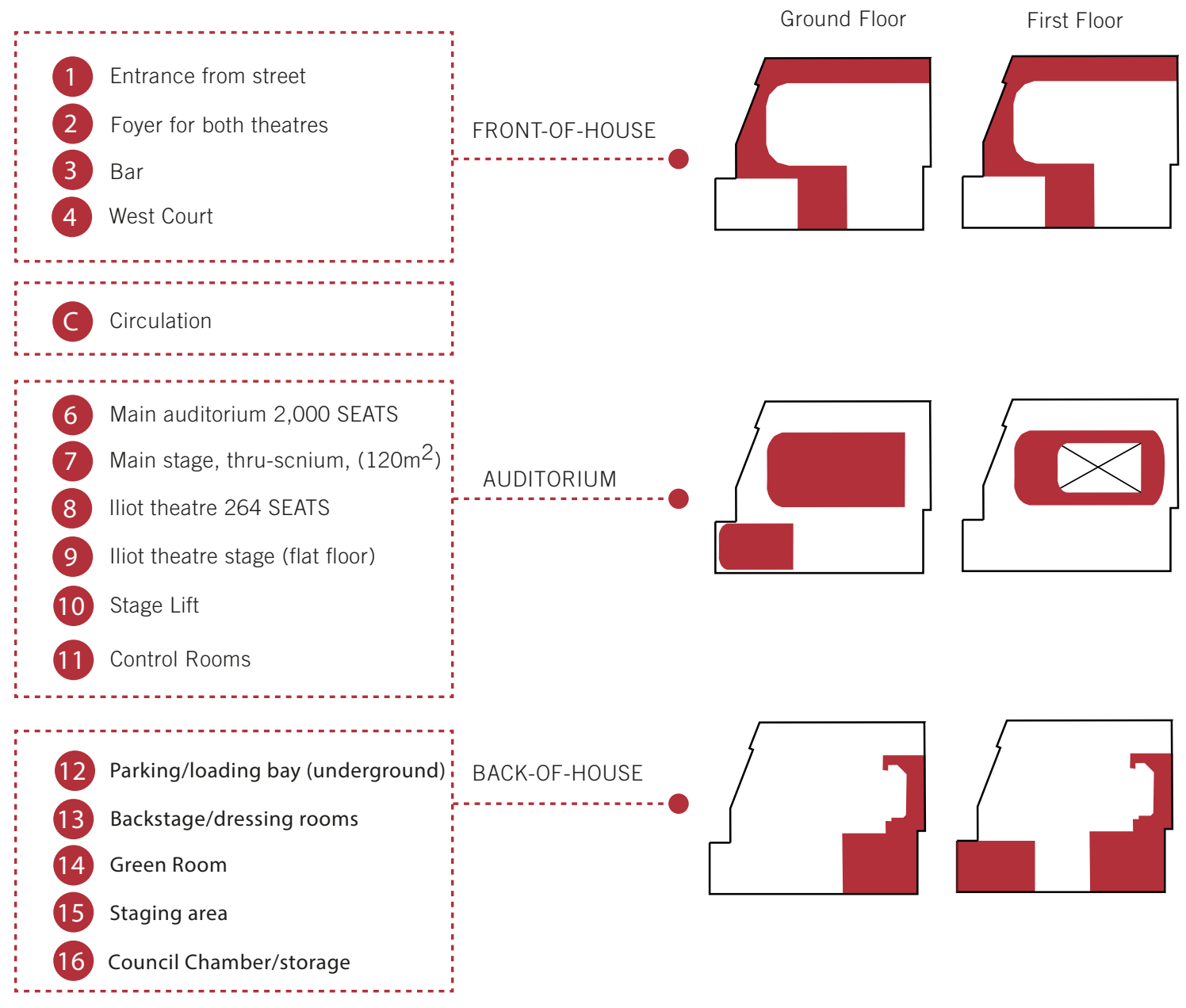

Front-of-house activities occupy approx. $30 \%$ of prgrammatic volume

Auditoria activities occupy approx. $40 \%$ of prgrammatic volume

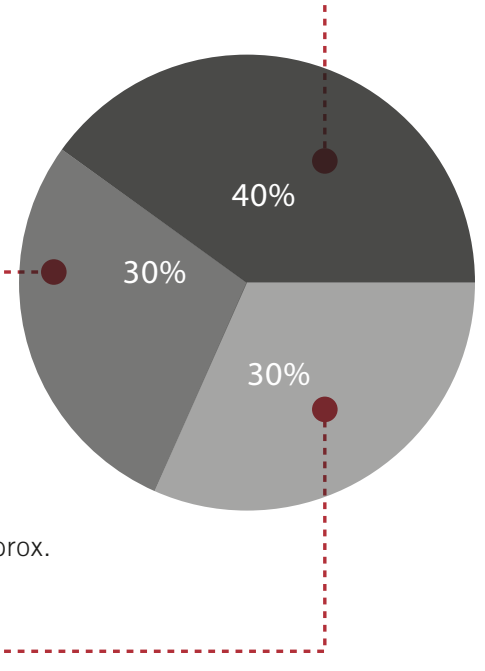

Back-of-house activities occupy approx. $30 \%$ of prgrammatic volume 
by an orchestra pit (see Figure 8.12). Additionally, there is limited space for $\mathrm{FOH}$ activity, only encompassing $20 \%$ of the overall theatre's floor area. Many of the $\mathrm{FOH}$ components are displaced by the theatre's location - Courtenay Place contains a variety of bars, restaurants and public gathering areas. Important requirements elucidated from this example include:

$\mathrm{BOH}$ : dressing room space for up to 50 performers; separate parking/loading bay; significant dressing area(s); storage underneath the stage

FOH: off-street gathering/overflow space in the entrance foyer; 15 separate toilet cubicles ( 5 male 10 female), 5 male urinals; a bar/cafe

AUDITORIUM: $250 \mathrm{~m}^{2}$ stage with adjacent staging wings; above/below backstage-staging area adjacency (circulation via a stair); linear staging/ stage adjacency; permanent seating arrangements (tiered)

From this exemplar several important programmatic factors arise. The medium-sized theatres in the design case study will look to improve on the $\mathrm{St} \mathrm{James}$ Theatre by providing: a flexible, front-on or side-on staging layout; sufficient $\mathrm{FOH}$ gathering spaces; and flexible spectator seating arrangements. Additionally, based upon the St James precedent, the medium sized event spaces can incorporate above/below adjacencies between the staging and backstage areas.

\section{Wellington Convention Centre/Town Hall}

Constructed in 1901 the Wellington Town Hall is a Category One Historical Places Trust Heritage Building. Recently, the Town Hall has been determined unsafe in light of the new earthquake code; from 31/08/2012, the estimated re-strengthening cost was $\$ 30$ million ( $\$ 10$ million more than expected). ${ }^{23}$ The Town Hall is the only example of a performance venue in Wellington that exhibits programme adjacencies and sizes for a specific venue capacity of $1500-2000$ seats.

Unlike the St James Theatre, the Town Hall exhibits a variety of design elements that make it a flexible space for events. Similar to the CIRCA theatre, the Town Hall has two stages (see Figure 8.13). The main stage is primarily front-on in format; however, the ground floor has no permanent seating and is therefore capable of hosting thru-scenium or traverse performances. Depending on layout, the main stage has a capacity of 2000 seats. The secondary stage (the Iliot Theatre) seats a capacity of 264 and is a rigidly front-on proscenium format. Programmatic adjacencies are distributed almost evenly (unlike the St James), as $\mathrm{FOH}$ and $\mathrm{BOH}$ volumes are shared between the two theatres. Important requirements elucidated from this example include:

$\mathrm{BOH}$ (distributed over two floors): dressing rooms; toilets, staging area are dressing room space for up to 70 performers; separate underground parking/loading bay; significant dressing area(s); storage underneath the stage.

$\mathrm{FOH}$ : multiple entrances to the main auditorium; large foyer/gathering/overflow space at the entrance; 20 separate toilet cubicles (4 male, 16 female), 10 male urinals; a bar.

AUDITORIUM: $120 \mathrm{~m}^{2}$ main stage with BELOW adjacent staging wings; multiple above/below/ photograph of 'Anvil House' adjacent backstage-staging areas (circulation via a stairs); linear staging/stage adjacency; permanent (first floor, Iliot theatre) and flexible seating arrangements (ground floor).

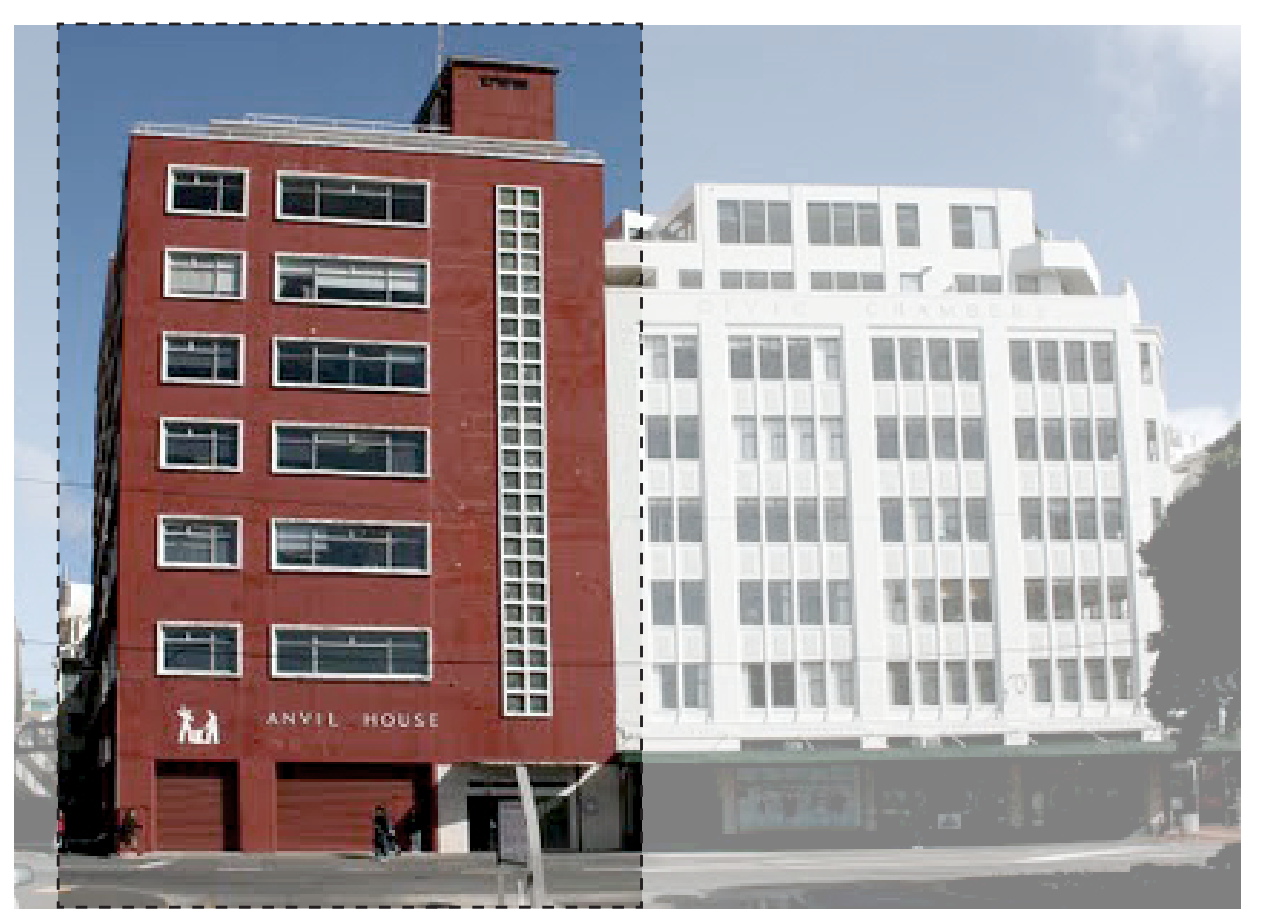


The Wellington Town Hall is a relevant example for the design case study as it implements facilities for two theatres with flexible staging and seating arrangements. Of particular importance are its shared $\mathrm{FOH}$ and $\mathrm{BOH}$ facilities, distributed in a non-linear format throughout the building. However, circulation limitations arise as the building structure is rigid and bounded, preventing publics from flowing between performance spaces with ease.

\section{An Exemplary Client: The NZIAF}

The New Zealand International Arts Festival (NZIAF) is a bi-annual series of cultural events that run from the $24^{\text {th }}$ of February until the $18^{\text {th }}$ of March, showcasing the works of international and local artists in 'outdoor spaces' and 'indoor stages' across Wellington. The 2012 festival was attended by 275,000 people, $30 \%$ of which were tourists, making it one of the city's marquee public events. ${ }^{24}$

Although the festival occurs once every two years for a four-week period, its size and scope require permanent administration staff and offices, located at Level 2, Anvil House, 142 Wakefield Street, Te Aro (see Figure 8.14). Permanent festival staffs (approximately 30-50 persons) occupy one floor of this building year-round. Based upon these figures, the NZIAF permanent staffs require $800-1000 \mathrm{~m}^{2}$ of office space. Additionally, Section 124 of The Building Act 2004 and the Wellington City Council 'List of Earthquake Prone Buildings' (released on 03/05/2013), posits that Anvil House has been marked as unsafe for occupation without strengthening.

Analysing the 2010 event programme reveals that nine venues are currently designated to host the NZIAF programme. The festival contains an itinerary that consists of various forms of performance and arts-based events (see Figure 8.15).

The flexibility of its programme, combined with the need for a new location for its private branch renders the NZIAF an exemplary client for the design case study, introducing a permanent physical, social, and economic infrastructure which can also support a variety of Wellington's other cultural events.

\section{Conclusion: Programme Brief}

As Chapter Six acknowledges, architectural programme is fundamental to the construction of positive socio-spatial design strategies. Building upon this argument, Chapter Eight has focused on how programme may contribute practical dimensions to an inversion of the rigid economic and social contexts of the Clifton Street Car Park thick edge condition. Whilst it is recognised that economic rigidity may manifest in various forms, the purpose of this chapter has been specifically to find a positive critical solution to the car park's current limited singular use.

Highlighting the need for cultural and economic diversity in Wellington's downtown, the 'cultural node' concept is proposed - taking advantage of Wellington's self-proclaimed identity as New Zealand's 'cultural capital'. Multiple events in Wellington's cultural calendar have been identified, drawing forth the conclusion that there is not only a lack of cultural activity within close proximity to the car park, but also, that many of the aforementioned events are currently lacking sufficient facilities to support them. Resisting the normative approach of constructing new event spaces, this thesis proposes that the Clifton Street Car Park is an ideal opportunity to introduce economic vitality to the area and to structure the flexible requirements of an events facility.

Upon elucidating the typological requirements and programmatic adjacencies of standard performance venues, a severe shortage of flexible, 500-1500 capacity venues exists in Wellington. Further analysing the programmatic requirements of such a facility, three local examples of various scale are discussed in detail. Additionally, the requirement for permanent occupation of the site, introducing public/private looseness, resulted in the selection of the New Zealand International Arts Festival as an exemplary client. This client allows the programme to contribute a permanent infrastructure and architectural design within the site - initiating year-round social activity, and engaging 
socio-spatial flows through the car park. Additionally, the programme contributes to a potential political redefinition of the site as a public and private space that is neither 'inner residential' nor confined to the 'central area' commerce.

This design process and analysis informs the core parameters for an events facility to successfully fulfil a local problem. Alongside the design strategies investigated in chapters five, six, and seven, this chapter posits a method for inverting the thick edge as a site of negativity, treating the car park as an opportunity for positive local development. Upon concluding this chapter, a programmatic brief is presented.

\section{Programmatic Footprint(s):}

Office (private)

$800 \mathrm{~m}^{2}$ permanent office space

$400 \mathrm{~m}^{2}$ flexible office space - able to be rented out as office space or artist studios during Arts Festival off-season

5-10 toilets (non-public)

$50 \mathrm{~m}^{2}$ communal space

\section{Auditoria (public)}

Six performance areas: three formal, three informal.

Formal:

Thrust/proscenium/thru-scenium theatre

$$
\text { Seating: 1000-1500 }
$$

Traverse/arena theatre

\section{Seating: 1000-1500}

Black Box/in-the-round theatre

Seating: $350-500$

Informal:

Open-air, covered, flat-floor performance space

$$
\text { Seating: 750-1500 }
$$

Open-air, covered, sound-shell/cinema space

Seating: $750-1500$
Open-air, covered, ad-hoc performance/

event spaces

Seating: flexible, up to 100

Front-of-House (public)

Separate circulation/overflow/gathering spaces for all of the main performance areas

Seating and recreation spaces

Art display areas

Advertising spaces

Café/Bar

Public Bookstore

Reception/coat check/ticket office

Toilets: male 10-15; female $10-15 ; 2$ paraplegic; parent room (s)

Back-of-House (private)

A recital/rehearsal space, able to be hired out to the public

Dressing rooms $\left(5-10 \mathrm{~m}^{2}\right)$ for up to 100 performers

\section{Toilets 5-10}

Storage $100-200 \mathrm{~m}^{2}$

Workshop 100-250 $\mathrm{m}^{2}$

Loading/delivery bay

Plant room

Circulation routes for performers and $\mathrm{BOH}$ staff

Back stage(s)

Staging area(s) for main theatres

\section{Programmatic adjacencies}

Within reason, theatres may share $\mathrm{BOH}$ facilities such as staging/back stage areas.

Loading areas and storage facilities must accommodate delivery equipment as well as circulation routes that allow for movement between theatres. of the 2010 New Zealand International Arts Festival. A events are hosted by 15002000 seat venues significant proportion of the 


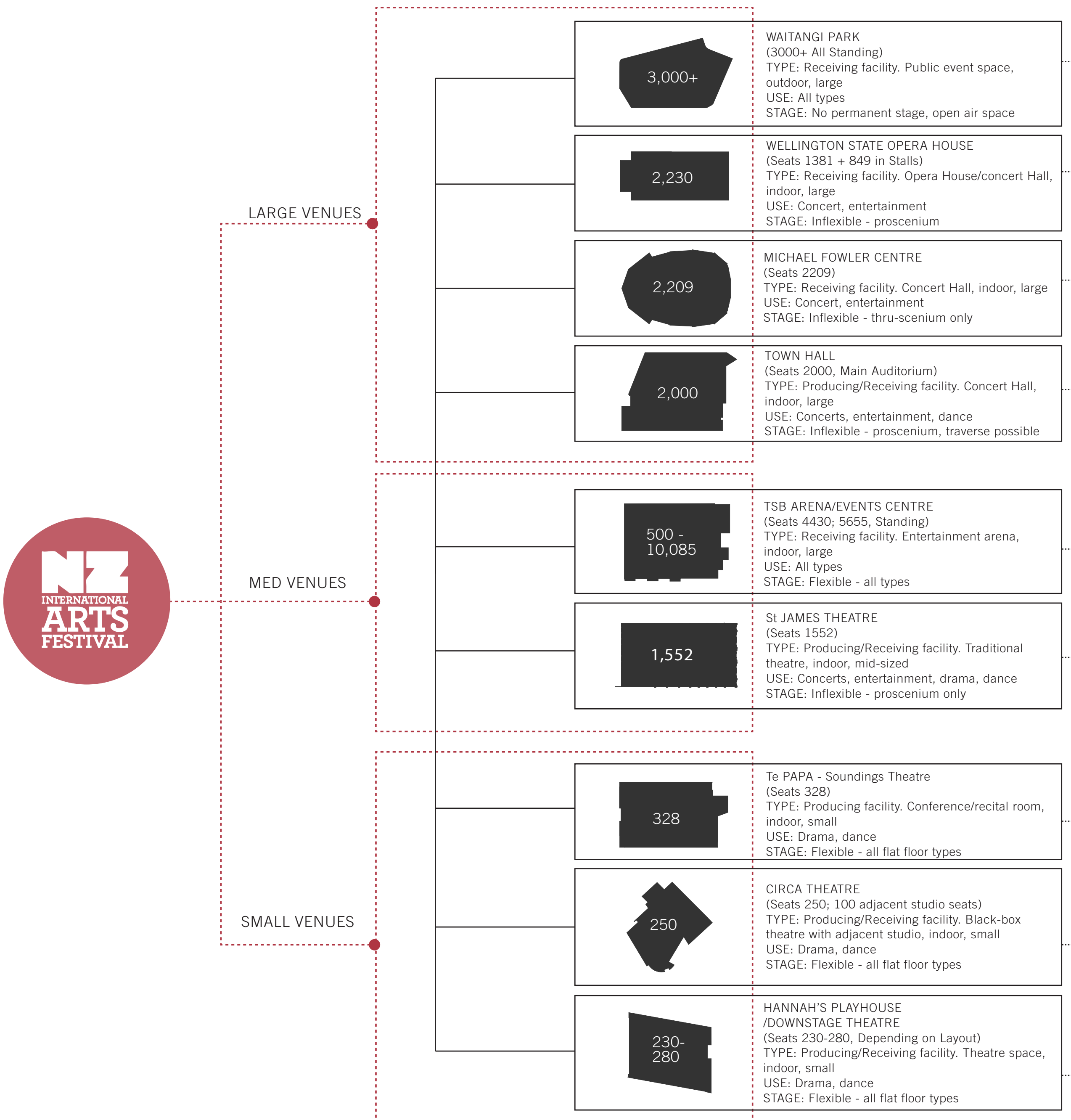




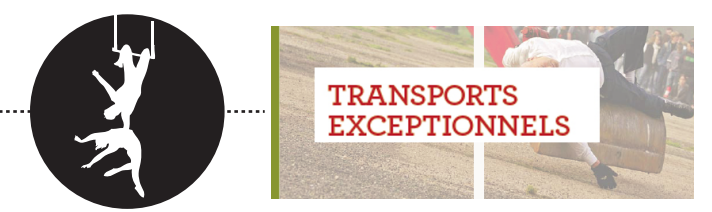

(2) тав валиа (2)

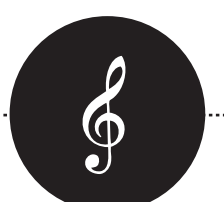

\section{FREIBURG BAROQUE ORCHESTRA}
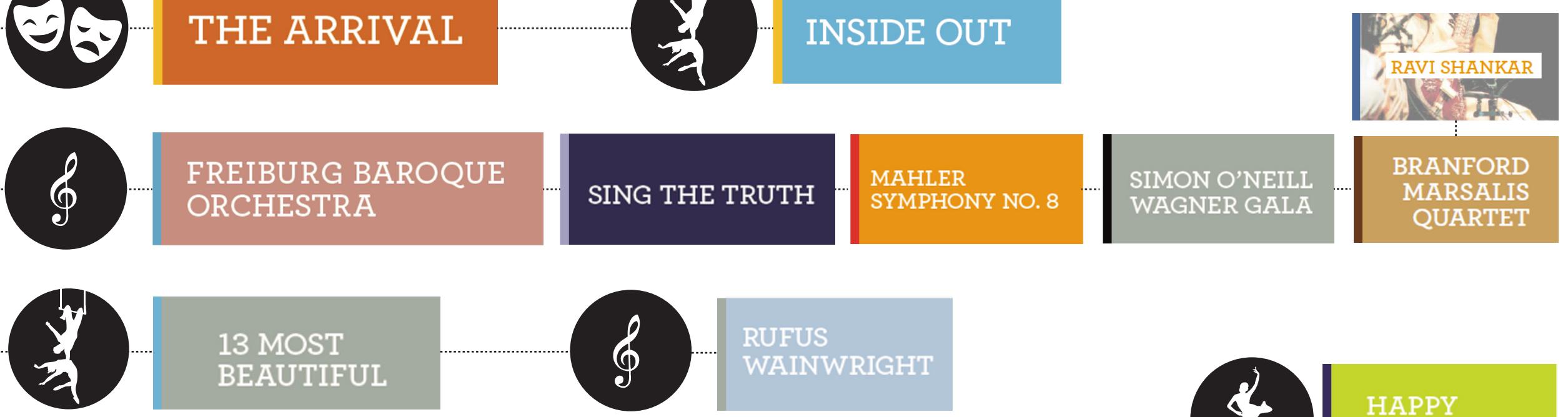

RUFUS

WAINWRIGHT

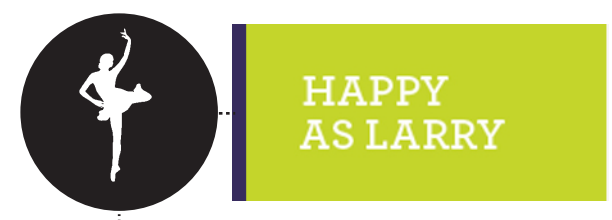

0

\section{SOUND OF SILENCE}

9

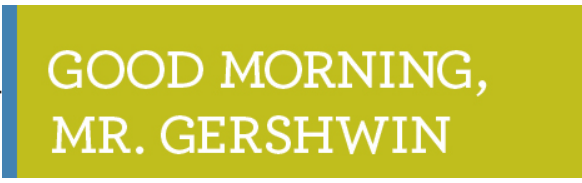

\section{SUTRA}

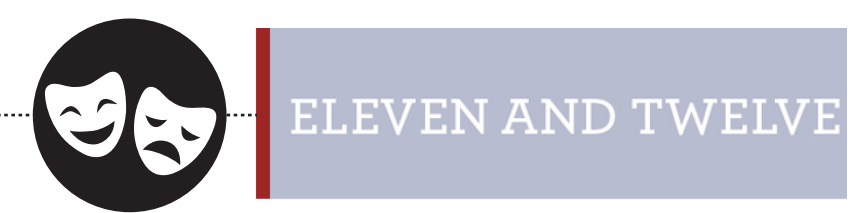

ECHOA

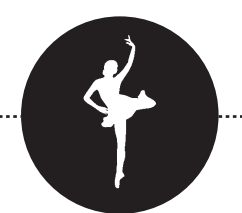

MTYLAND
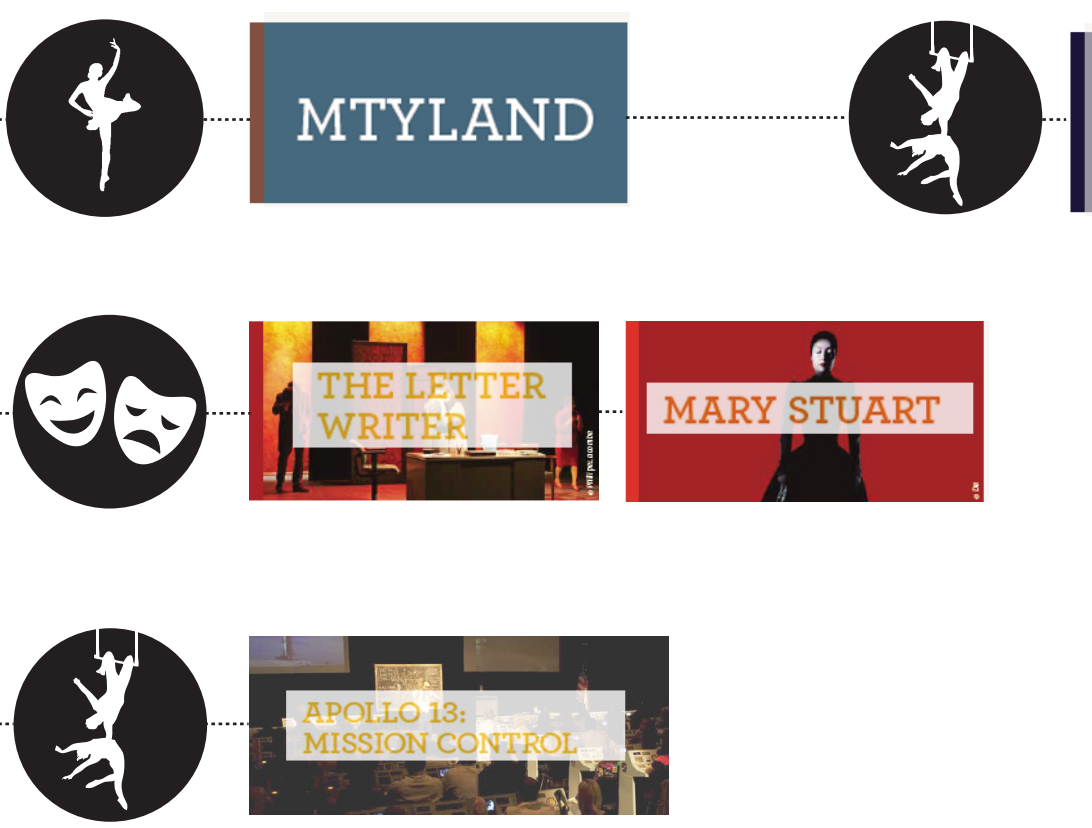


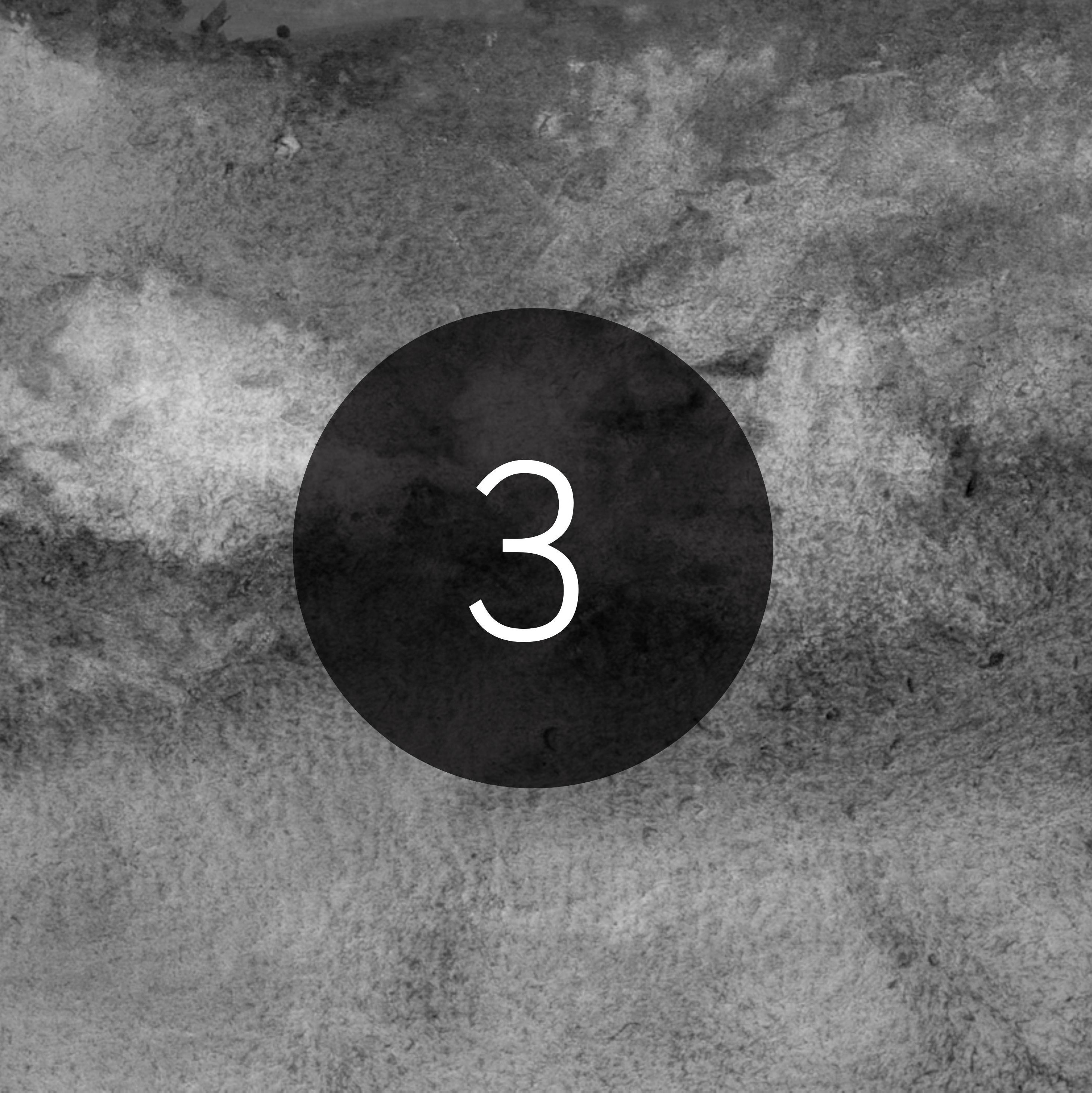


So far in this thesis, Section One investigated a discursive problem, an urban problem, and a site for design-research. Building upon Section One, Section Two developed the framework put forth in Chapter Two, further examining opportunities for critical architecture to engage specific cultural and contextual attributes of the Clifton Street Car Park, as an exemplar of an urban thick edge condition.. These contexts include: historical, economic, political, social, physical and visual.

Additionally in Section Two, through a specific analysis of three key authors - Nick Kaye, Kim Dovey and Gevork Hartoonian - and an investigation into related contemporary architectural case studies, the design-research revealed a number of strategies for critical spatial practices as they relate to Architecture's Tightrope. These strategies focus Rendell's framework further, translating the theoretical and discursive research questions into design approaches for the problem of urban thick edges as an urban social, spatial and cultural barrier condition.

The primary focus of these critical approaches is to invert the thick edge, and to elucidate strategies that can facilitate a polycontextual flow through the site. Upon identifying and analysing these contexts for the design case study, Architecture's Tightrope, is yielded through engagement with formal strategies of:

\section{Continuity}

Discontinuity

Addition
Subtraction

Verticality

Horizontality

Juxtaposition

The strategies listed aim to engender the design with dual intentions - to resist and critique the forces which have contributed to the formation of the thick edge condition; and to investigate / test contemporary applications of critical spatial practices. In doing so, critical spatial practices attempt to dialectically mediate between cultural place-production and formal imperatives, revitalising the original proposition of K. Michael Hays for a critical architecture.

Concluding Section Two, a programmatic imperative for the design has been established - a multi-functional performance facility that accommodates the bi-annual New Zealand International Arts festival, as well as a wide range of other festival activities and public park functions. The broader aims of this programme are: to facilitate a wide range of temporary events that occur throughout Wellington's cultural calendar; to generate a cultural node that economically and politically reconfigures the site into a positive public/private space; and to enhance social permeability through the thick edge. These programmatic priorities add to the structure and constraints of the design case study, introducing requirements for six performance spaces with flexible seating and staging arrangements:

\section{[Formal Staging]}

Thru-scenium 
Traverse

\section{Black Box}

[Informal Staging]

Open-air cinema/projection space

Flat-floor

Ad-hoc

In addition to the performance areas the facility will accommodate various public and private functions, attempting to create a non-deterministic, relational loose space for multiple publics that can operate throughout the day and evenings.

Section Three looks to further engage critical architectural design to test these parameters, developing a hypothetical proposal for the intervention of various architectures, spaces and programmes sited within the Clifton Street Car Park. The production and outcome(s) of this process are situated in Chapter Nine which discusses and presents the overall design case study in relation to pertinent programmatic, contextual, and strategic issues.

Following the presentation of the case study, Architecture's Tightrope critiques strengths and weaknesses that emerged as consequences of the design process. Chapter Ten concludes Section Three and the thesis, reflecting upon the implications of the design-research according to the discursive and urban issues. As Sarah Wigglesworth notes above, the intentions and outcomes of critical practice are frequently misinterpreted. Section Three attempts to create transparency between the aims of critical spatial practices and the outcomes as they manifest in this architectural design thesis.

\section{This section addresses the following} research questions:

Through the testing of critical spatial practices as a design research framework, is it possible to produce positive solutions to the urban thick edge condition? Further, how does this framework challenge the position of critical architecture in the contemporary context? 


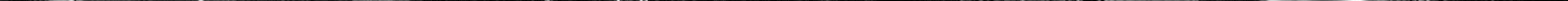




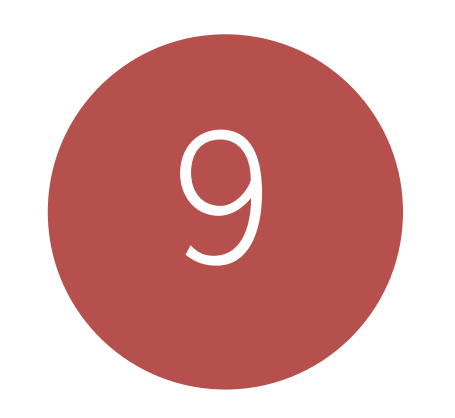

\section{DESIGN CASE STUDY:}

Architecture's Tightrope: A

Multifunctional Performance Facility

for the NZIAF and Multiple Publics

Introduction: Design Descriptions

Programmatic Structuration

Functions

Adjacencies

Circulations

Critical Strategies and Indices

Implementation of Strategies: Ground

Implementation of Strategies: Middle

Implementation of Strategies: Upper

NEXT PAGE > Figure 9.2: Rendered plan of the upper level(s) of the design (NTS). Longitudinally the design can be roughly divided into three parts: fine grain; 


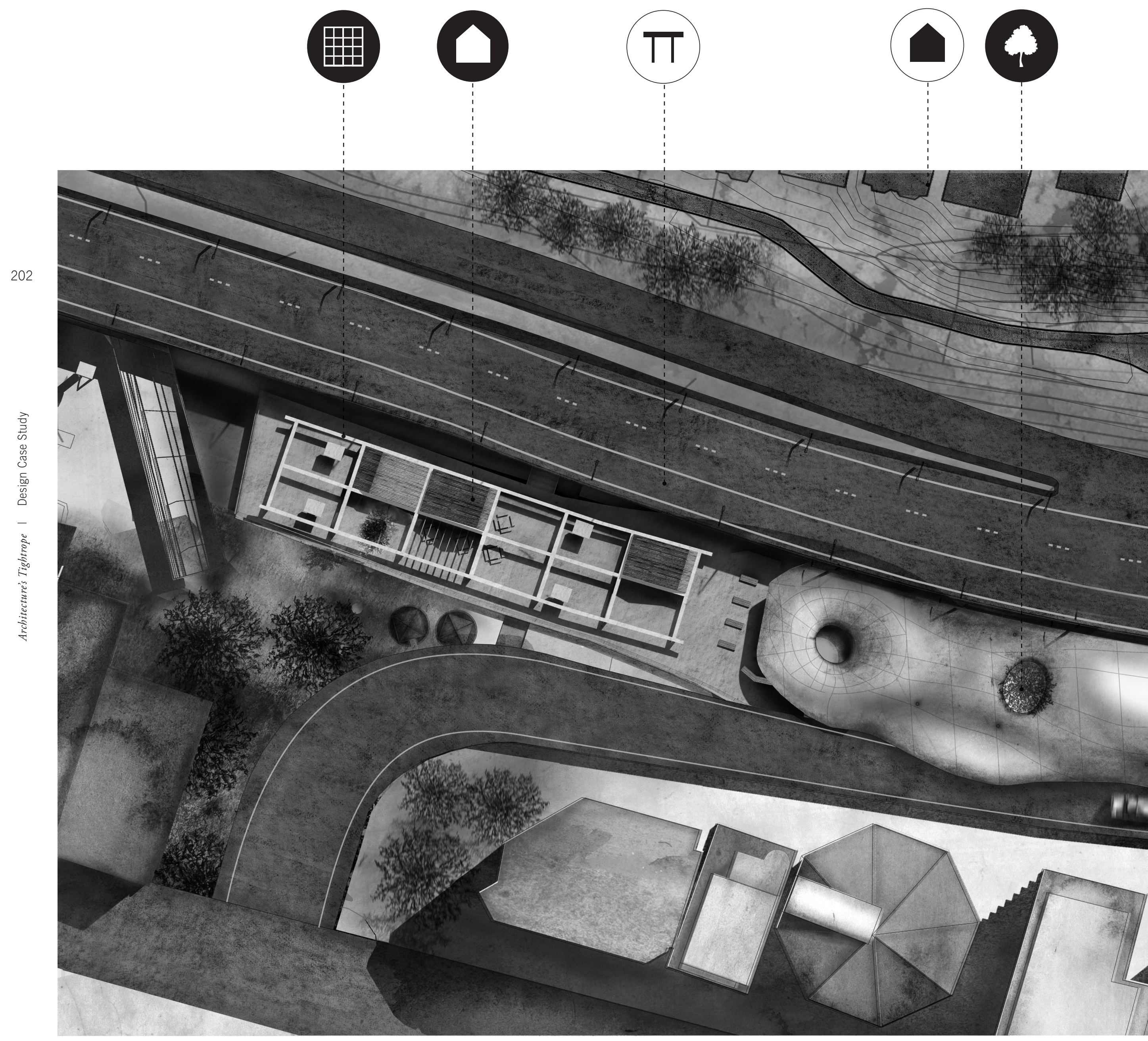



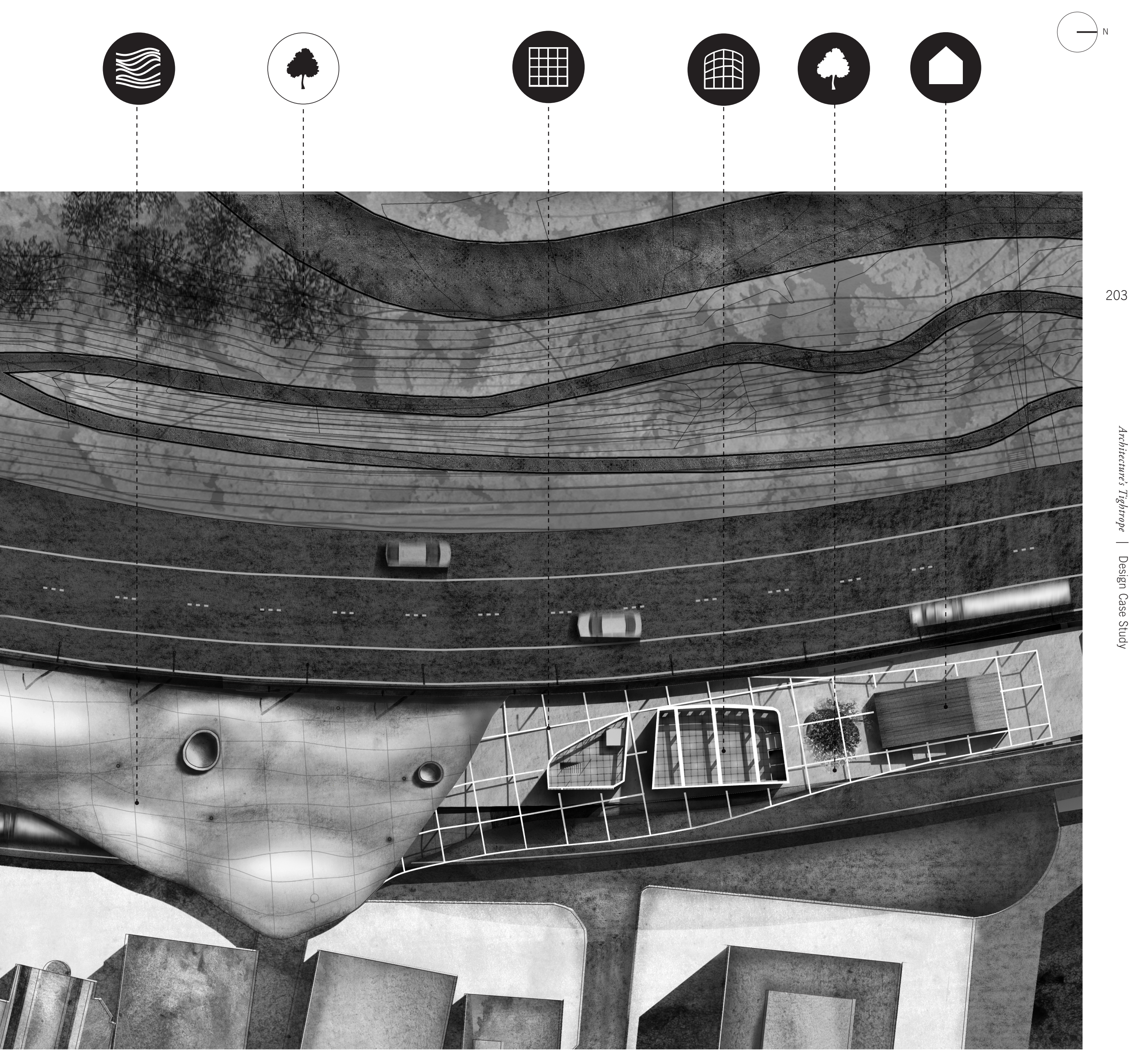


\section{Introduction: Design Descriptions}

Upper Levels / Level Two

Analysing the intervention in plan, as it is exposed to the wider urban context, reveals three distinct longitudinal sectors (sector 1,2 and 3) that negotiate with the existing structures (infrastructure, residential, commercial)(see Figure 9.2, 9.? and 9.?). Formally, the structures inserted in these three sectors are typologically identifiable as: existing rectilinear/ orthogonal; adjusted rectilinear; curvilinear/fluid; residential; and naturally occurring (see Figure 9.3).

The southernmost section, comprised of three small new architectural interventions contained within a timber grid (See Figures 9.6, 9.9 and 9.20), is pedestrian scale - the elements of the design closest to human scale. The rectangular grid isolates this section of the design both visually and physically - providing an overhead structure that frames the open-air public activity space. Depending on the user access point(s) this can either perform as a beginning or an ending to the experience of the facility.

Moving further north towards the site's central section, the framing transition into, but are nonetheless segregated from, the canopy. From above, the canopy takes the form of a fluid mass, tucked underneath the motorway to the west and bleeding out towards The Terrace and over the motorway off-ramp to the east. The continuity of the form is punctured four times (moving from south to north): a depressed funnel that forms a light well to the ground level; a large aperture that exposes a pohutukawa tree; and two vertically extruded cylindrical light wells

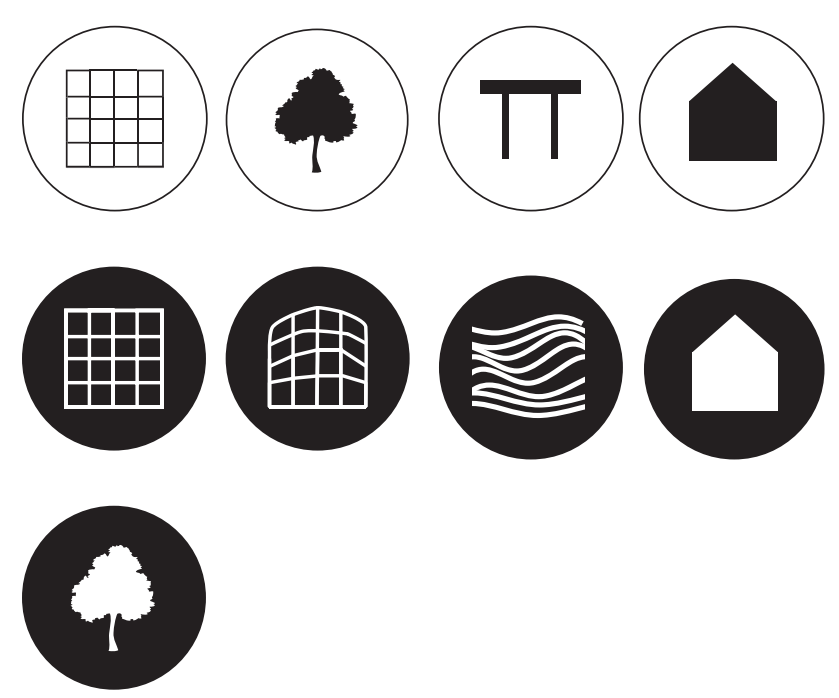

$<$ LEFT

Figure 9.3: Diagrammatic KEY of the formal typologies used throughout the design case study

TOP ROW (from left to right) existing structures/typologies: commercial, natural, infrastructure, residential.

BOTTOM ROW (from left to right) intervention typologies: rectilinear/orthogonal; augmented rectilinear; curvilinear/fluid; residential and natural (implemented)

that extend upwards. Beyond the last punctuation at the northern end, the canopy is 'torn away' and disappears beneath the motorway.

From this point another grid emerges, a white-painted steel frame that is axially rotated to run parallel to the motorway above, aligning with the greenbelt. Unlike the timber loggia, this steel grid (See Figures 9.6 and 9.23-9.25) is square in formation and connects directly to the canopy - suggesting a structural purpose. Moreover, the steel grid is bounded within a curvilinear frame that continues the lines of the canopy's central mass, swelling over the motorway off-ramp and finishing suspended above the top car park datum.

At the northernmost section of the design, there are three separate buildings that are isolated from each other and the steel canopy grid (See Figures 9.6 and 9.24-9.25). The first is a chamfered square in plan, echoing the angular tear of the canopy. This intervention encloses an elevated glazed lookout area that provides a $360^{\circ}$ aspect of the site 


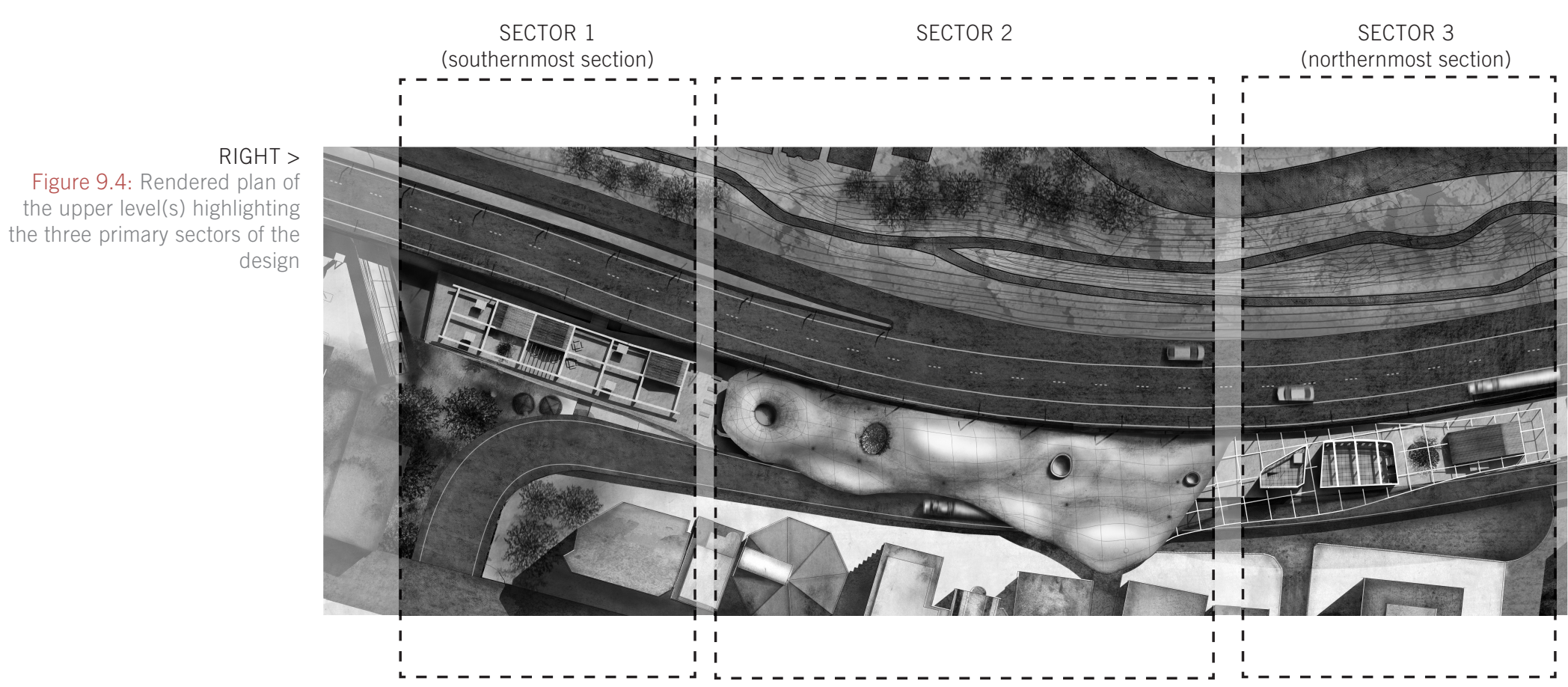

and its visual context. To the right of this is the second intervention of this section of the design, an augmented rectangular plan that is seven storeys in height. These two elements appear as if they were one building, split in plan by a diagonal void that reflects the axis into the site created by Shell Lane. The final design intervention in this sequence, beyond another punctuation revealing a pohutukawa tree (through Datum 2), is a narrower rectangular form with a gabled roof. This volume is significantly lower in height than the previous two, reducing its scale to a similar height to the buildings in the southern section.

\section{Middle, 'Public', Level / Level One}

Continuing in plan, and sectioning vertically downwards through the design, reveals that the canopy and motorway both provide outdoor cover to a variety of spaces - public, private, private-public, and performance (see Figures 9.5, 9.6 and 9.7). The structures appear 'loosely' positioned along the site; this approach establishes a variety of scales of public space(s) positioned according to functions, adjacencies and circulations. This strategically provides adequate of additional spatial typologies

From left to right: private; private-public: public; performance breakout lobby space for performances with very

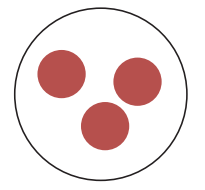

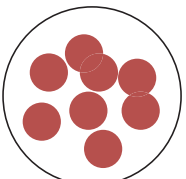

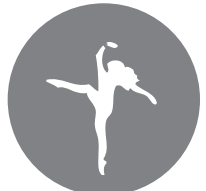

large audiences, while simultaneously providing smaller scale breakout areas for smaller audiences. This assortment of integrated covered outdoor public spaces not only mitigate the scale transition between The Terrace and Thorndon, but they also serve as public 'park' gathering spaces when performances are not in session throughout the day. As the new intervention is primarily a performance facility, much of the added built structure is orientated around the locations of the theatre spaces and the relationship between performer and spectator. The middle level contains two formal theatres - thru-scneium and traverse (see Figures 9.49 and 9.50) - held within the existing open volume underneath the Urban Motorway; and informal ad-hoc performance areas surrounded by public seating (see Figure 9.6).

Analysing Figure 9.7 from left to right (south to north), the design is anchored around a series of public/private/private-public and performance/ spectator relationships. As such, this level uncovers the human scale of the design, and presents Architecture's Tightrope as a primarily sociallyorientated facility. Punctuations in the canopy above reveal an alignment with spaces at this level - open areas which are readily accessible to the public and move along the datum as it swells outwards to accommodate the new intervention. Moreover, the formal languages developed at the urban level above find repetition in this central section - a combination of rectilinear, augmented rectilinear, and curvilinear forms are scattered across the car park's middle
NEXT PAGE > Figure 9.7: Rendered plan of the middle level of the design (NTS). At this level the design orientated around structuring fluid relationships between spectators, publics and/or performers

Datum 3 is the ground level at the top of the image, with Datum 1 below it 


\section{0:8 \\ (1)}

(2):8

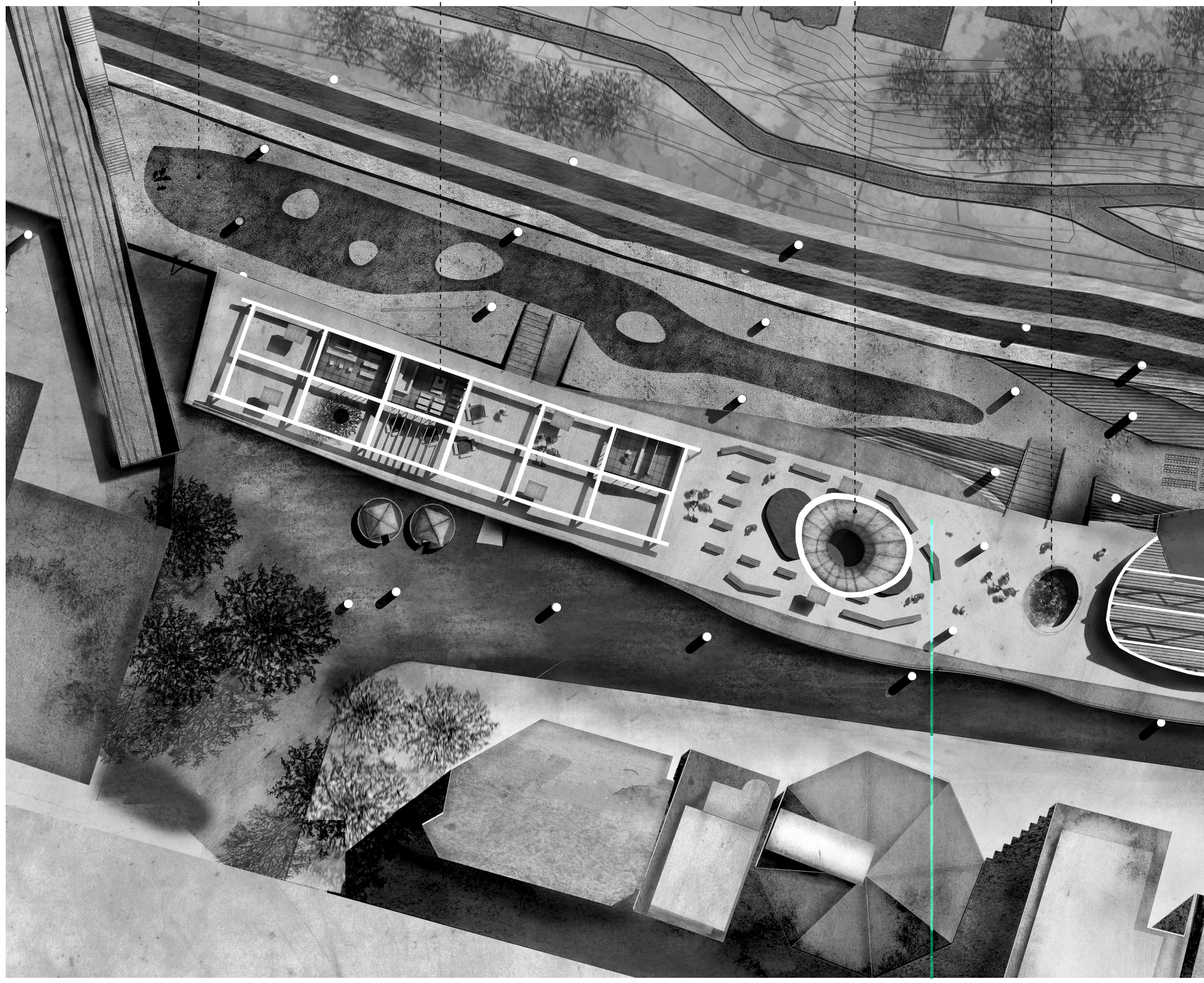


(웅

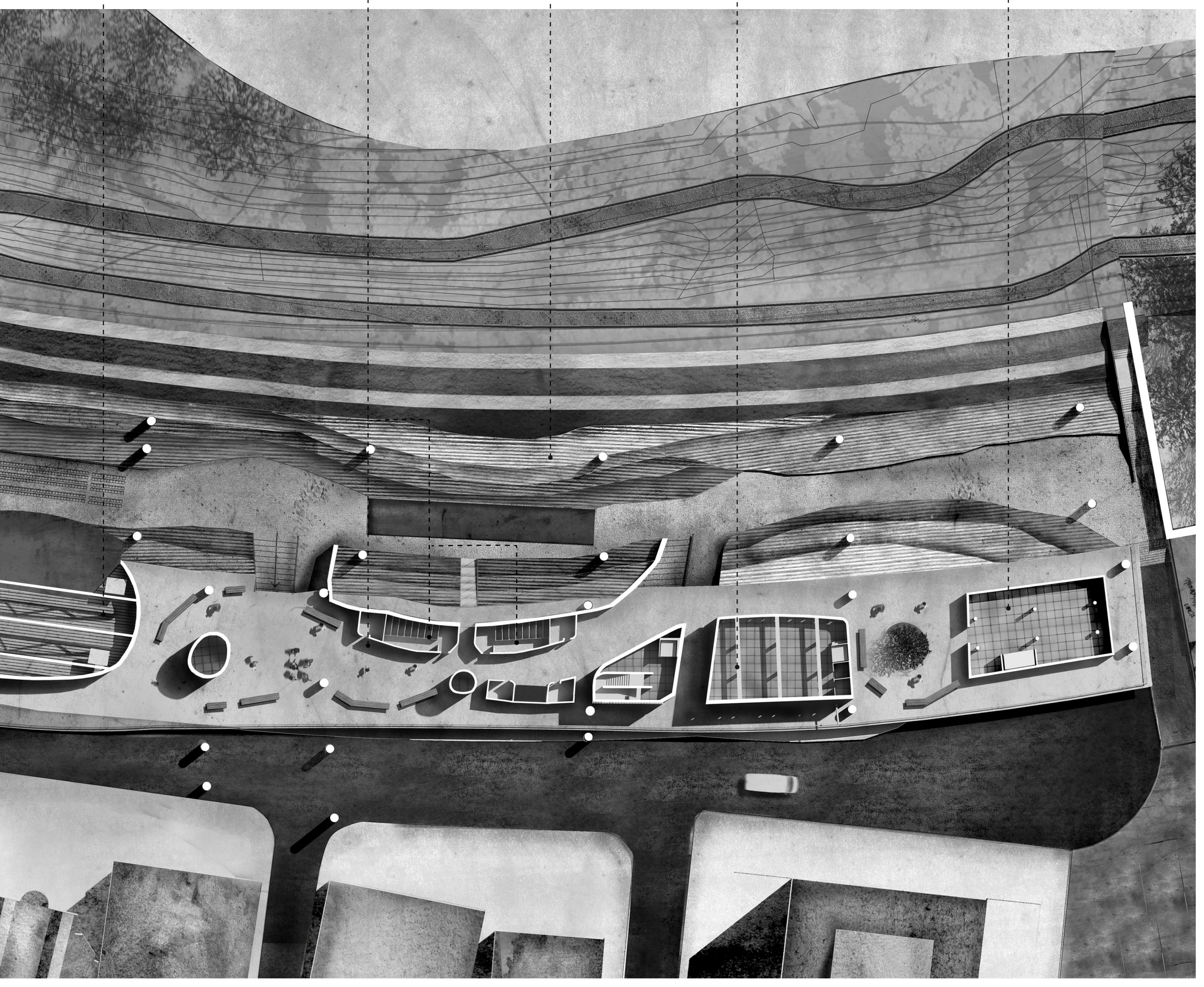


datum - becoming 'more curvilinear/fluid' within the middle section of the design (encompassed by the canopy). The southernmost and nothernmost ends of the site continue the rectilinear plans of the buildings above, creating orthogonal endings and 'tighter' spaces that are restrained by the two sets of grids (see Figure 9.7).

\section{Bottom, 'Service' Level / Ground}

Sectioning to the ground, the design introduces a level that is both performance and service based (see Figure 9.9). Beneath the public level, the operations of the facility occur. In addition to the service requirements of the facility, the black box theatre is contained at ground level (See Figure 9.49). This theatre repeats the formal language of the two theatres at higher levels, curvilinear in form and penetrated above by the canopy well to introduce natural light and continue the dialogue between canopy and performance.

The two ground levels (Datum 0 and Datum 3) also reveal site-specific, performative architectures.
An excavated section of gravel and soil greets publics as the enter the site along Datum 3, from the Cable Car, leaving behind four pads of concrete which are able to display public sculptures and works of art. This excavated space runs parallel to the grid structure that supports Datum 1, left unchanged to reveal how the containing grid directly above was drawn out.

Continuing along Datum 3, a number of public seating terraces (topped by timber decking) extend outwards from both the greenbelt and the threshold with Datum 1 (See Figures 9.48-9.50). The Terraces also run perpendicular to stage orientations of the thru-scenium and traverse theatres, creating a spectator/performance seating relationship that permanently occupies the design (see Figure 9.8).

The three levels highlighted and described so far in this section (upper/level two, middle/level one, ground) vertically structure the design, and will be used throughout the remainder of this chapter to analyse the strategies and outcomes of the design case study.

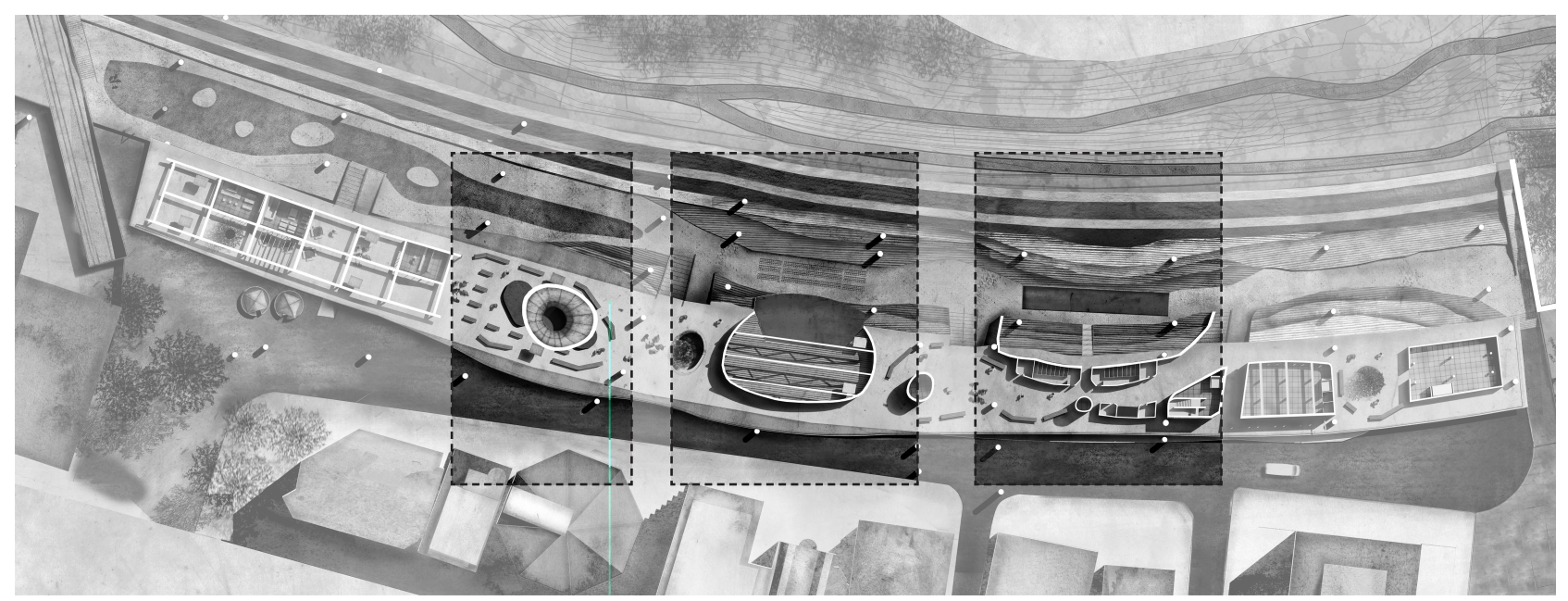

RIGHT > Figure 9.8: Rendered plan of the ground level highlighting the three theatre spaces along this datum as the relate to the seating terraces

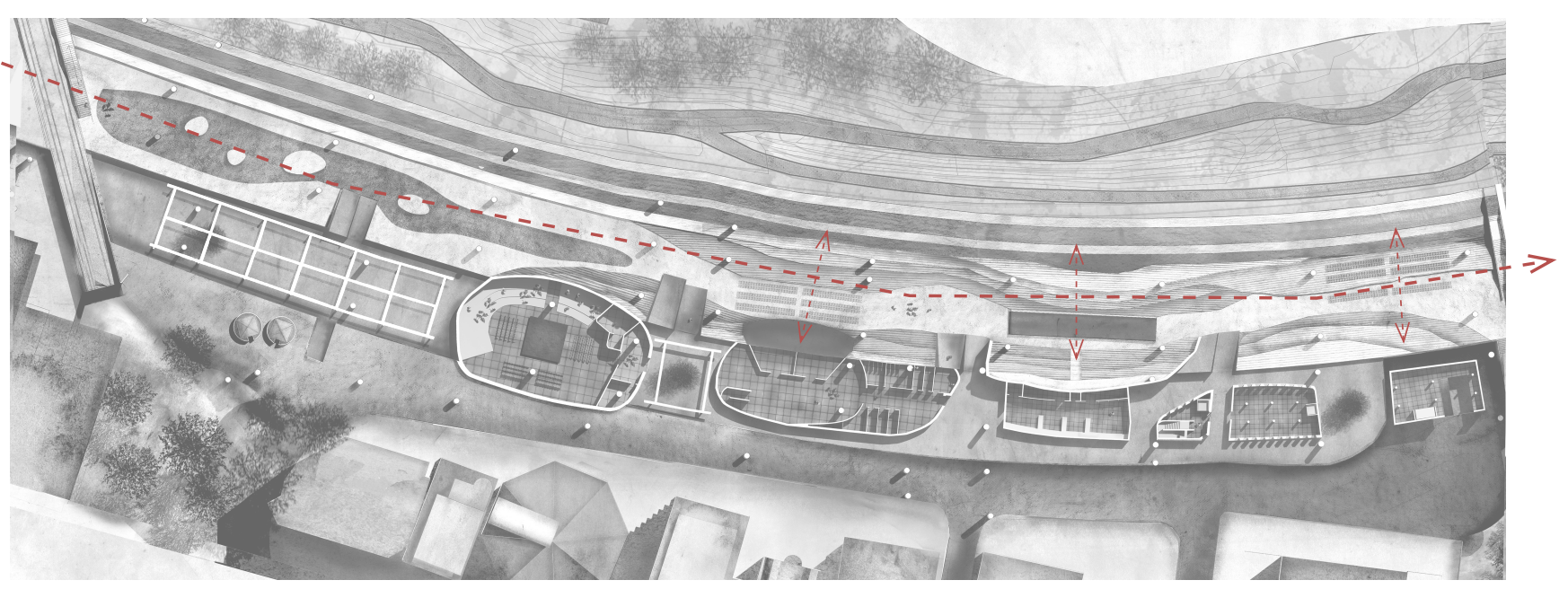


209

竞

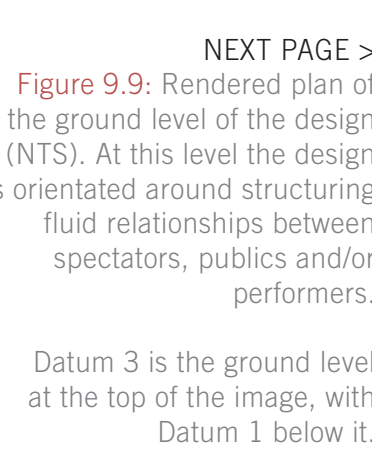




\section{궁 9 (18)

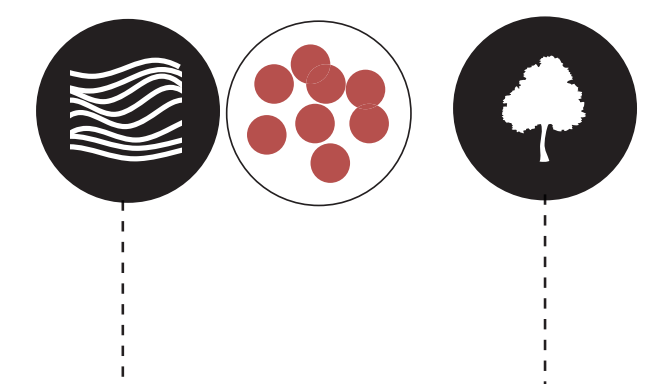

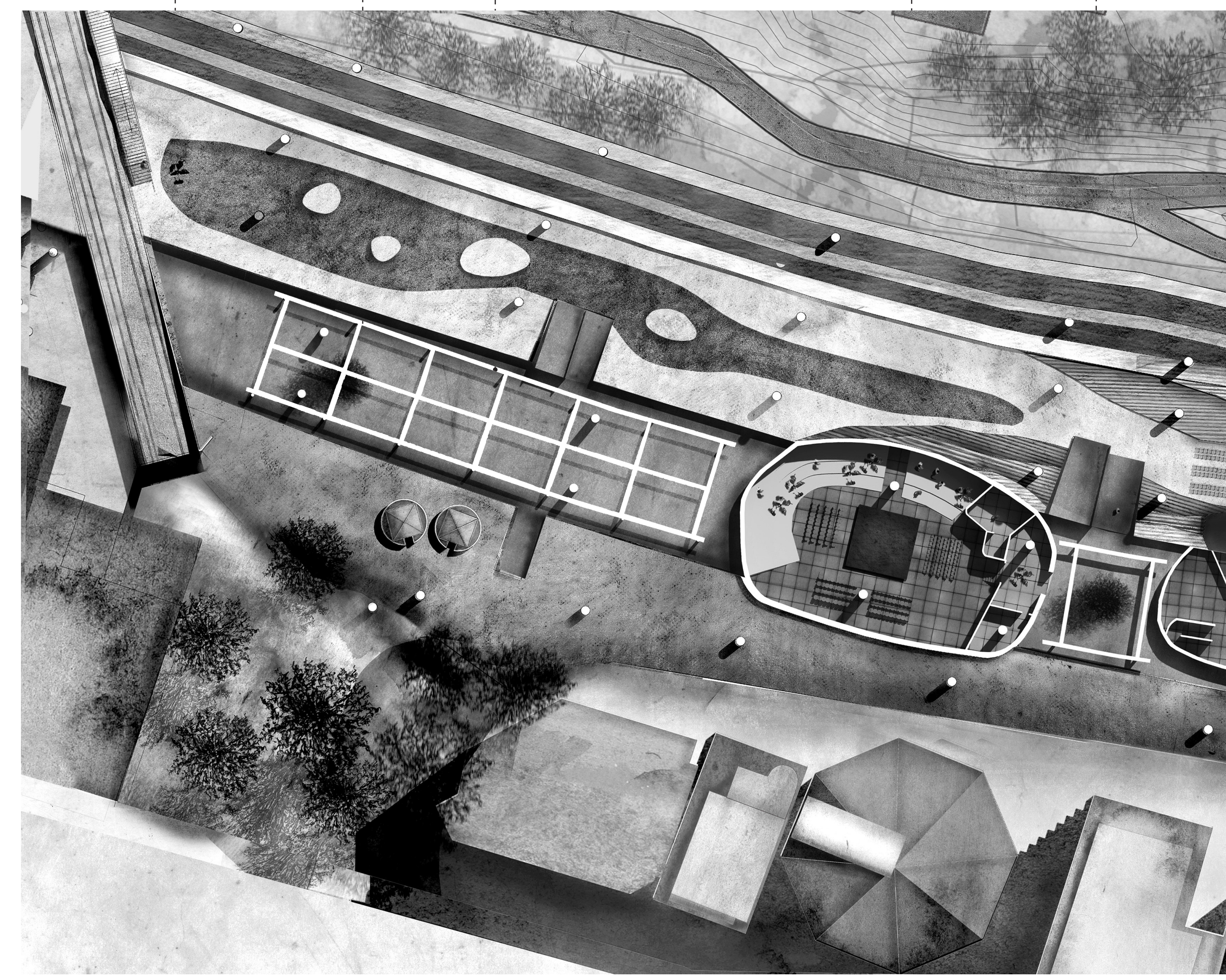


Programmatic Structuration: Functions, Adjacencies and Circulations

As outlined in Chapter Six, for Kim Dovey 'structuration' refers to the potency of architectural form in framing the relationship between spatial structure and social practices. Socio-spatial practices are either enabled or constrained according to the intervention of architectural design, informing social and political contexts that exist as a negotiation between tight and loose. The driving intention of this design is to introduce multiple programmes and activities to the site, introducing socio-spatial flows, and looseness. This intention is based upon the current thick edge condition as one of constraint - a tight space. However, a tension is necessarily maintained, and in Architecture's Tightrope, the tight/loose dialectic operates programmatically, configured according to the functions, adjacencies and circulations of particular spaces. 
2. For this thesis contextually normative' spatial practices a those which are related to the previous condition of vehicle-
3. This approach is based upon the Lefebvrian understanding that space produces and is produced by, social actions.

4. de Certeau, 1984, p. 11
The design functions are generated according to the programme brief outlined in Chapter Eight. The position and size of these functions are presented in Figures 9.10 and 9.11.

Building upon the brief, functions of the design (as a cultural node) are multiple and varied, inviting the surrounding publics to perform contextually non-normative spatial practices. ${ }^{2}$ Unlike the site's previous condition as a car park, publics are now encouraged to engage in activities such as: spectating art, cinema, performance, and other publics; walking along multiple circulation routes and paths; sitting on the sheltered and unsheltered public seating scattered throughout; playing in a reconfigured social site, one of 'openness'; eating at the café, bar; shopping at the bookshop; working in the office building for the NZIAF, artist studios, and a workshop; producing and performing on stages for ad-hoc public busking, announcements and appropriation as well as a rehearsal space that can be hired by the public.

Introducing these spatial practices contributes to a non-deterministic structuration insofar as the design is only attempting to 'open' relations across the site, rather than control the actions that take place within it. ${ }^{3}$ These design functions contribute significantly to shift the thick edge condition towards a de Certeauian notion of 'place', an ordering system which is socially and spatially 'practised', realising a larger range of possibilities for the location. ${ }^{4}$ 

Figure 9.10

GROUND LEVEL PLAN

KEY

1. Cable Car stop / southern entrance

2. Gravel 'river' / sculpture park

3. Black box permanent covered indoor seating

4. Black box performance volume / flexible seating area

5. Black box performer $\mathrm{BOH}$

6. Black Box coat check / recpetion

7. Spectator volume for thru-scenium theatre / covered open-air seating

8. Main performer BOH storage space $\left(100 \mathrm{~m}^{2}\right)$

9. Main performer $\mathrm{BOH}$ backstage volume $\left(450 \mathrm{~m}^{2}\right)$

10. Main performer $\mathrm{BOH}$ dressing/changing rooms $\left(350 \mathrm{~m}^{2}\right)$

11i. Thru-scenium terraced seating, permanent, 400-600 capcity

11ii. Thru-scenium terraced seating, permanent, $600-800$ capcity

11iii. Thru-scenium temporary seating area, flexible, 100-200 capcity

12. Workshop storage $\left(100 \mathrm{~m}^{2}\right)$

13. Workshop $\left(250 \mathrm{~m}^{2}\right)$

14. Circulation tower, stairwell and elevator

15. Plant room $\left(200 \mathrm{~m}^{2}\right)$

16. Loading bay

17. Delivery entrance and turn-around area

18. Open function space

C: Pedestrian circulation

E: Elevator

P: Partitioning curtains

S: Performance stage

$\mathrm{T}$ : Toilets (public)

V: Vehicle access

A - A': Traverse section 1 (See Figure 9.48)

B - B': Traverse section 2 (See Figure 9.49)

C - C': Traverse section 3 (See Figure 9.50)

D - D': Longitudinal section (See Figure 9.19

SCALE: 




\section{Adjacencies}

Where the implementation of various spatial practices presents an opportunity to introduce a reconfiguration of place from singular (non-place) to multiple (relational), the tangible adjacencies of these programmes are more pragmatic. The cohesive functioning of a multi-stage events facility requires specific connections between back-of-house $(\mathrm{BOH})$, front-of-house (FOH) and auditoria (performance) spaces. In addition to these adjacencies, Architecture's Tightrope has been designed to facilitate the NZIAF staffs, temporary offices for administrators of other festival events, and various spaces for hire (artist's studios, rehearsal spaces), introducing a commercial element to the design (see Figure 9.13).

Following the relationship(s) between the performance adjacencies outlined previously (Chapter Eight), a large majority of $\mathrm{BOH}$ functions have been implemented at ground level, creating a level divided into 'service' and 'performance' $\mathrm{BOH}$ spaces (see Figure 9.12). This decision, based upon progressive design-research experiments, was in response to several site-specific and programmatic parameters:

- The functions of $\mathrm{BOH}$ spaces require less natural light and are therefore suited to the volume between Datum 0 and 1 .

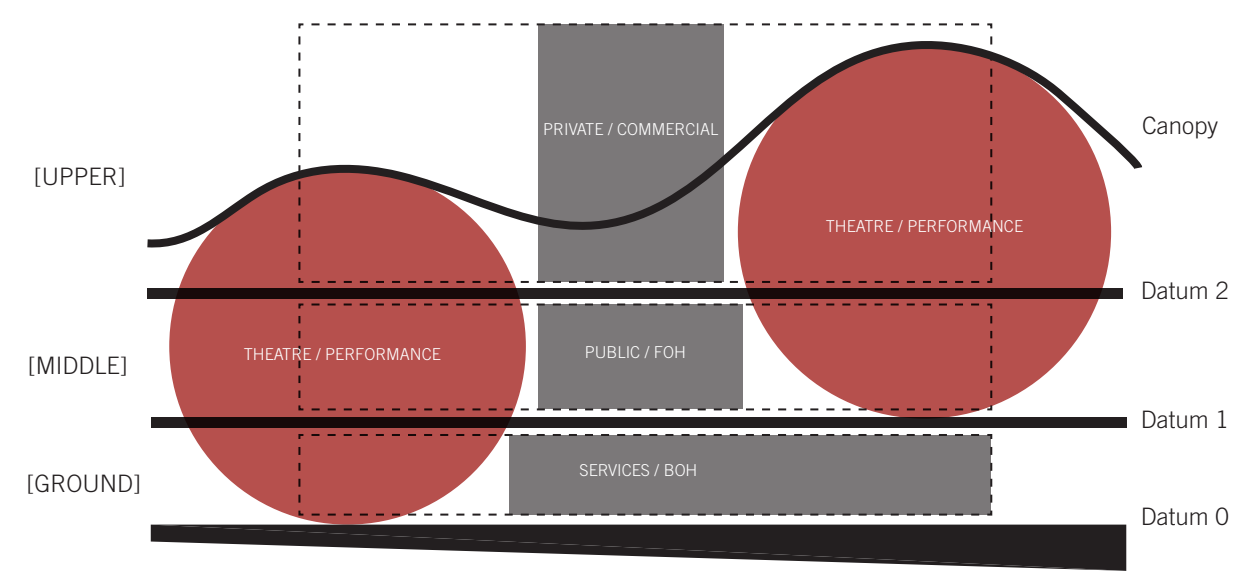

- Privacy from publics is necessary for certain areas of $\mathrm{BOH}$ facilities; positioning these spaces at ground level allows for easy public/ private segregation.

- Service $\mathrm{BOH}$ activities require vehicular access for staffs and deliveries (including, drop-off, loading and turning space), and therefore need to be as close as possible to the main vehicular entry points of the site, Dalmuir and Shell Lanes (see Figures 9.24 and 9.25);

- As performance $\mathrm{BOH}$ facilities may need be shared, they should be placed in close proximity to each other for ease of access.

- To minimise vertical adjacencies between all $\mathrm{BOH}$ spaces, informing a cohesive line of $\wedge$ ABOVE

igure 9.13: Diagram showing the various programmatic adjacencies within the design case study. These are divided into four categories: front-ofhouse (FOH); back-of-house $(\mathrm{BOH})$; performance; and

RIGHT > Figure 9.12: Diagram showing the back-of-house facilities divided into 'performance (red) and 'service' (grey).

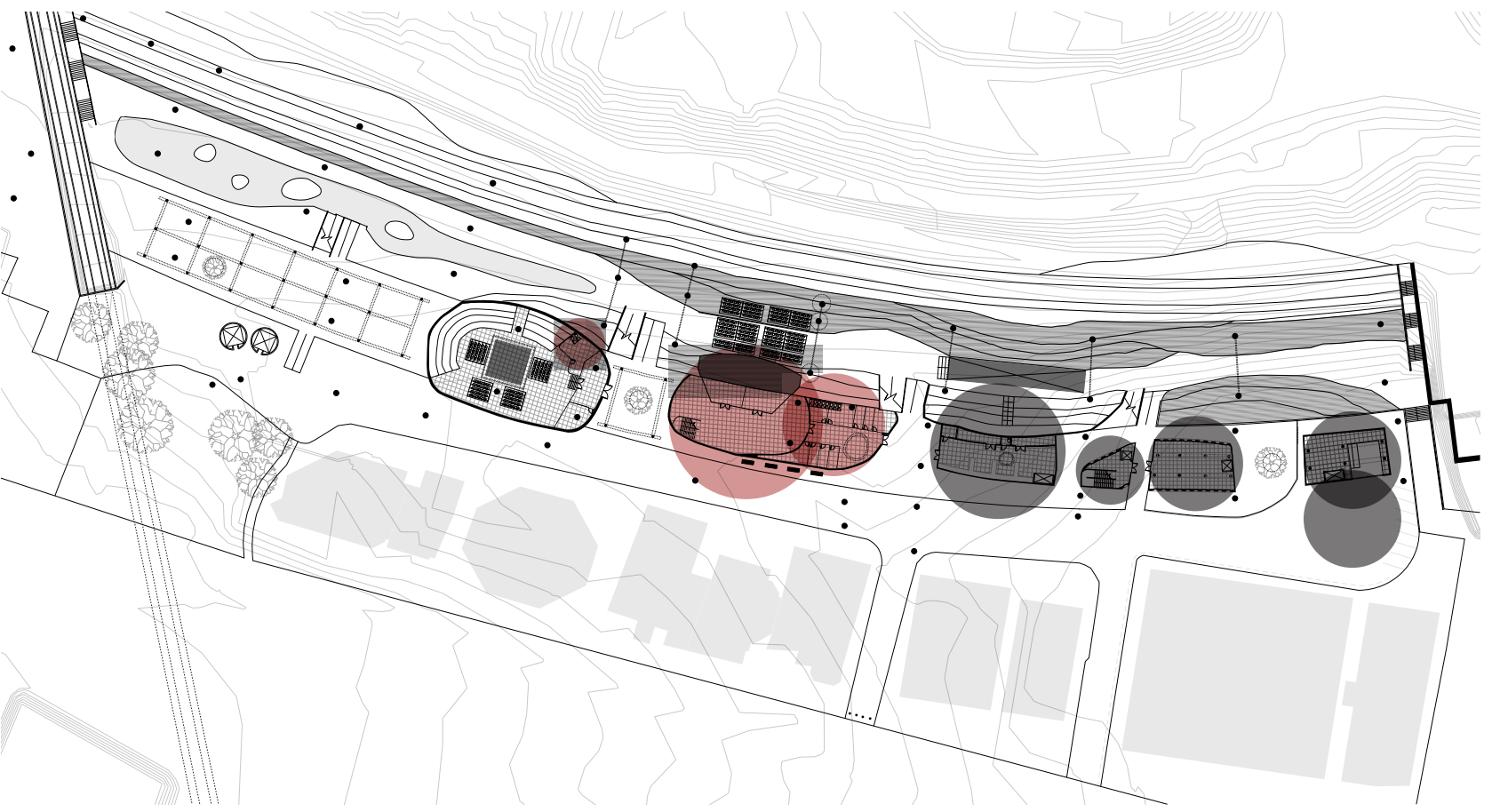




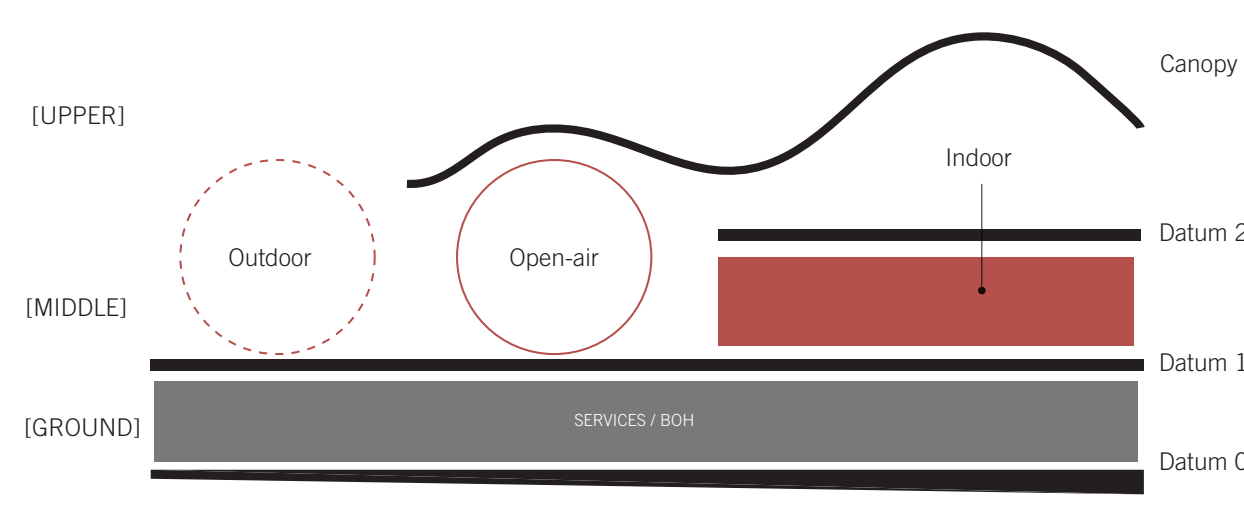

ABOVE ^

Figure 9.14: Diagran showing the various forms of shelter created by vertical arrangements

access;

- Adjacencies between $\mathrm{BOH}$ and auditoria spaces can be vertical, as is the case with the Wellington Town Hall and St James Theatre, allowing performance $\mathrm{BOH}$ to exist within a lower volume.

Additionally, positioning $\mathrm{BOH}$ facilities within the Datum $0 / 1$ volume allows the large open-air volume under the motorway to remain unstructured creating a spectator threshold that aligns axially with the performance $\mathrm{BOH}$.

Consequently, many of the $\mathrm{FOH}$ activities are positioned along Datum 1 (some between Datum 1 and 2, others contained by the canopy or openair). At this level, flow between publics is pivotal, informing an alternating relationship of adjacencies between performance and gathering spaces (see Diagram 9.16). Keeping the FOH structures to a minimal floor area, and positioning them axially along Datum 1, allows the design to achieve several imperatives:

- To maintain a linear performance/spectator relationship between the motorway volume and the $\mathrm{BOH} / \mathrm{FOH}$ design interventions;

- To produce a variety of spaces - open-air sheltered, indoor, outdoor (see Figure 9.14);

- To encourage pedestrian access from the cable car, northern stair, and underground path

- To allow adjacencies to be shared vertically between $\mathrm{BOH}$ and FOH (see Figure 9.15), in particular storage, plumbing, electricity etc.;

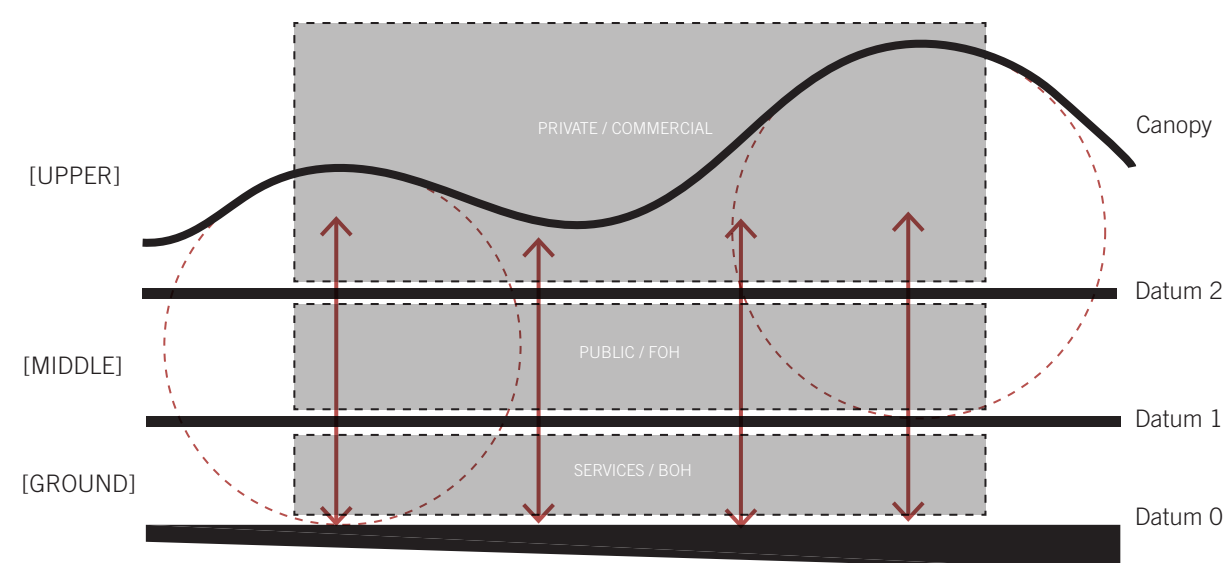

- To allow FOH facilities such as public toilets, retail and hospitality to be shared between publics attending performance and those who $\wedge$ ABOVE

Figure 9.15: Diagram showing how the $\mathrm{BOH}, \mathrm{FOH}$ and private are not.

services

Interwoven within, and also informing these $\mathrm{FOH} / \mathrm{BOH}$ adjacencies, is the auditoria positioning. The four large performance spaces (black box, thruscenium, traverse, and open-air/flat-floor) anchor the infrastructure on a curved line - creating the performance/spectator threshold axis. The black box theatre requires the least amount of seating as well as an intimate interior space, and has therefore been positioned separately, underneath Datum 1 towards the southern end of the site (see Figures 9.21 and 9.48). As the thru-scenium and traverse theatres are the largest in capacity and infrastructure, they have been positioned adjacent to one another (see Figures 9.49 and 9.50); this allows the design a certain economy as the theatres can share $\mathrm{BOH}$ facilities and overflow space. Additionally, placing the two largest auditoria in the centre of the site limits congestion as publics can flow from the north or south entrances, filtering through the various gathering points.

The flexible, open-air, flat-floor performance space (see Figures 9.10 and 9.52) has been designated to the northernmost section of the spectator volume. This space can be used for a variety of lowinfrastructure events such as: cinema, congregation, protest, concerts, markets, outdoor rehearsals and festivals, etc. For this reason, the design of this performance space does not impose an infrastructure beyond that of shelter and seating. 


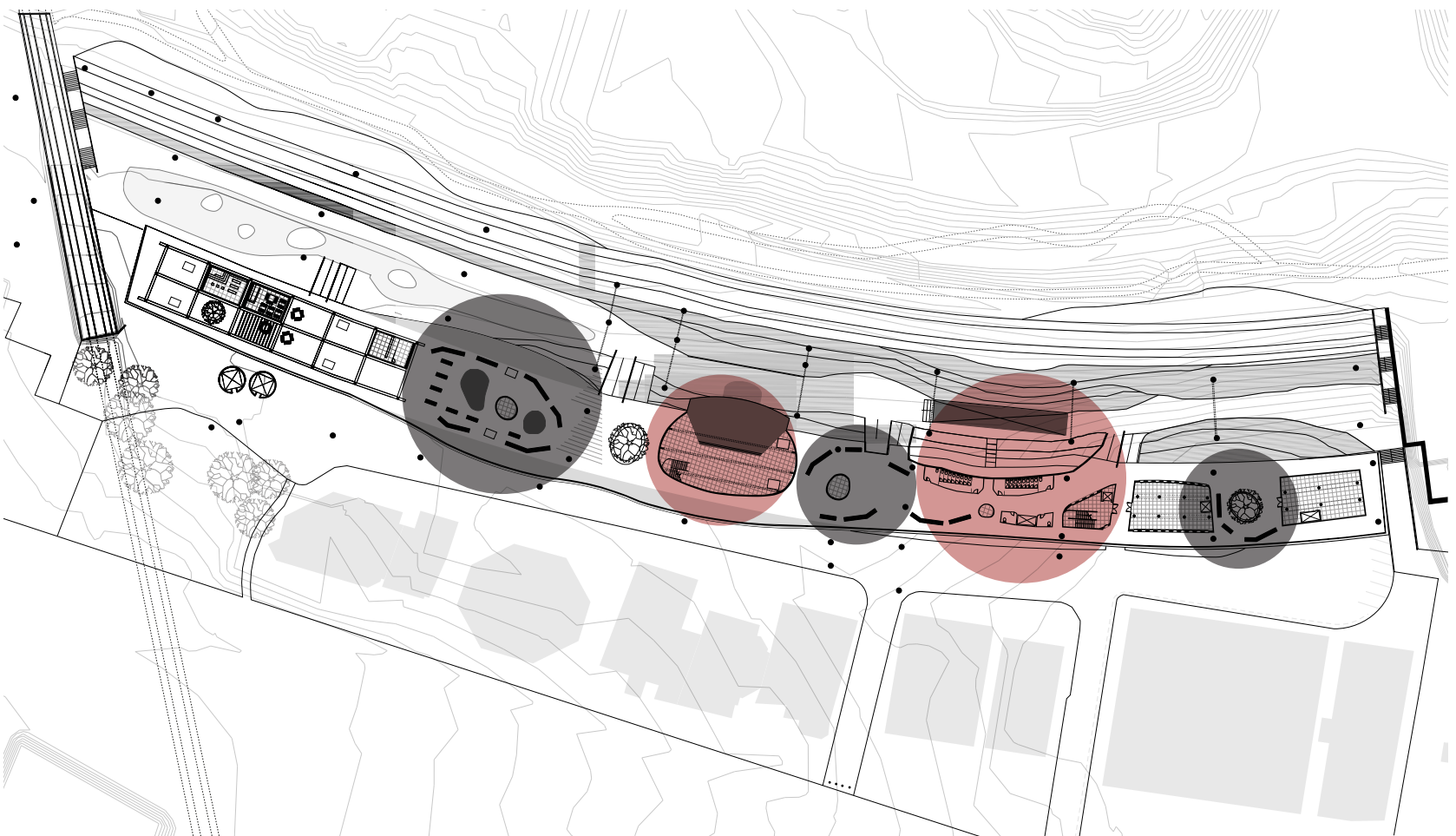

\section{Circulations}

Given the complex proposition of negotiating multiple, independent yet connected, spaces within one design, circulation is positioned as a crucial operation to achieve cohesiveness and flow. Beyond the practical requirements of access, circulation contributes to an overall language for the design, an effect, performing as a mediation between inside and outside, framing and dissolving and reframing public and private, performance and spectator.

The requirements and subsequent design of the facility's programmatic adjacencies have conditioned much of the pedestrian and nonpedestrian (vehicular) circulation throughout. These circulations operate both horizontally (paths, ramps and roads) and vertically (stairs, ramps, elevators, and light wells).

Horizontally, one of the major experiments and strategies of the design is to shift, and define, the emphasis of the urban function of the site from vehicular to pedestrian - conditioning a space that promotes social permeability over the existing rigidity of the car park. The design uses a simple but important gesture to initiate this change positioning four bollards at the entrance of Dalmuir Lane, transforming it into a pedestrian-only access way. In so doing, the facility requires service vehicle access to enter and exit down Shell Lane only, with a turnaround area adjacent to the loading bay (see Figure 9.17). This turnaround space has been enlarged to allow for large trucks to now enter the site. Complementing this line of vehicular access, Shell Lane has been extended through the site, enabling vehicles to drive equipment directly to the back of the traverse theatre and plant room if required to do so.

From a pedestrian perspective, a number of horizontal access points already exist within the site (see Chapter Four). One purpose of the design is to attract more pedestrians to the site by removing its vehicular functions. In so doing, it is expected that the Cable Car will perform a more intensive and diverse role as a transportation device - facilitating more than simply tourist movements. This is also a symbolic gesture - reducing the emphasis of 
automobiles to engage the site as pedestrian friendly. Moreover, given the day/night activity of the facility, the greenbelt path and northern stair (currently unfrequented at night) will be activated on a more frequent basis.

Within the facility, and between adjacencies, access is promoted through several up/down ramps. As these ramps have the original purpose of directing cars between parking datums, they are wide enough to allow for paraplegic access if necessary. The up/down ramps are positioned central to the public gathering spaces (and either side of the large auditoria), limiting congestion by directing publics into overflow areas.

Vertically, there are a number of means to transition between ground/middle/upper levels. Central to the design is the six storeys high circulation tower - encompassing an elevator and two fire stairs. This tower provides vertical access to all levels, and also transports publics to the viewing deck (See Figure 9.24) - a space which punctures through the canopy grid and overlooks the entire site. In addition to the circulation tower and up/down ramps, two large service elevators (one in the workshop and one in the loading bay) connect service $\mathrm{BOH}$ to the middle and upper levels. A standard passenger elevator also connects floors within the office building.

Although the large public ramps have been promoted over stairs and elevators, a large service stairwell connects the thru-scenium/traverse backstage to the staging area above.

Another crucial consideration for the design is the separation of performance areas within the spectator volume. As can be seen in Figures 9.10 and 9.11, floor-to-ceiling partitioning curtains (label ' $\mathrm{P}$ ') reach between the existing structural columns either side of the large performance spaces (see Figures 9.489.50). These can be either tied back or closed to allow or restrict access according to the activities of the facility at any point in time. Dividing curtains, rather than walls, allow for a flexible and easy transition from public space to private-public spectatorship.

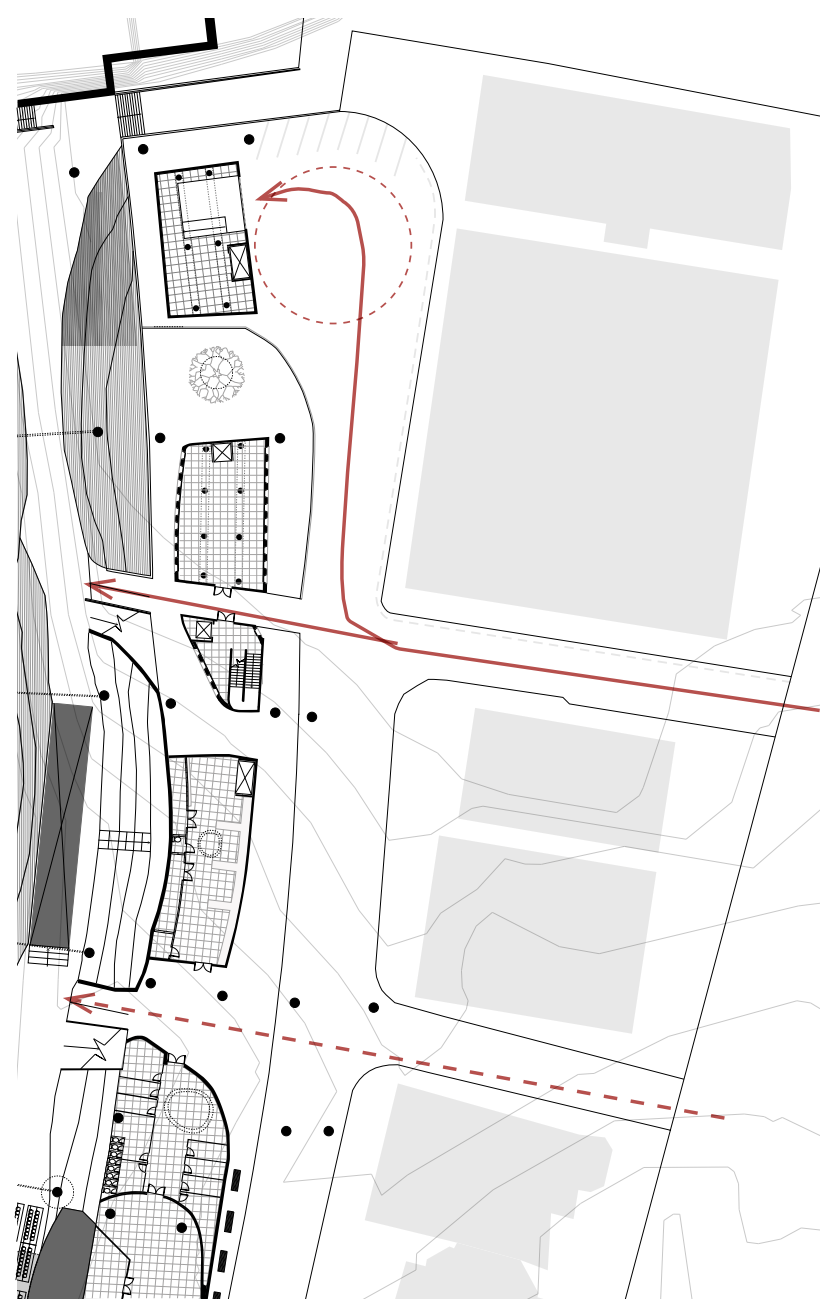


So far the description and structuration of Architecture's Tightrope has examined how the programme - and its associated spatial practices - has been articulated within the design process. Through this process, the appropriation of the existing space has been discussed, displaying an intention to create an overall structure of socio-spatial looseness for multiple publics.

This chapter now turns to specific examination of how this has been achieved formally, showing how design strategies for critical spatial practices, elucidated in Section Two, have been implemented in physical structures. To do so, the design is explored in two and three-dimensions, through a series of isometric, section, sectional perspective, and diagrammatic images. These images continue the logic of discussion used so far, referencing the design in relation to its ground, middle, and upper levels. The critical formal strategies implemented throughout the design (addition, subtraction, continuity, discontinuity, horizontality, verticality and juxtaposition) are to be examined through each of the major buildings and discussed according to their formal typologies, spatial dimensions, functions and materiality (see Figure 9.18).5

Following the performative understanding been designed relative to the operations that were deemed necessary to embed the design with critical potency, and therefore will display different strategies from each other Additionally, each building is numbered according to their position in Figures 9.11 and

9.12. of architectural forms argued for in Chapters Five and Seven, the design strategies operate according to four site-specific indices that are embedded in many of the design gestures: fluidity/flow, orthogonality/ rigidity, excavation and terracing. Here, fluidity and orthogonality are considered as formal and metaphorical opposites. Fluidity is a dual metaphor - it visually symbolises the latent curvilinear forms of the site's natural state -as a river bed for the Kumototo Stream and the undulating contours of the adjacent Thorndon green belt. Also, fluidity frames the production of dynamic spatial flows, informing looseness using similar spatial approaches to the Rolex Learning Centre or Metropol Parasol.

Conversely, orthogonality is symbolically related to the physical and visual context of the repetitive and homogenous geometries of the infrastructure and the commercial development of The Terrace. Orthogonality presents space as a rigid and tightly structured condition, a series of regular structures that constrain and restrict. As Bernard Tschumi imposes the grid as a formal logic within the design of Parc de la Villete, in Architecture's Tightrope the orthogonal represents an imposed global force and ubiquitous formalism - the imposition of the rectilinear masses of economically driven urbanisation. How these two indices manifest (in continuity, discontinuity or juxtaposition) depends upon their position and relationship to the other elements in the design. The adjacent Thorndon hillside is defined by contours that are fluid, while the adjacent site of The Terrace is orthogonal. This thick edge site, which lies between, is both. The car park / motorway is orthogonal in some areas, but also bends and banks (inclines) in some areas. The design case study acknowledges this and has incorporated the undulating canopy to accentuate this dual condition, drawing visitor focus and attention to the complex 'blurred' condition. This assists the design intervention in establishing a new, inverted thick edge that performs as a threshold between conditions, thus encouraging flow from one to the other.

Evolving the precedent set by the Igualada cemetery, terracing and excavation are indices that are tied to processes of tabula rasa construction/ destruction. In Architecture's Tightrope, excavation performs as an index of exposure and removal, correlating to the processes that were undertaken in the construction of the Urban Motorway and the subsequent repositioning of graves at the Bolton Street Cemetery. As with 'burial' in Miralles's design, excavation implies an action of removal whereby it moves physical material and also represents removal of earth, trees and graves historically - community memories. Excavation is a device of exposure, drawing out the history of the site that has been covered with concrete and jump cut urbanism.

Excavation works in tandem with the index of terracing, informing a continuity with the natural landscape. Terracing repositions the material that 
has been removed through processes of excavation and applies them in a functional way (seating, stages etc.) in the design case study. The dual reference of the action of terracing also relates to the site's historical trauma, the terracing of the greenbelt and The Terrace adjacent. The intervention of terracing reinforces boundary blurring along the perimeter of the thick edge condition. Applied in multiple ways, these indices:

- Contribute to the construction of a consistent overall 'language of boundary mediation' throughout the design - an ordering system that allows the individual elements of the intervention to be understood holistically as a critical narrative;

- Expand the notion of 'site' beyond that of a static and materially bounded entity, introducing flows of contexts beyond the limitations of a cadastrally 'fixed position';

- Inscribe the process with site-specific information (visual, historical, physical), layering the design with local referents that contribute to its identity as a contextually relational entity - reconfiguring the site towards notions of place (and away from its current condition as closer to that of non-place);

- Undermine the idea of a one-to-one relationship between architectural object and its symbolic meaning as each index 'acts out' its adjective signifier by performing functional operations. In so doing, each index refuses to submit to the superficiality of formal language as image, attempting to resist the culture of spectacle;

- Produce and are being produced by the architecture in a dialectical process mediating between the architectural form and its multiple contexts, and cultural place identity (engaging Hays).

The deployment of these indices becomes apparent as the design is experienced at human scale. Breaking down the existing large-scale homogeneous space into < LEFT smaller-scale spatial flow elements and paths provides this human scale to the site. This is evidenced in the longitudinal section, D - D' (see Figure 9.19).
Design dimensions pertinent to the discussion of strategies.

FORMAL TYPOLOGY: Rectilinear, augmented rectilinear, curvilinear,

IMPLEMENTED natural, residential

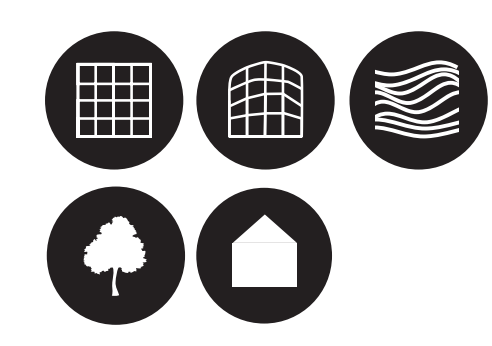

SPATIAL TYPOLOGY: Private, private-public, public

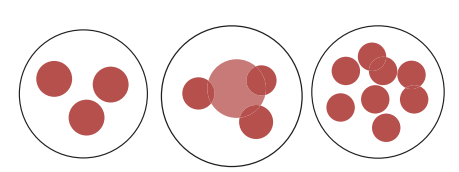

PROGRAMME TYPOLOGY: Performance, BOH, FOH, Commercial

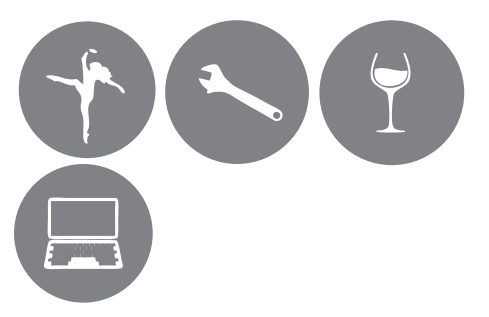

MATERIALS PALETTE

Brick, channel glass, earth/gravel

Plywood, ship-lap timber cladding, rough-sawn timber decking

Shuttered concrete, pour in-situ concrete

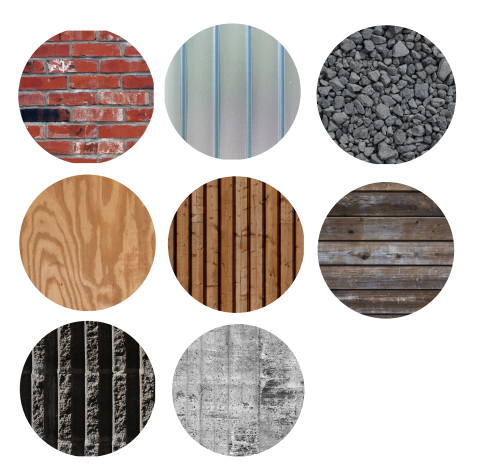


6 longitudinal cross-sections,

highlighting relevant section cuts and corresponding building functions.

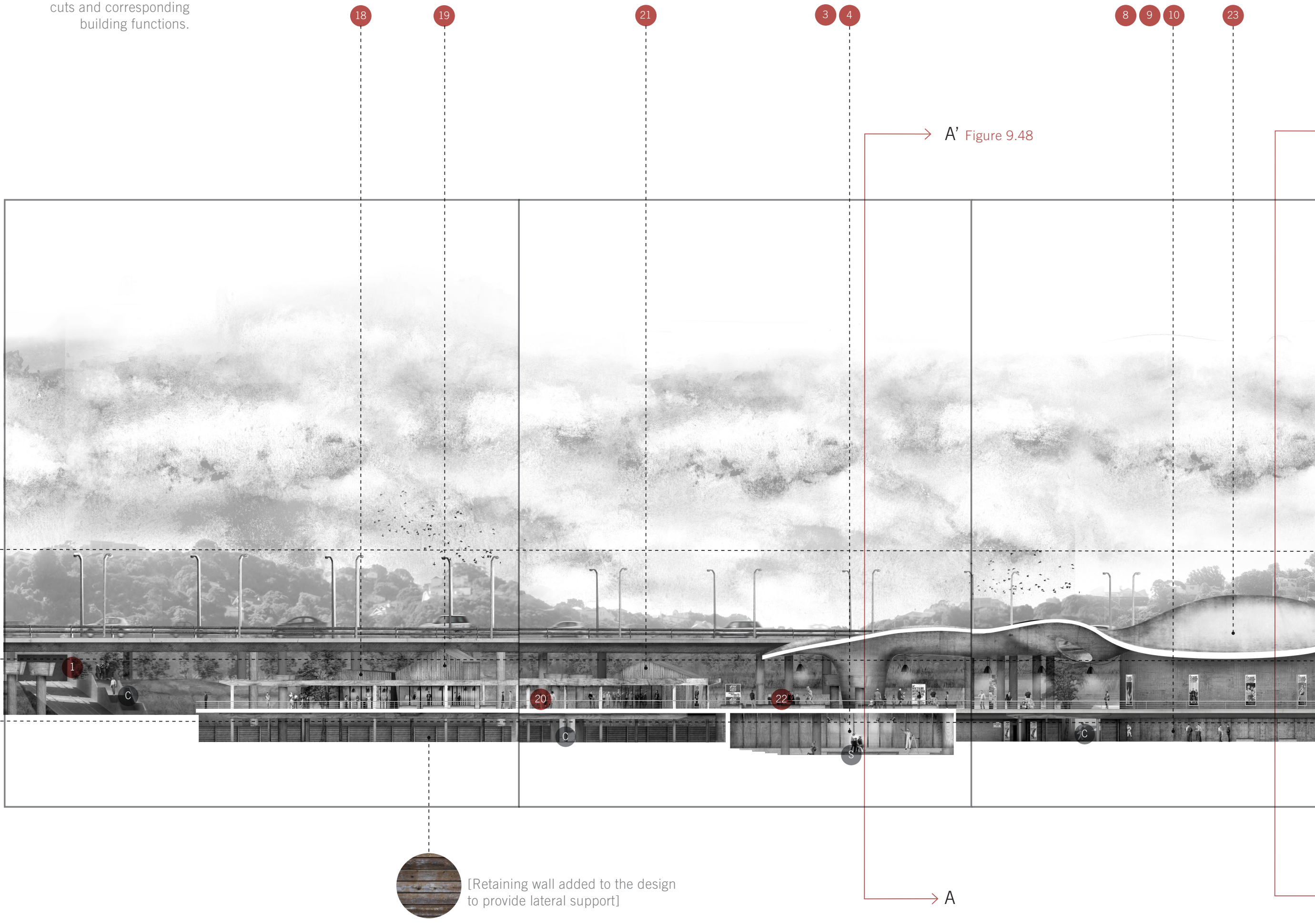

\section{D-D' ZONE ONE}

Figure 9.20: Southernmost

section of the site. This image

shows in detail the materiality and elevation form of the primary public $\mathrm{FOH}$ facilties. To the left is the site entrance via

the Wellington Cable Car
D-D' ZONE TWO

Figure 9.21: Moving north, this image shows a continuation of the $\mathrm{FOH}$ facilities as well as sectioning through the black box theatre. The canopy's downward depression is also visible, flooding the theatre with natural light.
D-D' ZONE THREE Figure 9.22: The first vertical punctuation through the canopy becomes apparent, revealing the pohutukawa tree below. The Thru-scenium theatre presents a strong, blank, volume which has flows of publics interacting around it. 


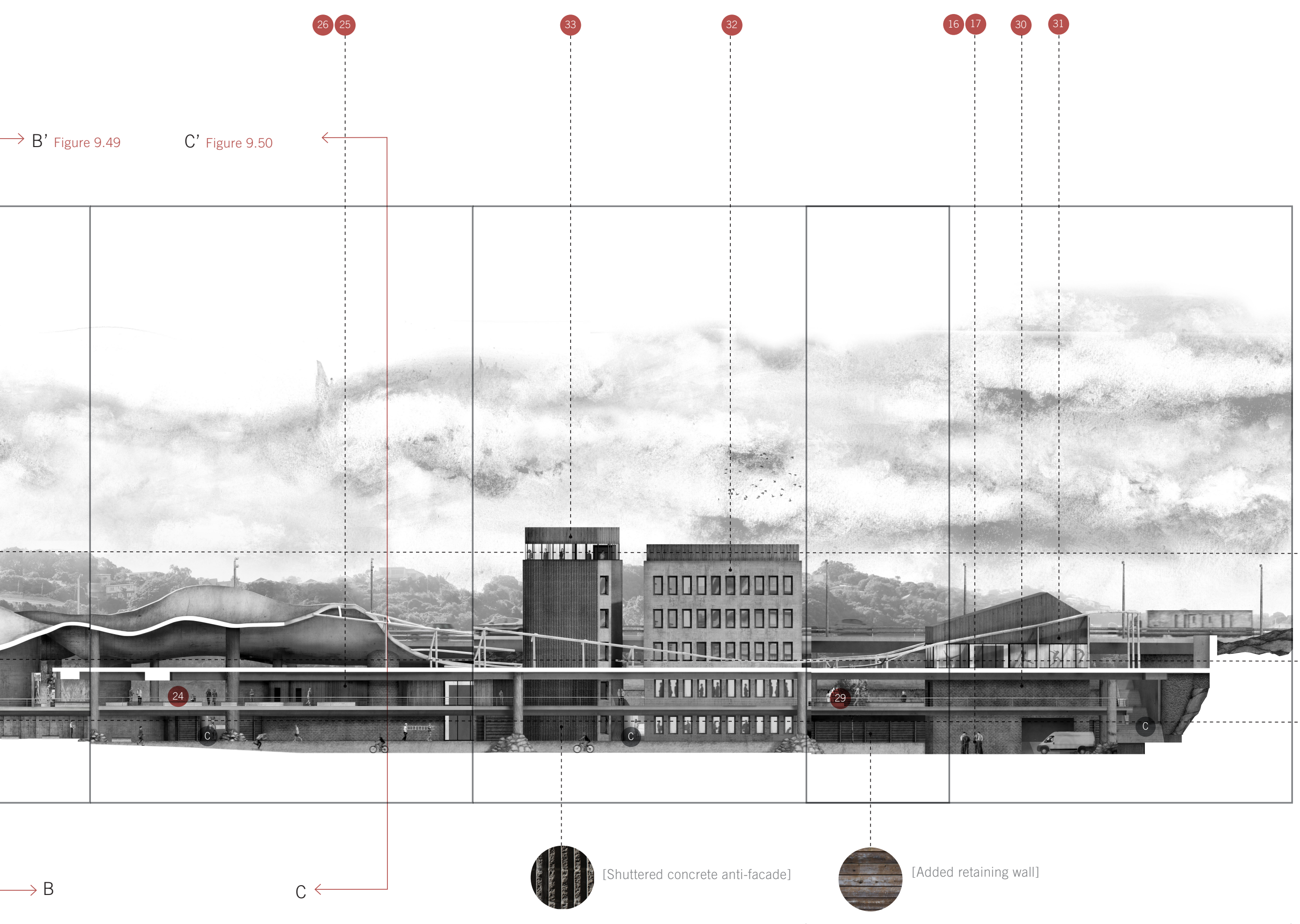

\section{D-D' ZONE FOUR}

Figure 9.23: The canopy lifts above Datum 2, framing the travserse theatre under the motorway. Two light wells or 'chimneys' penetrate upwards to allow natural light to enter the service $\mathrm{BOH}$ level. The gathering space supports public seating, adjacent to the toilet block.

\section{D-D' ZONE FIVE}

Figure 9.24: The canopy dissolves into a steel grid framing structure, punctuated vertically by the circulation tower and NZIAF office block.
D-D' SECTION SIX

Figure 9.25: This section image shows the 'end' of the design at the northern site boundary. The vehicular access (foreground) and northern stair are visible (background) 

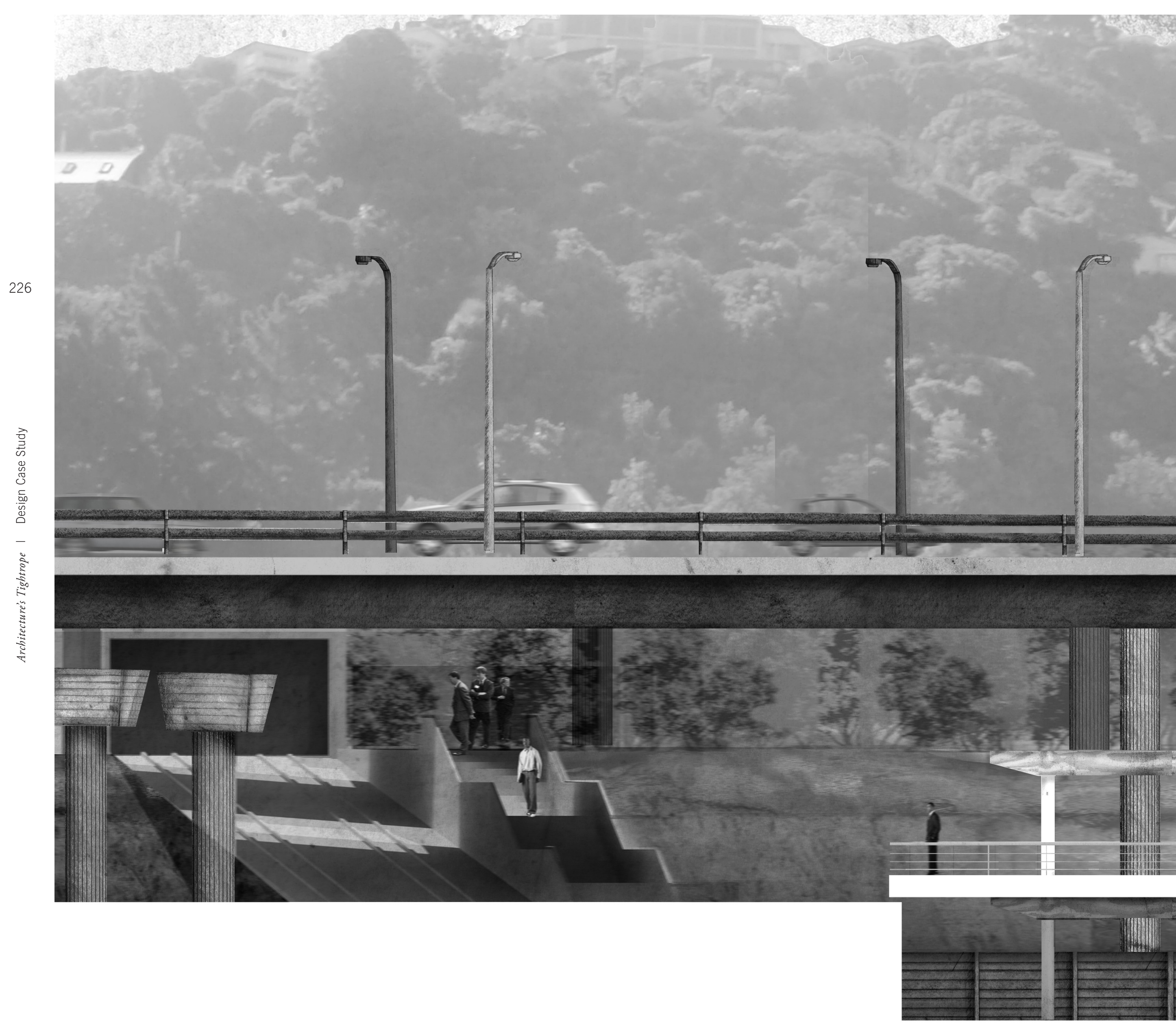

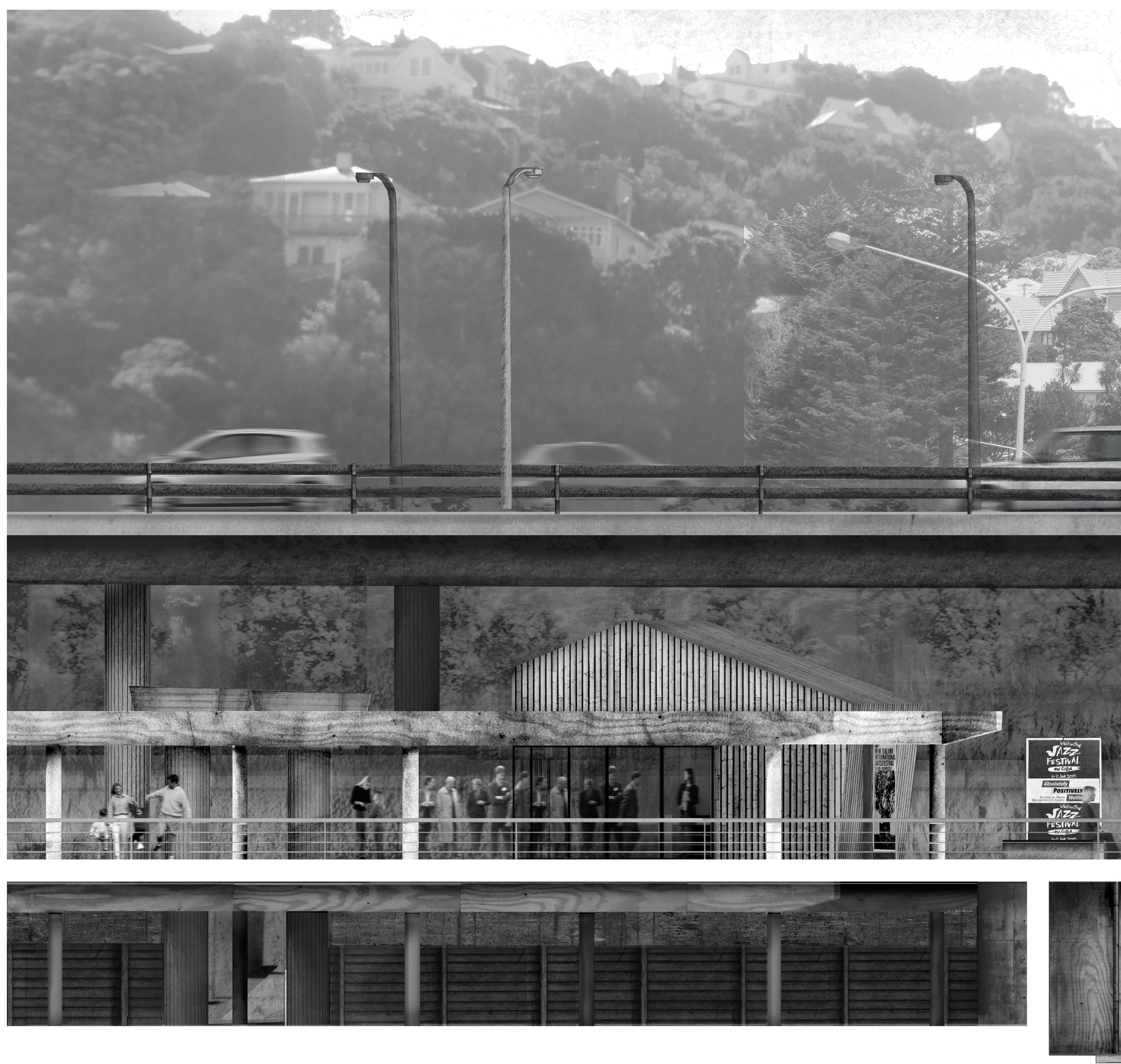

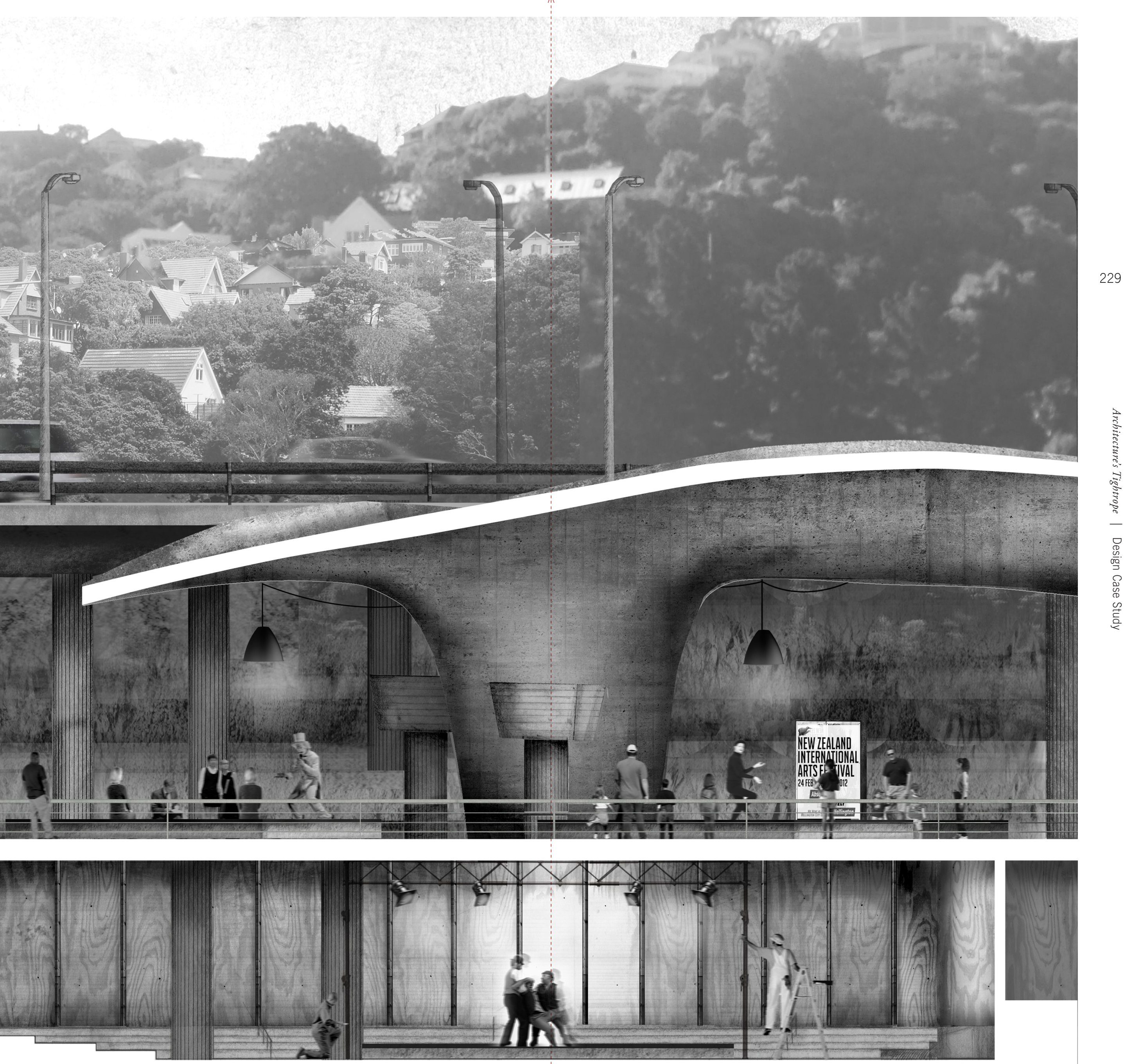

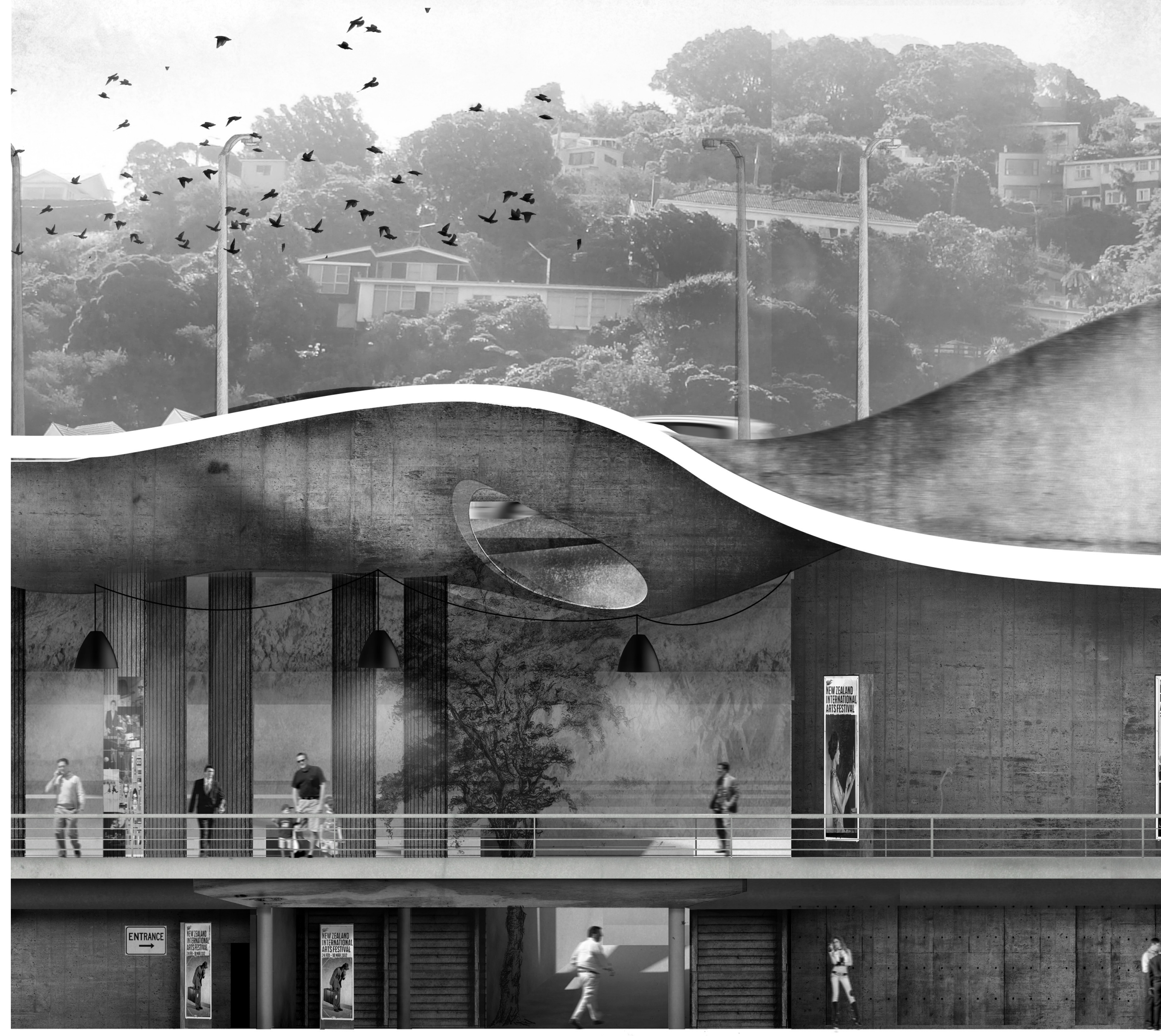

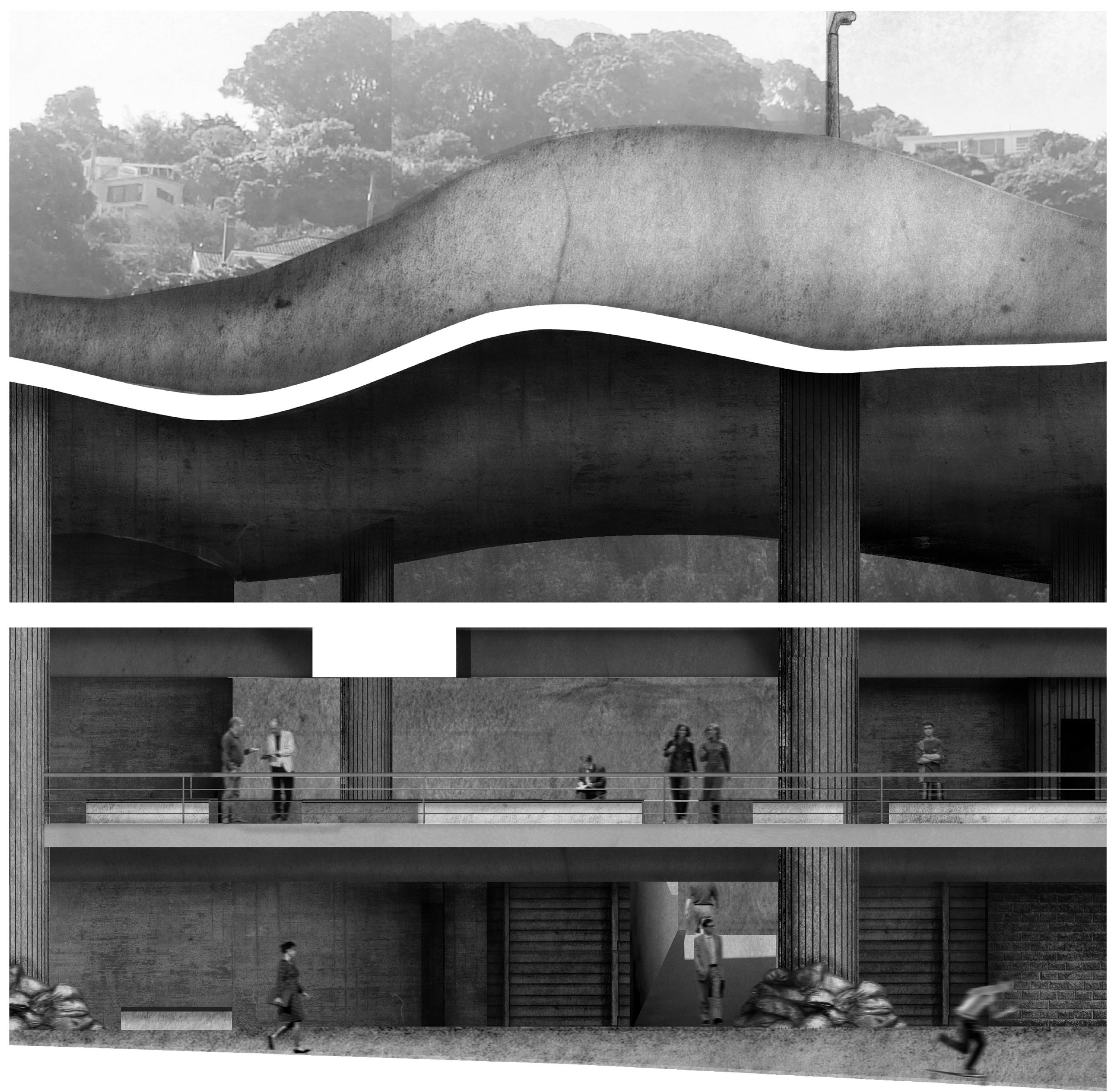


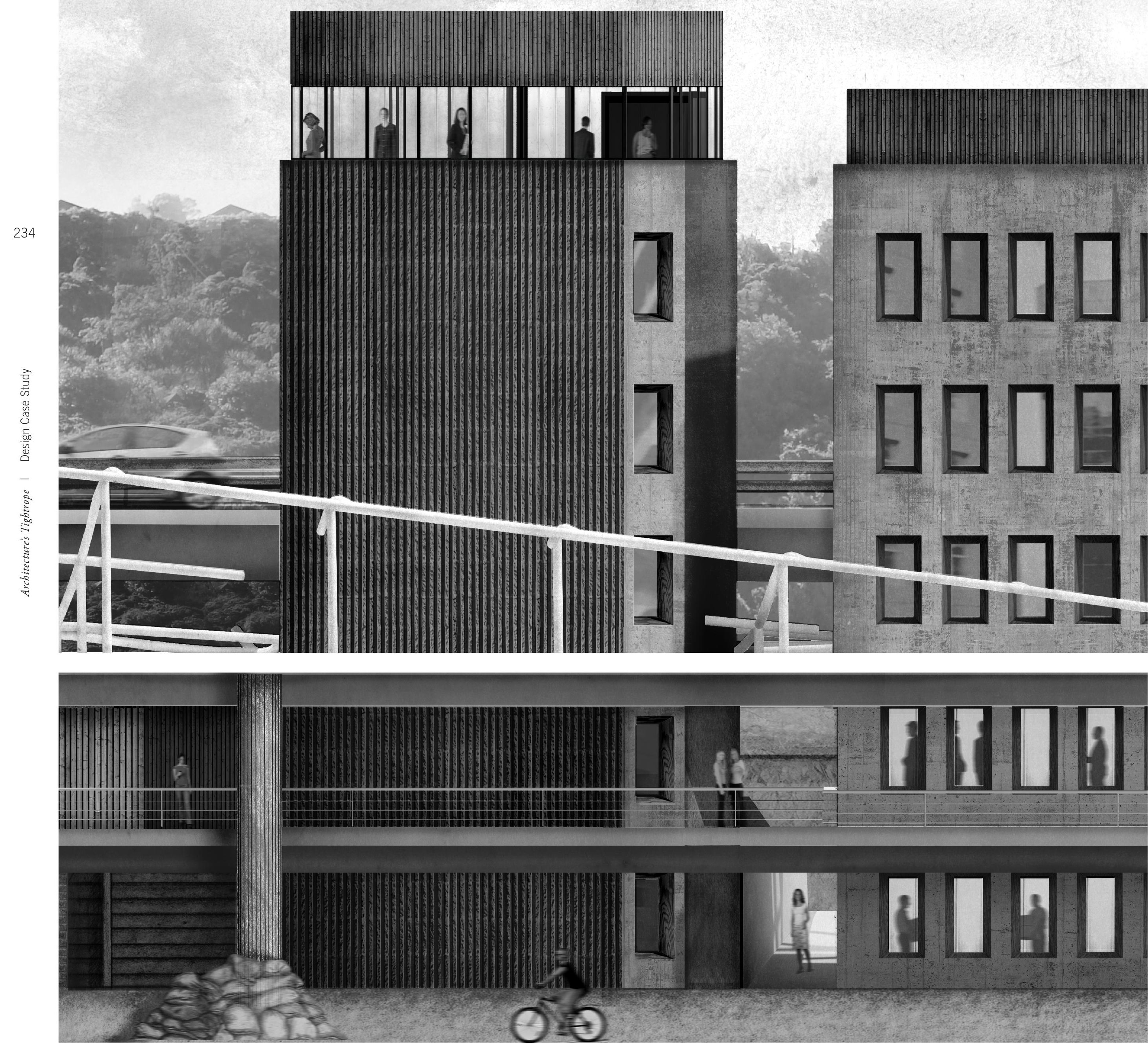




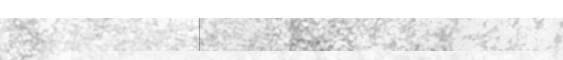
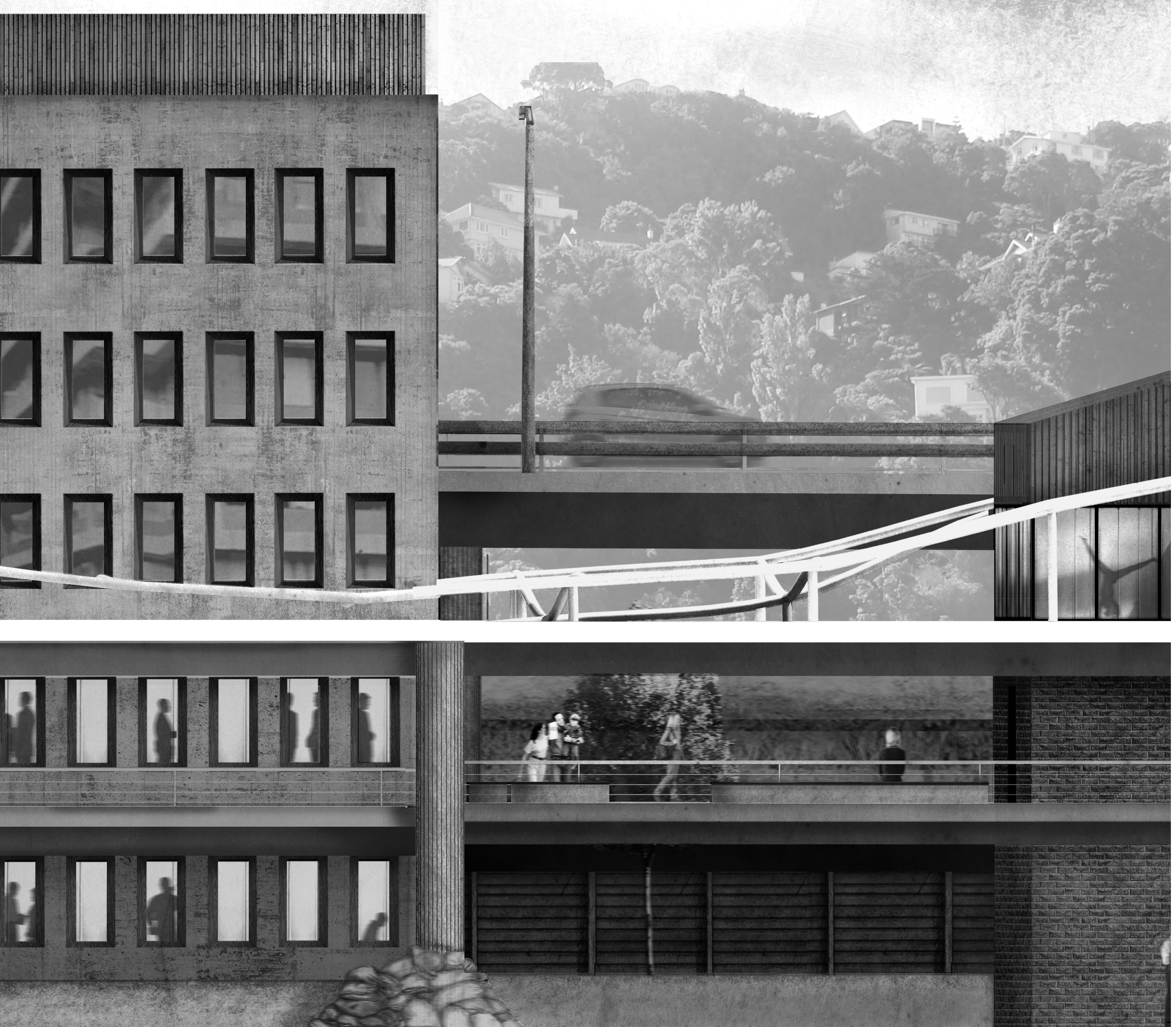

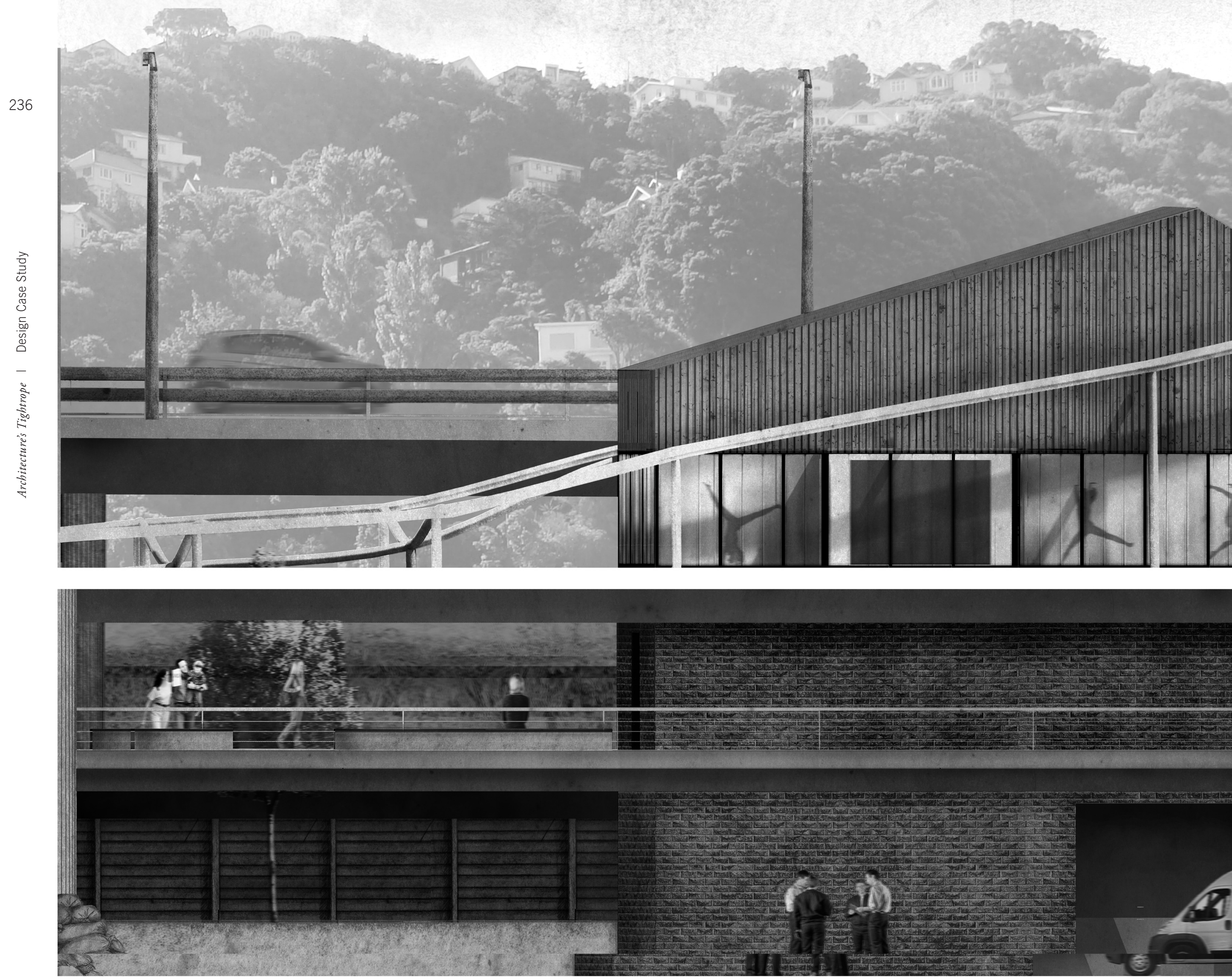


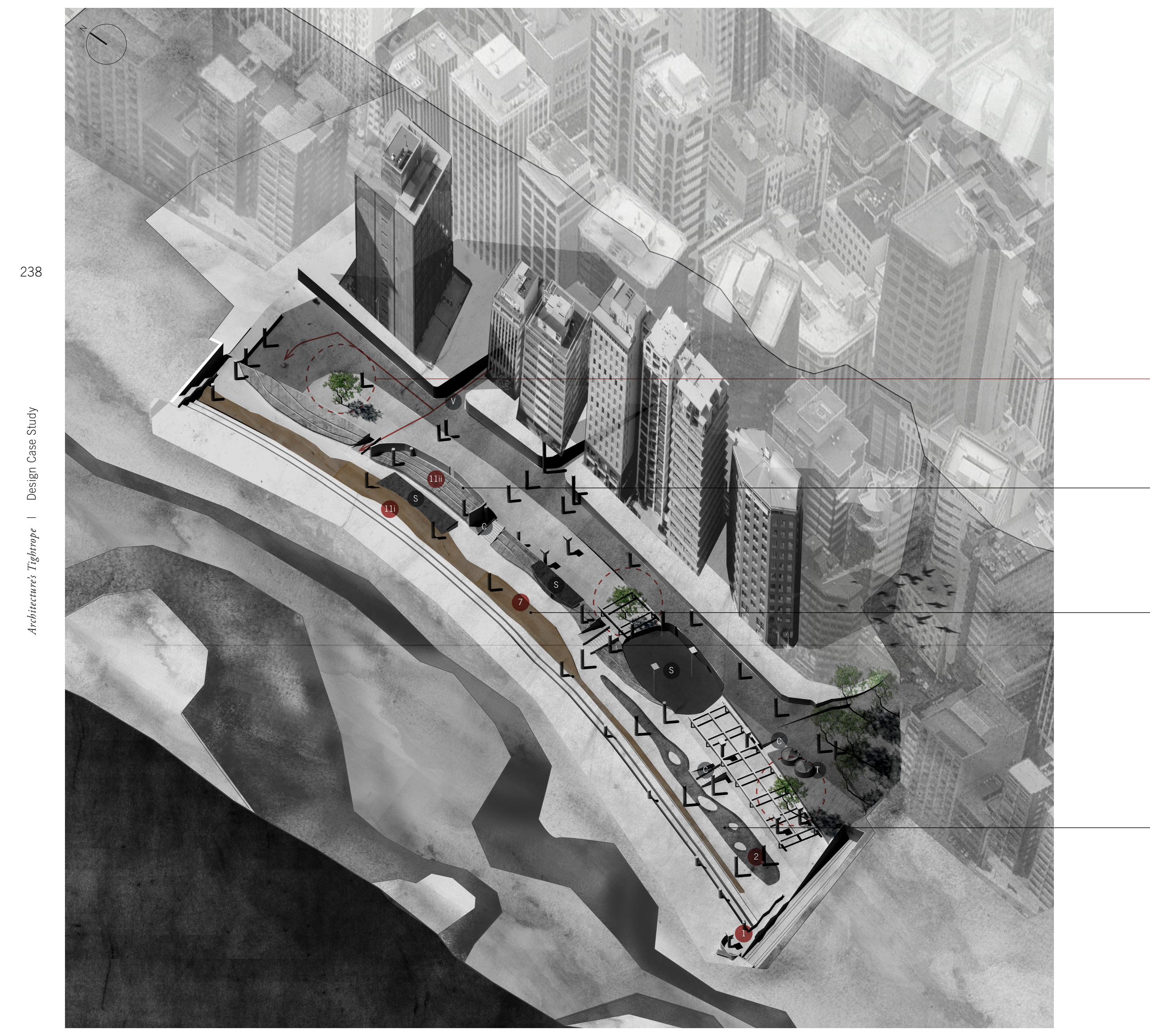




\section{Implementation of Strategies: Ground}

Analysing the ground levels, Datum 0 and Datum 3, in isometric view (Figure 9.26) reveals how the critical indices have yielded a performative infrastructure. Arriving at the site from the cable car (1) presents a full view of the timber terraces (7) and (11i) and and gravel/soil pit (2) that run along Datum 3 and the western boundary of the site. These elements create a material and formal continuity/discontinuity with the existing landscape and infrastructure. The timber starkly contrasts the shot-crete terraces of the western hill-edge of the site in a material discontinuity, positioning them as a distinctly inserted element. However, the form of these terraces - drawn from the topographical contours of the greenbelt - suggests continuity with the natural landscape, an extension of the existing geography. Thus the typology and materiality of The Terraces work to resist the tabula rasa techniques that have conditioned the thick edge. Shifting east towards The Terrace the language of The Terraces changes in materiality, introducing in-situ poured concrete - a gesture that signals a shift as one transitions through the design. The edge becomes less 'natural', more designed, as 'softer' timbers, soils, and gravel along the western site edgeway to the 'hardness' of concrete toward the eastern site edge. As The Terraces are also used for spectator seating, performances, and other public spatial practices they contribute to the addition of social space within the site.

The gravel pit (2) creates continuity with the natural landscape by 'exposing' the earth beneath. This method of grounding considers Datum 3 as a historically embedded layer, a local reference point. The pit is curvilinear in form, referencing (without replicating) the Kumototo Stream that has been

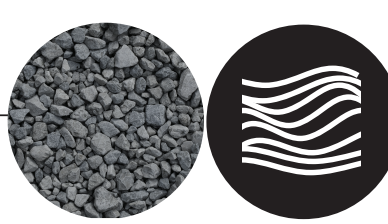
buried beneath the car park. The gravel and earth that are exposed in this 'river' initiate a questioning of how the ground has been previously treated - covered in concrete and left to the devices of infrastructure. The reconstitution of the material is a gesture that draws from the theoretical experiments of land art practices, re-locating the site as a historically latent medium. Unlike processes of excavation which shaped the site, here the subtracted earth is reconstituted to create The Terraces as continuous with the landscape. Additionally, excess soil is placed in bags and piled

up against the bases of columns, creating a dialogue
Figure 9.26: Isometric image of the two ground levels andscapes, Datum 3 and Datum 0 between the columns as elements imposed into the site and the removal of soil as intrinsically linked to them (see Figures 9.23-9.25 and Figures 9.49. 9.50). Within the gravel pit four concrete pads are left behind, as performance stages and sculpture sites. Placing public art upon these pads destabilises the identity of the concrete ground plane as one of parking and reconfigures it as a space for the addition of public art; identity is further destabilised by 'curating' art upon an unexpected contextual field. Much like Lacaton and Vassal, space for performance, continually changing public art, and public interaction is treated as paramount, and the singular programme of the site is shifted towards dynamic relations.

Three Pohutakawa trees are planted at specific points to introduce natural elements to the ground plane and to reference the historic kainga settlement. These trees inform punctuations through all of the datums above - to allow natural light and to emphasise the importance of natural and historical elements within the design (See Figure 9.22).

Exploding the buildings positioned along Datum 0 (Figure 9.27) demonstrates the density of $\mathrm{BOH}$ performance and service facilities at this level. Each of these structures contributes further to the strategic design of the facility. The language of each building as it relates to the overall system of indices is presented in Figures 9.28-9.32. design (see Figure 9.22). Generally speaking, commercial buildings have been designed as rectilinear (orthogonal index) and from concrete. These two operations create continuity with the existing infrastructure, contributing to the language that orthogonality is intrinsically related to the site's rigid commercially driven history. Conversely, performance buildings are designed as curvilinear (fluid index), a formal typology that is discontinuous with notions of rigidity (orthogonal index). The use of concrete in this manner may appear as a gesture of continuity; however, the radical forms of the performance spaces (further expressed by the undulating canopy) are intended to subvert its use as a rigid material, creating a new context from the dominant language of the existing context. Negotiating both of these languages, service buildings are constructed from brick - a traditionally industrial material - visually separating them from the concrete commercial/performance dialogues (see Figures 9.28 and 9.30). 

$<$ LEFT, OPPOSITE

Figure 9.27: Exploded isometric mage of the two ground levels with structures positined along Datum 0 .

RIGHT >

Figure 9.28: Isometric image of the loading bay. This is privately accessed space.

RIGHT > Figure 9.29: Isometric image of the plant room and circulation tower (ground level only).

The plant room is private access, the circulation is accessible to all publics.

RIGHT > Figure 9.30: Isometric image of the workshop including storage (underneath terraces) and separate toilet This is privately accessed space.
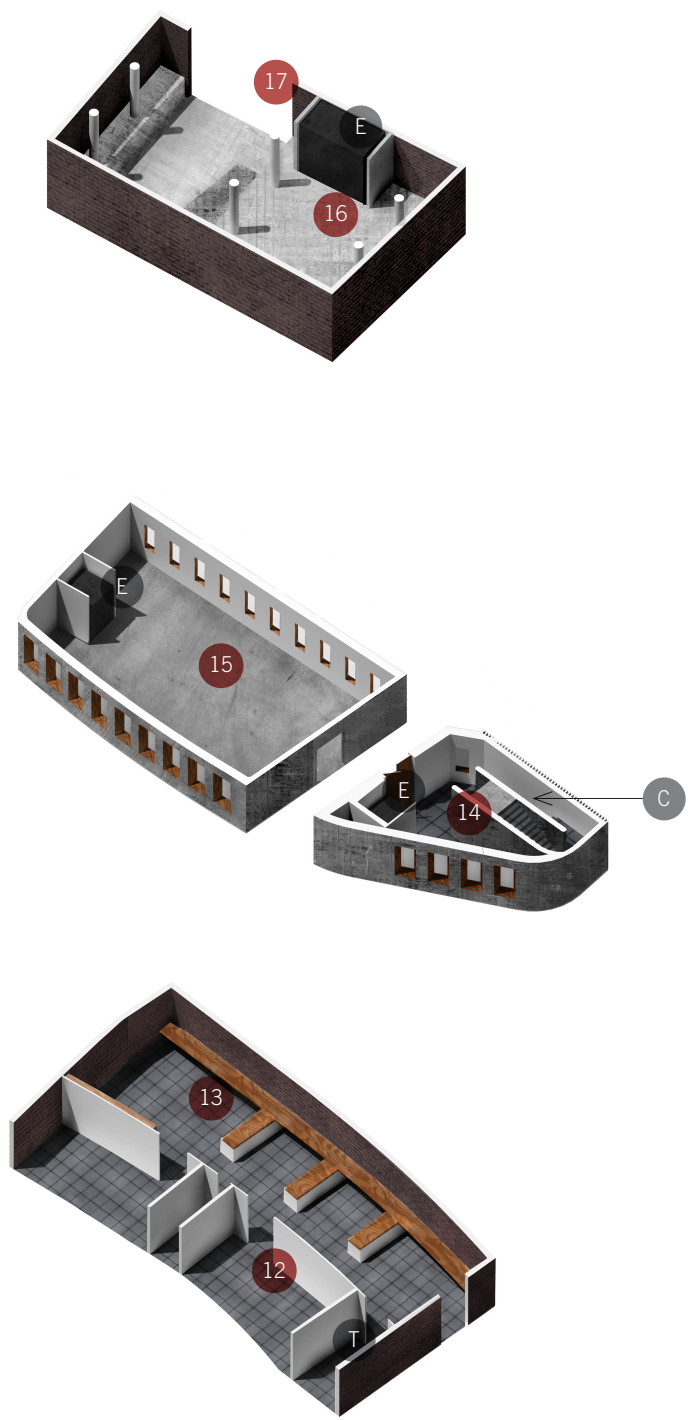

RIGHT > Figure 9.31: Isometric image of the major (shared) performance $\mathrm{BOH}$ facilities. This includes backstage, storage, dressing rooms, storage and private circulation. This is a privately accessed space.

RIGHT >

Figure 9.32: Isometric imag of the black box performance space. This is relatively self-contained and has been insulated to prevent sound bleed. Separate $\mathrm{BOH}$ and $\mathrm{FOH}$ are included in the design. This is a public-private space as access is limited to those attending the performances.

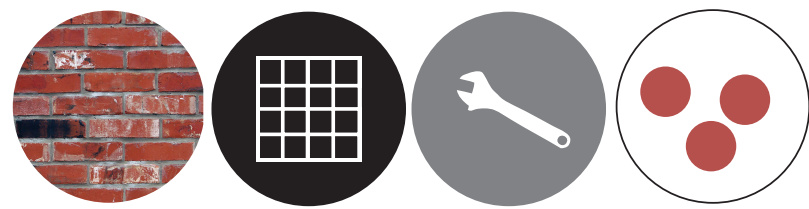

(17) Delivery entrance and turn-around area

(16) Loading bay

Brick, rectilinear, $\mathrm{BOH}$, private
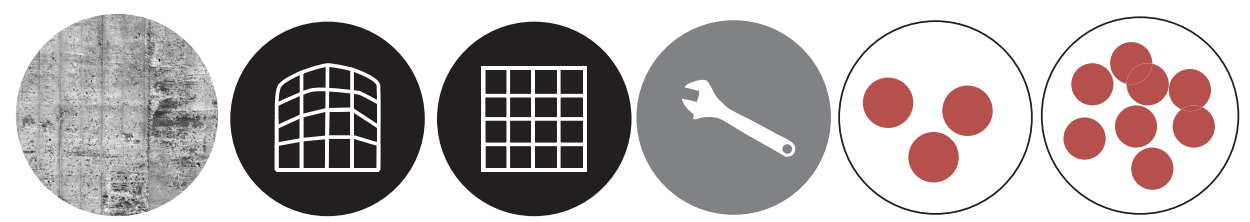

(15) Plant room $\left(200 \mathrm{~m}^{2}\right)$

(14) Circulation tower, stairwell and elevator

In-situ concrete, augmented rectilinear (tower) and rectilinear (plant room), $\mathrm{BOH}$, private and public

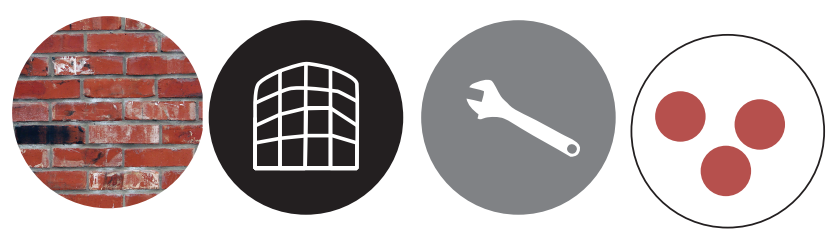

(13) Workshop $\left(250 \mathrm{~m}^{2}\right)$, timber work benches

(12) Workshop storage $\left(100 \mathrm{~m}^{2}\right)$, includes toilet

Brick, augmented rectilinear, private
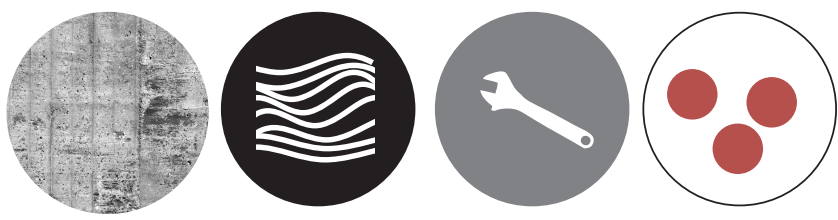

(10) Main performer $\mathrm{BOH}$ dressing/changing rooms (350 $\left.\mathrm{m}^{2}\right)$

(9) Main performer BOH backstage volume $\left(450 \mathrm{~m}^{2}\right)$

(8) Main performer BOH storage space $\left(100 \mathrm{~m}^{2}\right)$

In-situ concrete, curvilinear, $\mathrm{BOH}$ (performance and service), private
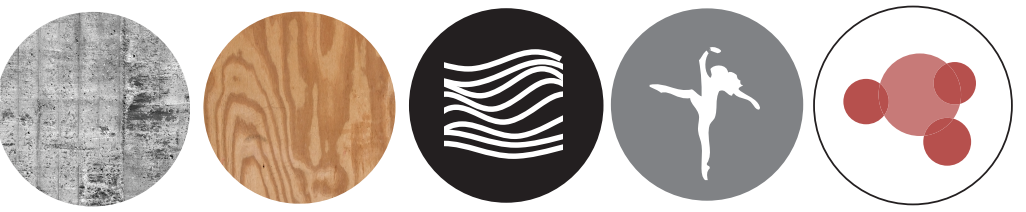

(6) Black Box coat check / reception

(5) Black box performer $\mathrm{BOH}$

(4) Black box performance volume / flexible seating area

(3) Black box permanent covered indoor seating

In-situ concrete, timber plywood lining, curvilinear, Performance (auxiliary $\mathrm{BOH}$ and $\mathrm{FOH}$ ), privatepublic (paid entry for publics) 


\section{Implementation of Strategies: Middle}

Moving upwards, the volume of space between Datum 1 and Datum 2 performs as the core section of public activity and gathering. Exploding the buildings in isometric view reveals that there is significantly more space framed for publics on this level (see Figure 9.34). The four overflow areas introduce a socio-spatial looseness to the structuration of the space, reinforced by permanent public seating, signage, and busking/performance 'pads' (see Figure 9.35). The seating areas are varied in shelter: (29) and (24) are sheltered between Datum 1 and 2; (22) and (20) are open with minimal protection from the canopy and loggia. Handrails border the datum and the punctuations through it.

The strategy of horizontality is crucial to the conditioning of looseness at this level as the designs are individually separated in plan between Datum's 1 and 2, allowing social flows between all of the spaces. Some of the spaces are necessarily private and closed off (such as the thru-scenium theatre); however, transparency and porosity is still important. The addition of further horizontal space was required to allow for this, which can be seen in the 'swelling' of the datum around the theatres, (22) and (23). The extension of the datum is marked with the traces of concrete formwork throughout, in a similar gesture to the use of concrete in the Palais de Tokyo. Physical subtraction is also revealed along the datum - the

punctuations are experienced at human scale with the vertical penetration of Pohutakawa trees occurring regularly where there is gathering space. The holes act as anchor points, as with the Rolex Learning Centre,

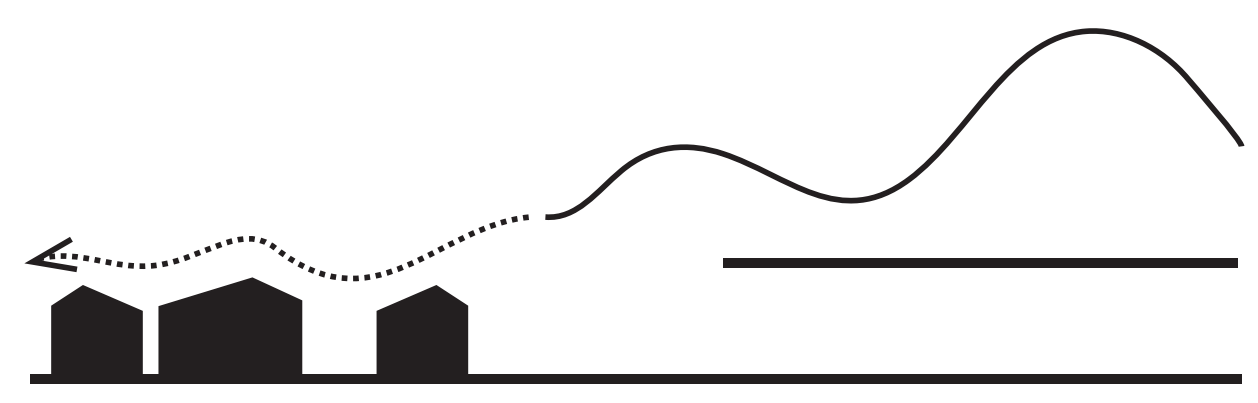

that focuses the public spaces around the re-insertion of natural elements.

A new formal and material language is also created with the introduction of $\mathrm{FOH}$ facilities that are designed for all publics (see Figures 9.36-9.40). These structures: the toilet block (25) and (26); ticket office (21); café/bar (20); and bookstore (19) introduce the use of timber and glass. All of these buildings are clad with a $100 \times 25$ ship-lap system, and are walled with large sliding glazed doors. This use of glazing allows maximum porosity and transparency between inside and outside of the public level (weather permitting).

The ship-lap cladding acts to create visual continuity throughout the designs at a human scaleidentifying them as publicly accessible buildings. The ship-lap is also a reference to the timber construction methods of the traditional villa, bringing into the design a visual language from the Thorndon suburb. This 'residential' typology is repeated in the asymmetrical gable roof lines of the ticket office, café, and bookstore (see Figures 9.11 and 9.20). The gables have been designed so that in elevation they visually continue the undulations of the canopy (see Figure 9.33). These gestures implicate the site's historical context, considering the re-insertion of villa typologies as referents to their politically charged destruction during the motorway incision.

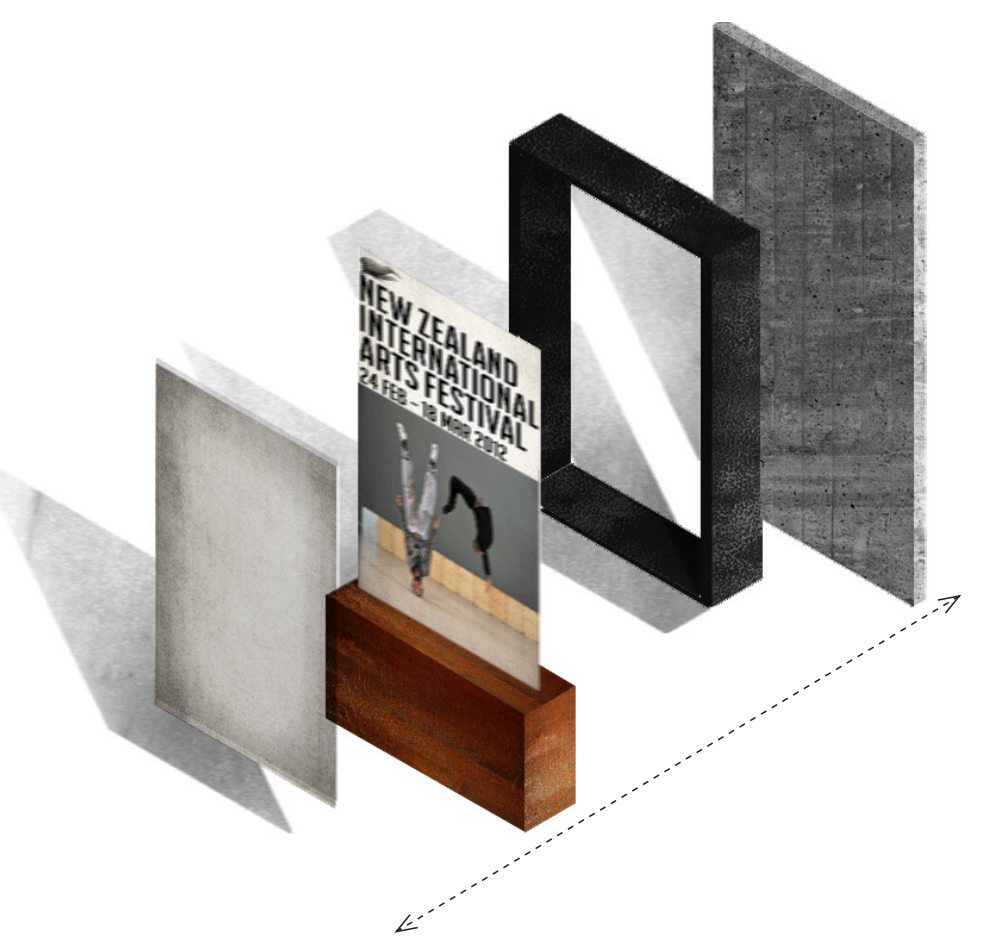

RIGHT, OPPOSITE > Figure 9.34: Exploded isometric mage of the middle level and structures positined along Datum 1 

RIGHT >

Figure 9.36: Isometric image of the storage / $\mathrm{BOH}$ overflow space. Storage is crucial to

the function of the facility. Additionally, in times of peak staff (during the Arts Festival) this space can be used as office/overflow. This is a private space as access is limited to those working for the facility

NZIAF.

RIGHT > Figure 9.37: Isometric image of the studios/hire space and circulation tower (middle leve

The hire spaces are publicprivate as access is limited to publics who wish to rent them; the circulation is accessible to all publics.

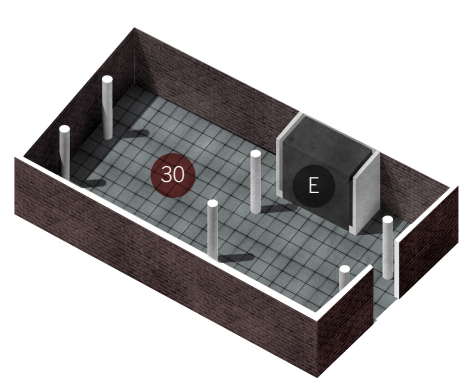

[FOR KEYS, REFER TO FIGURE 9.18]

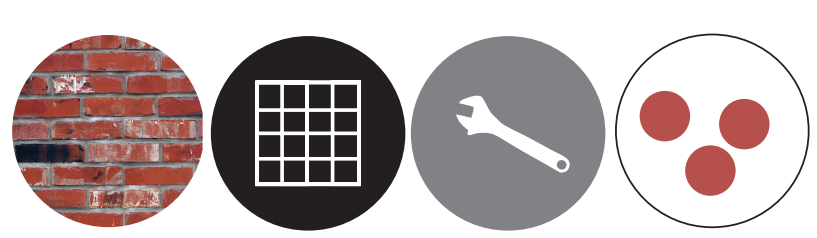

(30) Storage $\left(200 \mathrm{~m}^{2}\right)$ (Recital / rehearsal space above)

Brick, rectilinear, $\mathrm{BOH}$, private

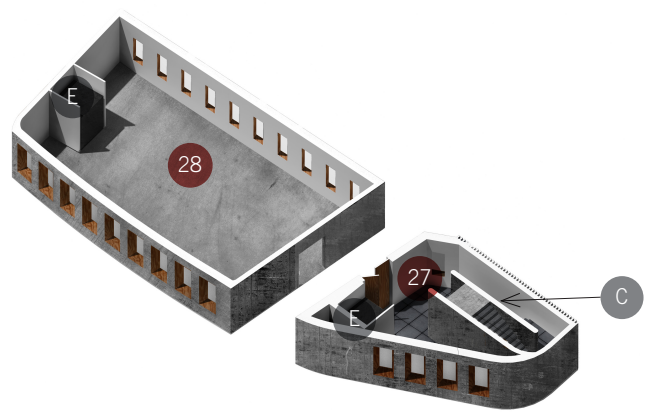

RIGHT >

Figure 9.38: Isometric image of the thru-scenium staging area. This includes lighting/ sound rig trusses above and a proscenium cutain that separates stage from staging.

This is a private space as access is limited to performers and service staff.

RIGHT >

Figure 9.39: Isometric image of the toilet block. The service elevator provides easy vertical access to/from the workshop, making delivery to the major theatres cohesive. The toilet area is located between the theatres so that the facilities can be shared. The toilets are accessible to all publics.

RIGHT > Figure 9.40: Isometric image of the primary $\mathrm{FOH}$ facilties at the southern end of Datum 1. These are accessible to all publics.
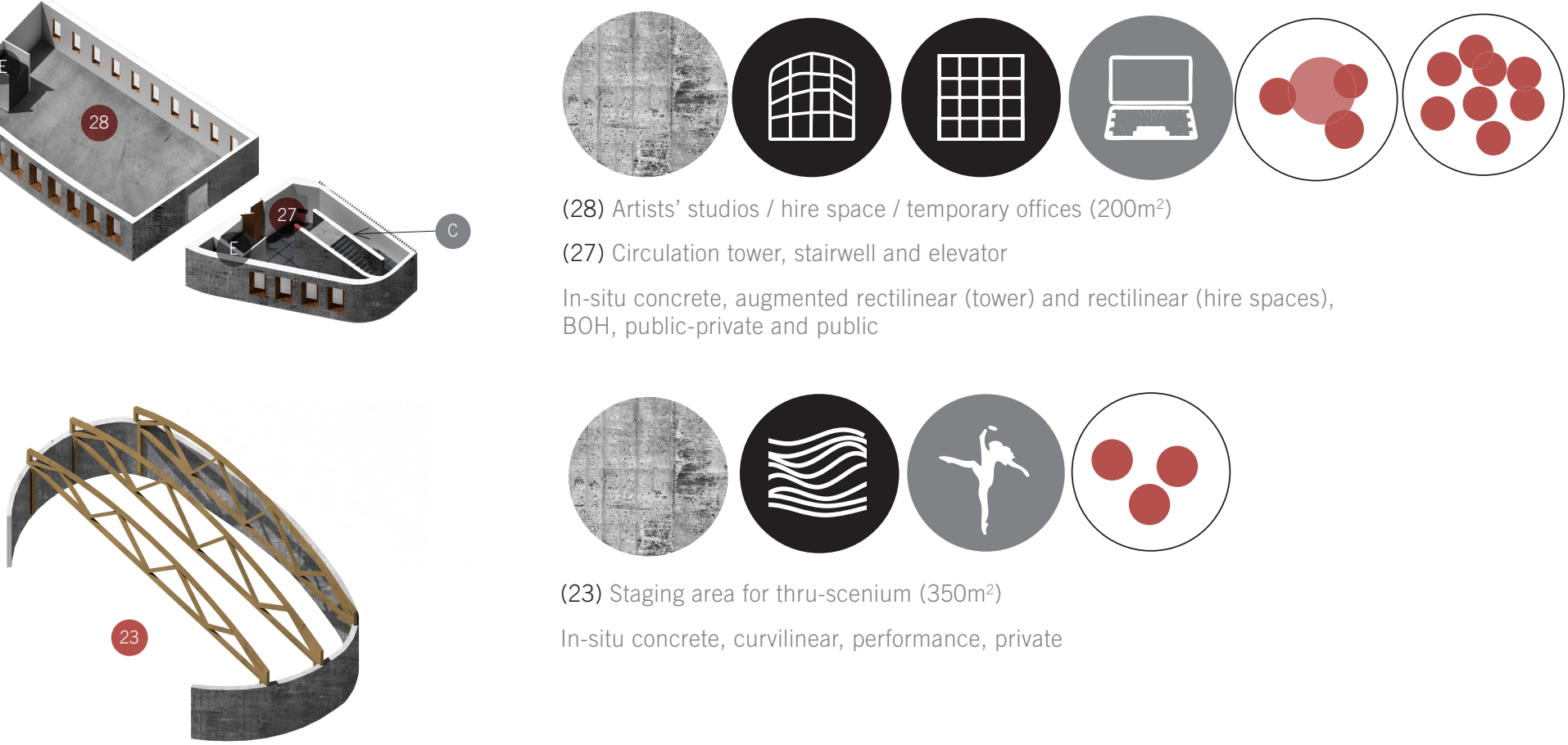

(28) Artists' studios / hire space / temporary offices (200 m²) (27) Circulation tower, stairwell and elevator

In-situ concrete, augmented rectilinear (tower) and rectilinear (hire spaces), $\mathrm{BOH}$, public-private and public
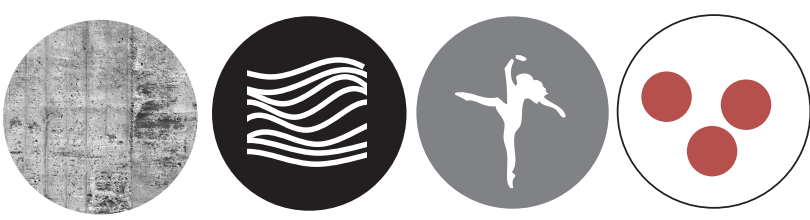

(23) Staging area for thru-scenium $\left(350 \mathrm{~m}^{2}\right)$

In-situ concrete, curvilinear, performance, private

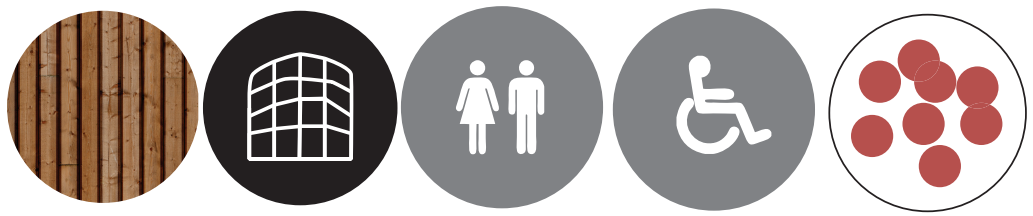

(26) Service / workshop elevator, paraplegic toilets (x2)

(25) Public toilet block ( 9 male, 9 female, 2 parents' rooms)

Ship lap timber cladding, augmented rectilinear, FOH, public

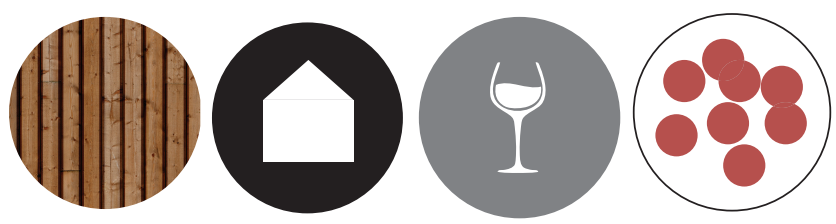

(21) Ticket office / information / coat-check $\left(75 \mathrm{~m}^{2}\right)$

(20) Cafe outdoor seating / loggia

(19) Cafe / Bar $\left(75 m^{2}\right)$

(18) Bookshop $\left(75 m^{2}\right)$

Ship lap timber cladding, residential, FOH, public 


\section{Implementation of Strategies: Upper}

As the beginning of this chapter touched upon, the upper levels of the design inform much of the urban physical and visual identity of Architecture's Tightrope. Strategies of horizontality, verticality and juxtaposition have been deployed within the design process to yield, test, and confirm these identities (see Figure 9.42).

Positioned above Datum 2, the NZIAF offices and circulation tower are the strongest vertical gesture of the intervention (see Figures 9.24 and 9.46). These two buildings are designed to create a visual logic that is in subversive continuity with The Terrace. The regular rectangular fenestrations are positioned symmetrically across the buildings' facades, facades which 'swell' outwards in plan from east to west. This shift from rigidly orthogonal to curvilinear gives the impression that the buildings visually 'soften' moving towards the motorway; simultaneously the interventions mediate between the motorway (as it bends to follow the curvilinear contours of Thorndon) and The Terrace (which aligns with the orthogonal urban grid). Moreover, this slight adjustment in the formal orthogonality of the buildings reveals deep timber casements that chamfer back into the interior - exposing depth of structure (see Figure 9.45). Thus from the outside the NZIAF offices attempt to suggest a subtle resistance to the rigid logic of the surrounding context. These gestures are further exaggerated in the design of the circulation tower which dissolves into an entirely curvilinear chamfer.

The gable roof and timber cladding of the recital room adds to the visual language created by the $\mathrm{FOH}$ buildings. However, given its functional requirement for privacy, the glazing that wraps around the space is constructed from industrial channel glass (see Figure 9.43). This opaque glazing system allows the space to 'glow' when in use, suggesting activity to the outside by projecting dynamic silhouettes and movement (See Figure 9.25).

All three of these buildings vertically 'puncture' through the canopy grid - and are purposefully segregated from its curvilinear language.
They continue, in one way or another, the material and typological languages established in the levels below. Conversely, viewed from the outside, they hint at the indices that are explored throughout.

The most immediately contrasting design element of Architecture's Tightrope is the canopy. The fluid-form concrete and steel structure interrogates several strategies as well as performing multiple pragmatic functions. Its design emerged from the index of fluidity - visually curvilinear and simultaneously framing the socio-spatial flows below. The canopy is derived from a flat horizontal datum (a replica of Datum 1) with a grid imposed upon it. Starting from a grid allowed the canopy to be manipulated at multiple points, horizontally and vertically, and to instruct the new elements using the language of the infrastructure. Its vertical axes are extrusions or depressions based upon the positioning of the performance areas, creating variable undulations across the datum (see Figure 9.41) that continually transform spatial scale to reflect high public performance spaces merging seamlessly with lower private gathering spaces. The verticality of the canopy has a similar framing effect as the Metropol Parasol, providing shelter and structure to the open aspects of the site and framing the performance areas which have specific volume requirements.

Horizontally the canopy spreads beyond the widths of Datum's 1 and 2, sliding underneath the Urban Motorway to provide sound panels for the thruscenium and traverse theatres. In so doing the canopy is framing social and performance spaces, expanding the existing cover of the site and opening it to flows of additional spatial practices. This is particularly evident sectioning across the site, explored in traverse sectional images A-A', B-B', and C-C' (see Figures 9.48-9.50). These perspective sections show how the canopy avoids symbolic arbitrariness through its performative fluidity - framing the theatre spaces and programmatic functions.

Visually and physically, the addition of another concrete datum purposefully places the canopy in a juxtaposed dialogue with the motorway 
246

and parking platforms, presenting an enhanced fluidity that reinforces the thick edge space as a transitional environment (threshold) between the orthogonality of The Terrace and the fluidity of the Thorndon hillside. The inserted design is a locally radical new typology, a contemporary form that has emerged through the innovations of parametric design developments - it drastically contrasts the existing fabric and demands the attention of inhabitants from the urban surroundings. However, it is not an iconic construction, nor does it submit to spectacle. Following the precedent of Daniel Libeskind's Jewish Museum Berlin, from human scale the canopy element cannot be grasped in an overall image. As such, it refuses to project a static identity or stable symbolic reproduction. The decision to critically avoid 'iconic' symbolism - combined with the programmatic opportunity to engage covered but open public spaces - also led to the experimental approach of designing a large urban intervention without a 'façade' to mark

RIGHT >

Figure 9.41: Exploded isometric diagram showing the points of verticality that have informed the canopy undulations

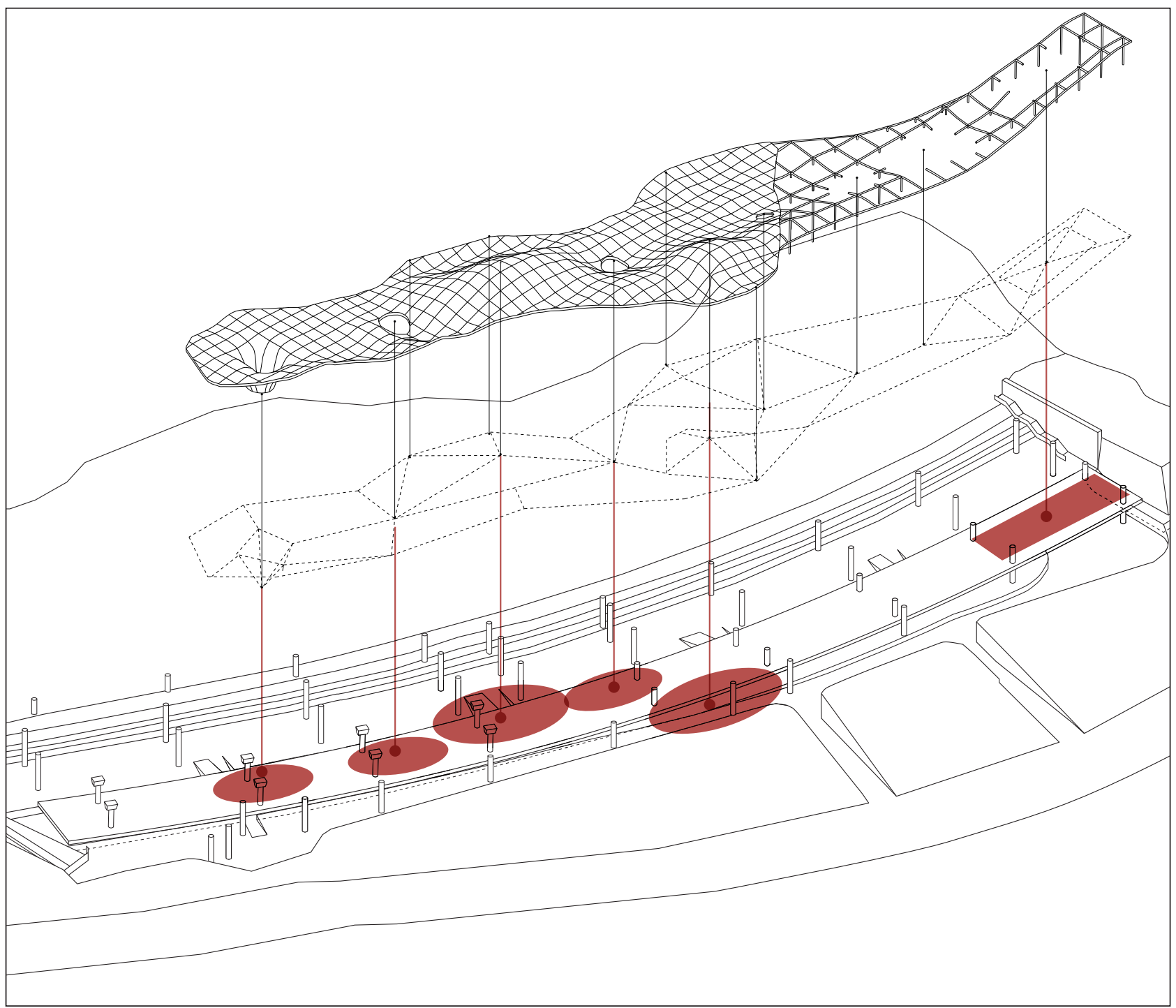

its 'identity'. This is also facilitated by the positioning of the site, as the intervention can only be captured visually in a series of threshold moments as one enters the facility (examined in Chapter Seven).

In these moments the canopy constructs a dialectical image through juxtaposition, deploying the language of the car park against itself. The existing orthogonal context (s) of the site are re-inserted through a manipulated architectural form, constructing a series of temporal images experienced throughout the design. These moments - juxtaposed images - draw attention to the contrast created by Architecture's Tightrope, and the strategies it has performed to implicate context (as 'then') in the present (as 'now'). These moments are explored by re-visiting the four threshold perspectives (see Figures 9.51-9.58).

RIGHT, OPPOSITE >

Figure 9.42: Exploded isometric image of the upper levels

(Datum 2 and canopy) and

structures 

FROM TOP LEFT > Figure 9.43: Assembly isometric diagram of the isometric diagram of the
channel glass system that wraps around the recital room.

Figure 9.44: Isometric diagram
of the recital/rehearsal space During busy periods this space may be used as overflow office space or exclusively for NZIAF rehearsal. This is a publicprivate space as access is limited to those who have hired its use.

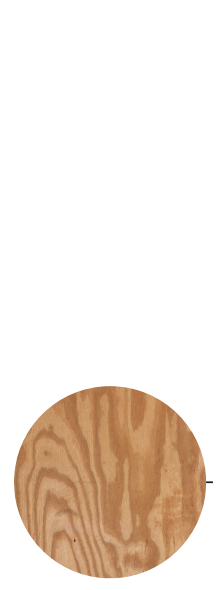

FROM LEFT > Figure 9.45: Assembly isometric diagram window casement system that regularly penetrates tha office building

Figure 9.46: Isometric diagram of the NZIAF office spaces. Each floor has a kitchenette and two unisex toilets. They are connected via an elevator.

The lookout area is enclosed by glazed walls and supported by a steel post-and-beam truss
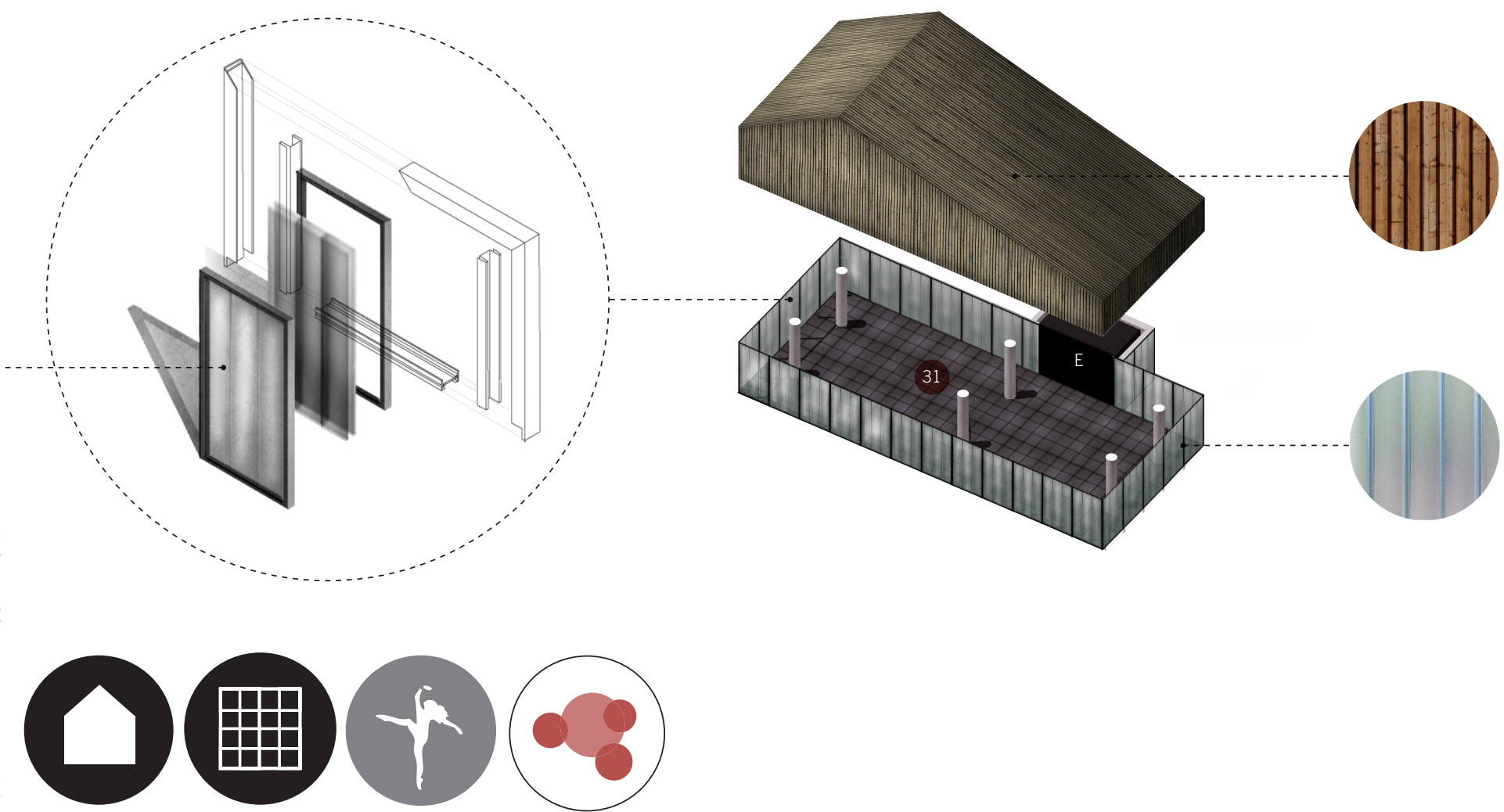

31) Recital / rehearsal room $\left(200 \mathrm{~m}^{2}\right)$

n-situ concrete, augmented rectilinear (tower) and rectilinear (hire spaces),

$\mathrm{BOH}$, public-private and public

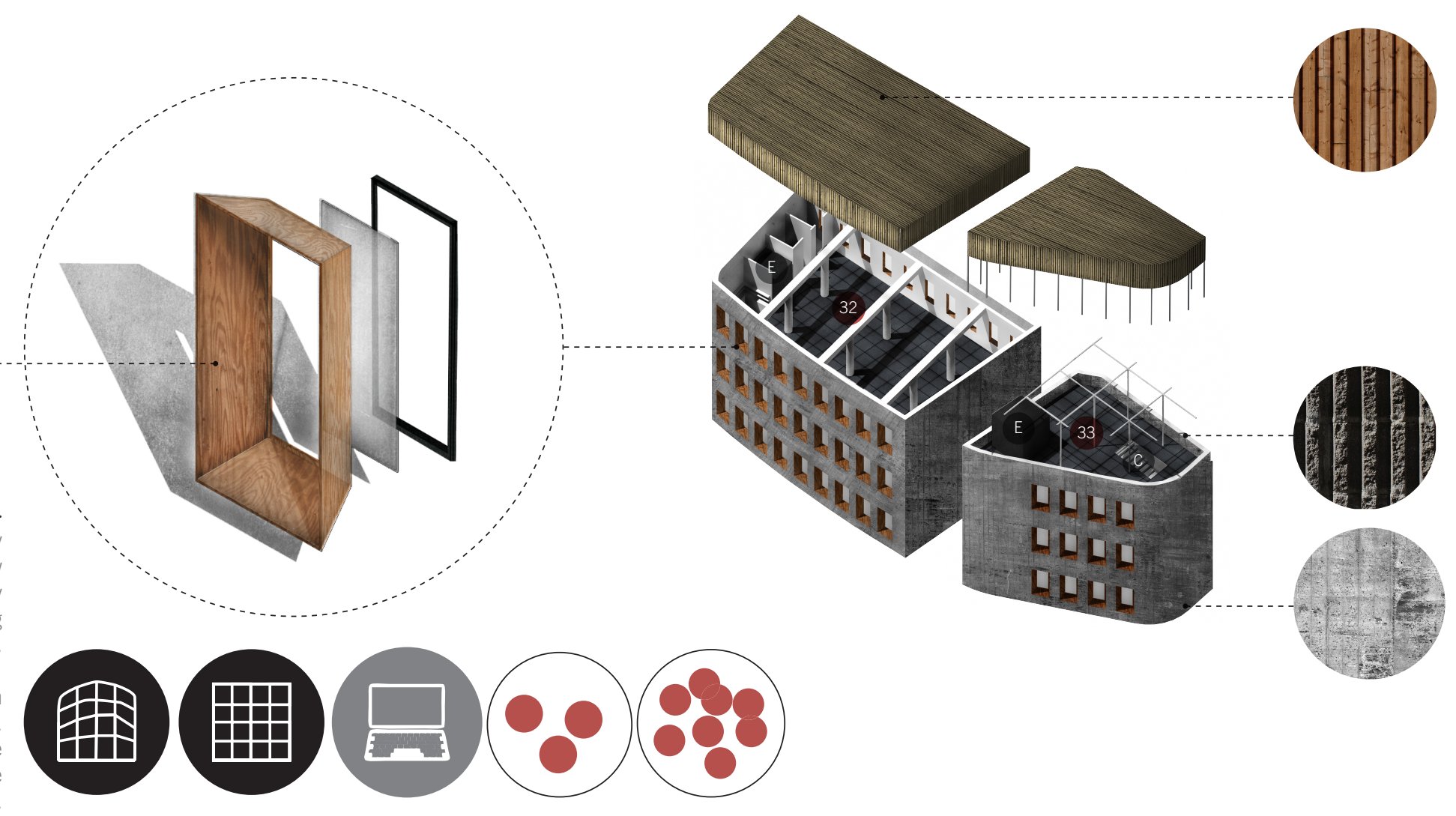

(32) NZIAF permanent offices, total 600m² (three storeys @ 200m²)

(33) Circulation tower lookout terrace

In-situ concrete, augmented rectilinear (tower) and rectilinear (offices), $\mathrm{BOH}$ private and public 


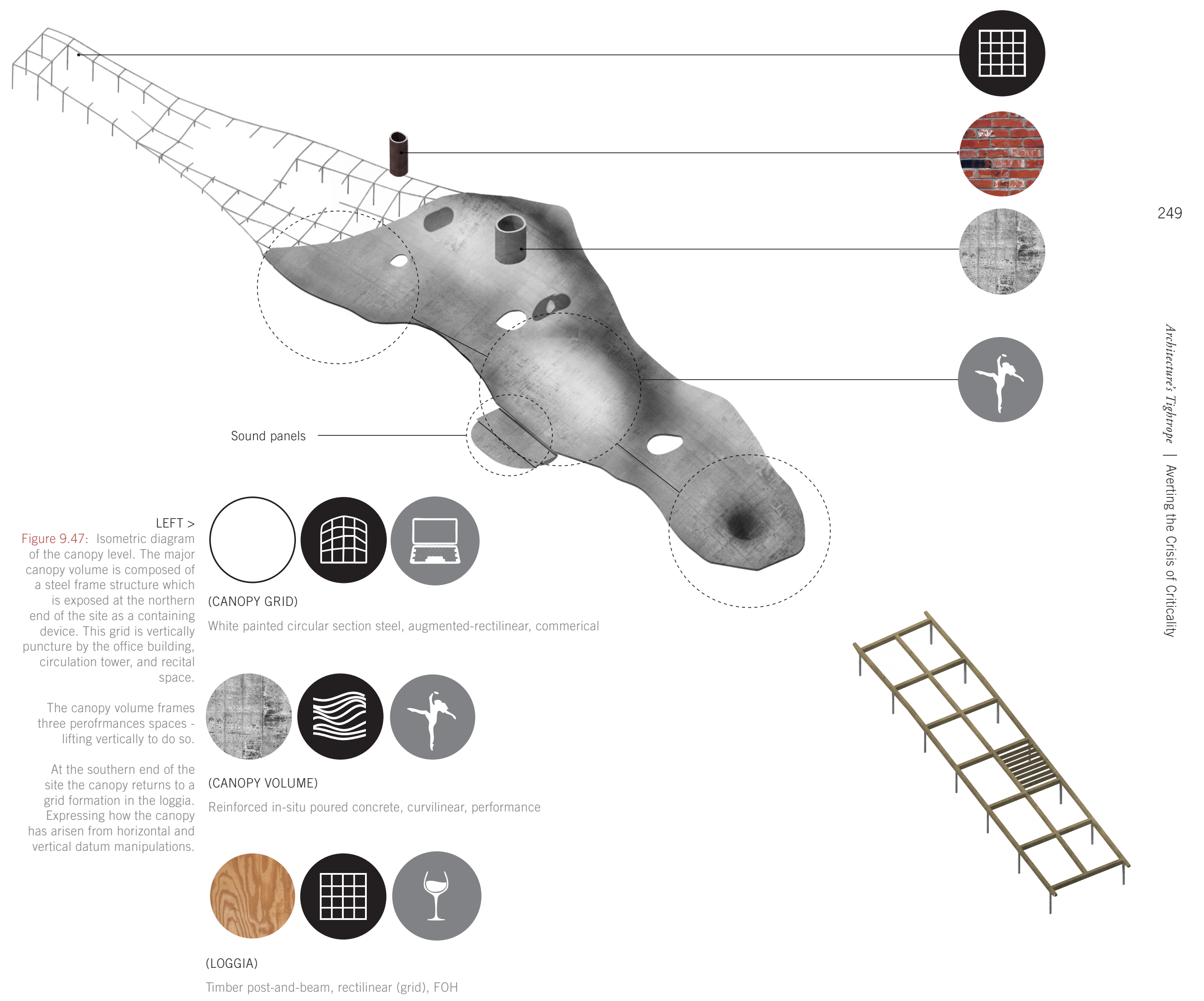




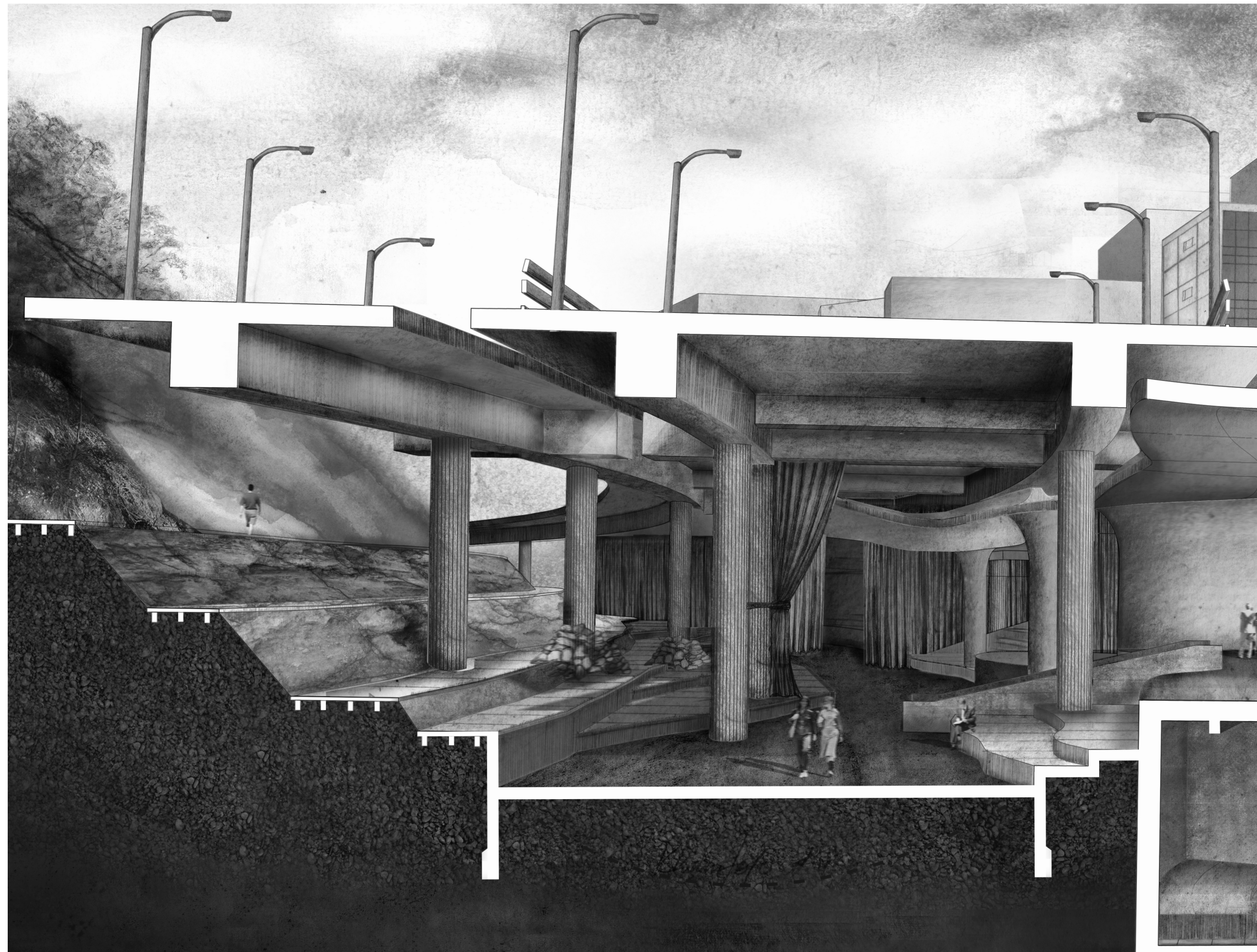

Figure 9.48: Traverse sectional perspective A-A' (looking north) showing the framing of the ck box theatre through the performative fluidity of the 


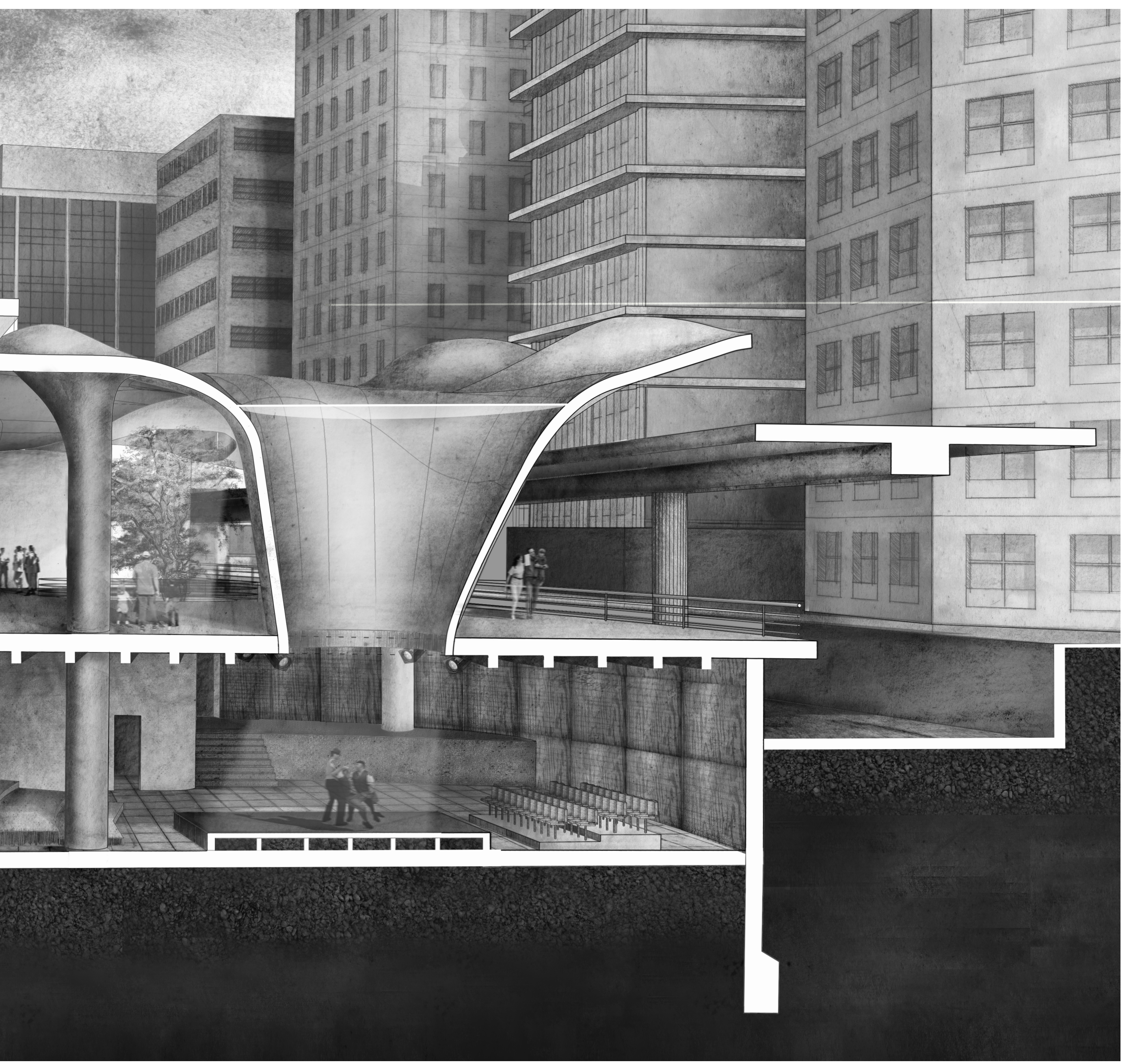




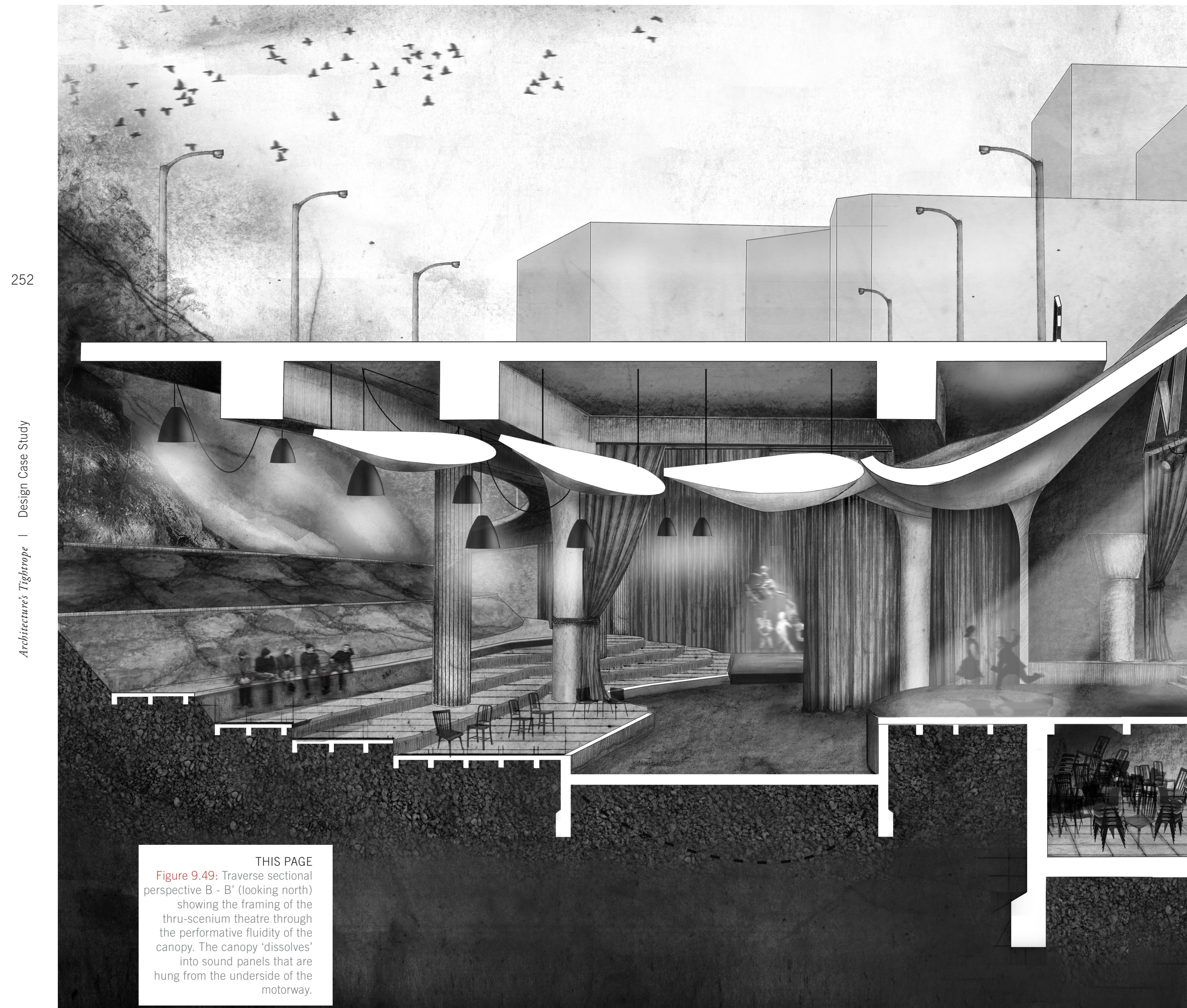




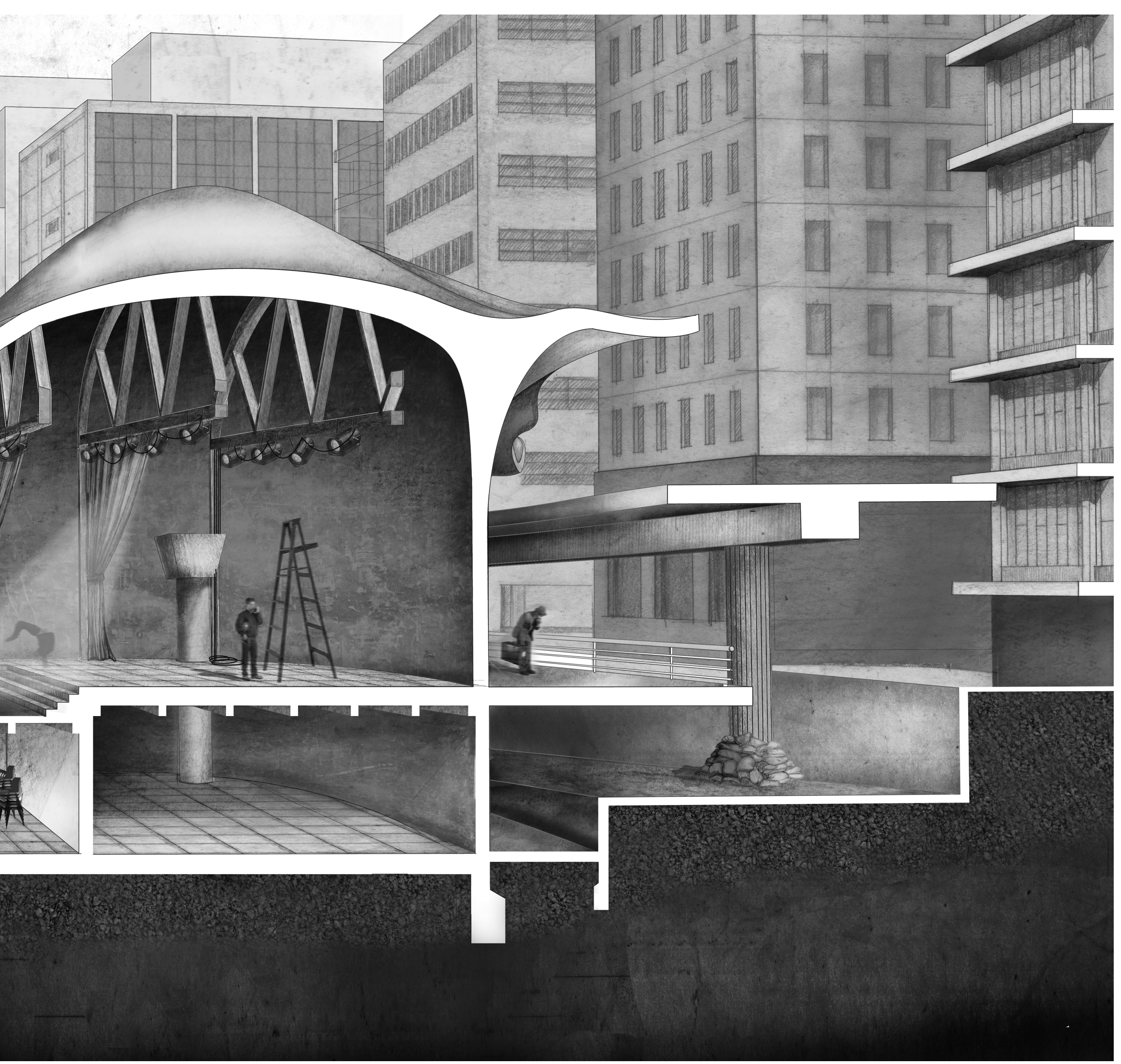




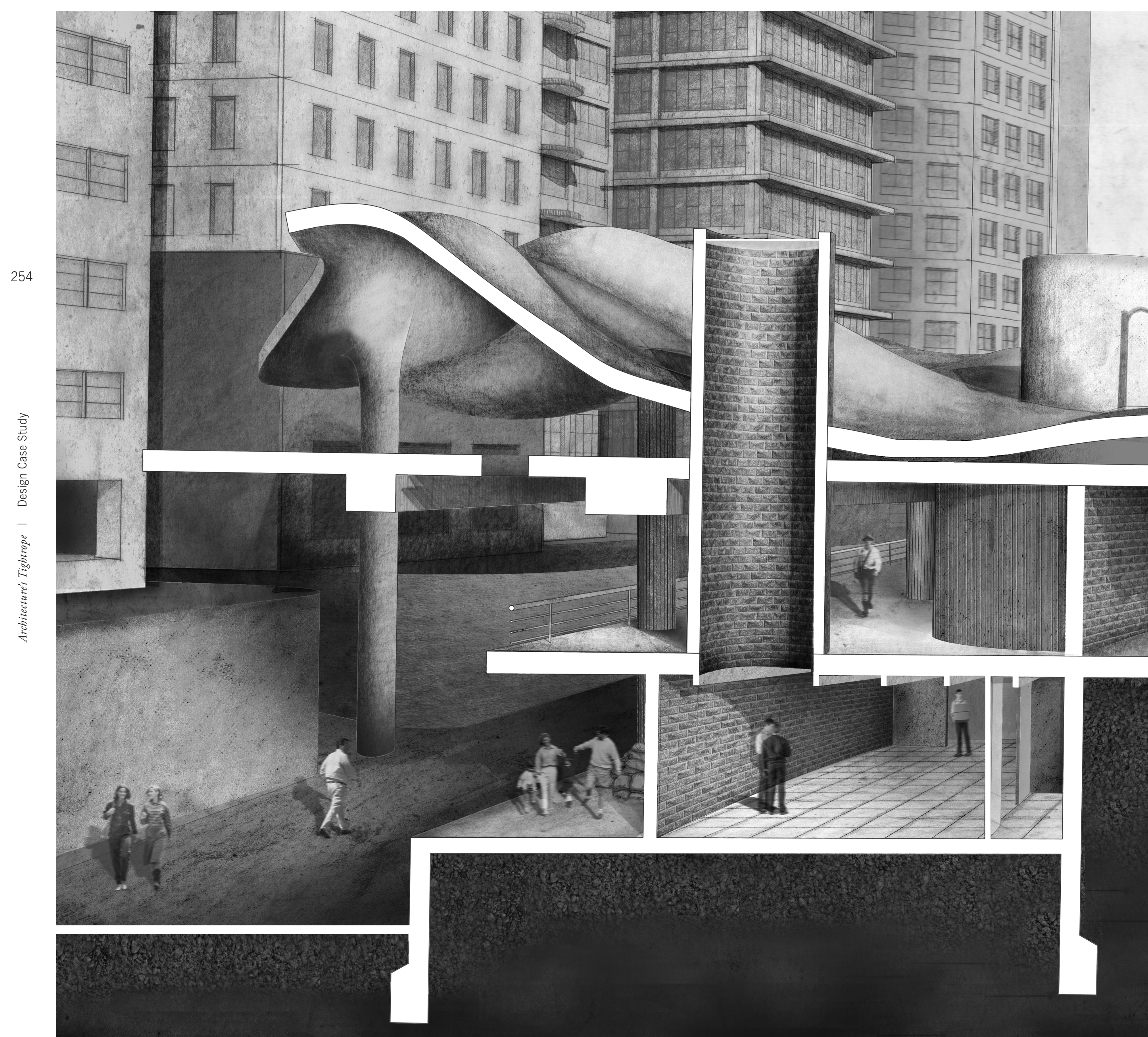




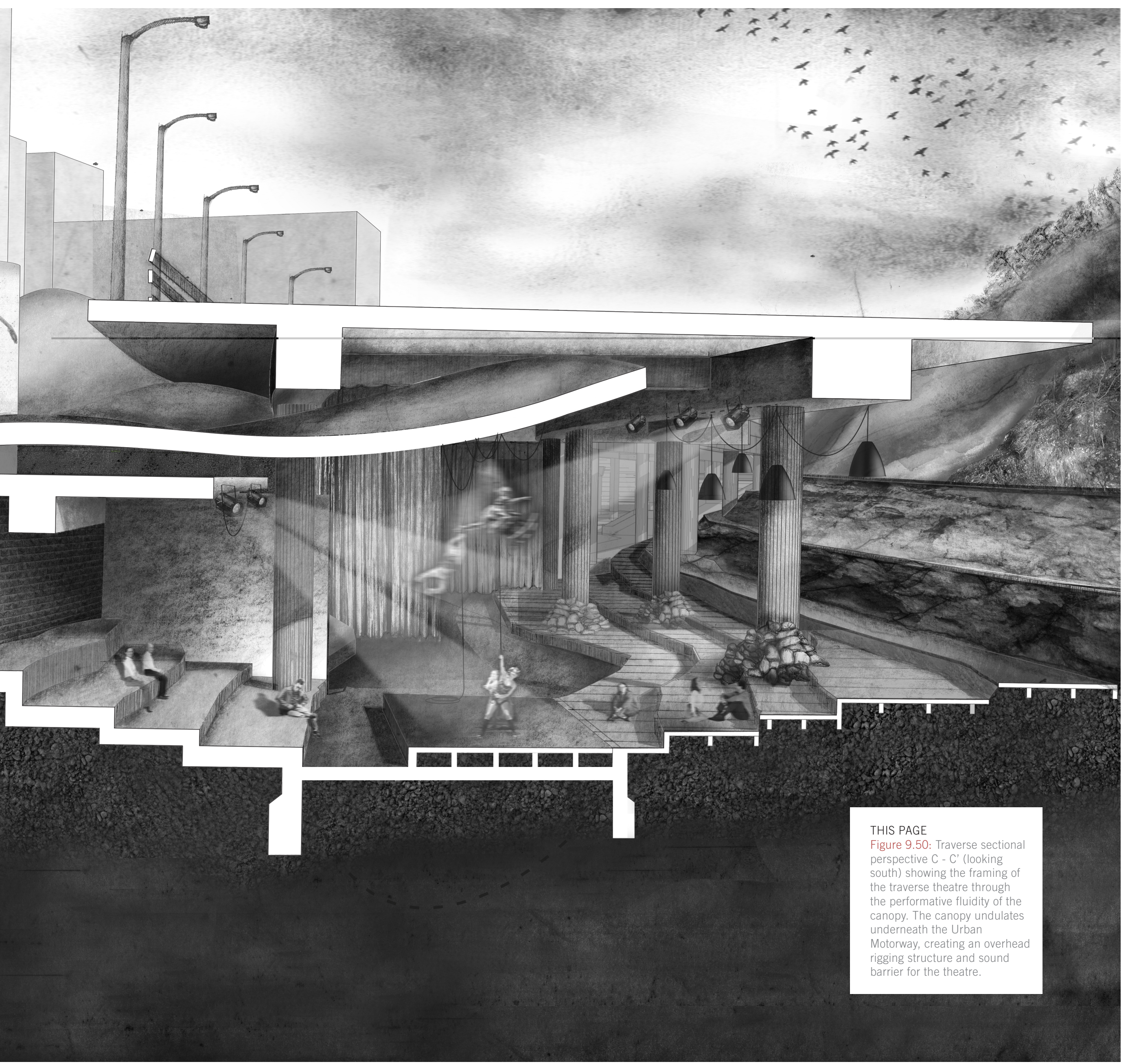




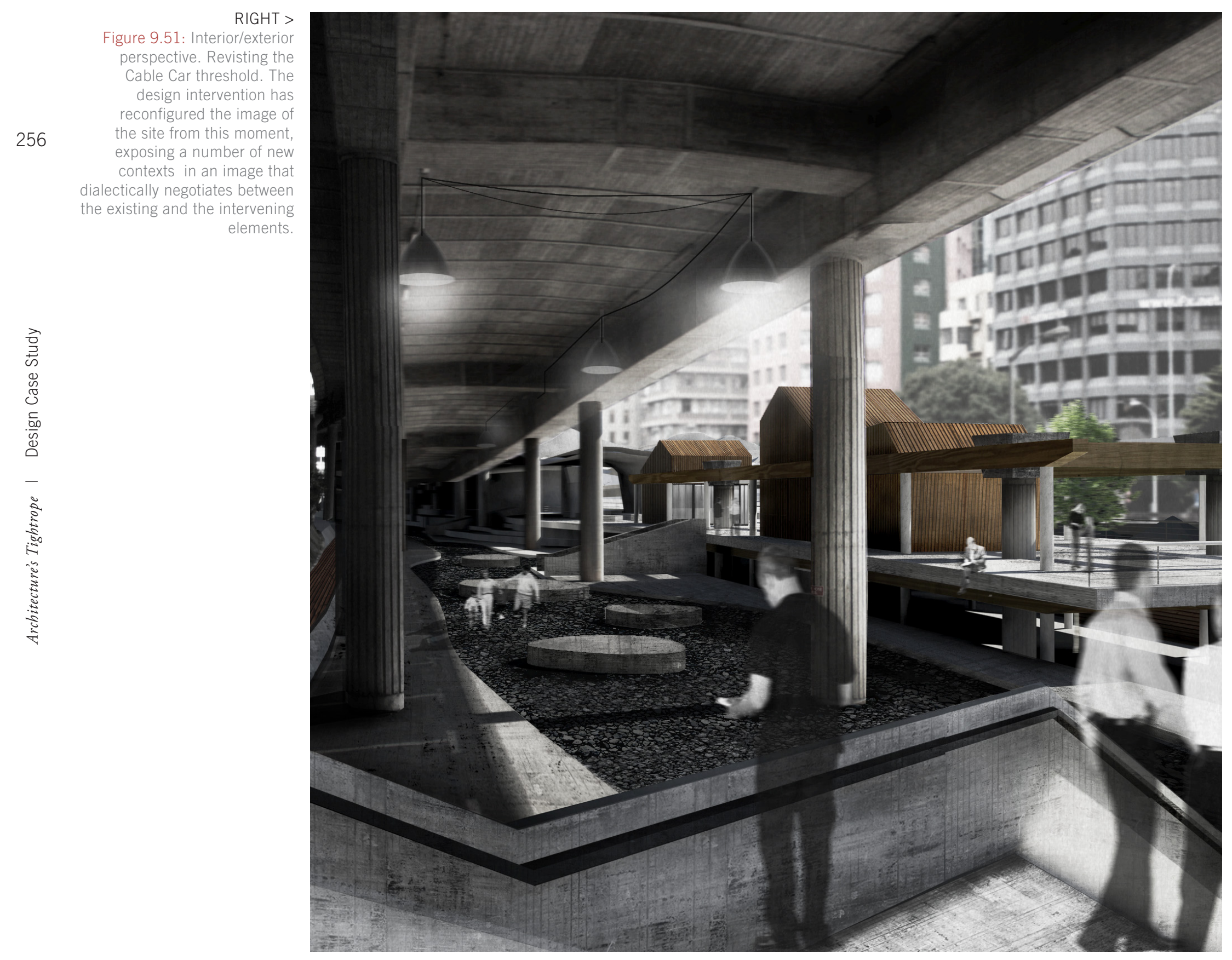




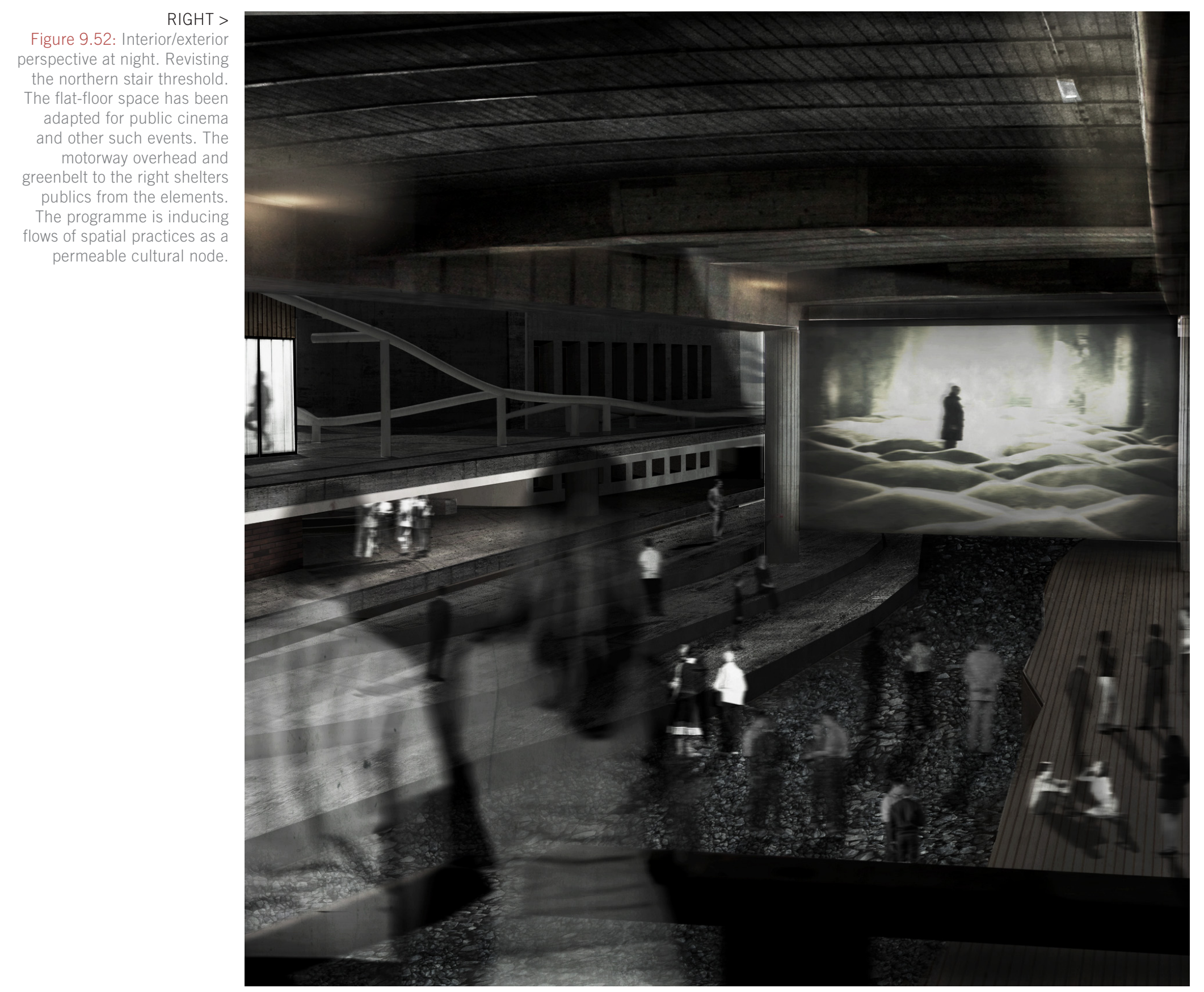



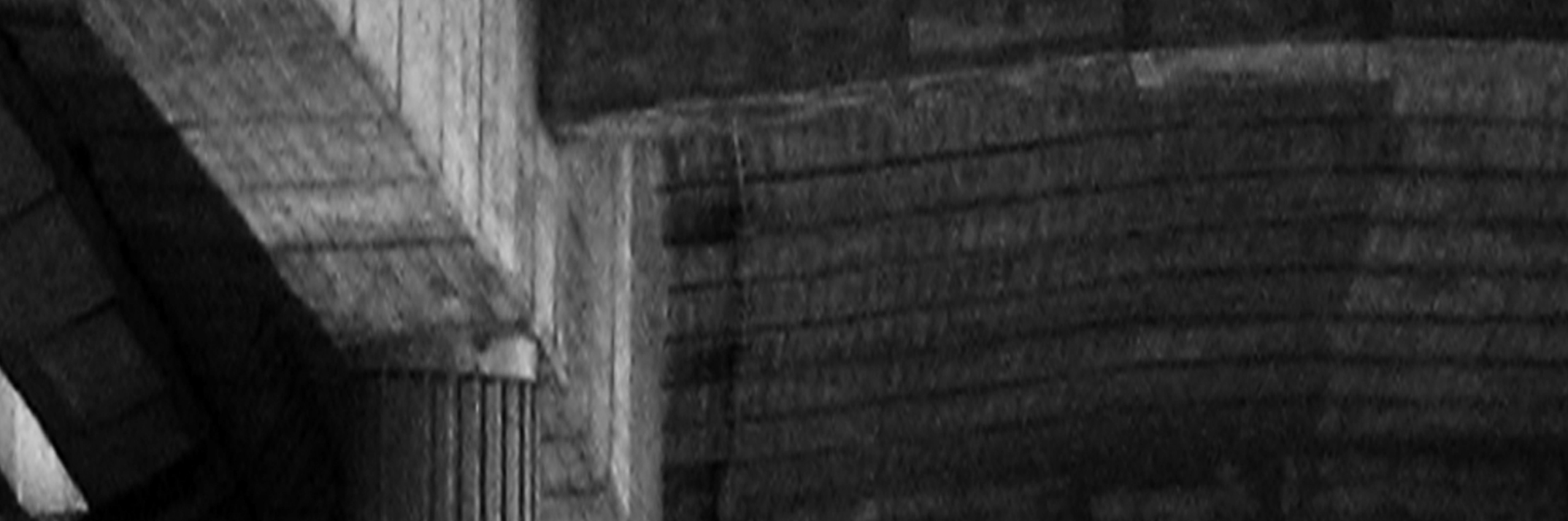

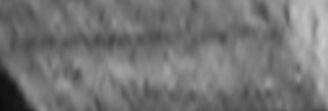
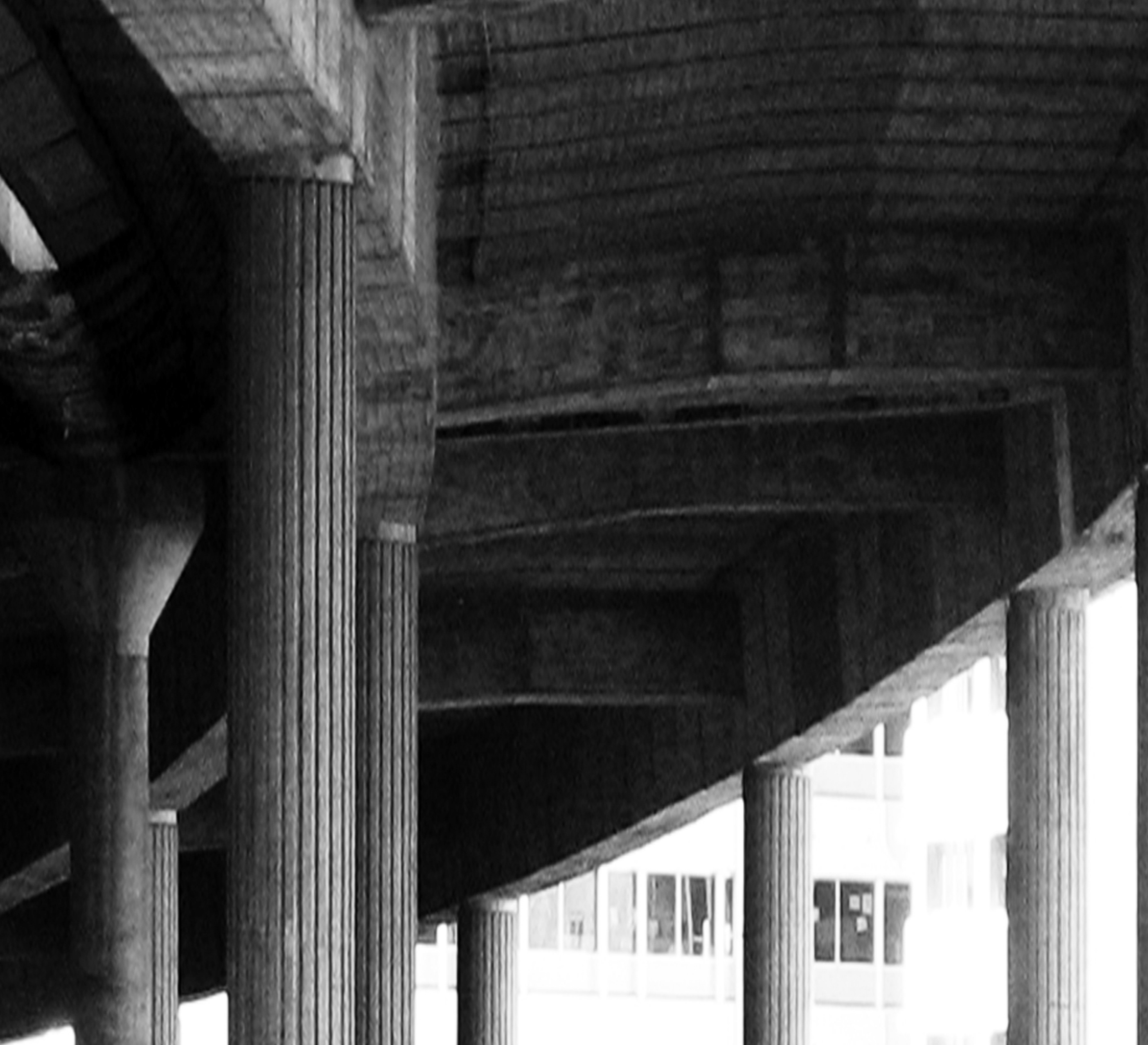
(4) 30

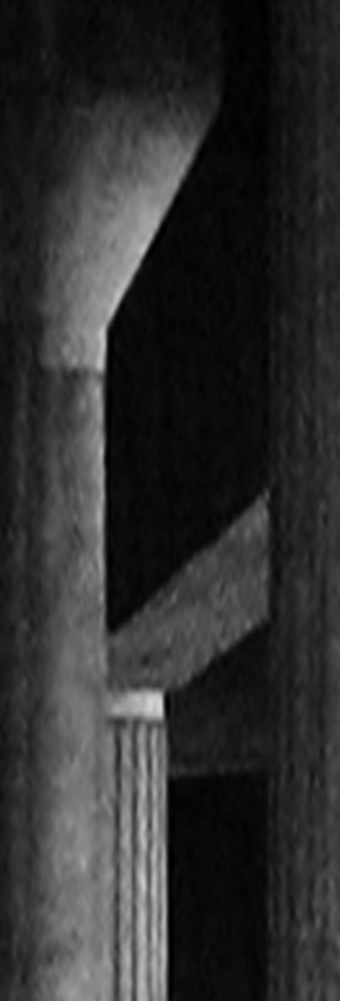

- 


\section{DESIGN-RESEARCH REFLECTIONS:}

Evaluations and Implications

Reflections on Design-research

Evaluations: Inverting the Thick Edge

Conclusion 


\section{Reflections on Design-Research}

The central aims of this design investigation was to test the potential of critical spatial practices, a framework for critical architectural design that is both culturally and formally engendered; and the possibility for such a framework to invert the negative conditions of urban thick edges through an engagement with multiple contexts. These design aims have been pursued in response to two contemporary problems - one theoretical (discursive) and another practical (urban). Concluding the design-research of this thesis, Chapter 10 reflects upon the processes engaged to interrogate the research aims, and the implications for theory, practice and further research relative to the thesis objective. Reflections on the thesis are structured according to the research questions put forth in Chapter One, followed by an evaluation of the design according to the thesis objective to propose a framework for urban development procedures that prioritises multiple contexts in the construction of place identities, re-establishing these thick edges as potent opportunities for critical architectural intervention.

[What are the conditions of a 'critical' architecture and what processes have contributed to its currently maligned position and a discursive 'crisis of criticality'?]

Discursively, an argument for a contemporary criticality has been established - emphasising the impacts of a post-critical position and thus the compounding importance and vitality of a focus for critical architectural design that re-engages Hays's dialectic mediation between formal and cultural production.

[What are the problematic implications of the postcritical position, and how can critical spatial practices provide a contemporary, flexible and resistant alternative?]

Throughout Chapter Two, the 'crisis' of criticality and instigating post-critical impasse has been contextualised, critiqued and deemed ineffective on a number of counts. Critical spatial practices was posited as a flexible, positive and culturally engendered framework for architectural design - an alternative to both the autonomous and post-critical positions. Consequently, practices that are site-specific, sociospatial and temporal, have been identified as a means for providing design processes with a framework for engagement with 'real' world concerns, as well as engendering architectural interventions with a critical positioning.

[What are the negative outcomes attributed to the proliferation of urban thick edges, and how can architectural intervention provide positive alternatives and approaches to this contemporary problem?]

Investigating critical spatial practices through a practical problem, the demand for new solutions to the conditions of urban thick edges has been identified. Thick edges were shown to manifest as a bi-product of 'jump-cut' urbanisation processes, created by the incisions of infrastructures such as the Wellington Urban Motorway. Contextually singular and rigid, 
these thick edges divide communities - dislocating previously congruent social groups and producing culturally disengaged sites. The tangible implications of this urban problem were investigated in Chapter's Three and Four, isolating the Clifton Street Car Park as an extreme thick edge condition that has emerged through development and urbanisation that prioritises vehicular concerns over visual, physical, social, political, economic and historical contexts. Consequently, a critical and polycontextual imperative was established as a means to challenge the current conditions and approaches look to superficially condition or disregard the thick edge as fertile spaces for architectural intervention.

[How can critical spatial practices manifest in the built environment, in particular through strategies of architectural design? How can these strategies engage multiple contexts to introduce positive flows to the urban thick edge? ]

Responding simultaneously to these two problems, Section Two of this thesis has examined the potential for critical spatial practices to engage multiple contexts as a directive for urban development and architectural intervention. Explored through Nick Kaye, Kim Dovey, Gevork Hartoonian and Walter Benjamin, a framework for design was constructed upon critiques that are site-specific, socio-spatial and temporal. Analysing pertinent case studies, strategies of continuity, discontinuity, addition, subtraction, verticality, horizontality and juxtaposition have been found to manifest within the built environment in a critical capacity. Operating within the limitations of this design-research thesis, the investigation of these critiques and strategies has been limited to one author. Expanding the scope of the research would allow a more detailed investigation of these principles and their relationship to architectural practices.

[Can the implementation of architectural programmes that have beneficial polycontextual implications facilitate the production of critical spatial practices within the thick edge?]

In addition to these strategies, architectural programme as a means to revitalise urban space has been investigated as a potentially critical design gesture
- facilitating the structuration of space and spatial practices for multiple publics. The revitalisation of the Clifton Street Car Park into an arts and performance base cultural node has been shown to facilitate the intervention of critical architectures by engaging the social, political and economic contexts of the existing thick edge condition. Framing the strategies elucidated in Chapter's Five, Six and Seven, Chapter Eight identified architectural programme as a crucial component to the reconfiguration of space (physically and socially) through architectural intervention

\section{Evaluations: Inverting the Thick Edge}

Embodying a critical intention, and testing Rendell's framework for critical spatial practices, Architecture's Tightrope inverts an urban thick edge condition by implicating a number of wider contextual concerns within the design to facilitate flow through the Clifton Street Car Park. The result is a design that is both contextually coherent and contradictory, both social and formal, both public and private. The identity of car park has been completely shifted, inverting the thick edge condition into one of permeability and polycontextual flows.

Architecture's Tightrope inscribes the Clifton Street Car Park with architectural insertions and interventions that are site-specific, socio-spatial and temporal: critically engaging the urban problem of thick edges from a historical, economic, political, social, physical and visual positioning. As the design is engendered with this critical positioning, it can now be evaluated according to its engagement and reconfiguration of these contexts.

\section{Social}

As highlighted in Chapter Six, the means by which architectural design frames and produces spatial practices through its programme is crucial to the creation of positive public sites. Sites of spatial looseness resist definition, they deny appropriation, and they enable multiple publics to perform spatial practices in a variety of ways. It is beyond the scope of this thesis to test whether the case study would in fact perform as a node for multiplicities of spatial 


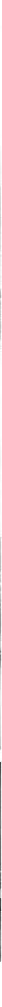

$\wedge$ ABOVE practice. Nonetheless, evolving the precedent of Figure 10.2: Longitudinal section of the design case

study

existing buildings such as the Rolex Learning Centre and the Metropol Parasol, the design cohesively provides permanent and temporary infrastructure for the framing of a number of different events in Wellington's cultural calendar. Aligning with the critical position of Kim Dovey, it has privileged an open, transparent, and porous public space that can be accessed and/or appropriated to inform a structuration of "movements and instability through which stable territories are erased and new identities and spatial practices become possible.".

The placement and configuration of the programme in the design test has holistically implicated the strategies of addition/subtraction and horizontality/verticality. Much like the Palais de Tokyo, these strategies have been employed spatially - where space is considered a luxury unto itself and inserted into an existing negative condition to invert its social rigidity. Therefore, the design can be seen as the addition of several social spaces, and the subtraction of the vehicle-orientated car park space, critically inverting the social context of the site by facilitating social flow.

In so doing, the design has created a landscape of public, private, and public-private spaces, drawing into the site publics who have previously been constrained from its use. Additionally, through much of the design (in particular the middle level) publics not only have unrestricted access, but are also encouraged to perform in a loose, non-determined fashion. Currently there is no outdoor gathering space near the 'upper city' except for Midland Park (which provides no cover in the event of rain). Architecture's Tightrope encourages continual daytime and nighttime outdoor activities as varied as skateboarding or busking $(22,24,29)$, drinking coffee $(19,20)$, reading quietly $(22,24,29)$, watching a outdoor performance, or simply commuting through the site (see Figure 9.11).

The social intention and execution of design is 'critical' insofar as it radically reconfigures the existing condition of the car park - resisting the thick edge by turning it inside-out - enabling a flow of various different activities to permeate the site. Beyond the programme of the facility, the design has shifted the car park towards the conditions and effects of a semi-unstructured and open space (such as Waitangi Park), whilst still recognising the potency of architectural intervention as a formal device to insert infrastructure and place identity. In different contextual conditions the design framework would have shifted the outcomes completely - perhaps 


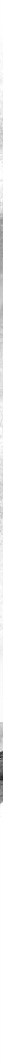

introducing publics to different activities with different emphases relating to those contexts, or even a higher degree of 'openness'.

\section{Political}

The political critique within the design has been a positive one, introducing an identity to a site that currently exists in a state of negativity. As identified in Chapter Four, the site exists between governmentally prescribed land-uses, a zoning tightrope that must straddle both commercial and residential definitions. This condition is not unique - it exists as the political situation of many sites redefined by new infrastructure in central Wellington. Architecture's Tightrope subverts this political nondefinition by saturating the site with contextual identities, creating a relational space that 'draws in' the commercial, residential, and historical identities of the surrounding area.

The design instigates a strong, unique, and public identity to the site. Resisting the superficial notion of place put forth by the Wellington City Council, the design creates a place that is relational and socially produced. Playing upon the notion that Wellington is New Zealand's 'cultural capital', the design structures a cultural node within an
Augé-ian non-place, appropriating the thick edge for positive political use. Thus one political outcome of Architecture's Tightrope is to offer a specific, opportunistic, solution to these residual sites presenting the council with an alternative proposition to the standard car park infill approach.

The political appropriation and re-definition of the site through architectural design is yielded from the specific conditions of this test. However - and particularly in that the design case study approach is polycontextual - in a different location outcomes would shift according to site and surrounding contexts. For instance, if critical spatial practices was instructing a design for a residential site, the design may look to question the political definitions of 'house', 'home' or domesticity in a locational and cultural context. If this design experiment were carried further, the important next step would be to conceive design solutions to sites within varied contextual conditions. By comparing the results, a set of common guidelines would emerge. Alternatively, emphasising a political critique through architectural design may look to resist common identities associated with the programme, questioning further what is entailed in the notion of 'cultural events' or 'performance'.

Economic 
The economic performance of the design is beyond the scope of this thesis, although the design approach specifically introduces capital ventures into the site to increase its economic value to the city. Nonetheless, the design-research has evidenced that cultural nodes introduce economic diversity and flexibility to a site that is singular in its production of income. The intervention of an events facility set within an array of outdoor public gathering spaces reconfigures the use of the site - primarily placing value in the social outcomes of architectural design rather than as a commodity. Positioning the social context above the economic in the polycontextual hierarchy of the design is a criticism of forces and imperatives that have shaped the Clifton Street Car Park as a thick edge. Additionally, the design resists the conciliatory practices that are purported by the post-critical, asking architecture to shift its focus towards multiple contexts, rather than 'going with the flows' of twenty-first century market forces.

This thesis does not discuss or investigate the financial cost or feasibility of constructing such a facility, as this is beyond the scope of the investigation. Given further design-research, it would be possible to explore alternative construction methods, materials, and design scales to test and enhance economic feasibility. This may involve re-visiting the critical gestures of some contexts (physical, visual). An example of this may be to use an ETFE membrane for the canopy to create a tent-like structure, reducing the construction and material cost. Another critical tactic may have been to redistribute the NZIAF offices within the buildings of The Terrace, spreading the identity of the intervention further into the city. However, as a design-thesis, these considerations were secondary to the investigation and exaggeration of strategies that test the potency of form, typology, and materiality in an un-constricted fashion.

\section{Physical, Visual and Historical}

The physical and visual dimensions of the design can be seen as both its strongest and weakest attribute(s). Deriving the formal outcomes of the case study from purely contextual and critical positions has created an intervention that is both radical (for
Wellington) and contemporary - contrasting the existing fabric from within itself. Engaging the formal, material and typological languages of the wider context has instructed an outcome that embodies strategies to critique the existing infrastructural and commercial contexts, as well as projecting a new, positive, architectural identity. These strategies collage the design with specific referents, a system of moments that 'act out' the formal and cultural resistance. As a result, the design has created flows of visual and physical engagement from multiple aspects and scales (urban, human, and vehicular).

Informed by an understanding of representation put forth by the key authors of this design-research investigation, a physical and visual language was tested that engages architecture as a discipline that is relational and subjectively interpreted by the individuals experiencing it. They are the authors of their own visual experiences of the urban environment. As such, the design is composed of elements that are in one sense narrative, yet never determined; the buildings can be either interpreted or 'misinterpreted' according to the occupant. The performative indices of the design are crucial in this regard, allowing the 'image' of the design to be axiomatically and consistently associated with its functioning as an events facility and public park. As a design framework, treating architecture as a physical and visual index allows a critical flexibility as the various indices will change with every site elucidating different strategies and therefore different design outcomes.

The indices used throughout the design also implicate the contextual histories associated with this car park site. Following the position of Walter Benjamin, the historical context is the physical and visual context; the what-has-been and the now are dialectically linked. In Architecture's Tightrope the indices of terraces, excavations, fluidity and orthogonality, resonate between history of the site and its positive, projective use. The design projects forward from an engagement with the past, rather than statically memorialising it or submitting itself to the spectacle of the present. 


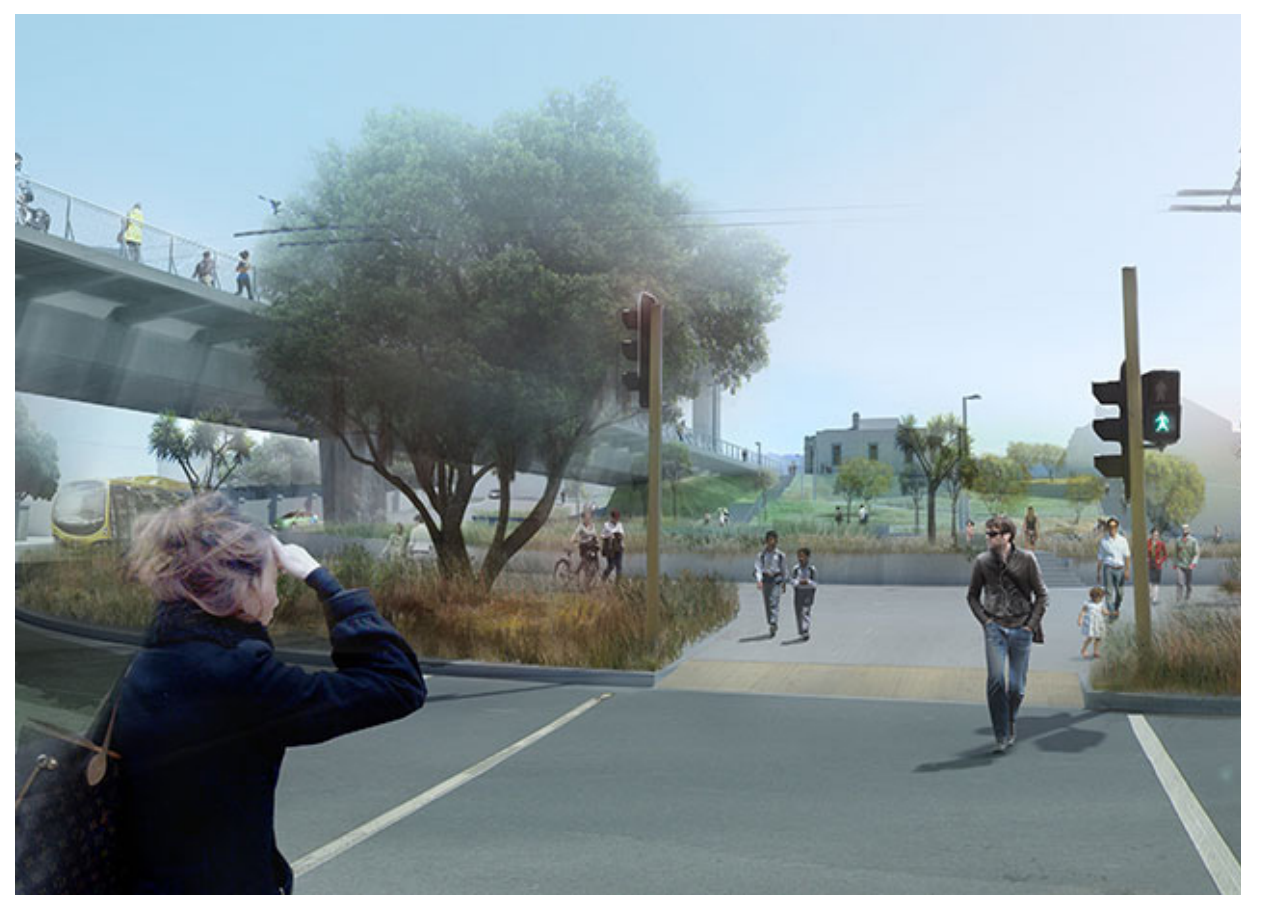

$\wedge$ ABOVE Figure 10.3: An architectural rendering of the proposal for the Basin Reserve Flyover

project.

However, drawing the design almost entirely from its surroundings has limiting implications as well. Designing in material continuity (and formal discontinuity) with the infrastructure datums has had an austere effect on the design - due in no small part to the fact that all of the existing visible 'architecture' was built as highway infrastructure. Further design experimentation may look to articulate a critical language that is less reliant on 'hard' materials such as concrete, timber and brick and could introduce new materials that juxtapose the context in materiality as well as form. In particular, the materiality of the curvilinear elements could be investigated as performing the fluid index in a clearer manner. Additionally, further design testing could explore a manipulation of the infrastructure as it exists deconstructing the orthogonality of the datums and columns to visually and physically 'open' the site even further.

Leaving the infrastructure intact and exposed, although theoretically pertinent, placed a number of restrictions on the design outcome. The ordering system of the car park has had the effect of 'inserting' the design within it; as such the performance spaces are not as articulated as they may have been on a blank site. The scale of the site has also produced challenges for the design; the size of the car park, $350 \mathrm{~m}$ in length, has situated the thesis within a scale that is distinctly urban yet investigating

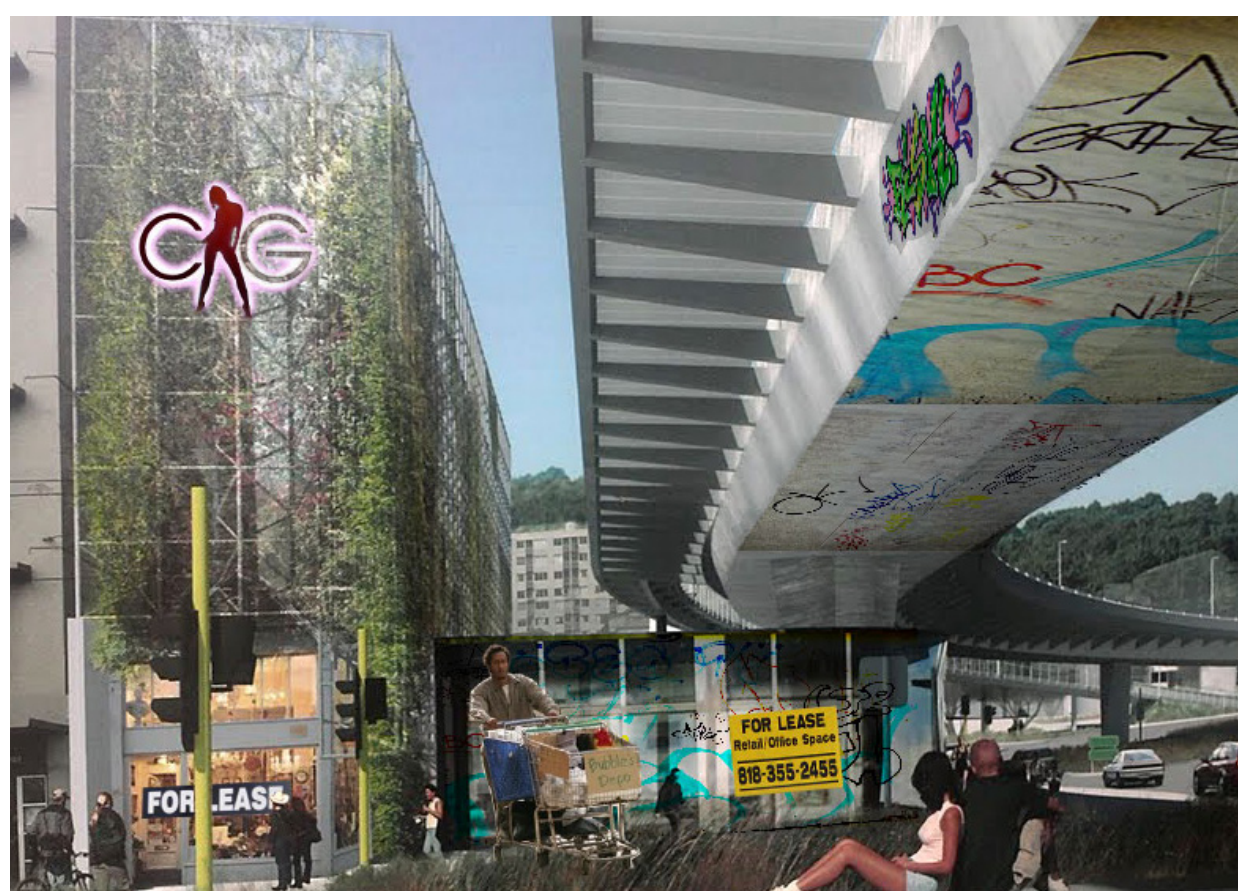

problems as they occur at human scale. Consequently, ^ABOVE the resolution and articulation of the multi-building image of the proposal for the design is less detailed than would Reserve Flyover project. The image has been digitally reconfigured by a member a smaller scale site and programme. Further design $\begin{aligned} & \text { reconfigured by a member } \\ & \text { of the public, suggesting the }\end{aligned}$ research would rigorously investigate and further impending negative social develop the detailing and junction relationships between the performance spaces and the car park, as well as look to the critical potential of the construction process. 
The persistence of the two-fold designresearch conducted by this thesis - a polycontextual engagement with the urban condition of thick edges that are created by new infrastructures; and critical spatial practices as a positive and flexible design framework - brings forth the importance of a critical approach to design intervention. The vitality of architectural strategies to resist, provoke and question dominant forces and popular modes of thinking; challenges architects to engage the discipline as both a formal and a culturally pertinent proposition. Importantly, the application of this approach is not limited to this thesis, it is self-perpetuating - the outcomes of this design-research are now reflexively open to critique themselves, allowing new approaches to emerge from it - evidencing its discursive flexibility.

In light of these understandings, following further research, the framework of critical spatial practices could be pursued further through additional case studies, or an investigation of alternative modes of critique beyond the site-specific, socio-spatial and temporal. Additionally, applied within a different context, critical spatial practices would yield different results - looking to wider contextual dimensions that provide opportunities to expose information that has been previously supressed or marginalised.

The compounding importance of such a flexible, critical position is evidenced by the current solutions (or lack thereof) to existing thick edges 'junkspaces' that have generally been disregarded, filled in by singular anti-social programmes, or unimaginatively and cosmetically revitalised according to a contextually limited framework. This thesis demonstrates that unsightly and undesirable infrastructures can not only be explored for public, social or cultural benefits, but that the may also stand as structures of critique - where their ideological forces of production are subverted and destabilised by architectural intervention. In the case of the Architecture's Tightrope, this critique has emerged as an opposition to the privatised forces of development that have shaped the fabric of Wellington's City centre, in particular through infrastructure and high-rise commercial buildings. Resisting this notion, the design intervention has appropriated the space for public and social concerns - a position that evidences the necessity for cultural engagement.

Current approaches to new infrastructure and urbanisation will continue to produce potentially problematic outcomes and the proliferation thick edges, evidenced by the new proposal for the Basin Reserve Flyover (see Figures 10.3 and 10.4). In contemporary society these developments are acknowledged to have negative and anti-social implications, yet design interventions continue to be limited to a narrow range of outcomes - a problem that will not be addressed as long as architectural ideology is apathetic towards contextual engagement, restricted to an internal disciplinarity, or entirely submissive to flows of capital. 


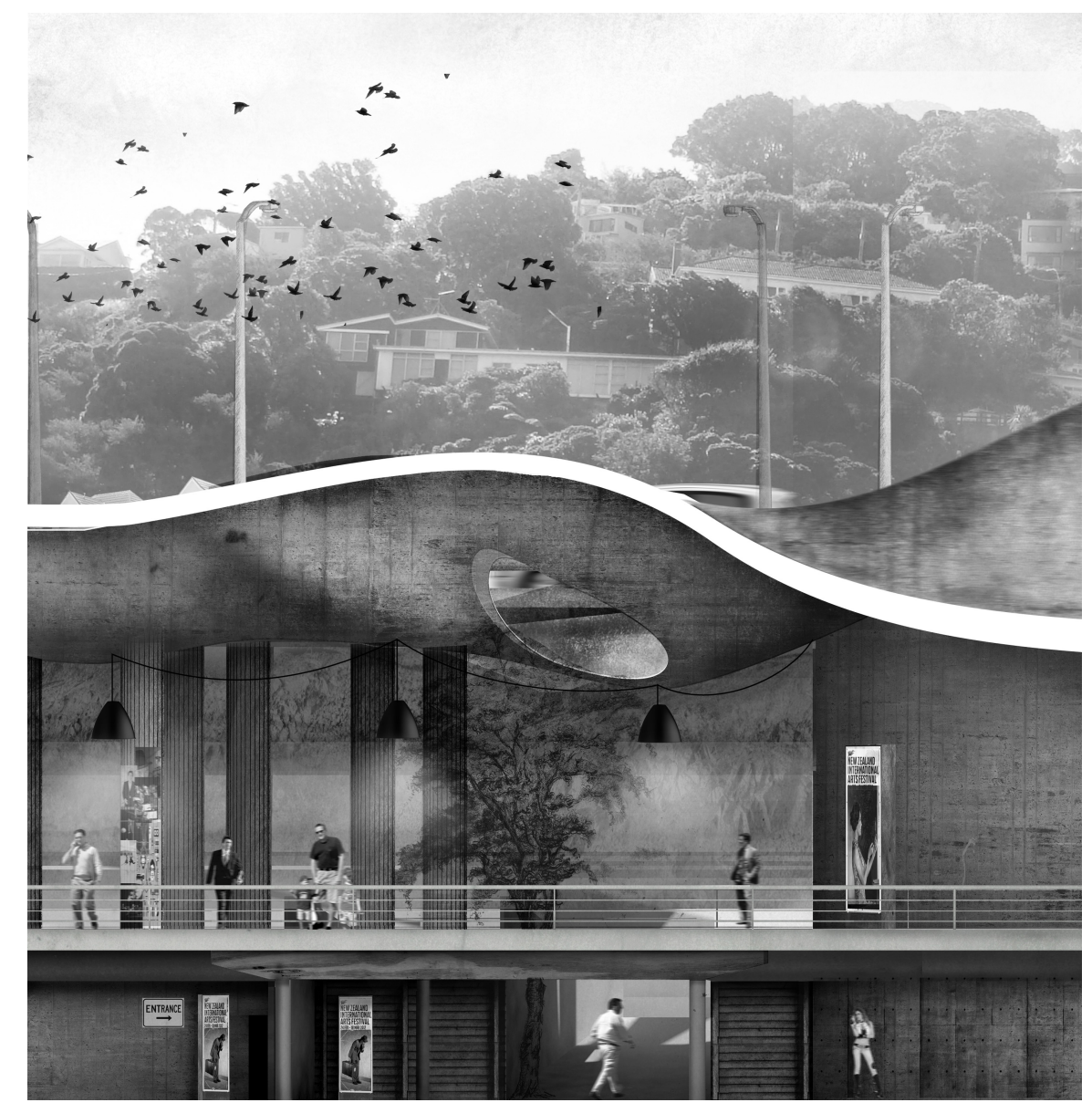

RIGHT >

Figure 10.5: Architecture's 


\section{BIBLIOGRAPHY}

Abbas, Ackbar. "On Fascination: Walter Benjamin's Images." New German Critique (1989): 43-62.

Abdelmonem, Gamal and Ruth Morrow. "Introduction: Transcending the Boundaries of Architecture." Abdelmonem, Ruth Morrow and Gamal. Peripheries: Edge Conditions in Architecture. London: Routledge, 2013. 1-8.

Adams, Phillip. "Forward." Charlesworth, Esther R. Cityedge: Case Studies in Contemporary Urbanism. Oxford: Architectural Press, 2005. xii-xiii.

Adorno, Theodore. Aesthetic Theory. London: Athlone, 1996.

Alington, Margaret $\mathrm{H}$. Unquiet Earth. Wellington: Wellington City Council Ministry of Works and Development, 1978

Allen, Stan. "SANAA's Dirty Realism." Idenburg, Florian and Iwan Baan. The Sanaa Studios 2006-2008: Learning from Japan : Single Story Urbanism. Baden, Switzerland: Lars Muiller, 2010. 58-67.

Amin, Ash. Post-Fordism, A Reader. Boston: Blackwell, 1994.

Appadurai, Arjun. Modernity at Large: Cultural Dimensions of Globalisation. . Minneapolis, Minn: University of Minnesota Press, 1996.

Arendt, Hannah. The Human Condition. Chicago: University of Chicago Press, 1998 [1958]

Argyriades, Marcia. Metropol Parasol: The World's Largest Wooden Structure. 28 April 2011. <http://www.yatzer.com/Metropol-ParasolThe-World-s-Largest-Wooden-Structure-JMAYER-H-Architects>.

Augé, Marc. Non-Places: An Introduciton to Supermodernity. Second. Verso: London, 2008 [1995].

Aureli, Pier Vittorio. The Possibility of an Absolute Architecture. Cambridge, Mass: MIT Press, 2011.
- "The Theology of Tabula Rasa: Walter Benjamin and Architecture in the Age of Precarity." Log 27, Spring 2013.

Ayers, Andrew. "Fun Palais." Architecture Review CCXXXI.1384 (2012): 44-51.

Bachelard, Gaston. The Poetics of Space. Trans. M. Jolas. Boston: Beacon Press, 1969.

Baird, George. "Criticality" and its Discontents." Harvard Design Magazine Fall-Winter (2004): 16-21.

Bakhtin, Mikhail M. The Dialogic Imagination: Four Essays. Austin: University of Texas Press, 2006 [1981].

Barber, Daniel A. "Militant Architecture." Rendell, Jane, et al. Critical Architecture. London: Routledge, 2007.57-66.

Barthes, Roland. Mythologies. New York: Hill and Wang, 1972

Bauman, Zygmunt. Liquid Modernity. London: Blackwell, 2000.

Benedikt, Michael. "The First Rule of the Architect." Owen, Graham. Architecture, Ethics and Globalisation . New York: Routledge, 2009. 105-113.

Benjamin, Andrew E. "The Architecture of Hope, Daniel Libeskind's Jewish Museum." Benjamin, Andrew E. Present Hope: Philosophy, Architecture, Judaism. London: Routledge, 1997. 103-118.

Benjamin, Walter and Rolf Tiedemann. The Arcades Project. 7th. Cambridge, Mass: Belknap Press, 1999.

Benjamin, Walter. The Arcades Project (1927-39). Trans. Howard Eiland and Kevin McLaughlin. Cambridge, Mass: Harvard University Press, 1999.

The Origin of German Tragic Drama. London: Verso, 2003 [1925]. 
"The Work of Art in the Age of Mechanical Reproduction." Benjamin, Walter. Illuminations. Ed. Hannah Arendt. New York: Harcourt, Brace and World, 1968. 217-253.

Benjamin, Walter. "Theses on the Philosophy of History." Benjamin, Walter. Illuminations. Ed. Hannah Arendt. New York: Harcourt, Brace and World, 1968. 253-264.

Bentley, Ian. Urban Transformations: Power, People and Urban Design. New York, NY: Routledge, 1999.

Berrizbeitia, Anita and Linda Pollak. Inside Outside: Between Architecture and Landscape. Gloucester, Mass: Rockport Publishers, 1999.

Betsky, Aaron. "Nothing But Flowers: Against Public Space." Bell, Michael and Sze T. Leong. Slow Space. New York: Monacelli Press, 1998. 457 170 .

Bishop, Claire. "Antagonism and Relational Aesthetics." October Fall.110 (2004): 51-79.

Black, Jane, Michael Kelly and Chris Cochran. Thorndon Heritage Project. Wellington: Wellington City Council, 2008

Bognar, Botond. "Towards an Architecture of Critical Inquiry." Journal of Architectural Education 43.1 (1984): 13-34

Bois, Yves Alain and Rosalind Krauss. Formless: A User's Guide. Cambridge, Massachusettes: Zone Books, 1997.

Bond, Anthony. "Swapping Public and Private." Parry, Ben, Sally Medlyn and Miriam Tahir. Cultural Hijack: Rethinking Intervention. Liverpool Liverpool University Press, 2011. 173-188.

Borden, Iain. "Thick edge: Architectural Boundaries in the Postmodern Metropolis." Borden, Iain and Jane Rendell. Intersections: Architectural Histories and Critical Theories. London: Routledge, 2000. 221-246.

Bourdieu, Pierre. The Logic of Practice. Stanford, California: Stanford University Press, 1990.

Bourriaud, Nicolas. Relational Aesthetics. Dijon: Les Presses du réel, 2002.
Braham, William W. and Jonathan A. Hale. Rethinking Technology: A Reader in Architectural Theory. London: Routledge, 2007.

Brain, David. "From Good Neighborhoods to Sustainable Cities: Social Science and the Social Agenda of New Urbanism." International Science Review 28.2 (2005): 217-238.

Bruner, Jerome S. Acts of Meaning. Cambridge : Harvard University Press, 1990.

Buck-Morss, Susan and Walter Benjamin. The Dialectics of Seeing: Walter Benjamin and the Arcades Project. Cambridge, Mass: MIT Press, 1989.

Bürger, Peter. Theory of the Avant-Garde. Manchester Manchester University Press, 1984.

Burns, Carol and Andrea Kahn. "Why Site Matters." Burns, Carol J. and Andrea Kahn. Site Matters: Design Concepts, Histories, and Strategies. New York: Routledge, 2005, vii-xxix.

Castiglioni, Luisa. "Tatzu Nishi: Discovering Columbus." 5 October 2012. Domus. <http:// www.domusweb.it/en/art/2012/10/05/ tatzu-nishi--em-discovering-columbus-em-. html $>$.

Caygill, Howard. Walter Benjamin: The Colour of Experience, London : Routledge, 1998.

Chapman, Katie. Spy Car Loss Blamed For Revenue Fall. 7 March 2013. 4 April 2013. <http // www.stuff.co.nz/dominion-post/news/localpapers/local-papers-directory/4988119/ Other-Local-Papers $>$

Charlesworth, Esther R. Cityedge: Case Studies in Contemporary Urbanism. Oxford: Architectura Press, 2005.

Chi, Lily. "The Problem with the Architect as Writer." Pelletier, Louise and Alberto Pérez-Gómez. Architecture, ethics, and technology. Montreal: McGill-Queen's University Press, 1994. 199 221.

Clark, Terry Nichols. "The City as an Entertainment Machine." Critical Perspectives on Urban Development. Chicago: University of Chicago, 2001. 1-17. 
Clayden, Andrew and Jan Woudstra. "Some European Approaches To Twentieth-Century Cemetery Design: Continental Solutions For British Dilemmas." Mortality 8.2 (2003): 189-208.

Colquhoun, Alan. Modernity and the Classical Tradition : Architectural Essays, 1980-1987. Cambridge, Mass: MIT Press, 1989.

Council, New Zealand Property. "The Future of our Capital." 8 April 2013. Property Council of New Zealand. 24 May 2013. <http://www. thewellingtoncompany.co.nz/pdf/The $\% 20$ Future\%20of\%20Our\%20Capital.pdf>.

Council, Wellington City. "1.3 The District Plan in Context." 12 September 2012. Wellington City Council. 12 May 2012. <http://wellington. govt.nz/ /media/your-council/plans-policiesand-bylaws/district-plan/understanding-thedistrict-plan/files/1-3.pdf $>$.

-. "Central Area Urban Design Guide." 12 September 2012. Wellington City Council - New Zealand. 05 2012. <http://www. wellington.govt.nz/plans/district/volume2/ pdfs/v2central.pdf $>$.

-. Central Area Urban Design Guide Appendix 1 - Pipitea Precinct. Wellington: Council, Wellington City, 2011.

-. Central City Framework: Approach to Implementation. Wellington: Wellington City Council, 2011.

-. Council Owned Car Parks. 2013. 15042012. <http://wellington.govt.nz/services/parkingand-roads/parking/council-owned-carparks $>$.

-. "Profile of Wellington." 2013. Wellington City Council. 13 February 2013. <http:// wellington.govt.nz/about-wellington/profileof-wellington $>$.

- Theatrical and Performance Venues in Wellington. 25 April 2013.7906 2012. <http://venueweb. co.nz/north/10_wellington/10-wellington. htm\#festival_venues $>$.

Cowan, Bainard."Walter Benjamin's Theory of Allegory." New Gerrman Critique (1981): 109-122.
Cowlishaw, Shane, et al. "Prime Minister Side-Steps 'Dying' Comment" 07 May 2013. Stuff.co.nz. May 2013. <http://www.stuff.co.nz/national/ politics/8639116/Prime-minister-side-stepsdying-comment $>$.

CreativeNZ. Contact Us, $<$ http://www.creativenz.govt. $\mathrm{nz} / \mathrm{en} /$ about-creative-new-zealand/contactus $>$.

Creswell, John W. Research Design. Second. London: Sage Publications Ltd., 2003.

Crinson, Mark. "Urban Memory - an Introduction." Crinson, Mark. Urban Memory: History and Amnesia in the Modern City. London: Routledge, 2005. xi-xx.

Crisman, Phoebe. "Inhabiting the Edge: Architecture and Transport Infrastructure Intertwined." Morrow, Ruth and Mohamed Gamal Abdelmonem. Peripheries: Edge Conditions in Architecture. London: Routledge, 2013. 115128.

Crotty, Michael. The Foundations of Social Research: Meaning and Perspective in the Research Process. Crows Nest, NSW: Allen and Unwin, 1998.

Cullinan, Nicholas. "From Vietnam to Fiat-Nam: The Politics of Arte Povera." October 124.Spring (2008): $8-30$

Cunningham, David. "Architetcure as Critical Knowledge." Rendell, Jane. London: Routledge, 2007. Print. Critical Architecture. London: Routledge, 2007.31-39.

Cunningham, Stuart. From Cultural to Creative Industries: Theory, Industry, And Policy Implications. Brisbane: University of Technology, 2002. <http://eprints.qut.edu. au/588/1/cunningham_from.pdf $>$.

Dahms, Harry F. The Vitality of Critical Theory. Ed. Current Perspectives in Social Theory. Vol. 28. Bradford: Emerald Group Publishing Limited, 2011.

Davis, Michael. "Has Globalism Made Architecture's Professional Ethics Obsolete?" Owen, Graham. Architecture, Ethics and Globalisation. New york: Routledge, 2009. 121-132. 
de Certeau, Michel. The Practice of Everyday Life. Berkeley: University of California Press, 1984.

-. "Walking in the City" During, Simon. r London: Routledge, 1993. Print. The Cultural Studies Reader. London: Routledge, 1993. 126133.

Deamer, Peggy. "Review of K. Michael Hays, Architecture's Desire: Reading "The Late AvantGarde' and Pier Vittorio Aureli, 'The Possibility of an Absolute Architecture." November 2011. Peggy Deamer, Architect. 15 October 2012. <http://www.peggydeamer.com/images/ aureli_hays.pdf $>$.

Derrida, Jacques. Of Grammatology. Baltimore: Johns Hopkins University Press, 1976.

Deutsche, Rosalyn. "Art and Public Space: Questions of Democracy." Social Text 33 (1992): 34-53.

-. Evictions: Art and Spatial Politics. Chicago, Illinois: Graham Foundation for Advanced Studies in the Fine Arts, 1996.

D'Hooghe, Alexander. “The Objectification of Infrastructure: The Cultural Project of Suburban Infrastructure Design." Stoll, Katrina, Scott Lloyd and Stan Allen. Infrastructure As Architecture: Designing Composite Networks. Berlin: Jovis, 2010. 78-87.

Dixon, John Morris. "Monumental Buildings in Context." Progressive Architecture 74.6 (1993).

Dorrian, Mark. "Criticism, Negation, Action." The Journal of Architecture (2005): 229-233.

Doucet, Isabelle and Kenny Cupers. "Agency in Architecture: Rethinking Criticality in Theory and Practice." Footprint (2009): 1-6.

Dovey, Kim. Framing Places: Mediating Power in Built Form (Second Edition). Second. London: Routledge, 2008.

-. $\quad$ “I Mean to be Critical, But."' Rendell, Jane, et al. Critical Architecture. New York: Routledge, 2007.252-260.

-. etal."Peripheries Dialogue: A roundtable debate on architecture." Abdelmonem, Mohamed Gamal and Ruth Morrow. Peripheries: Edge Conditions in Architecture. London: Routledge, 2013.9-24.
Dunham-Jones, Ellen. "The Landscapes of Globalisation: Free Trade Zones, Downtown Financial Cores, and Sprawl." Taylor, William $\mathrm{M}$ and Michael P. Levine. Prospects for an Ethics of Architecture. Abingdon, Oxon: Routledge, 2011. 17-29.

Duttlinger, Carolin. "Between Contemplation and Distraction: Configurations of Attention in Walter Benjamin." German Studies Review (2007): 33-54.

Eisenman, Peter. "Foreward." Grosz, Elizabeth. Architecture from the Outside: Essays on Virtual and Real Space. 2001.

-. "Introduction." Eisenman, Peter. Eisenman Inside Out: Selected Writings 1963-1988. New Haven, CT: Yale University Press, 2004. vii-xv.

-. "Notes on Conceptual Architecture: Towards a Defintion." Eisenman, Peter. Eisenman Inside Out: Selected Writings, 1963-1988. New Haven, CT: Yale University Press, 2004. 11-27.

-. "The End of the Classical, The End of the beginning, The End of the End." Perspecta 21 (1986): 154-173.

-. Ten Canonical Buildings: 1950-2000. New York: Rizzoli International Publications, 2008.

Elliott, Brian. Benjamin for Architects. New York, NY: Routledge, 2011.

Emmitt, Stephen. Architectural Technology. 2. Hoboken: Wiley-Blackwell, 2012.

Evans, Fred. "Deleuze, Bakhtin, and the 'Clamour of Voices.” Deleuze Studies 2 (2008): 178-188.

Fat. "It's Not Unusual: Projects and Tactics." Borden, Iain. The Unknown City: Contesting Architecture and Social Space. Cambridge, Mass: MIT Press, 2002.340-355.

Finlay, Steven. WETA: a Case Study. CANZ: Creative Advantage New Zealand. Wellington: Victoria University of Wellington, 2006. < http://www. victoria.ac.nz/som/researchprojects/researchprojects/competitive-advantage/documents/ weta_case_study.pdf $>$.

Fletcher, Angus. Allegory: The Theory of a Symbolic Mode. Ithaca, N.Y: Cornell University Press, 1964. 
Forcast.id. "Wellington City Council Population Forecasts." 2013. ID: The Population Experts. <http:// forecast.idnz.co.nz/Default. aspx $? \mathrm{id}=3668 \mathrm{pg}=5000>$.

Foster, Hal. Design and Crime (and Other Diatribes). London: Verso, 2004.

-. $\quad$ "Image Building." Artforum International 43.2 (2004): 270-273, 310-311.

Foucault, Michel and Alan Sheridan. The Archaeology of Knowledge. New York: Pantheon Books, 1972.

—. On Power. New York: Routledge, 2000 [1988].

Fox, Warwick. Ethics and the Built Environment. London: Routledge, 2000.

Frampton, Kenneth. "Brief Reflections on the Predicament of Urbanism." Tschumi, Bernard and Irene Cheng. The State of Architecture at the Beginning of the 21st Century. New York: Monacelli Press, 2003. 13.

-. Modern Architecture: a Critical History. London: Thames and Hudson, 1980.

-. "Prospects for a Critical Regionalism." Perspecta 20 (1983): 147-162.

-. "Ten Points on an Architecture of Regionalism." Center 3 (1986): 24 .

-. "Towards a Critical Regionalism: Six Points for an Architecture of Resistance." Foster, Hal. The Anti-Aesthetic: Essays on Post Modern Culture. Seattle: Bay Press, 1983. 16-30.

Franck, Karen A and Quentin Stevens. "Tying Down Loose Space." Franck, Karen A, and Quentin Stevens: , 2007. Print. Loose Space. London: Routledge, 2007. 1-35.

Frank, Suzanne S. Peter Eisenman's House VI: The Client's Response. New York: Whitney Library of Design, 1994.

Fraser, Murray. "Introduction" Rendell, Jane. Critical Architecture. London: Routledge, 2007. 249251.

-. "The Cultural Context of Critical Architecture." The Journal of Architecture (2006): 317-322.
Frieden, Bernard. The Future of Old Neighborhoods. Cambridge, Mass: MIT Press, 1964.

Ganoe, Cathy J. "Design as Narrative: A Theory of Inhabiting Interior Space." Journal of Interior Design (1999): 1-15.

Gardiner, Michael. "A Very Understandable Horror of Dialectics." Brandist, Craig and Galin Tihanov. Materializing Bakhtin: The Bakbtin Circle and Social Theory. Basingstoke: Macmillan, 2000. 119-141.

Gehl, Jan. Life Between Buildings. Copenhagen: Arkiektens Forlag., 1996.

Gentry, Regan."Subject to Change." 2009. Regan Gentry. <http://www.regangentry.com/featured/ subject-to-change/ $>$.

Giddens, Anthony. Central Problems in Social Theory: Action, Structure, and Contradiction in Social Analysis. Berkeley: University of California Press, 1979.

-. Modernity and Self-Identity: Self and Society in the Late Modern Age. Stanford, California: Stanford University Press, 1991.

Gillis, John R. Commemorations: The Politics of National Identity. Princeton, N.J: Princeton University Press, 1994.

Gilloch, Graeme. Myth and Metropolis: Walter Benjamin and the City. Cambridge Mass: Blackwell Publishers Inc., 1996.

Gleason, Philip. "Identifying Identity." Gillis, John R. Commemorations: The Politics of National Identity. Vol. March. Princeton, N.J: Princeton University Press, 1994. 27-40.

Glendinning, Miles. Architecture's Evil Empire?: The Triumph and Tragedy of Global Modernism. London: Reaktion, 2010.

Goodman, Robert. After the Planners. Harmondsworth, 1972.

Gottdiener, Mark. The Social Production of Urban Space. Austin, TX: Universtiy of Texas Press, 1997.

Gritzner, Karoline. "Form and Formlessness: Participation at the Limit." Performance Research: A Journal of the Performing Arts 16.4 (2011): 109-116 
Grosz, Elizabeth. Architecture from the Outside: Essays on Virtual and Real Space. Cambridge, Mass.: MIT Press, 2001.

Guess, Raymond. The Idea of Critical Theory. Cambridge: Cambridge University Press, 1981.

Hall, Peter Ruthven and Kate Burnett. 2D/3D: Design for Theatre and Performance. London: Society of British Theatre Designers, 2002.

Handler, Richard. "Is 'Identity' a Useful Cross-Cultural Concept." Gillis, John R. Commemorations: The Politics of National Identity. Princeton, N.J: Princeton University Press, 1994. 27-40.

Haralambidou, Penelope. "The Fall: The Allegorical Architectural Project as a Critical Method." Rendell, Jane, et al. Critical Architecture. London: Routledge, 2007. 225-236.

Harpham, Geoffrey Galt. "Architecture and Ethics: 16 Points." Taylor, William M and Michael P. Levine. Prospects for an Ethics of Architecture. Abingdon, Oxon: Routledg, 2011.33-39.

Harries, Karsten. The Ethical Function of Architecture Cambridge, Mass: MIT Press, 1997.

Hartoonian, Gevork. Architecture and Spectacle: A Critique. Surrey: Ashgate, 2012.

-. "Introduction." Hartoonian, Gevork. Walter Benjamin and Architecture. London: Routledge, 2010. 1-5.

-. "Looking Backward, Looking Forward: Delightful Delays.” Hartoonian, Gevork. Walter Benjamin and Architecture. London: Routledge, 2010. 23-38.

Harvey, David. Justice, Nature and the Geography of Difference. Cambridge, Mass: Blackwell, 1996.

-. Justice, Nature, and the Geography of Difference Cambridge, Mass: Blackwell Publishers, 1996.

- Paris, Capital of Modernity. London: Routledge, 2003.

-. The Condition of Postmodernity: An Enquiry into the Origins of Cultural Change. Oxford: Blackwell, 1990.

Hasegawa, Yuko. Kazuyo Sejima + Ryue Nishizawa: Sanaa. Milan: Electaarchitecture, 2006.
Haxthausen, Charles W. "Reproduction/Repetition: Walter Benjamin/Carl Einstein.” October 107. Winter (2004): 47-74.

Hays, K. Michael. Architecture Theory Since 1968. Cambridge, Mass: MIT Press, 2000.

-. Architecture's Desire: Reading the Late AvantGarde. Cambridge, Mass: MIT Press, 2010.

-. "Critical Architecture." Perspecta 21 (1984): 15 28.

Heidegger, Martin. Being and Time. New York: Harper, 1962.

Heizer, Michael. "The Art of Michael Heizer." Artforum (1969).

Held, David. Introduction to Critical Theory: Horkheimer to Habermas. Berkeley: University of California Press, 1980.

Hillier, Bill and Julienne Hanson. The Social Logic of Space. London: Cambridge University Press, 1984.

Hitters, Erik and Greg Richards. "The Creation and Management of Cultural Clusters." Creativity and Innovation Management 11 (2002): 234 247.

Holloway, Julian and James Kneale. "Mikhail Bakhtin Dialogics of Space." Crang, Mike and Nigel J. Thrift. Thinking Space. London: Routledge, 2000 .

IFLA50. "Kumototo" 12 April 2013. IFLA50: Shared Wisdom in an Age of Change. <http://www. ifla2013.com/landscape-architecture-projects/ kumutoto/>.

Ingersoll, Richard. Sprawltown: Looking for the City on its Edges. New York: Princeton Architectural Press, 2006.

Isenstadt, Sandy* "Contested Contexts" Burns, Carol and Andrea Kahn. Site Matters: Design Concepts, Histories, and Strategies. New York: Routledge, 2005. 158-183.

Iveson, Kurt. Publics and the City. Malden: MA: Blackwell Pub., 2007. 
Jacobs, Allan and Donald Appleyard. "Towards an Urban Design Manifesto." LeGates, Richard T. and Stout, Frederic. The City Reader. Ed. Fourth. New York: Routledge, 2007. 456-466.

Jacobs, Jane. The Death and Life of Great American Cities. : Vintage Books, 1992. Print. . New York: Random House, 1961.

Jameson, Fredric. Postmodernism, Or, the Cultural Logic of Late Capitalism. Durham: Duke University Press, 1991.

Jarvis, Simon. Adorno: A Critical Introduction. London: Routledge, 1998.

Kahn, Andrea. "Defining Urban Sites." Burns, Carol and Andrea Kahn. Site Matters: Design Concepts, Histories, and Strategies. New York: Routledge, 2005. 281-297.

Kaminer, Tahl. Architecture, Crisis and Resuscitation: The Reproduction of Post-Fordism in LateTwentieth-Century Architecture. Abingdon, Oxon: Routledge, 2011.

Kaye, Nick. Site-Specific Art. London: Routledge, 2000.

Keith, Michael and Steve Pile."Introduction Part 1: The Politics of Place..." Keith, Michael and Steve Pile. Place and the Politics of Identity. London: Routledge, 1993. 1-21.

Kelbaugh, Douglas. "Attention Getting." New York Times. New York, 12 August 2001. 4.

Kenniff, Thomas-Barnard. "Identity in Peripheries: Barking and its Others." Morrow, Ruth and Mohamed Gamal Abdelmonem. Peripheries: Edge Conditions in Architecture. London: Routledge, 2013. 40-53.

Kernohan, David. Wellington's New Buildings. Wellington: Victoria University Press, 1989.

-. Wellington's Old Buildings. Wellington: Victoria University Press, 1994.

King, Anthony D. Spaces of Global Cultures: Architecture, Urbanism, Identity. London: Routledge, 2004.

Kingwell, Mark. "Builiding, Dwelling, Acting." Owen, Graham. Architecture, Ethics and Globalisation. New York: Routledge, 2009. 40-49.
Klanten, Robert. Going Public: Public Architecture, Urbanism and Interventions. Berlin: Gestalten, 2012.

Klien, Kerwin Lee. "On the Emergence of Memory in Historical Discourse." Representations Winter.69 (2000): 127-150.

Knox, Paul L. "The Social Production of the Built Environment: Architects, Architecture and the Post-Modern City." Carmona, Matthew and Steve Tiesdell. Urban Design Reader. Oxford: Elsevier Architectural Press, 2007. 115-125.

Koolhaas, Rem. "Junkspace." October 100 (2002): 175190.

-. Small, Medium, Large, Extra-Large: Office for Metropolitan Architecture, Rem Koolhaas, and Bruce Mau. New York, N.Y: Monacelli Press, 1998.

Krauss, Rosalind. "Notes on the Index: Seventies Art in America Part 1" October 3.Spring (1977): 68-81.

"Notes on the Index: Seventies Art in America Part 2." October 4.Autumn (1977): 58-67.

- $\quad$ "Sculpture in the Expanded Field." October 8.Spring (1979): 30-44.

- The originality of the avant-garde and other modernist myths. Cambridge, Mass: MIT Press, 1985.

Krivý, Maroš. "Obselete Industrial Space in the Expanded Field." Morrow, Ruth and Mohamed Gamal Abdelmonem. Peripheries: Edge Conditions in Architecture. London: Routledge, 2013. 101-114.

Kroll, Andrew. AD Classics: Igualada Cemetery / Enric Miralles \& Carme Pinos . 13 January 2011. 11 August 2012. <http://www.archdaily. com/103839/ad-classics-igualada-cemeteryenric-miralles/ $>$.

Kromer, John. Fixing Broken Cities: The Implementation of Urban Development Strategies. New York: Routledge, 2010.

Lacan, Jacques. Seminar of Jacques Lacan: The Psychoses, 1955-1956. Ed. Jacques-Alain Miller. Trans. Russell Grigg. New York: W W NORTON \& CO, 1997. 
Lacaton, Anne and Vassal Jean-Phillipe. Palais de Tokyo 2010. <http://www.lacatonvassal.com/index. php?idp $=20>$.

Lahiji, Nadir. "Architecture Under the Gaze of Photography: Benjamin's Actuality and Consequences." Elliott, Brian. Benjamin for Architects. Abingdon, Oxon: Routledge, 2011. 75-91.

-. "Introduction" Lahiji, Nadir. The Political Unconscious of Architecture: Re-opening Jameson's Narrative. Surrey: Ashgate Publishing Ltd., 2011. 1-11.

- $\quad$ "Reloading Ideology Critique of Architecture." Lahiji, Nadir. The Political Unconscious of Architecture: Re-opening Jameson's Narrative. Surrey: Ashgate Publishing Ltd., 2011. 211234.

Lamster, Mark and Ezra Stoller. Guggenheim New York. New York, NY: Princeton Architectural Press, 1999.

Lawrence, Sam. "Clifton Street Car Park Capacity. Wellington City Council, n.d.

Leach, Neil. "Walter Benjamin, Mimesis and the Dreamworld of Photography." Borden, Iain and Jane Rendell. Intersections: Architectural Histories and Critical Theories. London: Routledge, 2000. 27-38.

Lefebvre, Henri. "Right to the City." Lefebvre, Henri. Writings on Cities, Cambridge, Mass: USA Blackwell Publishers, 1996.

- The Production of Space. Oxford: blackwell, 1991.

Lehtovuori, Panu. Experience and Conflict: The Production of Urban Space. Farnham, Surrey, England: Ashgate Publications, 2010.

Leinberger, Christopher B. Turning Around Downtown: Twelve Steps to Revitalization. Washington, D.C.: The Brooking Institution Center on Urban and Metropolitan Policy, 2005.

Libeskind, Daniel and Hélène Binet. Jewish Museum, Berlin. Amsterdam: G + B Arts International, 1999.

- "Between the Lines." Libeskind, Daniel. The Space of Encounter. London: Thames and Hudson, 2001.
Liggett, Helen and David C. Perry. "Spatial Practices An Introduction." Liggett, Helen and David C. Perry. Spatial Practices: Critical Explorations in Social/Spatial Theory. London: Sage Publications Ltd., 1995. 1-12.

- Urban Encounters. Minneapolis: University of Minnesota Press, 2003.

Lofland, Lyn H. The Public Realm: Exploring the City's Quintessential Social Theory. Hawthorne, N.Y: Aldine de Gruyter, 1998.

Luce, Kristina. "The Collision of Process and Form: Drawing's Imprint on Peter Eisenman's House VI." Getty research Journal 2 (2010): 125-137.

Lynch, Kevin. The Image of the City. Cambridge, Mass: MIT Press, 1960.

Mahoney, Maurice and Sir Miles Warren. The Supreme Court of New Zealand. n.d. 19 February 2013. <http://www.warrenandmahoney.com/en/ portfolio/supreme-court/>

Maier, Charles. "A Surfeit of Memory." History and Memory 5.2 (1993): 136-151.

Marin, Louis. Utopiques: Jeux D'espaces. Paris: Editions de Minuit, 1973.

Martin, A. D. "Wellington Urban Motorway - Shell Gully Parking Building - Interim report." Wellington City Archives Document. Wellington: Wellington City Corporation: City Engineer's Department, 26 March 1970.

Martin, Louis. "Frederic Jameson and Critical Architecture." Lahiji, Nadir. The Political Unconscious of Architecture: Re-opening Jameson's Narrative. Surrey: Ashgate Publishing Ltd., 2011. 171-210.

Martin, Reinhold. "Critical of What? Toward a Utopian Realism." Sykes, Krista. Constructing a new agenda: Architectural theory 1993-2009. New York: Princeton Architectural Press, 2010. 348-362.

Massey, Doreen. "Politics and Space/Time." Keith, Michael and Steve Pile. Place and the Politics of Identity. London: Routledge, 1993. 141-159.

Mayer, Jürgen H. Metropol Parasol. 2013. <http://www. jmayerh.de/19-0-Metropol-Parasol.html >. 
- . Metropol Parasol, Seville." Architectural Design (2012):70-73.

Merleau-Ponty, Maurice. Phenomenology of Perception. New York: Humanities Press, 1962.

Merriman, Peter. "Marc Augé." Hubbard, Phil and Rob Kitchin. Key Thinkers of Space and Place. Ed. Second. London: SAGE Publications Ltd., 2011. 26-33.

Miles, Malcolm. "1967: Why Tomorrow Never Dawns." Miles, Malcolm. Urban Avant-Gardes: Art, Architecture and Change. London: Routledge, 2004. 70-92.

-. "In Memories of Dark Times (1993)." Miles, Malcolm. Urban Avant-Gardes: Art, Architecture and Change. London: Routledge, 2004. 119-146.

-. "Participation and Provocation." Miles, Malcolm. Urban Avant-Gardes: Art, Architecture and Change. London: Routledge, 2004. 147179.

-. "Sustainabilities (2001)." Miles, Malcolm. Urban Avant-gardes: Art, Architecture and Change. London: Routledge, 2004. 180-208.

Miller, Richard Ogilvy. "The Wellington Urban Motorway: The Parts Played by the Planning Authorities and the Bolton Street Preservation Society" 1969.

Mitchell, William J. City of Bits: Space, Place, and the Infobahn. Cambridge, Mass.: MIT Press, 1995.

Montgomery, John. "Cultural Quarters as Mechanism for Urban Regeneration Part 1: Conceptualising Cultural Quarters." Planning, Practice \& Research 18.4 (2003): 293-306.

-. "Cultural Quarters as Mechanism for Urban Regeneration Part 2: a Review of Four Cultural Quarters in the UK, Ireland and Australia." Planning and Practice Research 19.1 (2010): 3-31.

-. "Dublin, Sheffield, Manchester and Adelaide: Cultural Quarters as Mechanisms for Urban Regeneration." Charlesworth, Esther R. Cityedge: Case Studies in Contemporary Urbanism. Oxford: Architectural Press, 2005. 84-101.
Muzaffar, Ijlal. "Book review: Eisenman Inside Out." Future Anterior 1.2 (2004): 67-73.

New Zealand, Statistics. "Interactive Population Pyramid for New Zealand." 18 February 2013. Statistics New Zealand. <http://www.stats. govt.nz/tools_and_services/tools/interactivepop-pyramid.aspx $>$.

Newman, Peter and Jeffrey Kenworthy. Sustainability and Cities: Overcoming Automobile Dependence. Washington: Island Press, 1999.

Newton, Katherine. "Where is NZ's Cultural Capital." 02 March 2009. Stuff.co.nz. 3 October 2012. <http://www.stuff.co.nz/entertainment/ arts/1761460/Where-is-NZs-culturalcapital>.

Nishat, Awan, Tatjana Schneider and Jeremy Till. Spatial Agency: Other Ways of Doing Architecture. London: Routledge, 2011.

Nishizawa, Ryue. Experience of Architectural Concepts Nobuyuki Yoshida. A+U (Architecture and Urbanism), 05 2013. 66-73.

-. "Lanscape-like Architecture, Verb-like Architecture." $A+U 511$ (2013): 8-9.

Nora, Pierre and Lawrence D. Kritzman. Realms of Memory: Rethinking the French Past. New York: Columbia University Press, 1996.

Norberg-Schulz, Christian. Genius Loci: Towards a Phenomenology of Architecture. New York: Rizzoli, 1980.

Nye, David.American Technological Sublime. Cambridge, Mass: MIT Press, 1996.

Ockman, Joan. "Criticism in the Age of Globalisation." Tschumi, Bernard and Irene Cheng. The State of Architecture at the Beginning of the 21st Century. New York: Monacelli Press, 2003. 7879.

O'Connor, Justin. "A Special Kind of City Knowledge": Innovative clusters, tacit knowledge and the 'Creative City' ". Media International Australia 112 (2004): 131-149.

Onions, C. T. The Shorter Oxford English Dictionary on Historical Principles. Oxford: Clarendon Press, 1973. 
Owen, Graham. "Introduction." Owen, Graham. Architecture, Ethics and Globalisation. New York: Routledge, 2009. 1-16.

Owens, Craig. "The Allegorical Impulse: Toward a Theory of Postmodernism Part 1." October 12. Spring $(1980) \div 67-86$

-. "The Allegorical Impulse: Toward a Theory of Postmodernism Part 2." October 13.Summer (1980): 58-80.

Parking, Tournament. About Us, n.d. 20032012. <http://www.tournament.co.nz/abouttournament/ $>$.

Parliament, New Zealand. "Order Paper and Questions." 08 May 2013. New Zealand Parliament. 12 May 2013. <http://www.parliament. $\mathrm{nz} / \mathrm{en}-\mathrm{NZ} / \mathrm{PB} / \mathrm{Bus}$ iness / QOA / 5 / a/1/50HansQ_20130509_00000002-2. Wellington-Economy.htm $>$.

Patke, Rajeev S. "Benjamin's "Arcades Project" and the Postcolonial City." Diacritics 30.4 (2000): 2-14

Pearson, Mike Parker and Colin Richards. "Ordering the World: Perceptions of Architecture, Space, and Time." Pearson, Mike Parker and Colin Richards. Architecture and Order: Approaches to Social Space. Londonः Routledge, 1994. 1-37.

Pechey, Graham. "Boundaries Versus Binaries: Bakhtin in/against the History of Ideas." Radical Philosophy (1990): 23-31

Pérez-Gómez, Alberto and Louise Pelletier. Architectural Representation and the Perspective Hinge. Cambridge, Mass: MIT Press, 2000.

-. Architecture and Ethics Beyond Globalisation. Hamburg: Material-Ver, 2003.

-. Architecture and the Crisis of Modern Science. Cambridge, Mass: MIT Press, 1983.

-. "Introduction." Pelletier, Louise and Alberto Pérez-Gómez. Architecture, Ethics, and Technology. Montreal \& Kingston: McGillQueen's University Press, 1994. 3-15.

-. "Introduction." Pelletier, Louise and Alberto Pérez-Gómez. Architecture, Ethics, and Technology. Montreal: McGill-Queen's University Press, 1994. 3-15.
Picon, Antoine. Digital Culture in Architecture: An Introduciton for the Design Professions. Basel Birkhauser, 2010

Pollak, Linda."Partially Open Spaces, Boundary Events, and Transitional Objects." Appendix: Culture, Theory, Praxis 4 (1999): 77-78

Pope, Albert. "From Form to Space." Cuff, Dana and Roger Sherman. Fast-forward Urbanism: Rethinking Architecture's Engagement with the City. New York: Princeton Architectural Press, 2011.143-209.

Porphyrios, Demetri. "On Critical History." Ockman, Joan. Architecture, Criticism, Ideology Princeton, N.J: Princeton Architectural Press, 1985. 16-21.

Porter, Michael E. "Clusters and the New Economics of Competetiveness." Harvard Business Review (1998): 77-90.

Projects, Theatre. "Types and Forms of Theatres." n.d. Theatre Projects Consultants. 21 May 2012 <http://www.theatreprojects.com/files/pdf/ Resources_IdeasInfo_typesandformsoftheatre. pdf $>$.

Reiss, Julie H. From Margin to Center: the Spaces of Installation Art. Cambridge, Mass.: MIT Press, 2001.

Relph, Edward. Place and Placelessness. London: Pion Press Ltd, 1976.

Rendell, Jane. Art and Architecture: A Place Between. New York: I.B Tauris and Co. Ltd, 2008.

-. "Introduction." Rendell, Jane, et al. Critical Archtiecture. London: Routledge, 2007. 1-8.

- Site-writing: The Architecture of Art Criticism. London: I. B. Taurus, 2010.

Rice, Charles. "Critical Post-Critical." Rendell, Jane, et al. Critical Architecture. New York: Routledge, 2007. 261-268.

Ricour, Paul. History and Truth: [essays]. Evanston: Northwestern University Press, 1965.

Riley, Terence. "The Global and the Local." Tschumi, Bernard and Irene Cheng. The State of Architecture at the Beginning of the 21st Century. New york: Monacelli Press, 2003.88-89. 
Robbins, Bruce. The Phantom Public Sphere. Minneapolis: University of Minnesota Press, 1993.

Roodhouse, Simon. Cultural Quarters: Principles and Practice. Chicago: Intellect Books, 2010.

Rosen, Charles. “The Ruins of Walter Benjamin." Smith, Gary. On Walter Benjamin: Critical Essays and Recollections. Cambridge, Mass: MIT Press, 1988. 129-175.

Rossi, Aldo. Architecture and the City. Cambridge, Mass: MIT Press, 2002 [1966].

Ruggeri, Laura. "The Poetics of Urban Inscription." Rendell, Jane, et al. Critical Architecture. New York: Routledge, 2007.103-111.

Sacco, Pier Luici. "The Economics of Quarters." Roodhouse, Simon. Cultural Quarters: Principles and Practice. Chicago: Intellect Ltd, 2010. 29-45.

Saint, Andrew. The Image of the Architect. New Haven: Yale University Press, 1983.

Sanger, Alice. "Venus of the Rags 1967, 1974." December 2009. TATE Modern. <http://www. tate.org.uk/art/artworks/pistoletto-venus-ofthe-rags-t $12200 /$ text-summary $>$

Saunders, William S. "Introduction." Saunders, William S. The New Architectural Pragmatism. Minneapolis, Minn: University of Minnesota Press, 2007. vii-xvii.

Savage, Mike. "Walter Benjamin's Urban Thought: A Critical Analysis." Environment and Planning D: Society and Space (1995): 201-216.

Schneider, Bernhard and Daniel Libeskind. Daniel Libeskind: Jewish Museum Berlin: Between the Lines. Munich: Prestel, 1999.

Schouten, Hank. "Wellington Town Hall earthquake strengthening looms." 31 August 2012. Stuff. co.nz. 01 Spetember 2012. <http://www.stuff. co.nz/dominion-post/business/commercialproperty/7588330/Wellington-Town-Hallearthquake-strengthening-looms $>$.

Scott, Allen J. The Cultural Economy of Cities: Essays on the Geography of Image-Producing Industries. London : SAGE Publications , 2000
-. Global City-Regions: Trends, Theory, Policy. . Oxford: Oxford University Press, 2001.

Selim, Gehan. "Re-imaging the periphery: The reproduction of space in Cairo." Morrow, Ruth and Mohamed Gamal Abdelmonem. Peripheries: Edge Conditions in Architecture. London: Routledge, 2013.151-169.

Semper, Gottfried and Harry F. Mallgrave. Style in the Technical and Tectonic Arts, Or, Practical Aesthetics. Los Angeles: Getty Research Institute, 2004.

Senie, Harriet. The Tilted Arc Controversy: Dangerous Precedent? Minneapolis, MN: University of Minnesota Press, 2002.

Shannon, Kelly and Marcel Smets. The Landscape of Contemporary Infrastructure. Rotterdam: NAi Publishers, 2010.

Sherman, William. "Engaging the Field" Burns, Carol and Andrea Kahn. Site Matters: Design Concepts, Histories, and Strategies. New York: Routledge, 2005.311-314.

Simpson, Bennett. "Public Relations: an interview with Nicolas Bourriaud." ArtForum (2001): 1-4.

Simpson, J A and E S. C Weiner. The Oxford English Dictionary. Oxford: Clarendon Press, 1989.

Smith, Cathy. "Between-ness: Theory and Practice within the Margins of Excess." IDEA Journal (2003): 1-14.

Smith, Terry+ "Daniel Amongst the Philosophers." Benjamin, Walter and Gevork Hartoonian. Walter Benjamin and Architecture. London: Routledge, 2010. 137-159.

Soja, Edward W. Post Modern Geographies: Reassertion of Space in Critical Social Theory. London: Verso, 1998.

Solomon, Jonathan D. 13 Projects for the Sheridan Expressway: A.k.a. Jump, Slump, Hump, Bump-Guide Specifications for a PostFordist Infrastructure. New York: Princeton Architectural Press, 2004.

Somol, Robert and Sarah Whiting. "Notes around the Doppler Effect and other Moods of Modernism." Perspecta 33 (2002): 72-77. 
Speaks, Michael. "Design Intelligence and the New Economy. Architectural Record January (2002): 72-79.

-. $\quad$ "Intelligence After Theory." Perspecta 38 (2006): 103-106.

-. "Which Way Avant-garde?" Assemblage 41 (2000): 78 .

Stacpoole, John and Peter Beaven. New Zealand Art: Architecture 1820-1970. Wellington: A.H. \& A.W. Reed Ltd, 1972.

Stanek, Lukasz. Henri Lefebvre on Space: Architecture, Urban Research, and the Production of Theory. Minneapolis, MN: University of Minnesota Press, 2011.

Stead, Naomi. "Criticism in/and/of Crisis." Rendell, Jane. Critical Architecture. London: Routledge, 2007.76-83.

Stern, Robert A. M. "Urbanism is About Human Life." Tschumi, Bernard and Irene Cheng. The State of Architecture at the Beginning of the 21st Century. New York: Monacelli Press, 2003. 21.

Stevens, Quentin. The Lucid City: Exploring the Potential of Public Spaces. London: Routledge, 2007.

Stivers, Camilla. "Reflections on the Role of Personal Narrative in Social Science” Signs 18.2 (1993) 408-425.

Stoll, Katrina, Scott Lloyd and Stan Allen. Infrastructure As Architecture: Designing Composite Networks. Berlin: Jovis, 2010.

Storper, Michael. The Regional World: Territorial Development in a Global Economy. New York: Guilford Press, 1997.

Strong, Judith. Theatre Buildings: A Design Guide. Abingdon, Oxon : Routledge, 2010.

Szondi, Peter. "Walter Benjamin's City Portraits." Smith, Gary. On Walter Benjamin: Critical Essays and Reflections. Cambridge, Mass: MIT Press, 1988. 18-32.

Tafuri, Manfredo. Architecture and Utopia: Design and Capitalist Development. Cambridge, Mass: MIT Press, 1976.
"Toward a Critique of Architectural Ideology (2000)." Hays, K. Michael. Architecture Theory Since 1968. Cambridge, Mass: MIT Press, 1969. 2-35.

Taylor, Mark C. "Co-evolutionary Disequilibrium." Tschumi, Bernard and Irene Cheng. The State of Architecture at the Beginning of the 21st Century. New York: Monacelli Press, 2003. 80 81 .

Taylor, William M and Michael P. Levine. Prospects for an Ethics of Architecture. Abingdon, Oxon: Routledge, 2011.

Tiedemann, Rolf."Dialectics at a Standstill: Approaches to the 'Passagen-Werk." Smith, Gary. On Walter Benjamin: Critical Essays and Reflections. Cambridge, Mass: MIT Press, 1988. 260-291.

Trancik, Roger. Finding Lost Space: Theories of Urban Design. New York: Van Nostrand Reinhold, 1986.

-. "What is Lost Space?" Carmona, Matthew and Steve Tiesdell. Urban Design Reader. Oxford: Elsevier Architectural Press, 2007.62-69.

Trust, Wellington Sculpture. "Regan Gentry: Subject to Change." 2009. Wellington Sculpture Trust. <http://www.sculpture.org.nz/engine/ SID/10007/AID/1245.htm >.

Tschumi, Bernard. Architecture and Disjunction. Cambridge, Mass: MIT Press, 1994

-. Cinegram Folie: Le Parc De La Villette. New York, NY: Princeton Architectural Press, 1987.

van der Rohe, Mies. "(Quote)." Neumeyer, Fritz. The Artless World: Mies van der Rohe on the Building Art. Trans. Mark Jarzombek. Cambridge, Mass MIT Press, 1991. 324.

Varnelis, Kazys. "Ethics after the avant-garde: the critical, the post-critical, and beyond" Owen, Graham. Architetcure, Ethics and Globalisation. London: Routledge, 2009. 148-157.

Venturi, Robert. Complexity and Contradiction in Architecture. New York: Museum of Modern Art, 2011 [1977]. 
Vesely, Dalibor. Wholeness, The Nature of the Modern Fragment and the Sense of. Fragments: Architecture and the Unfinished. London: Thames and Hudson Ltd, 2006. 43-55.

-. Architecture in the Age of Divided Representation: The Question of Creativity in the Shadow of Production. Cambridge, Mass.: MIT Press, 2004.

Vidler, Anthony. "Architecture's Expanded Field." Vidler, Anthony. Architecture between Spectacle and Use. Williamstown, Mass: Sterling and Francine Clark Art Institute, 2008. 143-154.

-. The Architectural Uncanny: Essays in the Modern Unhomely. Cambridge, Mass: MIT Press, 1992.

-. "Warped space: architectural anxiety in digital culture." Smith, Terry. Impossible Presence: Surface and Screen in the Photogenic Era. Chicago, IL: University of Chicago Press, 2001. 294-295.

Warf, Barney and Santa Arias. The Spatial Turn: Interdisciplinary Perspectives. London: Routledge, 2009.

Wasserman, Barry L, Gregory Palermo and Patrick Sullivan. Ethics and the practice of architecture. New York: Wiley, 2000.

Webb, Michael. Metropol Parasol by Jürgen Mayer H. Architekten, Seville, Spain. 27 May 2011. <http://www.architectural-review. $\mathrm{com} /$ metropol-parasol-by-jrgen-mayer-harchitekten-seville-spain/8615207.article>.

Webber, Melvyn. "Urban Place and Non-Place Urban Realm." Webber, Melvyn. Explorations in Urban Structure. Philadelphia: University of Pennysilvania, 1964.

Weber, Samuel. "Genealogy of Modernity: History, Myth and Allegory in Benjamin's Origin of the German Mourning Play." MLN 106.3 (1991): 465-500.

Whiting, Sarah. "Critical Reflections." Assemblage 41 (2000): 88-89. 24032012.

Wigglesworth, Sarah. "Critical Practice." Rendell, Jane. Critical Architecture. London: Routledge, 2007. 309-317.
Williams, Alastair. NewENPYusic and the Claims of Modernity. Ashgate: Aldershot, Hants, 1997.

Wirth, L. "Urbanism as a Way of Life." LeGates, R and F Stout. The City Reader. London: Routledge, 1996. 190-91.

Yska, Redmer. Wellington: Biography of a City. Wellington: Reed Publishing Ltd., 2006.

Zabalbeascoa, Anatxu. Igualada Cemetery: Enric Miralles and Carme Pinós. London: Phaidon, 1996.

Zimmerman, Michael E. "Globalisation, Multiculturalism, and Architectural Ethics." Owen, Graham. Architecture, Ethics and Globalisation. New York: Routledge, 2009. Print. . New York: Routledge, 2009. 158-170.

Zukin, Sharon. The Cultures of Cities. Cambridge, Mass: Blackwell, 1995. 
281

$\frac{\mid}{\frac{1}{2}}$ 


\section{LIST OF FIGURES}

Clifton Street Car Park, NZTA engineers plan placed in context. (Author's own image, 2013)
Fig. $i$

Fig. i

Fig, iii

Fig. iv

Fig. v

Fig. vi

Chapter One

Fig. 1.1

Fig. 1.2

Chapter Two

Fig. 2.1

Fig. 2.2

Fig. 2.3

Fig. 2.4

Fig. 2.5

Fig. 2.6

Fig. 2.7

Fig. 2.8

Fig. 2.8

Fig. 2.9

Fig. 2.10
Wellington Urban Motorway, Wellington. (Personal photograph by author, 2012)

Clifton Street Car Park, Wellington. (Personal photograph by author, 2012)

The Bolton Street Cemetery, Wellington. (Personal photograph by author, 2012)

The Neues Museum, Berlin. (Photograph courtesy of Tessa Ayling-Guhl. 17 Feb. 2013)

The Wellington Urban Motorway, Wellington. (Personal photograph by author, 2012)

Arch2o.com 2012-2013. Superkilen Urban Park, Copenhagen. <http://www.arch2o.com >

Diagram of overall thesis structure. (Author's own image, 2012)

Neue Nationalgalerie, Berlin. (Photograph courtesy of Tessa Ayling-Guhl. 17 Feb. 2013)

Diagram of argument structure. (Author's own image, 2012)

Diagram of argument structure. (Author's own image, 2012)

van der Rohe, Mies. Friedrichstrasse Project

(1922). (Reproduced from: Hays, Critical Architecture, 1984)

Judd, Donald. Minimal Myth, (1973). 16 Feb, 2013, <http://www.contemporaryartdailycom >

Eisenman, Peter. House III, (1972). 16 April 2012, (Reproduced from: Eisenman Inside Out: Selected Writings 1963-1988. By Peter Eisnman. New Haven: Yale University Press, 2004)

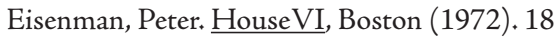
April 2013,<www.archdaily.com>

Eisenman, Peter. HouseVI, Boston (1972). 18 April 2013, <www.archdaily.com>

Diagram of argument structure. (Author's own image, 2012)

Gehry, Frank O. Guggenheim Musuem, Bilbao (1997). 10 March 2013, <www.archdaily.com>

Hadid, Zaha. Galaxy SOHO, Beijing (2012). 10 March 2013, <www.archdaily.com>
Fig. 2.11 Hadid, Zaha. Galaxy SOHO, Beijing (2012). 10 March 2013, <www.dezeen.com>

Fig. 2.12 Hadid, Zaha. Galaxy SOHO, Beijing (2012). 10 March 2013, <www.dezeen.com>

Fig. 2.13 Diagram of argument structure. (Author's own image, 2012)

Chapter Three

Fig. 3.1 Wellington Urban Motorway, Wellington. (Personal photograph by author, 2012)

Fig. 3.2

Allison, Noah. Los Angeles Freeways, Los Angeles (2012). 15 Oct. $2012<$ www.goodfoodpreservation.com>

Fig. 3.3 Le Corbusier. Model of Plan Vosin, Paris (1930) 15 Oct. 2012 <www.meganrolph.wordpress.com>

Fig. 3.4 Hilberseimer, Ludwig. Grossstadt Project, Berlin (1927). 1 Sept. 2012 <www.artic.edu>

Fig. 3.5 Diagram of jump-cut urbanisation. (Author's own image, 2013

Fig. 3.6 City Council, Christchurch. Central Christchurch Master Plan, Christchurch (2011). 1 Feb. 2013 $<$ www.stuff.co.nz>

Fig. 3.7

Diagram of thick edge condition. (Author's own image, 2013)

Fig. 3.8 Subarquitectura Architects. Alicante Tram Stop, Alicante (2008). 19 March 2013 <www.archdaily. com>

Fig. 3.9 Hargreaves Associates. Louisville Waterfront Park Louisville (1992). 19 March 2013 <www.pruned. blogspot.com>

Fig 3.10 Nouvel, John. Placa De Les Glories, Barcelona (2005). 19 March 2013 <www.arcspace.com>

Fig. 3.11 Diagram of wider local context, Wellington. (Author's own image, 2013)

Fig. 3.12 Diagram/map of central Wellington's infrastructure. (Author's own image, 2013)

Fig $3.13 \quad$ Figure/ground diagram/map of central Welling ton. (Author's own image, 2013)

Fig. 3.14 Diagram/map locating three thick edges in Wellington. (Author's own image, 2013)

Fig $3.15 \quad$ Bing Maps. Aerial photograph of Westpac Stadium Car Park. Wellington (2013). 23 March 2013 $<$ nz.bing.com/maps >

Fig, 3.16 Westpac Stadium Car Park, Wellington. (Photograph courtesy of Daniel K. Brown. 20 June 2013

Fig. 3.17 Westpac Stadium Car Park, Wellington. (Photo- 

graph courtesy of Daniel K. Brown. 20 June 2013)

Fig. 3.19 Bing Maps. Aerial photograph of Ballantrae Car Park. Wellington (2013). 23 March $2013<\mathrm{nz}$. bing.com/maps $>$

Fig. 3.20 Ballantrae Car Park, Wellington. (Photograph courtesy of Daniel K. Brown. 20 June 2013)

Fig. 3.21 Ballantrae Car Park, Wellington. (Photograph courtesy of Daniel K. Brown. 20 June 2013)

Fig 3.22 Ballantrae Car Park, Wellington. (Photograph courtesy of Daniel K. Brown. 20 June 2013)

Fig. 3.23 Clifton Street Car Park, Wellington. (Personal photograph by author, 2012)

Fig. 3.24 Clifton Street Car Park, Wellington. (Personal photograph by author, 2012)

Fig. 3.25 Clifton Street Car Park, Wellington. (Personal photograph by author, 2012)

Fig. 3.26 Clifton Street Car Park, Wellington. (Personal photograph by author, 2012)

Chapter Four

Fig. 4.1 Bolton Street Cemetery, Wellington. (Personal photograph by author, 2013)

Fig. 4.2 Photograph of Shell House, The Terrace, Wellington. Fearnley, Charles James, 1915-1988 :Photographs of New Zealand buildings. Ref: 1/4-075735-F. Alexander Turnbull Library, Wellington, New Zealand. <http://natlib.govt.nz/ records/23191046>

Fig. 4.3 Wellington Urban Motorway under construction at Shell Gully. Evening post (Newspaper. 1865-2002):Photographic negatives and prints of the Evening Post newspaper. Ref: PAColl-9150-24. Alexander Turnbull Library, Wellington, New Zealand. < http://natlib.govt.nz/ records/23249606>

Fig. 4.4 Lambton Quay, Wellington, looking south from railway tram terminus. Smith, Alan :Collection of negatives of buses and trams, 1963 to 1970. Ref: 35mm-02059-21-F. Alexander Turnbull Library, Wellington, New Zealand. <http://natlib.govt.nz/ records/23011875>

Fig. 4.5 Wellington motorway under construction at Shell Gully. Negatives of the Evening Post newspaper. Ref: 1/4-020395-F. Alexander Turnbull Library, Wellington, New Zealand. <http://natlib.govt.nz/ records/23250383>

Fig. 4.6 Lodge, Neville. Motorway Protest (1965). 20 March 2013, <http://www.teara.govt.nz/en/cartoon $/ 25751 /$ motorway-protest $>$

Fig. 4.7 Wellington Urban Motorway under construction at Shell Gully. Evening post (Newspaper. 1865-2002) :Photographic negatives and prints of the Evening Post newspaper. Ref:
PAColl-9150-24. Alexander Turnbull Library, Wellington, New Zealand. <http://natlib.govt.nz/ records/23249606>

Fig. 4.8

Clifton Street Car Park, Wellington. (Personal photograph by author, 2013)

Fig. 4.9 The Terrace, Wellington. Winder, Duncan, 1919-1970 :Architectural photographs. Ref: DW-2805-F. Alexander Turnbull Library, Wellington, New Zealand. < http://natlib.govt.nz/ records/22833036>

Fig. 4.10 City Council, Wellington. High City Low City. 12 Feb. 2012. <http://www.mfe.govt.nz >

Fig. 4.11 Diagram of The Terrace showing high city/low city structure. (Author's own image, 2012)

Fig. 4.12 Clifton Street Car Park, Wellington. (Personal photograph by author, 2013)

Fig. 4.13 Bing Maps. Map of Bolton Street Cemetery, Wellington (2013). 23 March $2013<$ nz.bing.com/ maps>

Fig. 4.14 View of motorway under construction from Bolton Street Cemetery, Wellington. Further negatives of the Evening Post newspaper. Ref: EP/1976/2436/28-F. Alexander Turnbull Library Wellington, New Zealand. <http://natlib.govt.nz/ records/23008277>

Fig. 4.15 Bolton Street Cemetery, Wellington. (Personal photograph by author, 2013)

Fig. 4.16 Bolton Street Cemetery, Wellington. (Personal photograph by author, 2013)

Fig. 4.17 Clifton Street Car Park, Wellington. (Personal photograph by author, 2013)

Fig. 4.18 Sign showing map of Wellington Cable Car, Wellington. (Personal photograph by author, 2013)

Fig. 4.19 City Council, Wellington. Land-use Zoning for Central Area. (Reconfigured by author) $12 \mathrm{Feb}$. 2012. <http://www.mfe.govt.nz >

Fig.4.19 City Council, Wellington. Land-use Zoning for Central Area. (Reconfigured by author) $12 \mathrm{Feb}$. 2012. <http://www.mfe.govt.nz>

Fig. 4.20 Diagram of property values adjacent to The Terrace. (Author's own image, 2012)

Fig. 4.20 Diagram of property values adjacent to The Terrace. (Author's own image, 2012)

Fig. 4.21 Diagram of social publics witnessed at site. (Author's own image, 2013)

Fig. 4.22 Isometric diagram of site ground levels. (Author's own image, 2013)

Fig. 4.23 Isometric diagram of site adjacent zones. (Author's own image, 2013)

Fig. 4.24 Isometric diagram of site Datum 1. (Author's own image, 2013)

Fig. 4.25 Isometric diagram of site Datum 2. (Author's own image, 2013) 
Fig* 4.27 Clifton Street Car Park, Wellington. (Personal photograph by author, 2013)

Fig. 4.28 Isometric diagram of site visual context 1. (Author's own image, 2013)

Fig. 4.29 Plan diagram of site visual context 1. (Author's own image, 2013)

Fig. 4.30 Photograph of Marsh Ltd. offices, Wellington. (Personal photograph by author, 2013)

Fig. 4.31 Photograph of Marsh Ltd. lobby, Wellington. (Personal photograph by author, 2013) photograph by author, 2013)

Fig. 4.33 Photograph of The Terrace, Wellington. (Personal photograph by author, 2013)

Fig. 4.34 Clifton Street Car Park, Wellington. (Personal photograph by author, 2013)

Fig. 4.35 Clifton Street Car Park, Wellington. (Personal photograph by author, 2013)

Fig. 4.36 Clifton Street Car Park, Wellington. (Personal photograph by author, 2013)

Fig. 4.37 Clifton Street Car Park, Wellington. (Personal photograph by author, 2013)

Fig. 4.38 Clifton Street Car Park, Wellington. (Personal photograph by author, 2013)

Fig. 4.39 Isometric diagram of site visual context 2. (Author's own image, 2013)

Fig. 4.40 Plan diagram of site visual context 2. (Author's own image, 2013)

Fig. 4.41 Lambton Quay, Wellington. (Personal photograph by author, 2013)

Fig. 4.42 Woodward Street, Wellington. (Personal photograph by author, 2013)

Fig. 4.43 Woodward Street, Wellington. (Personal photograph by author, 2013)

Fig. 4.44 Woodward Street, Wellington. (Personal photograph by author, 2013)

Fig. 4.45 Clifton Street Car Park, Wellington. (Personal photograph by author, 2013)

Fig. 4.46 Clifton Street Car Park, Wellington. (Personal photograph by author, 2013)

Fig.4.47 Clifton Street Car Park, Wellington. (Personal photograph by author, 2013)

Fig. 4.48 Clifton Street Car Park, Wellington. (Personal photograph by author, 2013)

Fig. 4.49 Clifton Street Car Park, Wellington. (Personal photograph by author, 2013)

Fig. 4.50 Isometric diagram of site visual context 3. (Au thor's own image, 2013)

Fig. 4.52 Lambton Quay, Wellington. (Personal photograph by author, 2013)

Fig. 4.53 Cable Car Lane, Wellington. (Personal photograph by author, 2013)

Fig. 4.54 Wellington Cable Car, Wellington. (Personal photograph by author, 2013)

Fig. 4.55 Wellington Cable Car, Wellington. (Persona photograph by author, 2013)

Fig. 4.56 Clifton Street Car Park, Wellington. (Personal photograph by author, 2013)

Fig. 4.57 Wellington Cable Car, Wellington. (Persona photograph by author, 2013)

Fig. 4.58 Botanical Gardens lookout. (Personal photograph by author, 2013)

Fig 4.59 Botanical Gardens lookout, Wellington. (Persona photograph by author, 2013)

Fig. 4.60 Botanical Gardens lookout, Wellington. (Personal photograph by author, 2013)

Fig. 4.61 Isometric diagram of site visual context 4. (Author's own image, 2013)

Fig. 4.62 Plan diagram of site visual context 4. (Author's own image, 2013)

Fig. 4.63 Clifton Street Car Park, Wellington. (Personal photograph by author, 2013)

Fig. 4.64 Isometric diagram of site visual context 5. (Author's own image, 2013)

Fig. 4.65 Plan diagram of site visual context 5. (Author's own image, 2013)

Fig. 4.66 Thorndon suburb. (Personal photograph by author, 2013)

Fig, 4.67 Greenbelt path (Personal photograph by author, 2013)

Fig. 4.68 Greenbelt path. (Personal photograph by author, 2013)

Fig, 4.69 Greenbelt path. (Personal photograph by author, 2013)

Fig. 4.70 Greenbelt path. (Personal photograph by author, 2013)

Fig. 4.71 Northern Stair entrance to Clifton Street Car Park. (Personal photograph by author, 2013)

Fig. 4.72 Greenbelt path. (Personal photograph by author, 2013)

Fig 4.73 Wellington Urban Motorway. (Personal photograph by author, 2013)

Fig. 4.74 Bolton Street Cemetery. (Personal photograph by author, 2013)

Fig. 4.75 Bolton Street Cemetery. (Personal photograph by author, 2013) 
Fig. 4.77 Bolton Street Cemetery. (Personal photograph by author, 2013)

Chapter Five

Fig. 5.1 Smithson, Robert. Asphalt Rundown, 1969,

Rome. History of Our World. 2 Feb 2010

$<$ http://historyofourworld.wordpress.

com/2010/10/18/robert-smithson/>

Fig. 5.2 Serra, Richard. Tilted Arc, 1981, New York City. Reproduced from Contemporary Art Daily. 2 Feb 2010, <http://www.contemporaryartdaily.com>

Fig. 5.3 Matta-Clark, Gordon. Cibachrome, 1975, Paris. History of Our World. 2 Feb 2010, < http:// historyofourworld.wordpress.com/2010/10/18/ robert-smithson/>

Fig. 5.4 Eisenman, Peter. City of Culture of Galicia, Galicia, (2013). 18 April 2013, <www.archdaily.com>

Fig. 5.5 Bing Maps. Aerial photograph of Paris, Paris (2013). 23 March 2013 <nz.bing.com/maps>

Fig. 5.6 Bing Maps. Aerial photograph of Parc de la Villette, Paris (2013). 23 March 2013 <nz.bing.com/ maps>

Fig. 5.7 Penarroyo, Cyprus. Exploded isometric of Parc de la Villette (1982-1983). 22 Sept $2012<$ <ww. archdaily.com>

Fig. 5.8 Penarroyo, Cyprus. Exploded isometric of Parc de la Villette (2013). 22 Sept $2012<$ www.archdaily. com>

Fig. 5.9 Penarroyo, Cyprus. Parc de la Villette, Paris, (1982-1983).22 Sept 2012 <www.archdaily.com>

Fig. 5.10 Penarroyo, Cyprus. Parc de la Villette, Paris, (1982-1983). 22 Sept 2012 <www.archdaily.com>

Fig. 5.11 Penarroyo, Cyprus. Parc de la Villette, Paris, (1982-1983).22 Sept 2012 <www.archdaily.com>

Fig. 5.12 Bing Maps. Aerial photograph of Igualada, Igualada (1982-1983). 23 March 2013 <nz.bing.com/ maps>

Fig. 5.13 Bing Maps. Aerial photograph of Igualada Cem etery, Igualada (2013). 23 March $2013<$ nz.bing. com/maps >

Fig. 5.14 Kroll, Andrew. Igualada Cemetery, Igualada, (1994). 2 April 2013 <www.archdaily.com>

Fig. 5.15 Kroll, Andrew. Igualada Cemetery, Igualada, (1994). 2 April 2013 <www.archdaily.com>

Fig. 5.16 Berrizbeitia, Anita. Igualada Cemetery, Igualada (1994). Inside Outside: Between Architecture and Landscape. Berrizbeitia, Anita; Pollak Linda. Gloucester, Mass: Rockport, 2003. Print.

Fig. 5.17 Berrizbeitia, Anita. Igualada Cemetery, Igualada (1994). Inside Outside: Between Architecture and Landscape. Berrizbeitia, Anita; Pollak Linda.

Gloucester, Mass: Rockport, 2003. Print.

Fig. 5.18

Fig. 5.19

Fig. 5.20

Fig. 5.21

Fig. 5.22

Fig. 5.23

Fig. 5.24

Fig. 5.25

Chapter 6

Fig. 6.1

Fig. 6.2

Fig. 6.3

Fig. 6.4

Fig. 6.5

Fig. 6.6

Fig. 6.7

Fig. 6.8

Fig. 6.9

Fig. 6.10

Fig. 6.11

Fig. 6.12

Fig. 6.13
Berrizbeitia, Anita. Igualada Cemetery, Igualada, (1994). Inside Outside: Between Architecture and Landscape. Berrizbeitia, Anita; Pollak Linda. Gloucester, Mass: Rockport, 2003. Print.

sometric diagram of ordering system. (Author's own image, 2013)

Isometric diagram of ordering system. (Author's own image, 2013)

image, 2013)

Plan diagram of ordering system. (Author's own image, 2013)

Isometric diagram of terracing. (Author's own image, 2013)

Isometric diagram of terracing. (Author's own image, 2013)

an diagram of design ordering system. (Author's own image, 2013)

Winstanley, Tim. Palais de Tokyo, Paris, (2011). 2 April 2013 <www.archdaily.com>

Solomon R. Guggenheim Foundation. BMW Guggenheim LAB, Berlin, (2012). 5 April 2013 $<$ www.bmwguggenheimlab.org>

Diagram of tight/loose space dimensions. (Author's own image, 2012)

Civic Square, Wellington. (Personal photograph by author, 2013)

Russell, Anne. Civic Square during protest. Wel lington (2013), <www.fightback.org.nz>

Capper, Phillip. Waitangi Park, Wellington (2007) $<$ www.flickr.com $>$

Capper, Phillip. Waitangi Park, Wellington (2007) <www.flickr.com>

Paredes Pino Architects. CAAC, Cordoba (2010) 28 March 2013 < http://www.archdaily.com>

Bing Maps. Aerial photograph of Paris, Paris (1982-1983). 23 March 2013 <nz.bing.com/ maps >

ing Maps. Aerial photograph of Palais de Tokyo Paris (1982-1983). 23 March 2013 <nz.bing.com/ maps >

Technical plan of Palais de Tokyo (Authors own image, 2012)

Winstanley, Tim. Palais de Tokyo, Paris, (2011). 2 April 2013 <www.archdaily.com>

Winstanley, Tim. Palais de Tokyo, Paris, (2011). 2 April 2013 <www.archdaily.com> 
Fig. 6.16

Bing Maps. Metropol Parasol, Seville (2013). 2 May $2013<$ nz.bing.com/maps $>$

Fig. 6.17 Alda, Fernando. Metropol Parasol, Seville, (2011) 2 April 2013 < http://www.yatzer.com>

Fig. 6.18 Unknown photographer. Car Park, Seville, (1960). Reproduced from: Mayer, Jurgen H.'Metropo Parasol, Seville'. Architectural Design, Sept/Oct 2012, ed. Alexander Eisenschmidt

Fig. 6.19 Alda, Fernando. Metropol Parasol, Seville, (2011) 2 April 2013 < http://www.yatzer.com>

Fig. 6.20 Technical plan of Metropol Parasol (Authors own image, 2012)

Fig. 6.21 Alda, Fernando. Metropol Parasol, Seville, (2011). 2 April 2013 < http://www.yatzer.com>

Fig. 6.22 Alda, Fernando. Metropol Parasol, Seville, (2011) 2 April 2013 < http://www.yatzer.com>

Fig, 6.23 Diagram of Metropol Parasol's strategies (Authors own image, 2012)

Fig. 6.24 Bing Maps. Site of EPFL, Lausanne (2013). 2 May $2013<$ nz.bing.com/maps>

Fig. 6.25 Bing Maps. Site of EPFL, Lausanne (2013). 2 May $2013<$ nz.bing.com/maps >

Fig. 6.26 Basulto, David. Rolex Learning Center/SANAA Lausanne, (2010). 21 May 2013 < http://www. archdaily.com>

Fig. 6.27 Bing Maps. Site of EPFL, Lausanne (2013). 2 May $2013<$ nz.bing.com/maps>

Fig. 6.28 Diagram plan of Rolex Learning Center's strategies (Authors own image, 2012)

Fig. 6.29 Diagram plan of Rolex Learning Center's strategies (Authors own image, 2012)

Fig. 6.30 Basulto, David. Rolex Learning Center/SANAA Lausanne, (2010). 21 May 2013 < http://www. archdaily.com>

Fig. 6.31 Basulto, David. Rolex Learning Center/SANAA Lausanne, (2010). 21 May $2013<$ http://www. archdaily.com>

Fig. 6.32 Diagram plan of Rolex Learning Center's strategies (Authors own image, 2012)

\section{Chapter Seven}

Fig. 7.1 Pistoletto, Michelangelo. Venus in Rags (1974). 2 May $2012<$ http://www.contempraryartdaily. com>

Fig. 7.2 Hadid, Zaha. Galaxy SOHO, Beijing (2012). 10 March 2013, <www.archdaily.com>

Fig. 7.3 Image reproduced from: Fairs, Marcus. Zaha Had-
Fig. 7.4

Klee, Paul. Angelus Novus (1920). 8 June 2012. Reproduced from <http://www. http:// plundered-art.blogspot.com $>$

Fig. 7.5 Supreme Courthouse of New Zealand, Welling ton. (Personal photograph by author, 2013)

Fig. 7.6 Ito, Toyo. Meiso no Mori Municipal Funeral Hall, Gifu (2009). 5 July 2012. Reproduced from <http://www. http://www.dezeen.com>

Fig. 7.7 Nishi, Tatzu. Engel, Basel(2002). Reproduced from Contemporary Art Daily. 2 July 2010, $<$ http://www.contemporaryartdaily.com>

Fig.7.8 Nishi, Tatzu. Discovering Columbus, New York (2012). Reproduced from Contemporary Art Daily. 2 July 2010, <http://www.contemporaryartdaily.com>

Fig. 7.9 Gentry, Regan. Subject to Change (2002). Repro duced from Contemporary Art Daily. 2 July 2010 $<$ http://www.contemporaryartdaily.com >

Fig. 7.10 Eisenman, Peter. Checkpoint Charlie Housing for the IBA, Berlin (1987). Reproduced from MIMOA. 2 July 2010, <http://www. http://www mimoa.eu>

Fig. 7.11

Libeskind, Daniel. The Jewish Museum Berlin, Berlin (1999). 21 Jan 2013 <nz.bing.com/maps>

Fig. 7.12

Libeskind, Daniel. The Jewish Museum Berlin Berlin (1999). 21 Jan 2013 <nz.bing.com/maps>

Fig. 7.12 Libeskind, Daniel. The Jewish Museum Berlin, Berlin (1999). 21 Jan 2013 <nz.bing.com/maps>

Fig. 7.13 Axonometric diagram of Jewish Museum Berlin (Authors own image, 2012)

Fig. 7.14 Jewish Museum, Berlin. (Personal photograph by author, 2013)

Fig.7.15 Jewish Museum, Berlin. (Personal photograph by author, 2013)

Fig. 7.16 Diagram/photograph of the Jewish Museum Berlin. (Personal photograph by author, 2013)

Fig. 7.17 Diagram/photograph of the Jewish Museum Berlin. (Personal photograph by author, 2013)

Fig. 7.18 Libeskind, Daniel. Jewish Museum Berlin, Berlin (1999). 10 March 2013, <www.archdaily.com>

Fig. 7.19 Diagram/photograph of the Clifton Street Car Park, Wellington. (Personal photograph by author, 2013)

Fig. 7.20 Diagram/photograph of the Clifton Street Car Park, Wellington. (Personal photograph by autho 2013)

Fig. 7.21 Diagram/photograph of the Clifton Street Car Park, Wellington. (Personal photograph by author, 2013)

Fig. 7.22 Diagram/photograph of the Clifton Street Car Park, Wellington. (Personal photograph by author, 
Fig. 8.1

Assembly Architect's. Folly for a Flyover, London (2011). Reproduced from Dezeen. 2 July 2010 , $<$ http://www.dezeen.com>

Fig. 8.2 Diagram of performance types. (Author's own image, 2012)

Fig. 8.3 Diagram of annual events in Wellington. (Author's own image, 2012)

Fig. 8.4 Diagram of performance adjacencies. (Author's own image, 2012)

Fig. 8.5 Diagram of performance adjacencies. (Author's own image, 2012

Fig. 8.6 Diagram of performance adjacencies. (Author's own image, 2012)

Fig. 8.7 Diagram of staging typologies. (Author's own image, 2012)

Fig. 8.8 Map of performance venues in Wellington. (Author's own image, 2012)

Fig. 8.9 Chart of performance venue sizes in Wellington. (Author's own image, 2012)

Fig. 8.10 Location of performance case studies. (Author's own image, 2012)

Fig. 8.11 Spatial analysis of Circa Theatre, Wellington. (Author's own image, 2012)

Fig. 8.12 Spatial analysis of St James Theatre, Wellington. (Author's own image, 2012)

Fig. 8.13 Spatial analysis of Wellington Town Hall, Welling ton. (Author's own image, 2012)

Fig. 8.14 Photograph of the Anvil House, Wellington. (Personal photograph by author, 2013)

Fig. 8.15 Flow chart of New Zealand INternaitonal Arts Festival 2010 programme, Wellington. (Author's own image, 2012)

\section{Chapter Nine}

Fig. 9.1 Clifton Street Car Park, Wellington. (Personal photograph by author, 2013)

Fig. 9.2 Rendered plan of design upper levels (Author's own image, 2012)

Fig. 9.3 Diagrammatic key of existing and intervening formal typologies (Author's own image, 2012)

Fig. 9.4 Key for rendered plan of design upper levels (Author's own image, 2012)

Fig. 9.5 Diagrammatic key of intervening spatial typologie (Author's own image, 2012)

Fig. 9.6 Key for rendered plan of design middle level (Author's own image, 2012)

Fig. 9.7 Rendered plan of design middle level (Author's
Key for rendered plan of design ground levels (Author's own image, 2012)

Fig. 9.9

Fig. 9.10 Technical plan of design ground levels (Author's own image, 2012)

Fig. 9.11

Fig. 9.12

Fig. 9.13

Fig. 9.14

Fig. 9.15

Fig, 9.16

Fig. 9.17

Fig. 9.18

Fig. 9.19

Fig. 9.20

Fig, 9.21

Fig, 9.22

Fig. 9.23

Fig. 9.24

Fig. 9.25

Fig, 9.26

Fig, 9.27

Fig. 9.28

Fig. 9.29

Fig. 9.30

Fig. 9.31

Technical plan of design middle levels (Author's own image, 2012)

Diagram of back-of-house adjacencies (Author's own image, 2012)

Diagram of performance, front-of-house and back of-house adjacencies (Author's own image, 2012)

own image, 2012)

Diagram of various shared services adjacencies (Author's own image, 2012)

Diagram of Datum 1programme adjacencies (Author's own image, 2012)

Diagram of design vehicular and pedestrian access (Author's own image, 2012)

iagrammatic key of design typologies - formal spatial, programmatic and material (Author's own image, 2012)

Rendered key of section D-D' with (Author's own image, 2012)

Rendered section D-D', part two (Author's own image, 2012)

Rendered section D-D', part three (Author's own image, 2012)

Rendered section D-D', part three (Author's own image, 2012)

Rendered section D-D', part four (Author's own image, 2012)

Rendered section D-D', part five (Author's own image, 2012)

Rendered section D-D', part six (Author's own image, 2012)

Isometric image of ground levels (Author's own image, 2012)

Exploded isometric image of ground level buildings (Author's own image, 2012)

Isometric image of loading bay (Author's own image, 2012)

image of plant room (Author's own image, 2012)

Isometric image of workshop (Author's own image, 2012)

image of performance back-of-house facilities (Author's own image, 2012) 

own image, 2012)

Fig. 9.33 Diagram section of canopy continuity (Author's own image, 2012)

Fig. 9.34 Exploded isometric image of middle level and buildings (Author's own image, 2012)

Fig. 9.35 Exploded assembly isometric image of signage detail (Author's own image, 2012)

Fig. 9.36 Isometric image of storage (Author's own image, 2012)

Fig. 9.37 Isometric image of studio/hire spaces (Author's own image, 2012)

Fig 9.38 Isometric image of thru-scenium theatre (Author's own image, 2012)

Fig, 9.39 Isometric image of public toilet facilities (Author's own image, 2012)

Fig, 9.40 Isometric image of front-of-house facilities $(\mathrm{Au}$ thor's own image, 2012)

Fig. 9.41 Exploded isometric image of canopy derived from major programmatic points (Author's own image, 2012)

Fig.9.41 Exploded isometric image of upper levels and buildings (Author's own image, 2012)

Fig. 9.42 Exploded isometric image of upper levels and buildings (Author's own image, 2012)

Fig 9.43 Exploded assembly isometric image of channel glass detail (Author's own image, 2012)

Fig. 9.44 Exploded isometric image of recital space $(\mathrm{Au}$ thor's own image, 2012)

Fig. 9.45 Exploded assembly isometric image of window casement detail (Author's own image, 2012)

Fig. 9.46 Exploded assembly isometric image of NZIAF office spaces (Author's own image, 2012)

Fig, 9.47 Isometric image of canopy and adjacent grids (Author's own image, 2012)

Fig. 9.48 Sectional perspective image A-A' of black box theatre (Author's own image, 2012)

Fig. 9.49 Sectional perspective image B-B' of thru-scenium theatre (Author's own image, 2012)

Fig. 9.50 Sectional perspective image C-C' of traverse theatre (Author's own image, 2012)

Fig. 9.51 Perspective image cable car threshold (Author's own image, 2012)

Fig.9.52 Perspective image northern stairwell threshold (Author's own image, 2012)

\section{Chapter 10}

Fig. 10.1 Clifton Street Car Park, Wellington. (Personal photograph by author, 2012) photograph by author, 2012)

Fig. 10.3 New Zealand Transport Authority. Basin Reserve Flyover, Wellington, (2013). 21 May $2013<$ http://www.nzta.govt.nz/projects/basin-bridge/ index.html>

Fig. 10.4 Brunton, Simon. New Zealand Transport Authority. Basin Reserve Flyover, Wellington, (2013). 22 May $2013<$ http://wellington.scoop. co.nz/?p=50715>

Fig. 10.5 Rendered section D-D', (Author's own image, 2012) 


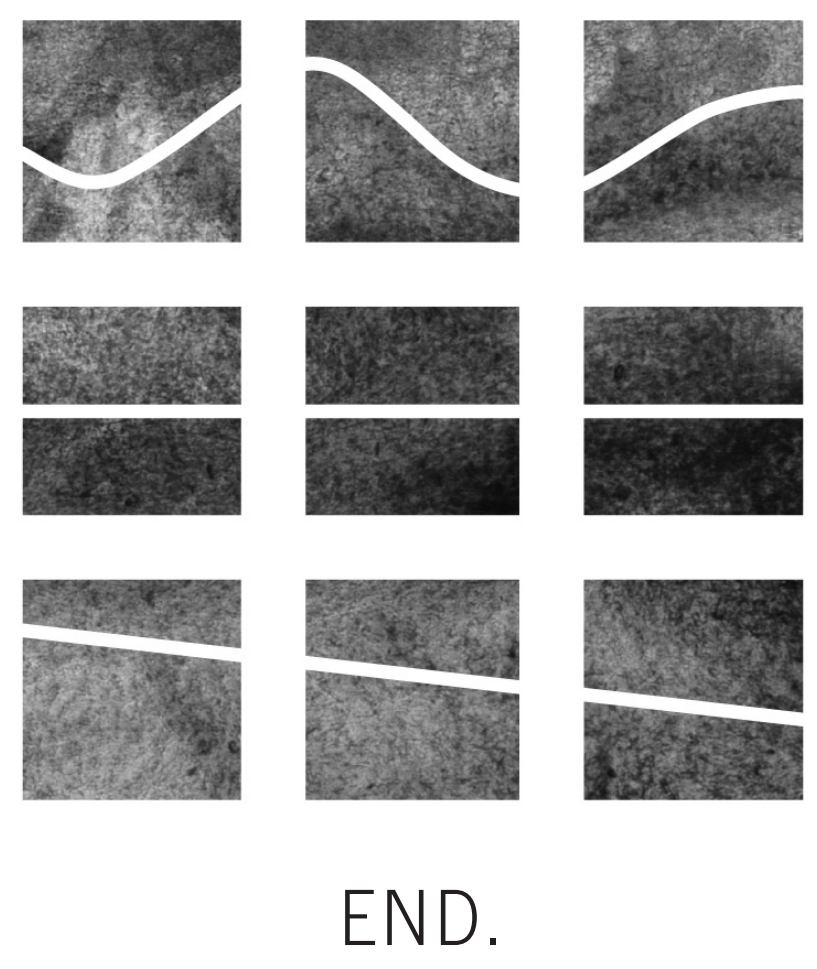

It's all about the Discipline.

A. Earl and G. Mills 